\title{
Hop Hill: Culture and Climactic Change in Central Texas
}

Joel Gunn

Center for Archaeological Research

Royce Mahula

Center for Archaeological Research

Follow this and additional works at: https://scholarworks.sfasu.edu/ita

Part of the American Material Culture Commons, Archaeological Anthropology Commons, Environmental Studies Commons, Other American Studies Commons, Other Arts and Humanities Commons, Other History of Art, Architecture, and Archaeology Commons, and the United States History Commons

Tell us how this article helped you.

This Article is brought to you for free and open access by the Center for Regional Heritage Research at SFA ScholarWorks. It has been accepted for inclusion in Index of Texas Archaeology: Open Access Gray Literature from the Lone Star State by an authorized editor of SFA ScholarWorks. For more information, please contact cdsscholarworks@sfasu.edu. 


\section{Hop Hill: Culture and Climactic Change in Central Texas}

Creative Commons License

(c) (i) (8)

This work is licensed under a Creative Commons Attribution-NonCommercial 4.0 International License 


\section{HOP HILL: CULTURE AND CLIMATIC CHANGE IN CENTRAL TEXAS}

\section{JOEL GUNN and ROYCE MAHULA}

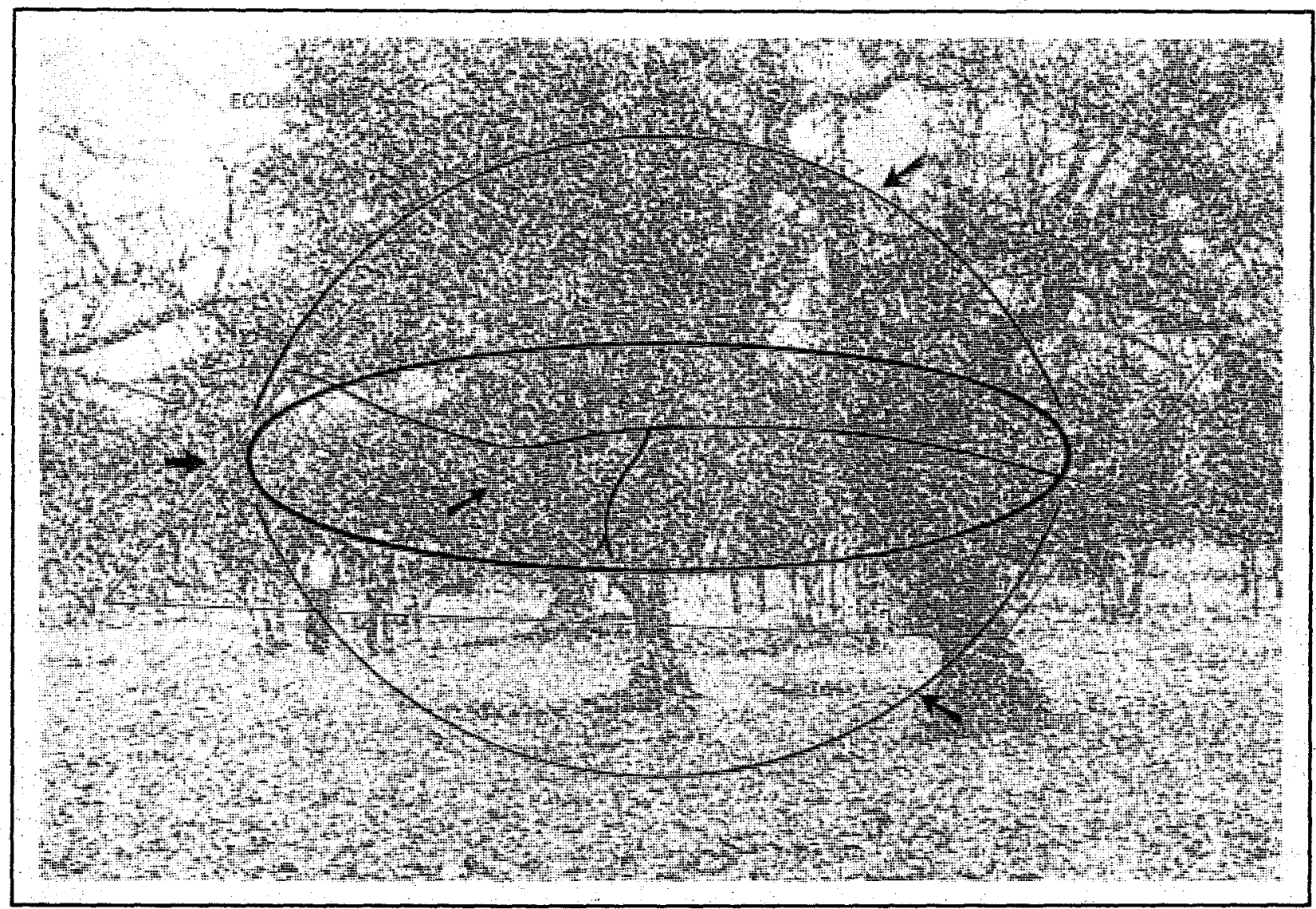

in association with

Kay Simpson, David Brown, Sue Turner

Clint Davis, Stacey Wright, Sarah Bielser

Ken Lord, James E. Ivey, Elizabeth Frkuska

Augustine Frkuska, Douglas R. White

T. C. Kelly, Max Witkind and James Escobedo

CENTER FOR ARCHAEOLOGICAL RESEARCH

THE UNIVERSITY OF TEXAS AT SAN ANTONIO

SPECIAL REPORT, NO. 5 

Center for Archaeological Research

The University of Texas at San Antonio

78285

Thomas R. Hester, Birector

Special Reports

Publications dealing with the archaeology of Texas and Mesoamerica.

No. 1 (1975) "Some Aspects of Late Prehistoric and Protohistoric Archaeology in Southern Texas: (By thomas R. Hester and T. C. Hi 1 1 , Jr. $)$. (\$2.00 +.10 tax for Texas residents).

No. $2(1976)$ "The Texas Archaic: A Symposium" (Edited by Thomas R. Hester). $(\$ 4.00+.20$ tax for texas residents $)$.

No. 3 (1976) "Papers on Paleo-Indian Archaedlogy in Texas: I" (Papers by T. R. Hester and W. W. Birmingham). $(\$ 3.00+.15$ tax for Texas residents).

No. 4 (1976) "Maya Lithic Studies: Papers from the 1976 Bel ize Field Symposium" (Edited by Thomas R. llester and Norman Hammond). ( $\$ 8.00+.40$ tax for Texas residents).

No. 5 (1977) Hop H111: Culture and Cl imatic Change in Central Texas" (By Joel Gunn and Royce Mahulal). 



\section{HOP HILL: CULTURE AND \\ CLIMATIC CHANGE IN CENTRAL TEXAS}

Joel Gunn and Royce Mahula

$$
\text { in association with }
$$

Kay Simpson, David Brown, Sue Turner, Clint Davis, Stacey Wright, Sarah Bielser, Ken Lord, James E. Ivey, Elizabeth Frkuska, Augustine Frkuska, Douglas R. White,

T. C. Kelly, Max Witkind and James Escobedo

Center for Archaeological Research The University of Texas at San Antonio Special Report, No. 5 
The archaeological investigations reported here were performed under the terms of Permit No. 153, Texas Antiquities Committee.

\author{
Joel Gunn, \\ Principal Investigator
}

\author{
Published by \\ Center for Archaeological Research \\ The University of Texas at San Antonio \\ August, 1977
}


TABLE OF CONTENTS

Page

List of Figures $\quad$ iii

List of Tables viii

Preface $x$

1.0 Introduction (Joel Gunn) 1

2.0 Ecosphere of the Hop Hil1 Locality (Kay Simpson) 9

3.0 Global Climate (Stacey Wright and Joel Gunn) 25

4.0 Atmosphere and Modern Climate (Sarah Bielser and Joel Gunn) 37

5.0 The Geosphere: Physiography, Stratigraphy and Soil Analysis
(Royce Mahula)

6.0 Site History, Physiography, Vegetation and Erosion (David Brown and Clint Davis) 67

7.0 Mollusks from the Hop Hill Site (Kenneth J. Lord) 103

7.1 Environmental Implications from Molluscan Analysis
(Royce Mahula)

8.0 Ethnohistory (Sue Turner) 107

9.0 Recovery Procedures (Royce Mahula) 127

10.0 Sollberger Distributions: Functional Areas of the Site
(Joel Gunn and Royce Mahula)

$11.0 \begin{aligned} & \text { Core Flaking Model (Linear Technospatial Analysis) } \\ & \text { (Joel Gunn) }\end{aligned}$

12.0 Face Flaking Model - Bifacing (Curvilinear Technospatial Ana7ysis) (Joel Gunn and James E. Ivey) 163

12.1 Anarys is of Arrow and Dart Points (Thomas C. Kel1y) 185

13.0 Hop Hill Lithics: Uniface Analysis (Elizabeth Frkuska and
Augustine Frkuska)

14.0 An Experiment in Stone Boiling (W. Max Witkind) 205

15.0 Clustering of Technological Types (Non-Linear Technospatial Analysis, Resov 4) (Joel Gunn and Douglas R. White) 209 
16.0 Analysis of Occupation Floors (Joel Gunn) 223

17.0 Flake Technology (Joel Gunn and Royce Mahula) 235

17.7 Lithic Raw Materials (James Escobedo) 253

18.0 Envirocultural System for Central Texas (Joel Gunn) 257

19.0 Conclusions (Joel Gunn and Royce MahuTa) 277

Acknowledgements 281

References Cited 283 



\section{LIST OF FIGURES}

FIGURES

Page

1.01 The Sphere of Research 2

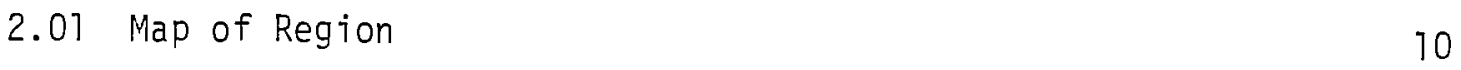

2.02 Subsurface Formation in the Study Area $\quad 17$

3.01 The Major Physical Components and Feedback Processes
Responsible for the Maintenance of Climate

3.02 Wind Circulation Patterns 28

3.03 Climatic Fluctuations as Illustrated by Several Paleoclimatic Indicators 31

4.01 Weather Systems Affecting Central Texas Climate 41

4.02 Changes in Fredericksburg Climate Between Period 1 (1940-1960), Period 2 (1961-1971) and Period 3 (1972-7976)

4.03 Decrease in Mean Temperature During 1972-1976 from the
1940-1976 Mean

4.04 The Five Seasons of Fredericksburg Climatic Change 45

4.05 Residuals Over the Last 37 Years Showing Weather Becoming
Unstable

5.01 Typical Stratigraphic Profile in Road 51

5.02 Midden Stratigraphic Profile 52

5.03 Principal Components Analysis of Soil Chemical Data: Plot
of Component Scores

6.01 Soi1 Types and Slope Zones Used to Code Erosion Study Data
Points

6.02 Vegetation Zones 68

6.03 Aerial Photo of Site Taken September 27, 1938

6.04 Aerial Photo of Site Taken December 3, 1948

6.05 Aerial Photo of Site Taken November 11, 1955 
6.06 Aeria1 Photo of Site Taken February 3, 1963

6.07 Aerial Photo of Site Taken January 31, 1974

6.08 Vegetation Inside Stock Pens 79

6.09 The Vegimeter 85

6.10 The STope-o-matic 88

7.01 Change in Proportions of Snail Types Through Time 105

9.01 Concepts for Excavation Control 127

9.02 Substratum Unit Recording Format 137

9.03 Mapping Format 132

9.04 Culture Unit Recording Format 133

9.05 Stratigraphic Profile of Fire Feature (FN557) 135

9.06 Horizontal Configuration of Fire Feature (FN557) 136

10.01 Py Plotted for the Sollberger Distributions and the Hop Hill Midden and Quarry 139

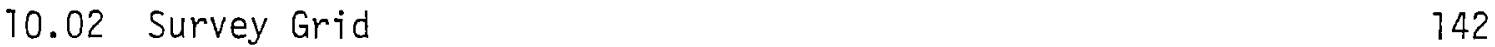

10.03 Photographs of Intensively Collected 10-meter Squares 143

10.04 Surface Lithic Densities and Most Nearly Related Phases of Sollberger Distribution for Each Collected 10-meter Squares

11.01 Flake Core Terminology 151

11.02 Flake Cores 153

11.03 Flake Cores, Rounded Platform 155

11.04 Block Reduction Moder $\quad 156$

11.05 Causal Model Describing Relationships of Causal Variables to Resultant Tool Form 158

11.06 Core Distribution 160

12.01 Facing Model, Concepts for Unifacing and Bifacing 164 
12.03 Pointed Bifaces

12.04 End Bifaces

12.05 Side and Rectangular Bifaces

12.06 Round, Thin Biface

12.07 Oval and Ovoid Bifaces

178

12.08 Causal Biface Relationships

180

12.09 Biface Distribution

12.10 Number of Points of Each Type and Their Time Ranges

185

13.01 Envirotechnical Model for Uniface Production

190

13.02 Polar Coordinate Grid for Uniface Measurement

193

13.03 Example of Component Score Plotting Along Bit-Haft Dimensions

13.04 End Trimmed Unifaces

13.05 Unilateral1y Trimmed Unifaces

13.06 Unilaterally and End Trimmed Unifaces

13.07 Bilaterally and End Trimmed Unifaces

200

13.08 Circular Trimmed and Notched Unifaces

202

15.01 Base and Range Concept of Site Locality

210

15.02 Food Collecting and Related Tasks

211

15.03 Meat, Skin and Fiber Processing

211

15.04 Loci Matrix for Nonlinear Distribution Analysis

215

15.05 Fundamental Operators of Entailment Analysis

218

$15.06 T_{1}$ and $T_{2}$ in Three Constructed Contingency Tables

219

15.07 Entailogram for Hop Hill Loci Matrix 
FIGURES

Page

16.01 Spil1-Fan Analytical Concept 224

16.02 Dccupation Fioor 2.10 226

16.03 Histogram of Rock Sizes in Hearth and Spil1-Fan 227

16.04 Activity Area in OF 2.10 228

76.05 Occupation Floor $2.20 \quad 229$

16.06 Schematic of Occupation Floor 2.20

16.07 Occupation Floor 2.30

16.08 Occupation Floor 2.40

17.01 Terminology for Flake Technology 235

17.02 Form for Coding Data on Flake and Blade Technology 236

17.03 Various Platform Shapes 238

17.04 Various Terminations 239

17.05 Measurement of Length and Width Thickness 241

17.06 Effects of Varying Force on Termination 243

17.07 Interaction of FORCE and RELATED VARIABLES 244

17.08 More Highty Specified Flake Production System 245

17.09 Universal Patterns in Hop Hil Tl Flake Technology,
Principal Components of All Floors

17.10 Knapping Pattern in Occupation Floor 2.10

17.11 Curvature Decreases as Inner Platform Angle Decreases 246

17.12 Knapping Patterns of Occupation Floor 2.20 247

17.13 Knapping Patterns of Occupation Floor 2.30

17.14 Knapping Patterns of Occupation Floor 2.40

17.15 Trajectories of Interaction Between Attributes Without
Space-Time Variables

17.16 Length and Width Components of Hop Hill Flakes from
Midden Occupation Floors 
FIGURES

Page

18.01 Biotic Provinces and Supraprovinces of Texas

18.02 Monsoonal Precipitation Gradient on an East-West

Transect Through Study Area

18.03 Climatic Variation During the Holocene

18.04 Nomadic Seasonal Round

271

18.05 Load Length to Various Substations

273

18.06 Relative Probability of Occupation at Various Geographic Localities

275

18.07 Transhumant Seasonal Activities

276 


\section{LIST OF TABLES}

TABLES

Page

2.01 Geologic Time

2.02 Climatic Norms of Edwards Plateau

2.03 Peak Stages (Pedernales River Near Johnson City)

3.01 GTobal Climatic Discontinuties and Central Texas Prehistory

3.02 Relationships of Zonal and Azonal Parameters

5.01 Soil Sample Descriptions

5.02 Soil Test Results

5.03 Rotated Component Matrix for Soil Chemical Analysis 61

5.04 Rotated Component Matrix for Soil Analysis 64

6.01 Variable Characteristics 87

6.02 Levels of Measurement 90

6.03 Land Surface Regression Analysis 91

6.04 Means of Treecover Values 92

6.05 Mean Percentage Leafcover 92

6.06 Means of Rillsize Values 93

6.07 Activity Regression Analysis 94

6.08 Best Step of Biweekly Regression Analysis 95

6.09 Comparative F Values 96

6.10 Climate vs. Significance 96

6.11 Varimax Rotated Components Matrix 97

6.12 Land Surface Change by Slopezone 99

6.13 Land Surface Change by Soilzone 99 
TABLES

Page

6.14 Land Surface Change by Vegetation Zone 100

9.01 Physiographic Position Indicators 123

10.01 Classes and Class Sizes 138

10.02 Sollberger Distributions 139

10.03 Distributions from the Excavated Midden and Quarry Areas
at Hop Hill

10.04 Lithic Photo Density and Flake Counts 145

12.01 Dimensions of Variability in Bifaces and States Each
Variable Can Take

12.02 Types and a Description of the State that Type will
Take on any Given Variable

$\begin{array}{ll}12.03 \text { FN's and Provenience of Types } & 170\end{array}$

12.04 Mean and Standard Deviation of Edge Arc for Types 1-3 182

12.05 Projectile Points by Terminal Date, Type, Field Number, and Coordinates

13.01 FN's and Provenience of Types 191

14.01 Boiling Stone Data 206

15.01 Rotated Principal Components Matrix for Loci Matrix 212

15.02 Hypothesized Tool Kits from Hop Hill 213

17.1.01 Chert Types from Hop Hill Quarry : 253

17.1.02 Breakdown of Artifacts to Rock Samples 254

18.01 Weir's Central Texas Phases 258

18.02 Summary of Weir's Findings, Variable Trajectories 


\section{PREFACE}

During June and JuTy, 1976, the Center for Archaeological Research carried out archaeological investigations at the Hop Hill site (41 GL 21). This research was conducted at the request of the Texas Parks and Wildiife Department, and was funded through an Interagency Contract (IAC-76-77-1162).

Field work was directed by Dr. Joel Gunn, utilizing Center staff members and participants in the UTSA Division of Social Sciences 1976 Field Course in Archaeology. This monograph presents the results of the field work and the multidisciplinary research activities that followed.

Thomas R. Hester

Director

Center for Archaeological Research 


\subsection{INTRODUCTION (Joel Gunn)}

As Shafer (1976a:8) has noted, the more cultural knowns one can obtain the more cultural unknowns it is possible to solve for. In the context of 1ithic analysis, Shafer was encouraging the consideration of normaliy ignored types of lithic debris in order to expand knowledge of past cultures beyond what projectile points alone show. Of course, this principle can be extended far beyond lithics. Much of the prehistoric content is normally ignored. In the following discussions the probable paleoecology of Central Texas will be elucidated as far as the present state of knowledge will allow. Global climatic variables and modern Fredericksburg weather data are used to obtain a better understanding of climatic change. At a more specific level, the environmental characteristics of Gillespie County are studied; and, finally, the environment and culture of the Hop Hill locality are conjoined to the whole. At times our attempts to discover unknowns are faltering or even abortive; but the results that appear as products of our finite efforts are presented in the spirit of defining the parameters of a cultural and ecological universe, not as the last word on what needs to be done.

Relative to discovering unknowns, two basic approaches are available to the researcher. The first is to collect materials and try to infer cultural and contextual information from them. The second is to infer the cultural and contextual parameters and then compare the fit between the inferred and those observed in the field. The first option is an "inside looking out" approach; the second is an "outside looking in" method. In the following study we have used the "outside looking in" technique as often as possibie for two reasons. At the beginning, it provides a double check on the course of archaeological events. The first check is to determine what should have been according to a set of assumptions. The second is to see by field work what did happen. The other reason, which is closely related to, or a consequence of the first, is that when a fit fails to appear between expected and observed events, the lack of fit automatically asks a question: "What is wrong and what other inferred model would better explain the relevant data?" This inherent questioning can quickly leäd to better solutions.

At the beginning, therefore, certain theoretical positions will be espoused which outiine in broadest terms the integrated relationships between environment and culture, the purpose and character of excavation and the prehistoric domain to which this research pertains. These theories represent the system of environment and culture which is studied and the methods used to study it. The quality of information obtained on prehistoric lifeways is inextricably bound not only to the way it was laid down by prehistoric people, but also to the way it is taken up by modern archaeologists. In this sense then - the information sense - Indians and archaeologists are part of the same system, and we will try to conceptualize that relationship.

Perhaps the most important unknowns to resolve at the beginning are the boundaries of the research project. In its broadest scope the Hop Hill project is concerned with anything that affected the inhabitants of the locality during its prehistory and history. As a matter of practicality we have attempted to define and concentrate on those dimensions of variability, or parameters, which appear to be most important to cultural stability and change at the 
site. We have conceived this more limited world as a sphere intersected by a plane, as illustrated in Figure 1.01. The plane is distorted by forces such as the Pedernales River which flows through the research sphere, and where those distortions are significant they provide unequal cultural advantage to segments of the plane. We assume, then, that the cultural plane is transformed in proportion to the ground plane. Unequal cultural advantage is in direct or indirect proportion to distortion depending on the cultural variable involved. For instance, if the cultural variable is ability-toobserve, the higher points are proportionally more important. Subsistence, on the other hand, would be favored by lower and flatter or less distorted points.

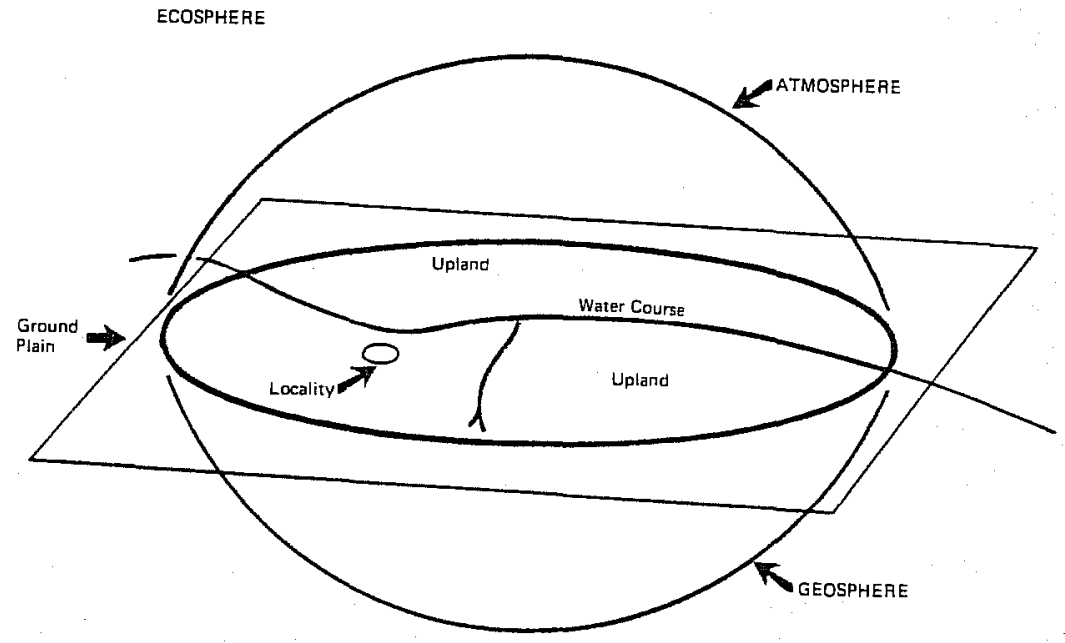

Figure 1.01. The Sphere of Research

The circumference of the ground plane is selected on the basis of the scope of the project. The excavation of a site with no related information would 
require a ground plane only a few meters in diameter. The study of a locality is a larger plane. A settlement pattern study might require a ground plane the size of the annual wanderings of a band. In this study the sphere of research is the Hop Hill locality and the distance which would be effectively worked for subsistence by a visiting band. Such ground plarie would be on the order of magnitude of $1-1.0 \mathrm{~km}$ in diameter.

Around the ground plane is the ecosphere in its atmospheric and geospheric aspects. The atmosphere is relatively unstable while the geosphere is relatively stable. The instability of the atmosphere in particular tends to alter the physiographic and biotic characteristics of the ground plane. Most of these transformations are effected through the media of moisture and temperature.

The ground plane is, in theory, an imaginary surface which has horizontal dimensions but no thickness or inequalities. In practice it is a plasma of biotic and cultural forces which diminish rapidly away from the plane into the geosphere and atmosphere. Since the biotic element of the plasma is the precondition for the cultural element, the cultural forces must be in balance with the biotic forces; but the reverse is not true. Therefore, all models which are constructed for this report are recursive; that is, we assume no feedback from culture to nature.

At the center of the ground plane is the site, which is a place where prehistoric people and archaeologists gather. It is about this point that we build a theory of recovery. In the broadest perspective it is a scenario of relationships between prehistoric men, historic men and the one-way glass of time which affords the latter some understanding of the former.

In 1838 the Morse code was devised, and for generations thereafter the image of the lonely teletype operator tapping away on a device which transmitted information to an unseen but attentive fellow operator became a part of the human experience. I have often thought of the similarity between these unseen partners and the archaeological process of excavation. The prehistoric person leaves a message written in the soil by patterning the symbols of daily activity: fireplaces, points, grinding stones, etc. Later an attentive archaeologists reads the pattern of those symbols and sees the broad outline of the message sent from former times.

Eventualiy Morse's tapping device evolved into more complex instruments of communication. In an effort to understand and improve the performance of such devices, scientists such as C. E. Shannin (Singh 1966) formulated a set of ideas calied "information theory." Information theory attempts to explain in an integrated manner the relationships between sending devices or "encoders," the medium through which a message travels; and "channels" and "decoders," devices which unravel the message coming through the channel and present it in a form which humans can comprehend.

In the terms of information theory the archaeological process is one in which prehistoric people are encoders. Time is the channel through which the message travels and archaeologists and their excavation and analys is techniques are the decoders. With both Indians and archaeologists there are different levels of competence of the encoding and decoding tasks and problems in the channel which tend to distort the message. The characteristics of competence and 
distortion determine how well the message is sent and therefore must be explicitly understood. The message or "signal" can be distorted by "noise" in the channel. The efficiency of the encoder, channel and decoder as each manages the signal and the noise which results from external sources are all mathematically interrelated. If the performance characteristics of each element in the communication system are known, then the amount of distortion introduced into the signal by noise while it is being transmitted can be estimated.

If the archaeological process were taken to be an information system and the characteristics of each element of the system clearly defined, we could judge the quality of information that would come from any given site provided that a specific set of excavation techniques were used.

First let us try to understand the content of the message or signal that comes from the past. Initially one might be inclined to say that the message consists of artifacts, patterns, etc. These, however, are the physical phenomena which in part symbolize the 1 jfeways of the people who left them. They are the encoding device, but it is something more that is being encoded. If we could choose we would rather have a movie of the occupation of a site than the artifacts because so much more would be apparent about the meaning of the artifacts and patterns. Even this, however, would be incomplete. It would leave unseen the motivating forces which cause the pattern, culture and environment in the broadest sense. The message, then, is the information content of the whole enviro-cultural system at the moment of encoding.

How much of the enviro-cultural system can be encoded in some soil containing a few rocks and ashes in a pattern? Some think that little can be learned from the archaeological record and they have therefore taken the position that it is a very inefficient encoder. Others have claimed more for it. The answer, of course, is unknown and hinges on our coming to grips with the problem of how much information there is in an enviro-cultural system and how much of that gets into the archaeological record. No one has measured either quantity and those who say they know are guessing. The impression of complexity could be real or spurious.

Probably the "site" concept is the best equivalent of the idea of an encoder, although the making of a site and the passage of time at a site do not necessarily overlap. The deposition of a site, for instance, can stop, reverse, etc., leaving only the passage of time as the channel of transmittance.

Similarly, the efficiency with which a given site serves as an encoder varies with its inherent characteristics. The most important of these quantities is the rate of deposition. If the rate of deposition is negative (erosional), the signal from any given moment is mixed with all other moments. This, of course, presents acute decoding problems which, if they can be dealt with at al1, require sophisticated analysis techniques. The problem with decoding will be discussed. The higher the rate of positive deposition the more discrete each pulse of the signal. As the matrix is telescoped much more specific data is transmitted per unit of time. 
Let us define archaeological information units as artifacts and ecofacts. The information content of a site (I) will then be summed ecofacts (E) and artifacts $(A)$.

$$
I=E+A
$$

The information load (L) is then calculable as the information content divided by the depth of the deposit in centimeters $(C)$.

$$
L=I / C
$$

The rate of deposition and the intensity of habitation are related to the resolution obtainable from a site. If the "occupation floor" is taken to be the desired unit of excavation, then the chance of separating discrete occupation floors is increased in direct proportion to the amount of sterile material added to the site between occupations. In a socjety that practices seasonal rounds, an annual increment of soil which would allow the discrimination of one annum from the last would be the most perfect and highly resolved encoding of the information. Less deposition or more frequent visits would produce a successively less resolved signal.

The channel involves space and time in the system. Space, the area of the site in square meters, is the room available to transmit the signal. The signal itself is the pattern of artifacts on an occupation floor, and the area of the site reflects how dispersed that pattern is. A more dispersed pattern is more easily perceived and therefore more resolved. The dispersion (D) may be calculated as the information load $(L)$ divided by the area $(A)$.

$$
D=L / A
$$

Pattern tests such as nearest neighbor analysis have been developed to determine if the dispersion of artifacts is clustered, or randomly or uniformly dispersed on a living floor (Whalion 1974). Such relationships are, however, only the most fundamental of camp patterns.

Time defines the trajectory of occupation habits at a site. Time is a place of unequal advantage where residues of the biotic and cultural plasma jell. It is the vehicle of transmission and it is also the medium through which noise, or. "disturbance," enters the record. Disturbance can come as erosional activity, or human activity such as digging storage and fire pits, pothunting, etc. A11 of these events disrupt the signal and cause problems with interpretation. The effect of disturbance is alleviated by "redundancy" in the encoding system. If a man habitually manufactures points in a specific segment of a site the passage of a rodent may destroy some of the evidence, but not all of it. Short of total disruption, then, disturbance adds error or unexplained variance to the pattern but does not necessarily destroy it.

If we take a generation of twenty years to be a unit of potential cultural stability and therefore useful as a time interval, the information load per qeneration $(G)$ can be calculated as the length of occupation in years (0) divided by 20 , the quotient of which is divided into the information load.

$$
G=\frac{L}{0 / 20}
$$


This estimate can be calculated for any unit such as a year, climatic period, or whatever, depending on theoretical objectives.

The final part of the process, decoding, is the only one over which archaeologists have any control. The resolution at which decoding was done has

progressed over the last two centuries from no resolution to excavation by living floors and the use of many techniques such as granulometry, chemical analysis, pollen analysis, etc. These have increased information recovered in absolute terms as well as the resolve with which that information is viewed. As will be discussed later, the theory of a living floor itself is a concept which requires some cautious understanding. Nevertheless, it does represent a remarkable refinement over formerly used arbitrary levels, and before that, trenching and test pits. Whatever information is encoded and survives the channel of time is destroyed in an instant by archaeological excavation. The resolution of the archaeologist's techniques has been and still probably does consist of inadequate mechanisms for the delicate pulses--taps of an ancient person's hand.

In practical terms, most of the discussion of excavation theory can be summed up by one term, "resolution." Resolution is the amount of information per unit of time. The levels of resolution, which are specified for each study done for the Hop Hill project, are as follows.

Resolution Level 1. An occupation floor that was occupied for one habitation event. It is a pulse with little redundancy. A habitation event is taken to be an overnight stay, a brief period of observation, or a similar incident of short duration. Artifactual remains could be any one or all of building a fire, making a few tools, butchering an animal or preparing vegetable food. After the habitation the living floor was stratigraphically sealed or otherwise saved from disturbance. Appropriate technique is microstratigraphic excavation and location of all artifacts and ecofacts to the centimeter $(\mathrm{cm})$ by Cartesian Coordinates. Patterns in the data represent a single and potentially idiosyncratic camp pattern.

Resolution Level 2. An occupation floor that was a stable surface for a period of time during which it was occupied by a number of habitation events by the same social group. Artifacts reflect repeated building of fires, food preparations and working of tools. Ecofacts will show a single climatic episode. After a finite number of visits the habitation was sealed. Appropriate technique is microstratigraphic excavation and recording the artifacts and ecofacts to the $\mathrm{cm}$. Patterns in the data are products of repeated, systematic occupation of the floor which reflect the customary camp habits of the group. There will be some error variance due to persons making exceptions to standard practice. The same effect could be achieved by deflating a series of Resolution 1 floors but ecofacts would disappear.

Resolution Level 3. A living floor that was stable surface and was occupied by more than one social group. Artifacts show a varied pattern of food preparation and workshop activity. Ecofacts may indicate more than one climatic regime thus explaining the change of culture. Appropriate techniques are microstratigraphic excavation with recording of artifact locations to with in $1 \mathrm{~cm}$. Since the occupation floor represents the occupation habits of more than one group the signal will have to be unscrambled using numerical 
techniques. In the linear instance, principal components anaiys is can be used to determine independent relationships between subsets of artifact characteristics and directions. Depending on diagnostic traits, correlated sets of artifacts can be assumed to delineate various camp patterns. Unexpiained variances will be higher than at Resolution 1 or 2 . The same effect could be achieved by deflating a series of Resolution 1 and/or 2 floors. Ecofacts such as pollen would disappear.

As using the "site" for the spatial unit suggests, an occupation floor is a conceptual unit of the size of the "site." The disturbance of any percentage of the area of a floor deprives the analyst of that much of the pattern. For the sake of insuring the presence of this factor in an analysis, let us establish that the level of resolution is the characteristic resolution of a series, of floors plus the percent of area disturbed. A series of Resolution 1 floors that are deflated in $18 \%$ of their area constitutes a site that is Resolution 1.18. A site that is occupied through several climatic periods and is fully deflated through more than one period is Resolution 4 (R3 $+100 \%$ ). Appropriate recovery techniques would be resolved less than $1 \mathrm{~cm}$. There is less information inherent in the pattern and it would be inefficient to operate at a high resolution recovery level.

If a systematic relationship is assumed between prehistoric persons and ourselves, it is equally important to understand the relationship between the prehistoric people under study and their contemporaries. The enviro-cultural milieu in which prehistoric Central Texans found themselves is obscure in many ways. In other aspects, however, it can still be observed or was observed ethnohistoricaliy.

Since relatively complex cultures developed to the east and west of Texas, perhaps the broadest theoretical question which needs to be addressed in order to properly set the scene for this study is, "Why did no complex cultures develop in Central Texas?" Typically, cultural ecologists have considered ecotonal situations to be favorable for the development of complex society due to opportunities for trade and other interactions between diverse and therefore mutually stimulating communities. Why Texas as an ecotone did not provide fertile soil for civil development can only be left to conjecture in specific terms. Perhaps, however, the broad and far-ranging character of the PlainsWoodland ecotone is substantively different from the narrow contrastive ecotones of Mesoamerica where these theories developed. The biotic plasma does not provide a favorable context for elaboration of the cultural plasma.

What did happen in the vicinity of the Plains-Woodland ecotone and its related ecozones can be understood as a function of the decidedly differing ecological preferences of primitive and complex cultures. Primitive cultures of North America have long been thought to be oriented toward broad spectrum hunting and gathering, which implies in some circumstances that ecotones are the best habitat. Once conjunctive archaeology began to reveal the character of postPleistocene cultural adaptations in the 1950s, a synthesis of Caldwell's Primary Forest Efficiency for the Eastern Woodiands and Jenning's and Norbeck's Desert culture for the arid west was readily amalgamated into an American Archaic Tradition with a total emphasis on broad spectrum subsistence whether the environment provides desertic or humid adapted resources. It is not 
surprising, therefore, that ecotonal Central Texas was for a very long time one of the hearthstones of primitive American cultures, even long after other areas had "gone modern." This is half of the pattern. It is left to the other half to explain why complex societies stayed away from Central Texas.

What cultural ecologists have in large part ignored is that complex societies are in essence specialized societies. While primitives thrive in habitats of wide abundance, complex societies are oriented to a few domesticated plants for the dominant portion of their livelihood. These plants are nurtured for generation after generation and in time the bond between human and plant is inseverable. The humans move where the plants will grow best. Ultimately, plants grow best at the centers of ecozones where conditions of moisture and soil are most reliable, not on the ecotones where conditions may be desertic one year and humid the next. In other words, complex cultures will tend purely on the basis of subsistence to gravitate to the center of ecozones. The unstable character of broad ecotones is fundamentally disruptive to complex social organization and sedentary lifeways.

This pattern is readily visible in regions adjacent to Central Texas. The epicenter of Eastern Woodland activity was in the climatically stable Lower and Middle Mississippi Valley. The more complex cultures developed in the heart of the arid west where conditions were adverse but relatively stable. Gravitation to the center of their respective zones left Central Texas and its fluctuating prairie margin to those who preferred it, in perpetuity.

Climatically stable regions could explain some, perhaps a great deal, of the problems with civil development. While more complex cultures developed in stable areas, especially Mesoamerica, but also the southwest and southeast, no dependable agricultural bas is of subsistence developed in Central Texas. The Tonkawa Indians, who were the potentially oldest inhabitants of Central Texas, had no native agriculture in historic times. Their mythology held that they were exhorted by the wolf, to whom they attributed their origin, to avoid planting and live as predators as he did. The tales also indicate that in former times when the Tonkawa were a strong people they planted crops for a time at the headwaters of the Brazos River (Sjoberg 1953).

While we have no way of knowing from what age this possible record dates, it is the expectable result of attempting agriculture in an area which, during times of drought such as that of the 1930s, is reported by local inhabitants to have been denuded of vegetation.

What follows is, at least in part, an attempt to document the environmental instability of Central Texas and assess the cultures that adapted to it. 


\subsection{ECOSPHERE OF THE HOP HILL LOCALITY (Kay Simpson)}

Introduction

Lyndon B. Johnson State Historic Park lies along the south bend of the Pedernales River, near the town of Stonewa11, Texas, (Figure 2.01). It is across Ranch Road 1 from the LBJ Ranch. The site being studied is within the park. and lies on the upland margin and valley slope of the river. In the area around the site various geological formations are found. The LBJ ranchhouse and airstrip, St. Francis Xavier Church and the Stonewall community lie on the Hense11 Sandstone of the Trinity group (Maxwe11 1970:135).

Also found within the park are San Saba and Morgan Creek Limestone members of the Wilberns Formation. From the park, hills can be seen in the distance which are "erosional remnants of the Edwards Plateau and are capped by Edwards Limestone. A few of the valleys are underlain by the Glen Rose Limestone" (Maxwe1 1 1970:135).

The site is on a recently acquired portion of the park, north of Highway 290 and west of the developed park vicinity. It is about midway between Fredericksburg, the county seat of Gillespie County, and Johnson City, the county seat of $\mathrm{Bl}$ anco County. The altitude of Gillespie County ranges from 335 to 686 meters, and the altitude of the site is about 457 meters (Texas A7manac 1964-65:222).

Rocks found on or near the surface in Gillespie County are mostly Cretaceous (Comanche Series). In the northwestern quadrant and along the eastern edge of the county, there are some Devonian, Silurian, Ordovician, Cambrian and Paleozoic (Table 2.07). A7so, some Ignecus (undifferentiated) rocks are found in the northeastern quadrant (Arbingast et al. 1973:9).

\section{Physiography}

The site is situated on the Edwards Plateau, directly south of the Central Mineral Region, or Llano area. Also nearby, to the northeast, is another physiographic region, the Grand Prairie. Because each of these environments has a bearing on the ecological resources of the early peoples who may have inhabited the site, they will all be dealt with in more detail.

\section{Edwards Platean}

The Hill Country region of West-Central Texas comprises the Edwards Plateau. On the south and east, this region has a physical boundary line, the Balcones Escarpment (Gould 1975:12). The escarpment is formed by "a belt of many subparallel fractures that separate the Lower Cretaceous rock in the plateau from the younger Cretaceous and Tertiary rock that underlies the Coastal Plain" (Maxwe11 1970:13).

The limestone formations dip gently toward the southeast, and on the plateau are found the headwaters of the Blanco, San Marcos, Guadalupe, Medina, Frio and Nueces rivers (Maxwe11 1970:13). 


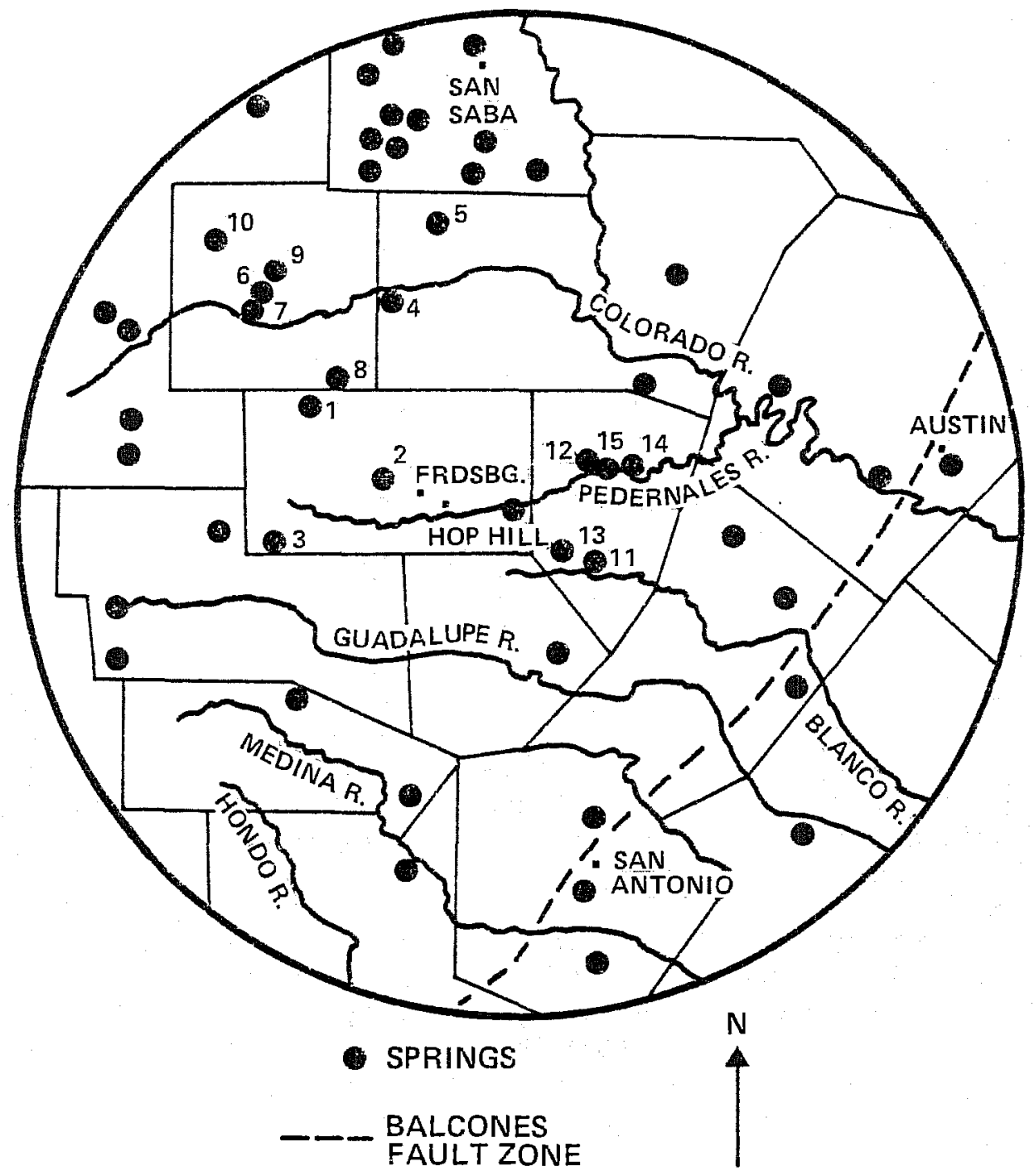

Figure 2.01. Map of Region.

(Numbered springs are mentioned in text)

1. Lange Springs

2. Guenther Spring

3. Fall Springs

4. Castell Spring

5. Valley Spring

6. Kothmann Springs

7. Mi11 Springs

8. Anderegg Springs
9. Gamel Spring

10. Pluenneke Springs

11. Koch Springs

12. Buffalo Spring

13. Rocky Creek Spring

14. Crofts Springs

15. Hobbs Spring 
Table 2.01 Geologic Time

(adapted from Maxwe11 1970:26)

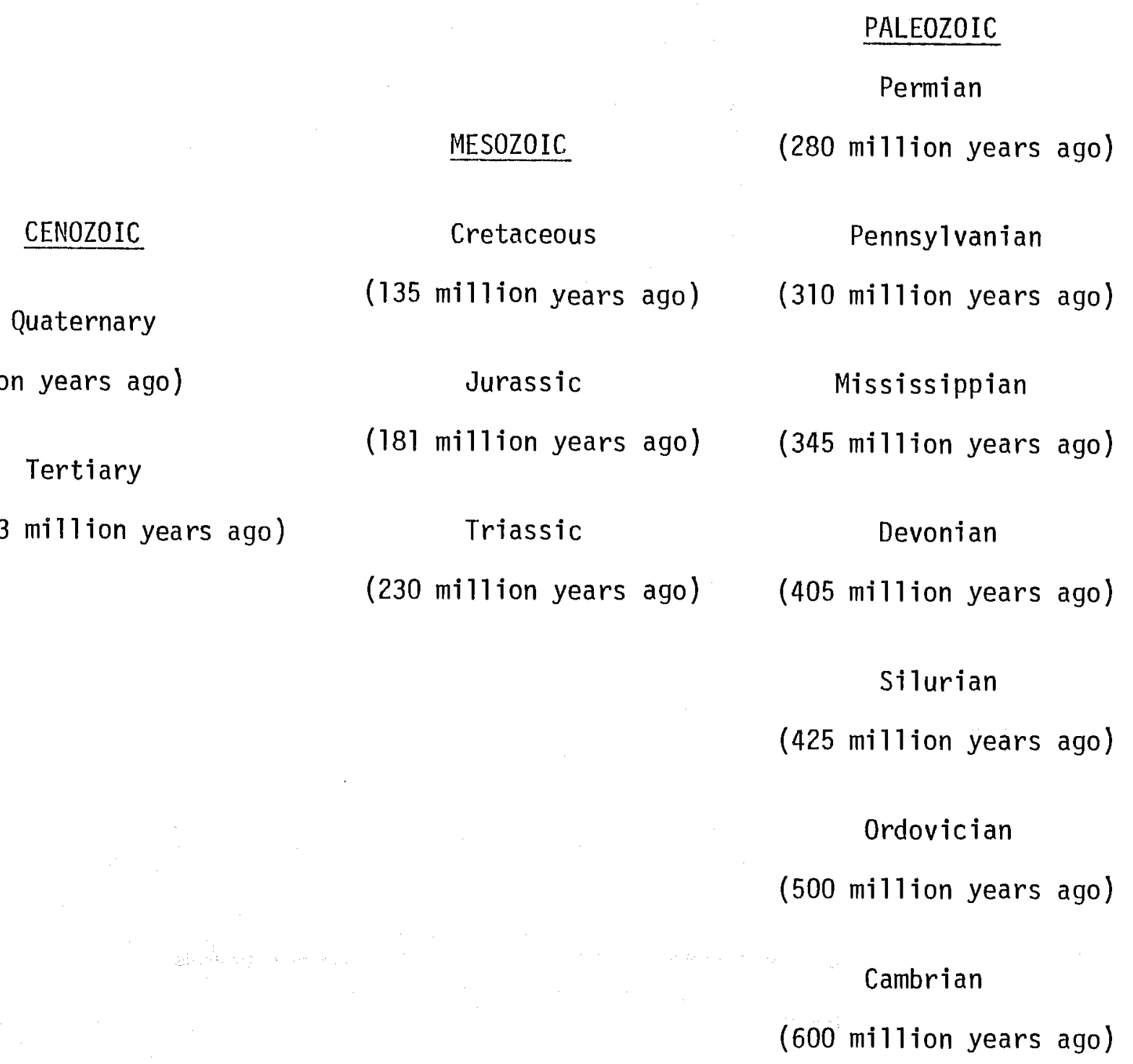


Vegetation of the area (see Section 6.0) can be described as a juniper-oakmesquite savanna (Arbingast et al. 1973:11). "Along the large streams, the vegetation varies from heavy deciduous forests in the lower valley bottoms to sparse and stunted scrub oak, cedar, and chaparral on the higher slopes..." (Maxwe11 1970:13).

While some parts of the plateau have a heavy cover, others are more prairielike, and here cattle-grazing is the leading industry. "Dark, calcareous stony clays and some clay loams" make up this area's soil content (Arbingast et al. 1973:10).

\section{Central Mineral Region}

Some of the oldest rocks in Texas are found in the granitic Llano area. Its central basin is 25 miles wide and 50 miles long, and contains mountains 400 to 600 feet in height (Maxwe11 1970:16).

A rim of overlying Paleozoic 7 imestone and sandstone formations 5 to 25 miles wide partly surrounds the area of the oldest rocks. A second rim, more irregular than the Paleozoic, is formed by Cretaceous limestone beds and shaly limestone (Maxwe11 1970:16). Soils of this region are general1y "reddish-brown to brown, neutral to slightly acid gravel1y and stony sandy 1oams" (Arbingast et al. 1973:10).

\section{Grand Prairie}

The Grand Prairie is underlain by Cretaceous rocks which resist erosion. "It is a limestone-based area, usually treeless except along the numerous streams, and adapted primarily to livestock raising and staple crop growing" (Texas Almanac 1964-65:294). The topography of this area ranges from plateau-1ike, such as is seen north and west of Fort Worth, to Lampasas, where the 1 imestone formations of the Grand Prajie and Edwards Plateau merge. On the western boundary is the Cross Timbers belt, with sandy soil and crops of peanuts, maize and peaches, and oak forests (Maxwe11 1970:13).

\section{Climate}

Texas

Location between the warm waters of the Gulf of Mexico and the high plateaus and mountain ranges of North America results in great diversity in weather within the state of Texas. "Winds from the Gulf of Mexico and the Atlantic transport moisture for most of the precipitation that falls in Texas" (Ladd 1957, Vol. 1:2-1911). Rainfa11 decreases from the eastern areas of the state toward the western regions. (Table 2.02 shows examples of temperature and rainfal1 for the Edwards Plateau.) The highest amount of rainfal1 occurs in May and September, and the Towest during mid-summer throughout most of the state. "The two maximum precipitation periods in May and September are characteristic of the interior, partiy 
because of convective thunderstorm activity and partiy because at these two times migrations of cooler air from the north have a good opportunity to encounter wel1 established moisture-laden winds from the Gulf of Mexico" (Carr 1967:11).

Table 2.02 Climatic Norms of Edwards Plateau

\begin{tabular}{lcc} 
& Rainfal1 & Temperature \\
\cline { 2 - 3 } January & 1.60 & 48.8 \\
February & 1.59 & 52.5 \\
March & 1.46 & 58.5 \\
Apri1 & 2.42 & 66.7 \\
May & 3.54 & 73.9 \\
June & 2.67 & 80.7 \\
July & 2.26 & 83.1 \\
August & 1.98 & 83.1 \\
September & 3.11 & 77.4 \\
October & 2.33 & 68.6 \\
November & 1.28 & 56.4 \\
December & 1.67 & 50.3 \\
Annual & 25.91 & 66.7 \\
& & (from Carr 1967:8)
\end{tabular}

Rainfall is erratic in Texas, usually with more years below than above average. Temporary or prolonged droughts are common, resulting in a reduction in vegetation and crops (Gould 1975:8).

Even during normal years, dry periods affect crops. One of the worst droughts recently began in West Texas in 1950 and spread until, by 1956, 244 out of 254 counties were classified as disaster areas (Texas Almanac 1964-65:97). 
During the winter, cold winds and little inland precipitation generally characterize the weather in Texas. "Wide plains and prairies and the lack of sheltering mountains and extensive forests allow the wind free play and permit sudden weather changes, expecially those associated with the passage of 'northers' across the state. The highest recorded wind was 84 miles an hour from the northwest, but the prevajiing winds are from the south" (Ladd 1957, Vol. 1:2-1911).

Cold fronts, or "northers," move from northwest to southeast Texas at a rate of 30 to 40 miles per hour, resulting in drops of 40 degrees or more within 12 hours in the northern part of the state. As they move southward, they become milder. Frequent "northers" may result in dry winters in the interior of the state. "In winter, visitations of cold air from the north may be too frequent for the winds to have had time to shift to the south and begin transporting moisture inland before a new cold visitation arrives" (Carr 1967:14).

\section{Gillespie County}

Weather in Gillespie County is generally mild the year round. Average precipitation per year is 27.44 inches in Gillespie County, and the period of greatest rainfall is during Apri1, May, June and September (A11ison et al. 1975:1). (See Section 4.0.)

Taking an average of the years 1931-1960, the mean annual temperature of Gillespie County is 64 degrees on the western half, and 66 degrees on the eastern half, where the site is located (Arbingast et al. 1973:15). "The growing season (freeze-free period) in Gillespie County averages 219 days. The average dates of the last occurrence of 32 degrees in spring and the first occurrence in fal1 are Apri1 1 and November 6, respectively" (A11ison et al. 1975:77).

$\underline{\text { Soi1 }}$

The general soil area is the Luckenbach-Pedernales-Heatly association with deep, sandy loams, gently sloping soils on upland and terraces (A17 ison et al. 1975: General Soil Map). "The soils in Gillespie County range from young to old. The younger soils have little profile development, and the older soils have wellexpressed soil horizons. Some of the soils along the Pedernales River are examples of younger soils. They are still recejving sediments as the river floods" (A11 ison et al. 1975:75).

Soils at the site, according to the soil survey, sheet 56 , are Hensley soils, 1 to 3 percent slopes (on the east side); Purves soils, undulating (on the west side); with some Pedernales fine sandy loam, 1 to 3 percent slopes, in between. (See Section 6.0.)

Hydrology

This area is drained by the Pedernales River, which is 106 miles long, and flows southeast from Kimble County to Travis County, where it meets the Colorado 
River (Texas Almanac 1964-65:310). "The Pedernales River watershed is near the eastern edge of the Edwards Plateau where much of the original plateau surface has been dissected by the streams in the river system" (Holland and Hughes $1964: 2)$.

Tributaries flowing into the Pedernales from the north include Live Oak, Barons and Palo Alto creeks, and from the south, Wolf, Bear and Meusebach creeks. "The streams derive their base flow from springs and seeps and generally flow throughout the year. During the summer months, however, irrigation from the streams and high rates of evaporation and plant transpiration cause many streams to become dry in some reaches". (Mount 1963:4).

During most of the period since May 1939, a recording gauge at the bridge on U.S. Highway 281 on the Pedernales, 1.2 miles northeast of Johnson City, has supplied a continuous record of the streamflow (See Table 2.03). The drainage area upstream from the gauging station, maintained by the Geological Survey, is 947 square miles.

"The average discharge for 30 years of record (water years 1939-69) was 153 cfs (cubic feet per second) or 110,800 acre-feet per year. During this period, the maximum discharge was 441,000 cfs on September 11, 1952; there was no flow at various times in 1951-52, 1954, 1956-57, 1963-64, and 1967-68. The flood stage of 42.5 feet on September 11, 1952, was the maximum since at least 1859. A flood in July 1869 reached a stage of 33 feet" (Follett 1973:8).

The Texas Water Development Board also operates a suspended-sediment sampling station at this location. By using bottles suspended a foot below the surface of the water, samples are collected and computations are made to equate the results with the streamflow. According to the Board, one acre-foot of streamflow $=1,361.25$ tons, and one acre-foot of sediment $=1,524.60$ tons. "No attempt has been made to estimate or compute that sediment which may be moved by a flowing stream along its bottom and commonly referred to as 'bed load' (Cook $1967: 2)$.

The Summary for the water year 1962 shows 47,528 acre-feet of streamflow and 40,943 tons (27 acre-feet) of suspended-sediment load. The figures for 1963 are 16,164 acre-feet of streamflow and 15,686 tons (10 acre-feet) of suspendedsediment load (Cook 1967:31).

water-Bearing Units

Principal water-bearing units in the Fredericksburg area are the Edwards and Comanche Peak Limestones, the Hensell Sand Member of the Shingle Hills Formation, the Ellenburger Group, and the Hickory Sandstone Member of the Riley Formation (Mount 1963:14). 
Table 2.03 Peak Stages

Pedernaies River near Johnson City
Water Year
Gauge Height (feet)

1869

1940

1941

1942

1943

1944

1945

1946

1947

1948

1949

1950

1951

1952

1953

1954

1955

1956

1957

1958

1959

1960

1961

33 (approximately)

17.53

12.83

14.10

14.25

26.10

13.96

9.40

9.57

8.78

8.74

5.79

10.18

42.5

14.70

7.22

10.67

2.91

24.80

17.45

17.00

26.1

11.08

(adapted from Patterson 1963:B-108)

Edwards and Comanche Peak Limestones

Limestone rocks, which intercept precipitation, supply water to streams and furnish recharge by downward leakage to other water-bearing formations (Mount 1963:14).

The Edwards Limestone group also contains dolomite and gypsum. It is not overlain by younger formations, and crops out on the surface at higher elevations in this area. The Comanche Peak Limestone has more clay than the Edwards, and is softer and more susceptible to erosion. It is also less permeable than the Edwards. The walnut Clay is a thin bed of yellow clay grading upward into limestone clay. Its very low permeability prevents water in the Edwards and Comanche Peak from percolating further downward, and establishes a "perched water table, perhaps as high as 50 feet above the base of the Edwards" (Mount 1963:74). 
MARBLE FALLS AND ELLENBURGER-SAN SABA LIMESTONES

EDWARDS-TRINITY PLATEAU

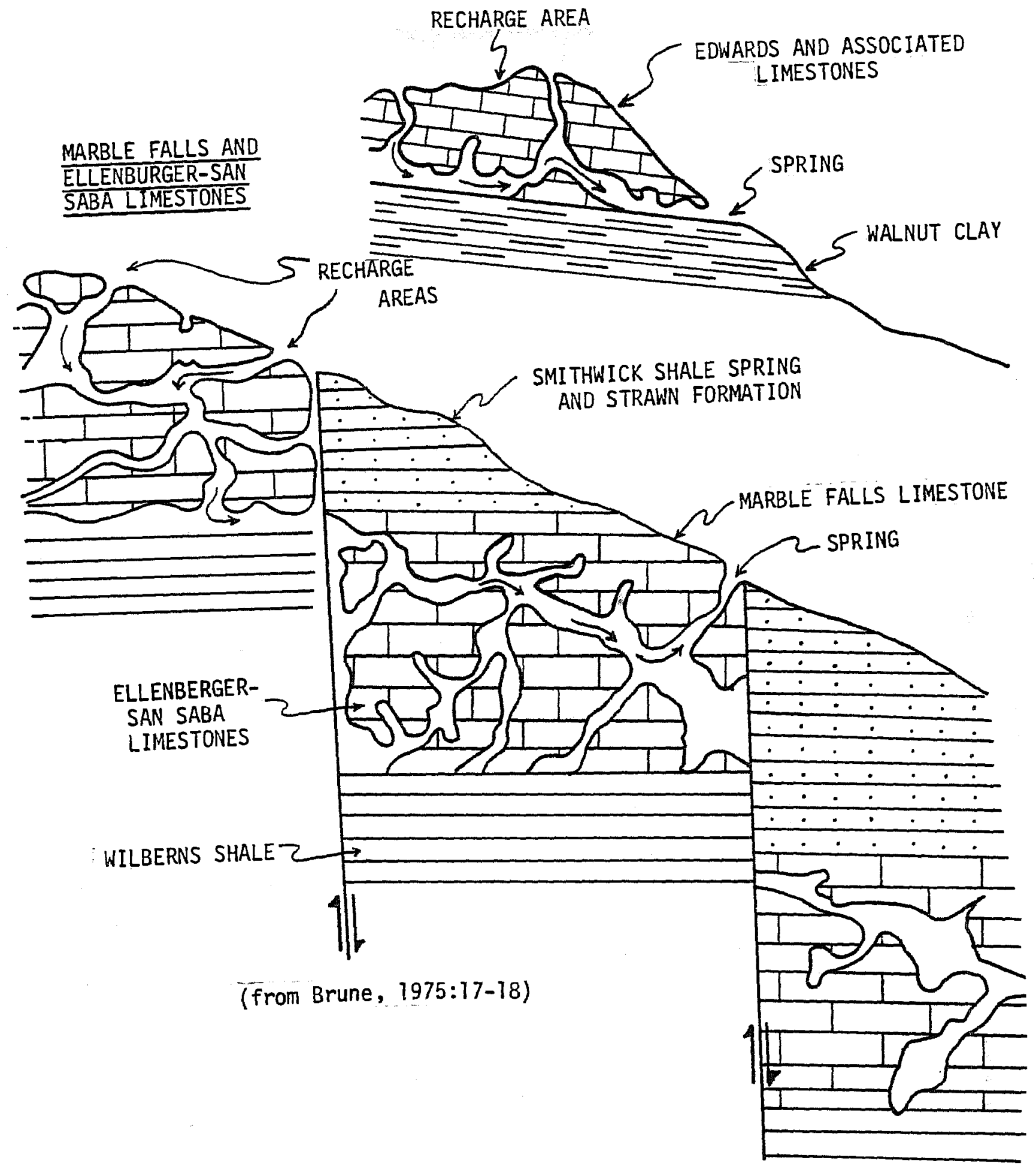

Figure 2.02. Subsurface Formations in the Study Area. 
Recharge occurs where Edwards and Comanche Peak outcrop when precipitation infiltrates down through openings in the rocks until it reaches the water table (Mount 1963:14). "In a typical case the recharge water percolates downward through the Edwards and associated limestones until it reaches the impervious clays of the Walnut Formation. Then it moves laterally, emerging as springs on the hillsides or in stream beds" (Brune 1975:13). "Rainfa11 absorbed on the Edwards outcrop is lost mostly by evapotranspiration but some water penetrates to the water table and eventually appears in springs and seeps at the base of the formation" (Holland and Hughes 1964:2). (See Figure 2.02.)

Other than discharge through springs and seeps, some water is also discharged artificially through "smal1-capacity wells for domestic and 1ivestock purposes" (Mount 1963:15). The springs do not have large amounts of discharge, but their flow has been sustained during periods of drought.

Hensell Sand Member of the Shingle Hills Formation

This unit has poorly-sorted sand, silt and clay, with the finer-grained material at the top and the conglomerate and coarse sand at the bottom. Its color is red, and it becomes gray and less sandy near the top as it grades into the overlying Glen Rose Limestone. Often the Hensell contains 1 imestone beds in its subsurface (Mount 1963:15).

"With few exceptions, the Hense11 is present throughout the Fredericksburg area. It crops out at the lower elevations, but at higher elevations the Hensell is covered by overlying strata" (Mount 1963:15).

Recharge occurs mostly from precipitation falling on its outcrop area and infiltrating the deep sandy soils. "Some recharge also occurs by infiltration from streams crossing the Hensell outcrop. Probably small amounts of water are contributed to the Hensell by downward leakage from the Edwards and Comanche Peak Limestones in the northern and western parts of the area" (Mount 1963:16).

Discharge occurs in a southeasterly direction through seeps along streams, emptying into the Pedernales River. It also occurs artificially through wells.

As the clay and sand beds of the Hensell are generally saturated with water, except in the upper few feet, the Hensell is a water-table aquifer. Water in its lower sands are under artesian conditions, however, because they are overlain by more impervious clay layers (Mount 1963:16).

\section{Ellenburger Group}

East and south of Fredericksburg, the Ellenburger Group is found in the subsurface below the Hensell sand. It consists of dolomite and limestone. This group is underlain by a similar unit, the dolomite of the San Saba Member of the Wilberns Formation (Mount 1963:19). 
"Water in the Ellenburger occurs in solution-formed openings in Timestone and dolomite and is generally under artesian conditions. Recharge to the Ellenburger is by downward leakage from the overlying Hensell Sand... Water is discharged naturally from the Ellenburger through small springs and seeps along the Pedernales River" (Mount 1963:19).

Five large-capacity wells near the Pedernales comprise most of the artificial discharge, as there are few smal1-capacity wells in the Ellenburger.

Hickory Sandstone Member of the Riley Formation

"In the Fredericksburg area the Hickory Sandstone occurs only in the subsurface. Because of displacements due to faulting, the Hickory is present at various depths below the Cretaceous, but in some places, such as in the city of Fredericksburg, the Hickory is not present because it was removed by erosion prior to the deposition of the Hense11 Sand" (Mount 1963:21).

The Hickory consists of fine-to-medium-grained sand or sandstone. It has beds of shale or clay in the upper portion, and sometimes has conglomerate at the bottom. Recharge occurs by downward leakage from the overlying Edwards and Comanche Peak Limestones. Water movement is probably disrupted by a series of faults, and locations of natural discharge are not known. There are only a few wells to create artificial discharge from the Hickory (Mount 1963:21).

\section{Springs}

Some important springs occurring in Gillespie County are:

Lange Springs. There are at least two springs at this location, two miles north of Doss. The springs occur in the Hense17. Sand aquifer. History of the springs includes use by Indians as indicated by pictographs in a nearby cave, establishment of Lange's corn, feed and saw mill in 1849, and use for irrigation. The springs, originally described as "deep, cold springs gushing from beneath shelving rock," had a discharge of 0.67 cubic feet per second in 1937 (Brune 1975:43).

Guenther Spring. This spring, about four miles west of Fredericksburg, also occurs in Hense11 Sand. In 1851, the Guenther corn, feed and saw mi11 was established here. By 1937, there was no flow at the spring.

Fal1 Springs. These springs occur four miles north of Camp Scenic in the Comanche Peak Limestone. "Indians of the Archaic Period left burnt rock middens, projectile points and stone axes at these springs." In March 1965, discharge was 2.2 cubic feet per second (Brune 1975:43).

In Llano County, northeast of Gillespie, there are two major springs:

Castell Spring. The town of Castell was founded around this spring in 1847 by a group of German settlers led by the Count of Caste71. Its aquifer is composed of Precambrian marble, gneiss and schist. 
Valley Spring. Located in the town of the same name, this spring comes from Hickory Sandstone. In 1845, 0. C. Phillips built a gin, grist mill and saw mill here (Brune 1975:53).

Mason County, northwest of Gillespie, has several springs:

Kothmann, Mil1 and Anderegg Springs. These are all groups of springs in Ellenburger Limestone.

Pluenneke Springs. A group of several springs in Ellenburger-San Saba Limestone.

Gamel Spring. Located in the citv of Mason and based in Hickory Sandstone, the spring issued through a fault. It is believed to have been a favorite campground of the Comanches.

"In 1847 the German settler John Meusebach met with Indian chiefs here preparatory to his important treaty with the Comanches... The spring supplied water to fort Mason in 1851." As recently as 1940 the spring supplied water to the Mason swimming pool; by 1971 there was no longer any discharge (Brune 1975:54).

Blanco County, on the eastern boundary of Gillespie, has a number of springs:

Koch Springs. Located in Blanco State Park, these springs are from the Glen Rose Limestone of the Edwards aquifer. Historically, they were situated beside an old Comanche trail (Brune 1975:34).

Buffalo Spring. Based in Morgan Creek and Cap Mountain Limestones, this spring is the source of Buffalo Creek. It is located five miles northwest of Johnson City.

Rocky Creek, Crofts and Hobbs Springs. These springs are a 11 based in EllenburgerSan Saba formations. Rocky Creek is three miles east of Hye. Crofts is four miles northeast of Johnson City, and Hobbs is two miles north of that town (Brune (1975:34).

Geology of Gillespie County

Rocks cropping out in the specific area of the Hop Hill locality are the Trinity Group in the Comanche Series of the Cretaceous Period. "The Comanche series was first named by R. T. Hill in 1887 from the town Comanche, Texas, where he first studied these rocks, and from the Comanche Indians, who inhabited the central denuded region of Texas" (Sellards et al. 1932:272). Also located nearby to the northeast are the Ordovician and Cambrian of the Llano region.

Formations appearing in the general area can be seen in the geologic maps of the Hye, Rocky Creek, Cave Creek School, and Stonewa 11 quadrangles (Barnes 1965a, 1965b, 1966a and 1966b). For a list of geologic time references, refer to Table 2.01. 
Paleozoic rocks include the San Saba Member of the Wilberns Formation (Upper Cambrian) and the Threadgi11 Member of the Ellenburger Group. Tanyard Formation (Lower Ordovician). The Ellenburger "...forms an important unit in the geology of Texas, its known extent in both surface and subsurface covering approximately one-half of the State" (Hendricks 1952:5-6). San Saba rocks in this area are mostly dolomite with some limestone. There is little chert in the San Saba and some chert in the Threadgill of the Ellenburger. Concerning Ellenburger chert, Hendricks wrote: "Three main textural groups found in the El lenburger residues have been designated as smooth, granular and chalky" (Hendricks 1952:18).

Mesozoic rocks in the Trinity Group of the Shingle Hills Formation (Lower Cretaceous) are the Hensel1 Sand Member and the GTen Rose Limestone Member. Other rocks, in the Fredericksburg Group, are Walnut Clay, The Comanche Peak Limestone and the Edwards Limestone.

"The Hensel1 varies widely in color and composition, is in general very poorly sorted, and becomes finer grained upward." Its composition ranges from boulders and cobbles to silt and clay. It is mostly gray, but is red in some places. Hensel1 is cultivated in some areas (Barnes 1965a:3).

Glen Rose outcrops in most of the area. "It consists of alternating beds of limestone, dolomite, clay, silt and sand. The beds vary in their resistance to erosion, producing a 'stairstep' topography." Most of the area of the Glen Rose is used for ranching, but there is some farming (Barnes 1965a:3).

"Walnut Clay flanks the Edwards Plateau lobe which crosses the southern part of the quadrangle; it also crops out on several plateau outliers... The Walnut clay Bench, typically barren of trees, is too narrow to farm." This unit is silty, and very calcareous. It is yellowish-gray clay and partly mottled yel lowish-orange (Barnes 1965a:4).

"Comanche Peak Limestone forms a steep slope that is identified by a thick growth of vegetation dominated by a narrow-leaf oak. The upper part is bedded, partiy honeycombed, and ranges from yellowish gray to white." The lower part of the Comanche Peak Limestone is massive, nodular and yellowish-gray (Barnes 1965a:4).

The Edwards Limestone in this area consists of 1 imestone, dolomite and chert. "Some of the chert in the Edwards Limestone is of a quality suitable for the manufacture of flint implements, and the chert in the Edwards is usually referred to as flint" (Barnes 1965a:5). The distinction between these two terms was explained by Girard, 1972:84: Geologists do not agree on whether chert and flint are two names for one variety of rock, or whether each is a separate variety. Some, however, now give chert a geological meaning and flint an archaeological meaning."

Cenozoic rocks of the Quaternary System include high gravel in the Pleistocene, and aT7uvium of Recent Series. "High gravel deposits in the vicinity of the Pedernales River are probably mostly stream deposited; however, some are probably remnants of colluvial deposits once much more widely spread." Gravel materials come from the Edwards, Comanche Peak, Hensell Sand and Paleozoic rock 
in the form of cobbles, pebbles and finer materials such as caliche (Barnes 1965a:5). The alluvium consists of sand and silt with coarser materials underneath, deposited along the river and creek beds. "Some of the alluvium is cultivated and some supports a growth of pecan trees" (Barnes 1965a:5).

Geology of LTano Region

Gillespie County is Tocated on the southern flank of the Llano Uplift. (ATlison et al. 1975:75). The center of the Llano Uplift is north of Gillespie County, in LTano County (Mount 1963:6).

"The LTano Uplift was formed by an upward bulging of the earth that brought to the surface rocks normally covered by several miles of younger rocks. These rocks exposed by uplift are Precambrian, a billion years or so old, as well as rocks a half billion years old and younger" (A77ison et al. 1975:75). During the Paleozoic Era, there was strong fauTting, and in addition to the updoming, the rim was jagged in outline (Stenzel 1937:3).

In Mesozoic times, the uplift remained elevated and was undergoing erosion throughout the Triassic and the Jurassic (Rainwater and ZinguTa 1962:98). "At the beginning of Cretaceous time the highest part of the Llano uplift was in the south, near Fredericksburg: (Stenze1 1937:3). "The Mesozoic history of the Llano region can be described in terms of gulfward tilting of the north flank of the Gulf of Mexico geosyncline and the sinking of the Gulf and its margins, resulting in the slow but continued transgressive flooding of the north flank of the Gulf geosyncline and hence that part of its northern flank known as the LTano uplift" (Rainwater and Zingula 1962:98). "The Cretaceous overlapped gradualiy on the older rocks and some of the higher peaks remained as islands for a long time" (Stenzel 1937:3).

Today, because of erosion and weathering, "...the uplift is topographically a basin and the Paleozoic forms a rim cuesta around the basin and high outlier mountain in the basin" (Stenzel 1937:3). "The Llano region includes an erosional basin in which rocks of pre-Cambrian age are exposed and a surrounding higher area of Paleozoic and Cretaceous rocks forming in part a dissected plateau" (PTummer 1943:8). The highest elevations are found between the towns of Fredericksburg and Mason, and the lowest is southeast of Marble Falls.

Early Studies of the Area

In 1767 the first scientific investigations of Texas lands was conducted in the Edwards Plateau by Nicolas de la Flora, captain of the Spanish Royal Engineers (Ferguson 1969:4).

In a two-volume work on the Republic of Texas published in 1841, William Kennedy described the geography of the land for prospective settlers. "What captured the attentions of the land speculators and immigrants was Kennedy's report that he had seen specimens of gold and silver ores from the granite hill country of Central Texas. This rumored metallic mineral wealth provided the incentive for the launching of the first geological survey in Texas" (Ferguson 1969:10). 
Ferdinand von Roemer was appointed by the Adelsverein to lead the survey. The Mainzer Adelsverein was the Society for the Protection of German Immigrants in Texas, which a group of German noblemen had founded in 1842 (Ferguson 1969:10-11). In 1847 Roemer first published descriptions of rocks of the L1ano Region. "Roemer, accompanying an exploring party of German colonists under the command of Count Muesebach, entered the region from the south, traveled on horseback from Fredericksburg northwest, crossing Llano River at a shallow ford, and thence northwest to an Indian camp site on San Saba River near the present site of Camp San Saba" (P1ummer 1943:12).

In 1861, B. F. Shumard added to the work begun by Roemer. Jules Marcou compiled a map of the region in 1855. In 1883 Walcott described the Cambrian rocks in the area, and R. T. Hi11 first published work concerning this area in 1887 (P1 ummer 1943:13). 



\subsection{GLOBAL CLIMATE (Stacey Wright and Joel Gunn)}

\section{Introduction}

Recently there has been a marked upsurge in the attention given to the problem of climatic change. This stems in part from progress in the development of dynamical models applicable to climatic questions through the use of computers, and in part from a growing concern with the environment in genera?.

Table 3.01 Global Climatic Discontinuities and Central Texas Prehistory (adapted in part from Wenland and Bryson 1974 and Weir 1976)

Major

Minor

Central Texas Archaic Climatic Episodes Discontinuities Discontinuities (Starting Dates BP)

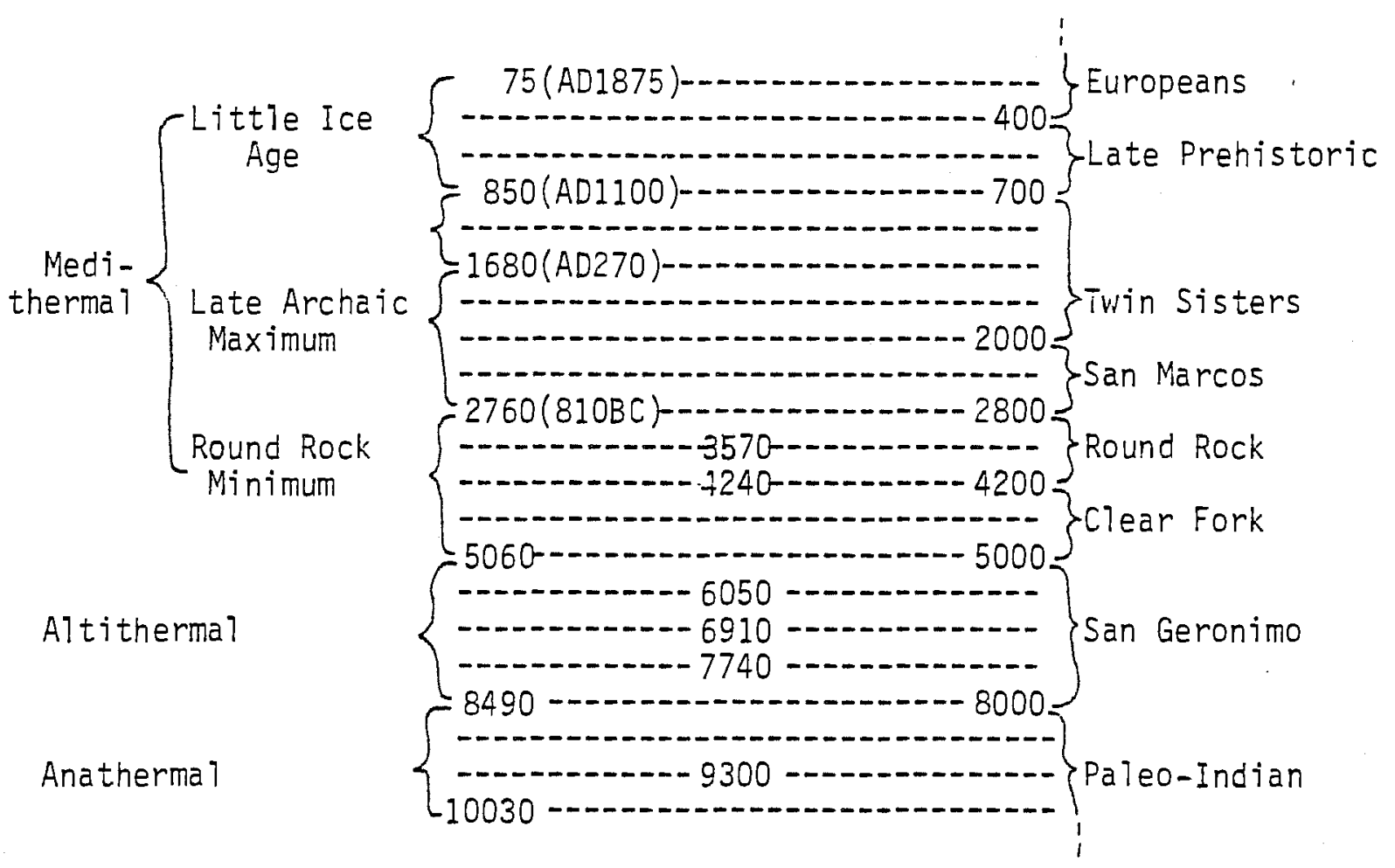

Relative to archaeological problems, Wenland and Bryson (1974) have convincingly demonstrated based on 815 botanical and 3700 cultural $\mathrm{C}^{14}$ dates ( 155 cultures) that cultures tend to begin and end in step with Holocene climatic episodes and that climatic episodes are globally synchronic. They argue that climatic episodes are periods of relative stability with abrupt changes or discontinuities from one state to the next. Culture changes generally lag about 50 years behind botanical changes which in turn lag about 700 years behind climatic events. Table 3.01 shows the results of Wenland 
and Bryson's very sophisticated study in $\mathrm{C}^{24}$ years of major and minor discontinuities in the pollen record. Also included are cross references to Weir's (1976) chronology for central Texas.

In view of the apparent correlation between the two records, taking into consideration the lag time and the expected error at increased time depths, a study of global climatic variation is in order as a means of controlling some of the impetus to culture change in the local archaeological record.

A review of global climate over the past million years shows interglacial periods, each lasting $10,000 \pm 2,000$ years and each followed by a glacial maximum which has occurred, on the average, every 100,000 years. The present interglacial interva?, which has now lasted for about 10,000 years, represents a warm climate that is relatively rare during the past million years, most of which has been occupied by colder glacial regimes. Only during about 8 percent of the past 700,000 years has the earth experienced climates as warm or warmer than the present.

The postglacial thermal maximum was reached about 6,000 years ago, and $c 1$ imates since then have undergone a gradual cooling, marked by three shorter periods of more marked cooling. Each was followed by a brief temperature recovery. During the Little Ice Age, from 1425 to 1800 , circulation may have been more meridional than at present and characterized in western Europe and western North America by short, wet summers and iong, severe winters (Global Atmospheric Research Program 1975). The well documented warming trend of global climate beginning in the 1880 s and continuing until the $1940 \mathrm{~s}$ is a continuation of the warming trend that terminated the Little Ice Age. Since the 1940s, mean temperatures have declined and are now nearly halfway back to the 1880 levels.

\section{Climate Theory}

Climatic theory has not evolved a universally acceptable model, al though many groups are focusing a large portion of their efforts specifically on developing models for climatic change.

Computer technology has become the primary tool in developing interaction models for climate prognostication. Two of the centers utilizing such computer research are the Ames Research Center in San Francisco and the Global Atmospheric Research Program (GARP) sponsored by the United Nations.

In the past, attempts were made to establish a single causative factor, such as solar radiation, solar particle emission, sunspot activity, terrestrial volcanic activity, ocean circulation, mountain building, and astronomical features of the earth's axis and orbit. However, no single indicator correlated exactly with statistical data, leading to the consensus that an interaction was indicated. The problem still remained to determine which factors were of primary significance.

The manifestations of weather have been defined as an interaction of temperature, rain, and snowfall, and the stirring of the atmosphere by wind which results from the flow of energy in and energy out over different parts of the spinning 
sphere (Lamb 1972). The earth sends energy back into space as a factor of the type of reflective surface, determined by cloud cover, snow and ice cover in proportion to 1 and cover, and the vegetational cover of the land surface.

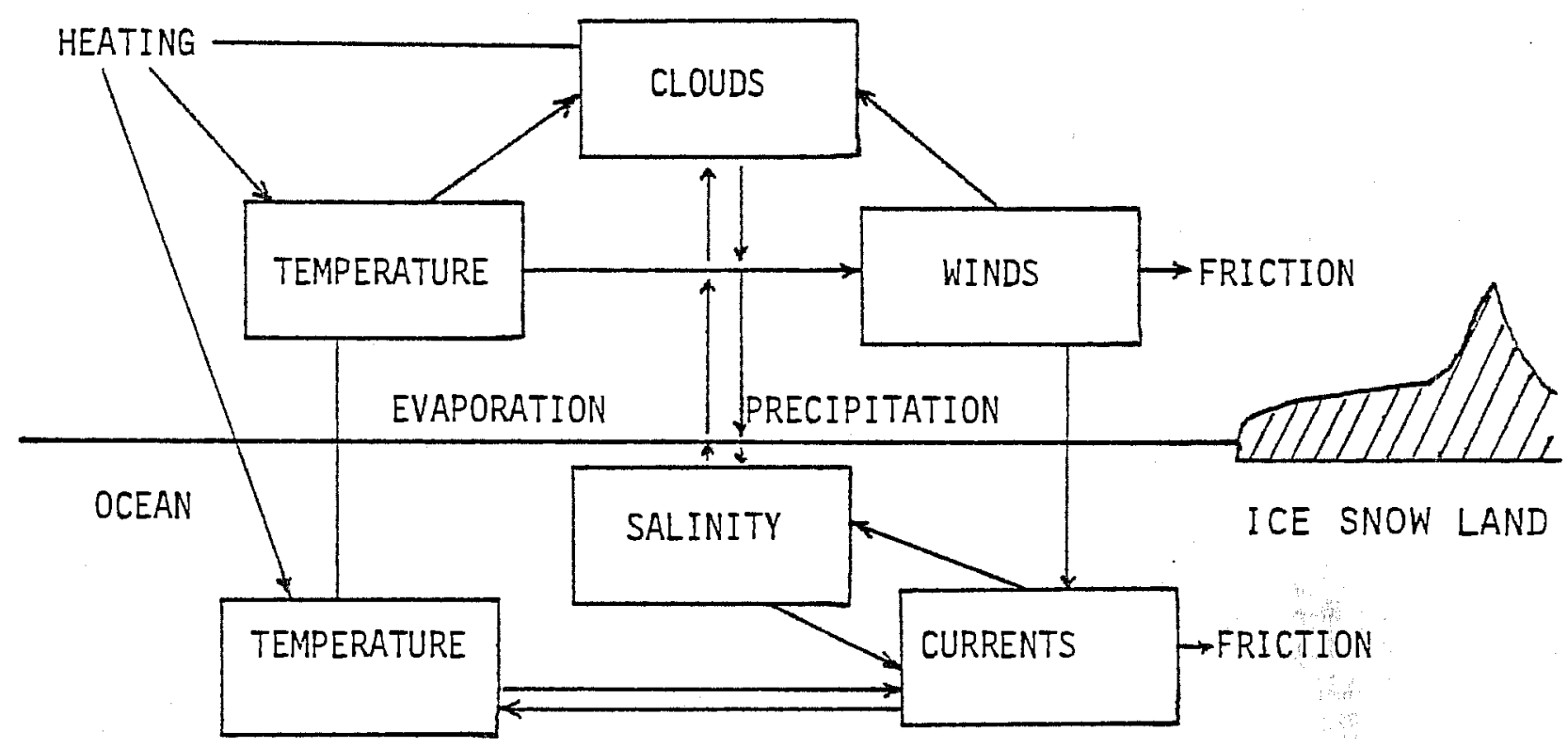

Figure 3.01. The Major Physical Components and Feedback Processes Responsible for the Maintenance of Climate.

The primary source of energy is the sun's radiation, and the transfer, transformation and dissipation of this energy by the giobal circuiation of the atmosphere and ocean involve all parts of the climatic system, as illustrated in Figure 3.01. In these processes a key role is played by watery substances in their various forms, and by the transfers of momentum, heat and moisture through the surface boundary layers of the atmosphere and ocean. Over land the hydrologic cycle's interactions with the surface are more complex, since part of the precipitation is re-evaporated, part is absorbed into the system of ground water, and part is used to nourish the surface vegetation. When precipitation falls as snow and accumulates, the surface albedo is raised and this changes the surface energy balance accordingly.

Unfortunately, many of the variables necessary to describe a single climatic state are unavailable, and therefore even the more recent climatic variations are not completely documented. 


\section{Radiation as a Determining Factor}

Climate theorists do not agree on which energy pattern is the determining system. Proponents of radiation define climete as the net gain or loss of heat in the form of radiation. This is determined by the amount of energy available as incoming solar radiation is modified by the given latitude, altitude, and the clarity of the atmosphere minus the amount of radiation reflected by the earth's surface at the prevailing temperature (Lamb 1972).

Therefore, it was theorized that if a variation of solar output could be determined and measured, the reactive processes of the earth could be correlated, possibly reflecting a lag time to the actual manifestation of climate change. Since the occurrence of variabie amounts of darkening of the sun's face in spots had long been observed, the early thinking that this should be the origin of climatic variations was obvious. However, evidence has not substantiated this theory. The sunspot record gives only a partial account of solar variation as it may have affected solar output and weather. A study of changes in the level of Lake Victoria in east central Africa, which was thought to correlate with maxima and minima years, was actually found to show bigger and more lasting changes occurring on two occasions apparently unconnected with solar phenomena (Shapley 1970).

Several climatologists are currently investigating the measurement of the solar constant. However, instrumentation has not been developed to capture more than the grossest variation. As reported by Roger Barry (1975), only a 2\% fluctuation of solar variation could result in the unstable growth or disappearance of the polar ice cap with resulting climate modification throughout the world.
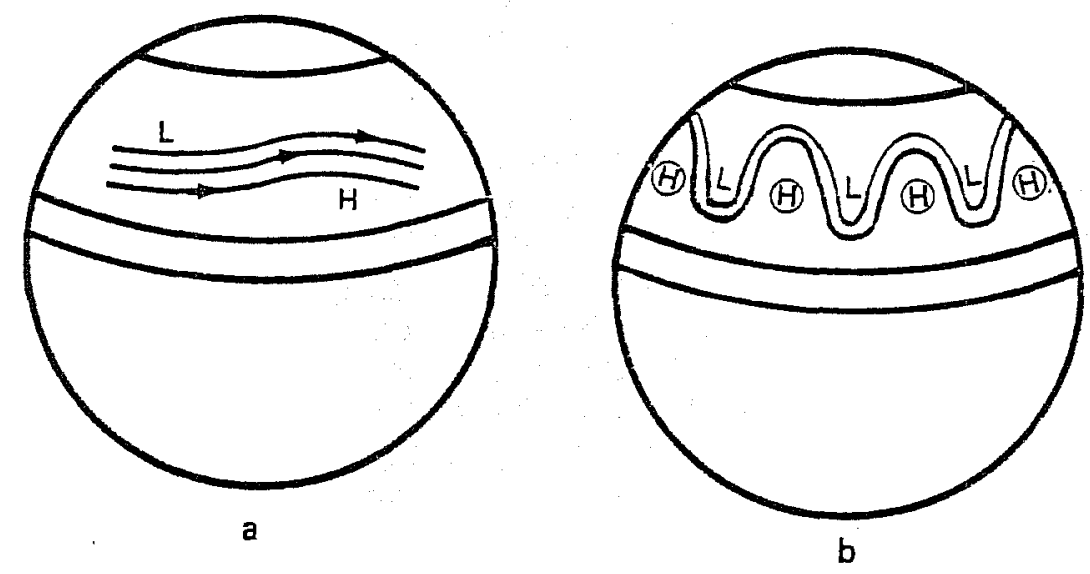

Figure 3.02. Wind Circulation Patterns. a, warm "zonal circulation" winds; b, cool "meridional circulation" winds (Douglas 1975). 
A significant part of the earth's annual radiation budget may occur through long term, periodic changes in the earth's orbit, the tilt of its rotation axis and the seasonal variation of its distance from the sun. Such epochs commonly change their character only slowly, over some thousands of years. But the changes are great, and some effects on climate appear inescapable, possibly including the causation of the ice ages and warm interglacial periods (Lamb 1972).

\section{Earth Processes as Determining Factors}

As air is warmed over tropical oceans, it rises and expands toward the poles. There it cools and descends, and high pressure systems form whose winds begin to circulate clockwise (in the Northern Hemisphere) because of angular momentum gained from the earth's spin. As these centers interact, the great movements of wind and weather result (Figure 3.02). The basis for global climate theory is founded on the fundamental equations of fluid dynamics and thermodynamics which specify the conservation of energy, mass and momentum. However, the actual manifestation of climate is further altered by the feedback mechanism of the nature of the ground surface, primarily determined by soil characteristics and vegetation cover.

\section{Climate Modeling for the Present}

Climate modeling must embrace the complete climatic system which is now recognized as involving not only the atmosphere, but the world's ice masses, oceans, and the character of the global land surface and its associated biomass, together with the coupled interactions which occur among them on a wide variety of space and time scales. These interactions are responsible for the characteristic complexity of climatic change. Numerical analysis of the various climatic indicators may help organize thinking about the subject of long term climatic change and perhaps help relate the global parameters to the local situation.

Paleoenvironmental interpretations are currently based on geomorphological features, soils, and animal and plant remains, which theoretically reflect the influence of vegetation and micro-organisms on soil development and the fact that climate thermally controls the rate of chemical reactions. Butzer (1974: 24) distinguishes between factors as zonal, or latitudinal associations; intrazonal, or local factors, e.g., drainage, regional climate; and azonal, which occur in any latitude, e.g., shoreline, tectonic activity. In Table 3.02 interrelated factors contributing to various environmental phenomenon are summarized.

A distinct problem is that much of the environmental data for model input and model verification is not available. Such details may be of great significance for localized climates.

In order to be useful, a data source must have a stratigraphic character, that is, the fluctuating values of a climatically sensitive parameter must be pre- 
served within the layers of a slowiy accumulating natural deposit or material (GARP 1975).

Table 3.02 Relationships of Zonal and Azonal Parameters

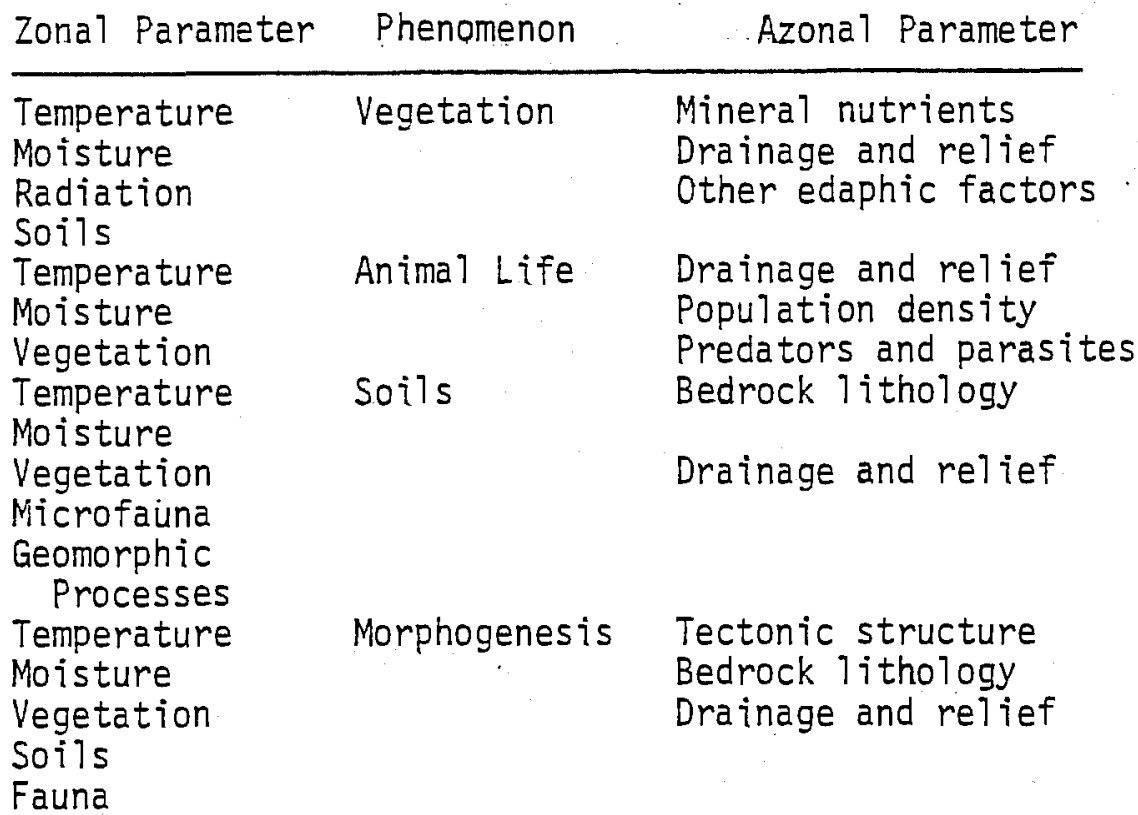

Each natural paleoclimatic indicator also has distinctive performance characteristics that must be understood if the data is to be interpreted correctly. For instance, the attainable sampling interval varies for particular paleocl $i$ matic indicators. Tree rings, varves, and some ice cores can be sampled at intervals of one year, while pollen or other sedimentary fossil samples only rarely represent less than about 100 years, and many geological series are sampled over intervals representing a thousand years or more. In addition, many indicators incorporate a lag time factor from climate-induced changes to the'expression of the measured factor. In a piant community, as reflected in pollen concentrations, for example, changes are relatively slow. The high frequency information is lost, but low-frequency changes are preserved. In contrast, tree-ring records and isotopic records in ice cores respond yearly and even seasonally in favored sites.

Each paleoclimatic record must then be calibrated or processed to provide an estimate of the climate. The composition of fossil assemblages in marine sediments and the width of tree rings, for example, are known to reflect the joint influence of several ecological factors. Here multivariate techniques can be used to obtain estimates of selected paleoclimatic parameters such as temperature and precipitation; however, such analysis is still in preliminary stages in most instances. 


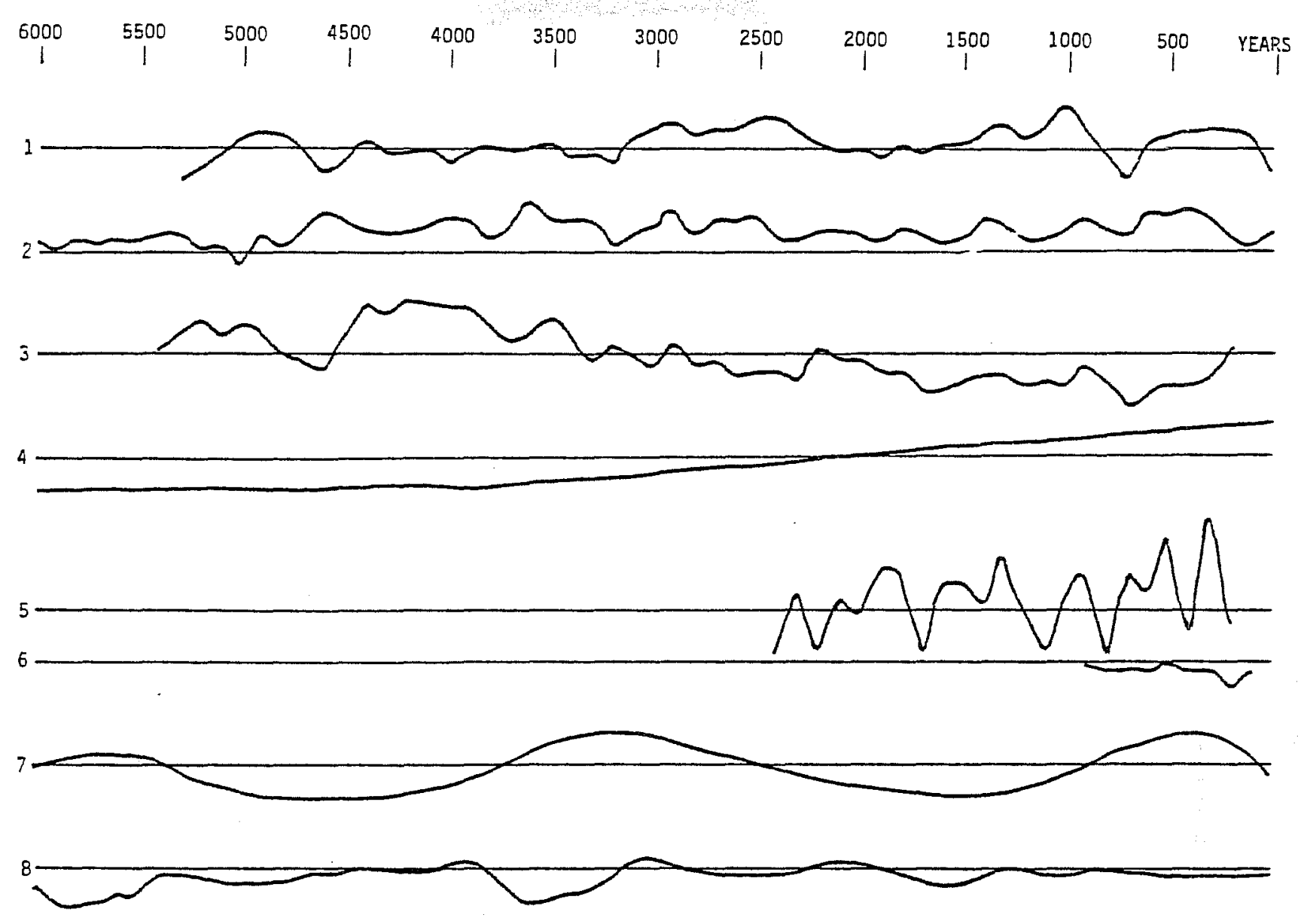

Figure 3.03. Climatic Fluctuations as Illustrated by Several paleoclimatic Indicatars (derived from measurements of respective graphs representing the following data).

1. Variation in growth width, tree rings, upper treeline limit, White Mountains, California (LaMarche and Fritts 1971).

2. Measurement of $D^{18}$ isotope in ice core sample from Camp Century, Greenland (Johnsen, Dansgaard, and Clausen 1972).

3. Measurement of $\mathrm{O}^{18}$ isotope in ice core sample from Byrd Station, Greenland (Johnsen, Dansgaard, and Clausen 1972).

4. Milankovitch compilation of orbital fluctuations as reinteroreted by Kukla based on the Autumn season (Kukla 1975).

5. Sunspot variation as interpreted by Schove, based on 11 year cycle (Lamb 1972).

6. Dust veil intensity due to volcanic eruptions in Iceiand (Lamb 1972).

7. Compilation of northern hemisphere temperatures, derived from tree rings, glacial recession, sea cores and pollen data (GARP 1976).

8. $C^{14}$ dates for increased lake volumes and/or stream discharge in the Sahara and East Africa (Butzer 1976). 
Paleoclimatic Indicators

A discussion of some available paleoclimatic indicators and their suitability to the present model follows (see Figure 3.03).

Deep Sea Cores. Sedimentation on the more level parts of the deep sea floor is limited to very slow accumulation. Part of the organisms found in these sediments are calcareous skeletons of one-celled Foramnifera. . During life, they inhabit the photosynthetic zone of the ocean's surface waters. They are consequently adapted to ocean surface temperatures which are, in their turn, in equilibrium with regional climates (Butzer 1974). As they accumulate on the sea floor, the faunal composition will reflect environmental conditions, particularly temperature, so that deep sea sediments may record stratigraphic successions of microfauna capabie of paleoecological study.

Analysis is made of the $0^{18}$ isotope content which is predominantly influenced by the isotopic ratio in seawater. It therefore reflects primarily the changing volume of polar ice, which upon melting releases isotopically light water into the oceans.

However, in both instances, the resolution of data is too broad for samples of the size necessary to study a 6,000-year period.

Pollen Analysis. As discussed previously, the resolution of most pollen data is not tight enough for this project. In addition, many data sources have not been effectively correlated to known climatic change. For example, closed basin lake sediments, available from the Great Basin, do not show significant correlation with the glacial record. This is further complicated by insufficient research devoted to the response of arid regions to glacial cycles, and the role of desert regions in the processes responsible for the climate of the earth.

Sea Level. Changes in sea level have been thought to indicate the volume of the ocean and thereby the volume of the ice caps. The temperature variations, as well as an albedo factor, could be deduced. However, sea level changes are also affected by 10cal crustal uplift and subsidence. In addition, the current sea level was reached 7,000 years ago, and data reflects a slow continuous increase during the last 7,000 years; this may result from the resolution. One suggestion is that the West Antartic ice sheet is unstable and has been disintegrating during the entire interval in question (GARP 1975). In any case, data is unusabie for a 6,000-year period.

Sunspot Frequencies. Althougn admittedly not an accurate representation of solar energy variation, it is at least a partial indicator of the energy input to the climatic system and is expected to exhibit correlation with other indicators. Schove estimated the relative magnitudes of sunspot maxima since 649 $B C$ (Lamb 1972). His data was then averaged for 100-year periods and entered at the beginning of each century.

Tree Rinos. "Biological evidence, multivariate analysis based on meteorological records, and comparison with other evidence for long-term temperature 
variations in the Northern Hemisphere all indicate that tree growth trends in this environment are positively related to temperature" (LaMarche and Fritts 1971). Data was derived from a graph reflecting mean ring widths at the upper treeline in White Mountains, California, from 3550 BC to AD 1950, averaged for successive 100-year intervals and centered on the first year of each century. This particular sample was selected due to the exhibited sensitivity to temperatures that any form of biota displays at its zonal boundary.

There are several periods, a century or more in length, during which departures from normal growth tended to persist at the site. These have been interpreted as indicating anomalies in temperature and precipitation. Conditions of high precipitation and low temperatures are inferred from positive growth departures and are referred to as moist climates. Conditions of low precipitation and high temperatures are inferred from negative growth departures and are referred to as dry climates.

Greenland Ice Core. Polar glacjers contain paleoclimatic information in the form of varying isotopic composition of the ice, specifically $0^{2 B}$. During the precipitation process, the heavy isotopic component condenses preferentially as the cooling proceeds. Consequently, at a given location snow and ice deposited in summers or in warm climatic periods have a higher concentration than snow and ice deposited in winters or in cold climatic periods. On this basis, continuous profiles along ice cores from the polar ice sheets have been interpreted in terms of seasonal or climatic variations.

For this study, data from the Byrd Station and Camp Century, Greenland Cores were utilized. Most if not all of the major climatic changes occuring within the past 100,000 years have been recognized as variations in the Camp Century Core. Johnsen, Dansgaard and Clausen (1972) suggest that climatic oscillations. appear to be caused by varying solar activity.

Milankovitch Radiation Curve. In the 19305, Milankovitch developed a radiation curve on the basis of astronomical theory at that time. The basic concept is one of constant total solar radiation with variations in time of the latitudinal and seasonal distributions. Utilizing the summer and winter seasons as indicators, his formulation has been alternately debated and lauded. G.J. Kukla (1975) investigated the summer-winter curves developed by Milankovitch relating to the earth's orbital fluctuations. He felt the theory was valid, but the sensitive zone and season had not been correctly identified. In his interaction model of $Q$, ground level irradiation; $T$, surface air temperature area of land with temperature below $0^{\circ} \mathrm{C}$; and $\mathrm{S}$, area of snow and ice cover, a seasonal asymmetry exists in the relationship of $Q$ to $S$. The snowline essentially follows the irradiation drop in autumn but it is substantially delayed behind insolation rise in spring. The greatest sensitivity of $S$ to $Q$ (and thus of climate to irradiation) is reached at the time when the snowline accurately follows the shifting insolation pattern resulting in zero change in $Q$ over the snowline. The conditions observed during the autumn in the interior of the continents of the Northern Hemisphere are closest to this situation, and seem to reflect no time lag. 
Lamb (1972) states that it seems reasonable to conclude that the effects of the orbital changes calculated by Milankovitch (utilizing summer curves) are of the right order of magnitude to cause ice ages and warm epochs, and there is evidence now from radiocarbon dating that the timing indicated for the last glaciation and the warmest epoch since then were about right, if a 5,000 year lag is accepted. Kukla says the correlation can be extended to at least 150,000 years BP simply by using conventional Milankovitch summer or winter half-year curves with timing shifted by 5,000 years. "According to my QTS-1 model, the autumn irradiation controls the global extent of cryosphere and through it the climate" (Kukla 1975:603). The autumn derivation by Kukla was calibrated in 100 -year intervals and included in the data as representative of irradiation at the top of the atmosphere.

Volcanism. Great volcanic eruptions of the explosive type sometimes create world-wide veils of finely divided dust which linger for some years in the high atmosphere. The increased particulate matter in the atmosphere serves to deflect a portion of incoming radiation, resulting in a marked lowering of prevailing temperatures. There is also a noticeable effect in the large-scale mean atmospheric circulation, primarily an enhancement of the strength of westerlies. It has been observed that many of the coldest, wettest summers in Britain (and it has been suggested for eastern North America and Japan as we11) occur in volcanic dust years. The radiation and surface temperature curves seem to indicate that the recovery to normal values takes from 3 to 7 years, depending on the extent of the eruption. Lamb (1975) concludes that cumulative effects at times when volcanic explosions are abnormally frequent can influence the prevailing temperature level and climatic pattern of a century or more.

The longest record currently available of volcanic activity is of the eruptions in Iceland for the past 1000 years (Lamb 1975). Admittedly, a more compre-hensive and longer series is desirable; however, the effect of this variable can be tested for the 1000-year period.

Average Temperatures for Northern Hemisphere. The Global Atmospheric Research Program has prepared a compliation of temperatures for the past 10,000 years utilizing tree rings from the upper treeline (LaMarch 1974), fluctuations in terminal recession rates of glaciers (Denten and Karlen 1973) and pollen shifts (GARP: Vanderhammen 1975). In addition, a record of winter severity in Paris and London for the past 1000 years (Lamb 1969) has been superimposed. Sampling for this project again followed the 100 -year interval model.

$\mathrm{C}^{14}$ Dates for African Lake Volumes. Butzer has assembled $\mathrm{C}^{14}$ dates indicating increased Take volumes and/or stream discharge in the Sahara and East Africa since $5000 \mathrm{BC}$. He interprets the data as reflecting Nile flooding. 100-year estimates were based on the 250-year samples in the data. 


\section{Discussion}

The ultimate goal of this project was to determine the best currently available indicator for past climatic variation. On the assumption that climatic changes are more than just random fluctuations, paleoclimatologists have sought evidence of regularities in records of the earth's climatic history. Many have found what they believe to be firm evidence of order and refer to the chronological patterns as "cycles." However, almost all alleged climatic cycles are questioned as being artifacts of statistical sampling as a result of loss of high-frequency information due to the 7 imited resolving power of indicators.

For example, many aspects of the global ice fluctuations during the last 700,000 years may be summarized in terms of a 100,000-year cycle. Each such period is marked by a gradual transition from a relatively ice-free climate (or interglacial) to a short, intense glacial maxima and followed by an abrupt return to ice-free climate. However, no two such cycles are the same in detail, and should not be construed as indicating strict periodicities in climate (GARP 1975). The cycle is thought to be induced by secular variations of the earth's orbital parameters, which are known to alter the latitudinal pattern of the seasonal and annual solar radiation received at the top of the atmosphere. This data is represented by the Milankovitch curve in the present model.

From 7000 to $5000 \mathrm{BP}$ evidence indicates that the climate was warmer than today with subsequent colder intervais every 2,500 years, with the most recent cold interval 300 years BP (Denton and Karlan 1973). In the current data, this period seems to be reflected in the fluctuations of the Nile River valley, and therefore attention was especially focused on the activity of this indicator.

Since climate has a cumulative response, that is, the current weather sets up a range of possibilities for succeeding manifestations of weather, it seemed logical to remove autocorrelation in order to derive cyclic patterning unaffected by the local conditions which contribute to each indicator.

Autoregressive effects were removed using the transformation:

$$
\text { Where: } \quad \begin{aligned}
Y_{t}= & Y_{t}-\left(c Y_{t-1}\right) \\
Y= & a c \text { imatic indicator } \\
t= & \text { time period } \\
C= & \text { an autoregressive constant determined by } \\
& \text { regressing an indicator against itself } \\
& \text { lagged one time period. }
\end{aligned}
$$

Two regression equations were calculated to determine if there was any coordination among the various indicators. First, Northern Hemisphere temperatures were regressed against tree rings, sunspots, volcanics and the Camp Century and Byrd ice cores. Sunspots, the Camp Century ice core and volcanics appear to respond to Northern Hemisphere temperature. 
In the second equation a construed variable called "cycles" was regressed against the same variables. Cycles is the Milankovitch curve for the last 6,000 years added to the variation in Nile flooding taken from Butzer. The record of Lake Volumes was used because it clearly represents a 2,500-year cycle.

Cycles showed relationships with Camp Century ice cores, tree rings and sunspots. At the preliminary level of this analysis, then, the high resolution indicator, Northern Hemisphere temperature, seems to relate to sunspots while the longer term "cycles" variable shows more response to the Camp Century ice core and tree rings. However, statistical significance scores were low in both cases. It may be that when autocorrelation is removed, the trend in the data is lost and comparability is lessened. This may not be in conflict with the data which, as representative of climate, is expected to be nonlinear and cumulative in effect.

Another method, spectral analysis, was applied to determine what periodicities appeared in the data. A uniform distribution of variance as a function of frequency (or "white noise") would imply a lack of predictability in the statistical sense or a lack of "memory" of prior climatic states. A "red-noise" spectrum, on the other hand, in which the variance decreases with increasing frequency, implies that some portion of the climatic system retains a "memory" of prior states (GARP 1975). In view of the relatively short memory of the atmosphere, it seems likely that this is provided by the oceans on time scales of years to centuries and by the world's major ice sheets on longer time scales. The results of this calculation were not statistically significant, but did illustrate the expected cycle for Nile Floods.

Analysis is admittedly inconclusive at this point, but seems to demonstrate that the Northern Hemisphere temperature may be the most reliable indicator for use in climatic testing and simulation. The data is well behaved and effects are observable. Eventually the combined "cycles" indicator may prove to be more accurate, but not enough is known at the present time to accept results based on its use.

For the ultimate purpose of localizing the global climate indicator, there may be a difficulty within the data concerning the period interval of 100 years. The period size should be large enough to capture the global character of climate, and at the same time be small enough to enable transformation to a specific area with prescribed localized conditions. In order to accomplish the second goal, coding the period may have to be adjusted to 50- or even 20-year intervals. This would be possible with most of the currently selected indicators, but would require acquisition of data from the primary sources, rather than published results of studies. 


\subsection{ATMOSPHERE AND MODERN CLIMATE (Sarah Bielser and Joel Gunn)}

Because of accurate recording of climatic variables such as temperature and precipitation over wide areas of the earth's surface for more than a century, short-term patterned changes in global climate can now be studied. Although archaeologists such as Reid Boyson have long been involved in climatology (Alexander 1974:92, Matthews 1976) for apparent ecologica1 reasons, only recently has climatic change become a matter of general academic and public interest. Some of the portents of cooler globsi climate are ominous, as the above cited articles indicate and as the wiriter just past bears witness. Locally, the effect of cooler summers in recent years has prompted San Antonio construction companies to install air conditioning systems to meet maximum temperatures $3^{\circ} \mathrm{F}$ less than previously thought necessary (John T. Saunders, personal communication). We hope in the course of the following discussion to discover the essential mechanisms of local climate and how the environment responds to changes in global climatic parameters. Eventually we will apply this knowledge to the interpretation of culture change in Central Texas and at the Hop Hill locality.

Most of the recent discussion of climatic change centers around the fact that the average annual temperature of the Northern Hemisphere rose steadily from about 1880 until the early 1940s. Since that time it has declined more or less steadily (National Academy of Sciences 1975). The rise and fall of global temperature has a noticeable effect on weather systems and it alters the amount of moisture availabie in any given place depending on how the weather systems move and effective moisture is influenced.

Central Texas weather is controlled at the most general level by three sets of factors. The first is monsoon air circulation between the sea and land; the second is convection cells which form over the land in summer; and the third is the interaction between a complex set of forces involving the moist westerlies, mountains to the west of Texas, and cold fronts from the plains.

Monsoon Circulation and Convective Circulation

At an areal level present Hop Hill weather is a part of North American monsoon circulation. The monsoon phenomenon occurs because of exchange of air flow between inland and ocean weather systems. Temperature and high and low pressure areas are tied together by the monsoon effect which in principle produces cool dry winters and warm moist summers.

The monsoon effect (Patterssen 1969) occurs because the conductive canacity of oceans is much greater than that of land masses. Annual variation o: temperatures is much greater over land than over the sea. Ocean water is uniformly heated by relatively constant solar radiation and the circulation of sea currents between equatorial and more northerly regions. Land, on the other hand, is subject to alternate cooling and warming.

During the spring half of the year the land warms much faster than the ocean, creating areas of low air pressure on land and highs at sea. The spring monsoon effect becomes apparent as warm, moist air moves to fill the lows. This results in precipitation. 
Similar conditions also occur in the summer but summer lows tend to decrease in intensity at higher altitudes such as those in the Fredericksburg area. Because of this decrease, summer precipitation as a result of monsoon circulation decreases. Other forces, however, are at work which bring relief to otherwise hot summers. These forces are called "convective currents," and they reflect the effect of temperatures on clouds (Palmer and Newton 1969). The amount of water vapor in the air varies. When there is no condensation the vapor behaves as a gas. The atmosphere, however, holds only a limited amount of water which is directly related to temperature. The higher the temperature, the more water the air can hold without saturation. Supersaturation occurs because of a variety of factors at given temperatures and condensation results.

As is typical of the monsoon effect in the Fredericksburg area, southerly winds near the ground bring moist air from the Gulf of Mexico into the Plateau. Warm, sultry temperatures generally prevail over the land surface, causing heating of an already conditionally unstable air mass (Orton 1954). As a result, a deep thermal low develops as the air rises in a column over the land. As the air rises it leaves the intensely hot and radiative land surface and encounters the naturally cooler upper reaches of the atmosphere. A high-pressure cell forms above the surface low. This termally produced pressure difference forces air outward at high levels. Simultaneously, the surface air converges on the low cell near the ground. As supersaturated air is pulled into the circulation, it cools near the top of the column and quickly returns to earth as thunderstorms and rain showers. These thermally engendered showers cool the land surface temporarily, moderating the summer heat and reducing the radiant effect of the land surface.

In the fall half of the year the land cools faster than the ocean, creating highs on land and lows at sea. Consequently, the monsoon air circulation reverses its course and moves toward the sea. The temperature difference between $l$ and and sea is even greater in the winter and this produces its own special effects. During winter there is intensive radiative cooling, especially at plateau levels. Cooling extracts heat from the overlying air which contracts verticaliy. Surfaces of constant pressure bend downward over the plateau. A continual high pressure center develops at the surface, above which hovers a low. Land and sea air circulation is reversed. Low level divergence results in an outward flow of air, sloping down to the sea. Upper level convergence of surrounding air masses replaces what was drawn downward, maintaining the circulation. The influx of high-level warm air will insure stability; hence, no condensation or rain will be associated with winter. Monsoon circulation usually reverses about every six months.

Moist Westerlies

Overlying and sometimes disrupting the circulation systems is the behavior of the moist westerlies which are moved about by several complex forces. The weather systems of the Western Hemisphere have been found to move northsouth in concordance. A study by Sanchez and Kutzbach (1974) shows that the general trend is for weather systems to be displaced southward by cooler winters. They noted that the decade of 1961-1970 was measurably cooler on the average than the period 1930-1960. Contoured temperature-change maps 
show that the Texas area was about $.5^{\circ} \mathrm{C}$ cooler during that period on an annual average and about 1.0 to $1.5^{\circ} \mathrm{C}$ cooler for mean January temperatures. The effect on precipitation in Texas was to depress it about $10 \%$ on an annual average. The January average, however, leaps up as much as $50 \%$.

Since the only reporting station in Texas appears to be Dallas, these figures are extrapolated from New Mexico, New Orleans, Dallas and a few surrounding stations. While the data may be generally correct, then, local precipitation studies could be of profit to local archaeological interests. Relative co weather trends, 1976-77 has been reported by the news media to be the raivest winter in 200 years and could be taken as a guide to the general effects of global cooling. A generalized analys is of the 1975-77 winter is not yet available but the period of instrumental observation is regarded to date from about 1880 . The coldest winter shown on graphs of annual mean temperatures for the Northern Hemisphere is the winter of 1884-85, during which temperatures were close to those of the bulk of the Little Ice Age (National Academy of Sciences 1975:148).

The effect of the 1976-77 winter on the moisture regime of South Central Texas was spectacular. According to Highway Department officials, lower temperatures reduced evaporation causing the countryside to become waterlogged. Among the results was a far more than normal disruption of asphalt paved roads. A rancher in South Texas (John T. Saunders, personal communication) reports that his ranch north of Laredo, which normally receives 18 inches of rain a year, was inundated with 50 inches of rain between July and December. Men who had hunted uninterrupted in South Texas for a quarter of a century found the area so sodden as to preclude entry by wheeled vehicle. (One who tried lost a carry-all and was unable to retrieve it for several weeks.)

Evidence of an eyewitness to the Little Ice Age corroborates these observations and suggests that the climate of South Texas was even harsher in the Little Ice Age. Jose Enrique de la Pena traveled with Santa Anna's army to the Alamo in the winter of 1836 (de la Pena 1975:26-37). His unit left Monclova, Mexico on February 13, 1836, and arrived in San Antonio on March 3, 1836. On the night after departure they were beset by "agonizing cold." At dawn the snow was knee deep. "The trees, totally covered, formed an amazing variety of cones and pyramids, which seemed to be made of alabaster" (de la Pena 1975:27). By eight o'clock the snow was turning to slush, adding to the misery of an army totally unprepared to cope with the rigors of these northern latitudes, which de la Pena compared to Napoleon's march into Russia. Over the course of the next few days de la Peria repeatedly complained against the rains and the sodden ground which slowed his progress first to Guerrero and then to San Antonio. On the 21 st near Guerrero they were struck by rain, hail, liginining and winds so strong as to make progress difficult.

On the 29th of February de la Pena came to the Frio River and he remarked that it was true to its name. Once again they were struck by bitter cold. They spent the night in the riverbed which provided some protection from the wind but not from the accompanying snow. In the morning they could not write messages because the ink was frozen. The next night a soldier died from the excessive cold. The story ended the following day as de la Pena entered San Antonio with his contingent of troops. 
While the excessive amounts of rain are suggestive in themselves, two snowstorms in two weeks is as many as present residents of the Rio Grande Plain see in a generation. Weather records from the Houston area (Hole and Wilkinson 1973:21-25) indicate expectably less frequent snow on the coast since the turn of the century.

There seems to be little agreement among weather scientists as to the effect the westerlies have on precipitation in Texas. We will assume as a working hypothesis, however, that the westerlies move south during colder winters in concordance with the jet stream and other weather systems. In warm years the westerlies do not seem to have a great deal of effect on precipitation as they cross Central Texas. The Sierra Nevadas of California comb the moisture out of air masses coming off the Pacific, resulting in the well-watered Sacramento Valley of California and the deserts to the east. There are no such high mountains in northern Mexico west of South Texas, however, so when the westerlies shift south during cold winters, they bring quantities of moisture across to South Texas. When the moist Pacific air masses collide with cold fronts off the plains the effect is to raise the precipitation of South Texas nearly an order of magnitude.

Fredericksburg Climate

Purely local conditions within the context of the larger circulation and westerly systems also have moderating effects on climate. Typical weather at present in the Fredericksburg-johnson City area is as follows. Temperatures are generally mild since it is far enough south to escape harsh winters and has a rather high elevation which contributes to cooler summers. Prevajling winds are southerly and humidity is relatively consistent during all months (Orton 1960).

If the year's weather were divided into the four conventional seasons, winter would be characterized by mild temperatures interspersed between one or two days of cold weather. Polar air masses traveling at different speeds move into the air from the northwest. These dry air masses are warmed considerably as they move south. If they advance rapidly through the area they disrupt the moist southerly monsoon air flow. These air masses are responsible for winters that are relatively drier than other seasons. If, however, warmed polar air masses move slowly enough into the area to be stalled by the moist southerly flow, precipitation in the form of light rain occurs.

The first months of spring are marked by cold fronts which occur less frequently as thundershower activity increases. From about May ist to the end of September, maritime tropical air from the Gulf of Mexico and southern North Atlantic source regions controls the weather (Orton 1964). This warm moist air is responsible for most of the shower activity since in moving across the hot land it becomes unstable and forms clouds. Summer in Fredericksburg is usually a period of warm days, cool nights and occasional showers.

Early Autumn is also marked by warm days and rather cold nights. Weather disturbances move out of the Gulf of Mexico and into the hill country causing 
increased precipitation. Cold fronts move into the area during October and November, which are usually dry months; Tow wind velocities and polar air masses are responsible for this dry trend. Precipitation is brought about in the Central Texas area by a variety of occurrences, one of the most frequent being cold fronts which are bogged down by southerly flow. Southwesterly winds, which occur mostly in summer, have a low precipitation corralation except when coupled with a northeasterly overrunning situation, which often brings rain (Knowles 1972). In addition to the larger effects engendered by the movement of these weather systems, temperature and precipitation are also affected by inconsistent terrain, vegetation and bodies of water.

Figure 4.01 is an attempt to visually summarize the foregoing concepts. Monsoon and convective circulation systems along with moistened or dried westerlies and winter cold fronts interact to control temperature and precipitation. Drawing from the previous chapter, we assume that each of these parameters reacts in its own way to global temperature changes and these reactions dictate changes in the interactive patterns of the local climatic parameters. The exact character of these changing balances between parameters will be dealt with in later sections.

\section{Fredericksburg Weather Data Analysis}

While the above discussion outlines the broad generalities of climatic behavior

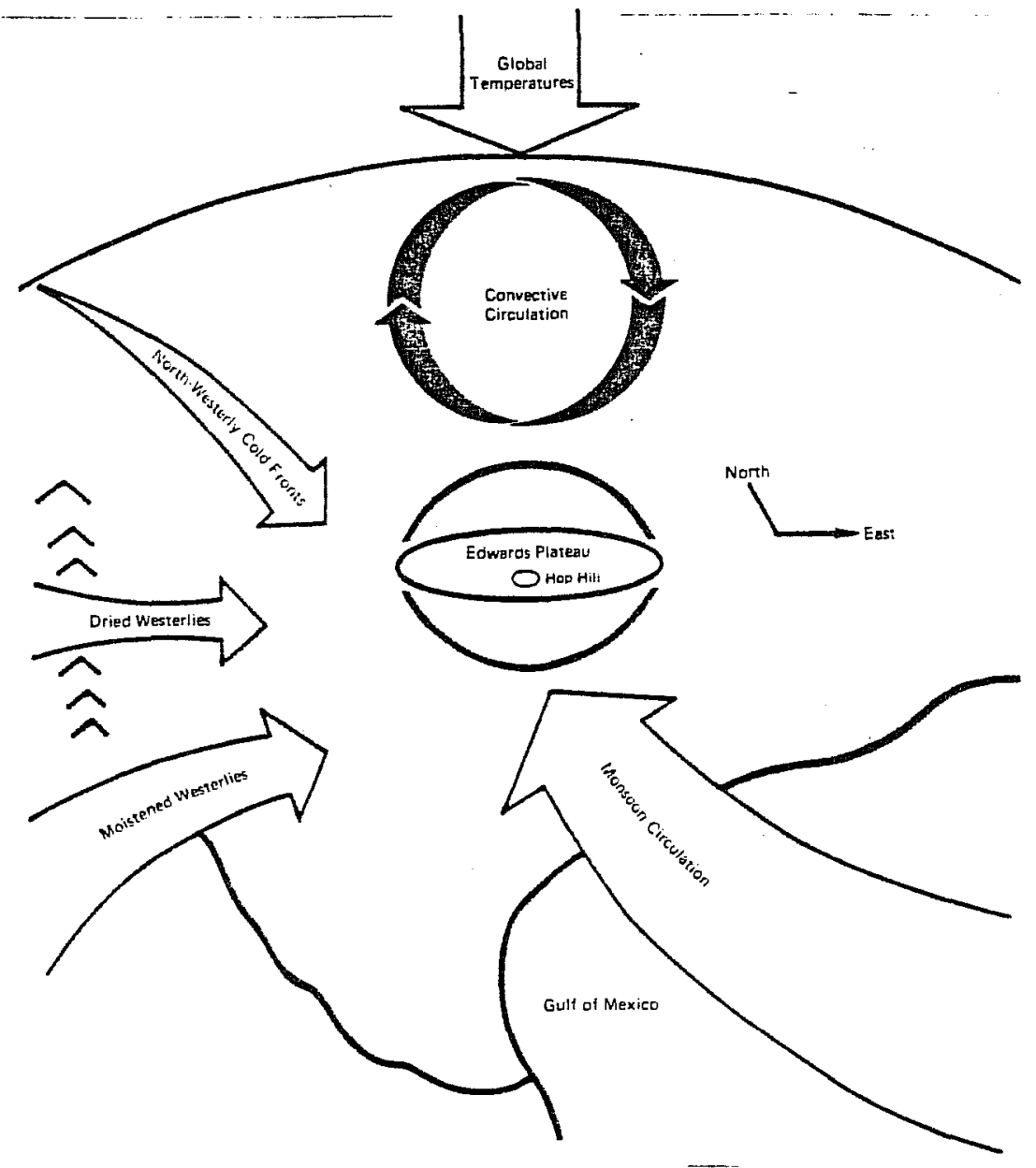

Figure 4.01. Weather Systems Affecting Central Texas Climate. 
in the region around Hop Hill, the finer details can be obtained only from an actual study of the course and character of weather over a period of time. In the following discussion data is collected on a number of climatic parameters and is analyzed for patterns which will ultimately be useful in modeling climatic variation in the Centrai Texas region.

\section{Data Collection}

Data for this project was obtained with some difficulty from a variety of sources. The U.S. Department of Commerce, Environmental Science Services Administration, offers climatological summary information by stations. Systematic recording of rainfall data by early German settlers began in August 1849 in Fredericksburg establishing one of the earliest climatological records in the state; the data between 1849 and 1896, however, was unavailable. Temperature observations were begun in August 1878 and continued with only short gaps until the station was closed in April 1915.

The earliest records were not found except for those of the period 1896 to 1915 , which were obtained from the National Weather Service Bulletin, published monthly by various governmental agencies over the years. A climatological station was reopened in 1939 and records from the National Weather Service

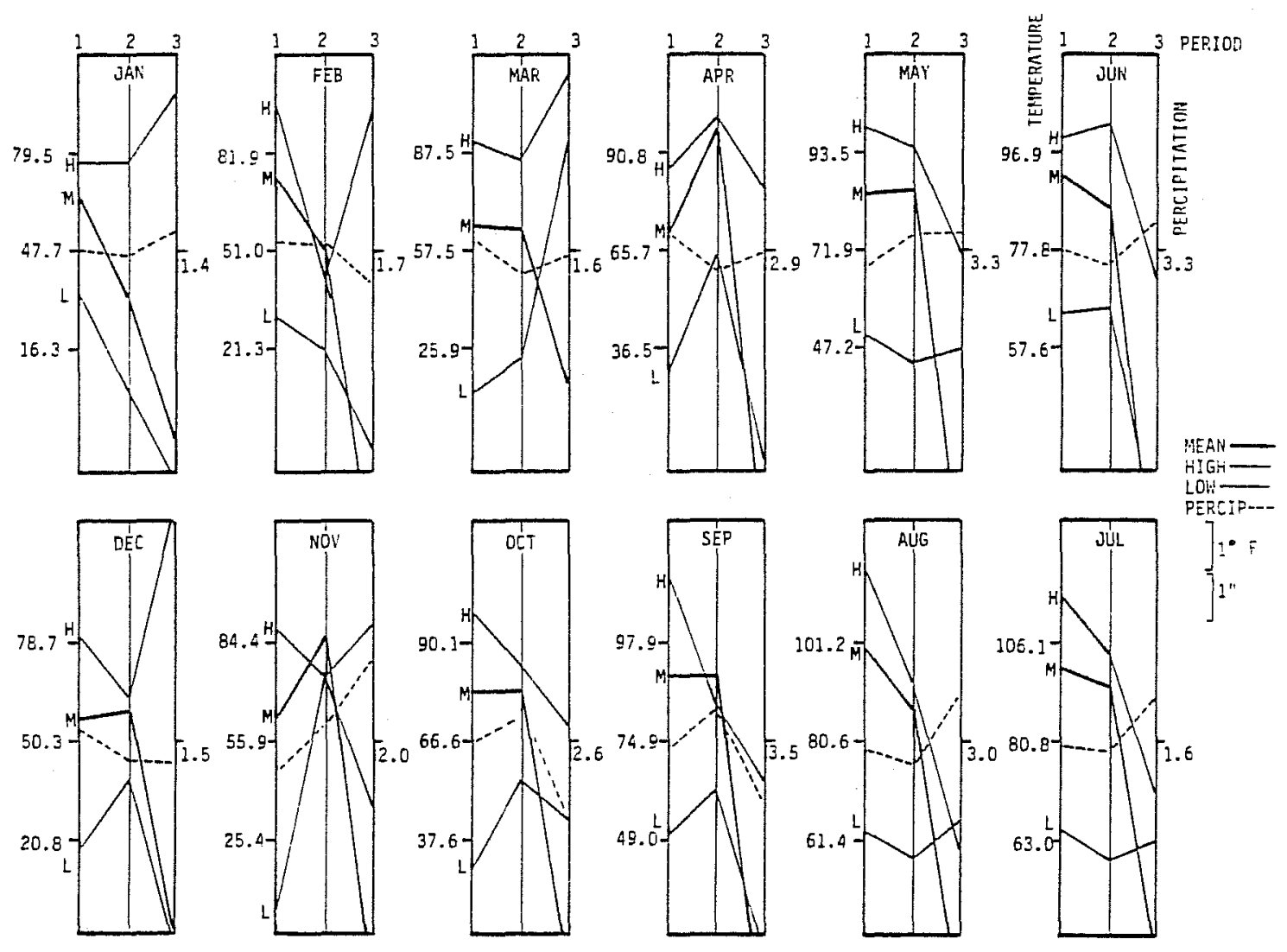

Figure 4.02. Changes in Fredericksburg Climate between Period 1 (1940-1960), Period 2 (1961-1961) and Period 3 (1972-1976). 
resumed in this year. These records were acquired from the Eugene $C$. Barker Texas History Collection Librery in Austin. Only temperatures and rainfal1 data for Fredericksburg were obtained from rainfall readings varying slightly from those in the National Weather Service Bulletin but for a longer time period were obtained from Norman J. Dietel, the current observer appointed June 1, 1954.

Data on yearly Northern Hemispheric mean temperatures was collected from the National Academy of Science report on Understanding Climatic Change. The data obtained from the National Weather Service Bulletin was found among detajled information on cloud cover, prevailing winds, frost, crops and much other valuable information of historical and meteorological value. However, due to the preliminary character of this study, only rainfall and temperature data was used.

Reliability

Early German settlers of Fredericksburg began collecting rainfall records in 1849 and temperatures in 1878. The weather station was closed in 1915 and reopened in 1939. From 1939 to 1975 readings were taken at a climatological station at the local airport by self-recording instruments. The weather station was moved several times but always to within a mile of its original location. Human error may be a problem in some of the earlier data. We found, however, that precipitation records collected manually and automatically correlated relatively well. Occasional correlations were obtained as low as .8.

\section{DEGREES}

- FARENHEIT

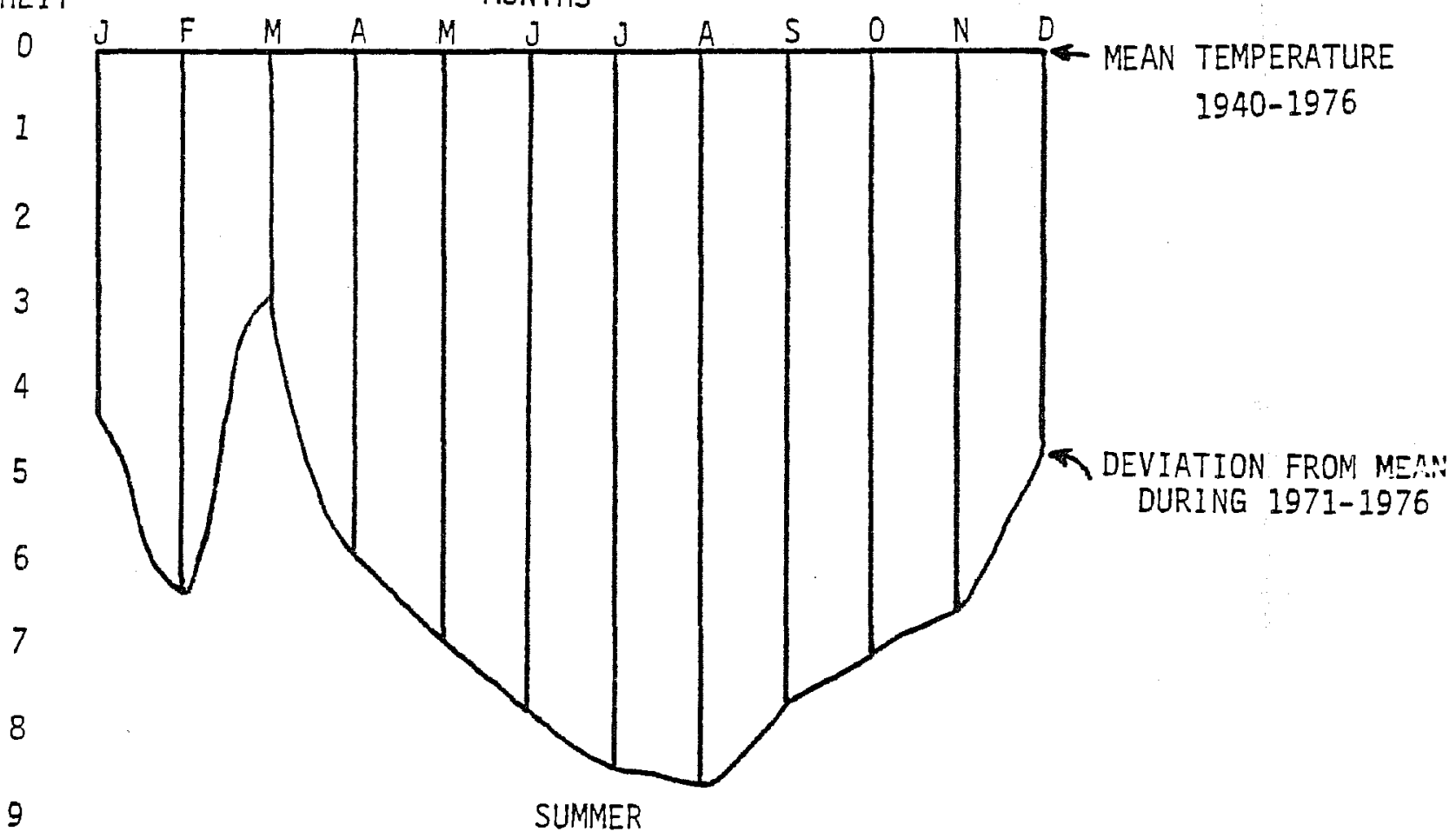

Figure 4.03. Decrease in Mean Temperatures During 1972-1976 from the 1940-1976 Mean. 
Such errors could easily have been a function of station location. In the interest of caution only the data automatically recorded since 1939 are used. Subsequent studies, however, could be profitably extended to the longer period and other variables.

Data Analysis

To stay within the format of the Sanchez and Kutzbach study as much as our data would allow we divided the time series into three periods: 1940-1960, 19611971, 1972-1976. For each of these periods the average high, low and mean temperature, and precipitation were calculated. The results of these calculations are illustrated in Figure 4.02 .

It is apparent that the pattern of the monthly highs, lows, means and precipitation varies on a seasonal basis and that the changes from period to period are related to seasons. The winter months (November-March), for instance, have had increased high temperatures in the most recent period while the summers (Apri1-October) have shown lower highs. Trends in low temperatures have been downward in December-June but have changed little in the warm and declining months (July-November). Without a doubt the most spectacular change has been in mean temperatures. All months have fallen but the summer months have seen means plunging as much as $9^{\circ} \mathrm{F}$. Figure 4.03 illustrates the relative monthly changes in means.

The relationship between precipitation and temperature does not appear to be strong. In general, however, the summer months (May-August) have precipitation in inverse proportion to the temperature while the winter months (SeptemberApri1) have precipitation in direct proportion to temperature changes. Precipitation has decreased in early spring and fall and has increased or stayed about the same in winter and summer.

The general decrease in temperature and particularly the plummeting means in the warmer months probably has the largest effect of any factor on the mositure regime. Cooler weather is accomapnied by a commensurate decrease in evaporation. Effective moisture, then, will have increased regardless of changes in precipitation. Cooler summers probably account for most of the recent trend toward greener summers in the Fredericksburg area, not to mention a lively and discomforting tick infestation.

If anything can be surmised from Figure 4.02 it is that the relationships between various aspects of the weather and climatic change are quite complex. The interaction of these complex forces is the substance of climatic change over the study period; so there is every reason to think the interactions must be understood before we can appreciate the effect that global climatic change has on the biota on which prehistoric people subsisted. Figure 4.04 illustrates an attempt to reduce the complexity of climatic change to manageable proportions. A principal components analysis was performed on the Fredericksburg weather data (autocorrelation removed before analysis). The five circles represent five sets of climatic variables which behave together. In a sense they are like five seasons of the year. There is, however, more information in each circle than one could pass on by simply saying "winter is colder." Most of the circles would be more closely approximated by phrases such as "spring and fall are alike" or "winter and summer are different." 

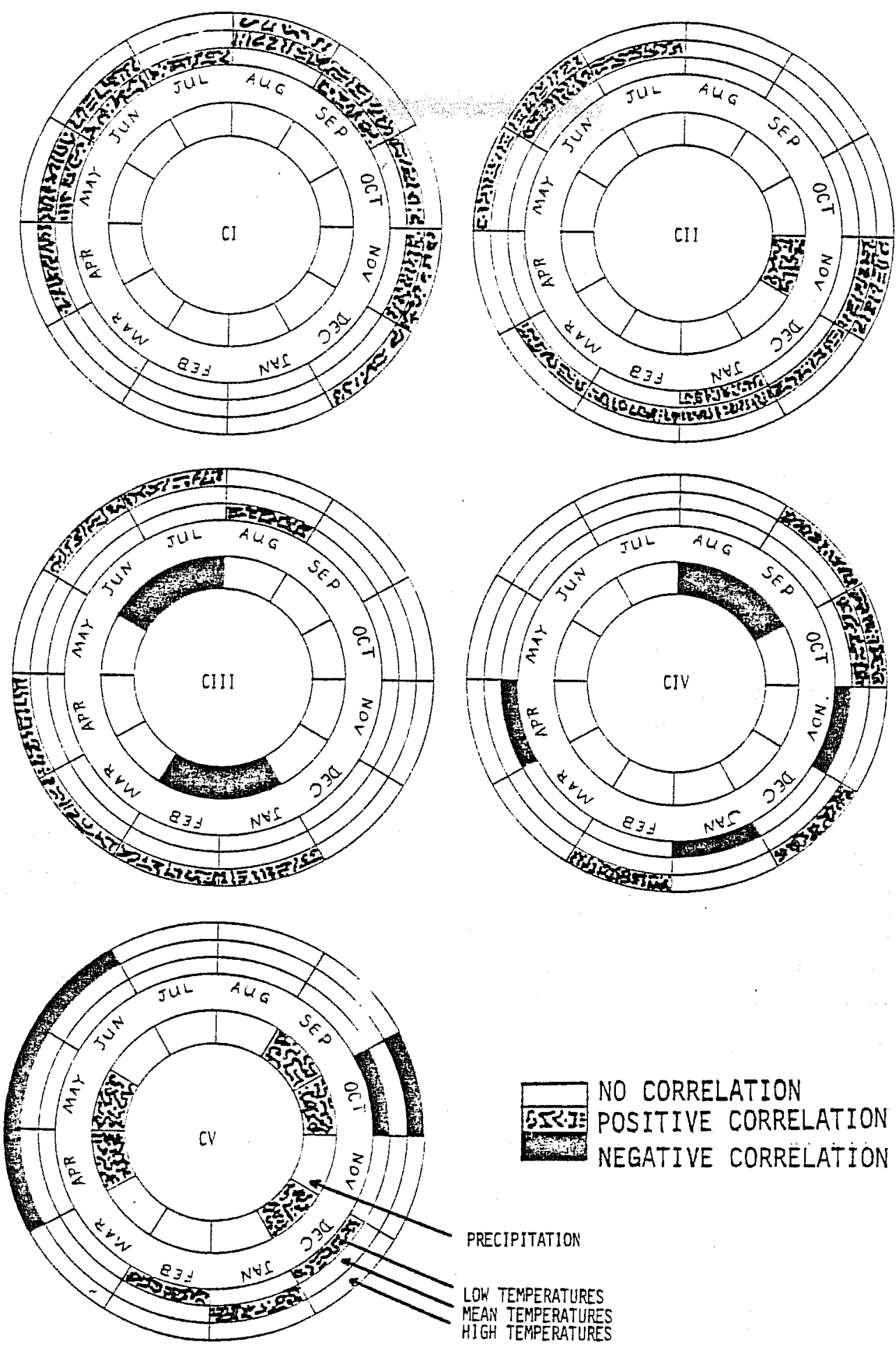

Figure 4.04. The Five Seasons of Fredericksburg Cimatic Change. Center circle is precipitation; outer three circles are low, mean and high temperatures. Textured months are positively correlated; black months are negatively correlated. 
Weather Circles I (Warmer $3 / 4$ Cooling) and II (Summer-Winter cooling)

Since we know temperatures have tended generally downward over the study period we discuss weather changes relative to that downward trend. Weather Circles I and II reflect the effect of downwerd trend in average temperatures in the Northern Hemisphere over the last 37 years. Circle I shows that lows and means during the warmer three-quariers of the year decreased in a correlated manner. The winter months are conspicuously absent from Circle I but appear on circle II as a separate system. Circle II contains elements of winter and summer temperatures which decreased together. Why there are two temperature regimes could be explained in principle by our climatic model. Winter temperature is dominated by northwesterly cold fronts and Arctic air while summer temperature is moderated by factors related to the sea. Beyond that, the research we have done does not allow an intelligent explanation of the cause of disjuncted summer and winter temperature patterns.

A number of hypotheses could be proposed and tested. For instance, are the monsoons losing out in a battle for control of the Plateau to more northerly influences? A study of prevailing wind patterns should answer the question.

Circle II shows that as November becomes colder less precipitation can be expected during that month. It is apparent from the manner in which November is shared between Circles I and II that it is a transitional month. November can be either summer or winter depending on when the transition is made.

In contrast to the fall transition which is variable but orderly enough to be correlated with one or the other, the spring months appear to be a study in confusion. March precipitation, and lows, are so unpredictable they never show up on any of the Circles as an orderly quantity. Both February and April appear to be of predictable behavior though, like November, they go with one season on some circles and with another season on another circle. A study needs to be made to see if this variability is related to changing climate.

Circle V (Spring-Fall)

Circle $V$ is mainly a spring and fall component. It shows that as maximum daily temperatures decrease precipitation increases. December is getting warmer relative to minimum temperatures and there is more precipitation. Circle $V$ is virtually unrelated to annual changes in global average temperatures.

Circle III (Ascending Half)

Circle III represents the first half of the year when temperatures are warming toward summer. It shows that rainfall increases as high temperatures decline in the later winter to early summer period. The solid black months in the circles are negatively or inversely correlated with the textured months. The changes in Fredericksburg weather represented by Circle III are significantly related to average temperature of the Northern Hemisphere, so global cooling is causing the lowering of maximum daytime temperatures and an increase in precipitation during the cold and early summer months of the year. This precipitation should significantly affect biota since it falls in the early part of the growing season. 


\section{Circle IV (Descending Half)}

Circle IV is predominantly the latter half of the year as temperatures cool toward winter. It is by far the most closely related to annual changes in average temperatures in the Northern Hemisphere. Highest daily temperatures are falling; August and September rain has increased. Notably, January and November lowest daily temperatures have increased as well. This circle as much as any shows that summer and winter are becoming more alike.

Except for that part of the precipitation which serves to recharge ground water, the rainfall represented by this component could serve little purpose since it does not occur during the growing season.

Several conclusions can be drawn from the Fredericksburg weather data. First, the transition from warmer to cooler global mean temperature is reflected in temperature readings at the Fredericksburg station by movement of mean temperatures in the same downward direction. The trend is detectable in all months but its most notable effect is in the summer. The consequence is presumed to be an appreciable increase in effective moisture due to reduced evaporation.

Yearly fluctuations in mean Northern Hemispheric temperatures have marked effects on the latter half of the year. Lowered hemispheric temperatures cause daily highs to be lowered and winter daily lows to be raised, and there is a strong, predictable increase in August and September precipitation. The same effects may occur in the winter-summer season but the relationship is only marginaliy significant.

Since the bulk of the effect that global temperatures have on precipitation occurs after the growing season, it can have little direct effect on biotic communities. The main difference that can be expected from lower temperatures is better retention of moisture due to reduced evaporation, i.e., more "effective" moisture.

Of course, these conclusions hold only so long as temperature trends stay within the range observed within the last 40 years. Should the trend continue to levels below that of any year included in this analys is these conclusions might not hold due to as yet unobserved effects. It seems reasonable to assume that if the trend continues the wet Texan Biotic Zone will expand westward at the expense of drier biotic zones to the west.

The decreasing precipitation in the coldest months indicates that cooling is intensifying the winter monsoon effect in the Fredericksburg area. Des anse of the increased effective moisture, however, there should be no drying in terms of the moisture budget during the winter season.

One other item of information that could be useful should be mentioned at this point. Figure 4.05 is a plot of the residuals from regressing the five components described above against mean Northern Hemispheric temperatures. It is apparent from the plot that the weather has become progressively more unstable from year to year. 


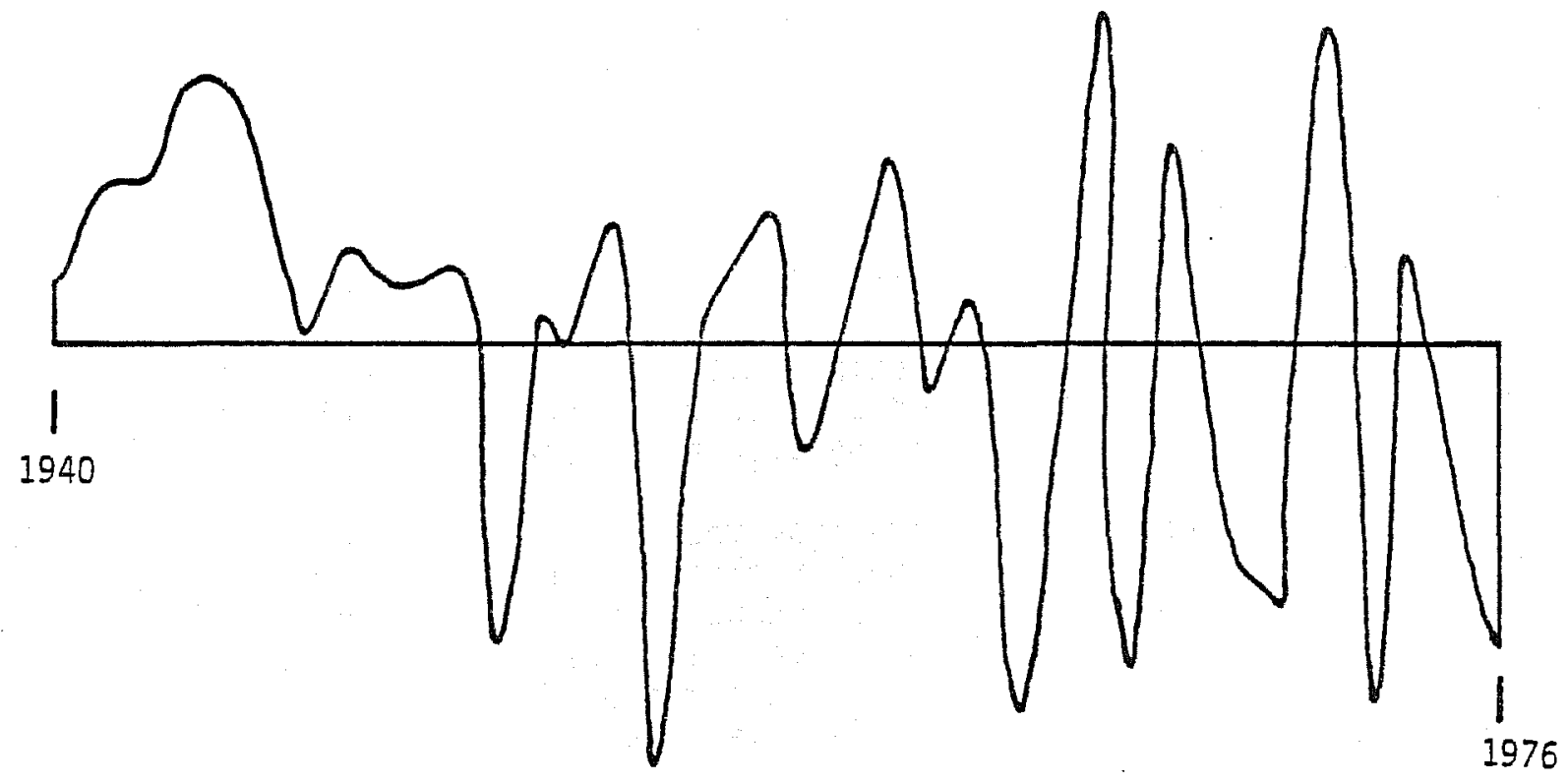

Figure 4.05. Residuals Over the Last 37 years Showing weather Becoming Unstable. 


\subsection{THE GEOSPHERE: PHYSIOGRAPHY, STRATIGRAPHY, AND SOIL ANALYSIS (Royce Mahula)}

Setting the physical parameters which govern excavation strategies are the physiographic boundaries and characteristics of a site. An understanding of the intricacies of these physical configurations which contain archaeological information is of critical importance in effecting a well-informed analysis of cultural remains within.

It is useful to consider the geosphere into which cultural materials have been deposited as an active geosystem--a system of geological elements bound together by sets of interrelationships which exchange matter and energy. These components are abstractions from larger lithological entities such as silt, sand, pebbles, cobbles, etc., to molecular and sub-molecular components. The elements of the geosphere constantly interact with one another through the catalyst components of the hydrosphere and the atmosphere and evolve to create constantly changing landforms and underlying stratigraphic and geologic units. A system state then would represent the unique configuration of these elements existing at a given time; for example, at time of human occupation. Decoding of cultural information stored in this system is aided by a thorough assessment of the state into which cultural information was encoded, through examination of physiography, geomorphology, geology, stratigraphy and chemical ana 1ysis.

Geology and some physiography and geomorphology have been presented in a foregoing section of this report. The following discussion will address zonal, stratigraphic, and some dimensions of chemical structure of the site.

\section{Zonal Structure}

Development of land forms in the Hop Hill locality is a direct function of the evolution of the Pedernales River system. The meandering river has inundated a substantial floodplain which rises rather strongly to a broad upland area. After examination of the site proper, the following zonal definitions were drawn (see Figure 10.02).

Zone 1: Upland. Flat fields to the south of the occupation area. The upland is probably very much as it was prehistorically, uneroded and grass-covered. Cultural materials consist largely of core tools.

Zone 2: Upland Margin. Southern sector of stock pen. Artifacts generally suggest an occupation area (i.e., manos, metates, flakes, boiling stones, etc.). Zone 3: Valley Slope. North sector of stock pen. Great quantities of lithic debris. Quarry-workshop area. Chert nodules, cores, flakes. Area of intensive erosion.

Zone 4: Floodplain. North of stock pen. Deposition of eroded soil from pig pen. Alluvial deposition from waterway. Assorted lithic debris, some cores, nodules. Since fence was built, alluvium has piled up to about $30 \mathrm{~cm}$ along fence.

Extraction of information through excavation and survey operations was governed by this zonal structure. 
Straticraphic Structure

It may be suggested that the natural stratigraphy of unoccupied areas of a site acts as a good reference against which cultural evidence may be more clearly recognized (this may be as readily recognized as an artifact or may be as obscure as altered physical and chemical structure of the soit in an occupation area). In this regard, a general stratigraphic sequence for the site was deduced.

Three soil types were in evidence across the site (Al1ison ct af. 1975:4850), grading from Pedernales (PeB) and a smâ $1 \mathrm{i}$ is land of Purves (PuC) soils in the east along the road, to Hensley (HsB) soils in the midden and village area in the southwest. These soil types are all variations on the same structure, with variations being increased sand in Hensley and increasing depth to bedrock from Hensley to Purves to Pedernales along roughly a southwest-northeast line.

The basic time-stratigraphic definition across the site was as follows:

Stratum 1: The most recent, historical episode. Thus, road building, massive sheet erosion and deposition and fence building activity are all confined to this stratum. Naturally most variant across site. In the roadbed the caliche surfacing of the road is Stratum 1. In general, it is a redeposited, sandy, clay top soil averaging $2-3 \mathrm{~cm}$ in thickness.

Stratum 2: Highly compacted clay loam.

Stratum 3: Red clay--often with sandy pockets and smal1 concentrations of limestone and quartz pebbles. Consistency very sticky and compacted. The red clay is a product of decomposition of highly ferric limestone bedrock. The oxidized iron coats clay-sized particles with its characteristic red color. Stratum 4: Eroded bedrock of limestone and chert with some inclusions of red clay and a fine-grained sand. Stratum 5: Fractured Timestone bedrock.

Stratigraphic Concepts

In order to facilitate excavation and discussion of results certain stratigraphic concepts must be defined. For the purposes of this excavation, the following definitions of units of stratigraphy obtained:

Stratum: A relatively undifferentiated vertical unit. A layer of sediment deposited during a time period which consists of any number of lithological facies.

Substratum: A discrete unit of vertical control within a stratum which may be of physical or cultural significance (i.e., a physical microstratum such as the brown sandy lens in the midden, a culturaily significant microstratum such as an occupation floor, or an arbitrary "level"). Level: For purposes of this excavation of $10-\mathrm{cm}$-thick unit of general control within a stratum which follows natural stratum contours. Levels were used only when unoccupied strata were being tested. 
Substrata Identification System

Each substratum was assigned a number which imnediately associated it with the stratum in which it occurred, established its sequential order in the substrata of that stratum and prescribed whether it is physically or culturally significant. For example, in Substratum 2.15 the 2 denotes Stratum 2 and the 5 identifies it as a physical microstratum occurring between cul tural substrata 2.1 and 2.2. The 1 shows that it occurs between the first (2.1) and second (2.2) culturally relevant substrata sequentially defined in Stratum 2.

\section{Area] Stratigraphy}

In order to more clearly demonstrate the unique physical and artifactual character of each stratum and substratum unit and their temporal and spatial relationships with one another, the following description of stratigraphy is presented in the context of progress of excavation within and throughout each excavation sector of the site. This excavation may be considered in three phases:

Phase I--Roadbed; Phase II--Midden; Phase III--Village.

HOP HILL (4IGL21)

NORTH-SOUTH PROFILE ON THE 1000 METERS EAST LINE FACING EAST

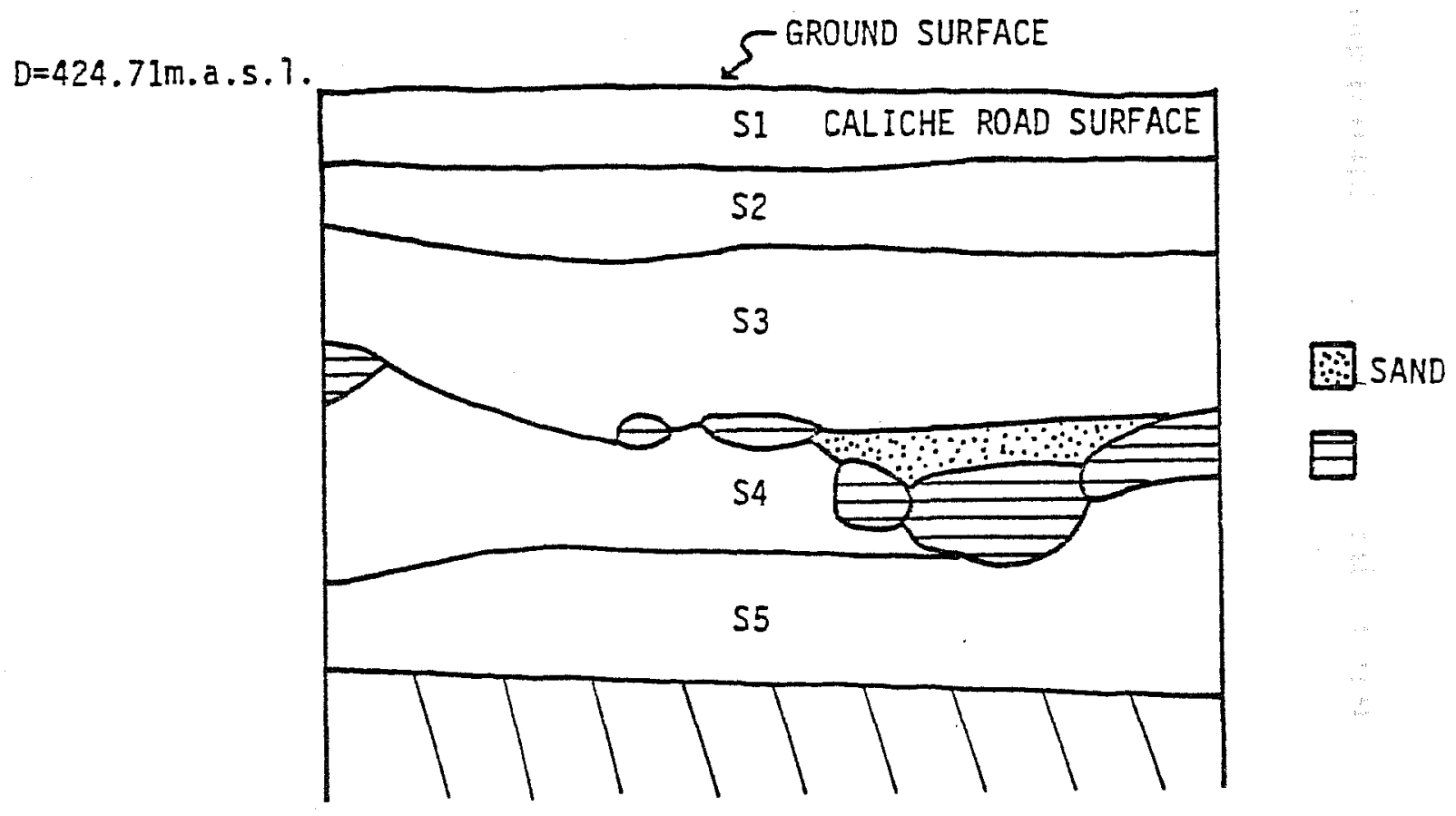

Figure 5.01. Typical Stratigraphic Profile in Road. 


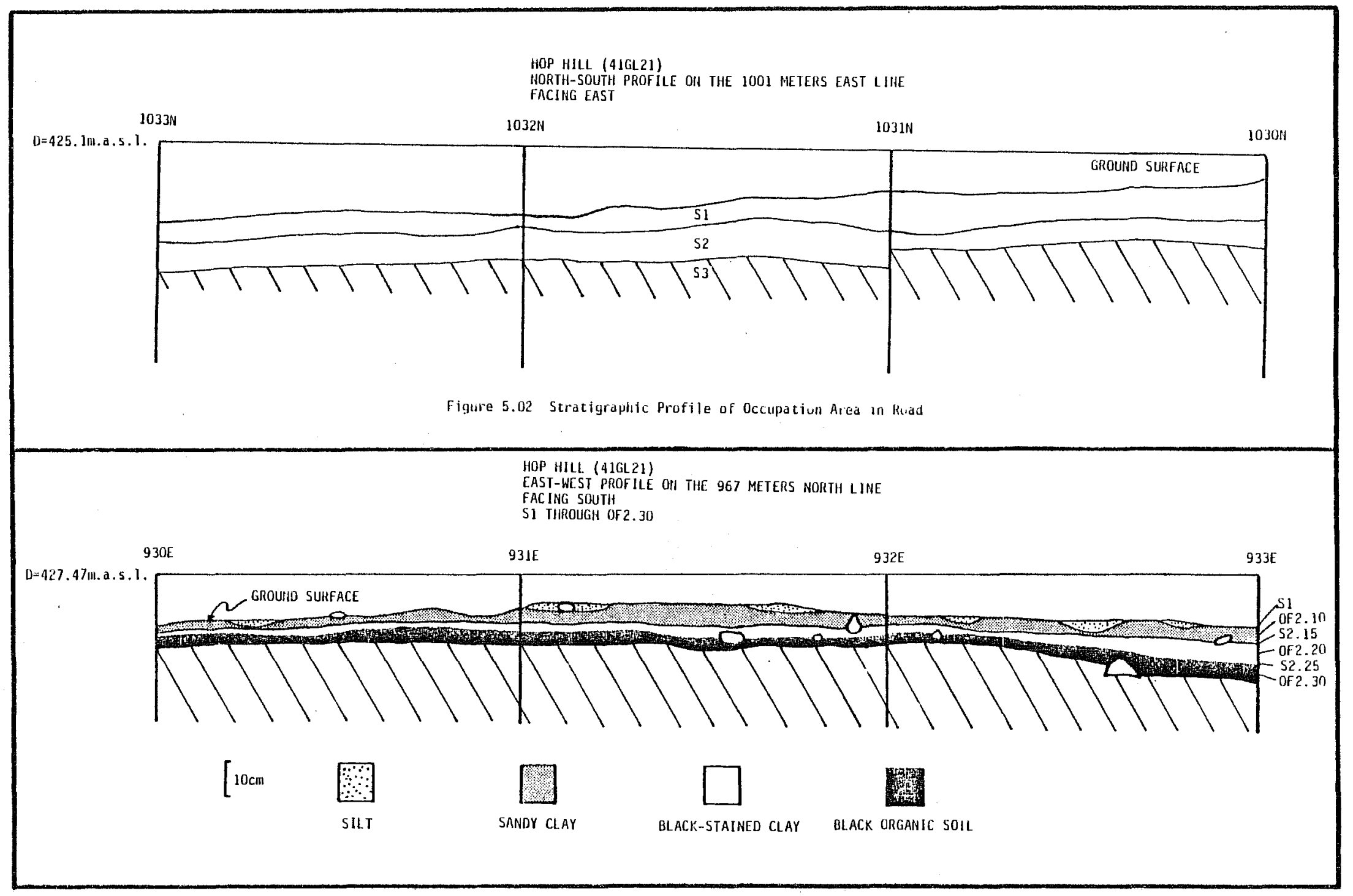

Figure 5.03. Midden Stratigraphic Profile. 
Phase I: The Road

Phase I of actual excavation began with the opening of the roadbed sector. A primary goal in this area was to determine the nature of the stratigraphy which, it was hoped, had been protected from erosional contamination by the presence of the road surface. Excavation units were opened at six-meter intervals down the base line, though modifications to the plan were necessary as new situations developed. During the course of excavation in each unit, the following stratigraphic descriptions and distributions of cultural materials emerged (see figure $5.01)$.

Stratum 0: Surface of road. Few isolated flakes deposited by erosional wash crossing road from adjacent field.

Stratum 1: Caliche road surface material containing pebble-sized pieces of Timestone. No cultural associations within this stratum were evident. Two substrata were identified (both excavated as a single layer).

Substratum 1.1: White crushed limestone, gravel and powdered limestone approximately $4 \mathrm{~cm}$ thick.

Substratum 1.2: Yellowish-brown clay ranging from 1 to 5-1/2 cm thick. Increasing in thickness south to north toward river.

From observations of eroded surfaces to the side of the road, it appears that both substrata were intentional road surfaces placed on top of the clay loam which is Stratum 2.

Stratum 2: Compacted dark clay loam directly underlying the roadbed gravel. By far the preponderance of cultural materials occurring in this sector of excavation occurred within this stratum. Though all levels throughout this stratum evidenced some artifactual material, the horizontal distribution of these artifacts was concentrated in certain units/areas. South of the $1019 \mathrm{~N} 7$ ine deposits contained primarily burned chert nodules, one burin, numerous flakes, pebble-sized quartz river rocks and a fire feature. The fire feature was encountered in the second and third levels of N1013 and N1014 and consisted of a pit-shaped depression filled with a red and yellow ash and various stages of carbonized wood in association with a few flakes. (Detailed description of this feature is included in a later section of this report). El000N1001 evidenced the unique occurrence for this sector of a level of flakes at quite a depth--40 to $50 \mathrm{~cm}$ into Stratum 2. Due to the verticaliy dispersed nature of these flakes no occupation floor was defined. The squares north of the 10197 ine contained what appears to be undisturbed prehistoric material immediately below the caliche roadbed, with the heaviest occupation concentrated between ElO0ON1030 and El000N1036. Two occupation floors were defined for Stratum 2 in this sector, and were exhibited in a 11 units north of the $1026 \mathrm{~N}$ line. (Figure 5.02 is a stratigraphic profile of this occupation area.)

Substratum 2.1: First encountered as a level of charcoal and reddened earth in association with fire-reddened limes tone cobbles, burned chert nodules and potlidded flakes approximately $3-4 \mathrm{~cm}$ into Stratum 2 in unit El000N1032. 
Substratum 2.2: Located just a few centimeters bejow Substratum 2.1 and exhibiting basicalTy the same character-- burned lithics,-reddened earth and charcoal flecks.

After excavation of this and neighboring units N]031 and N1030 a rectangular section composed of a $121 \mathrm{~m}^{2}$ grid units from N1030-N1032 to E997-E1000 was opened to investigate the extent of the occupation. Within this section evidence of occupation was most heavily concentrated along the baseline units and tapered off to nothing along the E9g7 line. No evidence of occupation floors below the first $10 \mathrm{~cm}$ of the stratum was encountered. One other feature was identified in Stratum 2. Within the top $10 \mathrm{~cm}$ a storage feature was exposed in association with a point, a few flakes and some carbonized seeds.

Stratum 3: Red clay with sand pockets and small concentrations of 1 imestone and quartz pebbles. Consistency is very sticky and compacted. (Though buried beneath a layer of roadbed gravel and alluvial soil on the road, this same ciay is highly visible in the stock pen area due west because of extensive erosion. This may be correlated with the clay out of which Plainview material was observed to be weathering near Austin.) Cultural material consisted of a few highly patinated flakes. of questionable cultural origin.

Stratum 4: Eroded bedrock--limestone and chert. Upper $10 \mathrm{~cm}$ of Stratum 4 are a mixture of red clay, caliche and chert fragments. Bottom 2-3 cm contain platy limestone cobbles on the interface. Also some evidence of a lens of coarse sand deposited between platy rocks. No cultural associations.

Stratum 5: Fractured 1imestone bedrock. A trash pit in Zone I dug by the former owner penetrated through the caliche and into a poorly consolidated sandstone-limestone bedrock below.

As pointed out above, beyond Stratum 2 cultural implications are few. Due to this finding, excavation in subsequent stratas was limited to E1000-N1026, N1036 and N1042 where the purpose was to define the stratigraphy. Upon the completion of excavation in these units operations in this sector ceased.

Phase 2: The Midden

The general midden area, located in Zone 2, is characterized on the surface by reddish sandy soil, which contains large quantities of lithics and debris. It is a zone of erosion (upland margin). Previous to abandonment as a hog farm the area was denuded of vegetation, and this resulted in massive sheetwash erosion, probably for a period of about ten years (see Section 6.0). Excavation began with the opening of $41 \mathrm{~m}^{2}$ units (E930-31N970-71) around a sandstone metate which was lying upon the surface. When this investigation encountered dark, greasy ash soil containing burned rocks and lithics in the top few $\mathrm{cm}$ of Stratum 2, eight additional $1 \mathrm{~m}^{2}$ units were opened, expanding this grid sector to a rectangle comprised of units E930-32 to N969-71. No further units were opened in the midden area due to land alteration during the last 10 years. A backhoe trench was dug years previous to the excavation just west of the excavation by the owner. To the east trees and an erosional incline also would have disturbed data. Stratigraphic descriptions (see Figure 5.02 for stratigraphic profiles) and distributions of artifacts in the midden area were: 
Stratum 0: Surface materials, various scattered artifacts, burned rocks, boiling stones and significant quantities of tiny flakes and flake fragments.

Stratum 1: A veneer of sand-size particles that have washed out of the sandy loam soil of the upland and is in the process of eroding downhill. Stratum 1 was excavated as a single unit, with thickness ranging from 1 to $2-1 / 2 \mathrm{~cm}$. Distribution of cultural material was homogeneous within this stratum throughout the midden area and consisted primarily of small flakes deposited by erosional wash.

Stratum 2: Black greasy ash soil containing limestone flecks, layers of burned rocks and 1 ithics and lenses of light brown sand soil separating various occupation episodes. Due to time limitations the vertical extent of this stratum was not determined in this sector, but within the time available 8 substrata were defined--all occurring in the top $10 \mathrm{~cm}$ of the stratum. Of these 8 substrata, 2 were physical definitions and 6 were cultural. Due to the topographic and erosional variation within the midden sector, the general sequence of substrata, while very pronounced in the majority of units, was interrupted in a few instances. Substratum 2.7 was exposed in only two units (E931N971 and E931N968), one as a result of an exploratory trench and the other due to the shallowness of Stratum 2 in that unit. Substratum 2.5 was fully exposed in one unit only and this as a result of the desire to investigate the sequence of occupation in at least one unit. The goal had been to excavate the entire midden sector to Substratum 2.5, but the time factor could not be overcome. (One soil sample was taken from Substratum 2.5 in another unit also for the purpose of soil, ci4, and microbiota testing.)

The physical substrata are two light brown sandy levels which evidently represent discrete depositional episodes. The occurrence of these substrata in the midden area was indeed fortuitous. Burned rock middens have long been an enigma to Texas archaeologists, being extremely difficult to excavate and interpret. The erosional situation in this case has served to present the excavator with the unique incidence of a stratified burned rock midden, if these brown sandy layers may be viewed as separating discrete occupations. The depth, sterility and relatively undisturbed character of the brown layers in the units in which they do exist would tend to indicate that some time did indeed elapse between these episodes.

Detailed horizontal distributions of artifacts within the following substrata for the midden will not be described here but will be discussed in a separate section of this report on computer analysis of distributional patterns of artifacts within the various sectors/zones of the excavation.

Substratum 2.1: Sandy clay soil stained brownish-black. This substratum lies directly on the surface of Stratum 2, is approximately $2-3 \mathrm{~cm}$ thick, and is characterized by flint chips, flakes and flake concentrations. It is partially eroded.

Substratum 2.15: The first of the light brown sandy layers which separates the first dark black occupation level from the second. Culturally sterile. This substratum, along with the second such layer, is most readily observable in the three westernmost units, though some evidence of them does occur in portions 
of other units, primarily the adjacent ones. The substratum is dispersed unevenly across the units with small pockets and shafts descending into the next substratum, no doubt characteristic of erosional wash and deposition.

Substratum 2.2: Also contained in a black stained clay sand horizon, this substratum with flecks of caliche is approximately $3 \mathrm{~cm}$ thick. It is characterized by a level of burned rocks and small flakes.

Substratum 2.3: Large burned limestone rocks and large chunky flakes contained in a black matrix similar to that of Substrata 2.1 and 2.2.

Substratum 2.35: The second of the brown sand levels, especially evident in E930N969 and E930N971. Culturally sterile.

Substratum 2.4: Very black, highly organic silt size soil approximately $3 \mathrm{~cm}$ thick containing a layer of vertical flakes and chunks with a few burned rocks.

Substratum 2.5: Same very dark, highly organic soil as Substratum 2.4. This occupation floor is represented by a heavy concentration of large burned limestone rocks. As noted before, this substratum was completely excavated only in E931N970, though soil samples were taken from it in E931N970, in the process of which a projectile point was recovered.

Substratum 2.7: This substratum was encountered only in two units and excavated in but one, E931N968, where its shallowness was due to the elevation of the unit and the heavy erosional activity. It is evidenced by quantities of large burned limestone rocks, numerous small flakes and one large biface. (An unconformity exists with overlying occupation floors eroded away.)

As the above sequence of substrata was defined this phase of investigation was completed.

Phase 3: The Village

This $1 \times 1$ meter unit (E925N959) was opened in the exposed red clay of the upland rim to investigate the possible Paleo-Indian cultural associations. The spot for the test unit was chosen on the basis of its position between two erosional channels and proximity to an ant bed which evidenced large numbers of small flakes about its mouth. Strata descriptions and cultural associations are as follows.

Stratum 0: Surface is redeposited sandy wash approximately $3 \mathrm{~mm}$ thick but containing fewer flakes and surface artifacts than occurred in the same stratum in the midden area. Color of surface material in the village area is much redder than in midden zone due to the mix of red sand and clay with the midden soil.

Stratum 1: Red sandy ciay soil containing quantities of charcoal flecks and small flakes, averaging 3-4 cm thick. No substrata were identified. 
Stratum 2: Similar in composition to Stratum 1 but much more compacted and of slightiy redder coloration containing similar amounts of lithics and charcoal flects. Small inclusions of the underlying gummy red clay of Stratum 3 are in evidence. Two probable substrata (2.7 and 2.2) were later noted but during excavation were removed without definition. A third substratum (2.3) was defined and was the interface between the sand of Stratum 2 and the red clay of Stratum 3. This substratum contained inclusions of red clay, flecks of charcoal and quantities of lithics.

Stratum 3: Thick gummy red clay. Complete excavation of this stratum was not accomplished due to time limitations but one substratum was encountered:

Substratum 3.1: A level of sma 11 flakes and chips which appear to represent an occupation floor occurring within the first $2-3 \mathrm{~cm}$ of Stratum 3.

Chemical Structure of Hop Hill Soils: An Investigation into Some Cultural Implications of Soil Constituents

Little systematic work has been done in the area of investigating the relationships between soil constituents at archaeological sites beyond the observances of an increased phosphate content and a reduced $\mathrm{pH}$ in areas of human occupation. Though some very complete chemical analyses of the soils in different strata and different areas of sites have been undertaken, (notably Pearson 1974), these analyses have largely ignored investigating the covariance of these constituents. Our purpose in the analysis of the soils from the Hop Hill locality was to pursue these interactions and determine if possible which relationships could be the result of cultural processes and which the result of pedogenesis, and to isolate these associations as possible future indicators of intensity of habitation.

The soil sample collection program has been described elsewhere in this report; but, to reiterate briefly, constant volume samples (2000 cc) were removed from the southwest corner of each substratum unit within each $1 \times 1$ meter square for control and to provide the bas is for postexcavation analyses. Representative samples were drawn from the constant volume specimens removed from the most diagnostic areas of the site. Both horizontal and vertical dimensions of the midden, roadbed and village sectors were sampled as well as control samples from a sterile area away from the site proper and several samples from another occupation/midden site in a nearby locality. (See Table 5.01 for sample descriptions.) These 28 samples were submitted to the Soil Testing Laboratory at Texas A\&M University and were subjected to the standard soil analysis (Agricultural Soil Test Report plus Micronutrients) provided by the Soil Conservation Service at a cost of $\$ 5.00$ per sample. Elements tested for in this analysis included $\mathrm{pH}$ (hydrogen-ion level), Ca (Calcium), Mg (Magnesium), $\mathrm{P}_{2} \mathrm{O}_{2}$ (Phosphorus), $\mathrm{K}_{2} \mathrm{O}$ (Potassium), $\mathrm{Zn}$ ( $\mathrm{inC}$ ), $\mathrm{Fe}$ (Iron), Mn (Manganese), and Na (Sodium). Soil test results of levels for the 28 samples are presented in Table 5.02.

With no more of a model to inform the analysis than that the phosphate content of more heavily occupied areas should be elevated, little of a sophisticated nature could be said about what could be expected of this data. Principal components analysis, which is generally a good exploratory tool, was chosen as a method of taking an initial look at the relationships between the variables. 


\section{Table 5.01 Soil Sample Descriptions}

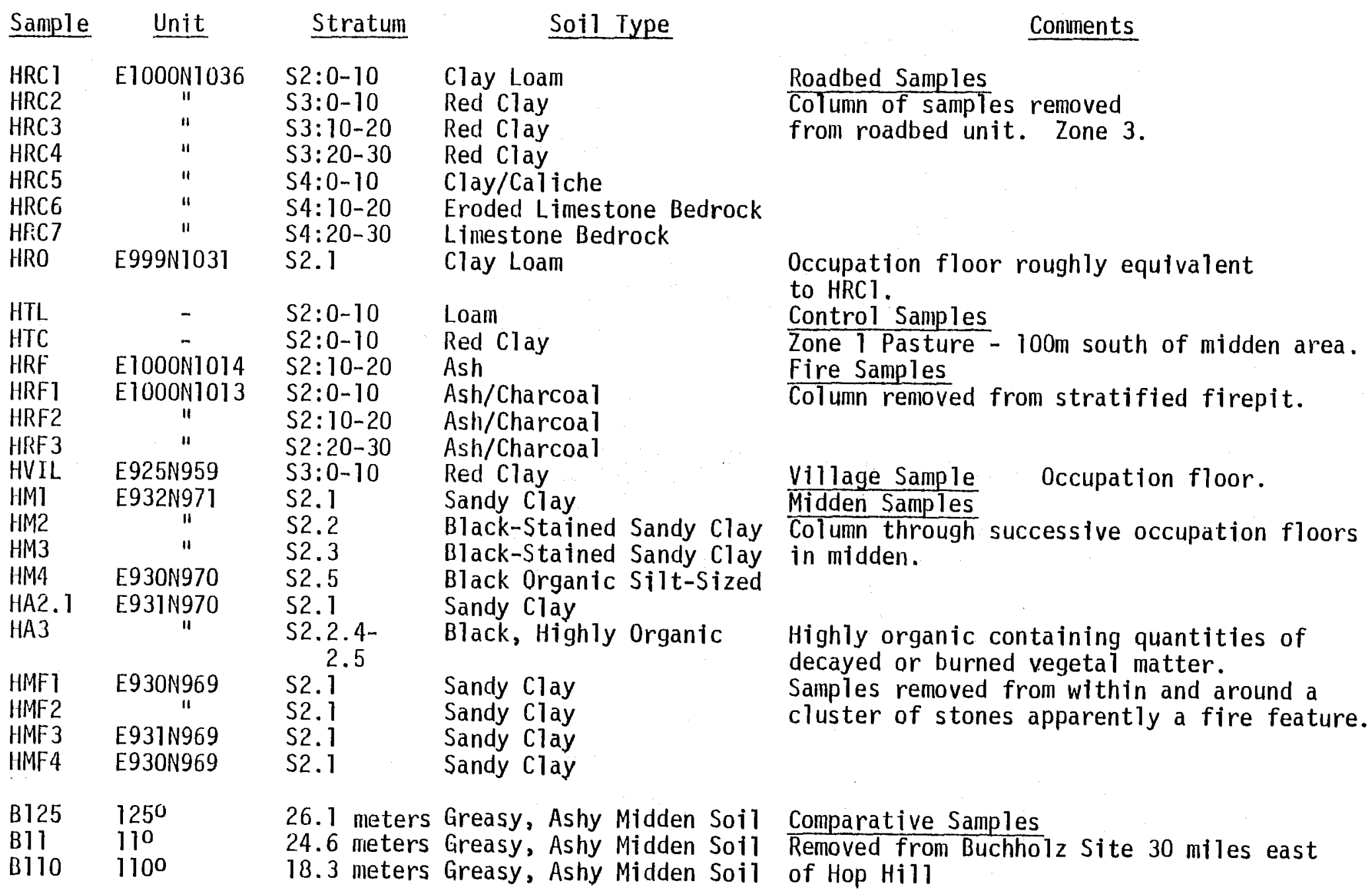

i Dash notation above represents arbitrary $10 \mathrm{~cm}$ levels.

Decimal notation represents culturally relevant substrata. 
Table 5.02 Soil Test Results

Sample $\mathrm{pH} \quad \mathrm{Ca}(\# / \mathrm{A}) \quad \mathrm{Mg}(\# / \mathrm{A}) \quad \mathrm{P}_{2} \mathrm{O}_{2}(\# / \mathrm{A}) \quad \mathrm{K}_{2} \mathrm{O}(\# / \mathrm{A}) \quad \mathrm{Zn}(\mathrm{ppm}) \quad \mathrm{Fe}(\mathrm{ppm})$

$\begin{array}{llll}\text { HRC1 } & \overline{6.6} & >6000 & \overline{350} \\ \text { HRC2 } & 6.7 & >6000 & 450 \\ \text { HRC3 } & 6.5 & >6000 & 475 \\ \text { HRC4 } & 7.3 & >6000 & 500 \\ \text { HRC5 } & 7.5 & >6000 & 310 \\ \text { HRC6 } & 7.9 & >6000 & 320 \\ \text { HRC7 } & 7.5 & >6000 & 325 \\ \text { HR0 } & 7.1 & >6000 & >500 \\ \text { HTL } & 8.2 & >6000 & 285 \\ \text { HTC } & 7.8 & >6000 & 340 \\ \text { HRF } & 7.2 & >6000 & 450 \\ \text { HRF1 } & 7.3 & >6000 & >500 \\ \text { HRF2 } & 7.3 & >6000 & >500 \\ \text { IIRF3 } & 7.0 & >6000 & >500 \\ \text { HVIL } & 7.1 & 5280 & 375\end{array}$

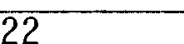

740

770

830

780

330

380

270

$>1200$

870

900

$>1200$

$>1200$

$>1200$

$>1050$

$>1030$

HMI $7.2>6000>500$

HM2 $\quad 7.6>6000 \quad 450$

HM3 $\quad 7.5>6000 \quad 400$

HM4 $7.8>6000 \quad 425$

HA2- $1>.2>6000>500$

$\begin{array}{llll}\mathrm{HA} 3 & 6.9 & >6000 & 425\end{array}$

HMF $\quad 7.2>6000 \quad 475$

$\begin{array}{llll}\text { HMF2 } & 7.5 & >6000 & 490\end{array}$

HMF $37.1>6000470$

HMF4 $7.8>6000 \quad 465$

B125 $7.7>6000>500$

B $11 \quad 8.2>6000>500$

B $110>7.8>6000>500$

$\# / A=$ pounds per acre

$\mathrm{ppm}=$ parts per milliliter
Mn (ppm) Na (hazard or \#/A)

$\overline{.28}-\overline{3.6}$

$.58 \quad 4.0$

$.68 \quad 4.6$

$.16 \quad 3.6$

$.24 \quad 2.2$

$.14 \quad 2.8$

$.12 \quad 2.2$

.50

.52

.22

.34

.30

.24

.16

1.16

$>2.00$

$>2.00$

$>2.00$

$>2.00$

$>2.00$

.64

$>2.00$

$>2.00$

$>2.00$

$>2.00$

.44

.12

.10

5.0

3.2

3.8

5.2

7.4

9.0

8.8

5.0

12.0

17.8

7.0

5.8

15.4

3.8

6.8

7.4

5.8

6.4

2.8

2.0

3.2

$\overline{10.0}$

none

330

5.8 none

8.2 none

7.0 none

5.4 none

4:4 none

10.0 none

10.0 none

7.8 none

$>10.0 \quad 430$

$>10.0$ none

$>10.0$ none

$>10.0$ none

8.8330

$>10.0 \quad 1100$

$>10.0 \quad 1100$

$>10.0$ none

9.9760

8.2 none

$4.8 \quad 1150$

$>10.0$ none

$>10.0 \quad 750$

8.9930

$>10.0$ none

$>10.0$ none

4.2 none

5.0 none

$\mathrm{Na}$ values are reported only when a salinity hazard exists. 
60

$\mathrm{pH}$

MANGANESE

NO MAGNESIUM

\section{PLOT OF COMPONENT SCORES}

- HRC6

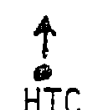

$$
T^{2.0}
$$

- HTL

- HM3

- HRC5

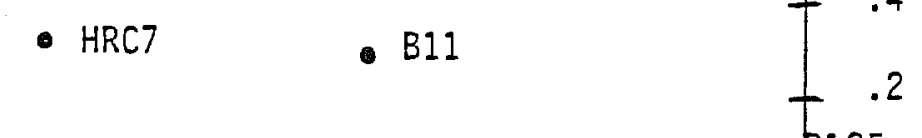

- B11

$+1.6$

1.4

$+1.2$

$-1.0$

8 HM4

.6

.

- HMF4

$\begin{array}{lllllll}-1.6 & -1.4 & -1,2 & -1,0 & -0 & -6 & -6\end{array}$

- B110

- HRCI - HRC4

- HRC2

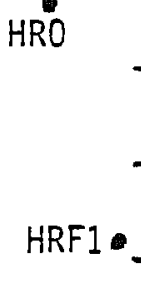

- HRC3

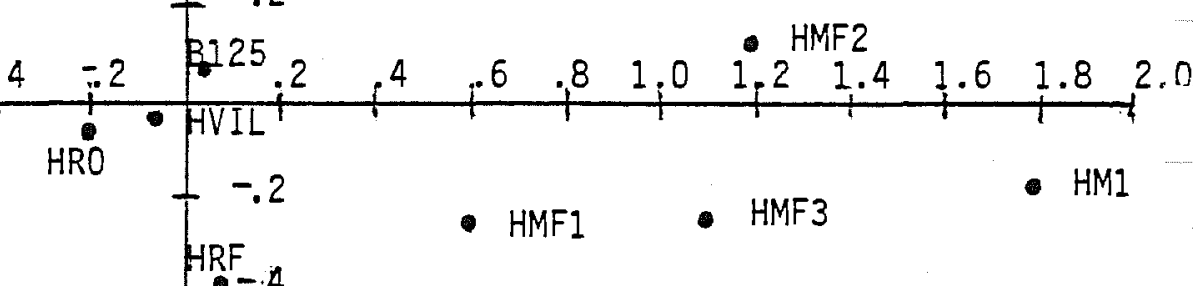

- HA2-1

PHOSPHORUS

POTASSIUM

IRON

SODIUM

MAGNESIUM

Figure 5.03. Principal Components Analysis of Soil Chemical Data: Plot of Component Scores. 
Given the proposition that increased phosphates is an indicator of intensity of occupation, principal components would show what other soil constituents are related to amount of phosphate and which behave independently of phosphate content.

To this end, correlations were calculated on 28 cases for the 9 variables listed above (SPSS, Version 6.6). Three principal axis components with eigenvalues greater than 1.0 were calculated for this matrix and the component matrix was rotated to varimax criteria. Component scores were calculated for each component. The rotated component matrix is displayed in Table 5.03.

Table 5.03 Rotated Component Matrix for Soil Chemical Analysis

\begin{tabular}{|c|c|c|c|}
\hline & I & II & III \\
\hline $\begin{array}{l}\mathrm{PH} \\
\mathrm{Ca} \\
\mathrm{Mg} \\
\mathrm{P}_{2} \mathrm{O}_{2} \\
\mathrm{~K}_{2} \mathrm{O}^{2} \\
\mathrm{Zn} \\
\mathrm{Fe} \\
\mathrm{Mn} \\
\mathrm{Na}\end{array}$ & $\begin{array}{l}-.06 \\
.02 \\
.47 \star \\
.86^{\star} \\
.83^{\star} \\
.08 \\
.79 \star \\
.04 \\
.68 \star\end{array}$ & $\begin{array}{l}.16 \\
.82^{*} \\
.26 \\
-.10 \\
-.15 \\
-.87^{\star} \\
.16 \\
.01 \\
-.02\end{array}$ & $\begin{array}{c}.73^{*} \\
.02 \\
-.58^{*} \\
.08 \\
-.14 \\
-.07 \\
-.12 \\
. .70^{\star} \\
-.03\end{array}$ \\
\hline
\end{tabular}

If phosphates are considered to be a cultural indicator, then the variables loading with phosphate on Component I are presumably related to occupation as we11. The fact that this "cultural component" accounts for some of the variance in magnesium $(.47=22 \%)$ and most of the variance in phosphate $(.83=69 \%)$, iron $(.79=62 \%)$ and sodium $(.68=46 \%)$ indicates that these elements covary with phosphate and thus may be explained to some extent by processes occurring as a result of human habitation.

What processes then could explain the covariance of these elements? Certain answers are more obvious than others. The high loading for potassium may be relatively easily explained by the greater occurrence of wood ash (potash) resulting from the burning of wood and other organic compounds which would naturaliy occur in occupation areas. The high loading for sodium is also fairly easy to foresee. The cultural connotation of sodium content in the soil from an archaeological site may be justified by the increased sodium resulting from the deposition and decay of soft animal tissue, which would also occur in greater abundance in an occupation area where bones still containing flesh and other animal scraps would be discarded about the area. Pearson (1974) also points out that sodium is often related to the presence of potash, especially so in areas of restricted rainfall (which does not, however, apply in this instance). The covariance of magnesium and iron with phosphates at first seemed a little obscure. Interpretation offered by our consultants ( $\mathrm{J}$. Morgan and R. McGehee, Division of Earth and Physical Sciences, UTSA, personal communication) suggests the following explanation. The phosphorus would be deposited in the soil of an occupation area in bone material in the form of $\mathrm{CaPO}_{4}$ (calcium phosphate). 
Within this same soil would be the ready availability of magnesium and iron, which due to their relative insolubility (Mg--.008 per $\mathrm{ml}$, Fe--registered as very slightly soluble) are easily precipitated out of ground water and deposited in the soil. An exchange of ions, replacing the $\mathrm{Ca}$ in $\mathrm{CaPO}_{4}$ with $\mathrm{Mg}$ and $\mathrm{Fe}$ $\left(\mathrm{CaPO}_{4}+\mathrm{Mg}=\mathrm{MgPO}_{4}=\mathrm{Ca}\right.$, and $\left.\mathrm{CaPO}_{4}+\mathrm{Fe}=\mathrm{FePO}_{4}+\mathrm{Ca}\right)$, would result in the new compounds $\mathrm{MgPO}_{4}$ and $\mathrm{FePO}_{4}$ in the soils, and the covariance of $\mathrm{Mg}$ and $\mathrm{Fe}$ with phosphates would be accounted for. Thus, the covariance of these variables can be derived from explained chemical activity generated by cultural refuse and does not contradict the assumption that Component I is culturally derived.

The validity of Component II as an analytical tool in this instance is extremely doubtful. It has no pivotal elements held in common with either of the other components. Also, there is a significant problem with the data. The figures on the calcium content of the samples are invariant with amounts $>6000$ units except in one sample. This invariance results in an ineffective metric for establishing correlations. As a consequence, for this analysis, this component will be discounted. However, the value of this element as a diagnostic implement is evident, especially in an area like Hop Hill where the bedrock is of 1 imestone. In future analyses, therefore, it is suggested that precise $\mathrm{Ca}$ content be explicitly requested.

Component III describes the interaction of the variables $\mathrm{pH}$, magnesium and manganese. This component picks up the rest of the variance in magnesium $(-.58=34 \%)$ and a 11 of the variance in $\mathrm{pH}$ and manganese and indicates that where the $\mathrm{pH}$ is low (acid), magnesium increases while manganese decreases, or where the $\mathrm{pH}$ is high (alkaline), manganese increases and magnesium decreases. These associations, however, are independent of the phosphate component of the data. This independence most possibly represents a chemical reaction occurring during the soil formation process which is related to the increased/decreased solubility of these elements in an acid/alkaline soil.

Examination of the component scores may serve in some way to clarify the situation. Figure 5.04 is a plot of component scores for components I and III. Component I, the phosphate component, is on the horizontal axis; component III, the $\mathrm{pH}$ component, is on the vertical. Plotting of the scores in this manner places each sample in the two-dimensional space defined by the two components. The significance of the relative position of points or clusters of points within this space may then be investigated.

Close scrutiny of the plot leads to several interesting conclusions. The eight roadbed samples cluster distinctly in two dimensions. The upper five samples from Strate 2 and 3 are located further to the right and thus rate higher in phosphate related elements. This may be substantiated by the fact that all cultural materials were retrieved from these strata. In addition to forming two distinct clusters along the horizontal axis, these same samples break into two clusters along the vertical pH component as we11, with the upper strata exhibiting a higher $\mathrm{pH}$ than the lower strata. This clear tendency for strata to group on Component III seems to lend validity to the suggestion that Component III is a soil phenomenon governed by increasing alkalinity caused by proximity to bedrock and is not culturally generated. The location of HTL and HTC seems to substantiate this presumption. The very high $\mathrm{pH}$ score is probably due to the fact that the soil over the caliche bedrock is only a few inches thick. 
One very interesting development is the relative location of the more heavily occupied midden scores. The uppermost occupation floor in the Hop Hill midden is the farthest to the right of any of the samples. The sample we expected to be indicative of the heaviest occupation period thus far excavated at Hop Hi11, HA3.0 (dark greasy ash), is located to the left of other midden samples. In addition, the samples from the Buchholz Site, known to have been intensely occupied, fall even farther to the left. At first glance, there is what appears to be an inverse relationship between phosphate content and intensity of occupation. Since all evidence points to higher phosphate content in areas of heavier human occupation, there must exist another explanation for the observed tendency. The higher phosphate content of the upper 2 midden floors may be accounted for by the fact that the midden is located within what was formerly a stock pen where animal food and wastes were deposited. These organic deposits would have increased the phosphate content of the soils to some undefined depth. One of the characteristics of phosphates which makes them valuable indicators of occupation is their relative immobility. Once deposited they travel little vertically or horizontally (Eidt 1973). The exact depth to which these newer phosphates are present is not clear from this analysis, but probably the lower floors are much less affected, thus falling further to the left on the plot. The intensity of occupation in the midden is still verified, however, by the position of the village sample. This sample also was within the confines of the stock pen, but does not approach the midden scores in phosphate content.

The presence of the Buchholz scores is puzzling when compared with the other occupation samples. It may be noted, however, that there are no control samples from the Buchholz site with which to compare other Buchholz samples. The chemical character of that site could be vastly different from Hop Hill with respect to these elements. This is confirmed when comparing the Buchholz samples with the control samples from Hop Hill. With the exception of B125, which was probably in the most heavily occupied area, the Buchholz samples evidence less phosphates than the control samples from Hop Hill. These findings emphasize the need for control samples from each area/site tested.

One final observation may be made about the component scores of the fire related samples. The fire samples from each area (column from road and sample from interior of fire pit) are located to the right and are thus higher in phosphates than associated material from the same area. Despite the fact that the fire pit was not located within a defined occupation floor, samples from this feature evidence higher phosphate related content than any occupation area in the road. This is possibly best explained by the accelerated release of potassium and phosphates through the burning of wood and bones.

The foregoing stage of the analysis served to define two sets of correlations. One set, the cultural component, consists of elements which are bound by some occupation related processes. The other set could represent a process of soil formation which operates independently of habitation, or a habitation generated condition which operates independently of those processes which encourage phosphates and related elements.

The next logical step in the investigation of the functioning of these processes was to study how they became evident at Hop Hill. In order to gain some insight into horizontal variance of the constituents, East-West and North-South coordinates of the samples were added to the list of variables. Vertical variance was 
likewise investigated through the addition of relative depth as a variable. Besides adding the three new variables, caicium was dropped from the analysis for reasons already explained. Principal components analys is was again applied and correlations were calculated on 28 cases for the 11 varjables. Four principal axis components with eigenvalues greater than 1.0 were extracted for this matrix and the components matrix was rotated to varimax criteria. This rotated component matrix is displayed in Table 5.04 .

Table 5.04 Rotated Component Matrix for Soil Analys is

\begin{tabular}{|c|c|c|c|c|}
\hline & $I$ & II & III & IV \\
\hline $\begin{array}{l}\mathrm{pH} \\
\mathrm{Mg} \\
\mathrm{P}_{2} \mathrm{O}_{2} \\
\mathrm{~K}_{2} \mathrm{O} \\
\mathrm{Zn} \\
\mathrm{Fe} \\
\mathrm{Mn} \\
\mathrm{Na} \\
\text { East } \\
\text { North } \\
\text { Level }\end{array}$ & $\begin{array}{c}-.12 \\
.60 \star \\
.79 * \\
.89 \star \\
.02 \\
.76^{\star} \\
-.05 \\
.83 \star \\
-.66^{\star} \\
-.30 \\
-.91 *\end{array}$ & $\begin{array}{c}-.86^{\star} \\
.40 * \\
-.38^{\star} \\
.15 \\
.03 \\
.10 \\
-.07 \\
-.35^{\star} \\
.60^{\star} \\
.64 * \\
.07\end{array}$ & $\begin{array}{r}-.09 \\
.29 \\
.18 \\
-.20 \\
.00 \\
.03 \\
-.95 * \\
-.11 \\
.12 \\
.67 * \\
.07\end{array}$ & $\begin{array}{l}-.16 \\
-.16 \\
.20 \\
.20 \\
.98 * \\
-.10 \\
-.05 \\
-.48 * \\
-.30 \\
-.10 \\
-.01\end{array}$ \\
\hline
\end{tabular}

As can be seen, the addition of the new variables and deletion of the calcium had little significant effect on the structure of either the phosphate or the pH component.

Component I retains $j$ ts strength in the occupation related variables and picks up even more variance in magnesium (22\% to $37 \%$ ). The significant new information imparted is the high negative loadings for East and Level. This locates higher concentrations of the occupation related elements to the west and shallower. This was, of course, expected. The midden occupations lie to the west of the less intensively occupied floors in the road. The fact that $83 \%$ of the variance in depth is associated with occupation related constituents represents the intensity of occupation of upper strata. In the road this is clear and is supported by artifactual evidence. Below the two occupation floors strata become increasingly sterile to bedrock. The indicated greater intensity of occupation of the upper midden floors is not, however, supported by either artifactual evidence or by the character of the midden soils, and is more probably due to the collection of animal wastes which is masking the true situation.

Component II, the $\mathrm{pH}$ associated component, picks up a strong directional element with high positive loadings for East (.60) and North (.64) and a high negative loading for $\mathrm{pH}$ itself $(-.86)$. This locates a tendency of increasing acidity along a line from the southwest to the northeast sector of the site. In addition, some small percent of variation in magnesium $(40=16 \%)$, phosphate $(-.38=14 \%)$ and sodium $(.35=12 \%)$ is associated with this tendency. This describes a situation of decreasing sodium and phosphate and slightly increasing 
magnesium in the milieu of a more acid soil. Our consultants suggest that the covariance of these elements is the direct result of the relative solubility of these elements in acid.

The association of $\mathrm{pH}$ with areas of increased occupation was one of the relationships which was of interest in this analysis. However, results of this investigation have revealed nothing to clarify this question. As observed, $\mathrm{pH}$ has no variance in common with the phosphate component of occupation related elements. This could mean that the $\mathrm{pH}$ factor acted independently of the processes which produce phosphates but still was related to culturally generated processes. If this were the case, the addition of directional elements would associate the pattern of increasing and decreasing $\mathrm{pH}$ with occupation areas, with lower $\mathrm{pH}$ occurring in the more heavily occupied areas in the southwest and greater $\mathrm{pH}$ occurring in the northeast. This, however, is not what is indicated by Component II. The $\mathrm{pH}$ component of the data must then have to do with soil-forming processes independent of cultural factors. One explanation for the northeast tendency of increasing acidity would be that it is the result of the increasing thickness of the $A$ and $B$ soil horizons to the northeast. $A$ quick referral to the component scores indicates that there is a trend of increasing alkalinity of samples closer to bedrock.

Component III loads heavily for manganese $(-.95=95 \%)$ and North $(.67=37 \%)$ and appears to be merely descriptive of soil conditions with an increase in the amount of manganese from north to south. This condition could be accounted for by the relative age of the soil deposits. Being stream deposits, the age increases from north to south. Older deposits could have amassed a higher manganese content.

Component IV represents an inverse relationship between zinc (.98) and sodium $(-.48)$. This correlation is a puzzling one. Our consultants were unable to suggest any inherent property in either sodium or zinc which would cause this negative variation. These two constituents are most probably, then, two dependent variables in a system, the causal element of which is undefined within the scope of the elements tested for by this standard analysis. One avenue of future research in this area would be to define a causal factor for an inverse sodium-zinc relationship and determine whether it has any cultural connotations. This possibility exists as sodium is a culturally related element. Other promising areas of investigation for future analyses would be levels of soluable salts, carbonates $\left(\mathrm{CO}_{4}\right)$ and nitrates $\left(\mathrm{NO}_{3}\right)$, the latter two of which bond with sodium and potassium and might be possible cultural indicators.

In summary then, the analysis of soils from Hop Hill seems to substantiate the proposition that phosphates and perhaps potassium, iron, sodium and magnesium are related to human habitation. Any tendency for decreased $\mathrm{pH}$ in areas of habitation at Hop Hill appears to be overridden by soil formation processes in relation to a limestone bedrock. The fact that low $\mathrm{pH}$ may be overpowered by other geologic factors would indicate caution and thorough knowledge of the geology of a site before attempting pH mapping. Once variant data is obtained for calcium, it will probably covary in some fashion with this component. Sampling in and outside of a firepit appears to indicate that the production of phosphates is related to some degree to fire use, though this relationship is in no way a complete one. For the future, further inquiries of this type, especially those which include investigation into other possible culturally-related ingredients, 
such as soluble salts, carbonates, and nitrates, can do much to refine soil constituent analysis as an interpretive tool in archaeology. 


\subsection{SITE HISTORY, PHYSIOGRAPHY, VEGETATION AND EROSION (David Brown and Clint Davis)}

Introduction

The discovery of the Hop Hill site, as with many other archaeological sites, was due to the exposure of artifacts through the process of soil erosion. While such erosion is a key factor in site discovery, it is equally important in the destruction of many sites. A few years of heavy soil erosion can destroy artifact associations laid down in thousands of years of soil accumulation. Stratigraphic records can vanish completely and horizontal placement can be radicaliy altered through unequal transport of artifacts and associated materials.

The basic processes of land surface change are generally slow, but they are continualiy present and occasionally surprisingly rapid. Archaeologists are usualiy cognizant of these basic geomorphological processes but do not always give adequate consideration to their effects on artifact relationships. Understanding the effects of rapid soil erosion is often crucial to a proper interpretation of the excavation or survey results. In spite of this fact few archaeologists have shown much interest in the phenomenon of soil erosion on archaeological sites.

This paper presents the results of an initial investigation of post-depositional land surface changes at the Hop Hill locality. Rather than attempting to explain artifact location or dislocation, we have been concerned with monitoring the progress of land surface change in the hope of defining areas of the site where such surface change takes place. Determination of these areas should then be useful in the interpretation of the results of the surface survey and in the determination of the locations of future test excavations at the site.

It is obvious that erosion has played a significant role in land form development at the site. Several gullies wind their way across the site; small rocks and artifacts have been left on pedestals as the result of raindrop impact. Tree roots are exposed and fence posts are buried in soil deposits. Even more dramatic evidence was provided by the condition of excavation pits after a few hours of rain--several centimeters of soil appeared on top of the plastic tarps used to cover the excavated squares while in other areas small piles of excavated soil disappeared. In these and other areas of the site, the activity of erosion and deposition is substantial; in other parts of the site activity appears to be negligible. Variability across the site is a question that will be dealt with and described in terms of the amount and kinds of activity that can be determined for specific microenvironments. In addition, certain inferences will be made concerning the dominant processes operating over the entire site.

While this study of land surface change will be directed toward the specific archaeological problems of Hop $\mathrm{Hill}$, the theory and techniques should be of significant interest to other disciplines. The results of this study, while of importance to the interpretation of the archeological evidence at the site, may also serve as a stimulation to future researchers interested in land surface change. Even though our own research is 1 imited in scope, we look 

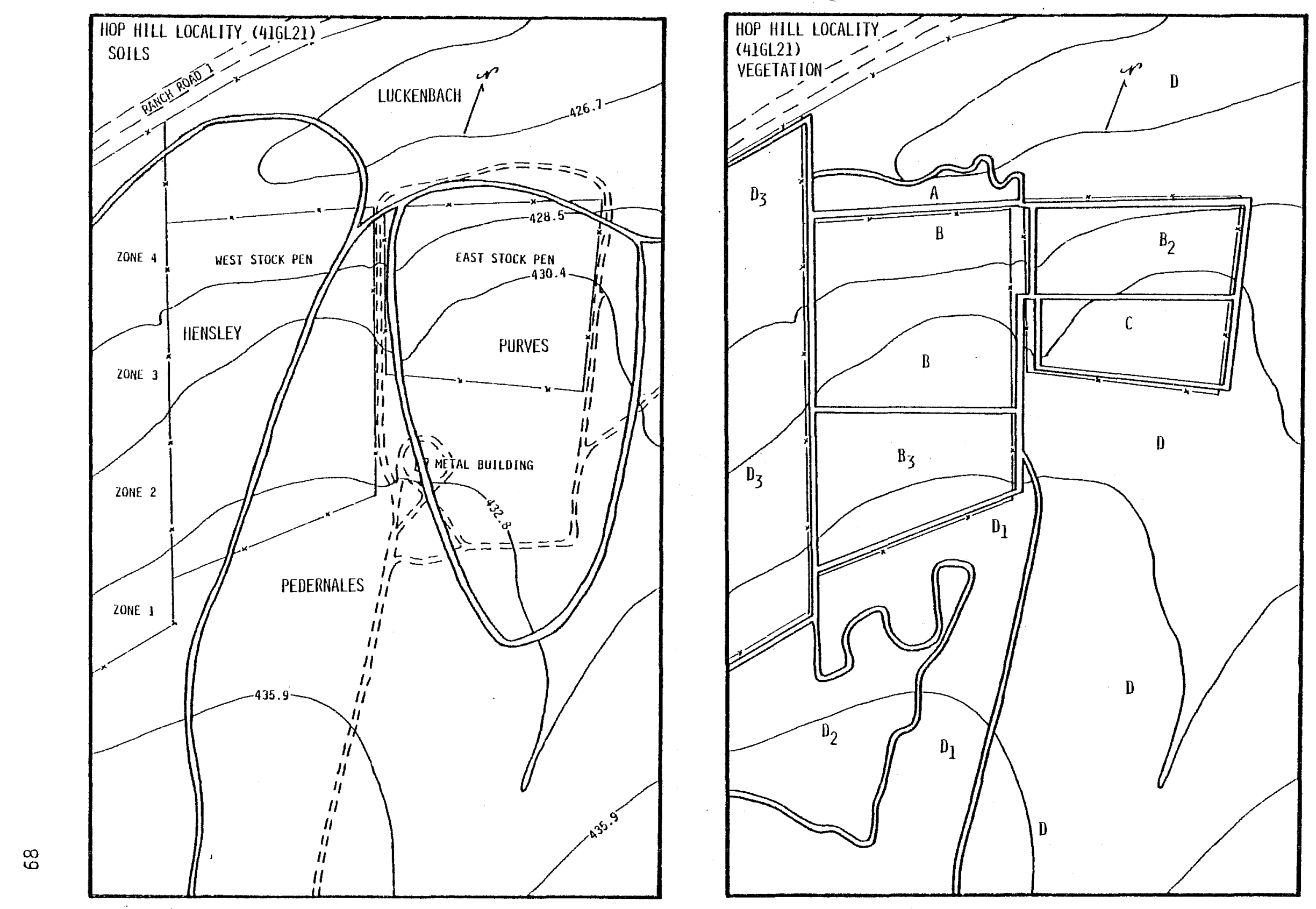

Figure 6.01.1 Soil Types and Slope Zones used to code Enosion Studey Data Points.

Figure 6.02. Vegetation Zones. (See text for explanation). 
forward to the possibility of future work which might enable archaeologists to better describe deposits on the basis of their geomorphological history.

\section{Site Physiography}

This section is concerned with a detailed description of those physical characteristics which we feel to be relevant to land surface change. These include soil types, topography, vegetation, rainfall and flooding.

\section{Soils}

According to the United States Department of Agriculture Soil Conservation Service (Allison et al. 1975), four soil series and accompanying phases are present or bordering on the site (Figure 6.01). These series are as follows: Hensley soils, 1 to $3 \%$ slope (HsB); Luckenbach clay loam, 1 to $3 \%$ slope (LuB); Pedernales fine sandy loam, 1 to $3 \%$ slope $(\mathrm{PeB})$; and Purves soils, undulating (PuC).

Classification by the 7th approximation (described in sequence from order through family) places the Hensley soil in the Alfisol; Lithic Rhodustalfs; clayey. mixed, thermic category. It was in this soil and the Pedernales fine sandy loam (an Alfisol; Udic Paleustalf; fine, mixed, thermic soil) that all of the excavations were carried out. Both soils have a moderate to high erosivity factor, with the Hensley soil having a low degree of permeability and water availability due to the tying up of water molecules in the clay. fraction and the fast drainage of water in the loam fraction of the lower profiles.

A large area of Purves soil is located on the eastern stock pen of the site (Figure 6.01) and was surface collected and surveyed during the excavations. Purves soil is a Mollisol; Lithic Vertic Haplusto 11; clayey, montmorilionitic, thermic. As indicated by the Soil survey, this soil is much more variable in composition while the presence of a montmorillonitic clay fraction makes it highly susceptible to shrink-swell, deep cracking and freeze-thaw characteristics. Because of these characteristics, Purves soils have a tendency toward surface and subsurface mixing while exhibiting a low permeability due to the clay fraction. Runoff would be induced by the low permeability but slowed by the surface cracking which tends to produce a well-drained soil.

The fourth soil, Luckenbach clay loam, borders the north end of the site and is classified as an Alfisol; Udic Haplustalf; fine, mixed, thermic soil. This soil type has a moderate erosional and water availability problem because of the clay fraction which decreases the permeability. Though susceptible to erosion, this soil is only present at the site in an area which is under a full vegetative cover and with almost no slope at all. Additionally, no surface collection was made in this area and no artifactual material was seen.

With the exception of the Purves soil with its montmorillonite clay content and subsequent erosion problems, these soils exhibit essentially the same characteristics in terms of erosivity, permeability, drainage and water 
accessibility. It is to be expected, therefore, that all four soils, with the possible exception of the Purves, will behave somewhat similarly under differing conditions of erosional pressure.

\section{Topography}

The topography of the site is fairly simple (Figure 6.01). The general direction of the slope of the floodplain is to the north toward the Pedernales River. Few of the slopes exceed a ten-percent gradient and most are under five percent. A naturally occurring creek runs north to the river and lies to the east of the site. It is now dammed in two places to form stock ponds and is not active in draining the major portion of the site. A caliche road cuts northward from Highway 290 bisecting the stock pens into eastern and western sections and encircling the eastern pens. Gullies which empty into the lower flat areas to the north of the site have formed on either side of the road. To the west of the site is a terraced field not presently under cultivation. The southernmost terrace of the field cuts across the site to the stock pens and the others drain through the northwestern corner of the site.

Initially the site was divided into four zones based on average overall slope configuration determined during preliminary investigations prior to excavation (Figure 6.01). Zone 1 is the grassy meadow which lies to the south of the stock pens and has slopes of approximately one to three percent. Zone 2 begins at about the line of the terrace south of the pens and extends downslope to ten meters north of the fence between the northwest and southwest pens. The average gradient of Zone 2 appears to be slightly greater than that of Zone 1 with some slopes ranging as high as five percent. Zone 3 is a narrow band of steeper slopes, ten percent or more in places, which extends from Zone 2 north to about twenty meters south of the extreme northern fence line. Zone 4 is a flat area lying at the extreme northern end of the site. Few slopes in this zone exceed three percent and many areas are flat or have a slight slope in the opposite direction.

Vegetation

Vegetation is perhaps the most important variable affecting the erosional process. For this reason it is given a fairly detailed treatment in this section. The natural vegetation of the site is short grass savannah with single trees in the drier areas and stands of trees in the wetter areas, a situation common to the Edwards Plateau. This natural vegetation has been disturbed in several areas through the effects of farming, grazing and road-building. The most notable disturbance occurs inside the stock pens where the grass has been most7y stripped and replaced by annual weeds or is not revegetated at a11. Cleared fields to the west of the stock pens have also been stripped of native grass and trees and then terraced for farming, but are now covered by long-stemmed sprangle grass (Leptochloa dubia) and biue-stem (Bothriochloa sacchroides). From the southwestern stock pens southward to Highway 290 lies a large clearing, either natural or cleared by man.

As with the slopes and the soils, vegetation was divided into zones which seemed to show distinct types. With the help of botanist Ed Zielinski, four 
major zones were recognized, some of which were further divided into subzones. These zones are as follows (Figure 6.02).

Zone $A$ is a twenty-meter wide strip running parallel to the northern border of the western stock pen, containing a dense concentration of Bermuda grass with only a few trees. This area is flat, low-lying and relatively wet, and is bordered on the north by a pocket of scrub thicket which extends almost to Ranch Road 7. Minor vegetation types include barnyard grass (Echinochola crusgal1i), silverleaf nightshade (Solanum eleoginfolium), Johnson grass (Sorghum halapense), giant ragweed (Ambrosia trifidia), Santa Maria feverfew (ITex nisteropherus), cowpen daisies (Verbesina encelioides), Mexican hats (Ratibida columnaris) and horseweed (Clyptocarpus vilalis). There are ten small elm trees (Ulmus crassifolia), three large pecan trees (Carya illinoensis), and one medium-size chinaberry (Melia azedabach).

Zone $B$ covers most of the area in the west and northeast stockpens, consisting mostly of annual weeds in nonhomogeneous clusters separated by patches of bare ground. Major plants include pigweed (Amaranthus retroflexus), Bermuda grass (Cynoden dactylon), and Johnson grass with only a few other unidentified species occurring relatively profusely. Minor plant groups include Santa Maria feverfew, sunflowers, horse nettle, cowpen daisies, snow on the prairie (Emphorbia bicolor), Texas croton (Croton texensis) and Verbena bipinnatisida. In

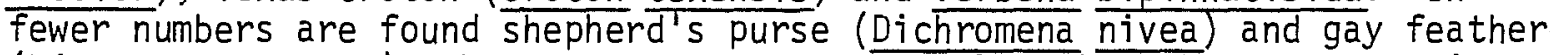
(Ljatrus mucronata). Trees are sparse and mostly oak with a few mesquite and pecan. This zone is differentiated from Zone A largely by its reduced cover, with Zone A having $100 \%$ cover and Zone B having about $60 \%$ cover.

Subzone B-1 differs only slightly from Zone B by its concentration of trees covering the low-lying area of the extreme north central part of the western stock pen. Its dimensions are ten meters by twenty meters, making it a very small part of Zone B, but peculiar not only for its concentration of trees but also for its lack of ground cover due to standing water which collects in the depression:

Subzone B-2, located within the confines of the northeastern stock pen but separated by a gully, is much like Zone B with the exception of having a rockier surface, more trees and several groups of cowpen daisies and sunflowers as a major group. Distribution of Zone B types across this area is less homogeneous.

Subzone B-3, at the southern end of the western stock pens, is an area approximately 30-40 meters wide which follows the southern fence line but stays inside the pen. It contains little vegetation and few trees, possibly due to the presence of numerous ant beds.

Zone $C$ is located in the southeastern stock pen where a concentration of 80$90 \%$ pigweed can be found with some sunflowers, clumps of bermuda grass, cowpen dajsies, horse nettle, Dakota vervene, and oak trees. This area contains a few bare areas as in Zone $B$ but is differentiated by the absence of most of the major vegetation groups of Zone $B$. It is bounded exactly by the south, east and west fences of the southeast pen but is transitional into Zone $B$ along its northern boundary. 
Zone $D$ is a tree-dotted meadow to the south of both stock pens. Its vegetation closely approximates the native vegetation of the area. The major vegetation is grass, predominantly sprangle top, three-awned and buffalo grass; minor vegetation includes Johnson grass, snow on the prairie, Texas croton, Mexican hats, Indian grass, agarita, side oats, silver blue-stem, bull nettle, and Gramma solanum. Trees in this area are almost exclusively oak (black jack or post oak) with a few elm and cedar. Some cactus may be found in the western half of Zone D.

Subzone $D-7$ is an area 50 meters wide, paralleling the western side of the caliche road from Highway 290 north to the western stock pen, with the same grasses as Zone $D$ but almost treeless.

Subzone D-2 lies south of the cultivated field. It possesses few trees and may represent a natural clearing or may have been cleared for installation of power lines.

Subzone D-3 is the cultivated field west of the western stockpen. It has been intentionally terraced and is now covered with annual grasses.

A11 other areas not described in the preceding zones and subzones are termed Zone $D$ and in most places represent $100 \%$ cover.

The vegetation types reported by Ed Zielinski and the authors are by no means a complete inventory of flora, but are meant only to represent the most common types and provided a basis for division. Of some interest is the fact that these vegetational zones are del ineated by fences and roads. All the major groups have been neatly partitioned off from the native grasses of Zone $D$ although in many places the boundaries are being challenged.

Rainfall and Flooding

According to information available from the work plan of the Gillespie County Soil and Water Conservation District (Gillespie County, 1967), the Hop Hill site lies in a region of extreme rainfa11, often of high intensity. Eighteen to twenty inches in twenty-four hours have been reported while yearly totals have fluctuated from 11.29 inches in 1956 to 44.04 inches in 1952 . These high intensity rainshowers generally occur during the month of April when thunderstom activity is at its peak (Allison et al. 1975).

Coincident with and partially a result of rainfall is the severity of flooding, not only of major rivers but over entire drainage basin areas. Streams are characterized by floods of high velocity and short duration due to their narrowness and generally impermeable soils.

At Hop Hill this regional picture is reflected in the lag deposits of the gullies and channels while pedestaling of many objects reflects an intense raindrop impact effect. 
Site History

The Hop Hill site, now part of the LBJ State Park, was part of a 400-acre parcel of land bought by Eugene Lindig's grandfather in 1874. The land was used for pasture where mostly sheep, some cattle and occasionally goats were grazed. According to Mr. Lindig, who owned the land prior to its acquisition by Texas Parks and Wildlife, the immediate area of the site was never cultivated or plowed for any reason, except for the terraced field to the west of the site which was first planted in the late 1940s or early 1950s.

In recent historic times two roads crossed the site, both apparently old wagon roads. Running from south to north was a road from Albert, Texas Austin highway which is now Ranch Road 1. The Albert road is the same one which was later resurfaced and now runs between the east and west stock pens. It also connected with the old wagon road from Johnson City to Fredericksburg which probably crossed from east to west through the northern stock pens. An east-west road which approximates this orientation is clearly visible in the 1948 aerial photograph of the site (Figure 6.04). The old Albert road is also visible in this picture.

Discussing the road, Mr. Lindig noted that the gully beside the north-south stock pen had always been there and was associated with the old Albert wagon road. This is the largest and best developed gully on the site and it is not surprising that it could be this old. While this gully is not clearly visible in any of the aerial views of the site (Figures 6.03-6.07), the major drainage to the east of the site can be seen. It is apparently free-running in the 1938 photo (Figure 6.07), but as early as 1948 (Figure 6.04) a dam had been built to the south of the site. The confluence of this small stream with the Pedernales River is also most easily seen in the 1948 picture.

The first and only major clearing of the land took place in 1946, though the cedars have been cut down several times. Even today only a few small cedars, most less than two feet high, can be seen in the extreme southeast and southwest corners of the property. This clearing seems obvious in the comparison of the 1938 and 1948 aerial photographs. Caution should be used in interpreting exactly which areas of the site were cleared because the 1938 photo was taken on September 27, before most trees would have lost their leaves, and the 1948 photo was taken on December 3, when only the evergreens would be prominent.

By the time of the 1955 aerial photograph (Figure 6.05), Highway 290 had been built to the south of the site, the field to the west had been plowed and a small tank built on the north end of the creek. This was after the major flood of 1952, one of the worst ever on the Pedernales; floodwaters reached all the way to what would have been the middle of the northern pens had they been there at that time.

The only evident change in the 1963 aerial photo (Figure 6.06) is a small rectangle under cultivation in the clearing to the northeast of the site. By about 1964, however, the stock pens were built and put into use. These pens were in more or less continuous use until the land was taken over by the park 


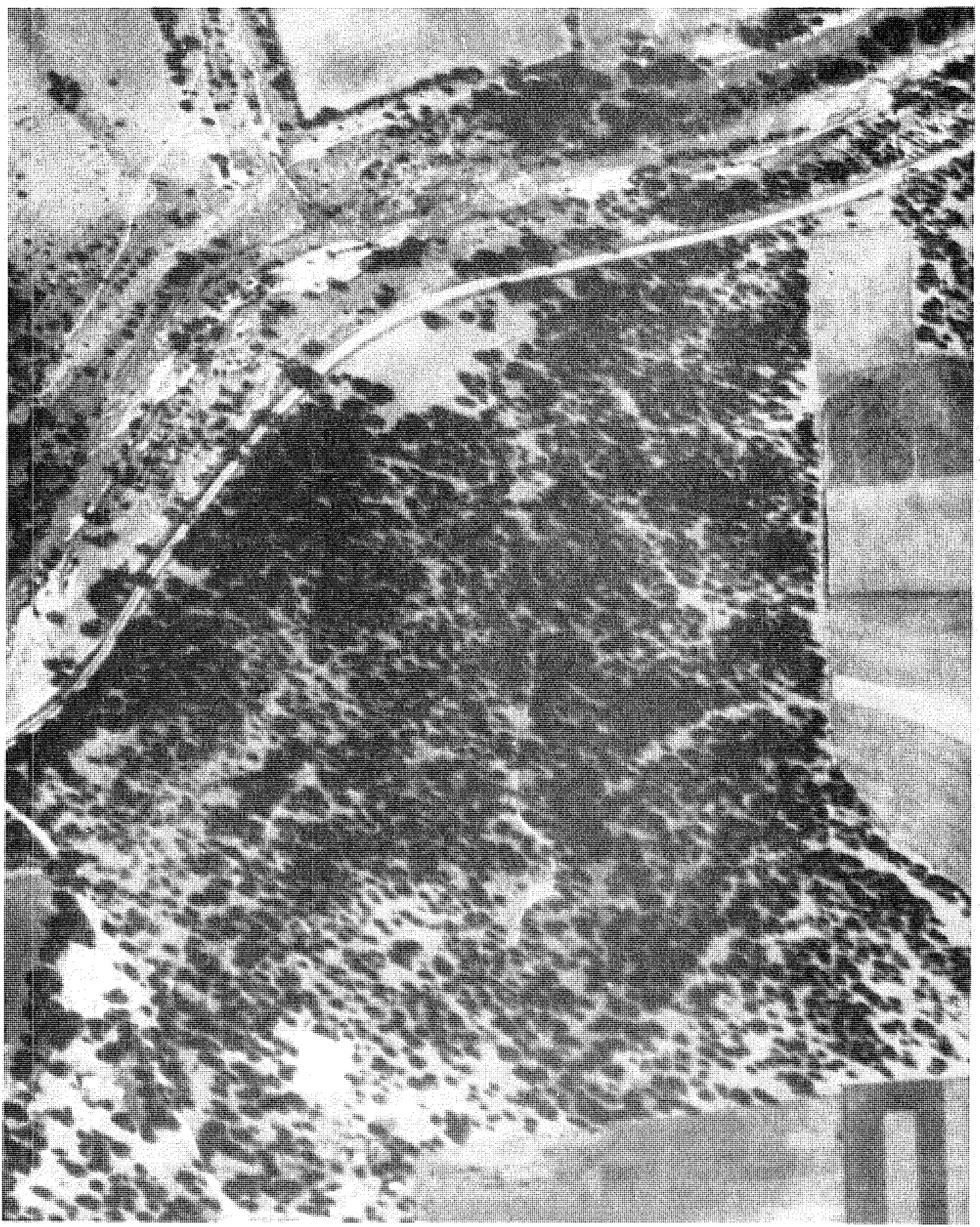

Figure 6.03. Aerial Photo of Site Taken September 27, 1.938. (Soil Conservation and Stabilization Service). 


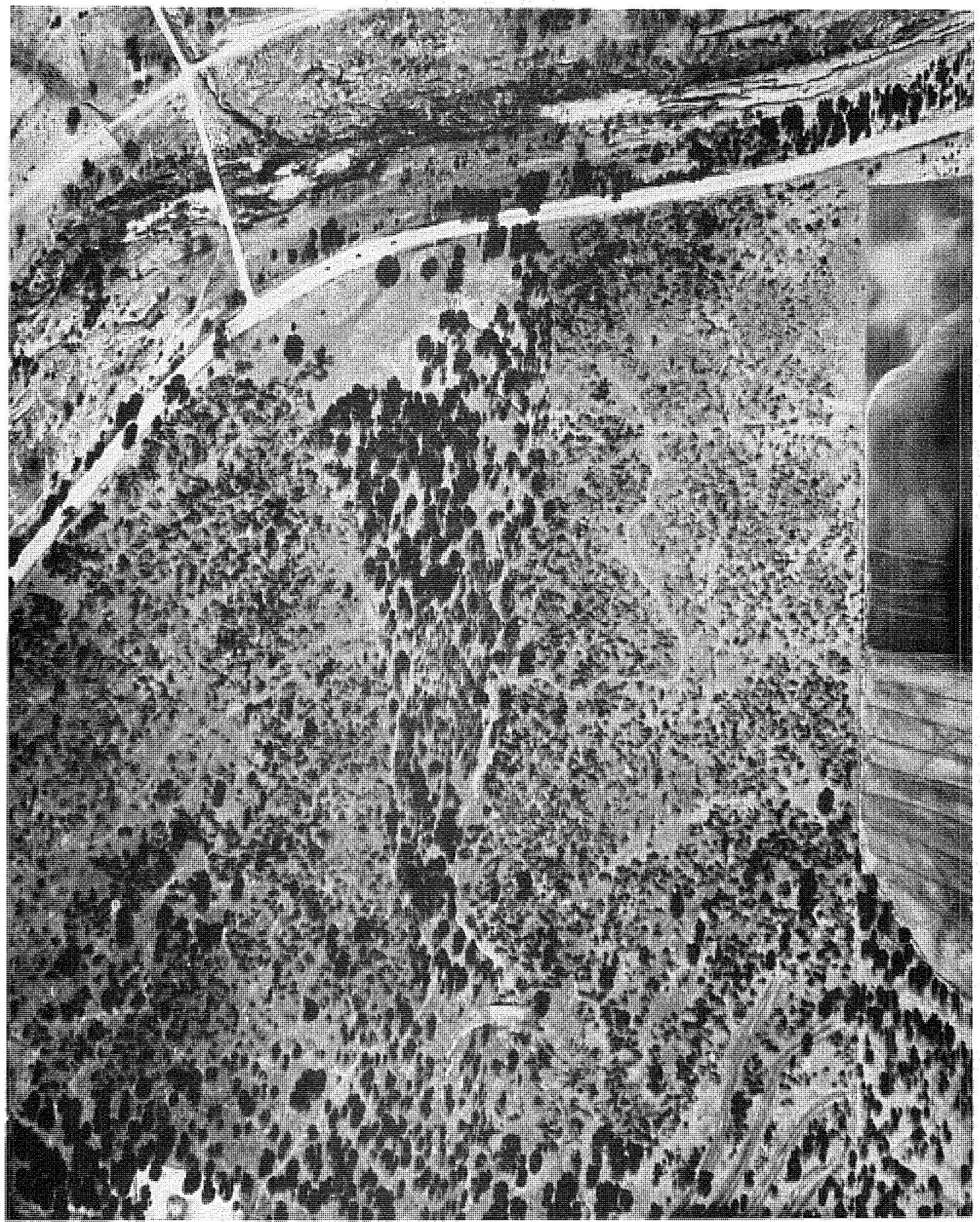

Figure 6.04. Aerial Photo of Site Taken December 3, 1948. (Soil Conservation and Stabilization Service). 


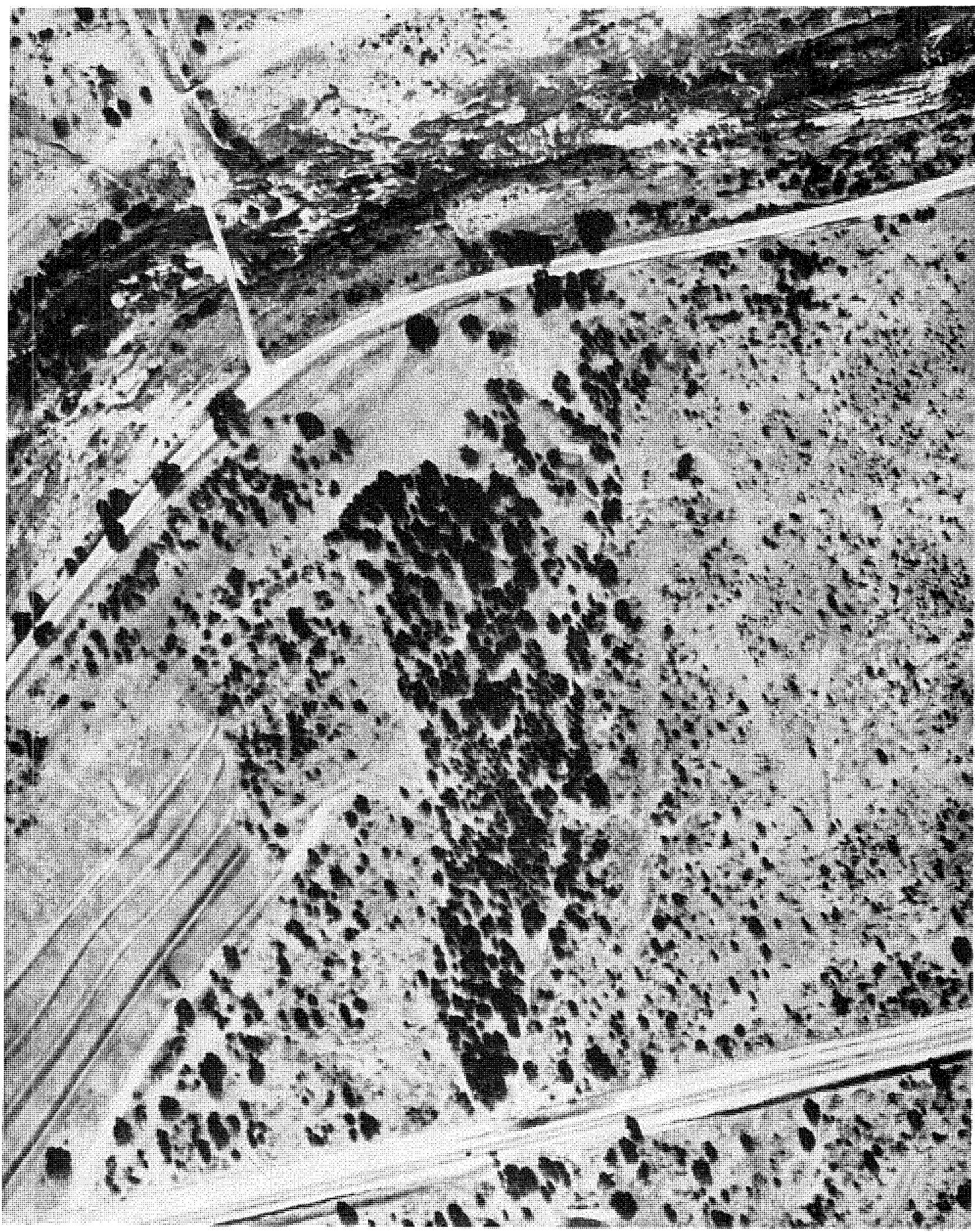

Figure 6.05. Aerial Photo of Site Taken November 11, 1955. (Soil Conservation and Stabilization Service). 


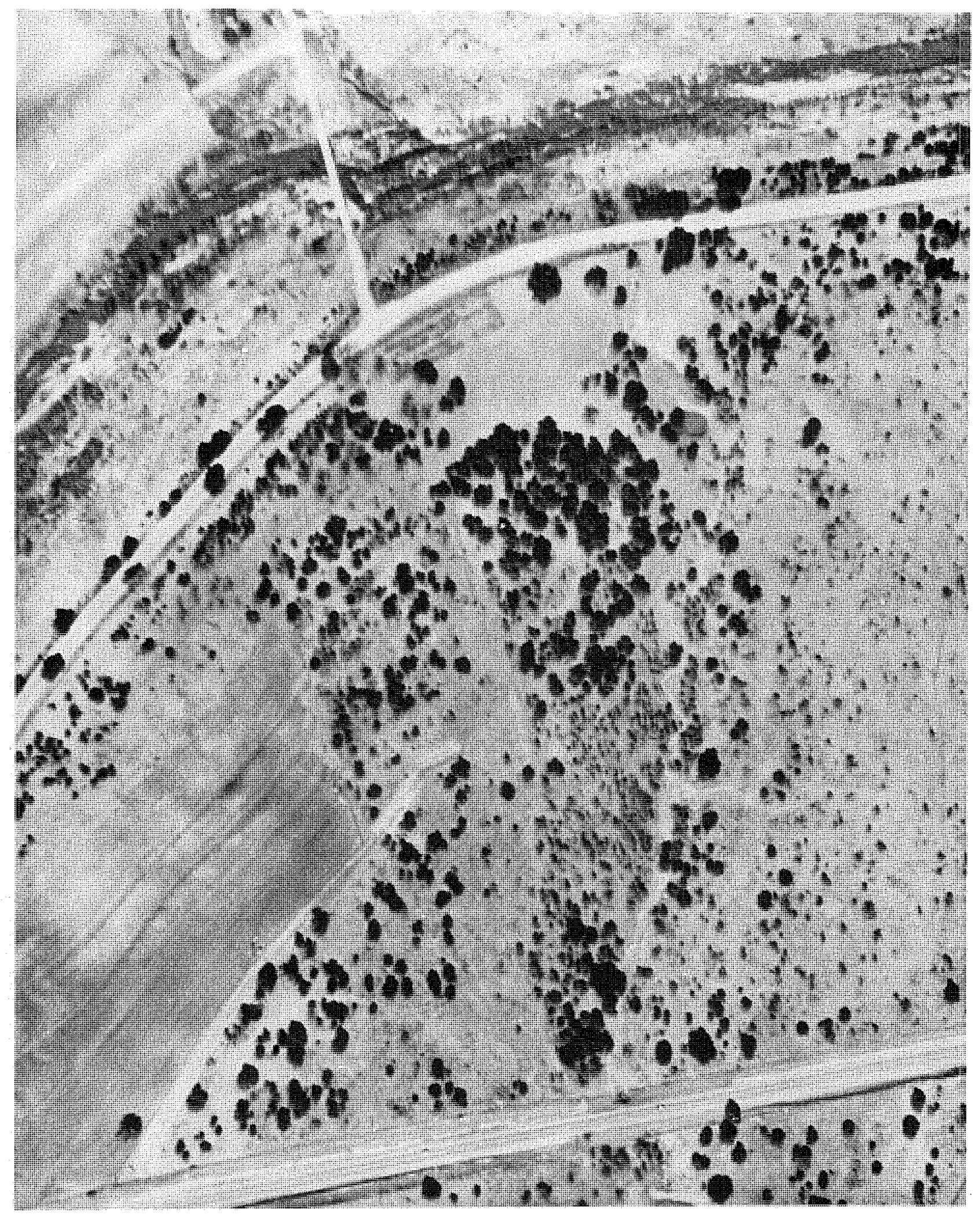

Figure 6.06. Aerial Photo of Site Taken February 3, 1963.

(Soil Conservation and Stabilization Service). 


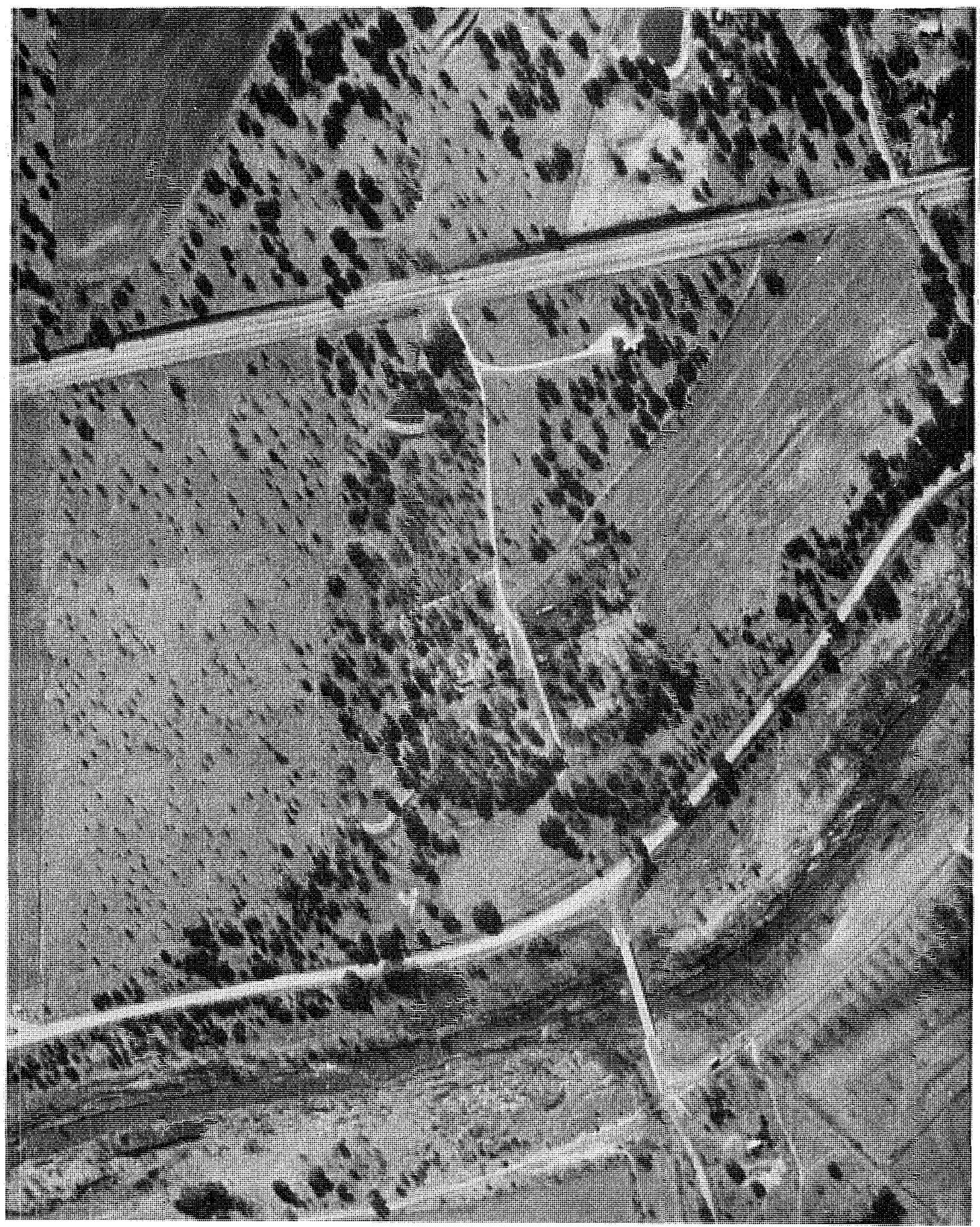

Figure 6.07. Aerial Photo of Site Taken January 31, 1974

(Soil Conservation and Stabilization Service). 

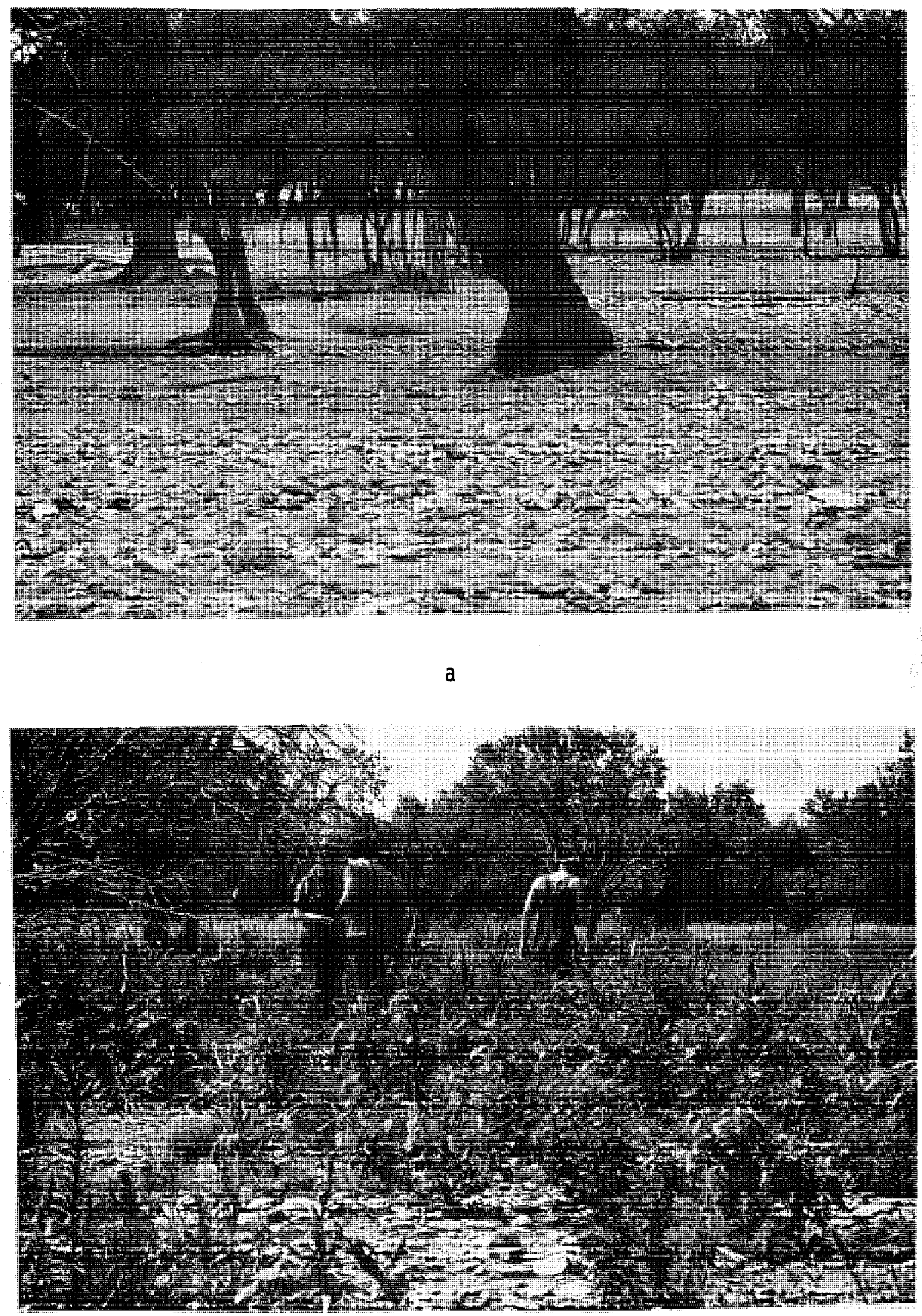

Figure 6.08. Vegetation Inside Stock Pens. a, photo taken December 1975 showing lack of vegetation in stock pens; b, photo taken in summer 1976 showing weed cover inside stock pens. 
in the summer of 1975. The road between the pens was surfaced in 1965 or 1966 when the lower (northern) tank was enlarged. Clay scooped out of the tanks was used on the road.

$\mathrm{Mr}$. Lindig stated that there was no differential use of the stock pens as the vegetation survey seemed to indicate, but felt that the difference was entirely due to the soil change between the pens, not only the distinction between the Purves soil of the east pens and the Hensley-Pedernales of the west, but also a soil change from bottom to top of the areas. The higher (southern) areas of the pens have red clay closer to the surface than the lower jying areas. The lower slopes of both pens are also somewhat rockjer. The different soil types noted earlier are distinguishable in the 1974 aerial photograph (Figure 6.07) as areas of darker and lighter shading.

The area of intense rock cover in the northwest corner of the site apparently does not continue into the plowed field, at least not superficially. In plowing the field to a depth of eight to ten inches, Mr. Lindig encountered almost no rocks (so few, in fact, that when he turned up a concentration of small rocks in a patch of blacker soil intrusive into the reddish clay loam he thought he had cut into an Indian burial.) The gully that drains the cultivated field shows rocks underneath the surface and it is difficult to say if the layer of rocks (many of them chert nodules) continue under the field at some depth or end there.

Other than the conditions created by the hogs, the only major disturbance in the pen area seems to have been when $\mathrm{Mr}$. Lindig used a tractor and blade attachment to scoop dirt out of the high places and fill in low areas around the fence lines which may have been eroding away. One area from which dirt was removed was just to the west of the occupation area excavation, which he had long noted as an "Indian fire." He feels certain that he did not disturb the excavated area.

When questioned about the erosion, he maintained that it was not a serious problem while the hogs were present to keep the soil well-compacted. He says that water would not penetrate the soil, but would wash over it without removing too much soil material. Hard packing would indeed make the soil less permeable but more susceptible to water erosion. Introduction of organic matter would, however, reduce erosional processes significantly in the stock pens. He noted that erosion appeared to be much greater after the hogs were removed. It is hard to imagine the stock pens in full operation without some erosion, but it is entirely possible that without the protection of the organic matter, and without the intentional protective measures of the farmer, soil erosion has proceeded at an increased rate.

It is possible then that much of the recent erosion has occurred since the site's abandonment in the summer of 1975 when the area of the pens was almost completely devoid of vegetation (Figure 6.08a) and the summer of 1976 when the vegetation began to return (Figure 6.08b). It seems certain, however, that the area had been eroding before, perhaps even before the time of the stock pens. 
The shallow depth of the red clay at the top of the pen area suggests this. Perhaps wash from the cultivated field had something to do with it, or perhaps erosion was serious in the great drought of the dust bowl years when, according to local residents, much of the natural vegetation was killed off.

\section{A Model of Erosion}

In order to understand the nature of land surface changes at Hop Hill, a model must be developed which would allow the causes and effects of those changes to be quantified and tested upon actual data from the site. Such a model, if it could be shown to be explanatory of the observed processes, could be used to predict future land surface change and to gain some insight into past changes which have left the site as it is today. Specifically, the excavator would be able to determine areas of differential disturbance so that excavations and surface surveys could be planned accordingly.

The logical place to start in choosing a model to work with is the accepted geomorphological model of land form change which involves detachment and transport of surface material. The basic element of this transport and detachment is, of course, gravity. Detachment of the land surface or surface particles (in a microenvironment) results from a movement of land form, air or rainsplash. Transport is then carried out by gravity, wind, or overland waterflow. Many variables are involved in this soij-removal process but the ones which appear to be the most relevant to the Hop Hill situation are those involved with the removal of surface materials by water. Wind erosion may play a role in the hot dry summer months but, as others have shown for similar semiarid environments, water is by far the prime mover of surface materials (Gregory and Walling 1973).

At Hop Hill, we are dealing with what is commonly called accelerated erosion (Hudson 1971:33) or the rapid removal of soil material due to the activities of man. While natural processes of leveling are undoubtedly a factor at the site, they are more difficult to measure and much smaller in scale than the erosion resulting from human activity at the site. That activity, which includes grazing, cultivation, and the intense surface modification by hogs, has disturbed and destroyed the vegetative cover and produced changes in the upper soil profiles. These changes have in turn led to greatly accelerated soil removal over parts of the site, exposing numerous previously buried artifacts, pedestaling large cement troughs and exposing tree roots.

Gully erosion at the site is noticeable and serious in places. The largest gully runs along the east side of the road after crossing from the loading dock area to the west of the road. It drains into the flat low area at the extreme south of the site. Several other small or incipient gullies can be mentioned, notably on the west side of the road and along the western fence line. But these gullies are not the main focus of the paper; the problems of excavation in gullies should be obvious, as should their location. What is more important is the remainder of the site where land surface change is less visible but nonetheless operating. 
At the Hop Hill site, as in any other small drainage basin, small rills or erosional channels carry water to feed the gullies. Water flows in any slight depression downhill. Often these are so small that it is difficult to see where water will flow until it actually rains, yet much soil material is carried downslope by these tiny channels. They are constantly changing and may even be completely obliterated and replaced by newer ones each year. It is this process of water erosion that we want to look at.

Water erosion is the result of two distinct processes, rainsplash and slope Wash, both of which possess a detachment component and a transpori coniponent. of these processes, rainsplash is the more efiective weathering force while the Tatter is more important as a mechanism of transport. The energy of the impact of raindrops has a cratering effect which loosens surface material, throwing smal1 particles as far as one meter away. Overland flow begins when the soil has been saturated; water sweeps down the hill, carrying away the smaller material that has been detached. As rain continues, overland flow picks up Targer and larger material and increases in abrasive force.

Vegetation acts initially to protect the soils from raindrop impact. Raindrops which hit leaves either roll harmlessly down the stem or fall off the leaf with only a fraction of their initial velocity. This may be the most important contribution of plants to soil protection but it is far from the only one. The stems of plants interrupt and divert overland flow protecting some areas. The roots bind together the soil into a proiected mat. Plants deposit organic matter in soils, helping to hold the soil together.

The soil itself plays a crucial part in the process: the nature of the soil, the lithic content, the moisture content, the clay content, all play vital roles.

Applying this information directly to the Hop Hill situation, it is easy to see the possibilities for erosion offered by the stripping of natural vegetation in the stock pen area. Throughout the rest of the site, the slopes are so gentle (less than one percent) that not a great deāl of soil surface movement will be seen where a natural grass vegetation remains. However, the removal of vegetation from the slopes of from approximately one to ten percent is open to serious erosion during intense rainfall conditions.

The rate of this erosional process is dependent upon a number of factors, the primary ones being soil type, vegetation density, slope gradient and rainfall conditions. Soil scientists and geomorphologists have long assumed that with the proper isolation of variables, soil erosion of various land surfaces could be predicted and measured with relative ease. One of the best known and most widely used attempts to predict soil loss is the Universal Soil. Loss Equation developed at Purdue in the late 1950s (Wischmeier et al. 1958). This equation predicts the annual soil loss in tons per acre when certain factors influencing soil erosion are known. These factors are storm intensity, soil type, slope, slope length, a crop management factor and a conservation practice factor. The equation was developed for use on agricultural land, so it is difficult to relate it directly to the abandoned stock pens at Hop Hi7l. Even if estimates could be 
made, it is not certain how the archaeologist would relate soil loss in tons per acre to artifact distribution on the surface. But the basic model provided by the equation is useful and can be considered here with some modifications.

Storm intensity as it relates to erosion is not dependent on the amount of rainfal1 but upon the energy of any given storm. One measure of erosion is based upon the theory that only rains in excess of a certain amount will saturate the soil and cause overland flow and thus erosion (Carson and Kirkby 1972:216). The factor used in the Universal Soil Loss Equation is measure of the "...greatest average intensity experienced in any 30-minute period..." during a given storm (Hudson 1971:64). This then is summed for individual storms to give a yearly total for a particular locale. As wischmeier has shown in his experiments, this maximum 30-minute intensity shows the strongest correlation with erosion.

Soil type is another important factor in erosion. Certain soils are more resistent to erosion than others and this is partially due to the structure or size of aggregates, bonding factors such as anion and cation exchange capacity, texture and organic matter content (Carson and Kirkby 1972:213-214).

Measures of a soil's resistance to erosion commonly use soil texture, specifically the silt and clay content in combination with other soil properties, usual7y organic matter content. (Carson and Kirkby 1972:215) In the Universal Soil Loss Equation the soil factor is determined from known runoff values on experimental plots (Hudson 1971:182).

Slope gradient is a rather obvious factor in the erosion phenomenon; the steeper the slope, the greater the erosion. The soil loss equation uses two factors, the slope itself and the length of the slope from the crest of hill to the bottom of the plot under consideration. The steeper the slope, the higher the velocity of water moving down it; and the longer the slope, the faster the water runs the farther it moves downslope.

An additional topographic factor which the Soil Loss Equation takes into account under the heading of conservation practices (and which is not all that important in determining the soil loss from a huge plowed field but can make a difference in natural areas) is the microtopography of small sections, including tiny concavities and bumps on the land surface as well as the nature and direction of small channels draining water into larger gulties.

What the Universal Soil Loss Equation considers a crop management factor can be best translated to a natural vegetation factor of any area which is not directly under cultivation. Vegetation is a complex factor which is difficult to measure directly, especially in diverse microenvironments such as those present at the Hop Hill site; but as we have shown earlier, is extremely important to erosional processes. 
Research Design

Archaeologically, erosion at Hop Hill is significant for what it can tell us of disturbances in the land surface which may reflect disturbances in artifact location. Knowing the loss of soil in tons per acre from the entire site would be useful to the geographer but would tell the archaeologist little. As archaeologists we are more interested in converting that loss in tons per acre into a dimension which reflects the vertical lowering of the land surface.

Another figure which is an integral part of soil removal is the total amount of material moving across the site, a difficult item to measure without sediment traps, but one which should give the researcher an idea of how much disturbance he can expect in any given area from movement of material.

Total vertical and total horizontal movement for the entire area are both useful variables, but even more so when the site is broken down into smaller areas having differing characteristics and thus different amounts of erosion. The ideal situation would be to determine the exact amount of erosion at every point, which at a site this size would be physically impossible. Therefore, a selected sample of points was studied as a reasonably accurate sample of the actual land surface movement in any given area.

In 211,149 points were selected to provide a representative sample from each soil zone and slope zone. A few of these points were selected to insure a statistically workable sample of points in association with rills. Except for the few lines of points that were set across rills, all locations were chosen on paper first and then measured into place so that the effects of other variables would be randomily distributed.

Although we felt certain that the distribution of factors which influence erosion was a close approximation of the total influence of those factors at the entire site, it was decided to note the mocroenvironment of each point as a means of assessing the relative importance of these erosional parameters. While it was not possible to measure all of the variables which influence erosion at any given point, we were able to record a number of relevant microenvironmental characteristics. Measurement of these factors, all of which were felt necessary to complete analysis, was often done on the basis of educated observation, but the final data set is as accurate and complete as could be under the circumstances.

Each point was coded for the slope zone in which it lay, using the site map prepared by Dr. Gunn. Then the four soil types were coded from the Gillespie County Soil Survey (Allison et al. 1975). These two were later subdivided on the basis of field observations; in both cases the added variables represented borderline or transitional categories. A third zonal variable, vegetation zones, was coded from the field survey of the vegetation types. These three variables represent the only obvious basis on which to divide the site into areas which might have significantly different erosion. 

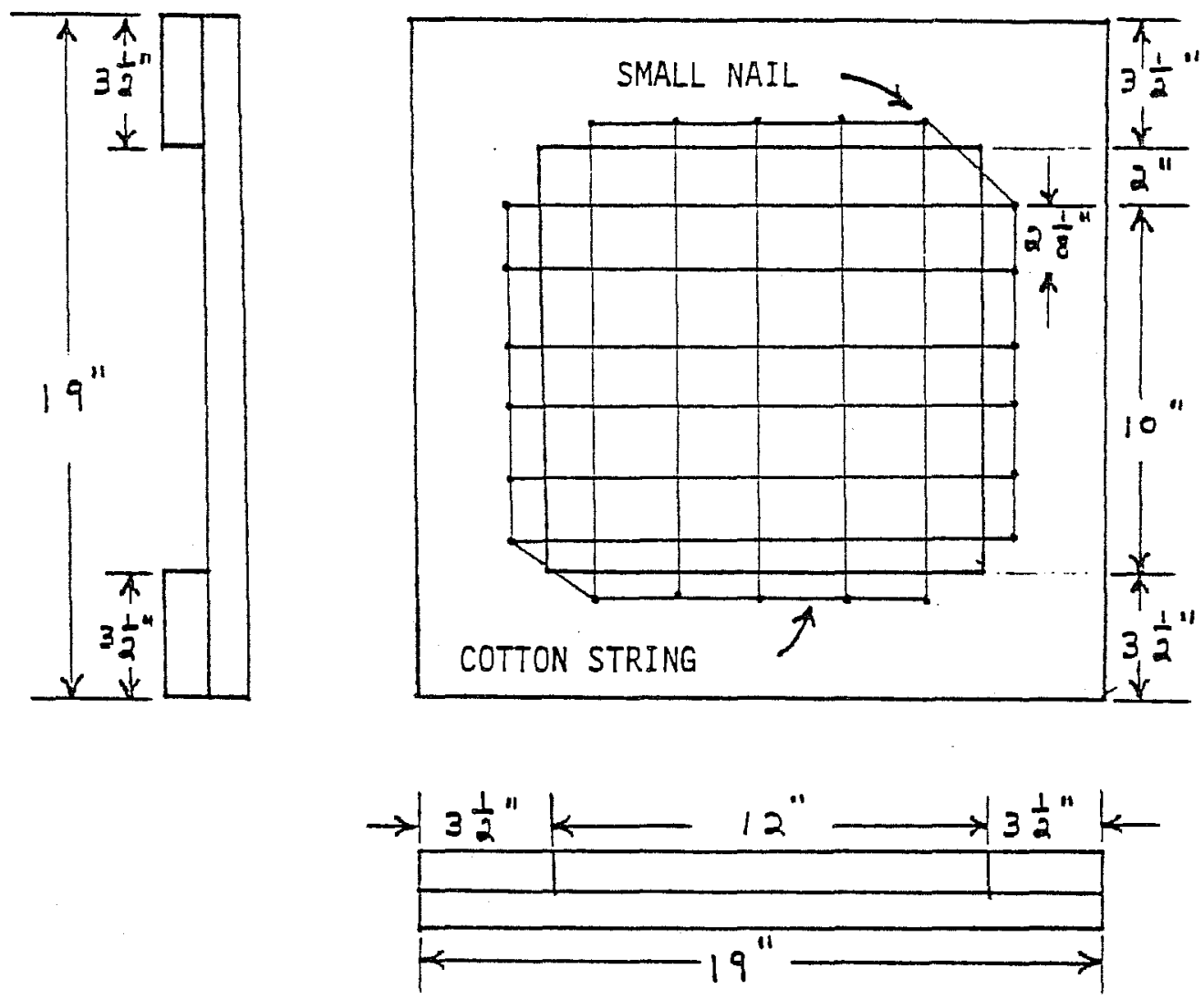

Fioure 6.09. The Vegiemeter. (drawn by Clint Davis). 
A sighting grid (Figure 6.09 ) was constructed and for each point the percentage of leafcover, basal cover (or stem cover) and rock cover was recorded. While it was difficult to decide on the proper size of area to measure in each case and the accuracy of the sighting device is not perfect, these variables must stand as the best estimates possible and are probably within at 1east $15-20 \%$.

One variable which was simply estimated was the amount of tree cover; one code for no trees, one for partial coverage and one for complete cover. The distinction between the last two was often difficult to make and was almost worthless because only twelve points or $8.7 \%$ of the total were either fully or partially covered. Another observed code was rillsize, in which one code stood for no rill, another for the edge of a small rill, another for either the center of a small rill or the bank of a gully (a miscombination, but the sample was so small as to make separation impossible) and the last code for the center of a gully. Although our intentional inclusion of several rills helped make the distribution of the first two codes comparable, there were still too few cases in the last two values to be of real use--only 7.3 points or $8.7 \%$ were in the center of rills or gullies.

One local variable which was carefully measured was the microslope, or the slope of a one-meter strip with the nail at the center. Because most of the flow of water is downhill or north toward the Pedernales, this variable was arbitrarily chosen to be measured entirely to the north even though in a few cases the slopes were greater in other directions. Some points on small south facing slopes were measured as negative microslopes.

The kind of root and the approximate amount of root material in the soil were estimated with the help of Ed Zielinski, who divided the roots into three types: fibrous, fleshy fibrous and tap root systems. Dominant and secondary codes were later combined into a single measure for economy in the statistical analysis; two variables, root type and root frequency, were used.

Erosion measurements were taken from both wooden stakes and nails set into the ground; a tape measure was used at the stakes and a vernier caliper at the nails. Due to the difference in scale of measurement between the tape and calipers, a variable was established which would distinguish between the two to test for possible measurement error in the statistical analysis (Table 6.01).

One of the most useful breakdowns of these variables was the separation of zonal, or areal, codes such as soilzone from individual or microareal nail codes such as treecover. Separation of semi-permanent conditions such as rockcover or microslope from seasonal and temporary variables such as leafcover or root type was also useful. Table 6.01 shows the breakdown of variables in this way. Treecover does not fit neatly into either individual code because it is at the same time more permanent than the smaller vegetation and yet also has seasonal effects. Nailcode is only a means of testing our measurement error at each point and does not properly belong in the table. 
Table 6.01 Variable Characteristics

\begin{tabular}{|c|c|c|c|}
\hline \multicolumn{3}{|c|}{ Semi-Permanent } & Seasonal \\
\hline ZONAL & \multicolumn{2}{|c|}{$\begin{array}{l}\text { Soilzone / Soilcode } \\
\text { Slopezone / Slopecode }\end{array}$} & Vegetation zone \\
\hline \multirow[t]{2}{*}{$\begin{array}{l}\text { MICRO } \\
\text { Areal }\end{array}$} & $\begin{array}{l}\text { Rillsize } \\
\text { Microslope }\end{array}$ & Treecover & $\begin{array}{l}\text { Leafcover } \\
\text { Basalcover }\end{array}$ \\
\hline & Rockcover & & $\begin{array}{l}\text { Root type } \\
\text { Root frequency }\end{array}$ \\
\hline
\end{tabular}

Field Measurements

Measurement of the soil erosion parameters of Hop Hill was divided into two categories: those taken at specific points (riails) and those taken to sample specific areas (zones) of the site. Point measurement included land surface change, relation of points to rills, microslope, rockcover, leafcover, basal cover, treecover, root type and root frequency. Area measurements included determination of soil type zones, slope zones and vegetation zones.

Field measurement of microenvironmental areas was done through the exact location of a number of points within the site. One hundred and forty-nine nails were located or placed in the ground at predetermined locations along a 7 ready established grid lines. Points were also placed in periphera 1 areas of the site both using transit and tape as well as lensatic compass and distance pacing. At some grid points wooden stakes were present instead of or in addition to nails. It was hoped that the location of these nails and stakes would effectively sample the microenvironmental areas of the site.

The main body of data includes biweekly measurement of the surface at nail and stake points. Data was collected by measuring the distance between nail head and ground surface with vernier calipers. and by measuring the distance from the top of stakes to the ground surface with a meter tape. The vernier caliper measurements were good to a twentieth of a millimeter while tape measurement was to the nearest millimeter. The data was collected on a biweekly basis over a twenty-week period from mid-July to mid-December of 1976. In severa 1 cases weather conditions did not permit a fourteen-day period, as indicated in Table 6.10.

While points were coded for the presence of rills on the basis of a visual estimate, the microslope variable was more accurately coded. A one-meterlong board with a level mounted on top and a meter stick attached perpendicular to one end (Figure 6.10) was centered over the nail and pointed in an 

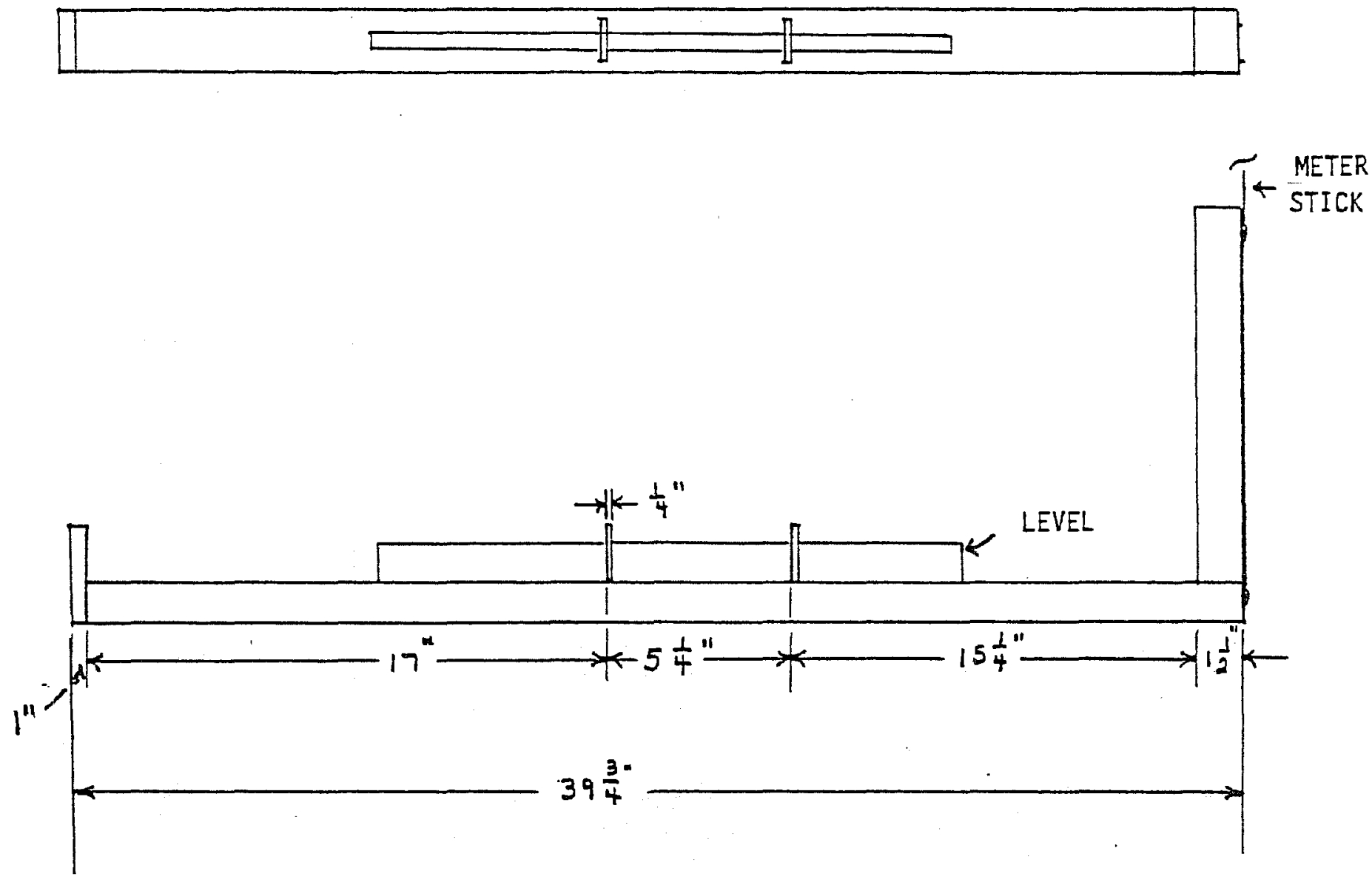

Figure 6.10. The slope-o-matic. (Drawn by Clint Davis).......... 
approximate northerly direction. When the board was level the rule was lowered and the distance from the bottom of the board to the ground taken as the percent grade or the number of centimeters the land surface drops in one meter of distance.

Leafcover for each point was calculated through the use of our vegiemeter (Figure 6.09) and recorded as percentage cover. The vegiemeter was held at a constant height above each point and the number of string intersections in the line of sight with vegetal cover were counted. A similar technique, the point quadrant method, is used in quantitative botany (Greig-Smith 1964).

Basal cover was also calculated using the vegiemeter except that the sighting device was held closer to the ground for a better determination of the percentage of stems. In addition, the vegiemeter was used to measure the percentage of rocks on the surface; any intersection with a rock of pebble size or larger was counted.

Root types around each point were visually analyzed by Ed Zielinski and designated as either fibrous, fleshy fibrous or tap including determination of either dominant and secondary types. Root frequency was done in the same manner and recorded as a percentage.

Soil type data was collected by dividing the site into soil zones on the basis of the Gillespie County Soil Survey (Alijison et al. 1975), then given a geographic designation by zone (Figure 6.01). Slope zones were also determined by dividing the site geographically into areas with approximately equal slope angles through the use of topographic maps and actual site examination. Vegetation zones were determined by field observation and mapping by Ed Zielinski and David Brown.

Al1 data was coded in the field on computer coding sheets which were later checked and then keypunched onto cards for later computer evaluation of the data.

Preliminary examination of the data indicated that very few nails showed a consistent direction of vertical surface change over more than three or four measurement periods. There are three possible explanations which account for this variability: one, from week to week different causal factors (e.g. rainno rain) brought about different erosional effects; two, we were not measuring permanent change at each point but the wavelike movement of soils downhill; and three, much of the vertical fluctuation was due to measurement error.

There is some evidence that land surface change is not linear under various types of vegetation. (Carson and Kirkby 1972:215). The relationships of various slopes and soil types to the causal factors of erosion might be equally nonlinear. The wave movement theory, difficult to demonstrate from the data alone, is supported by casual observations at the site; lighter colored alluvium would be deposited by a nail after a rain only to be washed away by the next rain. 
If all factors affecting erosion were held constant, rainsplash erosion would tend to lower a hillslope evenly over a long period of time. The effect of raindrop impact may not be as even at the micro scale. Between two points a meter apart, one raindrop crater more or less could make a difference in erosion.

The 1ast hypothesis, measurement error, finds support in the small scale of most of the weekly readings, despite the fact that our technique, modified somewhat, has been used very successfully by other researchers (Bridges and Harding 1971:67; Emmett 1965). Because of the apparent nonlinear character, it was decided that the cumulative change in land surface would be the most useful indicator of erosional activity.

A11 availabie microenvironmental variables (excluding duplicates such as soilzone and soilcode) were analyzed in a stepwise linear regression (SPSS Regression procedure; Nie et al. 1975) with the cumulative land surface change as the dependent variable. Characteristics of the variables are shown in Table 6.02.

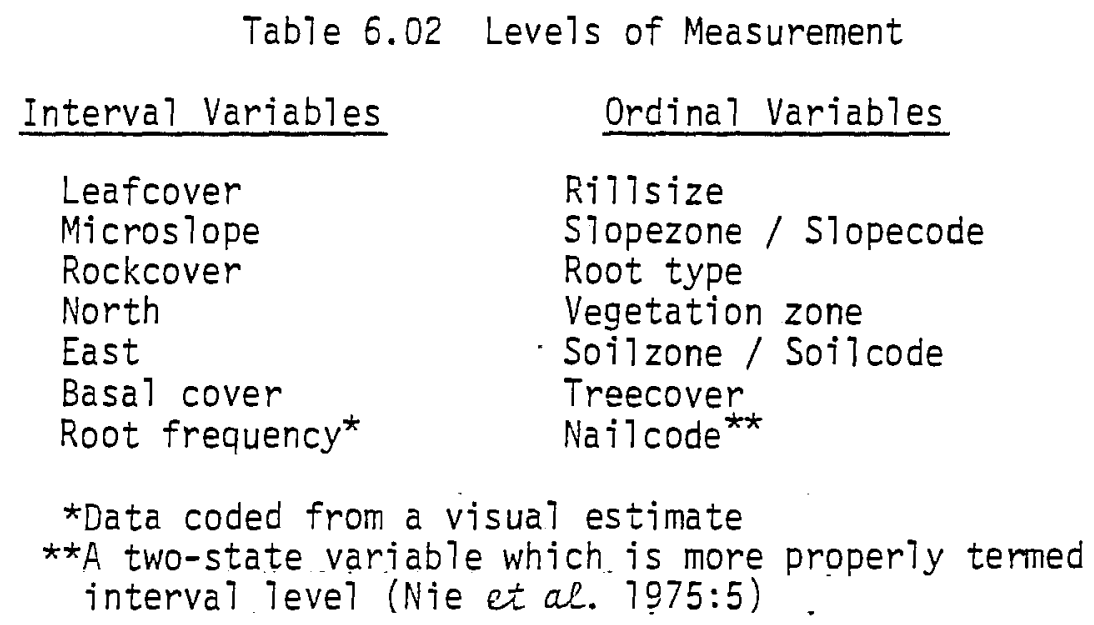

Several problems were anticipated in this technique: interactions between the variables were not entered in the equation; and certain of the variables were slightiy collinear. We assume with random measurement error will tend to reduce the $R^{2}$ value, a measure of the strength of correlation, and the corresponding $F$ value, a measure of statistical significance based on sampling, rather than increase them. Therefore, no spurious results will come from random measurement error. (Table 6.03).

The results of the stepwise regression of the cumulative land surface change with the environmental codes are shown in Table 6.03. Of the fourteen variables used in the equation only root type had an insufficient $F$ value for entry. The equation is significant at the five-percent level through the third step, the step which also has the largest adjusted $R^{2}$ value, $a$ measure of the amount of variance in the dependent variable explained by the 
Table 6.03 Land Surface Regression Analysis

\begin{tabular}{|c|c|c|c|}
\hline Variable & Step & R Square & Overall F \\
\hline $\begin{array}{l}\text { Treecover } \\
\text { Rillsize } \\
\text { Slopecode } \\
\text { Microslope } \\
\text { Rockcover } \\
\text { Root frequency } \\
\text { Leafcover } \\
\text { Nailcode } \\
\text { Basalcover } \\
\text { Soilcode } \\
\text { Veg. zone } \\
\text { North } \\
\text { East }\end{array}$ & $\begin{array}{r}1 \\
2 \\
3 \\
4 \\
5 \\
6 \\
7 \\
8 \\
9 \\
10 \\
11 \\
12 \\
13\end{array}$ & $\begin{array}{l}.0302 \\
.0689 \\
.0822 \\
.0904 \\
.0967 \\
.1058 \\
.1155 \\
.1233 \\
.1286 \\
.1301 \\
.1306 \\
.1354 \\
.1355\end{array}$ & $\begin{array}{l}3.0557 \\
3.5871 \\
2.85658 \\
2.3593 \\
2.0135 \\
1.8342 \\
1.7164 \\
1.6003 \\
1.4763 \\
1.3313 \\
1.2017 \\
1.1349 \\
1.0370\end{array}$ \\
\hline
\end{tabular}

independent variables, and the smallest residual standard deviation, a measure of the amount of variance left unexplained by the independent variables. The most useful prediction equation contained here would then be:

$$
\begin{aligned}
\text { EROSION }= & -364.80111+253.76955 \times \text { TREECOVER }-185.41856 \\
& \times \text { RILLSIZE }+37.376983 \times \text { SLOPECODE }
\end{aligned}
$$

The emphasis on treecover came as a surprise. Upon closer analysis it actually displayed a reversed relationship from its postulated effect. Using our model of erosion one would expect less erosion underneath trees because these points were protected from the full impact of raindrops. Yet Table 6.04 indicated that erosion was greater under trees than away from them. In tropical areas trees are high enough to allow raindrops to reattain their teminal velocity and thus cause erosion on bare ground after a few minutes of heavy rain (Young 1972:65); the trees at Hop Hill are seldom taller than four or five meters.

Since the sample of tree-covered points is quite small we feel that this may indicate sampling error. It may also represent a compounding of variables; one possibility that the authors have observed in other contexts is that tree cover might cause a noticeable reduction in ground vegetation cover because of shading.

Table 6.05 presents a comparison of mean leafcover percentages for the entire site and for full and partially covered points. While the partially covered points show about the same mean as for the site 
Table 6.04 Means of Treecover Values

$\begin{array}{llrrr}\text { Value } & \text { Explanation } & N & \text { Mean } & \text { St. Dev. } \\ & & & & \\ & & & & \\ 1 & \text { Full tree cover } & 7 & -0.25 & 4.98 \\ 2 & \text { Partial tree cover } & 4 & -2.44 & 2.47 \\ 3 & \text { No tree cover } & 99 & 2.50 & 5.58 \\ - & \text { Total } & 110 & 2.14 & 5.54\end{array}$

Table 6.05 Mean Percentage Leafcover

\begin{tabular}{|c|c|c|c|}
\hline Points & Location & Mean & St. Dev. \\
\hline $\begin{array}{r}149 \\
7 \\
4\end{array}$ & $\begin{array}{l}\text { Entire site } \\
\text { Full tree cover } \\
\text { Partial tree cover }\end{array}$ & $\begin{array}{l}63.22 \\
35.43 \\
63.00\end{array}$ & $\begin{array}{l}35.85 \\
33.18 \\
45.42\end{array}$ \\
\hline
\end{tabular}

as a whole, the fully covered points do exhibit what appears to be significantly less cover. Further checking reveals that of the four partially covered points, which show greater erosion than either of the other categories, two are near small rills.

The second variable in the stepwise regression solution, rillsize, was no surprise; we had long felt that this was a crucial variable in the erosional process. It was meant to be roughly ordinal with the lowest value representing no small channel nearby and the largest representing the center of gullies where it was felt that there would be the greatest erosion. As is evident from Table 6.06, the interior values exhibit a reverse relationship, and the eleven cases in the last two values are too small a sample to be statistically significant.

Slopecode, the next variable, is also a topographic variable, though not at the microenvironmental level. Its high position would seem to indicate that zonal approximations of average slope, especially where those areas are correlated with slope length as at Hop Hill, would be useful in a general prediction equation. However, the actual slope of a particular segment of hillslope, along with the length from the hill crest and the shape of the slope (concave, convex), would be far more useful. But the strength of even this very general variable shows the overall importance of slope in the erosional process. 
Table 6.06 Means of Rillsize Values

\begin{tabular}{|c|c|c|c|c|}
\hline $\begin{array}{l}\text { Value } \\
1 \\
2 \\
3 \\
4\end{array}$ & Explanation & $\underline{N}$ & Mean & St. Dev. \\
\hline \multirow[t]{2}{*}{$\begin{array}{l}1 \\
2 \\
3 \\
4\end{array}$} & $\begin{array}{l}\text { No small channels } \\
\text { Edge of small channels } \\
\text { Center of rill, edge of gully } \\
\text { Center of gully }\end{array}$ & $\begin{array}{r}67 \\
33 \\
7 \\
3\end{array}$ & $\begin{array}{r}3.15 \\
0.34 \\
2.70 \\
-7.82\end{array}$ & $\begin{array}{l}5.71 \\
4.22 \\
6.25 \\
9.38\end{array}$ \\
\hline & Total & 110 & 2.74 & 5.55 \\
\hline
\end{tabular}

Microslope, another individual topographic variable, reduces the effectiveness of the whole equation but oniy slightly. The adjusted $R^{2}$ value drops only .0015 and the residual standard deviation increases by .0043 , while the significance of the entire equation is stil1.059. We feel that its position in the equation marks it as a potentially useful variable, though its meterlong measurement of slope may be too long to represent the micro-relief of a few centimeters suspected to be important in certain kinds of erosion (Young 1972:67). But both microslope and the next step, rockcover, suggest the importance of the micro-areal, semi-permanent variables noted in Table 6.01.

The presence of three significant steps in the equation may indicate that measurement error does not play a large role in the data, but we were concerned that two of the three variables had questionable codes. In addition, the cumulative variable, though it might have the least measurement error, is not especially subtie. On the other hand, the activity variable, which is the absolute total of the bi-weekiy change, would be more susceptible to measurement error and at the same time more sensitive to the other possibilities. Absolute activity was therefore substituted for cumulative land surface change in the same regression equation.

As Table 6.07 shows, the regression equation is statistically significant to the thirteenth step, a much better result than the cumulative variable. The best prediction equation occurs at the eighth step where the residual standard deviation is 10.7256 compared to the standard deviation of the activity variable, which is 11.5016 . If measurement error were a greater factor in activity, we should expect a less significant rather than a more significant equation; w'e conclude that measurement error is not a factor in our data, and that various weekly changes in conditions are possibly more important than the overall cumulative.

An analysis of the relative positions of the variable shows that leafcover, not significant or highly ranked in the cumulative equation, shows up as the most important overall variable, suggesting the importance of rainsplash erosion which it was primarily designed to measure. No easy distinction can be made in this solution between the categories of Table 6.01; but rillsize, another individual variable, shows up second suggesting that, again, individual codes are best. 
Table 6.07 Activity Regression Anaiysis

\begin{tabular}{|c|c|c|c|}
\hline Variable & Step & $\underline{\text { Square }}$ & $\frac{\text { Overa } 11}{\underline{R}}$ \\
\hline $\begin{array}{l}\text { Leafcover } \\
\text { Rill size } \\
\text { Nailcode } \\
\text { Slopecode } \\
\text { Soilcode } \\
\text { North } \\
\text { Basalcover } \\
\text { Microslope } \\
\text { Root type } \\
\text { Root freq. } \\
\text { Treecover } \\
\text { East } \\
\text { Rockcover } \\
\text { Ver }\end{array}$ & $\begin{array}{r}1 \\
2 \\
3 \\
4 \\
5 \\
6 \\
7 \\
8 \\
9 \\
10 \\
11 \\
12 \\
13 \\
14\end{array}$ & $\begin{array}{l}.0679 \\
.0923 \\
.1354 \\
.1506 \\
.1632 \\
.1768 \\
.1895 \\
.2007 \\
.2064 \\
.2103 \\
.2117 \\
.2134 \\
.2147 \\
.2157\end{array}$ & $\begin{array}{l}7.1413 \\
4.9343 \\
5.0101 \\
4.2119 \\
3.6664 \\
3.3278 \\
3.0735 \\
2.8555 \\
2.6007 \\
2.3075 \\
2.1482 \\
1.9664 \\
1.8089 \\
1.6699\end{array}$ \\
\hline
\end{tabular}

On the third step a particular kind of measurement error does stand out. Nailcode, which we expected to show some explanation of the variable of the data, was controlled for and can be separated from a final solution. Slopecode is again important, and this time, soilcode shows up in a higher ranked significant location.

The use of either cumulative land surface or absolute activity makes the interpretation of temporal trends in the data difficult. Many of the microenvironmental variables, especially the seasonal ones, should show some variation in their influence through time. For this reason each of the biweekly. land surface change values was substituted for the cumulative value in ten different regression equations and the results compared. Instead of slopecode and soilcode the ten-week regression solution and the principal components that follow use slopezone and soilzone. Actually, both were used in the first two regressions with similar results, the codes being only slightly more significant. For all practical purposes the two appear to be interchangeable, so it was decided to switch to a variable with fewer cases to suffer ordinal transposition. Table 6.08 shows the best step and order of the variables for each period.

Except for periods 3 and 8, a 17 the equations have a significant prediction equation at some step, though the order of the variables is continually shuffing. Table 6.09 shows the means of the $F$ values of the fourteen variables compared to the $F$ values from the land surface and activity regressions. Rillsize appears to be consistently the best predictor, with slopecode and microsiope not far behind. Leafcover and the other seasonal individual variables are low, but this may reflect their seasonal importance. Vegetation zone, on the other 
Table 6.08 Best Step of Biweek7y Regression Analyses

\begin{tabular}{|c|c|c|c|c|c|c|c|}
\hline Period & Step & $\underline{F}$ & Sig. & $\frac{\text { Adjust. }}{\underline{\mathrm{R}}^{2}}$ & $\frac{\text { Resid. }}{\text { st. Dev. }}$ & St. Dev. & Variables in Stepwise Order \\
\hline 1 & 5 & 3.31 & .009 & .125 & 3.279 & 3.505 & $\begin{array}{l}\text { Rillsize, East, North, Soilzone, } \\
\text { Root frequency }\end{array}$ \\
\hline 2 & 9 & 2.74 & .008 & .162 & 2.460 & 2.688 & $\begin{array}{l}\text { Slopezone, Leafcover, Basal cover, } \\
\text { Soilzone, East, Micros1ope, } \\
\text { Nailcode, Vegetationzone, North }\end{array}$ \\
\hline 3 & 7 & 1.68 & .128 & .055 & 2.247 & 2.312 & $\begin{array}{l}\text { Naflcode, Soilzone, Vegetation } \\
\text { zone, North, Microslope,Basal- } \\
\text { cover, Leafcover }\end{array}$ \\
\hline 4 & 10 & 2.82 & .005 & .188 & 4.550 & 5.035 & $\begin{array}{l}\text { Ril1size, Nailcode, Rockcover, Mi- } \\
\text { croslope, Soilzone, North, Basa 1- } \\
\text { cover, Root frequency, Roottype, East }\end{array}$ \\
\hline 5 & 4 & 2.61 & .042 & .074 & 3.481 & 3.617 & $\begin{array}{l}\text { Rockcover, Treecover,Micros lope, } \\
\text { Rootype }\end{array}$ \\
\hline 6 & 11 & 3.13 & .002 & .224 & 2.914 & 3.308 & $\begin{array}{l}\text { Microslope, Soil, Rootype, Basalcover, } \\
\text { Leafcover, East, Rillsize, Rockcover, } \\
\text { Root frequency, Vegetation zone, } \\
\text { North }\end{array}$ \\
\hline 7 & 8 & 3.73 & .001 & .212 & 2.813 & 3.170 & $\begin{array}{l}\text { Soilzone, Vegetation zone,Micro- } \\
\text { slope,Slopezone, Basalcover, Root } \\
\text { frequency, North,Rillsize }\end{array}$ \\
\hline 8 & 4 & 1.74 & .149 & .035 & 2.667 & 2.715 & Nailcode,S1opezone,East,Basalcover \\
\hline 9 & 1 & 8.27 & .005 & .082 & 3.611 & 3.770 & Vegetation zone \\
\hline 10 & 5 & 7.07 & .000 & .273 & 1.843 & 2.161 & $\begin{array}{l}\text { Vegetation zone, Slopezone, Nailcode, } \\
\text { Microslope, Rilistze }\end{array}$ \\
\hline
\end{tabular}


Table 6.09 Comparative $F$ Values

\begin{tabular}{lccc} 
Variable & Biweekly $F$ & Cumulative $F$ & Activity $F$ \\
\cline { 2 - 3 } Veg, zone & & & \\
Rilisize & 3.187 & .048 & .107 \\
Microslope & 2.505 & 4.024 & 2.610 \\
Soilzone & 2.271 & .853 & 1.267 \\
Nailcode & 2.139 & $.151^{*}$ & $1.411^{*}$ \\
Slopezone & 1.922 & .812 & 4.777 \\
North & 1.579 & $1.394+$ & $1.707+$ \\
Basalcover & 1.303 & .479 & 1.531 \\
East & 1.178 & .548 & 1.451 \\
Root freq. & .959 & .016 & .186 \\
Leafcover & .936 & .944 & .443 \\
Rockcover & .913 & 1.009 & 7.147 \\
Root type & .827 & .664 & .150 \\
Treecover & .792 & .010 & .650 \\
& .523 & 3.056 & .151 \\
& *Soilcode & tSlopecode &
\end{tabular}

Table 6.10 Climate Vs. Significance

\begin{tabular}{|c|c|c|c|c|c|c|c|}
\hline Period & $\underline{F}$ & Sig. & Rain & Days & - DaiTy & Storms & Frost \\
\hline 1 & 3.37 & .009 & $\frac{1.79}{1.79}$ & 14 & $\frac{\mathrm{nam}}{.057}$ & 0 & 0 \\
\hline 2 & 2.74 & .008 & 0.00 & 14 & .000 & 0 & 0 \\
\hline 3 & 1.68 & .128 & 0.11 & 14 & .008 & 1 & 0 \\
\hline 4 & 2.82 & .005 & 5.69 & 27 & .271 & 0 & 0 \\
\hline 5 & 2.67 & .074 & 0.35 & 7 & .050 & 0 & 0 \\
\hline 6 & 3.73 & .002 & 1.33 & 14 & .095 & 3 & 0 \\
\hline 7 & 3.73 & .007 & 3.19 & 21 & .152 & 0 & 1 \\
\hline 8 & 1.74 & .749 & 0.00 & 7 & .000 & 0 & 0 \\
\hline 9 & 8.27 & .005 & 0.72 & 14 & .057 & 0 & 3 \\
\hline 10 & 7.07 & .000 & 0.16 & 14 & .017 & 0 & 7 \\
\hline Cumula. & & & & & & & \\
\hline tive & 2.86 & .047 & 12.26 & 140 & .088 & 4 & 11 \\
\hline
\end{tabular}

Climatic data furnished by Norman Dietel of Fredericksburg Radio-Post 
Table 6.11 Varimax Rotated Components Matrix

\begin{tabular}{|c|c|c|c|c|c|c|}
\hline Variable & $\frac{\text { Comp. }}{\underline{I}}$ & $\frac{\text { Comp. }}{I I}$ & $\frac{\text { Comp. }}{\text { III }}$ & $\frac{\text { Comp. }}{\text { IV }}$ & $\frac{\text { Comp. }}{\underline{v}}$ & $\frac{\text { Comp. }}{\text { VI }}$ \\
\hline $\begin{array}{l}\text { East } \\
\text { North } \\
\text { Leafcover } \\
\text { Rockcover } \\
\text { Treecover } \\
\text { Basalcover } \\
\text { Microslope } \\
\text { Rillsize } \\
\text { Veg. zone } \\
\text { Root freq. } \\
\text { Root type } \\
\text { Nailcode } \\
\text { Soilzone } \\
\text { Slopezone } \\
\text { Frost } \\
\text { Storms } \\
\text { Daily Rain }\end{array}$ & $\begin{array}{r}.912 \\
.691 \\
.796 \\
.025 \\
-.093 \\
.861 \\
.203 \\
-.023 \\
-.474 \\
-.007 \\
.068 \\
-.122 \\
.051 \\
.282 \\
-.383 \\
.122 \\
-.104\end{array}$ & $\begin{array}{r}.062 \\
.131 \\
-.246 \\
.386 \\
-.206 \\
-.216 \\
.064 \\
.839 \\
-.116 \\
.905 \\
.464 \\
.139 \\
.276 \\
-.267 \\
-.172 \\
-.017 \\
.937\end{array}$ & $\begin{array}{r}-.163 \\
-.188 \\
.119 \\
-.223 \\
-.140 \\
-.136 \\
.362 \\
-.229 \\
.829 \\
-.156 \\
-.330 \\
.542 \\
.136 \\
.845 \\
.817 \\
-.264 \\
-.028\end{array}$ & $\begin{array}{r}.089 \\
-.451 \\
.082 \\
.804 \\
.924 \\
-. .770 \\
.044 \\
-.155 \\
-.178 \\
.017 \\
.436 \\
-.167 \\
-.259 \\
-.119 \\
-.099 \\
-.095 \\
.077\end{array}$ & $\begin{array}{r}-.230 \\
.312 \\
.492 \\
.305 \\
-.116 \\
.375 \\
.868 \\
-.246 \\
.054 \\
.274 \\
.651 \\
.017 \\
.346 \\
-.247 \\
.064 \\
.936 \\
.052\end{array}$ & $\begin{array}{r}-.007 \\
.054 \\
.068 \\
-.210 \\
.057 \\
.128 \\
.227 \\
-.247 \\
.004 \\
.272 \\
.009 \\
-.766 \\
.839 \\
.090 \\
-.309 \\
.075 \\
.089\end{array}$ \\
\hline
\end{tabular}

hand, also a seasonal influence, comes out the strongest of al1, due to its high $F$ value (13.89767) in period 10--recorded during the first two weeks of December, a time when vegetation influences should not be overly great on erosion. It is also difficult to decide if the individual semi-permanent variables show up strongly here, though two of the best four are of this type.

Analysis of the average values does not allow the interpretation of temporal change in climatic patterns which might affect erosion in various ways. Differing amounts and intensities of rainfall could account for the variation from period to period. Table 6.10 shows the significance of each best step compared to available climatic data. The most significant $F$ values seem to correlate with either amount of rainfall or frosts; a definite influence of climate seems to be indicated.

Several problems are present in the interpretation of this data. One of the most important is that Fredericksburg is at least twe lve miles from Hop Hill and we have no way of knowing how closely this data fits the site. Data from other researchers indicates that a high variable of rainfall intensity occurs across as smal1 an area as a half acre (Wischmeier et al. 1958). Inferences made from the Fredericksburg rainfall data must be considered as rough guidelines only. Another factor which we have not attempted to measure in the equation is the influence of wind erosion. While we have no way of measuring the amount 
of wind at any given time, the hot, dry period 2 during the first two weeks of August shows some signs of this.

The data presented so far suggests that the erosion recorded at the site is fairly accurate and that different climatic conditions have brought about different amounts of erosion under different microenvironmental situations. As a measure of this effect, a principal components analysis (SPSS Factor procedure; Nie et al. 1975) was performed using the $F$ values for each of the fourteen variables at the last step of each bi-week $7 y$ regression solution in combination with the average daily rainfal1, the number of thunderstorms and the number of days with below freezing temperatures in each period. Six principal components had eigenvalues greater than 1.0. Table 6.11 shows the component matrix after varimax rotation with Kaiser nomalization.

Factors one, four and six are simply correlations within the $F$ values themselves, with no associated climatic data. Component two combines rainfali with both root type and root frequency as well as rillsize, factors which we might well expect to influence erosion under different conditions of rainfall. Component five associates storms, of which there were few, with microslope and leafcover, as well as root type again. Heavier rains associated with storms might make leafcover a more active variable, while sheetwash under such conditons might make microslope more important than rillsize, since rills would be overrun. Factor four combines frost with slopezone, vegetation zone and nailcode. The heaving ordinarily associated with frosts would naturally act differently on stakes and nails and cause this variable to become significant; vegetation zones (which are somewhat collinear with slopezones at Hop Hili) might provide differential protection to soils under frost conditons.

Climate, as should be expected, appears to be responsible for much of the variability in the bi-weekly erosion. This supports our previously stated hypothes is of differing effects under different microenviromental conditions.

None of the other hypotheses can be entirely discounted, but the analysis does not strongly support them. While the anaiysis could be carried further and a refined prediction equation derived, we feel that the rapidly changing environment of Hop Hill would make a normaliy complex prediction next to impossible. Further analysis will be confined to the zonal breakdowns.

\section{Conclusion}

The previous analysis has shown some of the major influential factors in erosion. In the components analysis, both rain and storms were shown to be related to individual variables, both seasonal and semi-permanent, while frost was associated with the zonal variables, specifically vegetation and slope zones. The soilzone variable was not associated strongly with any particular climatic factor; in addition, soilzone was negatively associated with nailcode, and may be less susceptible to error of this kind. A11 three zonal variables appear to be significant factors in land surface change and might be useful in 
delimiting erosional activity areas. Tables $6.12,6.13$, and 6.14 present the breakdowns of these variables. Because of missing values at some points, many of the sample sizes are too small to be taken seriously, but we feel that the larger groups, with 25 or more points, are probably an approximate average of any area.

Table 6.12 Land Surface Change By STopezone

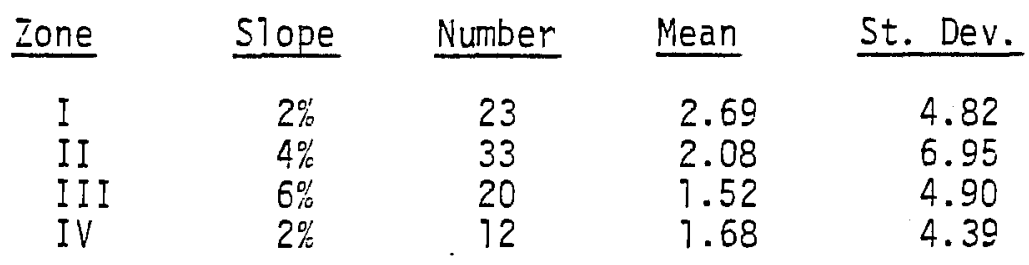

Mean land surface change in millimeters

Percent grade is rough estimate

Looking at mean land surface change in the slope zones, we find that while no zone shows erosion, the least deposition is in Zone III with the highest slopes. The greatest deposition is in Zone I, the grassy meadow to the south of the stock pens. Only Zone IV, the grassy strip at the north, does not seem to fit, but all the measurements in this area were taken at stakes which, as noted before, are more susceptible to error. The sample size of this area is also quite small (Table 6.12).

Table 6.13 Land Surface Change by Soil Zone

$\begin{array}{lrrr}\text { Luckenbach } & 2 & -0.50 & .71 \\ \text { Purves } & 21 & 2.46 & 5.96 \\ \text { Pedernales } & 41 & 1.90 & 4.59 \\ \text { Hensley } & 46 & 2.33 & 6.29\end{array}$

Mean land surface change in millimeters

Soilzone, because of its negative correlation with nailcode in the components analys is and because it was not associated with any particular type of climatic effect, may be the best dividing variable for the site (Table 6.13). Luckenbach soil with two cases is obviously too small, but the others, especial7y the Pedernales and Hensley soils, should be a fair measure of the differences between these areas. In this case Hensley soil shows to be less susceptible to erosion than Pedernales while Purves has the most deposition of a11. In the montmorillonitic clay of the Purves soil nails might tend to sink, thus recording a false deposition, but this area does seem to be higher than the rest of the site. 
the artifact, the material would move downslope slightiy. In this case larger material would break down its pedestals faster and probabiy roll farther downhill (depending on its shape) due to the action of gravity. In the rills, pedestaling would be less prominent and the concentration of flow should move smaller flakes first and bigger pieces last if at all. The rate of movement would depend on the amount of flow and size of the rill. At any rate, some movement can be expected at all areas of the site which have a noticeable slope; in most cases this movement will involve sorting of artifacts by size, weight and shape, creating a recognizable distribution pattern.

A full correlation of the distribution of material from the surface of the site with various erosional parameters could prove useful. This would in turn be aided by studies of the distribution pattern itself. Any site activity analysis should include these two tests to carefully assess the possibility that the activity might be geomorphological as well as human.

With particular reference to Hop Hill, we feel that the results of the surface survey should be interpreted carefully. It would be wise to assume that all material in a rill is not in its original position and that material found close to a rill should be suspect. With the movement of soils downhill, some redeposition is possible in low-lying areas and in small hollows. Therefore, even material which appears to be eroding out of the ground, or material that is found in the first few centimeters of soil, should be suspect.

With regard to any future work at the site, the suggestions are obvious though the exact needs of a given research problem will dictate how much disturbance can be tolerated. Excavation in the Purves soil of the eastern pens should be avoided because of its montmorillonitic clay content; artifacts are susceptible to vertical mixing. Slope Zone III appears to have seen the greatest erosion, but the northwest corner of the site through which the terraced field has drained for years may also have seen serious erosion. All major rills should be avoided.

We think, however, that in any area other than the partial vertisol Purves, that the disturbance caused by erosion, deposition and hogs' feet was confined to the first few centimeters below the surface and relationships beneath this point should be intact. 
seems little doubt that large amounts of soil material were removed and lithic remains disturbed or removed completely. At this time there was probably little or no vegetation in the pens, although observations at other stock pens show that preferential use by hogs of certain areas can leave others covered with brush and small trees. If the soil was at least partially saturated part of the time then erosion would proceed quickly during rainstorms.

After the site was abandoned, the pens remained completely devoid of vegetation for some time. As late as December of 1975 there was no vegetation whatever in the pens (See 10.0). During this period it might be surmised that erosion and disturbance were at a maximum (without the aid of the hogs' trampling, of course). Toward the summer a weed cover sprang up over much of the pen area though a lot of the area was still bare. One of the rainiest julys on record caused what appeared to be huge quantities of erosion at the same time as rapid plant growth. By. October the pen area was fairly thickly covered in many places; it had reached a maximum. In December of 1976 some of the vegetation had died off but the site was not bare as in the year before. The pen area was obviousiy returning to a wild state. This would suggest less erosion and this is what the figures show.

Two problems exist in this definition of deposition. One, more intense rains in spring and early summer may cause more erosion than all other times together. Two, whatever error there is outside of measurements is probably most reflected in nails being stepped on by animals, stray tourists, and unwitting archaeologists. The trend here would show deposition where it is not happening. This cannot really be checked out using our method; only the vigil network system where the nail heads are shot in by transit (Emmett 1965). would eliminate such error, but this is costly and time-consuming. In the later measurements frost heaving may have some effect on the nails but the vigil network survey found this to be negligible.

Although we came up with a value for each point and an average for the whole site, we do not feel that such measurement would be useful at every site with erosion problems. We do, however, feel that it is important to keep in mind such factors when making a surface survey.

Another interesting discovery is that al though the total land surface movement was not particularly great, the absolute activity variable (if it is a valid measure of what is moving through the points) shows that many times more soil is moving through than is actually deposited. Much of this movement is probabiy due to rainsplash erosion, which, though it has a net downs lope component, probabiy expends a great deal of energy and moves much soil to push just a little downhill. It is also possible that some of this movement indicates wash from erosion higher up the hill, but from where is uncertain.

It is not easy to predict how erosion would affect artifact location but there are several possibilities. Where no rill is present, water transport of any collectible lithic material larger than medium-sized flakes should be regligible. The rest of the material would be stood up on pedestals from rainsplash erosion, and when they broke down due to frost or simpiy under the weight of 
Pupoides albilabris is à very smal1 species no larger than $1 / 8$ inch in length. It is most abundant in limestone areas where it may be found under rocks, in grass roots and under leaf litter in sparse woodlands (Cheatum and Fullington 1973).

The gastropod fauna of Hop Hill suggest an environment of grassland prairie to open or sparse woodlands in a limestone area. Although moisture requirements are not known, all species are drought-resistant, suggesting at least a seasonally dry environment. This would not be significantly difierent from the habitat and rainfall of the area at present.

The total count of species from general provenience is as follows: 91 shells were identified as Helicina orbiculata tropica, 28 were Polygyra texasiana texasiana, 21 were Rabdotus dealbatus cf. mooreanus, 15 fragments were

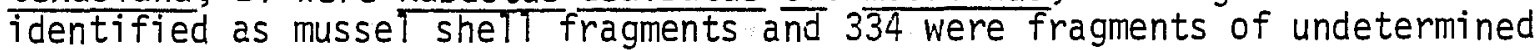
nature.

Two unsifted matrix samples were also screened through $1 / 4-i$ nch mesh and 1/32inch mesh. These samples were water-screened. The sample size was approximately $2000 \mathrm{cc}$. The first sample from Zone" 2 (932E,-971.N,) Occupation Floors 2.2 , and contained 2 Rabdotus dealbatus cf. mooreanus, 15 fragments albilabris and 100 unidentifiable fragments. The sample from below 2.0 in the same square contained 2 Helicina orbiculata tropica and 68 fragments. The results of the fine screen process yielded no snails in any quantity sufficient to permit a further refinement of the above habitat requirements.

\subsection{ENVIRONMENTAL IMPLICATIONS FROM MOLLUSCAN ANALYSIS (Royce Mahula)}

As noted in the foregoing analysis of mollusks from the Hop Hill site, land snails may be a source of information about prehistoric environments and change in those environments through time. This information is generally considered to be in the nature of preferred vegetation and habitat rather than on actual precipitation conditions due to the fact that the snails are resistant to drought. For this reason climatic change inferences have largely not been made except indirectly through changes in vegetation.

Though it is recognized that all species found at Hop Hill are drought resistant and do occur regularly in the same locality, there are some recognized differences between species in preferred climate. Helicina orbiculata tropica (Type 1) and Polygyra texasiana texasiana (Type 2) tend to prefer a slightly moister environment while Rabdotus dealbatus

(Type 3) prefers a more arid one (Ken Lord, personal communication).

It was our contention that, taking these preferences into consideration, changes in the proportions of each type through time could be an indicator of change in environment/climate. It is not necessarily the number of mollusks per type per level, but rather the change in proportion of snails per type per level which may be diagnostic of climatic variation. 


\subsection{MOLLUSKS FROM THE HOP HILL SITE (Kenneth J. Lord)}

The molluscan fauna from the Hop Hill site (41GL21) have been examined from both matrix samples collected from the site and from general provenience. The molluscan remains include both aquatic and terrestrial species. While the aquatic species have not been identified due to their fragmentary nature, four species of terrestrial gastropods were identified.

The terrestrial gastropods are all species which are found in the area at present. Land snails can be used to give rough environmental information about an area. The information generally is a geographic range and a preferred habitat. This information, when used in a stratigraphic context, can indicate changes in habitat through time. The species generally have preferred plant cover and to a lesser extent, moisture requirements. The moisture problem is most difficult since in times of drought, the majority of the resident species seal their apertures to prevent water loss and in effect become dormant. Species identification, for this reason, does not give us precipitation information. However, the preferred plant cover of the snails does give an indication of predominant vegetation and habitat which can in turn yield precipitation figures.

The four species of 1 and snails from the Hop Hill site are: Helicina orbiculata tropica, Polygyra texasiana texasiana, Rabdotus (Bulimulus) dealbatus $c$ f. mooreanus and pupoides albilabris. These species vary greatly in the numbers identified, which may be due in part to recovery techniques in two cases (Polygyra and Pupoides are smal1 and may pass through $1 / 4$ inch hardware mesh) and differences within the community structure itself. All species are very drought-resistant.

Helicina orbiculata tropica is a species which ranges statewide over Texas with the exception of extreme west Texas. Its fullest development comes in the Cretaceous terrain of Texas. Its habitat includes the weeds and grasses in pasture lands as well as protected woodland regions. In the Dallas region, the species has been observed ascending trees, usually of hackberry (Celtis occidentalis) and cedar elm (U1mas crassifolia) (Pilsbry 1948). It is extremely resistant to drought.

Polygyra texasiana texasiana is a species which occupies a variety of habitats ranging from deep woodlands to open prairies. It is usually found under rocks and other objects. This species is drought-resistant by sealing its aperture to prevent water loss (Cheatum and Fullington 1973).

Rabdotus dealbatus cf. mooreanus is abundant in central-southern Texas, particularty in the region around San Antonio. This species lives in vast numbers in mesquite chapparal but can be found less commonly in a prairie habitat. The species normally ascends bushes, adhering to the bark. Growth is rapid in wet weather and suspended during the dry season. In general the solidity of the shell is greatest in regions of low rainfall (Pilsbry 1948). 
Summing number of snails per depth indicates that the midden level ( $\left.D_{3}\right)$ contained the greatest concentration of snails (70 specimens). Occupation floors inmediately above the midden ( $D_{2}$ ) have fewer (48) specimens in spite of a larger excavated area; and finally, Stratum 1 (DI) contains only 16 specimens. This trend is to be expected since middens support a large snai] population due to the presence of the abundant organic refuse upon which they feed (Ken Lord, personal communication). However, it may be noted that Type 3 (Rabdotus), which is most commonly noted in middens, is not most highly represented in the midden occupation, but rather in the occupation floors directly overlying the midden.

\section{Conclusions}

Analys is of the proportions of mollusks by depth at Hop Hill reveals evidence for climatic variation through time at the site. Types 1 and 2 (Helicina orbiculata tropica and Polygyra texasiana texasiana) behave together and prefer a slightly moister environment, while Type 3 (Rabdotus dealbatus) behaves alone and prefers a more arid one. Change in proportions between Types 1 and 2 and Type 3 is inverse and suggests a switch to a more arid climate during the upper occupations (OF 1.0, 1.5 and 2.0). Also, the increased number of snails in $D_{3}$ (midden) may be correlated with the genera] character of middens, which contain a high concentration of refuse and thus attract large snail populations. 


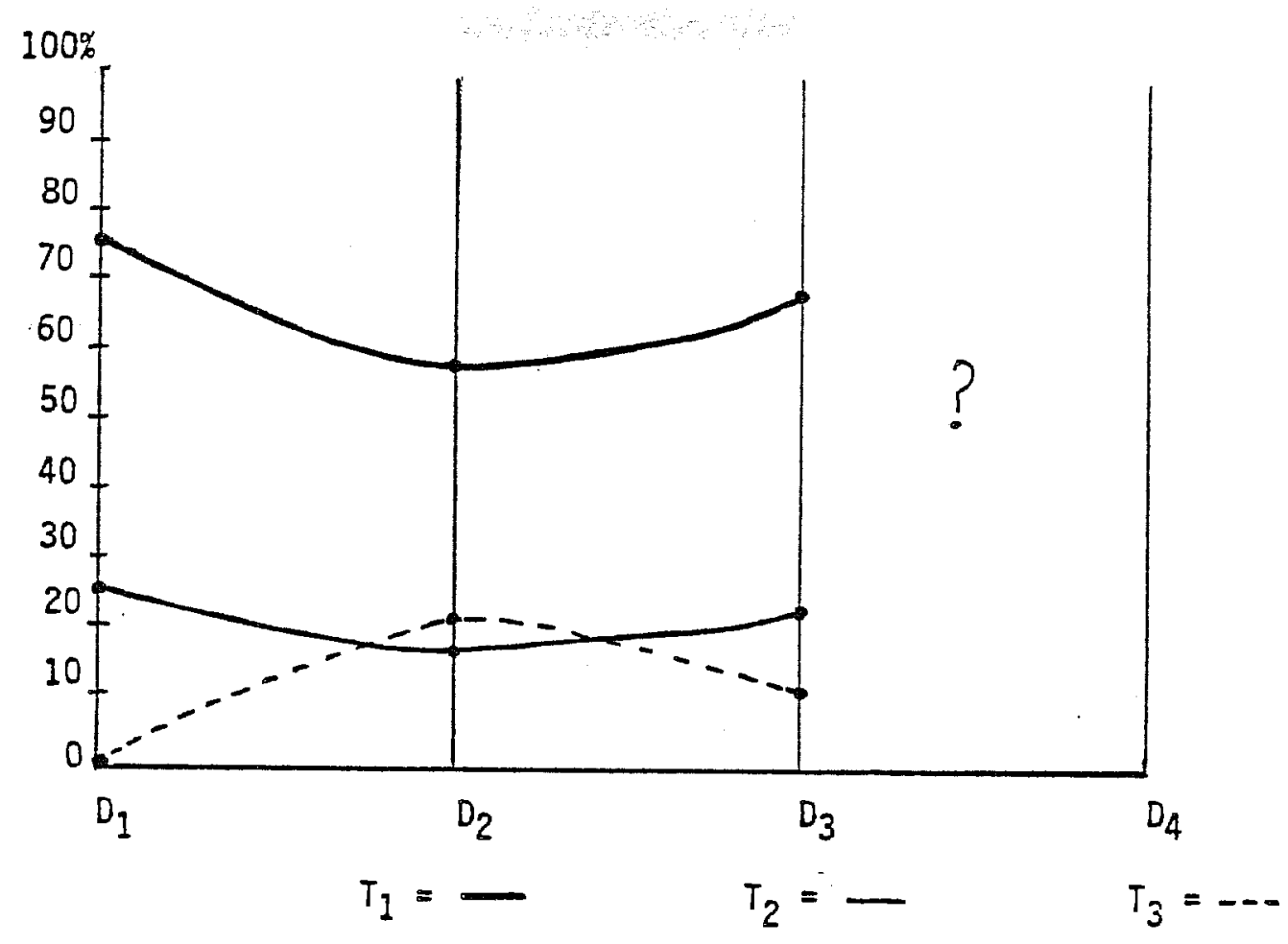

Figure 7.01. Change in Proportions of Snail Types Through Time.

To pursue this possibility the percentage of the total snail popuation represented by each type by depth was calculated and plotted as shown in Figure 7.01. D 1 represents the surface and Stratum 1; $D_{2}$, the upper occupation floors (OF 1.0, 1.5 and 2.0); $D_{3}$, the midden proper (OF $2.5,2.7$ and $2: 0-10$ ); $D_{4}$, Stratum 3. However, since only 1 snail (Type 1) was recovered this depth was not included in the interpretation.

Examination of the graph reveals interesting interactions between the three predominant types. First, it may be noted that Types 1 and 2 behave together: as the percentage of one increases or decreases, so does the percentage of the other. This is substantiated by Ken Lord (personal communication). The two species are usually associated with one another and often "travel" together. Type 3, on the other hand, behaves in an opposite manner. As the percentage of Types 1 and 2 increases, the percentage of Type 3 decreases; as the percentage of Types 1 and 2 decreases, the percentage of Type 3 increases. Since, as described earlier, Types 1 and 2 prefer a moister environment while Type 3 prefers a more arid one, the inverse relationship between these types is evidence of some change in $\mathrm{climate}$ through time. The upper occupations of $\mathrm{D}_{2}$ apparently were characterized by a somewhat more arid environment than either the preceding midden $\left(D_{3}\right)$ or the later levels $\left(D_{1}\right)$. 
We saw children who had been nursed by their mothers until their third year, leave their mothers' breast, jump on a horse, and light a cigarette.

For many years it was assumed that the Indians obtained abandoned or lost horses from the DeSoto or Coronado expedition in 1541. Even if the Indians had acquired a few strays, it is unlikely that they would have known what to do with them. The skill and technique of riding, and the care and training of horses, are not learned casually. It was the early 17th century Spanish settlements in New Mexico, particularly in the neighborhood of Santa Fe, that were the centers from which the horse complex spread to the Indians (Newcomb 1961:86). In 1598, the future governor and Captain General of the colony of New Mexico, Don Juan de Onate, rode into the Southwest. He brought some 400 soldiers, priests and families, and with them were 7,000 animals including 300 mares and colts. The Pueblo Indians were forced to take care of the horses. Soon the Indians were escaping from the Spaniards and taking with them the horses and considerable equine know-how. These escapees were the most obvious bearers of horses and equestrian art to the Indians. By 1659 , the Spanish settlements of New Mexico were being raided by Apaches from the northeast. In 1680, the Pueblo Indians revolted, and the Spanish settlers were driven out or killed, leaving the Indians heirs to large herds of sheep, cattle and horses. The Pueblo Indians were sedentary gardeners, but the horses they acquired were eagerly sought by wandering tribes. By the end of the 17th century, al1 Texas Indians had become familiar with horses and their source was like a magnet, drawing al1 sorts of Indians anxious to acquire this wonderful new anima 1 (Newcomb 1961:86-88).

In 1705, the Comanches, in company with their linguistic relatives the Utes, appeared in New Mexico. "They came begging peace, but on departing they stole some horses, thus marking the arrival of the greatest horse thieves of them al1" (Thomas 1935). By the 18th century the horse was spreading far beyond the borders of Texas, and by 1750 horses were common through the Plains, even as far north as Saskatchewan. Indians of diverse origins still held the land in Texas, but their culture had been pervaded by foreign influences for more than two hundred years. Exchanges between cultures occurred almost immediately upon contact: smallpox and measles for syphilis, smoking tobacco for whiskey and rum. Horses from the Spanish, guns from the French and iron from both were acquired by some of the Indians, and these added greatiy to Indian technology, resulting in a change of subsistence and social organization as well (Skeels 1972:xv,xvi).

Prior to the introduction of the horse, there were few nomadic Indians on the Plains proper. The coming of the horse to the Southwest, and the westward displacement of tribes pressured by eastern Indians armed with guns, changed all this at the beginning of the 17 th century. Walter Prescott Webb said that "Steam electricity has wrought no greater changes in our culture than did the horse in the culture of the Plains Indian." They went from infantrymen to fierce cavalrymen, and became dangerous warriors and insatiable raiders. With increased mobility, they mastered the buffalo and expanded their trade. Their superb nomadic horsemanship and dauntless military prowess enabled them to guard vast semi-arid lands that they won and claimed as their own (Wallace and Hoebel 1952:34-35). 


\subsection{ETHNOHISTORY (Sue Turner)}

The earliest historically recorded inhabitants of the Central Texas region were primitive cultures (Sjoberg 1953) belonging to Coahuiltecan and Apachean language stocks. They probably are the closest analogues to the prehistoric populations and are discussed in other sections of this volume. These people were eventually displaced by groups with more complex cultures which were either directly or indirectly of European derivation. The mounted Plains cultures such as the Comanches and the Europeans, especially Germans, e.tc., constitute this group.

As diverse as the background of these various groups were, they all eventualiy faced the same problem: how to bend the resources! of the Pedernales River Valley to their own benefit. The following is an account of those efforts.

\section{Indians of Texas}

The Plains tribes vitally associated with Texas in the 1700-1800s were Kiowa Apaches, Kiowas, and especially Comanches. The Kiowa Apaches, close cultural relatives of the Lipan Apaches, joined the Kiowas at some unknown date. These confederate tribes, along with the Comanches, are often thought of at the "real" Texas Indians. In many respects their position is justified, yet the Comanches and Kiowas were not indigenous to the state and did not appear within its borders unti1 after 1700 (Newcomb 1961:21-22). The Kiowas, Comanches and Kiowa Apaches were the fiercest of the Plains Indians. They were highly mobile and organized for one objective, the buffalo hunt. They lived in tipis which could be easily packed and moved. The Comanches apparently migrated from their fellow Shoshones in Wyoming, and came south to be near the buffalo herds in New Mexico, Texas and 01d Mexico. They forced many of the Plains Indians into the Trans-Pecos country. The Kiowa-Comanche alliance lasted until they were subdued by the white men in the late 19th century. The Kiowa Apaches were Apaches who attached themselves to the south Plains Kiowas, but retained their own language (Newcomb 1961:195-200).

For centuries the indigenous Indians had stabilized their lives using local resources which served their culturally important goals with technologies available to them. This balance was first upset by Europeans in the 16th century bringing diseases which devastated their ranks.

During the first half of the 16th century, three parties of Spaniards entered Texas. Cabeza de Vaca crossed the area in the 1530s. Early conquistadores left their mark on the future of the region although they were small exploration parties crossing the land in search of treasure, and seldom seeking permanent habitation. When Coronado returned to Mexico in the spring of 1543, he left behind in Texas and Oklahoma most of the horses and cattle his band had brought. The question of how and when the Indians acquired horses has always been an intriguing one, especially since many Indians were bewildered and terrified by the first horses they saw. Several centuries later, these same tribes were among the finest, most skilled equestrian people the world has ever known. In an old issue of the Magazine of Literature for Abroad, "Meusebach's Expedition into the Territory of the Comanche Indians," January 1847, the author claims when referring to the riding skills of the Indians: 
Under vegetation zone (Table 6.14), we find that both Zone $A$ and Zone $C$ have few points. Zone A almost directly corresponds to Slope Zone IV and exhibits the same low deposition, while Zone $C$ is in the Purves soil area where, as we have noted, deposition may not be as high as recorded. Otherwise, Zone $B$ and Zone $D$ show the relationship one would expect, that is, less deposition in the sparser cover of Zone B.

Over the entire site, the ten-period average for 124 points is 1.656 milijmeters of deposition with a standard deviation of 5.317 . Projected to a year, without any consideration of climatic change, this would give a figure of 4.377 millimeters of deposition per year. It is likely that changes in erosive potential of rainfall during the year could alter this estimate in either direction but it is a good guess for the entire site over the last year and possibly a year or two in the future. Farther back in time the site was almost completely barren in the pen area and this figure would have certainly not been as high. And at some point in the future the stock pens will become totally covered by the natural grass vegetation (assuming no disturbance by the park) and deposition may increase.

$\begin{array}{cccc}\text { Tabie } 6.14 & \text { Land Surface Change by Vegetation Zone } \\ \text { Zone } & \text { Number } & \text { Mean } & \text { St. Dev. } \\ \text { A } & 10 & 0.85 & 4.23 \\ \text { B } & 50 & 1.94 & 6.37 \\ \text { C } & 11 & 4.65 & 6.84 \\ \text { D } & 31 & 2.09 & 4.40\end{array}$

Mean land surface change in millimeters

The reliability of this figure can be insured by comparison with estimates of soil formation quoted elsewhere. Though the process is quite complex, Hudson notes that under undisturbed conditions, it takes an average of 300 years to produce 25 millimeters of top soil (Hudson 1971:35) and on 7 y 30 years where the soil has been disturbed by tilling. At the rate we have measured it would take 57.9 years to form that 25 millimeters of soil. It seems possible that the disturbance caused by the hogs could have had a similar effect to tillage in the speeding up of the process, or it might be that some soil is still moving downhill from a location we have yet to measure. One such possible location which we suspect is still eroding fairly rapidly is the large bare patch just inside the southwestern stock pen. This area remains bare because of the numerous large red ant beds, noted as a factor in erosion (Young 1972:67) by geomorphologists.

Before the stockpen was put in only grazing disturbed the site, as well as occasional flooding or drought related erosion. It seems likely that soil was formed at least as fast as it was removed except in unusual circumstances like overgrazing during a drought. While the stock pen was on the site there 
By 1836, the Comanches claimed and occupied al1 of the country from the Arkansas River on the north to the Mexican settlements on the south, and from Grand Cordellera on the west to the Cross Timbers on the east according to the report of the U.S. Indian agent for the Osages.

The Comanches and their Kiowa allies preferred to camp near a running stream in open timber. Their villages were found in creek valleys at the headwaters of larger streams where there were abundant canyons, arroyos, and breaks for protection. Here too were abundant grasses for their horse herds, ample buffalo and antelope for food, and sweet water for drinking (Wallace and Hoebel 1952:15). The hides of buffalo, flesh side out were stretched over a framework of 12-30 poles on their tipis. Hides were tanned by soaking the skin on the hair side with a mixture of wood ashes and water which produces lye. Lime obtained from burned rock was sometimes used in place of ashes (Wallace and Hoebel 1952:93). They had no clan organization, but moved in autonomous units of family bands and groups. However, a 17 Comanches, whatever their band were "Of the People." Through their dress, speech, thought and action, the Comanches held a common bond of identity and affinity that set them off from all Indians, from all the rest of the world (Wallace and Hoebel 1952:22). When Meusebach and Roemer visited the Comanche village to negotiate the Peace Treaty in 1847, Roemer spoke of a troop of twenty warriors that passed their tent. He said it was a beautiful sight; "Their shields and lances in addition to their peculiar headdresses reminded them of days of Knighthood." They were awesome hunters as well as warriors. A bullet would not always penetrate the shaggy skin of the buffalo, but the Comanches could shoot their bois d'arc bow and arrows with such force that the arrow emerged on the opposite side of the buffalo. The long iron points on their spears and arrows often consisted of a toledo sword blade several hundred years old (Roemer 1935:275).

The Indians, primarily the Comanches, remained in conflict for parts of Texas until the last quarter of the century. In the 1830s the Comanches numbered about 20,000, but the Anglo-American Texans were eventually victorious, and the Texas Indian cultures were obliterated by the onrushing frontier. By 1911 , there were on 1y 1,171 survivors.

Spanish Settlement on the San Saba River

Prior to 1820, Texas was a part of Mexico under Spanish rule, and before the first white settlements in the state which were of any real significance, the Catholic Missions were established for the purpose of converting the Indian tribes who until then held undisputed possession of the 7 and. In 1757 Father Terreros and fourother Franciscans, accompanied by nine families of Christianized Indians from Coahuila, and one hundred troops under the command of Colonel Diego Ortiz deParilla, founded the Mission San Saba de la Santa Cruz on the south bank of the San Saba River near the present Menard and about $100 \mathrm{~km}$ north of the Hop Hill locality (Figure 2.01). Upstream, Colonel Parilla built the massive stone presidio San Luis de las Amarillas. The priests were able to persuade many Lipan Apaches to visit the mission, and in June 1758 some three thousand Lipans were encamped around Santa Cruz. Before the end of the summer, most of them had left on a buffalo hunt, and that winter a few returned with rumors of a Comanche plan to attack the 
Spaniards in the spring. Parilla urged the priests to seek safety in the presidio, but they refused. In March of 1759, two thousand Comanches suddenly surrounded the mission and easily gained admission into the stockade under the pretext of friendship. They burned the buildings and killed all of the mission personnel except for four men who survived the smoldering ruins. The Spaniards successfully defended the later attack on the presidio.

A massive punitive expedition led by Parilla was sent from San Antonio against the Comanches. The expedition was a fiasco. No army--Spanish, Mexican, Texan, or American--could force the mounted nomads of the Plains into pitched battle in their home territory. At the approach of a substantial force, the Indians simply scattered and regrouped later. They had no permanent villages that could be attacked and no supply lines to be cut. Spain never again attempted a military expedition against the Comanches (Connor 1971:36-41).

German Settlers of the 19th Century

The Germans were the largest groups of European immigrant farmers that settled in 19th century Texas. Nature had given the Southwest vast wealth, and the Germans shaped their lives in response to the obstacles that nature put in their way.

The most critically important resource in the Southwest is water. The relatively low rainfall and relatively hot.climate are conditions generally unfavorable for farming (Ladd 1957 (1):1-1501, 1-3301, (2):8-2081-2082).

The first half-century was the crucial period in the development of the German immigrants' agricultural: way of life. During this forty- to fifty- year period, all major adaptations and changes were accomplished, and those aspects of their old World farming heritage that were to survive in the homeland met their great testing time. By the outbreak of the Civil War, the idea was well established that German farmers in Texas were something special (Jordan 1966: 40-45). After the War of Independence, and the establishing of the Republic of Texas, immigration increased significantly. The government was liberal in granting land to the immigrants, and as a result many settled in the interim.

German nobility became the organizers of the German emigration to Texas. They were mostly officers, who through widespread publicity of Texas and her War of Independence, got the idea to sponsor this colonization endeavor, though they were not the best qualified (Wisseman 1869:14). Count Castell, an officer in the Austrian garrison in the federal fortress of Mainz, initiated the movement. The result was the organization of the Mainzer Adelsverein, a society whose membership was composed entirely of titled Germans who were interested in overseas colonization for both economic and philanthropic reasons. Under the supervision of Prince Carl von Solms-Braunfels, and later Baron Ottfried hans von Meusebach, they went about the task of colonization. They obtained the right to settle Germans on a vast tract of 1 and in west central Texas known as the Fisher-Miller Grant (Jordan 1966:42-43).

The directors had no conception of the true nature of the contract. They believed they had actually purchased land which was suitable for agriculture, 
when in reality the grant provided free land given by the state of Texas on the condition that a specific number of families were to be settled within a given period of time on the land designated by the contract. Less than eight months remained to transport the first one-third of the required number of families. Further, the land grant was located in the western part of the state in undisputed possession of hostile Indian tribes, and the government of Texas was under no obligation to disperse the Indians or to provide protection for the settlers. Fisher and Miller never owned any land in Texas (Wisseman 1869:15-16). Neither Fisher and Miller nor the Society of Noblemen had any legal claim to the land. The German immigrants brought by the Adelsverein numbered 7,380 in the period from 1844-46. By 1847, the Adelsverein was bankrupt due to improper management and inadequate planning. However, in the early 1850s, the titles of German immigrants to land in the defunct grant were recognized by the state government which legally owned the area.

In 1845, Baron von Meusebach arrived from Germany to succeed Prince Solms as administrator. He not only proved himself capable of handling the chaotic situation, but without his vigorous and sane efforts the colonization project of the Society would undoubtedly have collapsed. After disposing of the most urgent business matters, he began his search for a site for another way station along the route of the grant. The most suitable place he could find was near the Pedernales River, about halfway to the grant. He purchased 10,000 acres of "head rights" on credit, since as usual the treasury was empty, and the area was divided into townlots and ten acre farms. These were allotted without cost to the colonists of 1845 and 1846 as temporary compensation for their claims in the land grant which could not yet be reached (Wisseman 1869:22-24).

The first wagon train of colonists left New Braunfels for Fredericksburg on April 23, 1846. Approximately 120 men, women and children took 16 days to make the trip (Wisseman 1869:16). When the wagon train approached the Pedernales River, a company of Delaware Indians approached with signs of friendliness. The immigrants caught sight of a large camp of Indians, but the Indians seemed friendly and they hurried on to their destination where they could find temporary shelter. As they were fording the river, a shot rang out. Their anxieties were assuaged when they found that the Immigration Society's soldier, John Schmidt, had shot a large bear on the bank of the river. Soon after the trooper, Conrad Merz, killed a panther. The meat from the panther and bear provided the first communal meal at the new settiement of Fredericksburg under gigantic post oak trees (Wisseman 1869:14).

Less than two weeks later the management of the Adelsverein in New Braunfels received word from Colonel Henderson, Governor of the State of Texas, advising them against the expedition to the Pedernales, declaring that he had reason to believe that the Comanches would prove hostile toward the settlers. Twelve young men volunteered to make the dangerous trip to Fredericksburg to inform the settlers of the Governor's letter and escort them back to New Braunfels should they care to return. The mounted company of volunteers found the colonists busily engaged in the building of their houses. The settlers decided to remain and depend upon their own means of protection (Roemer 1935:156-159). 
The site chosen by Meusebach was between two flowing creeks. The land drained to the Pedernales River, five miles distant, and the valley, encircled by seven hills, had a natural beauty. The town was named for Prinz Frederick of Prussia. For temporary shelter, the immigrants set up brush shelters and tents of linen sheets. They used the native grass, almost half the height of a man, to cover the roofs of their first huts. Covered with this grass, the roofs were rain proof (King 1967:20-25). In April of 1847, two houses of stone construction and about 150 log cabins were seen in Fredericksburg, but nine years later only stone houses were being built in the town (Jordan 1966:166). The Germans regarded their initial dwellings as temporary necessities of pioneer life to be discarded as quickly as possible. After the first few years, they built houses displaying many construction methods brought from Germany including half-timbering (Fachwerk), casement windows, exterior plastering and white-washing, and thatched roofs. From the rockribbed hills they were bringing limestone--the stone about which Meusebach had written the previous year that "hardens in the air, and would be excellent for houses." Artisans using the stone and the sand and gravel at hand would soon bring an indigenous style of building to the colony. They used only hewn logs for building. The log framework filled in with mortar, reeds and sage grass formed the distinctive "Fachwerk" construction (King 1967:108). Because of solid construction, many of the original stone and Fachwerk houses are still standing and in use, and their unique blend of native architecture and European construction methods wi 11 continue to be prominent in the cultural landscape of the German hill country for years to come.

Much of the lumber that the settlers used came from the sawmill in the Mormon community of Zodiac. In 1847 a group of migrating Mormons had established a settlement on the Pedernales about four miles east of Fredericksburg. The forty families, under the leadership of Elder Lyman Wight, taught the inhabitants of Fredericksburg when and what to plant, introduced corn and other grains to the area, and built a water-powered saw and grist mill and a woodturning lathe on the Pedernales River. Meusebach welcomed the Mormons when they established their settlement. They were excellent builders, and furnished lumber and furniture to their neighbors in Fredericksburg. The community left for Utah in 1853, but the son of Elder Wight returned temporarily some years later and supervised the construction of the old Vander Strucken Flour Mill. The German colonists were indebted to the Mormons who taught them what and how to plant at a time when they were near starvation (Wisseman $1869: 21-22$ ).

The settlers at Fredericksburg chose to cultivate one large communal field during the first year, delaying distribution of farms until 1848. The settlements were planned and laid out by surveyors. The most striking differences from the German prototype in the settlement plan were the great width of the streets, and the geometric pattern. The exhaustion of natural forage in the vicinity of the town caused by both excessive numbers of cattle and unwise prairie burning led many settlers to disperse in search of better grazing areas. It was soon apparent that the settlers were not going to be satisfied with their small ten-acre farms, and the perimeter of settlement extended farther from the town. The danger of Indian attack, which might have provided enough force to preserve the farm villages, was nullified in 1847 by the treaty between Meusebach and the local Comanche Chiefs. The farmers 
kept possession of their town lots, and later built "Sunday Houses" to use when they came to Fredericksburg for weekend shopping and church-going (Jordan 1966:150-161).

The winter of 1845-46 had exceptionally heavy rainfall and some of the immigrants dug caves in the ground, lacking better shelter facilities, in Indianola and Galveston. Disease broke out which took a heavy toll of the unacclimated immigrants, and the new arrivals carried the diseases to the colony. Nearly 850 persons died in New Braunfels and Fredericksburg (Wisseman 1869:24-25). In Fredericksburg the immigrants subsisted almost entirely on cornmeal, beef and coffee, with no fresh vegetables. One hundred and fifty-six deaths out of six hundred settlers occurred the first year, mostly from cholera, dysentery and "stomacacae" (Roemer 1935:234).

Dr. Ferdinand Roemer was a German geologist who visited the Texas colonies from December 1845 to April 1847, and his observations were published in a book which was translated from the German by Oswald Mueller in 1935. Roemer accompanied Meusebach to the Comanche winter quarters on the San Saba when the Peach Treaty with fifteen to twenty Comanche Chiefs and Santana was negotiated. Meusebach knew that the settlement could not survive unless peace was made with the Comanches, whose land the town occupied. After a note to the Comanches, Meuscebach was invited to the Indian camp, and on March 1, 1847, he entered the camp of five or six thousand Comanches with a company of forty men including Major Neighbors, and the Delaware Chief Jim Shaw who acted as interpreter. He asked only that the Indians let them farm along the Pedernales in peace. In return Meusebach assured the Indians that they would be welcome in Fredericksburg at any time. He promised that in times of hunger the town would provide the Indians with grain and other necessities in return for game, honey, and bear fat. The Chiefs expressed agreement with his proposals and vigorous embracing and feasting followed. A company of Comanches under Santana with their families accompanied them all the way to Fredericksburg. Rejoicing followed the safe return of Meusebach and the group, because the Peace Treaty would make anticipated settlements by Germans in the fertile and beautiful San Saba possible (Wisseman 1869:23).

The raising of corn was a matter of 1 ife and death to the colony. All had agreed to enclose several hundred acres with a fence, and each family would be assigned a proportionate share of land for raising corn (Roemer 1935:223). For European immigrants, it was a new and unfamiliar crop that was virtually absent in nineteenth-century Germany. The speed and degree of German acceptance of corn is therefore, a little surprising. Cornbread was adopted by the Germans as a major part of their diet from the very first, and virtually al1 immigrant farmers raised corn in the 1850-1880 period, and in 1880 corn accounted for about $36 \%$ of the total cultivated acreage of the Germans (Jordan 1966:66). During the winter of 1847-48 the immigrants subsisted on bread, meat, and no vegetables. Scurvy took a great toli. A cart driven by oxen was busy all day carrying corpses. The Comanches supplied families whose husbands were away finding work with buffalo, bear and deer meat and honey for very smal1 compensation. In 1848 the vegetables and corn were harvested and the sickness gradually abated (Wisseman 1869:30).

The Apaches and a few other Indian tribes ground the mesquite pod to a mea? from which they prepared the much prized "pinole." Mesquite was of limited 
value to the colonists, but the presence of mesquite trees was regarded as a sure sign of fertile soil, and they felt assured without further investigation that the soil was suitable for growing corn and other cultivated products. Mesquite trees never form a continuous forest, but are found singly scattered in the prairie. The tender mesquite grass so relished by cattle and horses is usually found where mesquite trees grow (Roemer 1935:115). The Pedernales River was small, rapidly flowing and clear with tall pecan trees bordering its banks, which were used as grazing for the livestock. The oak forest began on the other side of the river and extended beyond Fredericksburg for many miles along the river. The soil here was light and sandy; this probably accounted for the prevalence of post oaks which prefer a dry sandy soi1 (Roemer 1935:226).

Shawnees were camping in the vicinity of Fredericksburg and supplied bear meat and particularly bear fat for sale to the settlers. Fat was in liquid form and clear. It was kept in deer skins, and a gallon of fat or bear oil sold for a dollar by the Indians or for the equivalent in goods. Colonists came with all manner of vessels to buy, since this oil was suited for various purposes in the home in place of lard or oil. All food was cooked with bear oi1, and at the time of Roemer's visit to Texas, it was used in lamps instead of regular oil. That numerous bears were near Fredericksburg was attested by the fact that each Indian often had sixty gallons of such fat for sale (Roemer 1935:232).

In the summer of 1849, wagon trains en route to California and the gold rush passed through Fredericksburg. Cholera was brought into the colony and took many lives. Even the colonists' good friend Santana was a victim of cholera and with him the connection with the comanches came to an end. The comanches grew more hostile with the movement of so many whites across their country, but the Comanches were gradually moving westward after the arrival of soldiers. There was a correlation between the number of troops stationed on the frontier and the extent of marauding operations of Indians (Wallace and Hoebel 1952:302-308).

The picture of adaptation and the desperate struggle to survive as Indians and as colonists is a story interwoven with the environment, a multiplicity of cultures, disease, technology, faith and avarice (Skeels 1972:viii). Sacrifices to overcome what seemed unsurmountable difficulties drew the immigrants together. Coming from similar backgrounds, they found strength and comfort from each other, and were able to work out solutions for their problems.

Early Settlement in Stonewall

In 1860, four or five families were living in log cabins near the Pedernales River in the area now known as Stonewall. The original land grants were made before 1837. A widow, Justa Flores, held a land certificate for approximately 4,600 acres of land on the day of Declaration of Independence from Mexico. She sold the land to William Richardson of Galveston for $\$ 800.00$ in May of 1837. The land changed owners and the Flores grant was divided into smaller tracts (Stonewall Centennial). In 1845 Casper Danz and his wife came to the United States, and about the year 1850, he bought three sections of land (2,200 acres) along the Pedernales River. In June of 1869 he sold 188 acres to Frederich Sauer, and in March of 1872 he sold 209 acres to Henry 
Behrens. Danz kept the homestead property consisting of 250 acres which his youngest son, Richard Danz inherited. This is all now part of LBJ State Park (Lindig 1970:76).

In 1870, I. M. Nunez established a stage station about two miles south of the present Stonewall. Mail for early settlers was left with Nunez. When a post office was established, Nunez insisted that it should be named Stonewall in honor of Stonewall Jackson, under whom he had served in the Civil War. In 1870 Major Nunez laid out a town site called Miliville along the Pedernales River. He laid out 15 acres in town lots which he sold to people who intended to build a home or establish a business. When the stage station was discontinued and the post office moved to Miliville, the settlement was renamed Stonewal1 (Stonewall Centennial:5-10). Former President Lyndon B. Johnson was born August 27, 1908 on a farm three miles east of Stonewa11.

The first lime kiln in Gillespie County was built by Andreas Lindig, a relative of Eugene Lindig, former owner of the Hop Hill locality property. Andreas Lindig was trained in his native Germany in quicklime making. On his homestead he found rock which he hauled by ox-wagon, using seven loads of rock for each "batch" of 1 ime. Post oak wood, burned in a cooking bed, produced the high heat required to produce the lime. The rocks were carefully placed into the oven to permit the intense heat to convert the limestone into quicklime. It took one hundred hours at approximately 1,000 degrees of heat to convert the limestone rocks. Post oak was burned outside the oven, and only the live coals were shoveled into the bottom. After the oven reached its peak of heat, it had to be sealed airtight, and left in that condition for six days which included time to cool off. The quicklime or powdered lime when covered with water was called slaked lime. Mixed with sand and water, the slaked lime was used for mortar and plaster in the building of rock houses. The settlers learned the art of lime making from Lindig, and began building their own ovens and making their own lime. A two-story rock house using lime from the Lindig kiln and built in 1897 by Willie Meier is presently part of the ranch home of former President Lyndon B. Johnson on the LBJ Ranch near Stonewal1. The rock walls on the old homestead of Hugo Weinheimer were 18 inches thick, and the lumber for the rafters was sawed at the grist mill in Stonewa 11. The cypress shingles came from Comfort, where many trees on the Guadalupe River furnished the cypress for the area (personal interview with Hugo Weinheimer, 89 year-old lifetime resident of Stonewal1).

Everyone had a hand-dug well about twenty-five feet deep or more which furnished enough water for the household including a hog and a horse. Hugo Weinheimer described how the well was dug when his parents decided to dig a well close to the house instead of getting water from the creek with a 50-gallon barrel on a sled:

The well was five feet in diameter and the first six or eight feet was easy digging with a shovel. A frame was then built around the well and a strong post was set on each side of the frame with a gap in the posts to place the one-foot-thick log. This log had tapered ends and a wooden handle attached. To this $\log$ (windlass) a rope was fastened to 
raise and lower the strong wooden box. The person on top of the ground would hoist it up. He could move it to the side of the well and empty it. During this time the person in the well would dig some more dirt and rocks before the box was lowered to be filled again. Two men could remove four feet of dirt in a day, but when they struck rock which meant blasting, they could only dig one or two feet a day. The well on the old Weinheimer homestead was approximately 50 feet deep. They found a layer of two feet of hard rock at 35 feet. This was blasted with gun powder. Below this formation of rock it was easy digging (Weinheimer 1973:5-6).

The residents of Stonewall measured the miles travelled on the roads to Austin and Fredericksburg by notching posts. They measured the miles by tying a rag in the front wheel of a wagon and counting the rounds. So many rounds made a mile, they stopped, set a good cedar post and made a notch: the first mile one notch, the second mile two, etc. The measurements were very accurate (Lindig 1970:16).

One of the favorite Indian incidents remembered by the residents of Stonewall pertains to Joe Stahl who lived at Cave Creek. He was killed by the Indians while hunting a calf on Richard Immel's place in 1861. Every man joined in the search, and in the battle that ensued an Indian was killed. The Indians returned that night to bury their dead brother and killed a horse on his grave. For years the bones lay on the grave and were a topic of conversation for young and old.

Weinheimer said that the Indians frequently stole horses; consequently, the early settlers often chained and locked the horses in sheds at night or put them in the pasture with cows because the Indians wouldn't go for them there (Hugo Weinheimer, personal communication). Otto Lindig's mother and three little children were alone one day and the Indians stole a horse and a mule. The Indians ran into trouble with white men, and the mule was not fast enough. The next morning the mule was back, but the horse was never returned (Lindig 1970:17).

Adam Nebgen had a one-stand cotton gin at Stonewa11, and the cotton was taken in baskets and fed by hand to separate the lint from the seed. The bales were pressed with horsepower. In the first years the whole family worked in the fields, and the cultivation of cotton began to yield good revenue, especially for those families who were blessed with many children and needed no hired help. By 1890 every farmer had a Mexican family on his place; these families lived the year round in tents. Ninety-five percent of all the land in Gillespie County was cleared with a grubbing hoe and axe (Lindig 1970:37). At the end of the century wheat's place of importance in the area was challenged by cotton, but wheat survived while cotton disappeared from the scene (Jordan 1966:129).

Experiments with wheat began as early as 1848, and during the great expansion of wheat culture from 1850-1860, it became almost the universal crop among the Germans of Gillespie County. One German settler in Fredericksburg experimented with 110 different samples provided by the federal government in 1859 . 
They began to shift production to the winter to avoid the drought season and cattle and horses were pastured on the fields during the cold months to prevent the grain from heading too early in the spring. Lange's Mill, a stone structure in northwestern Gillespie County is still standing after more than a century (Jordan 1966:126-127).

Every farmer had sheep and the wool for socks, gloves, underwear and bedcovers was spun with a spinning wheel, then knitted by the women (Lindig 1970:46). Among German settlers, sheep raising has always been combined with cattle ranching, and the rise of this unique combination of ranching economy is characteristic of this part of Texas. Hog production soon became a major activity for market for many of the German farmers, and the animals thrived on the abundance of acorns and pecans in the area. The large volume of beef and pork production among the Germans encouraged the survival of sausage making, an art brought from Europe by the immigrants and virtually unknown to the southern Ang10-Americans (Jordan 1966:150-153).

The taste of water was strange to the settlers, so they substituted wine made from mustang grapes that grew wild on the farms; the wine was inexpensive to make. Wine-making among the Germans can be attributed to their agricultural heritage, but they had to abandon the domesticated grapes and winemaking methods they knew in Germany because the imported European vines would not grow properly in Texas. Once they mastered the technique of adding large amounts of sugar, they gathered the native wild mustang grapes and berries and utilized them. Most of the wine was made for home consumption, but the Texas Almanac in 1867 reported that some of the farmers around Fredericksburg were sending "as much as ten to twenty barrels of wine" to market (Jordan 1966:141).

The settlements were characterized from the very first by numerous kitchen gardens, in which a great variety of vegetables was grown including not only those found in Anglo-American gardens, but many brought from Gemany such as kohlrabi, mustard, parsley and leek. It did not take the Gemans long to realize that two garden plantings were possible each year in the mild Texas climate, and plantings were made in February and late summer. As a result some fresh vegetables were available all winter. Gardening was primarily the work of women, leaving the men available for more strenuous chores (Jordan 1966:134-135). Food was kept in dirt cellars four feet deep and two feet above ground. The cellars were made of post oak logs, and the roof from post oak boards was split by hand (Lindig 1970:19). Many local plants and shrubs were also utilized. Meusebach was found of agarita, a native shrub which resembles the Christmas holly. Frau Meusebach made delicious jellies from the berries of the agarita, and an eyewash from the roots. She used the roots of the yucca for a shampoo which gave her curls a beautiful sheen (King 1967:164).

Experiments were carried out with many trees common in Europe such as the apple, cherry and pear, but notable success was obtained only with peaches and to a lesser degree with native pecans, plums and figs. As early as the $1850 \mathrm{~s}$ it was evident that the peach tree offered the best possibilities (Jordan 1966:139); the community of Stonewall is now one of the largest peach producing areas in Texas. 
In the first critical year at Fredericksburg, the German farmers resorted to communal fencing, but later entire families including small children labored for months or even years to construct stone fences from the 1 imestone and sandstone of the area. In the Hill Country, the stone fences were used to enclose not only fields, gardens and corrals, but also pastures. At Live Oak settlement in Gillespie County, one German family enclosed 1,200 acres with a stone fence. Barbed wire was introduced in the $1880 \mathrm{~s}$ but miles of stone fences are sti11 in use (Jordan 1966:163-165).

After Roemer's sojourn in Texas in 1846-47, he deemed it a duty toward emigrated compatriots to make clear the chances for success of the contemplated colonization of the grant obtained by the Mainzer Adelsverein for the protection of German immigrants. His evaluation was that the land in question on the right bank of the Colorado and the region north of Llano were not the proper place for settlement by the Germans. His reasons were:

1. There are no extensive areas in the whole region which are of the same fertility as in the lower accessible areas of Texas.

2. Distance from inhabited section of Texas is too great.

3. Comanche Indians will become, if not dangerous, at least very annoying to any settlement north of Llano (Roemer 1935:286).

Although it was late, and many sacrifices had already been made, Roemer said

It would be to the interest of the Mainzer Verein or the Society which had taken over the rights and responsibilities of their German compatriots to abandon plans for colonization of the grant (Roemer 1935:286).

As Roemer left Fredericksburg, he thought of the many who had hoped to find gratification of many desires, but who instead found in this remote outpost only a place offering privations, hardships and sickness. The neat houses enclosed carefully with fences which German industry had called forth from the wilderness so far removed from the habitations of civilized human beings saddened him. He only hoped as he returned to Germany that the prospects for the settlement would prove less unfavorable than then, and that German industry and frugality could conquer these obstacles (Roemer 1935:289).

Most of the settlers' imported agricultural traits which survived were modified somewhat to meet local conditions. The production of wheat and rye came to serve primarily forage functions; haymaking was adapted to utilize unimproved prairie grasses; intensive application of labor was channeled toward the double-cropping of garden vegetables and the perfection of dryfarming methods; kitchen orcharding was modified to utilize trees uncommon in northwestern Europe; the traditional stability of the rural population was directed toward the purchase of 7 and and fencing of the open range; and German construction methods were adapted to local architectural styles and building materials (Jordan 1966:197-199). 
The German-American farmers of Texas have by no means lost their cultural identity in the century or more that they and their forefathers have lived in the state, and they still retain a measure of distinctiveness today. Over $90 \%$ of the farms and ranches in the county are owner-operated and average in size from 100- to 4,000-acre units. The farmers overcame untold hardships to win their soil and make it productive. The colonists wrote to their friends in Germany saying:

The taste of freedom is sweet; He who comes here penniless is richer than a German nobleman; Here, no one is master - there is no German yoke; Here, each one is what the other one is (King 1967:134).

One of the avowed purposes of the Adelsverein was to enlarge the sphere and influence of Germany. Certain members expected the idea to be obtained by a feudal entity, a German state or a German colony. A more desirable result came ultimately in the formation of a German community, a community which has retained $i$ ts unique quality through the years. 



\subsection{RECOVERY PROCEDURES (Royce Mahula)}

Located in Gillespie County on land recently purchased by the Texas Parks and Wildlife Department adjacent to LBJ State Park, the Hop Hill locality (4lGL21) rests on the valley slope and upland margin of the Pedernales River floodplain approximately 300 meters south of the river.

Excavation and survey of the site was contracted for by The University of Texas at San Antonio, Center for Archaeological Research, and was executed through the Summer Archaeological Field Course of The University. Under the direction of Joel Gunn, 23 undergraduate and graduate students participated in the excavation, which exposed them not only to proper field techniques but also to methods of retrieving and recording data for subsequent computer analys is of artifactual and physiographic evidence. Each student received some training in all aspects of the operation--survey techniques, stratigraphic concepts, excavation theory and procedures, recording, mapping, etc. Following the initial excavation a course in laboratory analys is of the artifacts recovered was conducted at The University to further analyze material.

\section{Initial Appraisal of Site}

The site was discovered eroding out of the red clay on the upland rim when the area was being surveyed by Parks and Wildlife personnel in preparation for the establishment of a camping area next to the park. In recent times the land had been in use as a swine farm. The denuding of the land of vegetation by domesticated animals led to severe erosion of the sandy clay topsoil, thus exposing quantities of lithics within the barren confines of the hog pens.

A preliminary surface survey by UTSA in November, 1975, identified a large quarry/workshop area north of the stock pen and also located an occupation area where such artifacts as a metate, manos, boiling stones, scrapers, points and other lithics were in evidence. This appears to be a unique occurrence in that the quarry/workshop and occupation area are proximate to each other. Although little work has been done in Texas on prehistoric chert quarries, present evidence suggests that there was little or no activity beyond removal and cursory preparation of lithic materials from these localities. Archaeological remains apparent at Hop Hill, however, appear to be in direct contrast to this pattern. Aboriginal inhabitants of this area of Central Texas were apparently drawn to this locale by the abundance of not one but many valuable resources.

The Grid System

A grid system of $20 \times 20$ meter units was superimposed over the four zones emanating from a "base point" located at the south end of the roadbed in the Upland Margin. It (the base point) was arbjtrarily. set as E1000-N1000. The vertical Datum 1 was established at the same point and is at an elevation of $432 \mathrm{~m}$ (1418 feet) above sea level. The grid was oriented $26^{\circ}$ west of north. Within this gross grid system, a more refined grid of $1 \times 1$ meter "squares" was drawn for each discrete excavation site within the locality (quarry, 
midden, and village). In essence, the $20 \times 20$ meter grid units functioned primarily for survey purposes while the $1 \times 1$ meter units served as the basis for excavation. Excavation was conducted in Zones II and III to show the character and diversity of occupation in those areas.

Quarry: Selected units along a line running 50 meters grid north from the base point and corresponding to the trajectory of the road. Squares were excavated every sixth meter. A rectangular section from E997 - E1001 by N1030 - N1033 was subjected to intensive excavation in Stratum 2 to investigate the character in Zone II, generally a quarry area.

Midden: A rectilinear section of 12 one-meter squares in the heaviest occupation area (metate area), E930 - E933 by N968 - N972.

Village: One meter square on red clayey sand (E925N959).

During the course of excavation as the grid was expanded, the following additional data points were established:

Datum 2: Najl in southwest corner of individual one-meter squares.

Datum 3: Nail in fence post adjacent to occupation floors in roadbed excavation. Elevation - 431.26 meters above sea level.

Datum 4: Nail in tree at northeast corner of midden excavation area. Elevation - 433.80 meters above sea level.

Survey Goals

Though survey procedures, like other excavation procedures, must be necessarily geared to the time and personnel at hand, the ultimate goal of the survey should be to collect the most representative sample of artifactual material in consonance with the time and manpower resources available. This was the ultimate goal at Hop Hill. Due to the extensive area of the site proper and the large concentration of Tithic materials scattered about the surface, survey sampling was in most cases necessarily selective. In order to devise a surveying program which would provide, in essence, this most representative sampling, the basic character of each zone was analyzed and a regime of surveying which would best preserve this character was devised. Surveying strategies for the four zones of the site basically were:

Zone 1: Upland - Large core tools. Relatively sparse distribution of artifacts. Dense vegetation. Walking survey by all personnel. Area crosswalked by full crew. Large core tools were to be flagged, shot in by transit and subsequently collected.

Zone 2: Upland Margin - Heavy occupation area. Intensive surface survey in conjunction with excavation of midden area. Random sampling of $10 \times 10$ meter southwest corners of $20 \times 20$ meter grid units, with total recovery of artifacts in these 10-meter units and a selected sample of significant artifacts from the $20 \times 20$ meter units. 
Zone 3: Valley Slope - Large quantities of lithic debris. Area of erosional deposition. Due to the density of materials full collection would be difficult to manage and probably of Tittle utility. Selected random sample of 20-meter units with total sampling of $10 \times 10$ southwest corners.

Zone 4: Floodplain - Alluvial deposition. Cultural connotations obscured by al7uvial and erosional deposition of artifacts. Not collected.

In addition to the aforementioned goals, a representative sampling of topographical features which would describe the erosional situation at the site was also undertaken.

Survey Techniques

Once units for sampling had been determined a standard survey-collection program was implemented. Each unit was subjected to close scrutiny during which time artifacts or concentrations of artifacts (i.e., lithic concentrations--primarily flakes, nodule samples, and reddened stone concentrations) were flagged. Following this initial location of artifacts, actual collection of materials and recording of data were begun. At Hop Hill, two methods of gathering this data were utilized: transit and surface surveyor. The transit method was implemented during the first half, and the surface surveyor during the last half of the five-week period. (A comparative evaluation of the effectiveness of each method will be forthcoming in a later section of this report.) A short explanation of each technique follows.

\section{Transit}

Artifacts which had been flagged were located with a transit by shooting an azimuth from known points, getting a distance by stadia and noting an elefation for each artifact. New grid markers and benchmarks were established as necessary to cover the entire site in this manner. Four to five people were required to keep this process running smoothly--two to locate, collect and code artifacts, one to run stadia, one to operate transit and one to record at the transit.

\section{Table 9.01 Physiographic Positions Indicators}

1. Bottom of Wash

2. Upper Edge of Gulley or Wash

3. Fence Post

4. On Fence

5. Center of Road

6. Edge of Road

7. Bottom of Borrow Pit
8. Tree

9. Pond

10. Bedrock

11. Flat Upland Surface, Sheet Wash

12. Slope Sheet Wash

13. Alluvium

14. Concrete S1ab

Surface Surveyor

This is a surveying method devised by Bandy (1975) for rapid collection. It consists of a circular wooden disc upon which has been superimposed a compass dial annotated in degrees and half degrees. The disc is then slipped over a 
non-magnetic stake which is driven into the ground. By the attachment of a compass to this apparatus the dial may be oriented to grid north. A line attached to the stake when extended across the face forms a needle by which to read the dial. To the end of this 7 ine is attached a 50-meter tape which measures distance of the artifact from the base point into which the stake is driven. With this method, only the initial base point upon which the surface surveyor is situated need be shot in by transit. All subsequent locations of artifacts within the 50-meter radius of the surface surveyor need only be recorded by angle and distance readings from the exact elevation of each artifact. Though it is not impossible to get a gross elevation by dropping a line from the tape to the artifact, this was not attempted at Hop Hill. The position indicator (Table 9.01) was some control for changes in elevation within the unit surveyed. Artifacts were retrieved in $90^{\circ}$ arcs at succeeding one-meter radij. This operation could be efficientiy run by two to three persons--one to pick up and bag and one or two to read the dial and record.

Surface Surveyor us. Transit

For our purposes at Hop Hi11, the surface surveyor far surpassed the transit in effectiveness as a surveying technique. The transit, while slightly more accurate in some aspects, took vastly more time and personnel to be kept running efficiently, and this in spite of the fact that the computer took over much of the computation which must ordinarily go on in the field. Considerable time was spent just setting the points and more time was spent just reading the data. Since the transit operators and the collection crew were often separated by some distance, just coordinating their efforts was timeconsuming. The surface surveyor, in contrast, was extremely quick and simple to set up and operate. Fewer personnel were needed and the proximity of a11 elements of the operation greatiy increased efficiency.

We have computed an efficiency index comparing the number of artifacts collected per person per day with each technique. Mean number of artifacts per day with the transit was 58.0. This figure divided by the number of personnel required to run the operation (5) yields 11.6 artifacts per person per day. Mean number of artifacts per day with the surface surveyor was 125.3. This figure divided by the number of personnel (3) yields 47.8 artifacts per person per day. Number of artifacts for surface surveyor (47.8) divided by number of artifacts for transit (17.6) yields an efficiency index of 3.600 or 3.6 artifacts collected by surface surveyor for every 1 collected by transit. The figures speak for themselves and for the efficiency of the surface surveyor for our purposes. Again, the methods must suit the needs of the situation. Possibly for a small concentration of artifacts scattered across a large area where exact provenience (elevation) is of the ultimate importance, the use of the transit might prove more beneficial. Hop Hill, however, is an eroded site with heavy concentrations of artifacts throughout a large area. The surface surveyor was without a doubt the more valuable method of surveying and collecting this type of site and allowed for a significant increase in the amount of data retrieved. 
Survey--Data Collection

With either method the same essential data was derived and recorded on a standard Survey Format. Such information as Format number, type of reading (i.e., back or foresite, transit or surface surveyor location, transit or surface surveyor reading), artifact $F N$, benchmark, elevation, azimuth (in degrees and minutes), distance, position indicator, cultural or non-cultural determination, cultural type and top hair reading (transit) was coded and entered on this form. Physiographic features could be shot in by either method and the position indicators of artifacts provided additional topographic information for computer-generated maps. In addition to recording these features, rebars were inserted at critical points for the erosion study to follow. Finally, artifacts were bagged and stapled to $3 \times 5$ cards containing the field numbers assigned to them and additionally bagged by provenience of survey unit.

Once this data was keypunched, it was submitted to a complex data management system developed by Richard Yoe to automatically standardize all of the provenience information to Cartesian Coordinates.

\section{Excavation Goals}

The principal goal of every excavation must be to extract the maximum amount of cultural information with as little distortion of data as possible, given the personnel and time available. This premise implies, therefore, the need for an organized set of goals, taking into account the unique physical and cultural characteristics of the site, and an equally organized plan for execution of these goals. The following scheme was devised for Hop Hill.

Since the erosional situation at the site was one of the primary factors influencing interpretation of cultural materials, the field season was formulated with this problem uppermost in mind. Surface distribution of artifacts across the site had been distorted by erosion and redeposition. In order to study the site in proper perspective, migration of archaeological materials would have to be controlled by mathematical means and field observation of the energy of the locations from which artifacts are collected. Thus, during surveying, collecting and excavation, erosional factors were constantly analyzed, and evaluated, and major efforts were made to collect erosional data which would allow reconstructing the original distributional patterns across the site within some acceptable error factor.

Because of the size, functional diversity, and eroded nature of the site, the primary thrust of the research effort was to define the various zones, both horizontal and vertical, within the site; and, in view of the erosional factors, to devise a plan of action which would allow for the least contaminated retrieval of data from each area. In line with this purpose, the following goals were proposed and eventualiy effected.

(1) Definition of the major zones within the site--basically by environmental criteria (upland margin, valley slope, floodplain)--and determination of their characteristic artifacts. 
(2) Definition of stratigraphy in terms of the erosional situation. Excavation of the roadbed to stand as a control area where normal erosional processes were impeded by the laying of the road surface. (A gravel road was built at the time the stock farm was in operation and it was hoped that excavation of this area would provide an uncorrupted stratigraphic reference.)

(3) Surface survey and stratified random sample of all zones for broad definition of distributional patterns and representative sample of artifacts occurring in each zone.

(4) Excavation of midden/occupation area to expose enough living floor area to properly analyze cultural characteristics of artifact distribution in this sector.

(5) Small excavation in the red clay to determine extent of occupation there. (Plainview material had been discovered eroding out of this same Pleistocene clay deposit near Austin. Ralph Robinson is working on a report on the site.)

(6) Collection of a broad sample of lithics across the site to investigate the level of lithic technology evident in this locality for further testing with a lithic production model.

(7) Compilation of pertinent data on erosional and topographic features of the site for computer display of these factors.

(8) Recovery of projectile points for additional chronological control and for the Center for Archaeological Research's Automatic Point Classification System.

\section{Excavation Concepts}

At the onset of the excavation it was imperative that students be instructed in proper excavation procedures which would govern information recovery. As is always the case, due to the unique situation of each excavation square area, techniques must be sculptured to fit the special problems at hand. Though excavation procedures in the road differed somewhat from procedures in the midden area, certain basic principles were adhered to in either case. Every effort was directed toward recovery of artifacts in as highly refined a stratigraphic context as possible, hoping that these high-resolution techniques would return the maximum amount of cultural information. Certain procedural guidelines gave direction to the excavation from the beginning, and there was a set of excavation skills which those participating in the excavation were required to master.

Trowels, or smaller tools if the situation demanded, were used during the entire operation (with the exception of testing bedrock in the roadbed and partial removal of the caliche road surface in later units along the road, which was accomplished by careful use of rockhammers). Participants were instructed to move their tools horizontally in conformity to the strata at all times in preference to punching down through it. Results of each movement were removed to a bucket by means of a dustpan. Excessive brushing-off of exposed strata was discouraged because this tends to distort the texture and color of the exposed surface. Economy of motion was stressed on the premise that the less 
disturbance of surface, the less the actual character of that surface will be distroted.

Utmost care was taken at all times to insure that excavation did not violate the integrity of presumed living floors. Once a living floor was defined, records were kept on its provenience across the site. Participants were instructed to move across living floors most efficiently and accurately by proceeding with a "control face"--a small vertical wall which extends across the 100-cm dimension of the excavation unit (Figure 9.01). The control face is exactiy as thick as the distance between occupation floors or between an occupation floor and a helpful, culturally sterile microstratum. In either case these are defined as "substrata" (physically or culturally relevant microstrata or arbitrary 10-cm levels) in strata where there is no occupation. The criteria defined by the excavator to recognize the substratum appear on the vertical section of the control face. The criteria of the next substratum appear on the lower horizontal surface. The contrast between these two surfaces serves to keep the excavator in constant awareness of the difference between what is and is not being excavated.

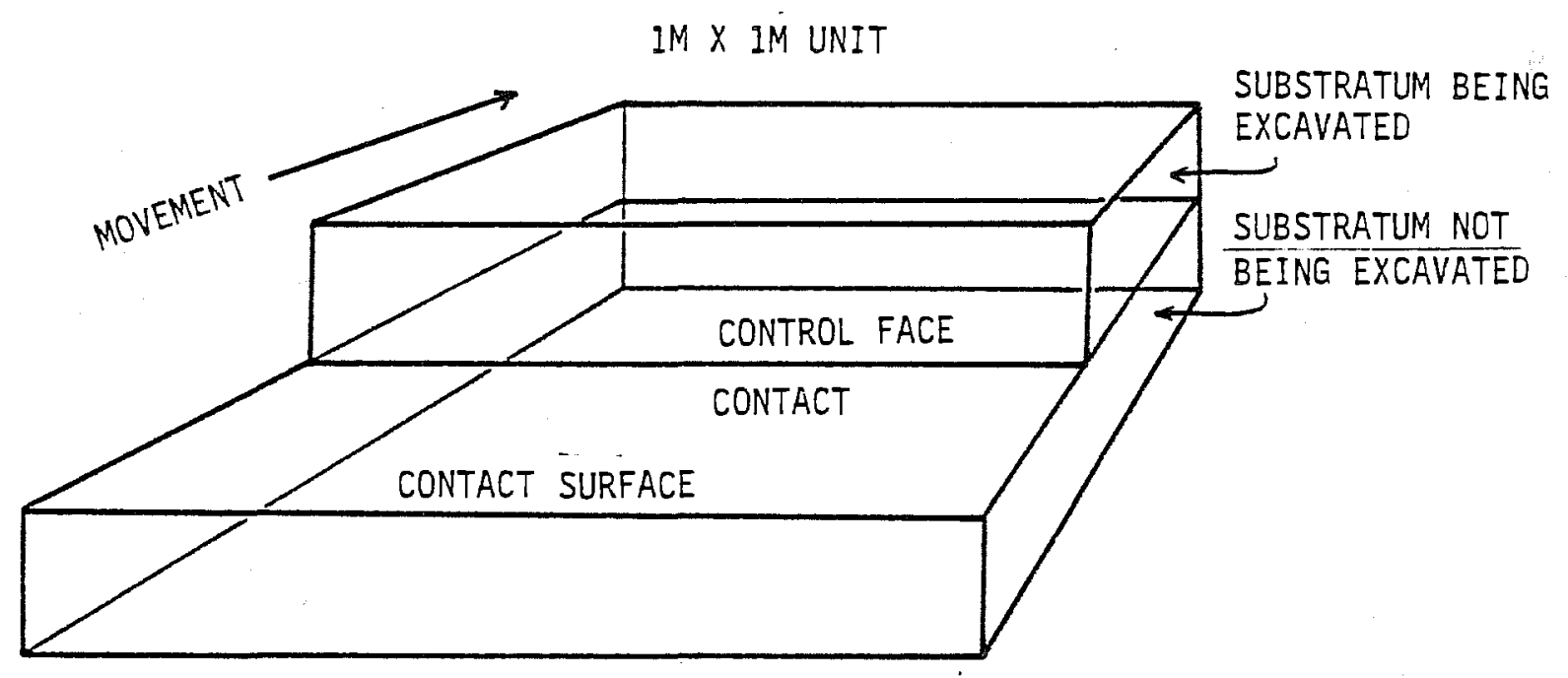

Figure 9.01. Concepts for Excavation Control.

The point at which the vertical and horizonial surfaces meet is called the "contact." Properly following the contact is of primary importance. When the contact is lost, control is lost. If this program is adhered to correct$7 y$ each horizontal motion is in conformity with the stratigraphic context and the control face is maintained straight and clearly visible. Supervisors need only check the control face and contact surface to determine the correctness of a given excavator's work. 
One important concept which was stressed to excavators was the fact that flat, smooth surfaces are in direct contrast to what occurs in nature. A unit with a nice flat excavated "floor" has obviously distorted the actual depositional character of the stratigraphy. Creation of an "occupation floor" occurred when early occupants placed and dropped materials upon an already irregular physiographic surface. Proper excavation of this living floor is accomplished by excavating the floor as the plane upon which rocks and artifacts rest, and this necessitates following and preserving the character of this original surface. In following the dips and bumps of the contact, the excavator is exposing the true nature of the "floor." In a purely physical context, the same holds true. Any unit of vertical control, no matter how minute, has an uneven depositional contour, proper excavation of which would not yield a smooth surface.

Definition of living floors as it is done here is admittedly a heuristic device. We understand that there is a chance of the data becoming confused through the ages, through excavation foul-ups, etc. It is possible, however, to model the alternatives and use this model as guide to reliability. The highest level of resolution is a brief period of occupation, perhaps a few hours, which is sealed above and below by sterile levels adequate in thickness to insure the integrity of the floor during excavation; the lowest level of resolution is a disturbed floor. The details of this resolution scheme are defined in the introduction.

As with most things archaeologists do, the way they approach occupation levels in a site is guided by a model. More often than not this model is implicit. The concern here is to explicate a system of concepts which will indicate the level of resolution at which that social organization is being observed. Archaeologists in the past who excavated in arbitrary levels were looking at blocks of occupation floors, lumping several floors with each arbitrary ievel. Since they were primarily concerned with chronological problems, they obtained the information they needed to form battleship curves of various artifact types.

More recent problem orientations have led to a concern for lateral as well as vertical analysis of trait distributions in order to discover composition of tool kits, plans of camp layout, etc. The most favorable situation for the archaeologist so involved would be to excavate each night's occupation of a site as a discrete occupation floor. A more likely situation is to get a series of nights or an occupation episode. An equally likely situation is a series of occupation episodes, such as when a group recurns to the same site for a series of seasons. The theory of occupation floors assumed here is that an occupation floor is a surface projected through a site which has a concentration of cultural materials near it. This concentration is understood to contrast with the situation physically above it in some perceivable dimension, either density of cultural material or stratigraphic indications.

The physical delineation was done according to a set of criteria arranged by priorities. Most desirable was to find an occupation floor between sterile substrata. Often, however, the floors were more closely spaced and required 
more flexible recognition criteria. More often than not, especially in the midden, the tops of large debris on one floor protruded up into the floor above. In this situation the bottoms of all artifacts were taken to represent the surface on which they were discarded. Thus, at the beginning of excavation of a new floor the field workers were instructed to excavate to the bottom of the next level of rocks and artifacts. When this was done a 11 materials that could be readily picked up without wrenching them from the ground were mapped, catalogued and removed. Any artifacts that were either wholly or partly in the ground were left to the next occupation floor down. The field numbers (FN) were written on the flint in pencil. Rocks were mapped and corresponding numbers placed on the map and on the rock with a marking pen.

\section{Screening Procedures}

Screening operations were geared at highest recovery taking into consideration the character of the area being excavated. The material from the midden and village sectors, with their quantities of small flakes and flake fragments, was a 77 passed through $1 / 8^{\prime \prime}$ mesh screen. Though with this system flakes smaller than the $1 / 8^{11}$ screen apertures would be lost, a recent study of 1 ithic debris from a flintworking demonstration by J.B. Soliberger suggests that a large percentage of these flakes are the products of simultaneous propagation, i.e., they are detached at the same time that larger daliberate flakes are removed (Gunn, Mahula and Sollberger, 1977). In this case, the amount of significant information lost by use of the $1 / 8$ " screen would be negligible. Because of the nature and size of the artifacts and debris being recovered from the roadbed sector--larger decortication flakes, chunks, nodules, etc.--the $1 / 4 "$ screen was initially implemented here. When the occupation floors and features were encountered, however, the $1 / 8^{\prime \prime}$ screen was immediately employed.

All artifacts--lithics, snails, bone, seed--in short, any cultural material recovered was placed in plastic bags, labeled as general provenience ard identified with a card stamped with the field number of the substratum unit from which it was recovered.

\section{General Mapping Procedures}

Among the purposes of mapping in an archaeological excavation are organization of physical areas/units into a comprehensive whole and re-creation of the cultural occurrences in their correct perspective and context for subsequent analysis and evaluation. At Hop Hill several types of mapping were performed. An overal1 map was drawn for the entire site describing the grid layout for al1 four zones. The status of each unit in that grid was continually recorded and updated. Stratigraphic profile maps were drawn on both east and north faces of each unit along the base line and around the perimeter of the occupation sector in the road as well as around the perimeter of the midden excavation. Isolated features found in test pits were well documented with a sequence of plan and profile maps. As a control reference, complete profile maps were drawn for each strata in a locality removed from the site proper. Control 
profile maps as well as feature profile maps were Munsell color-coded and Munsell readings are also available for roadbed profiles. As will be described below, each substratum unit was mapped. Joining horizontal unit maps into a continuous plan allows re-creation of cultural or physical patterns in areas opened broadly enough to reveal occupation floors. In such areas artifacts such as fire features are treated as parts of floor patterns, not as items surrounding artifact patterns. A broadly integrated mapping system such as this provides a multidimensional representation of previous conditions at a site.

\section{Excavation of Vertical Units}

As indicated before, the system in effect at Hop Hill for organizing and annotating vertical units was essentially the breaking down of each gross unit of vertical control, i.e., the stratum, into further refined substratum units-a stratum being defined as a relevant unit of either physical or cultural significance. This system proved to be very flexible in handing every eventuality. For every such substratum which was encountered, a substratum unit format (Figure 9.02) was opened and data such as coordinates of unit, depth to top of substratum, datum point, and stratum in which occurring were recorded. Before initiating excavation, a soil color was read by Munsell color chart and recorded, and a 2000-cc constant volume sample (corresponding in depth to the thickness of the substratum) was removed and bagged both as a control and for further high resolution analysis such as micro-snails, hydrogen peroxide flotation, etc. Ideally, a sample should be taken and analyzed from the southwest corner of every excavated unit in the grid. In practice a systematic sample was taken at grid intervals which match problem orientation and laboratory resources.

After excavation of the substratum unit, determinations such as consolidation, grain size of soil particles, presence of charcoal, shell, bone and chipped stone were made and noted and the unit was subsequently mapped (Figure 9.03). The mapping of substrata across a site in this case would result not only in the tracing of the horizontal pattern of occupation floors, but in the case of a physical unit such as the brown sandy layer it could result in a topographical view of an erosional or depositional episode. Artifacts, burned rock and any other cultural or pertinent physical occurrence were plotted and depth measurements recorded. The map number was in turn recorded on the cultural unit format of each artifact associated with that substratum unit. In addition to mapping, excavators documented more novel characteristics of the substratum unit with written accounts of conditions within their units. These notes are taken on standard continuation sheets and filed with the substratum unit format.

\section{Artifact Recovery}

Having a sound procedure for handling an artifact once it has been discovered is crucial for proper documentation. Excavators must be well briefed on the proper techniques for measuring and recording artifacts. The method employed at Hop Hill required careful attention to maintaining the purity of the provenience data in order for computer analysis to be most effective. 
CENTER FOR ARCHAEOLOGICAL RESEARCH

THE UNIVERSITY OF TEXAS AT SAN ANTONIO

\section{SUBSTRATUM UNII' FORMAT}

*Fill all blanks, Zero = Missing data*

I _ - - - Caro-Site \#

- - - - FieId Number of Substratum Unit

meters centimeters

-

$\overline{-} \overline{-}-\bar{z} \cdot \overline{\text { North }}$

- - - - Depth to Top

- Datum

- Thickmess

Excavators

( Thickess

Photo $\# 5$

Section $\overline{\pi s}$

$$
\text { - - - Substratum }
$$

before excavation

$$
\text { color }
$$

- Hue

- $(Y=1, Y R=2, R=3)$

- - I - Value and Chroma

after excavation

$$
\begin{aligned}
& \text { Consolidation ( } 1=\operatorname{sof} t, \geqslant 2=\text { hard) } \\
& \text { - Grain Size ( } 1=\operatorname{silt}, \geqslant 2=\operatorname{san} d) \\
& \text { - Charcoal ( } 1=\text { flecks, } 2=C-14 \text { ) } \\
& \text { - Shei1 }(9=\geqslant 9) \\
& \text { - Chippea Stone } \\
& \text { - - Bone }(9=\geqslant 9) \\
& \text { - Extent of Excavation ( } 0=n o, 1=N, 2=E, 3=S, 4=W \text { ) } \\
& \text { - Inspected } \\
& \text { - Tagged } \\
& \text { - Mapped } \\
& \text { - Day } \\
& \text { - Month } \\
& \text { - - Year }
\end{aligned}
$$

Comments:

Figure 9.02. Substratum Unit Recording Format. 
*Fill all blarks, Zero = Missing data

$$
\begin{aligned}
& \text { 3 - - - Card-Site }{ }^{*} \text { - Field Number of Substratum Unit } \\
& \text { - - Map Number } \\
& \text { - - Substratum } \\
& \text { - Depth Measurements (I=taken) } \\
& \text { - - Plan Photo } \\
& \text { - - Inspected } \\
& \text { - Day } \\
& \text { - Month } \\
& \text { - Year }
\end{aligned}
$$

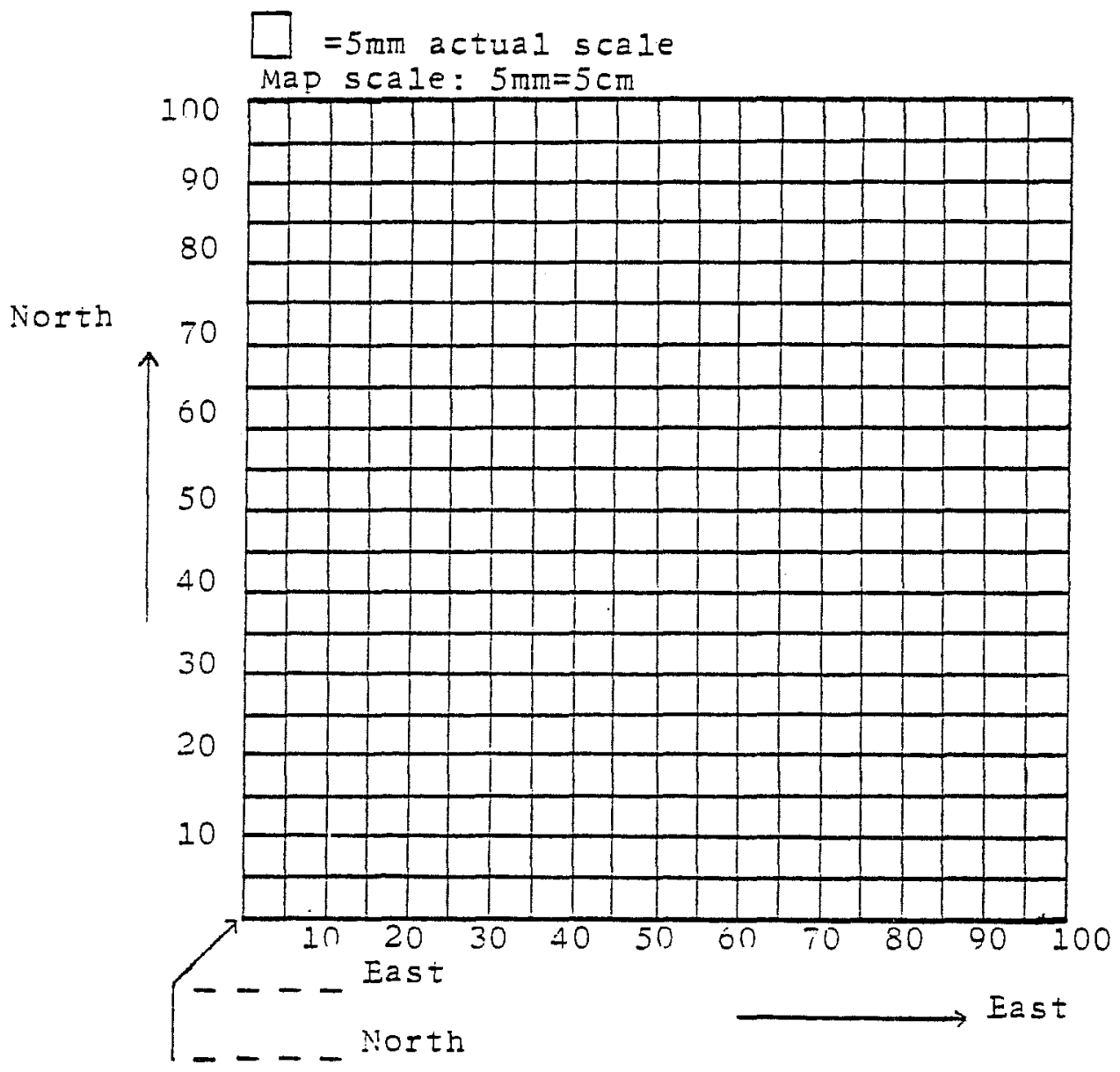

Measurer

Recorder

Figure 9.03. Mapping Format. 


\section{CULTURE UNIT FORMAT}

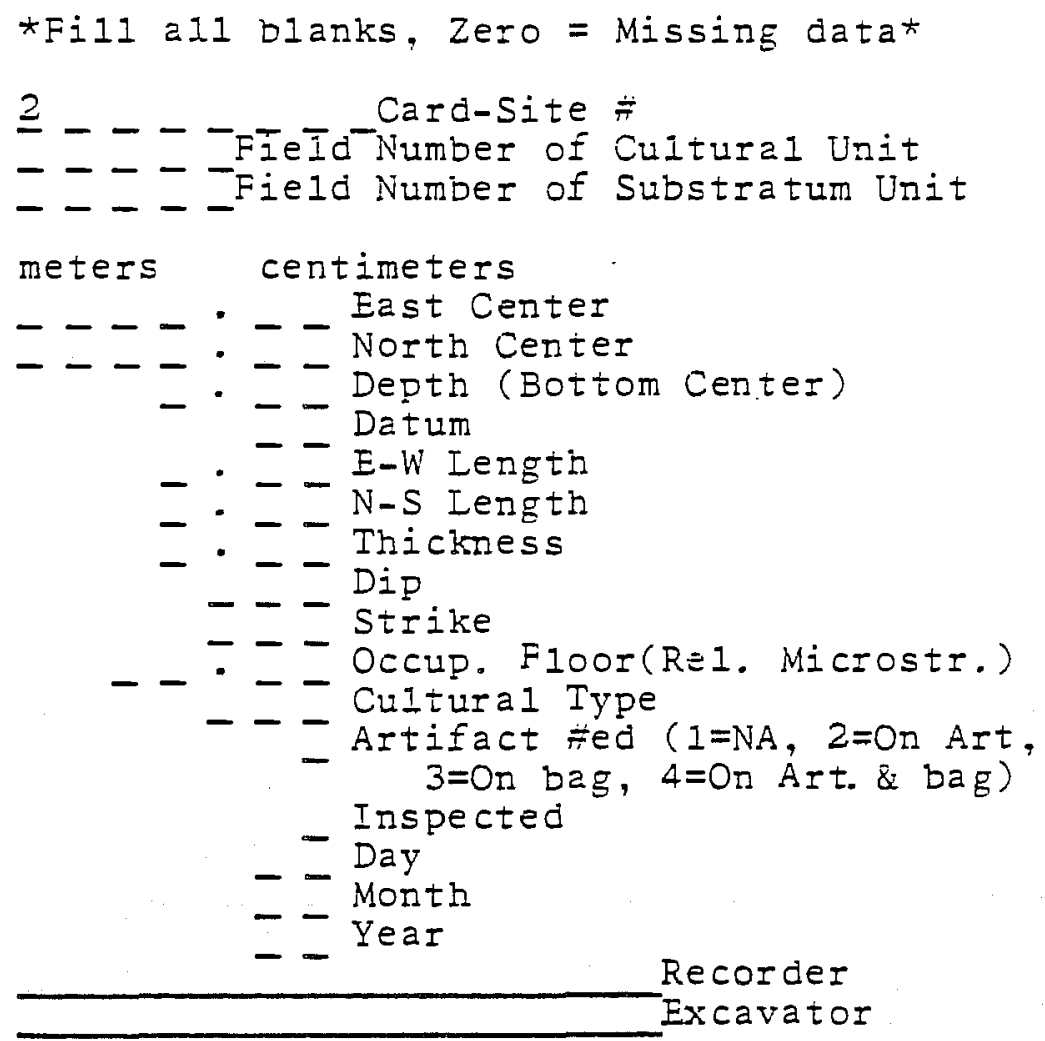

CUITURAI TYPES(3SEP76)

8 Charcoal

10 Fire Feature

11 Rediened Rock

12 Reddened Stone Concen.

13 Burned Iimestone

14 Burned Sandstone

15 Boiling Stone

20 Storage Feature

30 Hammerstone

31 Chip

32 Chunk

33 Core

34 Core, Fragment

35 Core, Eoge-Altered

36 Flake, Decortication

37 Flake

38 Flake, Pressure

30 Flake Retouched

40 Flake, Edge-Aitered
41 Flake, BiI. Thin.

42 Flake, Concen.

43 Heat SpalI

50 BIade

51 Blade, Fragment

52 Blade, Retouched

53 Blade, Edge-Altered

60 Quarry Blank

ó Chopper

62 Biface

ó3 Biface, Fragment

70 Point

90 Scraper

91 Scraper, Side

92 Scraper, End

93 Scraper, Side \& End

94 Scraper, Ovate

95 Scraper, Circular

96 Scraper, Thumbnail

97 Scraper, Miscellaneous

98 Uniface

110 Perferator

120 Engraver

130 Burin

131 Burin Spaul

140. Notch

150 Denticulate

160 Vlear Fork Gouge

170 Metate

171 Metate Fragment

172 Mano

173 Mano Fragment

180 Ceramics

200 Glass

300 Rock

301 Sandstone

302 Iimestone

310 Nodule

311 Nodule Concentration

400 Shell, Mussel

401 Shel1, Snail

500 Bone

501 Post-Skelotal

$510 \mathrm{Skin}$

520 Turtle Carapace

000 Metal

gog Other

Figure 9.04. Culture unit Reconding Format. 
When in the process of excavation an artifact was encountered, a culture unit format (Figure 9.04) was immediately opened, recording in situ information such as exact provenience- $N-S$ and $E-W$ coordinates (to the $\mathrm{cm}$ ) and depth (to bottom of artifact)--and orientation readings such as $N-S$ and $E-W$ length and thickness. These measurements allow the computer to replace the artifact not only with respect to exact location, but also with respect to its orientation within the unit. Angles at which artifacts are found in a site can be diagnostic information and could denote erosional periods (for example, pedestal effect on ariffacts undermined by erosional activity). In addition to the above information a preliminary determination of the artifact's cultural type (Figure 9.04) was made and recorded. Upon completion of coding, the artifact was numbered with its $F N$ in pencil, placed in a plastic bag and a $3 \times 5$ card stamped with the FN stapled to it. This bag was subsequently placed with other artifacts in an artifact bag for that substratum unit. If the recovered artifact was associated with an occupation floor, its location was flagged for mapping and later visual and photographic assessment of the distributions for that floor.

\section{Photography}

In order to have the most complete documentation possible, an integrated photographic record of the excavation was undertaken, from broadest aspect--zones-to most restricted--individual artifacts. First, each zone was photographed to document the basic character of each. Zones were photographed on first inspection of the site in the fall of 1975 when the site was denuded of vegetation as well as in the summer of 1976 before excavation began. Broad views of each discrete excavation area, i.e., roadbed (quarry) and midden (occupation), were taken as well as individual shots of each unit in the grid. Photographic records of the survey area were likewise compiled. A shot of each $10 \times 10$-meter sampled southwest corner of the $20 \times 20$-meter survey grid units was taken plus photographs of selected artifacts or concentrations of artifacts throughout the survey area. In addition to the basic program described above, photographic studies were made of various other conditions affecting interpretation of the site, namely (1) a photographic exposition of the microenvironmental biota of the Pedernales River, (2) a study of the swine farm across the road to get some perspective on "pig erosion" factors and (3) a study of runoff patterns occurring during heavy rains. Other general aspects of the excavation such as excavation procedures and techniques also were photographed--in short, a complete photographic record of the entire excavation.

Further Site Records--Site and Survey Logs

In addition to the other previously described record-keeping devices, both a site and a survey $\log$ were implemented. Each crew member was encouraged to record any and all pertinent observations in these logbooks. Such observations ranged from the recognition and discrimination of some fine distinction of topography or stratigraphy, to the unique nature of an artifact or artifact concentration within their field, to general comments about well-being of crew and character of the weather. In short, any significant bit of information of which any crew member was cognizant was noted. These documents served as an invaluable backlog of information which aided in subsequent re-creation, analysis and interpretation of findings. 
FN $557--$ Fire Feature

FN 557 (E1000N1014) represents the only fire feature, other than the stonelined hearth excavated in the midden area, which was identified at Hop Hill. It is recognized that each archaeological occurrence should be analyzed within the context of associated cultural materials and distributions. This feature, however, does not occur in direct association with any identified occupation area. It is therefore, being discussed separately.

\section{Excavation}

Evidence of burning was initially observed in the first $10 \mathrm{~cm}$ of Stratum 2 as bits of ash, charcoal, burned red clay and rock were revealed. Upon encountering this evidence, a $10-\mathrm{cm}$ trench was opened along the north-south axis of the west edge of the square. As layers of burned materials were encountered within the profile of this trench, a control face was initiated and followed across the east-west face of the square. The feature was excavated in this manner to a depth of approximately $37 \mathrm{~cm}$ during which various microstrata of white ash, red ash, and burned clay, all with inclusions of charcoal and burned wood, were identified and recorded (Figure 9.05). Little artifactual material was found in association. Only a few small flakes were recovered. Some small percentage of the feature was left unexcavated in the adjacent unit to the east.

HOP HILL (4IGL2I)

NORTH-SOUTH PROFILE ON THE 1001 METERS EAST LINE FACING EAST

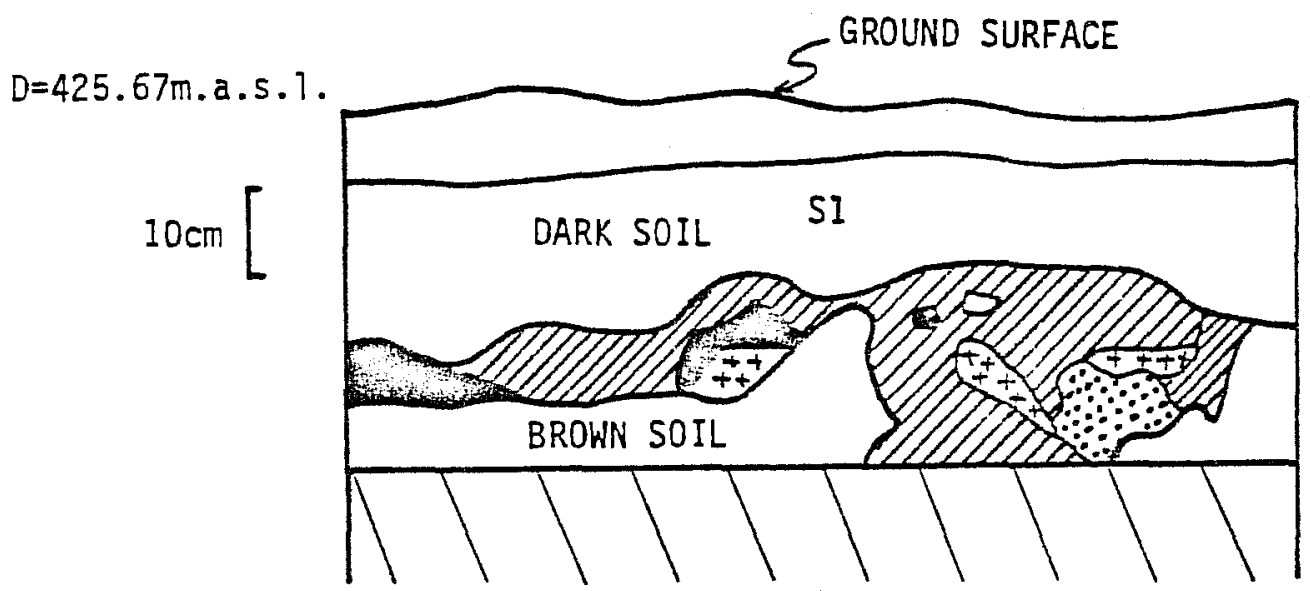

CHARCOAL (7.5YR2/0)

RED DEPOSIT(2.5YR3/6)

FWHITE ASH (5YRT/2)

BROWN-BLACK SOIL (5YR2.5-1)

Figure 9.05. Stratigraphic Profile of Fire Feature (FN557).

This feature appears to be a burned tree stump rather than a cultural feature.

Vertical contour evidences a basin shape, while horizontal configuration is splayed with "fingers" of burned materials radiating outward (Figure 9.06). Other such occurrences associated with Late Archaic occupations have been described by Elton Prewitt at the Loeve-Fox Site (Prewitt, 1975:6-7). The C14 assay is $230 \pm 70 \mathrm{BP}(\mathrm{TX} 2482)$. 
FN 55

\section{FiRE FEATURE}

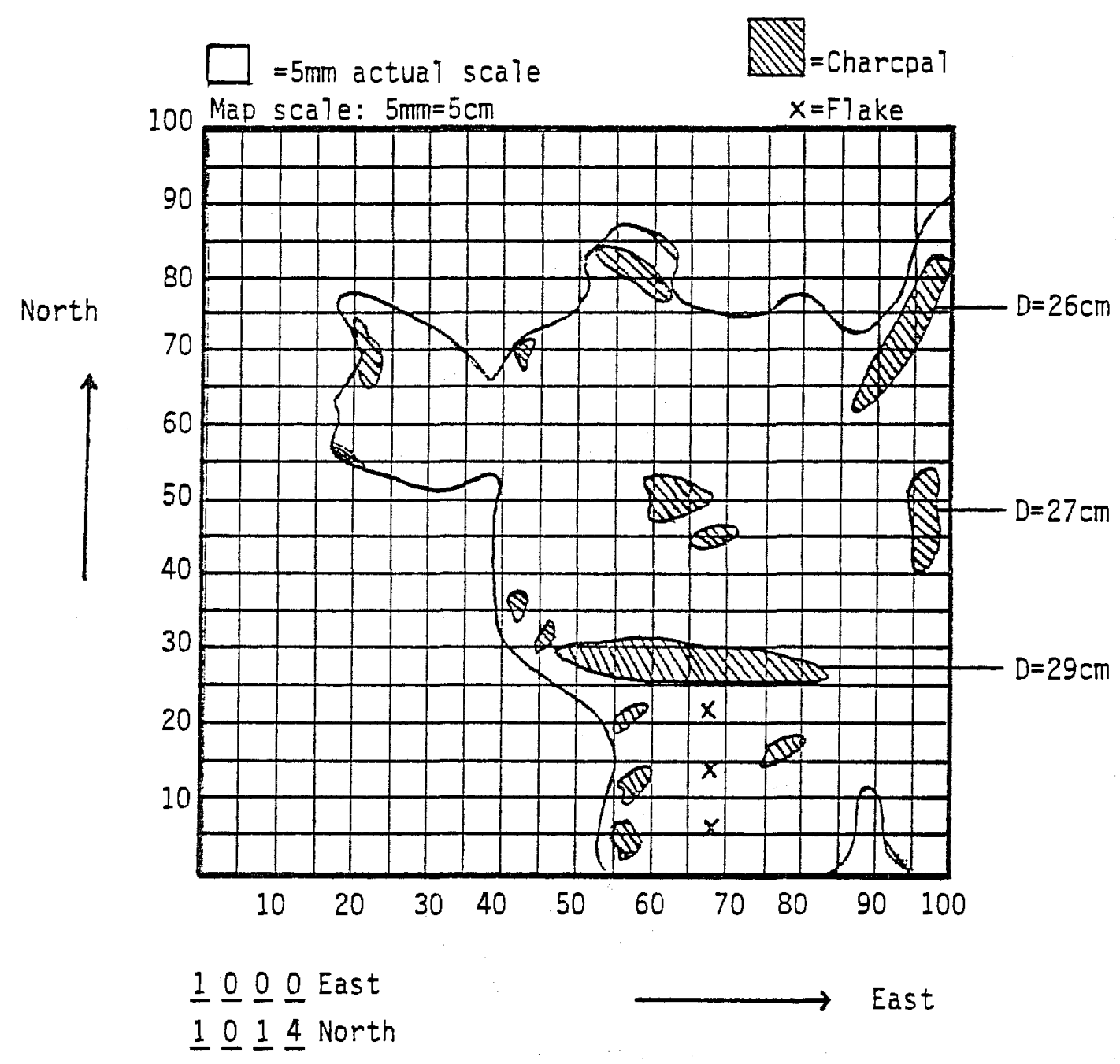

Figure 9.06. Horizontal Configuration of Fire Feature (FN557). 


\subsection{SOLLBERGER DISTRIBUTIONS: FUNCTIONAL AREAS OF THE SITE} (Joel Gunn and Royce MahuTa)

When we accepted the responsibility of survey, excavation, and analysis of the Hop Hill locality, J. David Ing of the Texas Parks and Wildlife Department and Elton R. Prewitt of the Texas Archeological Survey suggested we test the area for functional variability. It was believed at the time that within the large eroded area of the stock pen more than one type of habitation was represented, specifically habitation, midden, and quarry areas.

Since the locality is completely lithic in technology and, as will be shown, the working of lithics was in large part by the bifacing technique, we decided to use the products of bifacing to test the hypothesis. The assumption we make is that the various stages of bifacing are performed in different areas. We divide bifacing into three phases: (1) decortication and performing; (2) shaping and thinning; and (3) sharpening and reshaping (Gunn, Mahula and So11berger 1976). The fact that quarry sites can be recognized by decortication flakes and occupation sites by secondary flakes is fairly well established (Ke11y and Hester 1975:13). What has not been established are the distributional characteristics of these phases.

Since phases are used it seemed to us that each phase probably was represented in the minds of prehistoric knappers by a removal of ideal size and technical characteristics. Since size can be measured very efficiently by a device suggested by Paul Katz, comparing flakes to a series of graded rings (Katz 1976:115; Gunn, Mahula and Sol1berger 1976), our initial attempt was based around size.

The phase distribution model of bifacing presumes that the flakes of each successive phase will be progressively smaller. Further, since we assume an ideal sized flake for each phase, the sizes of flakes should be normally distributed around the mean for that phase.

To obtain the size distributions of the various phases, the aid of J. B. Sollberger was enlisted. He agreed to manufacture a biface breaking the process at critical points which would resemble the phases outlined. The debris from that phase was collected at each break and bagged separately. Later, the flakes produced in this demonstration were coded on several attributes and subjected to analysis.

Classes in which flakes belonged were determined by checking which of a series of graded circles each flake fit into. Table 10.01 shows the classes and the diameter of the circle. See Gunn, Mahula and Sollberger (1976) for illustrations and further explanation. 
Table 10.01 Classes and Class Sizes

$\begin{array}{lc}\text { Class } & \text { Circle Diameter (Maximum Measurement) } \\ 1 & 80 \mathrm{~mm} \\ 2 & 40-80 \mathrm{~mm} \\ 3 & 20-40 \mathrm{~mm} \\ 4 & 10-20 \mathrm{~mm} \\ 5 & 4.49-10 \mathrm{~mm}\end{array}$

Examination of the distribution for each phase showed that they were all skewed to the right. Since the bulk of the flakes in each phase distribution were smaller than would be struck intentionally we believe the smaller ones to be a product of "simultaneous propagation," that is, small flakes struck simultaneously with larger flakes. If we assume simultaneous propagation to be an exponential process (an experiment designed to test this hypothes is is in progress; Doug Land has made a biface collecting material after each blow, but analysis is not complete), the effect of simultaneous propagation can be removed by multiplying each class by an exponential function.

$$
y=p^{c} X
$$

Where:

$$
\begin{aligned}
& X=\text { the number of platformed flakes in a size class } \\
& Y=\text { the transformed value of } X \\
& P=\text { the average percent of change from class to class in the overall } \\
& \text { Sollberger Distribution (slope function) }=.32 \\
& c=\text { the class number (see Table } 10.01 \text { for classes) }
\end{aligned}
$$

Table 10.02 shows number of flakes for each class in the Sollberger Distribution and its various phases, the transformed value and the percent of the total transformed values $(P y)$. Figure 10.01 is a $Y$ value and it shows that when transformed in this manner the three stages show that sequential modes of flakes become smaller. This percentage can be used to compare the distribution of flakes from the field with Sollberger's distribution. 
Table 10.02 Sollberger Distributions

\begin{tabular}{|c|c|c|c|c|c|c|c|}
\hline \multicolumn{2}{|c|}{ Sollberger } & \multicolumn{2}{|c|}{ Overall (50) } & \multicolumn{4}{|c|}{ Sollberger Phase I (SI Decortication) } \\
\hline Class & $x$ & Y & $P_{y e}$ & Class & $x$ & Y & $P_{\text {ye }}$ \\
\hline $\begin{array}{l}1 \\
2 \\
3 \\
4 \\
5\end{array}$ & $\begin{array}{r}3 \\
23 \\
46 \\
101 \\
\frac{349}{522^{\star}}\end{array}$ & $\begin{array}{l}.96 \\
2.36 \\
1.51 \\
1.06 \\
1.17\end{array}$ & $\begin{array}{l}14 \\
33 \\
21 \\
15 \\
17\end{array}$ & $\begin{array}{l}1 \\
2 \\
3 \\
4 \\
5\end{array}$ & $\begin{array}{r}3 \\
23 \\
30 \\
48 \\
93 \\
97\end{array}$ & $\begin{array}{r}.96 \\
2.36 \\
.98 \\
.50 \\
.31\end{array}$ & $\begin{array}{r}19 \\
46 \\
19 \\
10 \\
6\end{array}$ \\
\hline
\end{tabular}

Sollberger Phase II (SII Shaping)

$\begin{array}{crcr}\text { Class } & X & Y & P_{\text {ye }} \\ 1 & 0 & .00 & 0 \\ 2 & 0 & .00 & 0 \\ 3 & 16 & .52 & 34 \\ 4 & 38 & .40 & 26 \\ 5 & \frac{184}{238} & .62 & 40\end{array}$

CLASS

$\begin{array}{lllll}1 & 2 & 3 & 4 & 5\end{array}$

so

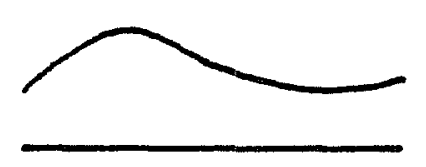

SI

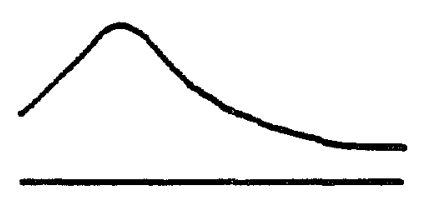

SII

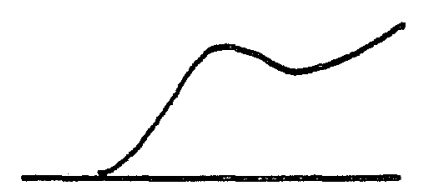

Sollberger Phase III (SIII Resharpening)

Class $x \quad Y \quad P_{y \in}$

$\begin{array}{rrrr}1 & 0 & .00 & 0 \\ 2 & 0 & .00 & 0 \\ 3 & 0 & .00 & 0 \\ 4 & 15 & .16 & 40 \\ 5 & \frac{72}{87} & .24 & 60\end{array}$

SIII

CLASS

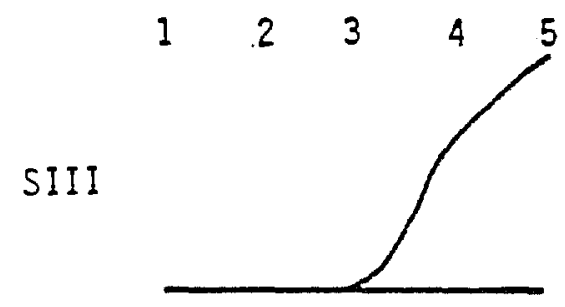

MIDDEN

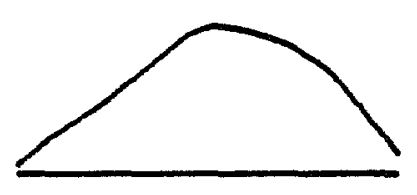

QUARRY

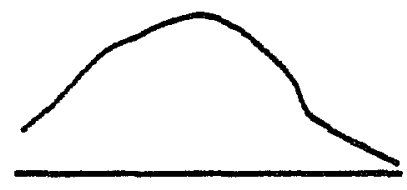

Figure 10.01. P Plotted for the Sollberger Distributions and the Hop Hill Midden and Quarry. 
Excavated Functional Areas

Now we turn to the use of the Sollberger Distributions as an instrument for determining the functions of various areas at the Hop Hill locality. First we counted the number of flakes with platforms in each class from each of the areas in question. Table 10.03 shows the counts $(x)$ from the two excavated areas of the site.

Table 10.03 Distributions From the Excavated Midden and Quarry Areas at Hop Hill

Midden

Transformation

\begin{tabular}{crrr}
\multicolumn{4}{c}{ Midden } \\
& \multicolumn{3}{c}{ Transformation } \\
\cline { 2 - 4 } C1ass & \multicolumn{1}{c}{$X$} & $Y$ & $P_{\text {ye }}$ \\
& & & 3 \\
1 & 8 & 1.28 & 3 \\
2 & 80 & 8.19 & 20 \\
3 & 524 & 17.17 & 42 \\
4 & 1193 & 12.51 & 30 \\
5 & 629 & 2.11 & 5 \\
& $\frac{}{2430}$ & &
\end{tabular}

Analysis

Distri-

bution

SO

SII

SI

SIII

$S$
14.4
14.8
17.2
26.0

The same calculations are performed on the field data as on the Sollberger distributions in the "transformation" section of Table 10.03. The "Analysis" section below shows the summed, absolute difference between classes divided by the number of classes, $\mathrm{S}=$

$$
\frac{\mid \text { Pye-Pyo } \mid}{c}
$$

for each area with each Sollberger distribution. The $S$ is an index of similarity between expected Sollberger distributions and observed field data. The lower the index the more similarity there is between distributions. The distributions are ranked on the index. 
By adapting an equation from Sokal and Rohlf $(1969: 607,386)$ a value, $t_{s}$, can be calculated which approximates the probable significance of $S$ :

$$
t_{s}=\frac{\arcsin \sqrt{s}}{\sqrt{820.8 \frac{1}{n_{1}}+\frac{1}{n_{2}}}}
$$

Where: $\begin{aligned} n_{1} & =\text { sample size of Sollberger Distribution being tested } \\ n_{2} & =\text { sample size of field distribution being tested }\end{aligned}$

(These calculations are most easily done on a Texas Instruments SR-51)

In the case of the midden, the $t_{s}$ for the lowest value is 863.67 which is clearly beyond $a p=.007$ as determined from areas under the normal curve. It is therefore unnecessary to calculate $t_{s}$ for larger deviations.

The quarry, on the other hand, has smaller n's and the results are more questionably different from zero, which is the nul1 hypotheses being tested. Even so, the smallest deviation is clearly beyond the .00l level of probability. In the case of both the quarry and the midden, $t_{s}$ indicates that the closest fitting Sollberger distribution is significantly different according to the $t_{s}$ criterion which involves considerations of sample size and the distributional characteristics of percentages. The utility of $t_{s}$ in this case is marginal since sample sizes are glaringly large and the probability of insignificant differences deceiving interpretations is remote. The fact that a 11 of the Sollberger distributions are significantly different from the field distributions perhaps suggests a note of caution. Whatever the cultural processes were that generated the field distributions, the Sollberger processes are significantly different. This is no surprise since we never deceived ourselves into thinking there were no artificialities in asking Sollberger to replicate a collection of flakes representing different stages of the bifacing process. The intention was to set a standard, however artificial, to test field distributions against until something better came along. The significance test indicates that, indeed, there remains work to be done toward finding a correct and/or useful distribution model. Also, the fault may not be completely with the Soliberger Model. The counts of flakes were originally made on the assumption that the assemblage was essentially a bifacing technology. Since then it has been found that a component of core flaking technology appears late in the sequence (see Section 17.0). There is every reason to think that inclusion of materials from core flaking technology in the counts would pervert the field distributions relative to the purely bifacial Sollberger distribution.

The usefulness of $t_{s}$ will be more apparent when questions arise from small samples and meanwhile, when it comes to test more advanced models, it can serve to show the relative closeness of fit between the various test runs. 


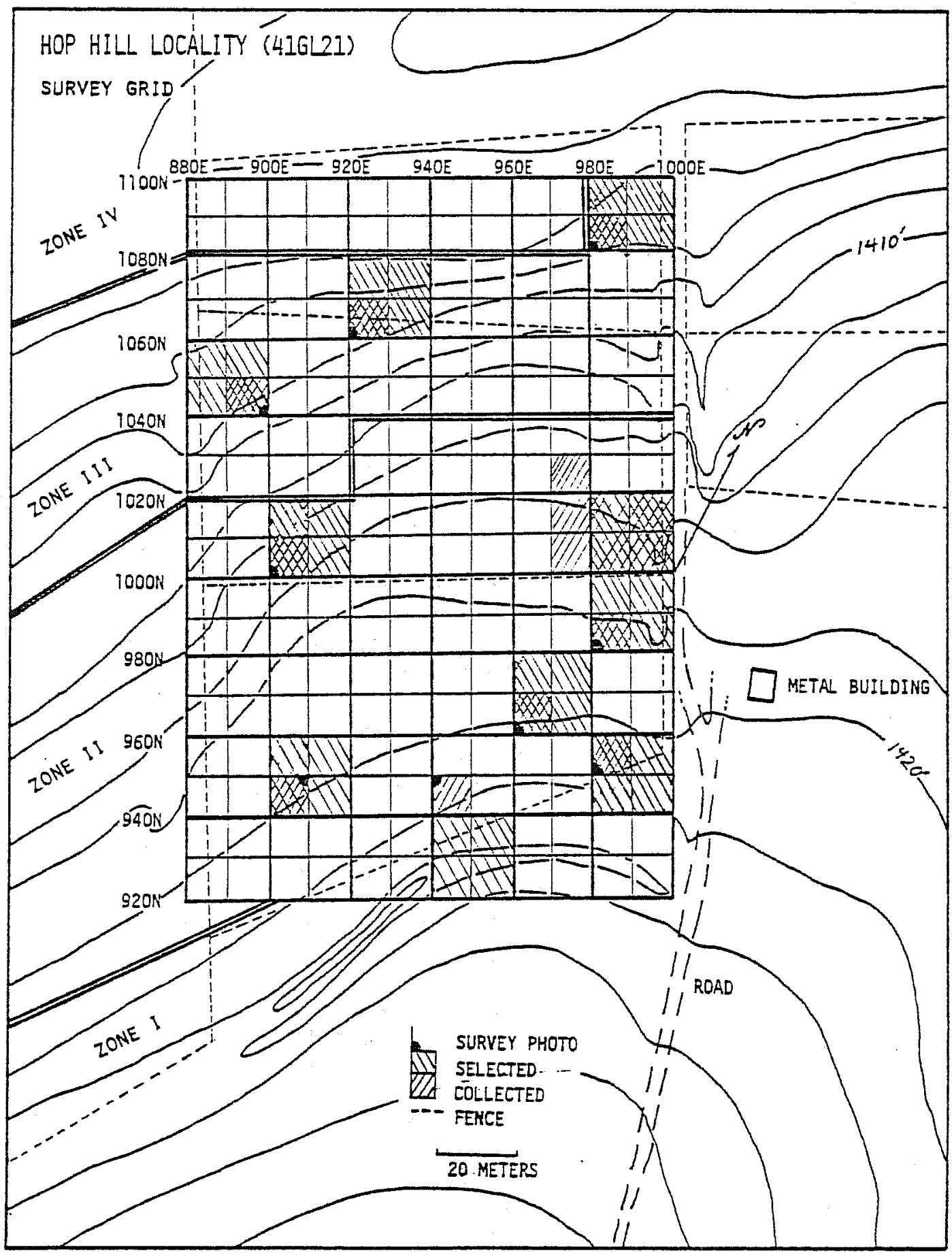

Figure 10.02. Survey Grid. Shaded 20-meter squares represent 20\% stratified random sample. Crosshatched squares represent intensiveiy collected 10-meter squares. 

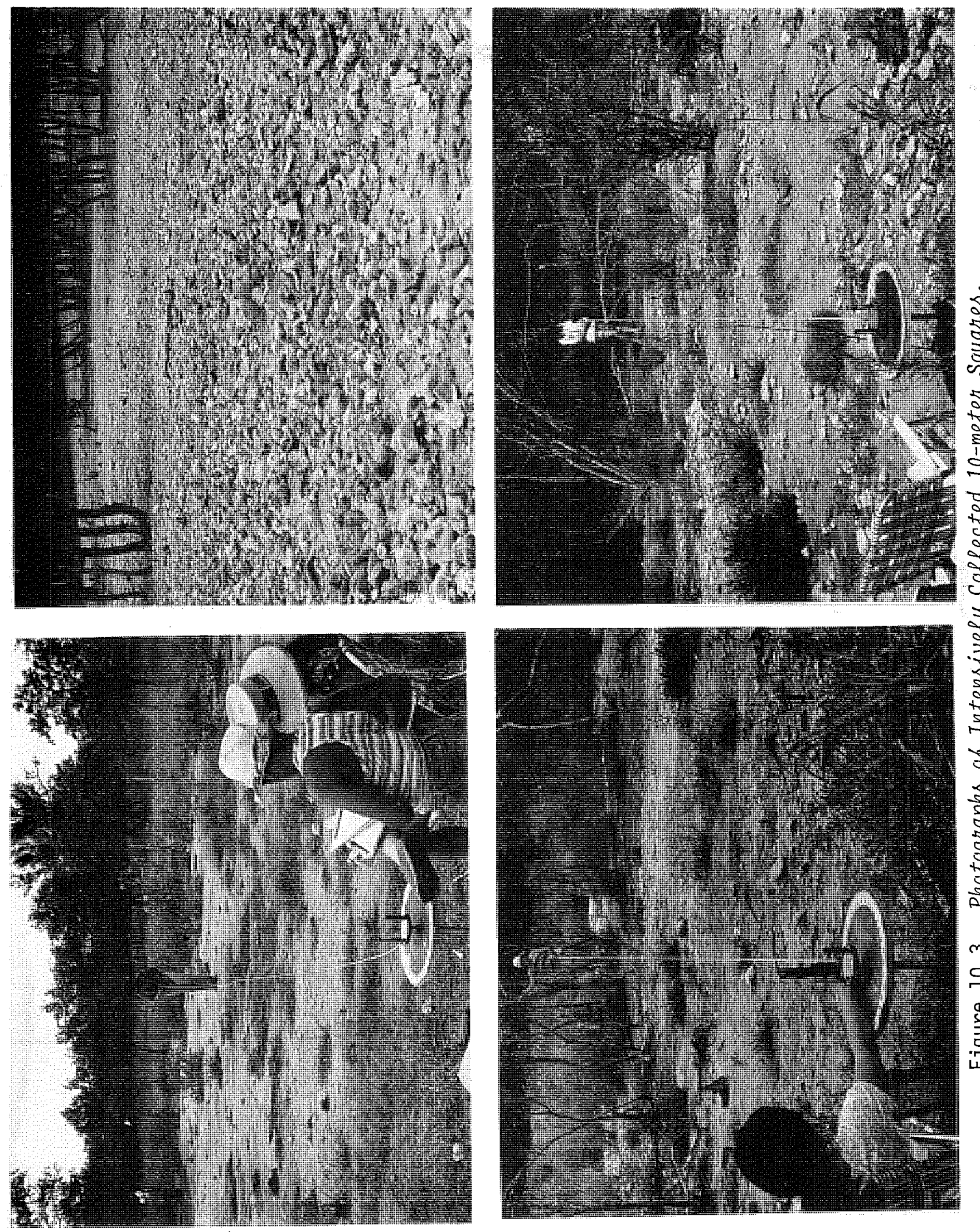


\section{HOP HILL LOCALITY (41GL21).}

\section{SURVEY}

$1100 N^{880}$

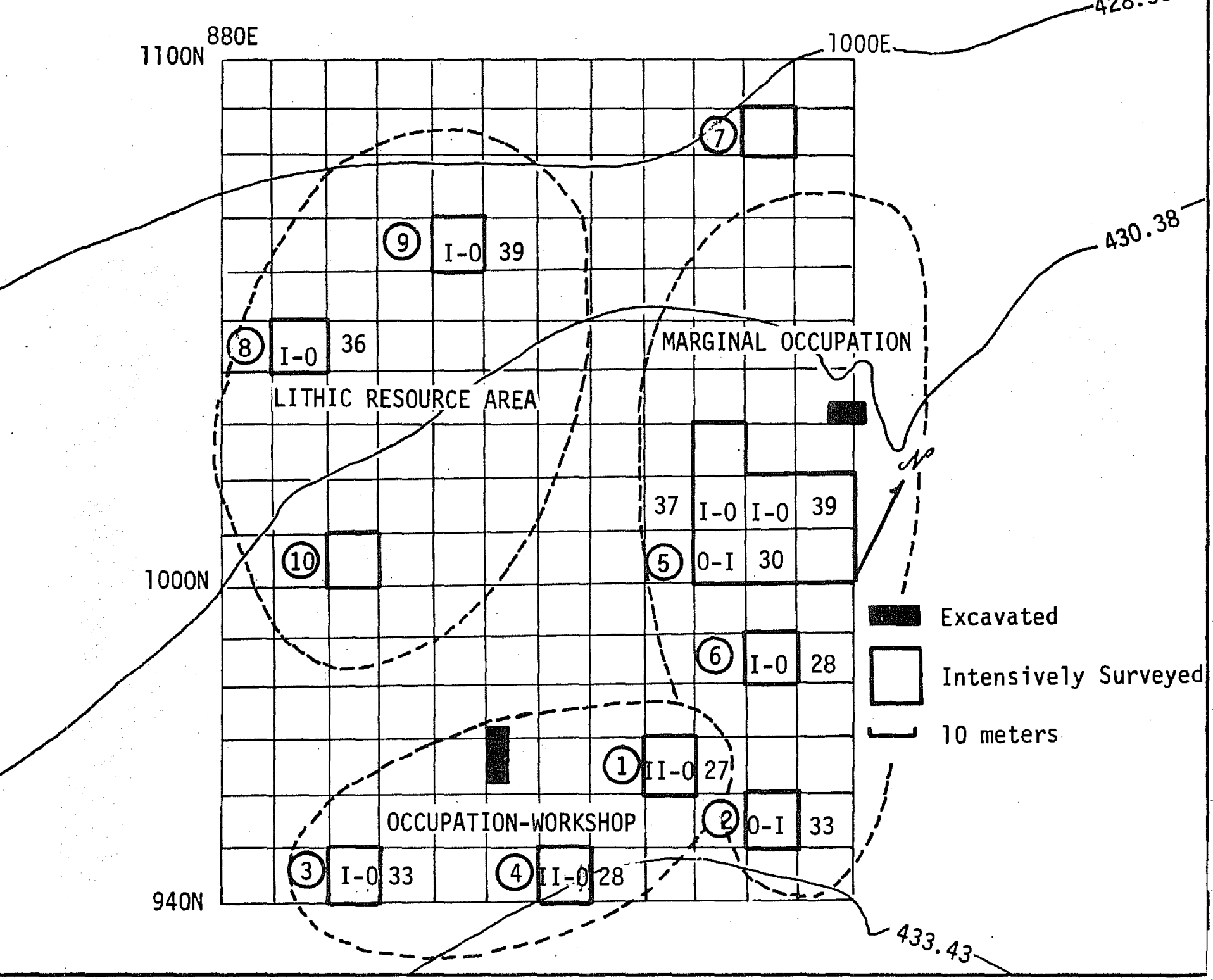

Figure 10.04. Surface Lithics Densities and Mast Nearly Related Phases of Sollberger Distribution for Each Collected 10-meter Square. Circled numbers indicate rank of 1 ithic densities. "Sollberger Phases are given in order of importance. 
Both the midden and the quarry are most Tike Sollberger's Overall Distribution. In both cases, however, the close second is the distribution which was expected. The midden is like the shaping phase while the quarry is like the decortication phase. From these rankings we surmise that both areas of the site were used for a broad range of knapping activities. Shaping, however, dominated in the midden and decortication was the main activity in the quarry.

\section{Surveyed Functiona 1 Areas}

The initial plan for survey of the Hop Hill locality was to intensively survey a $20 \%$ stratified random sample of the west stock pen (see Figure 10.02). The east stock pen exhibited far fewer artifacts on the surface. It appears to be outside the main occupation areas and/or less susceptible to erosion since it is in the tougher Purves soils (see Section 6.0 ). A $20 \%$ sample of the 20-meter squares from Zones II and III was selected by random number (Figure 10.02). It soon became apparent that a sample of ten 20-meter squares could not be collected at the level of intensity necessary to make meaningfur lithic technology studies in the length of time available. As a consequence a mixed strategy was adopted. One-quarter of each sample unit, a ten-meter square, was selected for intensive survey with the intention of analyzing flaking debris for differential distribution over the locality. In addition, a general reconnaissance of a 71 the stock pens and Zone I was conducted for bifaces, unifaces, points, cores, etc., to gather a more specialized data base for examination of tool distribution.

Figure 10.02 shows a grid map of the locality with the zone outlined and seTected and collected squares crosshatched. As a part of the survey procedure a photograph of each 10-meter square was made at the Tocation and orientation shown on the map. Examples are shown in Figure 10.03. In the lab these photos were ranked according to the density of visible debris displayed. Table 10.04 shows the ranking of surveyed units on debris apparent on the surface.

Table 10.04 Lithic Photo Density and Flake Counts

Unit Density Photo N Platformed Flakes

T. $E 960-N 960$

Least Dense

674

2. $E 980-\mathrm{N} 950$

19

3. $\mathrm{E} 900-\mathrm{N} 940$

348

4. $\mathrm{E} 940-\mathrm{N} 940$

576

5. E980-N1010

13

6. $E 980-\mathrm{N} 980$

$10.3 a$

$10.3 b$

24

7. $E 980-N 1080$

59

$10.3 c$

Most Dense

$10.3 d$

10. E900-N1000 
The rank of lithic density on the surface is noted in Figure 10.04 by the circled numbers to the left of the intensively surveyed units. The order of ranking shows that the lowest density of debris was found on the surface in the upland margin. Density increases as one moves north down the valley slope and finally achieves its highest values on the valley slope to the west side of the locality. The type of soil at various sampling points (see Section 6.0 ) is the reason behind the surface litter. The soils to the east are erosion resistant. Our excavation in the east shows that there are naturally occurring nodules near bedrock.

Soils to the west, on the other hand, are more erodable and as a consequence quantities of chert nodules were exposed on the surface. The situation was probably similar, though not so extreme prehistorically, so the area has always been an area of readily available chert material. The contour map (Figure 10.2) shows the west side of the locality to be the steepest area in the site and in some places the soil is eroded to bedrock. The slope is occasional7y precipitous so some erosion to bedrock probably existed prehistorically'.

As can be seen in Table 10.04, the occurrence of platformed flakes is inversely related to the amount of natural surface debris. Units with virtual7y no natural surface debris, ranks 1, 3, and 4, had on the order of 500 platformed flakes each while the most cluttered unit of all had none.

Within each unit the two Sollberger Distributions to which the flakes are most closely related are shown in the order of importance. 0 represents Overal1 Sollberger Distribution, I = Phase I and II = Phase III. Since Phase III was always least related the order of the third relationship can be inferred.

The only units which relate to Phase II, shaping and thinning, are in the flaking workshop area on the upland margin. A7j other units in the sparsely utilized area and in the lithic resource area are related to decortication, Phase I.

The main area of lithic workshop activity is shown by two bits of evidence. First, rank units 1 and 4 have the highest amounts of flaking debris. The amount of debris drops off appreciabiy to the east. Our excavations show that there is no more debris below the surface to the east than above it. The pattern is therefore not a product of differential erosion.

The amount of debris is down by about half to the west in rank unit 3 . Rank unit 3 may be approaching the limits of the site but we have no hard evidence. Since the field to the west was terraced it is unlikely the information is there to be recovered.

The midden is in the northwest and is an apparent occupation area. It is very near, within 20 meters of the north extent of the occupation-workshop complex. A grassy field lies south, Zone I. A drainage ditch was cut about $10 \mathrm{~m}$ south of rank unit 4. There is some cultural material but not a great deal. The most heavily utilized sector of the site, then, probably does not extend over 10-20 meters beyond the dashed line around the occupation workshop complex. 
A second notable trend is for the Phase III, sharpening and resharpening, to become more important in the workshop area. The number to the right of each unit is the S value for Phase III. Similarity is generally in the $30-40 \%$ range. In the heart of the workshop area, however, it drops into the $20-30 \%$ range. Rank units 5 and 6 also have good similarity to sharpening. They have few artifacts and may represent small resharpening flakes which are in the process of washing out of the main workshop area. 


\subsection{CORE FLAKING MODEL (Linear Technospatial Analys is)}

(Joel Gunn)

Introduction

Various persons have attempted to develop methods of analyzing the scattered debris on occupation floors. The most complete and recent review of this literature is to be found in Brose and Scarry (1976). Most of the methods have been attempts to determine statistically the significance of aggregations of tools and debris. All require the assumption that the debris is unmixed with pieces from other times and from strata above and below. Many methods require constraining devices such as the shape of the excavated floors or lumping of types by grid squares. All of these properties are undesirable in the give-and-take of the archaeological analysis. The most carefully excavated occupation floors can be assumed unmixed under the rarest of circumstances. More often the debris are surely mixed as in the case of deflated areas.

The procedure developed in this section is designed to generate hypotheses of functional variation over occupation spaces. The only assumptions are that the functional variates are non-randomly associated with space and that association is linear. Linear is taken to mean that the functional variation can be described by a straight line equation or some higher order equation which fits curved lines. An equivalent nonlinear analysis technique is developed elsewhere (see Section 15.0).

Linear Spatial Analysis is an attribute based analysis in the extreme. Both the occupation floor and the occupation debris on it are represerited as measurements of functional or technological variation.

In the case of the occupation floor functional dimensions appear, at least on the surface, to be apparent. Occupation floors, camp arrangements, etc., are in essence planes on which artifacts are discarded during habitation. The extent of these planes and the pattern of debris is defined by the Cartesian Coordinates (Gunn 1970) of the artifacts. Inherent in that pattern is the functional arrangement of the tools.

The technological and functional dimensions of the occupation debris are defined with much more difficulty, hesitation and misgiving. Personally I think it is more interesting to define a model, usually a systems related model, which explains how a class of occupation debris was manufactured and used. From this logical construct theoretically functionally relevant dimensions of variability are defined. For instance, edge angle on bifaces and unifaces is thought to be a functionally relevant attribute (Wilmsen 1970). The presumption is that tools used for scraping, chopping, etc., require tough, supported edges which are best realized in high edge angles. In contrast, tools that are used for cutting require more grace than strength and low edge angles are favored. 
Linear Spatial Analysis takes advantage of the continuous scale properties of Cartesian Coordiantes and technofunctional measures to determine if there are any directional tendencies in the pattern of occupation debris. So far, edge angle coordinates have been suggested. Suppose the edge angles of tools occurring on a floor and the east and north coordinates have been measured. The correlation between east and edge angle approaches zero; the correlation between north and edge angle, however, is very high. Our interpretation of these relationships is that nothing that is functionally relevant to edge angle was happening on the east-west dimension of the floor. On the north-south dimension, however, there is a distinct tendency for large edge angles to occur to the north and lower edge angles to the south. The inhabitants of the floor, then, were chopping and scraping to the north and cutting to the south. Reference by various means to a map of the floor will show the exact location of the activities.

It should be noted that classes of tools analyzed can only be as varied as the functional attributes are generally applicable. Edge angle is a very broadly applicable attribute. Both unifaces and bifaces have an equivalent edge angle. Weight is a very generalized attribute and probably functionally relevant. Notch width on points is not a generalized attribute as it applies only to that class of artifacts. It could be used in a Linear spatial Analysis but the sample would have to be confined to points. The relationship on an occupation floor between notch width and edge angle would have to be surmised by visually comparing two different analyses.

In the example above only one attribute was examined for $i$ ts relationship to space. A more complex analys is can be devised in which the artifacts from an occupation floor are measured for several attributes. A matrix of these attributes and Cartesian Coordinates can then be treated by some linear forms of analysis. Factor analysis, principal components, multiple regression, canonical analysis and discriminant function are among those commonly available, and the method selected will depend on the goals of the analysis.

The following pages spell out an analytic process for determining the applicability of Linear Spatial Analysis and for applying it in specific terms. The sample selected is a relatively small group of cores from the Hop Hill locality near Stonewall, Texas. The material was collected as a stratified random sample during the 1976 field season.

The analysis is effected in four steps. First, the assemblage is technologically described and the important dimensions of variability are hypothesized. Second, the core manufacturing process is conceptualized in a systematic fashion and a measurement scheme derived from that model. Third, the important dimensions of variability are tested to determine if a linear understructure crosscuts a 11 types of cores. Finally, the relationships between the understructure and space is determined and interpreted. 


\section{Core Flaking Model}

"Core is defined somewhat narrowly here as an item which served as a source of flakes, has a platform(s) with a relatively high platform angle $\left(>60^{\circ}\right)$ and is clearly not a biface. The intention is to conservatively estimate nonbifacial activities since bifacing is the dominant technique used at the site. Items that are marginal to both the core flaking and bifacial flaking models will be forced into bifacial categories. This tactic, hopefully will give an unobscured view of how techniques, aside from bifacing, were effected. The terminology used in the discussion is illustrated in Figure 11.01 .

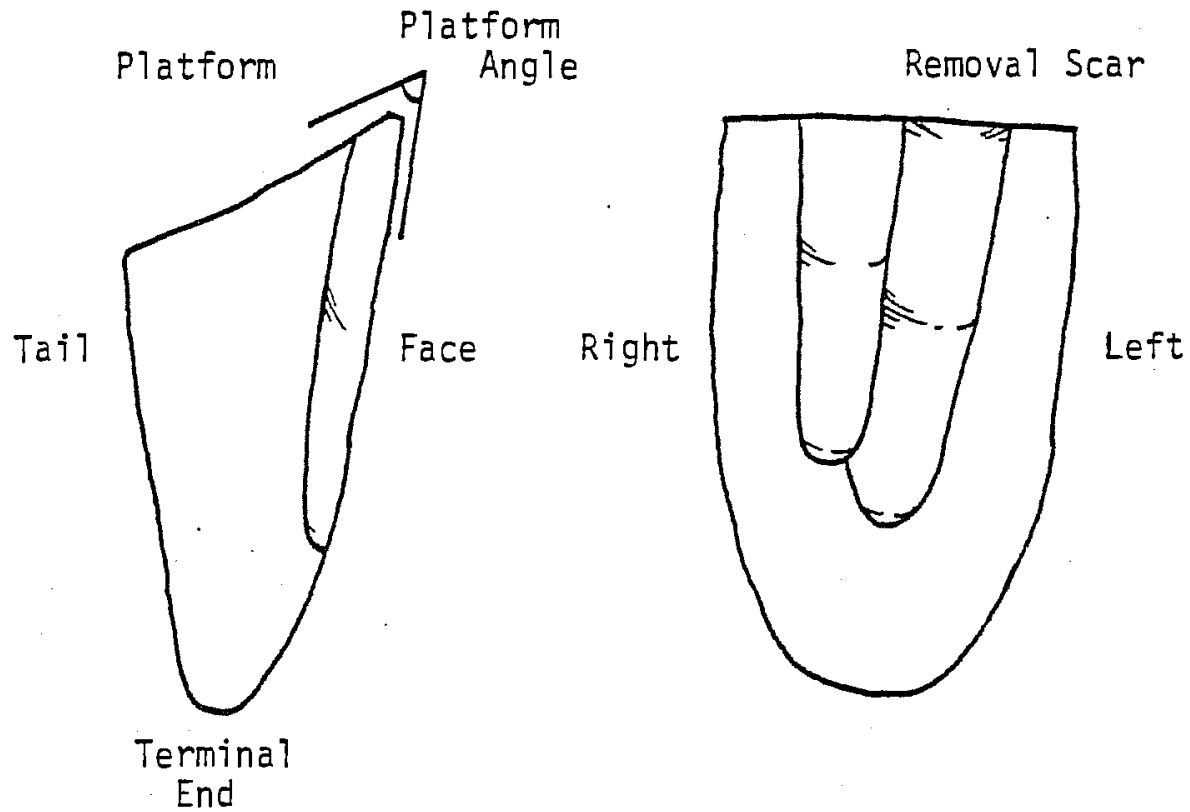

Figure 11.01. Flake Core Terminology.

"Bifacial cores" are classed with bifaces. This differs from the normal procedure of grouping bifacial cores into cores in general. The reason for doing so is that bifacing and core flaking are two distinct processes or models. Core bifacing and bifacial thinning, on the other hand, are clearly variations on the same process. As will become apparent, the model designed to explain core flaking would not be appropriate for bifacial flaking. 
Core Descriptions

Only twenty-seven pieces were identified as cores. The relatively small numbers reflect the fact that core flaking was a decided sideline at Hop Hi11. All but one core are from the surface.

1.0 Flake-blade Cores $(N=2)$

FN718(S:944-801), 741-1(S:1026-1014)

Weir (1976:74) calls these unidirectional blade cores. He notes that he observed no true prismatic blade cores in the seventeen sites from the Central Texas Archaic he studied for his dissertation. These are likewise not true blade cores.

Some true blades have been reported from Archaic contexts (Sollberger and Patterson 1976:527-530) and Toyah Phase sites (Green and Hester 1973). Also, there is a late prehistoric coastal blade core industry (Hester and Shafer 1975; Hester 1975). As will be shown, the two Hop Hill cores appear to be made by modifying essentially bifacing techniques to meet the demands of purpose. They are designed to yield flake-blades more than twice as long as they are wide and more or less parallel-sided in outline. The two cores as quite similar in concept. The platform angles are nearly the same $\left(718=60^{\circ}\right.$, $\left.741-1=57^{\circ}\right)$. They are so nearly expended they are reduced to long, thin slabs.

FN718 (Figure 11.02a) is made of a low-grade chalcedony which is predominantly fine-grained in spite of numerous inclusions. It is white with a purplish cast and white inclusions <l mm in diameter. The tail of the core has cortex which is pitted, perhaps by dripping water.

The platform is faceted though it has been reduced to a thin line along most of its length. On the face the last, centermost flake-blade hinged out about $1 / 3$ along the core length. The platform was probably too weak to carry any adequate blow to bring the blank to a successful termination. The face is unusually flat and wide for a flake core which probably indicates a design aimed at relatively broad as well as long removals. There are signs of shaping along the sides which serve to produce a convex removal face much like the Levallois core preparation technique. This technique is called Grand Presigne in the European Neolithic (Gunn 1971).

The left terminal edge of the core is modified for use. Closest to the end is a large notch $(17 \mathrm{~mm}$ wide). Next along the edge is a denticulated edge $32 \mathrm{~mm}$ long.

FN741-1 (Figure 11.02b) is made of fine grained grey chert. It is vaguely banded with reddish-white bands about 1-2 mm wide. The blocky structure of this core remnant suggests the parent block was of tabular rather than nodular chert. About $1 / 3$ of the back and the left side are covered with cortex. The rest of the cortex is trimmed away by careful core preparation. 

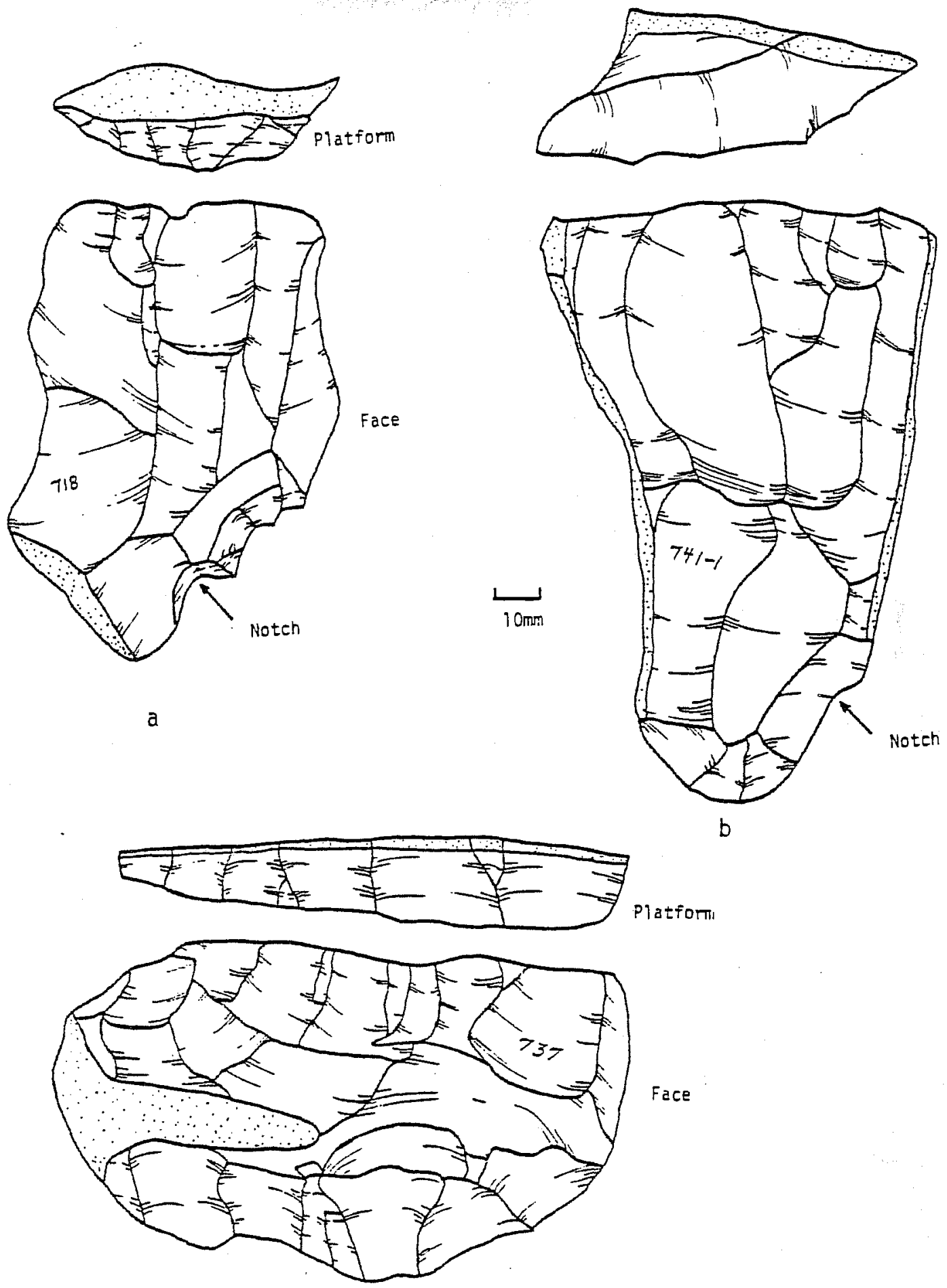

$c$

Figure 11.02. Flake Cores. $a-b$, flake-b7ade cores; $c$, flake core, straight platform. 
The platform was made by a single flake which originated on the right side of the core and terminated on the left. The platform edge was carefully shaped and ground just previous to the last removal.

The face shows a long history. Early flake-blades hinged out about $15 \mathrm{~mm}$ from the present end of the core. These obstructions were removed by bifacial working of the terminal end of the core. The reworking was to no avail, however, because the next two removals hinged out halfway down the face. After the second hinge the effort was abandoned. By standards of FN718 the knapper stopped very early; it is twice as thick. The problem was some coarse inclusion in the right side of the face which apparently caused the debilitating hinge fractures. The core was probably abandoned because it would be necessary to shorten it by half to get rid of the hinge fractures. This was too short for whatever use the flake-blades were to be put.

There is a notch at the left terminal edge as with FN718. There is no denticulated area.

Both cores show the workmanship of a skilled person(s) who was familiar with bifacing techniques. They were probably made by someone who ordinarily used bifacing to make flakes but who wanted flake-blades longer than can be produced from a bifacial core. One of the flake-blades from FN747 was $109 \mathrm{~mm}$ long which rivals products of the various punch blade techniques.

\subsection{Flake Cores, Straight Platform $(N=7)$}

FN737(S:1024-1081), 1383-2(S:963-965), 1500(S:902-931), 1502(S:983-951), 1523(X:988-954), 1712(E:931.69-968.17-2.3), 1970(S:906-1042).

Seven cores were classified in this category because of virtually straight platforms confined to the long edge of the nodule (Figure 11.02c). They appear to be opposite in intention to the flake-blade cores. Removals are short and broad. Most of the flake scare appear to be approximately square. The platform angles are high, $\bar{x}-81.8, s=8.4$. Taking such flat removals is something of a feat. That most of these cores were discarded with battered platform edges and hinged faces is mute evidence to the end of a difficult task. All of the cores are made of fine to very fine grained, grey or brown cherts.

FN1712 is the one excavated core. It is from occupation floor 2.20 in the Midden. FNT500 has two platforms. The additional platform is a standard, more rounded platform. FN737 (Figure 11.02c) and FN1523 have two straight platforms.

There is nothing to indicate that the cores were utilized. They are in more battered condition than other core types but the battering appears to be a result of problems with the method of manufacture. 


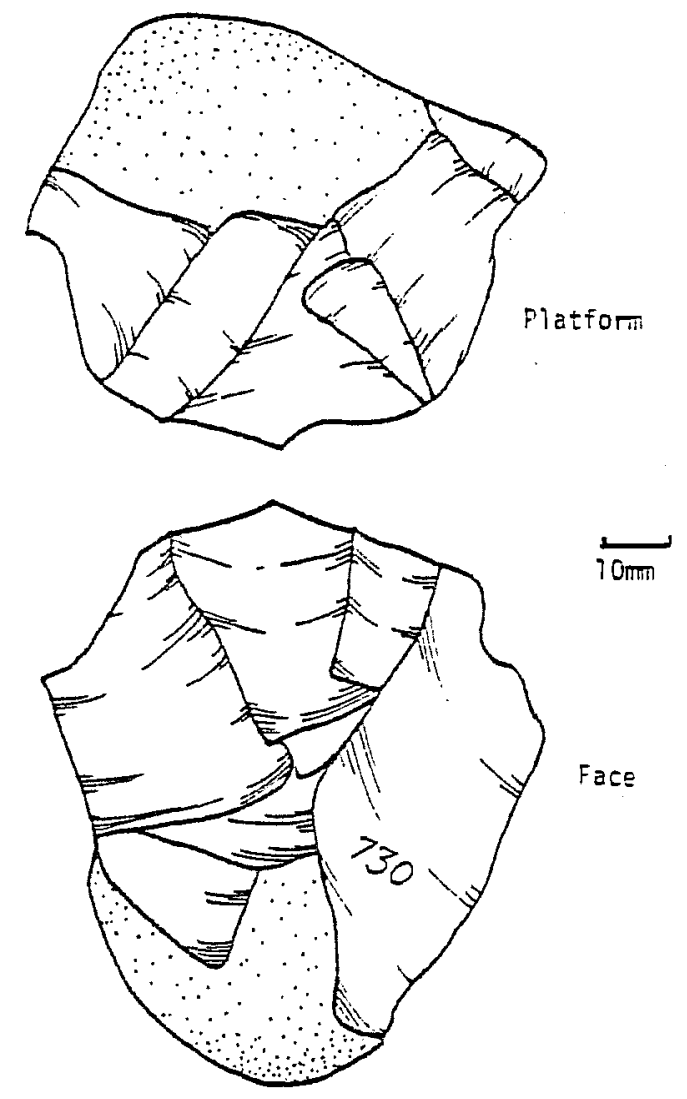

a
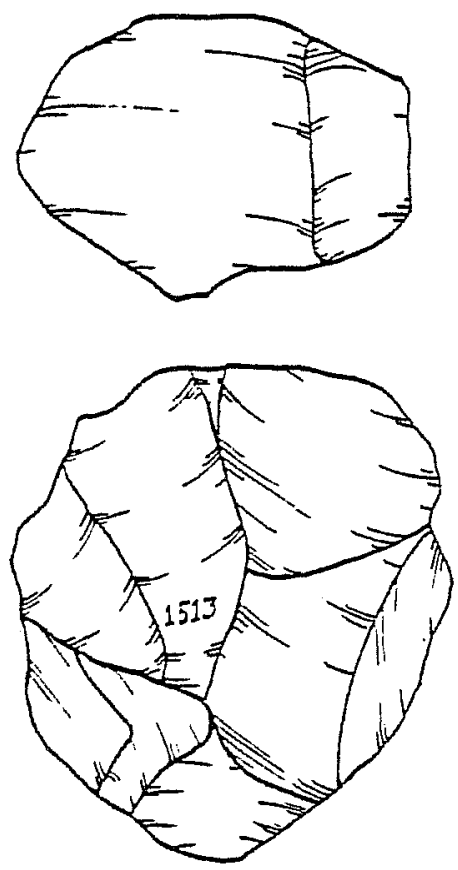

b

Figure 11.03. Flake Cores, Rounded Platform.

\subsection{Flake cores $(N=14)$}

FN104(S:991-1010), 216(S:975-1001), 675(S:958-1034), 730(S:951-953), $788(5: 910-947), 789(\$ 1: 904-943), 822(5: 935-933), 1061-1(5: 967-967)$, $1390-5(5: 968-962), 1406(5: 969-964), 1513(5: 981-950), 1808-2(5: 932-1067$, $1973(S: 905-1041), 1966(S: 908-1051)$.

These are standard flake cores (Figure 11.03a-b) with a normal, rounded and easy-to-use platform. Most are made on nodular chert and as a consequence many are basically spherical. Chert grain ranges from medium to very fine. only in a couple of instances was more than one platform established. Platforms were usually prepared with more than one large flake. Flake scars on the faces are usually slightly longer than they are wide but not twice as long as wide. Four specimens were heat-treated, judging by reddened cortex. Platform angles tend to be relatively low: $x=71.4, s=11.9$. There is nothing to suggest they were utilized; however, no microscopic examination of the platform edge has been done to test this assumption. 


\subsection{Flake cores, Multiplatform $(N=3)$}

FN1428(S:970-963), $2007(S: 905-1043), 2017(S: 942-950)$.

Three cores had no particular platform orientation. Any surface that was available and provided the correct angle for a platform edge was used. Al1 three are of fine-grained chert. Two are of the usual grey or brown material. One (1428) is tan with red "veins" in the material. FN2007 was retouched (nibbling) along a $16 \mathrm{~mm}$ section and used to a polished edge.

\subsection{Core Fragments $(N=13)$}

Thirteen pieces loosely conform to the category of core fragments. Whether they are nature-facts or the product of humble workmanship is open to question. Some, however, are of fine-grained material.

Block Reduction Model

A conceptual device for core reduction systems is appropriate for this category of artifacts. The Block Reduction Model (Gunn 1971) treats all cores as cubic blocks (Figure 11.04a) from which removals are made with the intention of preparing and shaping the core or detaching blanks. Whatever the shape of a core, it is reduced to six surfaces for purposes of analysis.

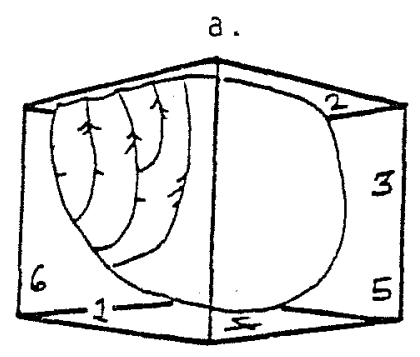

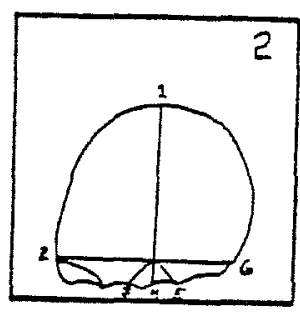

b.

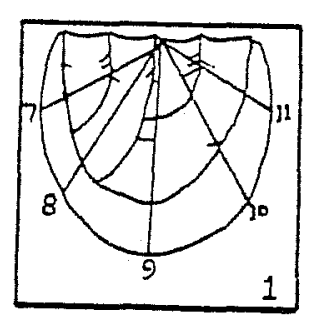

c.

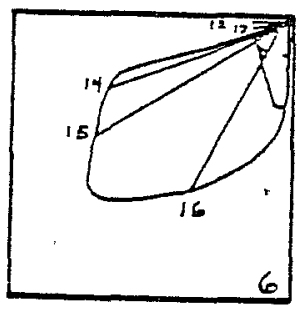

d.

Figure 11.04. Block Reduction kciel. a, 211 surfaces represented; $b$, top surface of model with polar coordinates superimposed on a core, polar coordinates $1-6$; c, face surface of model with polar coordinates superimposed on a core face, polar coordinates $7-17$; , right side surface of model with polar coordinates superimposed on core side, polar coordinates 12-16. 
Options available to the Knapper are:

1. Order of surface reductions--the artisan is at liberty to remove material from any surface in any direction. Ordering is constrained by unfavorable platform edges, or non-platform edges. A good knapper, however, can prepare a core so it has the correct angle at any edge by a series of reductions from a favorable edge. Ordering is determined by examining the margins of flake scars for fissure lines, intersecting ripple marks, etc.

2. Orientation of reduction--reduction of the block can be made either parallel to a surface or at an angle to a surface. The orientation of the reduction blow is causally related to the resultant platform angle (Speth 1975).

Each surface and edge of the block is the conceptual equivalent of some generaliy accepted term relative to cores. The "face" of the core is a constant plane or Surface 1 (Figure 11.04a). All operations are analyzed relative to the face. The upper edge of the face is the "platform edge." The surface above is the platform, Surface 2. The tail of the core, opposite the face, is Surface 3. The terminal end is Surface 4 . Surface 5 is the left side, as one looks down the core from the knapper's point of view, and the right side is Surface 6 .

Relative to the block, core surfaces are either conformant or nonconformant with a surface. If a core surface is nonconformant with a block surface the relationship between the theoretical and actual surface is a transformation of the plane. Sometimes these transformations can be described by a simple measurement. For instance, if a platform angles downward from the platform edge, the transformation from plane to platform is the complement of the platform angle. More complex surfaces require proportionally more complex measurement techniques. One method used in this study was to superimpose a polarcoordinate grid on the core conformant with the block. Three arrays of polar coordinates describe the shape of cores adequately, one each for the platform (Surface 2, Figure 11.04b), the face (Surface 1, Figure 11.04c) and the side (Surface 6, Figure 11.04d).

It can be surmised from the descriptions of core types that they key dimension of variability in the Hop Hill core assemblage is the length-width ratio of scars from core faces. The motivation behind this variability is most likely multiple tasks which require products of multiple core reduction strategies to meet specific functional requirements. Removals for tasks requiring flakes from one to two times longer than they are wide were made from loosely designed and nonspecialized Flake Cores. Deviation from these normal cores with normal flakes required more effort and more specialized techniques. Flakes longer than two times the width were produced from Flake-blade Cores. Flakes shorter than one times the width were produced from Straight Platform Flake Cores. Thus, the type of core flaking technique used is a linear function of the length/width ratio of the desired blank. 
The descriptions also suggest that there are other configurations covarying with the shape of the blank. The platiorm angle, for one, is low in the Flake-blade Cores and high in Strajght Platform Flake Cores. An analysis of variance between types and platform angle is significant at the .013 level of probability. Also, the relative breadth of the platform appears to differ from type to type. ANOVA confirms this observation with an F-value significant at the .004 level of probability. As a check on the idea that length/width ratio of the blank varies with type, ANOVA was also run on that combination of variables. The'significance is greatest of any at .001. It is hard not to believe that these parameters were manipulated through the platform angles. It seems possible that the breadth of the platform was manipulated to produce blanks as EFFICIENTLY as possible from available material. Taking a short flake off the long axis of a core simply wastes most of the core. Taking a short flake off the short axis, on the other hand, allows repeated and efficient use of the core.

a.

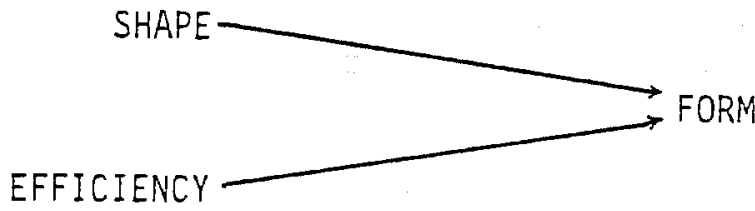

b.

Platf. Angle

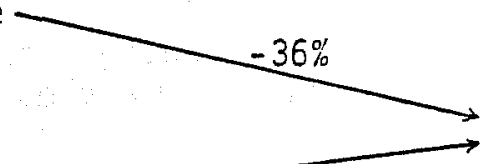

Blank Length/Blank Width
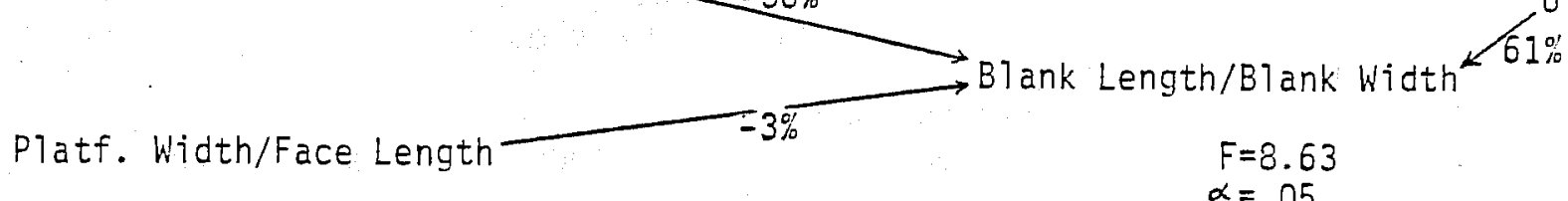

$\alpha=.05$

c. $V .=3.38$

Platf. Angle

d. $f .=2 \times 24$

c.

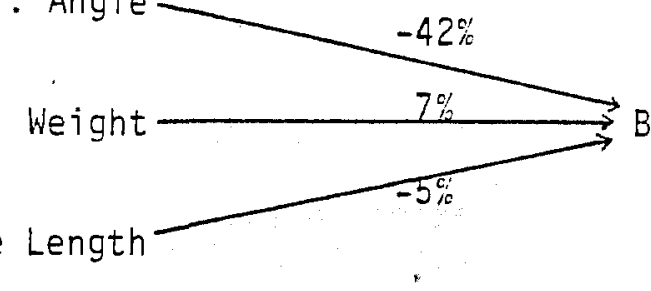

Blank Length/Blank Width $/ 46 \%$

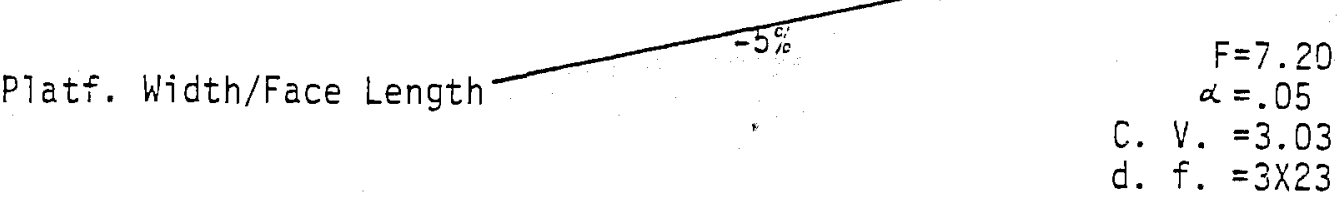

Figure 11.05. Causal Model Describing Relationships of Causal Variables to Resultant Tool Form. a, proposed causal relationships between technological motivations for desired SHAPE and EFFICIENT use of material and the final FORM of an artifact; $b$, indicators for the system and percent of variance accounted for; $c$, the system controlling for gross SIZE of the cores. 
We are presumably, then, dealing with two unknown quantities which motivated core flaking technology at some point in the prehistoric past. One is the desire to have blanks which meet specific tasks effectively. The other is to use materials as efficiently as possible. If the intentions are as clear as we theorize them to be we would expect the postulated tendencies to show up as deiectable levels across the entire assemblage of cores. The two specialized types of cores have pointed the way to this hypothesis; but was the tendency so pervasive that it holds in the nonspecialized Flake Cores as well? Is the whole assemblage the product of one set of rules, or were there three sets of rules?

Figure 11.05a shows a causal model derived from the foregoing discussion.

Desired tool SHAPE and EFFICIENT use of raw materials are taken to be the causal variables behind the resultant tool FORM. The desired SHAPE is assumed to be reflected in the angle at which the platform was set by the artisan at the beginning of the reduction operation. The choice for EFFICIENT use of the material is thought to be indicated by the placement of the platform relative to the long axis of the core. The width of the platform edge divided by the length of the core face was taken to be an indicator of this trait. An indicator of FORM was constructed in the following way. The longest successfully terminated removal on the face of each core (not hinged) was measured for maximum length and width. Since the usefuiness of a core is usually ended by a series of hinge fractures across the face, it was often necessary to extrapolate across these hinged areas to previous generations of scars, the evidence for which is still to be found toward the terminal end of the core.

The null and alternative hypotheses are as follows:

$H_{0}$ : If a linear relationship between the indicators chosen to test the model is statistically significant, then there is no evidence in the data to suggest that the model is not correct. The position will be supported that ali of the cores were made by linear variations of the same set of rules.

$H_{1}$ : If a linear relationship between the indicators is not statistically significant, then the evidence in the data suggests that there are more than one set of rules. The implication is that there are as many sets of rules as there are types of cores.

Figure $11.05 \mathrm{~b}$ shows the result of regressing blank length/blank width with platform angle and platform width. The percentage points next to the arrows are the squared beta coefficients. "U" stands for the unknown or unspecifier variables in the system. In this case $61 \%$ of the behavior in blank FORM is accounted for by unknown forces. Sixty-one percent is calculated as 1.0-R2. 
Relative to SHAPE and EFFICIENCY, SHAPE clearly is a close correlate of FORM. It accounts for 36\% of the behavior in FORM. By comparison EFFICIENCY is not very much involved. The implication is probably that even if efficiency is involved in the two specialized Flake cores and, since they form the bulk of the population, EFFICIENCY disappears as an important factor in the overall core-making process. Efficiency would tend to be a more significant variable where a shortage of quality resource material existed. This is not the case at the Hop Hill locality. Perhaps the abundance of chert material in Central Texas fosters a less strict attitude toward conservation of cherts (T. R. Hester, personal communication). This is not so with SHAPE or platform angle. It is done enough in whatever type that it appears as an important consideration across the entire group of cores.

From this analysis, then, it appears that there is probably one underlying process behind al1 core production and that process involves adjustment of the platform angle to achieve proper blank FORM.

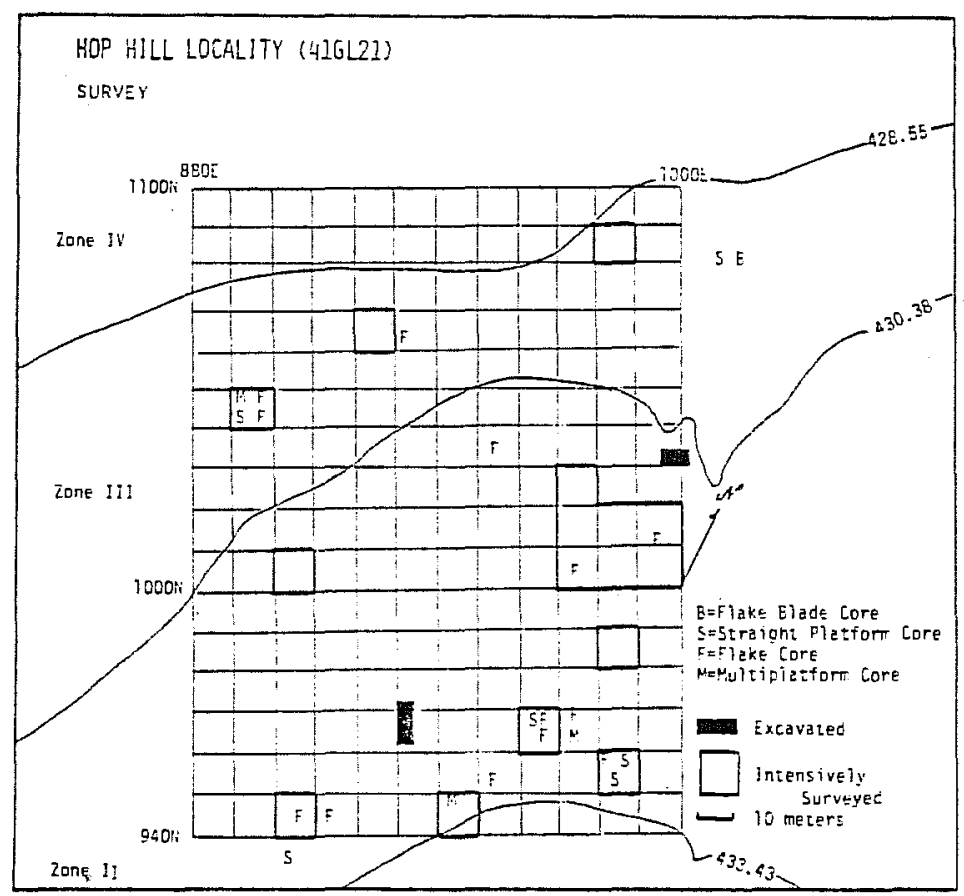

Figure 11.05. Core Distribution.

As a precaution a second equation was run. It seems possible that the sheer size of a core could influence the size and perhaps the shape of the blank. Since the size of the core is only partly controlled by the knapper it is a noncultural influence on the system. If the effect of core size could be removed and the relationships between SHAPE, EFFICIENCY, and FORli were examined 
free of core size it would assure that the relationships are not spurious. To control for SIZE the weight of cores in grams was added to the mul tiple regression equation. This automatically removes the effect of SIZE from the other two relationships. The results are shown in Figure $11.05 \mathrm{c}$. The effect of removing the influence of SIZE was favorable to both relationships. Removing the adverse effect of Mother Nature's lack of standards when she produces flint nodules increased the validity of our inferences slightly.

Visual inspection of the distribution map (Figure 11.06) of Hop Hill shows that the more specialized core type, Flake Core, Straight Platform is restricted to Zone II (the upland margin) except for one piece. The type is also located in the village area rather than the midden. The unspecialized Flake Core type, on the other hand, is more dispersed through both Zone II and II (Valley Slope). It is apparent from the map that cores in genera 1 were found in the areas of intensive survey and few were picked up in the causal collecting sorties.

Counting only the cores from intensively collected $10 \mathrm{~m}^{21} \mathrm{~s}$, the density per unit area is slightly higher in Zone III than in Zone II $(1.67$ and 1.00 cores per $10 \mathrm{~m}^{2}$, respectively). Thus, the higher overall concentration of cores in the zone thought to represent quarry activities and therefore nonspecific chert working is supported. Also, the more generalized type of core dominates in that area.

Zone II is somewhat different, with more specialized cores in addition to the nonspecialized pieces; this complies with the view that Zone II is a villageworkshop complex.

Principal components analysis of the interval and ordinal scale attributes taken on the cores along with their respective east and north coordinates shows several linear relationships between core attributes and space. The strongest of these is a tendency for cores with wider removals and higher platform angles to appear in the west. Conversely, items with narrow removals and low platform angles tend to be in the east. In other words, the flakeblade technology and cores most like it were practiced more in the eastern part of the site. The largest group of cores along the east coordinate appear around coordinates 980-960. This is east of the midden and locates the nexus of the "workshop" area.

A separate and independent tendency from this flake-type technology factor is the tendency for there to be more evidence of fire and heat treating to the south and, at the same time, heavier and more robust cores (weight and polar coordinates 1,8 and 15) to occur in the north. The firing of chert in the south again points to a workshop area on the hill and in Zone II. The heavier, probably less worked and finished cores, meanwhile, remain in the quarry area. Also, coarser-grained materials occur in the north. This reflects the coarser-grained local material being worked in the quarry as 
as opposed to the finer-grained and probably imported materials which show up in the workshop. Finally, the polar coordinates show that wider cores tend to occur in the north. This combination of attributes and spatial tendencies very interestingly, and hopefully adequately, describes the contrasts between village and midden on the east-west direction and between workshop and quarry on the north-south axis of the site.

Spatial autocorrelation has not been dealt with in this section. It needs to be studied before the results are accepted with confidence. 


\subsection{FACE FLAKING MODEL-BIFACING (Curvilinear Technospatial Analysis) \\ (Joel Gunn and James E. Ivey)}

Face flaking, either unifacial or bifacial, is in concept simpler than core flaking. Bifaces are biconvex objects whose edges generally describe a plane. Thus, where the core process requires six planes, the bifacing process can be explained by one plane (Figure 12.01a). The biconvex surfaces are here simplified to a diamond which can be rotated through the plane to describe the surfaces at any given point. The diamond is also the direction of a bifacing blow. Rotating the diamond around the origin or center of the plane will describe the direction of any blow. The surfaces of a biface, its shape, are a transformation of the diamond along any given trajectory (Figure 12.01b), and the effect of the biface shape on the blank resulting from a blow will be a function of that transformation. The angle at which a blow is delivered (Figure 12.01b) can also be described relative to the bifacing plane.

The tactics available to the knapper are:

1. Lateral movement--the knapper can move laterally along the edge of the bifacing plane placing blows at any point along the plane and orienting them toward either the upper or lower surface. (The model is potentially a unifacing model, also. The only change is to limit orientation to one direction.)

2. Orientation of blow angle--the angle at which a blow falls on the bifacing plane will control the length of the flake. If bifacial thinning flakes (flakes traveling more than halfway across the surface) are desired, the angle is adjusted low enough to propagate a long, thin flake and followed through with the energy necessary to complete the removal. A higher angle will produce shorter flakes that do not cross the center of the surface and will impart a biconical outline characteristic of bifacial cores.

The shape of a biface is measured by imposing a polar coordinate grid on the bifacing plane (Figure 12.01c). In this study the convention of orienting the bit and/or narrow end of the biface toward the lower end of the grid is adopted; the number of rays to the lower end is greater to describe those features in more detail.

Two sets of relevant dimensions are derived within the context of the model which account for the technological and functional variance in bifaces. The technological variables are ultimately of functional importance, but they are the first, fundamental choices that have to be made about how an artifact is to be produced. Edge Arc is the number of degrees of arc along the modified biface edge as measured from the center of the bifacing plane. As the Edge Arc increases the length of the functional edge increases. A pick, then, has a very short arc, a cleaver more, a chopper more still, while a knife will probably have an Edge Arch of about 180 degrees. A preform is modified all around so it has an arc of 360 degrees. Through the process modified edge increases at the expense of unmodified edge.

The Bifacing Mode describes the angle of incidence of the bifacing blows. It is not called edge angle here because the variable is more complex than that. Whether a bifacing flake has the effect of thinning or merely flaking depends on edge preparation, such as the strength of the platform, surface character- 


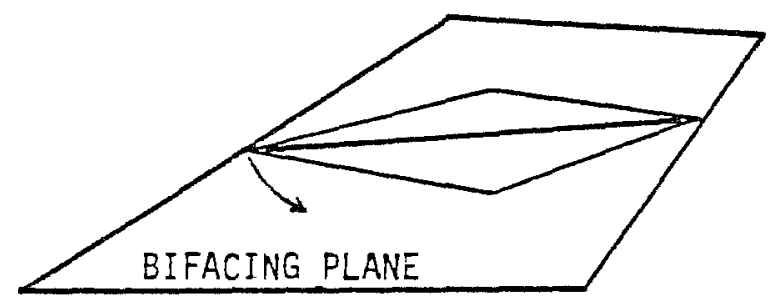

a.
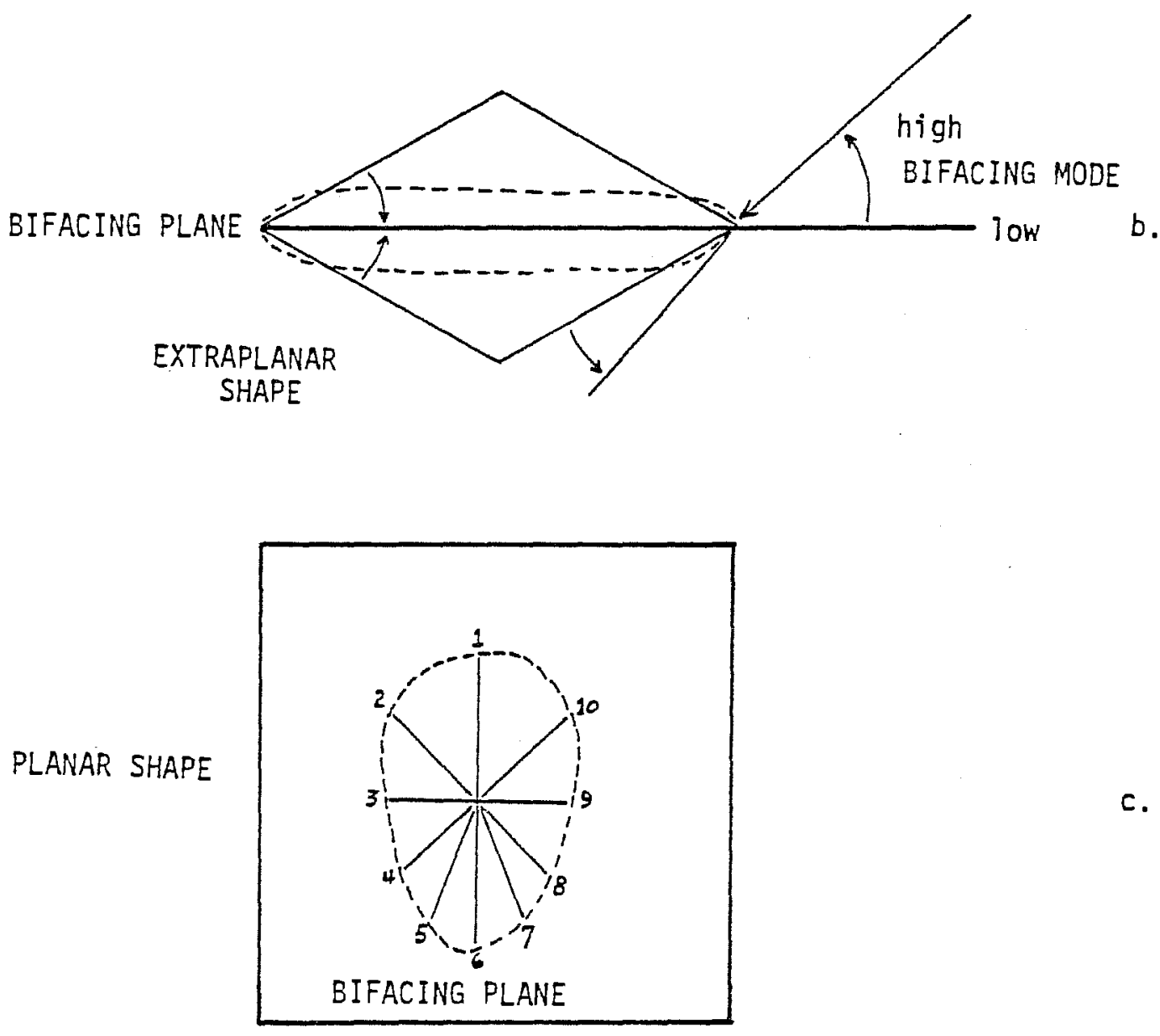

c.

Figure 12.01. Facing Model--Concepts for Unifacing and Bifacing. 
istics, such as spines, and a complex set of inward and downward forces controlled by the knapper as the blow is delivered. We will assume for now that most of the variance generated by this complex set of forces is accounted for or estimated by the measurement of edge angle. Relative to the bifacing model, the bifacing plane bisects the edge angle so the blow angle will be $1 / 2$ the edge angle (Figure 12.01b). The term "Bifacing Mode" is used to insure that edge angle is only an indicator which may some day be replaced by a better indicator.

The results of varying Bifacing Mode are roughly these:

1. A high angle will result in flakes that do not cross the center 1 ine, and is therefore, not a bifacial thinning flake. High angle bifacing is appropriate for bifacial cores and bifacial tools such as choppers.

2. A low blow angle is a component of the complex motions necessary to remove a flake which extends over halfway across the biface surface and as a consequence thins the center of the piece (Crabtree, Sollberger, personal communication). Low blows are appropriate for tools which are necessarily more effective with a thin cross section. Counted among these are projectiles and knives.

The size and shape of a biface is in large part a matter of functional consideration and these attributes are controlled by the knapper through the expedient of material selection. Size is a function of distance from the origin of the bifacing plane to the edge of the biface. The accuracy of a size estimate increases with the number of radii measured from the origin of the bifacing plane to the edge and/or surfaces of the object measured. A diameter is more accurate than a radius. Orthogonal diameters are still more accurate; ultimately, the weight of the object is equivalent to an infinity of radij and is the virtual size indicator.

Among the unhafied tools, larger sizes are appropriate for functions which require more force generated by momentum. Small sizes are more applicable to precision functions. This relationship is modifiable via the addition of mass by hafting which allows an increase of energy by momentum as in the case of a projectile, or by sheer force as with a knife. These relationships are approximated in Figure 12.02a.

Shape is a matter of circumscribing lines around the origin. In this study shape is defined by imposing a polar coordinate grid in the bifacing plane with its center at the origin (Figure 12.01c). Shape is defined by the ratios of radii with different orientations. A specific shape is produced by adjusting the ratios of selected radii to the correct ratios. Figure $12.02 \mathrm{~b}$ illustrates the description of a point which is generated by the ratio of $y$ to $x$. In a Cartesian Coordinate system 1 ine $A-B$ is generated by a -1.5 unit change in $x$ for every 1.0 unit change in $y$. A similar function could be devised for the polar coordinate system.

The method by which artifacts will be classified into shapes is, in practice, very similar to the approach outlined by Johnson (1967:41-51). The derivations of the ideal forms are somewhat different, however. Johnson's ideal forms 


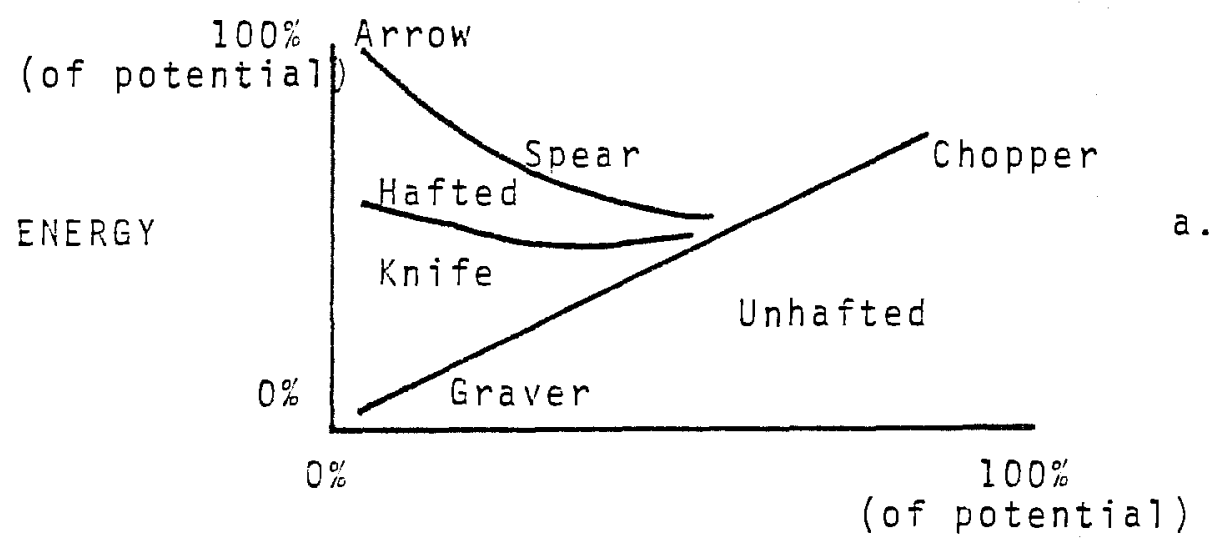

MASS

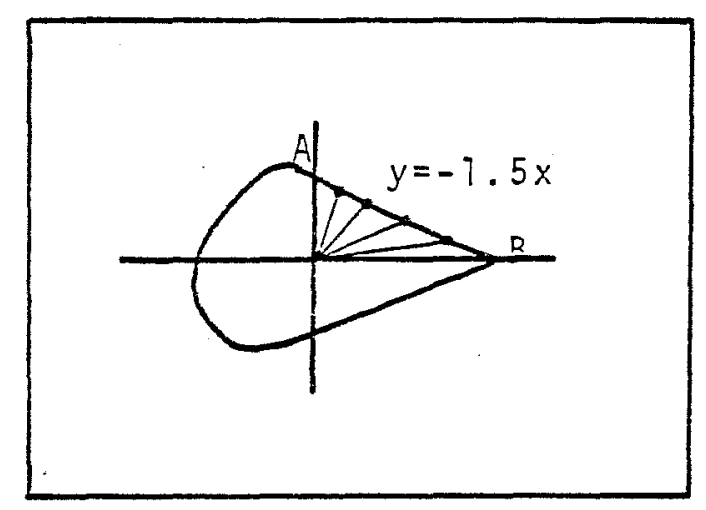

b.

Figure 12.02. Estimators of Size and Shape. a, mass-force relationships in stone tools; b, shape transformations with facing plane.

are empirically surmised from the range of lithomorphic outlines in his sample. The ideal forms in this study are theoretically derived in the following fashion. First it is posited that all shapes are bounded planes which bisect or intersect an artifact in a technologically crucial relationship. The bifacing plane defined earlier is such a plane, and the reason it is technologically crucial is that it is the focus of so many decisions and operations for making and using bifaces.

Second, three ideal or primitive shapes will be used. These are the square, rectangle and triangle. The further assumption is made that by appiying a set of rules to any one of these forms, any other form can be generated from it. A rectangle, for instance, is derived from a square by the process of "symmetrical reduction." Two opposite sides are reduced in a coordinated manner while the other two are kept relatively long. A triangle is derived from a 
square by "asymmetrical reduction." One end is reduced faster than the other. Notice that in both examples the strategy for deriving one shape from another involves the taking away of material, not the addition of material. The process of derivation, then, always involves the loss of area in the bounded plane.

Since prehistoric knappers seldom produced such basic shapes it will be helpful to define a set of terms for shapes generated from the primitives by the process of "rounding." Rounding is taken to mean the reductjon of angles to curves. "Circular" is derived froma square by rounding. "Oval" is derived from a rectangle and "ovoid" from a triangle.

Aside from defining systematic relationships between shapes, this scheme bears an apparent relationship to the strategic processes a knapper has to go through to alter raw material to a predetermined tool type. Normally a knapper starts with a circular solid (sphere, nodule or chert) and works it toward an oval or ovoid form. Alternatively, the raw material may be in the form of a rectangular solid (tabular chert) and the final tool is some transformation of that form. Resharpening and reshaping are resumptions of the process.

An index showing which of these ideal shapes an artifact is most like can be determined by subtracting its polar coordinate values from those of an ideal shape measured in the same system and summing the differences. The measurements must be normalized to that of the primitive shape before subtracting

$\left(f=A_{T} / P_{i} ;\right.$ Index $=\sum_{i=2}^{n} f a_{i}-P_{i} ; n=$ number of polar coordinate rays, $A=$ artifact measurements, $P=$ primitive shape measurements).

Shape outside the bifacing plane, or "extraplanar shape," will be assumed to be basically diamond in outline and some exact transformation of a diamond. The relative thickness of the diamond can be estimated by calculating the area of the bifacing plane from the polar coordinates (Area=Ray $X$ - Ray $Y$ - sin Angle between $X$ and $Y$ ) and dividing the weight by this quantity. Since this variable is in part a linear transformation of size, it is not good practice, though the results are not invalid. Several attempts to develop an alternative estimate of extraplanar shape failed to produce a better indicator. If the study is repeated it should be initiated with this problem in mind.

To this point we have defined a geometric concept which will hopefully help us to manage and explain the variability in biface production and use. From this model dimensions of variability were derived which are hypothesized to be relevant to the processes of manufacture and use: Edge Arc, Bifacing Mode, Size and Shape. All of these were defined so they would be continuous dimensions of variability underlying the range of biface productions. We also suspect that there are categorical breakdowns of these variables which will correspond to types. 
Table 12.01 Dimensions of Variability in Bifaces and States Each Variable Can Take

\begin{tabular}{llll}
\hline & 1 & 2 & 3 \\
Edge Arc & Sma11 & Medium & Large \\
Bifacing Mode & Low $\left(30^{\circ}\right)$ & High $\left(31^{\circ}\right)$ & \\
Size & Sma11 $(0-33 \%)$ & Medium $(33-66 \%)$ & Large (66-100\%) \\
Shape (Planar) & Triangular & Rectangular & Square \\
Shape (Extraplanar) & Thin $(<\bar{x})$ & Thick $(>\bar{x})$ &
\end{tabular}

The next step of this project will be to classify the Hop Hill bifaces by these criteria and test the resulting classification for statistical validity. Eight general types were defined among the 121 non-point bifaces. The first criterion for ordering types is Edge Arc. Lower type numbers have lower Edge Arc. Varieties within types are defined by adding decimals to the general type number. Table 12.01 shows the five dimensions of variability and the bounded ranges these states can take. With the number of states indicated 108 types could be generated which is probably a more than adequate level of typological resolution. Table 12.02 shows an outline of the various dimensions of variability developed above and the eight types which will be described in the following pages. A functional interpretation is given in the $c \& 11$ which is judged to be the most relevant to the function of the type.

\section{Biface Descriptions}

The bifaces recovered at Hop Hill with very rare exceptions, were not of such quality that they would be prized by anyone but the most dedicated archaeologist. They are, however, not without perceivable categories of form and technology. The following pages contain largely technological and lithomorphic descriptions of the broader categories. These descriptions are intended to acquaint the reader with the character of the range of bifaces in the collection without foregoing the presupposition that there are universal processes that underlie all bifacing which can be measured in terms of continuous variables.

Several runs of discriminant function using subtypes as prior groups showed size to be the best overall discriminator between groups. For this reason some of the characteristics of weight are given for each subtype. The mean and standard deviations are noted to show the range that an artifact of a given type is most likely to fall into. The coefficient of variation $(c v=s / \bar{x})$ is also recorded to indicate how extreme the variation within the class is relative to other types.

\subsection{Pointed Bifaces $(\mathrm{N}=7)$}

All specimens are trimmed to a bifacial point on one end and completely or relatively untrimmed on the other. As a consequence of the limited trimming the Edge Arc is relatively low, generally less than 180 degrees. 
Table 12.02 Types and a Description of the State That Type W111 Probably Take on Any Given Vartable

\begin{tabular}{|c|c|c|c|c|c|c|c|c|}
\hline $\begin{array}{l}\text { Varfable } \\
\text { Indicator }\end{array}$ & $\begin{array}{l}\text { Edge Arc } \\
\text { edge arc }\end{array}$ & $\begin{array}{l}\text { Brfacing } \\
\text { Mode } \\
\text { edge angle }\end{array}$ & $\begin{array}{l}\text { Stze } \\
\text { wetght }\end{array}$ & $\begin{array}{l}\text { Shape } \\
\text { (Planar) } \\
3 \text { Indices }\end{array}$ & $\begin{array}{l}\text { Shape } \\
\text { (Extraplanar) } \\
\text { wt/BF area }\end{array}$ & Weir (1976) & Prewitt (1974) & Hester (1971) \\
\hline Type 1 & $\begin{array}{l}\text { smal } \\
\text { (pick, } \\
\text { cleaver) }\end{array}$ & high & $\begin{array}{l}1.1 \text { med fum }(47 \%) \\
1.2 \text { small (17\%) }\end{array}$ & ovate & thick & & & \\
\hline 2 & $\begin{array}{l}\text { med lum } \\
\text { (chopper) }\end{array}$ & high & $\begin{array}{l}\text { medium to } \\
\text { large }\end{array}$ & rectangle & thick & & $\begin{array}{l}\text { Groups XIV } \\
\text { and XVI } \\
\text { (p. 101) }\end{array}$ & $\begin{array}{l}\text { core } \\
\text { choppers } \\
\text { (p. 86) }\end{array}$ \\
\hline 3 & $\begin{array}{l}\text { medtum } \\
\text { (chopper) }\end{array}$ & high & med lum & oval & thin & $\begin{array}{l}\text { Tateral } \\
\text { cortex } \\
\text { (p. 65) }\end{array}$ & & $\begin{array}{l}\text { pebble } \\
\text { choppers } \\
\text { (p. B6) }\end{array}$ \\
\hline 4 & large & htgh & medtum & rectangular & thin & $\begin{array}{l}\text { thick } \\
\text { ovate } \\
\text { (p. 51) }\end{array}$ & & \\
\hline 5 & large & high & $\begin{array}{l}5.1 \text { small } \\
5.2 \text { medium }\end{array}$ & circular & thick & $\begin{array}{l}\text { thick } \\
\text { ovate } \\
\text { (p. 51) }\end{array}$ & & $\begin{array}{l}\text { Group V } \\
\text { percussion } \\
\text { bifaces (p. 90) }\end{array}$ \\
\hline 6 & large & high & medium & oval & thick & $\begin{array}{l}\text { thick } \\
\text { ovate } \\
\text { (p. 51) }\end{array}$ & $\begin{array}{l}\text { Group XIII } \\
\text { (p.98) }\end{array}$ & \\
\hline 7 & large & low & $\begin{array}{l}7.1 \text { small } \\
7.2 \text { medium }\end{array}$ & ovate & thin & $\begin{array}{l}\text { thick } \\
\text { and thin } \\
\text { ovate } \\
(p .51)\end{array}$ & $\begin{array}{l}7.1=\text { Group II } \\
\text { (p. 92) } \\
7.2=\text { Group XVII } \\
\text { (p. 101) }\end{array}$ & $\begin{aligned} & T .1= \text { Groups } I, I I I, \text { and } V I(p .88) \\
& \text { thinned bifaces } \\
& 7.2=\text { Group } V=\text { percusston } \\
& \text { bifaces (p.90) }\end{aligned}$ \\
\hline 8 & large & low & small & triangle & thin & $\begin{array}{l}\text { thintriangutar } \\
1.5 \mathrm{~cm} \\
\text { (p. } 51 \text { ) }\end{array}$ & & \\
\hline
\end{tabular}


Table 12.03 FN's and Provenience of Types

\begin{tabular}{|c|c|c|c|c|c|c|c|c|c|}
\hline Type & FN & $\underline{S-E}$ & East & North & 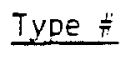 & FN & $\underline{S-E}$ & East & North \\
\hline \multirow[t]{3}{*}{1.1} & 265 & 5 & 974.00 & 1006.00 & 5.1 & 2034 & $s$ & 944.00 & 948.00 \\
\hline & 664 & 5 & 1010.00 & 1036.00 & & 2202 & $s$ & 950.00 & 949.00 \\
\hline & 1359 & 5 & 961.00 & 960.00 & 5.2 & 715 & 5 & 964.00 & 873.00 \\
\hline \multirow[t]{4}{*}{1.2} & 760 & 5 & 947.00 & 949.00 & & 794 & $s$ & 890.00 & 935.00 \\
\hline & 768 & $\mathrm{~s}$ & 924.00 & 945.00 & & 802 & $\mathbf{s}$ & 886.00 & 979.00 \\
\hline & 798 & $\mathrm{~s}$ & 892.00 & 936.00 & & 816 & $s$ & 930.00 & 933.00 \\
\hline & 1401 & 5 & 969.00 & 961.00 & & 819 & $s$ & 931.00 & 937.00 \\
\hline \multirow{4}{*}{2.1} & 711 & 5 & 959.00 & 823.00 & & 841 & $s$ & 947.00 & 941.00 \\
\hline & 746 & 5 & 1047.00 & 1062.00 & & 1101 & s & 906.00 & 937.00 \\
\hline & 1509 & 5 & 935.00 & 929.00 & & 1151 & $\mathrm{~s}$ & 890.00 & 1038.00 \\
\hline & 1966 & $s$ & 899.00 & 1043.00 & & 1939 & 5 & 889.00 & 1043.00 \\
\hline \multirow[t]{5}{*}{2.2} & 668 & 5 & 1016.00 & 1050.00 & & 1956 & 5 & 893.00 & 1050.00 \\
\hline & 701 & $\mathrm{~s}$ & 980.00 & 828.00 & 6.1 & 270 & s & 976.00 & 1004.00 \\
\hline & 708 & 5 & 939.00 & 846.00 & & 799 & $\mathrm{~s}$ & 887.00 & 933.00 \\
\hline & 709 & $\mathrm{~s}$ & 948.00 & 827.00 & & 813 & $\mathrm{~s}$ & 923.00 & 928.00 \\
\hline & 736 & $s$ & 1024.00 & 1077.00 & & 2378 & e & 932.44 & $970.59 ?$ \\
\hline \multirow[t]{2}{*}{2.3} & 712 & 5 & 959.00 & 819.00 & & 2442 & $\mathrm{~s}$ & 922.00 & 964.00 \\
\hline & 808 & 5 & 913.00 & 928.00 & 6.2 & 756 & $s$ & 1007.00 & 1029.00 \\
\hline \multirow{9}{*}{2.4} & 16 & e & 1000.97 & 1014.04 & 6.3 & 854 & 5 & 914.00 & 968.00 \\
\hline & 404 & 5 & 970.00 & 1013.00 & 6.4 & 697 & $s$ & 995.00 & 880.00 \\
\hline & 661 & $s$ & 1010.00 & 1035.00 & & 747 & $s$ & 1051.00 & 1067.00 \\
\hline & 710 & 5 & 959.00 & 827.00 & & 2475 & e & 932.74 & 968.13 \\
\hline & 716 & 5 & 948.00 & 810.00 & 7.1 & 50 & $e$ & 1000.22 & 1026.74 \\
\hline & 744 & 5 & 1025.00 & 1092.00 & & 395 & $s$ & 937.00 & 1014.00 \\
\hline & 831 & 5 & 940.00 & 937.00 & & 667 & $s$ & 1025.00 & 1059.00 \\
\hline & 1313 & 5 & 978.00 & 965.00 & & 691 & $s$ & 954.00 & 962.00 \\
\hline & 1954 & $s$ & 888.00 & 1044.00 & & 762 & $s$ & & \\
\hline \multirow[t]{18}{*}{3.0} & 328 & $s$ & 978.00 & 1012.00 & & 766 & $s$ & 923.00 & 946.00 \\
\hline & 612 & $s$ & 989.00 & 1015.00 & & 782 & $s$ & 955.00 & 949.00 \\
\hline & 675 & 5 & 968.00 & 1050.00 & & 783 & $s$ & 950.00 & 938.00 \\
\hline & 705 & s & 953.00 & 876.00 & & 790 & $s$ & 890.00 & 940.00 \\
\hline & 717 & $s$ & 934.00 & 816.00 & & 805 & $s$ & & \\
\hline & 721 & $s$ & 982.00 & 839.00 & & 811 & $s$ & 920.00 & 928.00 \\
\hline & 733 & $s$ & 953.00 & 953.00 & & 839 & $s$ & 948.00 & 940.00 \\
\hline & 777 & $s$ & 924.00 & 960.00 & & 842 & $s$ & 953.00 & 940.00 \\
\hline & 797 & $s$ & 887.00 & 932.00 & & 846 & $s$ & 979.00 & 974.00 \\
\hline & 804 & $s$ & 892.00 & 921.00 & & 1507 & $\mathrm{~s}$ & 938.00 & 927.00 \\
\hline & 1339 & $s$ & 955.00 & 969.00 & & 1934 & $s$ & 891.00 & 1041.00 \\
\hline & 1535 & $s$ & 988.00 & 955.0 & & 2114 & e & 931.55 & 967.35 \\
\hline & 1817 & s & 928.00 & 1063.00 & & 2438 & $s$ & 917.00 & 938.00 \\
\hline & 1957 & $s$ & 890.00 & 1049.00 & & 2540 & e & 932.12 & 969.99 \\
\hline & 1994 & $s$ & 909.00 & 1049.00 & 7.2 & 683 & $\mathrm{~s}$ & 973.00 & 903.00 \\
\hline & 1997 & 5 & 906.00 & 1050.00 & & 719 & $s$ & 936.00 & .00 \\
\hline & 2005 & $s$ & 903.00 & 1044.0 & & 752 & 5 & 972.00 & 995.00 \\
\hline & 2388 & 5 & 921.00 & 959.00 & & 757 & $s$ & 1026.00 & 1006.00 \\
\hline \multirow[t]{3}{*}{4.0} & 666 & $s$ & 1017.00 & 1054.00 & & 786 & $s$ & 934.00 & 952.00 \\
\hline & 748 & $s$ & 1061.00 & 1037. & & 818 & 5 & 930.00 & 933.00 \\
\hline & 1898 & $s$ & .00 & 1043. & & 843 & $\mathrm{~s}$ & 963.00 & 949.00 \\
\hline \multirow[t]{13}{*}{5.7} & 250 & $s$ & 976.00 & 1003. & & 998 & e & 930.10 & 968.34 \\
\hline & 258 & $\mathrm{~s}$ & 978.00 & 1006.00 & & 1328 & 5 & 960.00 & 965.00 \\
\hline & 724 & 5 & & & & & $\mathrm{~s}$ & 963.00 & 962.00 \\
\hline & 793 & s & 885.00 & 934.00 & & 1425 & 5 & 969.00 & 963.00 \\
\hline & 807 & 5 & 894.00 & 914.00 & & 1503 & $s$ & 904.00 & 930.00 \\
\hline & 829 & $s$ & 941.00 & 936.00 & & 1800 & 5 & 927.00 & 1066.00 \\
\hline & 1188 & e & 930.75 & 967.24 & & 1909 & s & 900.00 & 1048.00 \\
\hline & 1774 & $s$ & 921.00 & 1060.00 & & 2200 & 5 & 950.00 & 948.00 \\
\hline & 1826 & $\mathrm{~s}$ & 922.00 & 1065.00 & & 2373 & e & 932.44 & 970.59 \\
\hline & 1916 & $s$ & 900.00 & 1050.00 & 8.0 & 662 & 5 & 1010.00 & 1038.00 \\
\hline & 1935 & $\mathrm{~s}$ & 890.00 & 1042.00 & & 740 & $s$ & 1035.00 & 1076.00 \\
\hline & 2015 & $s$ & 942.00 & 948.00 & & 828 & $s$ & & \\
\hline & & & & 871.00 & & & & & \\
\hline
\end{tabular}


1.1 Poirted Bifaces, Pick ( $N=3$, Wt $\bar{x}=409, s=39, c v=10 \%$ )

See Table 12.03 for FN's and provenience.

Bifacing comprises a few blows to decorticate one end of a nodular piece of chert. In spite of definite bifacing blows all three were shaped to a planoconvex configuration (Figure 12.03a). The average weight is $47 \%$ of the weight of the largest piece in the collection so the subtype is a medium size and, as the low coefficient of variation shows, they are all of about the same size.

The picks are on medium- to fine-grained chert. FN 265 and 664 are nodules while 1359 is tabular. FN 1359 is potlided and reddened. FN 265 is heavily patinated.

1.2 Pointed Bifaces, Cleaver ( $N=4$, Wt $\bar{x}=140, s=124, c v=89 \%$ )

See Table 12.03 for FN's and provenience.

The subtype is much like picks except for an intentional reshaping of the point, apparentiy to give a broader area of cutting edge at the tip. While cleavers resemble picks in broad outline there are technological characteristics which distinguish them. The preferred material was flat nodules of chert which gives a much thinner cross section to the cleaver. The picks tend to be almost spherical. There is in all cases some attempt to thin the butt where there was no thinning in picks. Perhaps the cleaver was a hafted tool while the pick was hand held. The bit of the cleaver is lenticular rather than plano-convex. The tip was prepared with a few (two to four) unidirectional blows which gives a slightly broader tip than there is on a pick. The cleaver resembles the "truncated hand axes" (Crabtree 1972:52) which are common in Africa but the tip is made with several flakes rather than one oblique flake. Sollberger (1968:101) has used the term carcass-cleaver for similar pieces. These are not the finely made "fist axes" he had in mind but would be functionally equivalent so they would be included here.

The average size is much smaller than picks at $16 \%$ of potential. Also, the variation is very broad as the cv shows. The smallest cleaver is the smallest non-point biface in the collection. All of the cleavers were made on fine grained nodular chert, but as noted before, the nodules are all flat and probably selected for that reason.

\subsection{End Bifaces $(\mathrm{N}=20)$}

This type of biface is shaped by chipping a bit at one end of a generally rectangular and usually tabular piece of chert. Subtle variations in the Edge Arc are produced by chipping the bit on the narrower or wider end of the rectangle and sub-types are defined on these options. Bits are generally prepared closer to one surface or the other suggesting that they could as well have been platforms or chopper bits. Sizes range from 92 grams to the standard setting FN 1966 which weighs 873 grams. 

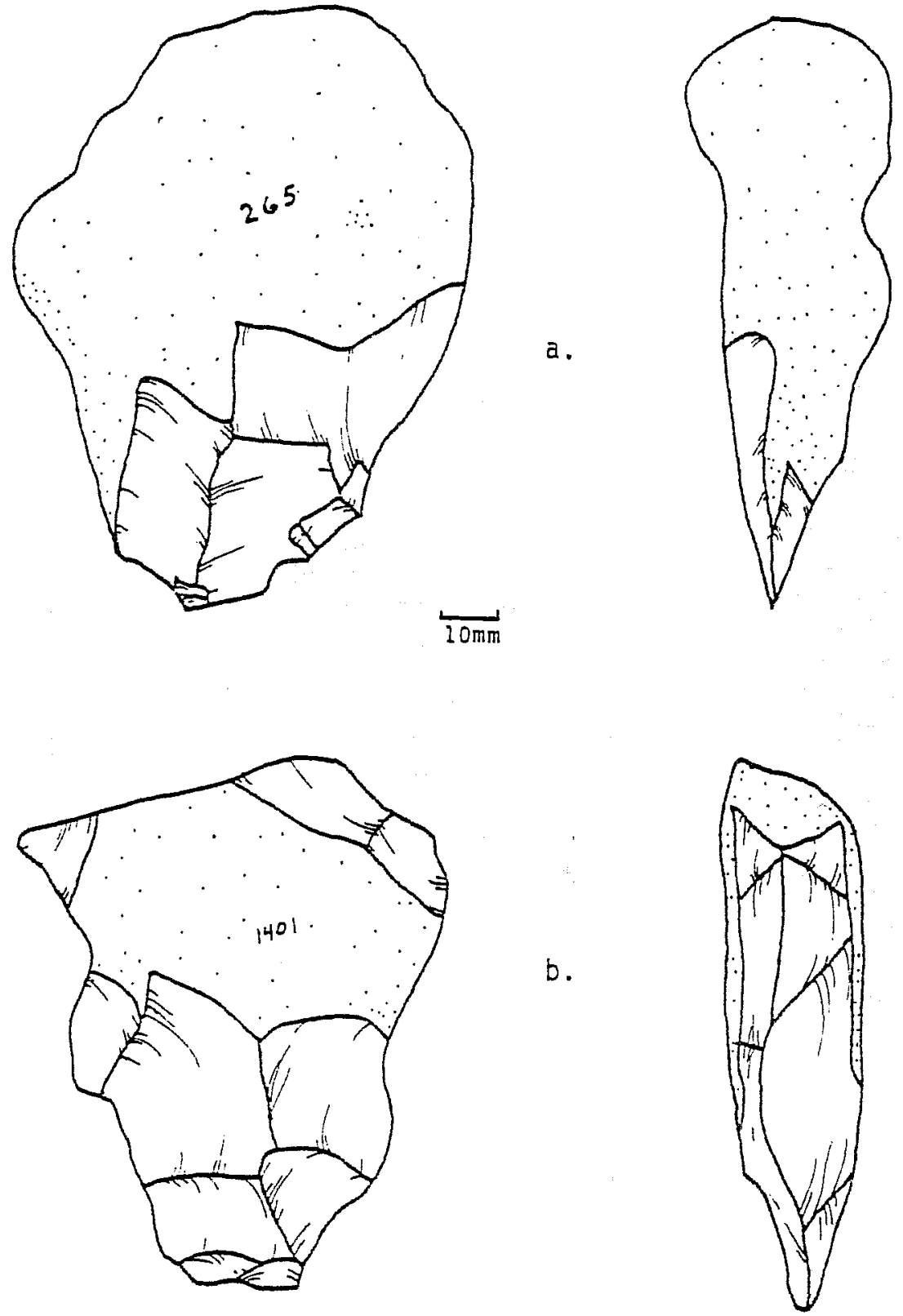

Figure 12.03. Pointed Bifaces. a, pointed biface (pick); b, pointed biface (cleaver). 
2.1 End Biface, Large Chopper ( $N=4$, wt $\bar{x}=543, s=361, c v=62 \%)$

See Table 12.03 for FN's and provenience.

These largest of the choppers are made on big, tabular blocks of chert. Figure $12.04 a$ is the smallest of the group. They are all distinctly rectangular or square. There is no trimming except on the bit. The Bifacing Mode is clearly too high to thin so the only purpose could have been to create a bit or remove flakes.

The sizes are the largest in the collection as the mean shows and the range of variation is, as is typical of most large tools, relatively wide. Materials range from fine- to coarse-grained.

2.2 End Biface, Oblique Chopper ( $N=5$, wt $\bar{x}=463, s=114, c v=25 \%$ )

See Table 12.03 for FN's and provenience.

Oblique choppers are made on blocks of tabular chert or nodules. The outline is generally rectangular but the bit is oblique to the longer dimension of the piece. Sizes are in the medium range $(53 \%)$ and the range of variation is relatively small. Cherts are generally fine- to medium-grained.

2.3 End Biface, Curved Chopper ( $N=2$, Wt $\bar{x}=482, s=163, \mathrm{cv}=34 \%$ )

See Table 12.03 for FN's and provenience.

The pieces are distinctiy members of the rectangular, end biface category. They are, however, shaped with a curved rather than straight bit across the end. They are of medium size (55\%) and of medium-grained chert.

2.4 End Biface, Celt ( $N=9$, Wt $\bar{x}=332, s=208, c v=38 \%$ )

See Table 12.03 for FN's and provenience.

Ceits are end bifaces in which the bit is prepared on the wider end of a nearly rectangular block of raw material. The pieces are technically between subrectangular and subtriangular in outline. In most cases the outline of the piece appears to be a result of carefully selecting raw material with the right outline. In other cases some effort was made to produce the trapezoidal outline by trimming on both ends. The larger the items are the more likely they are to be shaped by preselection of raw material. The smaller ones are more frequently shaped by casual bifacial shaping. Figure $12.04 \mathrm{~b}$ is an example of three almost identical pieces of the smal1 variety. It is these that most resemble the normally defined celt (see Weir 1976:64). Sizes are in the medium range while cherts are medium- to fine-grained.

3.0 Side Bifaces, Choppers ( $N=18$, Wt $\bar{x}=262, S=121, c v=46 \%$ )

See Table 12.03 for FN's and provenience. 

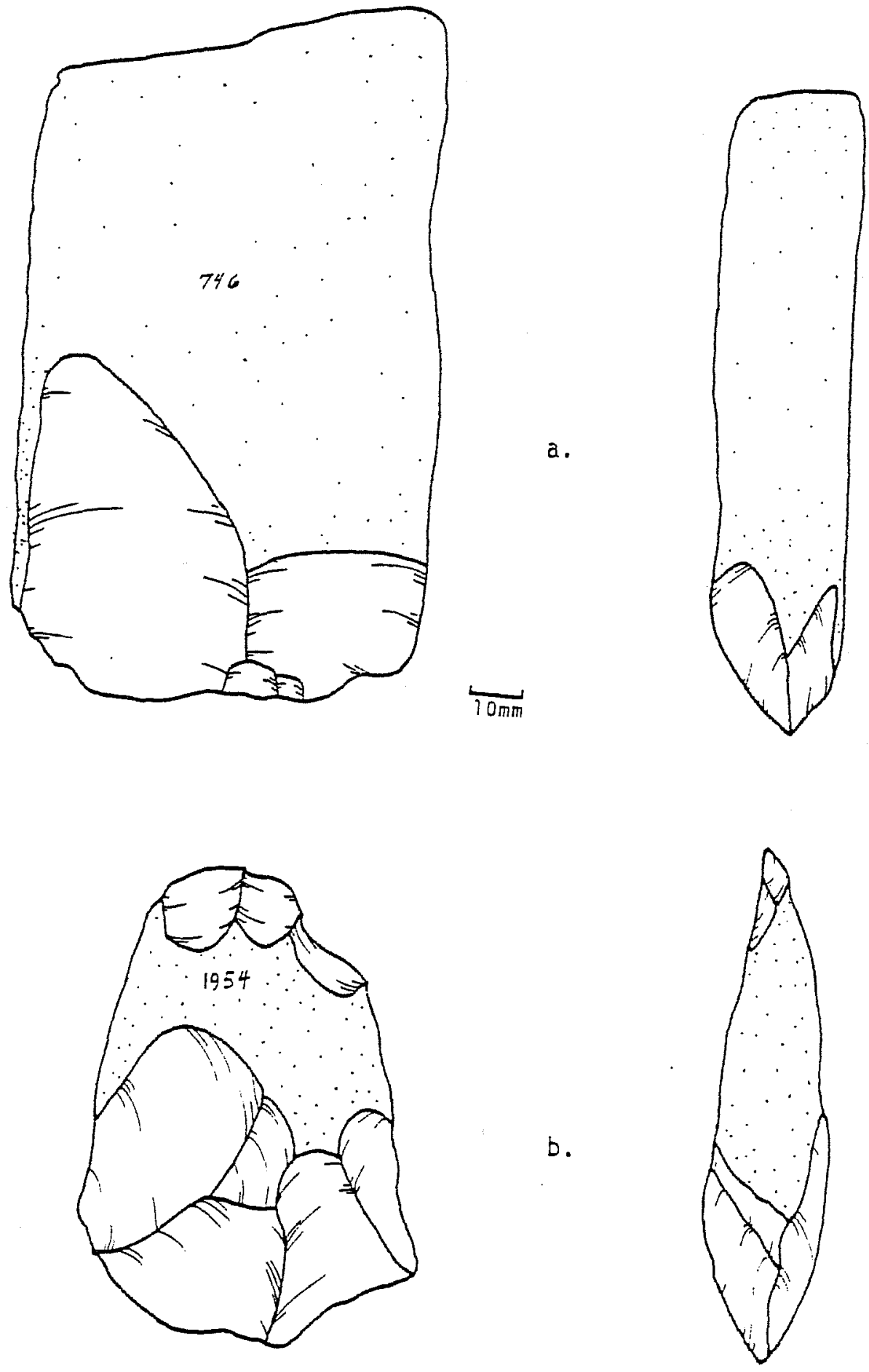

Figure 12.04. End Bifaces. a, end biface (1arge chopper); b, end biface (celt). 

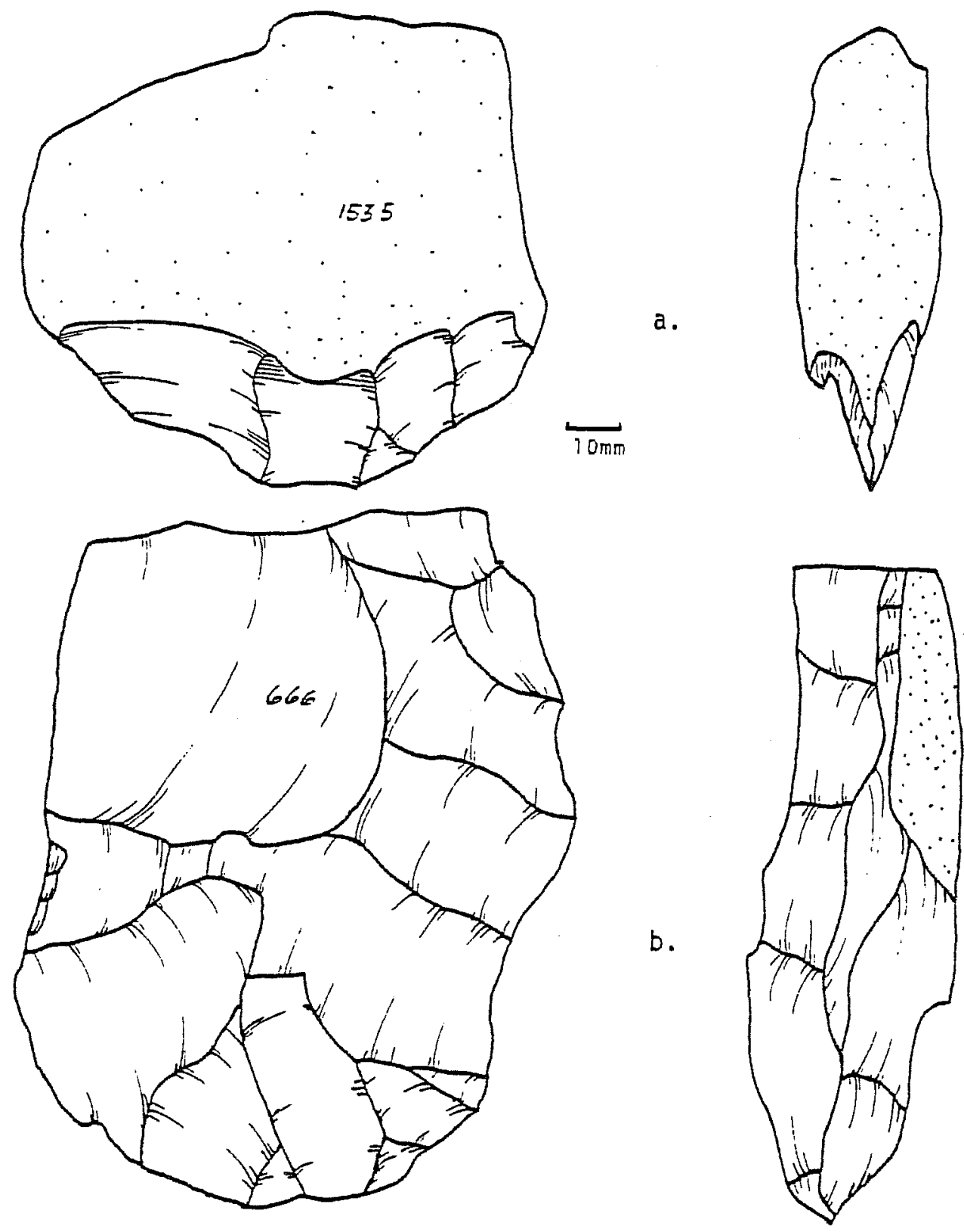

Figure 12.05. Side and Rectangular Bifaces. a, side biface (chopper); $b$, rectangular biface. 
Side bifaces are thin and subrectangular (Figure 12.05a). Bits are placed along one of the long sides of the rectanguloid while leaving the bulk of the cortex untouched. Weir calls them lateral-cortex bifaces (1976:65). The configuration suggests a wide range of potential functions. Naturally backed knives, or small choppers for preparing roots, etc., are possibilities. The resemblance to the Eskimo ulu is intriguing.

Size in the small range and materials are from coarse to fine-grained.

\subsection{Rectangular Bifaces ( $N=3$, wt $\bar{x}=266, s=113, c v=42 \%$ )}

See Table 12.03 for FN's and provenience.

Type 4.0 pieces are large, subrectangular bifaces. They a11 have cortex on at least one side, and one side is thinned off in two cases. They are all trimmed around three sides and one narrow side left unretouched. The great similarity between the three, two of them apparently adjusted for thickness, would lead one to suspect a tool or special purpose core. Figure 12.05b illustrates the largest of the specimens.

Size is in the small range. Cherts are medium-grained in all cases.

\subsection{Round Bifaces $(N=25)$}

The type 5.0 bifaces are generally round though in a very loose and jagged sense. They do not appear to have been worked with the intention of being thinned. This impression is supported by relatively high edge angles and remnants of cortex on virtually all surfaces. Most do not have the standard biconical profile of bifacial cores although there are a few superlative examples of biconical specimens.

5.1 Round Bifaces, Thin ( $N=15$, wt $\bar{x}=98, s=52, c v=53 \%$ )

See Table 12.03 for FN's and provenience.

This subtype is distinguished by relative thinness in comparison to subtype 5.2. As a consequence there are only two pieces that resemble very low profile biconical cores. Figure 12.06 illustrates a more run-of-the-mill example. The average size is very small (11\%) and all of the specimens are of fine to very fine grained cherts, chalcedonies and jaspers.

\subsection{Round Bifaces, Thick ( $N=10$, wt $\bar{x}=295, s=115, c v=39 \%$ )}

See Table 12.03 for FN's and provenience.

Type 5.2 pieces are thicker than 5.1 items and as a consequence about half of them are clearly interpretable as biconical cores. The others generally resemble larger versions of Figure 12.06. Their large size is also reflected by a medium range of potential size. Cherts range from coarse to very fine. 


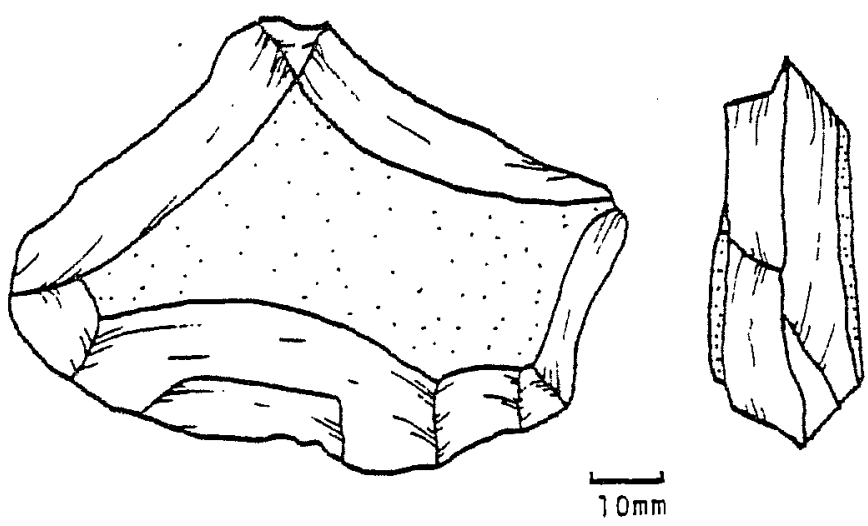

Figure 12.06. Round, Thin Biface.

\subsection{Oval, Thick Biface $(N=10)$}

The members of the Type 6.0 category are oval to nearly rectangular in outline and are in most cases nearly as thick as they are wide. Four specimens have some flaking at one end, note especially Figure 12.07a, which makes them appear to be stemmed. The suggestion of a stem may indicate a hafted tool of some description. Alternatively, the pieces may be the work of inexperienced novices who have not mastered the art of thinning.

6.1 Oval, Thick Bifaces ( $N=5$, Wt $\bar{x}=139, s=49, c v=35 \%$ )

See Table 12.03 for FN's and provenience.

This group of bifaces contains most of the seemingly stemmed items including FN 747 illustrated in Figure 12.07a. They are $16 \%$ of size potential and the chert is generally medium to fine-grained.

6.2 Oval, Thick Biface, Guadalupe Tool ( $N=1$, Wt $\bar{x}=135$ )

See Tabie 12.03 for FN's and provenience.

The item is a Guadalupe tool (Hester 1976). It is $15 \%$ of size potential and made on the fine-grained chert.

6.3 Oval, Thick Biface, Clear Fork Gouge $(N=1, W t \bar{x}=62)$

See Table 12.03 for FN's and provenience. 

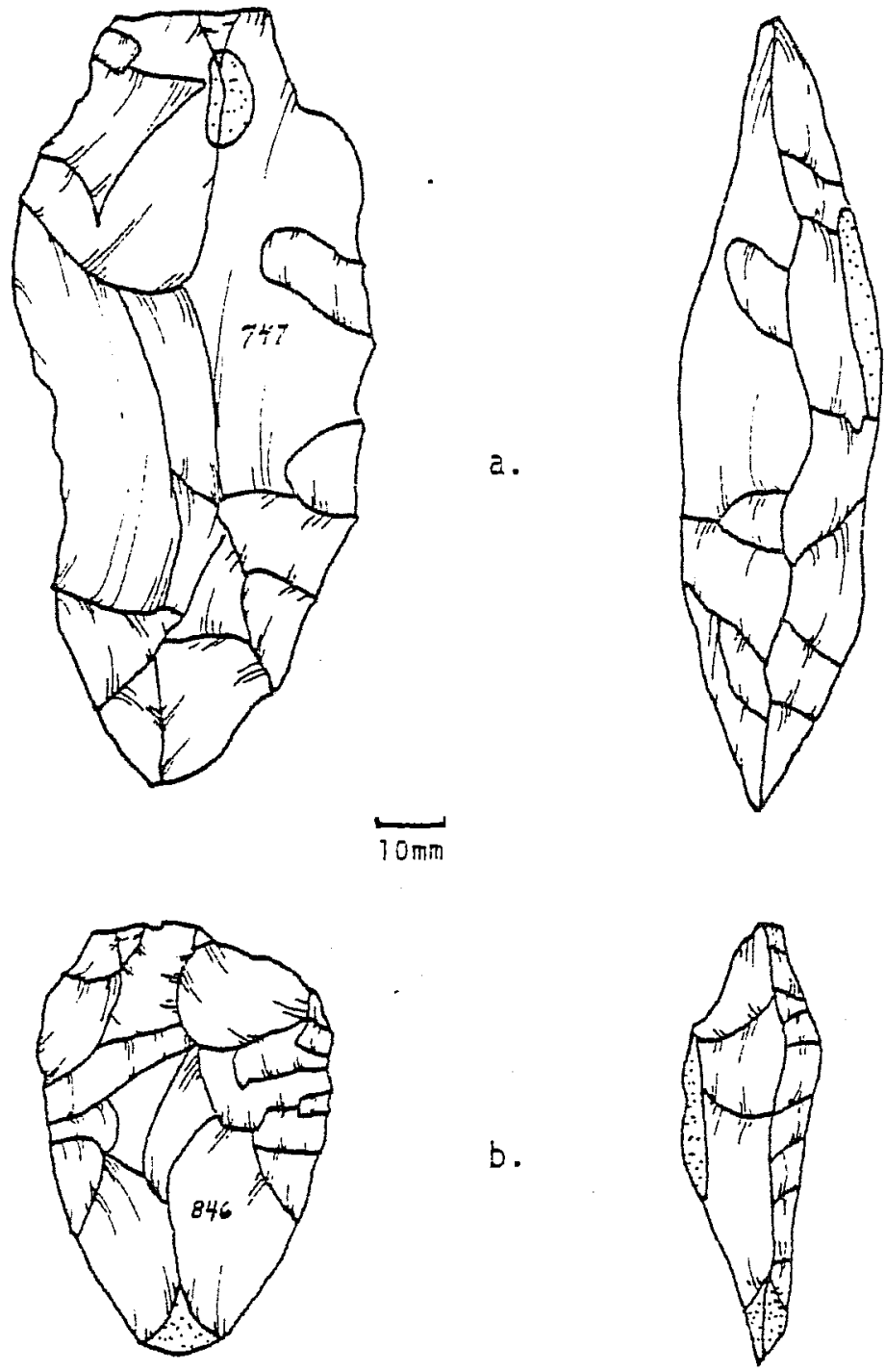

Figure 12.07. Oval and Ovoid Bifaces. a, oval, thick biface; $b$, ovoid biface, small thinned. 
FN 854 appears to be a broken Clear Fork Gouge (Ray 1938, Hester, Gilbow and Albee 1973). It is plano-convex in cross section even though the shape was achieved by bifacing. Two-thirds of the left side of the bit was removed by a transverse blow from the right (bit oriented downward). The removal is so large and the blow apparently so heavy that it must have been intentional rather than a product of utilization. Size is $7 \%$ of potential and the material is medium-grained chert. The material has a reddish cast to it which suggests heat treatment.

6.4 Oval, Thick bifaces, Irregular $(N=3, W t \bar{x}=129, s=9, c v=7 \%)$

See Table 12.03 for FN's and provenience.

Subtype 6.4 is a residual category for thick bifaces that appear to be incomplete or uncompletable but have the thick cross section which makes them inescapably members of the type.

\subsection{Ovoid Bifaces $(N=35)$}

Ovoid is defined somewhat narrowly here as subtriangular (Johnson 1967:49), or in othe words an asymetrical ova?.

7.1 Ovoid Bifaces, Small Thinned ( $N=19$, wt $\bar{x}=27, s=15, c v=56 \%)$

See Table 12.03 for FN's and provenience.

Variety 7.1 is sma 11, thinned bifaces (Fig. 12.07b) that could be preforms for more refined bifaces and points. Most of them have knobs that would have precluded finishing. The size is very smal1, 3\%, and the materials range from very fine- to medium-grained cherts. One specimen, FN 691, is obliquely truncated across the narrow end by steep retouch.

\subsection{Ovoid Bifaces, Large, Thick ( $N=16$, Wt $\bar{x}=109, s=80, c v=73 \%$ )}

See Table 12.03 for FN's and provenience.

Subtype 7.2 is composed of ovoid bifaces which are larger and thicker, and as the cv indicates, more varied than most of the types otherwise defined in this exercise. The specimens are distinctly cruder than subtype 7.1 and most of them appear to be cores. They probably are functionally equivalent to subtype 5.2 (round, thick bifaces). They are in the sma 11 size category at $12 \%$ of potential. Most of the cherts are medium- or fine-grained.

\subsection{Triangular Bifaces, Small ( $N=3$, wt $\bar{x}=11, s=7, c v=64 \%$ )}

See Table 12.03 for FN's and provenience.

Three pieces are more or less perfectly shaped, sma11, triangular bifaces. Two are about twice as long as the base is wide. One is an equilateral triangle. Size is about $1 \%$ and al1 are made on fine-grained chert. In two cases the bifacial thinning is excellently executed.

In analysis subsequent to modeling and description of the bifaces, it quickly became apparent that the types defined are not as neatly analyzable in terms of linear models as the cores. A discriminant analysis using subtypes as prior groups, for instance, was successful at classifying only $47 \%$ of the specimens 
into the correct subtypes. This relative failure to classify into groups reflects a great deal of overlap in the variables which were used as indicators to the model and the relative similarity of the various types as viewed through the simplifying scope of five measurements.

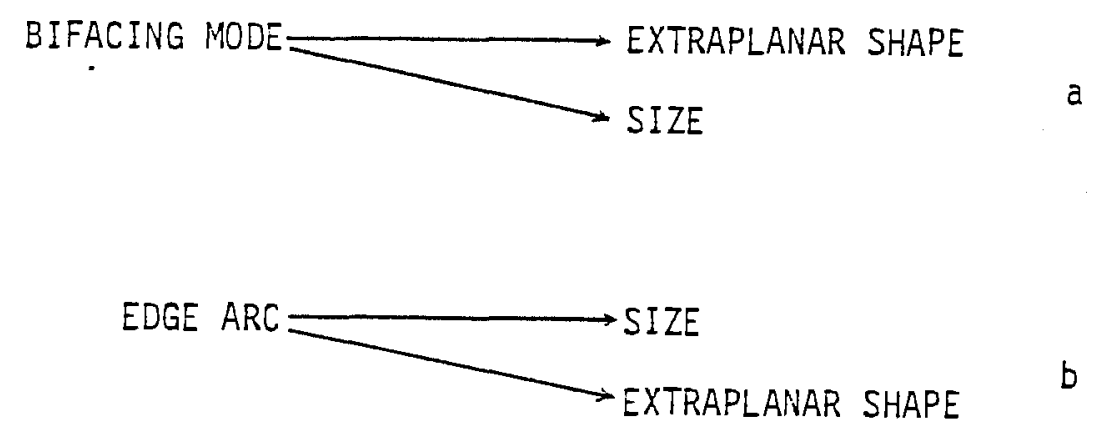

EDGE ARC $\longrightarrow$ PLANAR SHAPE $\quad C$

Figure 12.08. Causal Bifacing Relationships.

Factoring the model indicators showed three independent relationships in the data which largely support the structure of bifacing as modeled above. These relationships are illustrated in Figure 12.08. We have refrained from assigning numerical values to these relationships. It would be misleading to do so considering the bias introduced into the relationships by multicollinearity of extraplanar shape and size. Inspection of the collection, however, supports the general reliability of the relationships between variables. First, varying the Bifacing Mode produces a relatively thicker extraplanar shape (Figure 12.08), confirming the contrast between the thinned biface and the bifacial core.

The second relationship indicates that Edge Arc is negatively correlated to size. In short, larger pieces have more confined Edge Arcs (choppers) while smaller pieces are thinned more completely around their circumferences.

Finally, Edge Arc is adjusted negatively to the shape of the piece in the Bifacing Plain. Items which are triangular or subtriangular have more Edge Arc (ovoid bifaces) while rounder, squarer objects have less Edge Arc.

Each of these trends accounts for a specific percentage of the overall interaction between variables in the Bifacing Mode System. Bifacing Mode accounts for $72 \%$ of the variance while the other two represents $10 \%$ and $4 \%$ respectively. The unbalanced proportion of variance accounted for by Bifacing Mode suggests that it was the most carefully controlled aspect of bifacing.

The search for linear tendencies in the surface distribution was tested by including the east and north coordinates with the bifacing model variables. The initial test was very disappointing. East and north appeared together on 


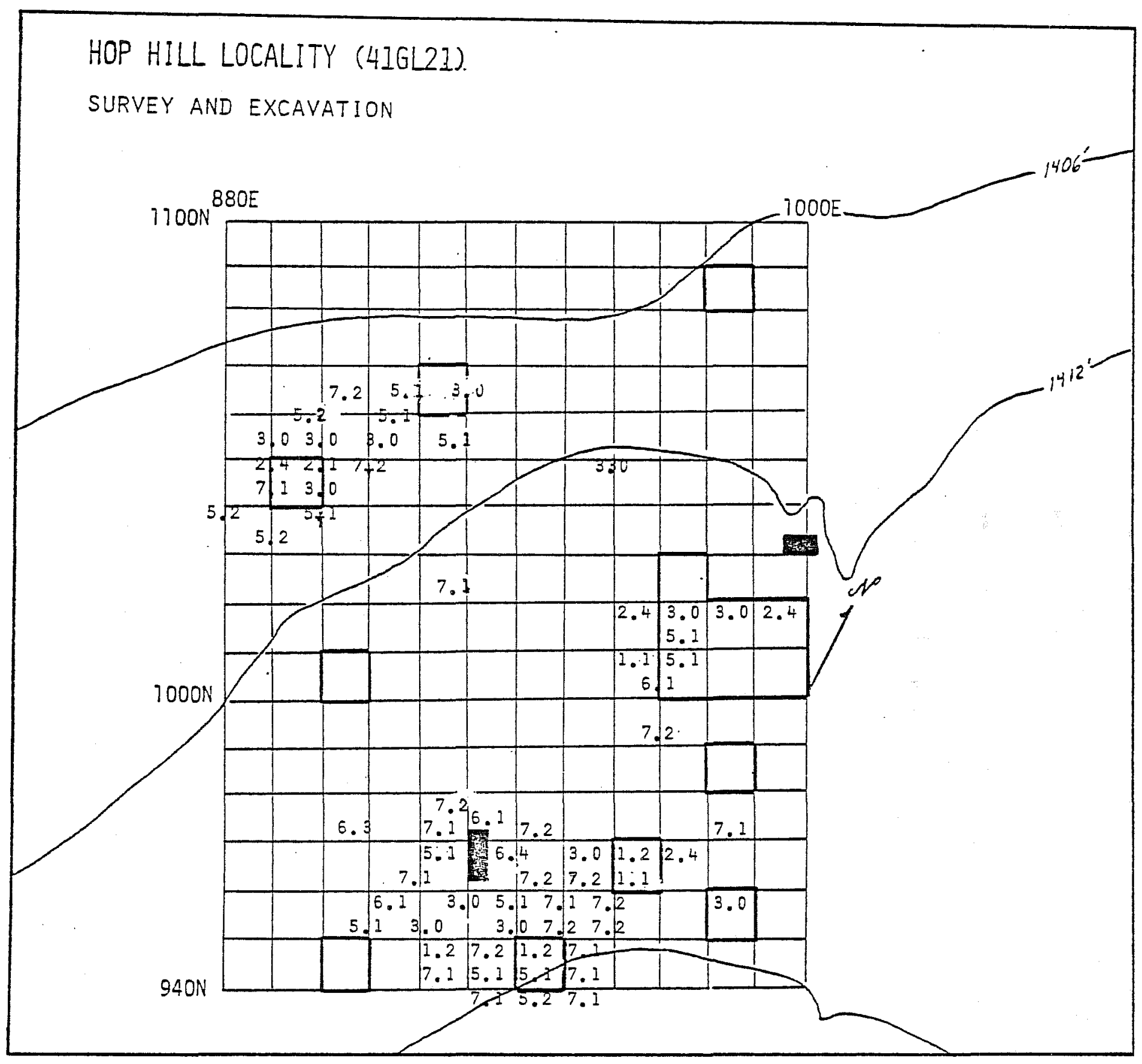

Figure 12.09. Biface Distribution. 
one component correctly indicating the southwest to northeast trend of the artifact distribution. Only planar shape loaded with the coordinates and that at a questionable -.37. From this relationship we can surmise that there is a slight tendency for round and square pieces to be located to the southwest and more triangular pieces in the northeast. Otherwise there are no overall relationships to space.

In the light of unexpected negative results it was decided to push the analysis further. Examination of scatter plots of the distribution showed that the southwest to northeast scatter of artifacts was slightly curvilinear (Figure 12.09). Since the correlation between a curvilinear variable and a linear variable tends to zero, we sumised that this could be the root of the problem. To remove the effect of curvilinear distribution the novel idea of a curvilinear principal components analysis was suggested to us by James Wadington of The University of Texas of San Antonio faculty. The analysis can be performed by standardizing and squaring the cartesian coordinates and including both the untransformed and transformed coordinates in the analysis. Since the original coordinate and its square are linear and curvilinear respectively, there is no problem with multicollinearityas long as there are enough points along the distribution to accurately represent the curved line.

In spits of our hopes for curvilinear principal components analysis, results again failed to appear. As a final attempt, we decided the complexity represented by such diverse types as choppers and preforms could be masking the distributions; therefore different segments of the population were analyzed separately. More satisfying results were obtained.

First, types 1-3 were analyzed together since they represent the various kinds of choppers. Three of four components showed relationships to direction.

Bifacing Mode appears negatively correlated to north squared. The implication, then, is that thinned pieces are farther north while flaked pieces are farther south. This is the relationship which was originally expected because the artifacts recovered from Zone 1, the open pasture to the south, were almost all large choppers while more refined items such as celts were found in Zone II, the upland margin. In addition, a strong relationship between Edge Arc and east appeared.

Table 12.04 Mean and Standard Deviation of Edge Arc for Types 1-3

\begin{tabular}{lrr} 
Type & $\bar{x}$ & $s$ \\
\cline { 2 - 3 } 1.1 & 168 & 42 \\
1.2 & 180 & 141 \\
2.1 & 207 & 119 \\
2.2 & 158 & 74 \\
2.3 & 234 & 25 \\
2.4 & 212 & 71 \\
3.0 & 184 & 69
\end{tabular}


As Table 12.04 shows, Edge Arc tends to be highest in the large choppers (Type 2) so the choppers must appear to the east while side choppers and picks tend more toward the west. If the side choppers have been properly assessed as food preparation tools then large end choppers are for woodworking. The bifaces support the cores in indicating a workshop area on the eastern portion of the upland margin. The third factor is a duplicate of the original analysis showing that planar shape is related to a southwest to northeast trend with triangles to the north and squares to the south.

In summary, then, the distribution of chopper-like bifaces shows a contrast between large implements to the south and small, more refined ones to the north in the village and workshop areas on the upland margin. On an eastwest axis, along the upland margin there is a food-processing area to the west and a workshop area to the east.

A separate curvilinear analys is of Types 4-8 was much less patterned. Only Edge Arc appeared related to the directional. Edge Arc increases in a northeasterly direction. Most of the specimens in Types $4-8$ have a $360^{\circ}$ Edge Arc. The subtype with the lowest mean Edge Arc is subtype $5.2\left(\bar{x}=316^{\circ}\right)$. These are the round, thick bifacial cores so Edge Arc in this analys is is probably just a function of crudity. The interpretation to be placed on the analysis is that, of specimens in types 4-8, the cruder ones are to the southwest while the more refined pieces are to the northeast. A cluster of small, well-formed bifaces across the road to the east is probably largely responsible for this trend. Relative to other dimensions the group is scattered more or less randomiy over the surface.

An additional observation should be made from the scatter plots of bifaces. No biface occurs on the side of the hill in Zone III. This could either mean that bifaces were susceptible to erosion and ended up at the bottom of the hill in the colluvium or there was little bifacing work in the quarry zone. The knappers preferred to move to the workshop area east of the quarry and village to practice their craft. 


$$
\text { . }
$$




\subsection{ANALYSIS OF ARROW AND DART POINTS (Thomas C. Kelly)}

Sixty-two bifaces from Hop Hill were classifiable points. Of these 62 specimens, two were recovered during excavation. The Montell point (FN 1184) was recovered from S2:10-20, in the quarry area. Cultura'] association included several flakes. It was not on a defined occupation floor.

The Wells point (FN 123) was excavated from $52.3,5 \mathrm{~cm}$ into the midden occupation. Cultural associations included burned 1 imestone, chert nodules and flakes. Presence of these points suggests a middle to late Archaic occupation for both contexts.

The remaining 60 points were collected during surface survey of all units of all zones of the site or by Texas Parks and Wildlife personnel during site survey previous to the 1976 field season. The majority of the points date to the late Archaic and include Marshall, Castroville, Monte 1l, Uvalde, Frio, Wells, Ensor, Yarbrough, Darl, Edwards and Fairland. The Fairland points in this sequence are indicative of the transitional period from dart to arrow points. They are lighter, smaller and thinner than most illustrated from the type sites (Suhm and Jelks 1962:191).

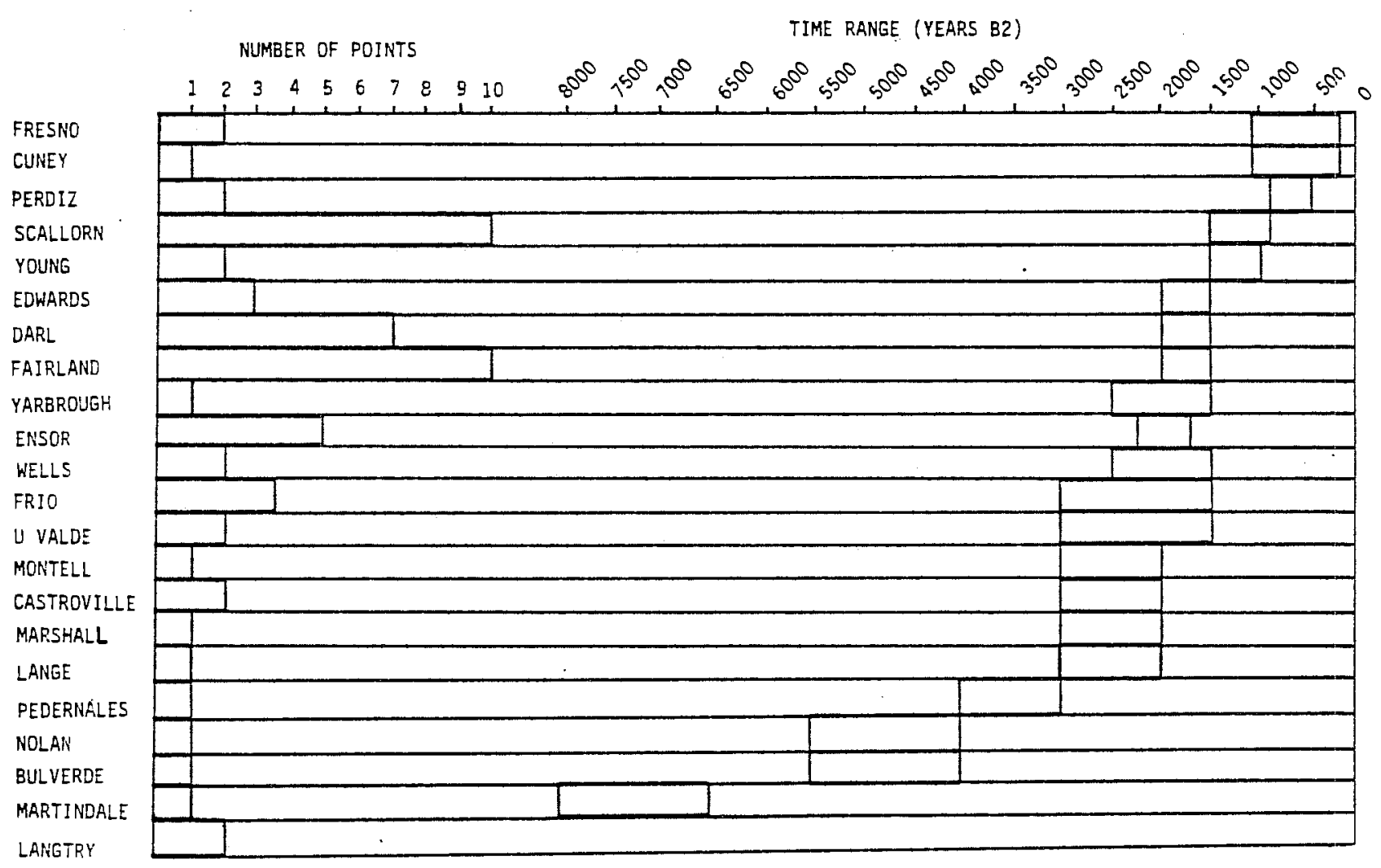

Figure 12.10. Number of Points of Each Type and Their Time Ranges. 
Table 12.05 Projectile Points By Terminal Date, Type, Field Number, and Coordinates

Terminal Date

Years B2

(Before AD2

Field No.

East North

Classification

\begin{tabular}{|c|c|c|c|c|}
\hline $200 \mathrm{~B} 2$ & $\begin{array}{l}0696 \\
6012 \\
6009\end{array}$ & 977 & 955 & $\begin{array}{l}\text { Fresno } \\
\text { Fresno } \\
\text { Cuney }\end{array}$ \\
\hline $500 \mathrm{B2}$ & $\begin{array}{l}6013 \\
1129 \\
6011 \\
2389 \\
2041\end{array}$ & $\begin{array}{l}905 \\
920 \\
933\end{array}$ & $\begin{array}{l}936 \\
958 \\
945\end{array}$ & $\begin{array}{l}\text { Perdiz } \\
\text { Perdiz } \\
\text { Edwards } \\
\text { Edwards } \\
\text { Edwards }\end{array}$ \\
\hline 800 B2 & $\begin{array}{l}1852 \\
1123 \\
2211 \\
2207 \\
2185 \\
2205 \\
1.505 \\
1126 \\
0776 \\
0859\end{array}$ & $\begin{array}{l}906 \\
893 \\
932 \\
942 \\
936 \\
937 \\
905 \\
933 \\
923\end{array}$ & $\begin{array}{r}935 \\
951 \\
961 \\
945 \\
961 \\
926 \\
936 \\
956 \\
1001\end{array}$ & $\begin{array}{l}\text { Scallorn } \\
\text { Scallorn } \\
\text { Scal lorn } \\
\text { Scal lorn } \\
\text { Scal lorn } \\
\text { Scallorn } \\
\text { Scallorn } \\
\text { Scal lorn } \\
\text { Scal lorn } \\
\text { Scallorn }\end{array}$ \\
\hline & $\begin{array}{l}1262 \\
2209\end{array}$ & $\begin{array}{l}965 \\
912\end{array}$ & $\begin{array}{l}964 \\
961\end{array}$ & $\begin{array}{l}\text { Young } \\
\text { Young }\end{array}$ \\
\hline $1500 \mathrm{~B} 2$ & $\begin{array}{l}0853 \\
0863 \\
0062 \\
0858\end{array}$ & $\begin{array}{l}909 \\
958 \\
998 \\
931\end{array}$ & $\begin{array}{r}973 \\
957 \\
1031 \\
999\end{array}$ & $\begin{array}{l}\text { Frio } \\
\text { Frio } \\
\text { Frio } \\
\text { Frio }\end{array}$ \\
\hline & $\begin{array}{l}6005 \\
6006 \\
6004 \\
0815 \\
0835 \\
0778 \\
2206 \\
0830 \\
6019 \\
2384\end{array}$ & $\begin{array}{l}928 \\
949 \\
919 \\
933 \\
942 \\
894\end{array}$ & $\begin{array}{l}931 \\
939 \\
951 \\
959 \\
935 \\
945\end{array}$ & $\begin{array}{l}\text { Fairland } \\
\text { Fairland } \\
\text { Fairland } \\
\text { Fairland } \\
\text { Fairland } \\
\text { Fairland } \\
\text { Fairland } \\
\text { Fairland } \\
\text { Fairland } \\
\text { Fairland }\end{array}$ \\
\hline & 657 & 1014 & 1086 & Yarbrough \\
\hline
\end{tabular}


Table 12.05 - Continued

Terminal Date

Years B2

(Before AD2000)

Field No.

East North

Classification

1500 B2

Cont'd

0832

1152

6003

6002

0061

1184

1562

1243

6001

1469

1878

6000

6007

0833

1100

0690

1855

0060

6008

2000 B2

670

739

1856

2443

921

940

892

937

Ensor

Ensor

Ensor

Ensor

Ensor

$1000 \quad 1042 \quad$ Montel1

$964 \quad 1018$

970

Wells

Wells

931

Darl

Dar1

Dar1

Darl

Dar

Dar 1

Darl

$\begin{array}{lll}940 & 934 & \text { Dar }\end{array}$

963

954

Uvalde

Uvalde

Langtry

Langtry

953

1032

Marshal1

Lange

Castroville

$964 \quad$ Castroville

$3000 B 2 \quad 0676$

1850

Pedernales

4000 B2

1851

Nolan

Bulverde

$7000 B 2$

2212

935

959

Martindale

Total Points

Classified

62

NOTE: $0060,0061,0062$ are from field NW of 41GL21 surveyed by Kelly 27 June 1976. 
The middle Archaic is represented by one Pedernales point while Buiverde, Nolan and Martindale specimens represent the early Archaic.

Figure 12.01 enumerates al1 points successfully classified with terminal dates and field numbers.

Dating sequences are from Sorrow, Shäfer and Ross (1967:142) and Prewitt (1974: 32).

There is a hint of Paleo-Indian presence in 2 lanceolate preforms (FN 2210 and FN 1153) but no conclusions may be drawn from them.

Figure 12.02 illustrates number of points per type and time range encompassed. The results of this brief study indicate that Hop Hill saw continuous light occupation from 5000 B2 to 2500 B2, heavier occupation from 2500 to 800 B2, followed by scanty occupation up to historic times. 
13.0 HOP HILL LITHICS: UNIFACE ANALYSIS (Elizabeth and Augustine Frkuska)

A "uniface" is defined as an artifact that has been flaked on one face only (Crabtree 1972:97). All unifaces share the above characteristic but all unifaces are not functionally or morphologically the same. This study proceeds with a series of measurements and observations to locate the definitive quality of a specific uniface or subgroup that makes it different from another specific uniface or subgroup. Many archaeologists have identified and created categories for these subgroups of unifaces; for example; side scrapers, end scrapers, snub nosed end scrapers, etc..., but unfortunately the means of analysis has been based on arbitrary divisions. Some of the categories have been divided accurately, however of ten the ultimately important attributes are masked and most of these subgroups have no true definition of clustering. Further subdivisions of subgroups may provide very important information.

The Model

Unlike the Core and Bifacial Flaking Models, the model used for this study does not explain knapping techniques.

Since a uniface is a tool which is utilized for some purpose (scraping, sawing, cutting, etc.), it follows that the task the uniface must perform is a response to a network of environmental and cultural demands activated by a chain reaction. The model (Figure 13.01) outlines this techno-environmental relationship.

The environment acts as the trigger which activates the system by forcing cultural adaptations in the manufacture and use of the uniface. Cultural idiosyncrasies will further limit the type of environmental exploitation a culture can undertake. Thus, the unique character of a particular environment should in some manner be encoded in its associated tool types. Each tool type represents a cluster of artifacts which are specific to an environmentally generated task. If a culture performs a certain task often, a specific uniface type will be used. These commonly utilized types form the largest cluster of artifacts. If, however, an unusual or seldom performed task needs to be done, the tool type will be sporadic and outside of the larger clustered tool types.

The knapper's skill and mental template will further limit the tool style since the knapper may create a functionally adequate tool before he has satisfied his mental image of what the tool should be like. After the tool has been made it may be rejected and never used or the tool can be used and retouched or modified until it also is discarded. Both the sporadic tool and the utilized or non-utilized "sub-ideal" may then constitute outliers to the clusters of more normative artifacts. The model suggests, then, that these functional groupings of unifaces, when analyzed in terms of the specific environment can provide ethnographic-like information if uniface morphology, wear patterns, and organic data are consistently documented. This study completes only step one, that is, morphological groupings. 


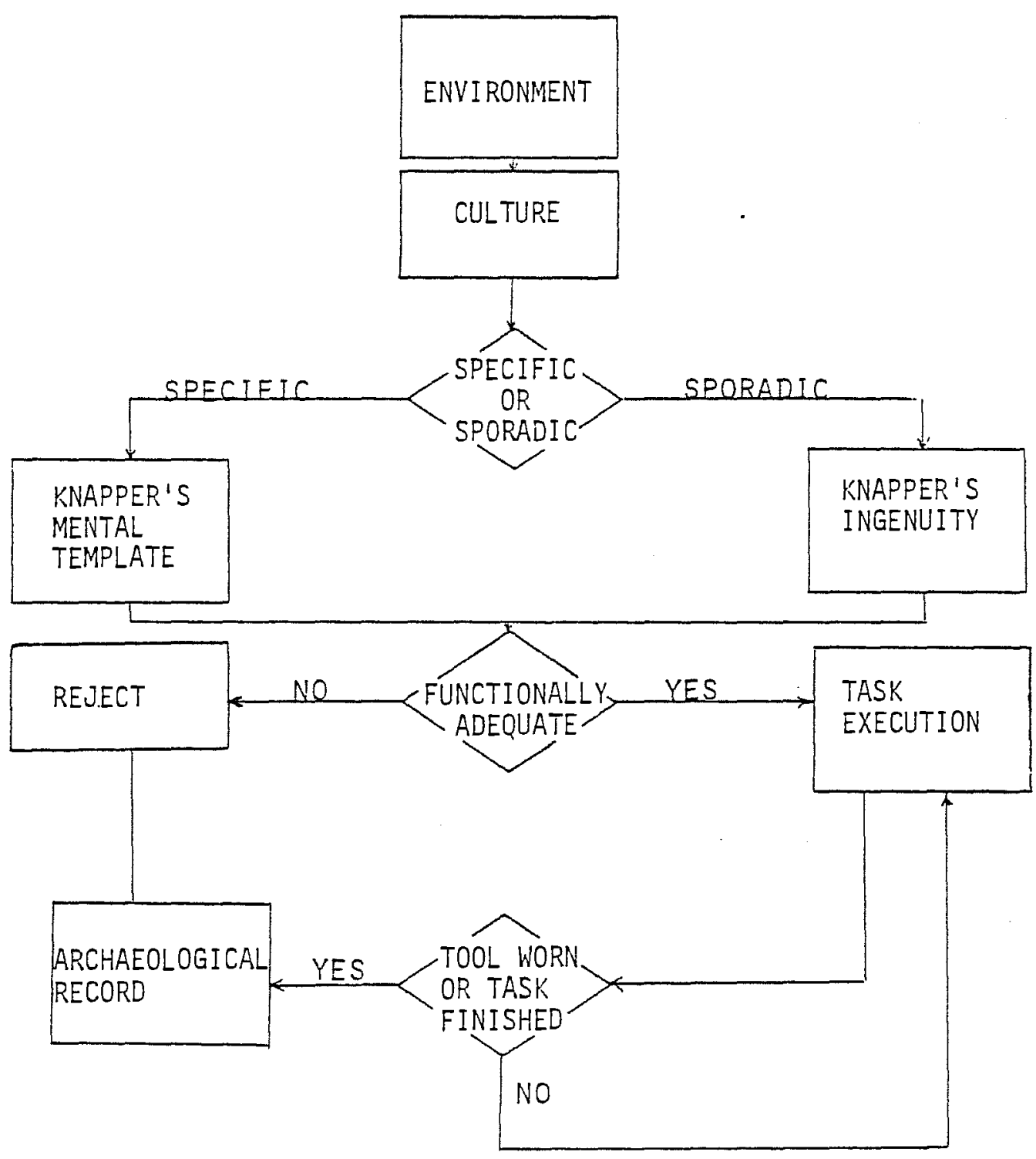

Figure 13.01 Envirotechnical Model for Unifiace Production. 
Table 13.01 FN's and Provenience of Types

\begin{tabular}{|c|c|c|c|c|c|c|c|c|c|}
\hline Type \# & FN & SE & East & North & Type \# & FN & $\underline{S E}$ & East & North \\
\hline \multirow[t]{2}{*}{1.1} & $\begin{array}{l}1104 \\
1917\end{array}$ & $\begin{array}{l}\mathrm{s} \\
\mathrm{s}\end{array}$ & $\begin{array}{l}906.42 \\
900.42\end{array}$ & $\begin{array}{r}938.17 \\
1050.18\end{array}$ & \multirow[t]{2}{*}{2.3} & $\begin{array}{l}723 \\
742\end{array}$ & $\begin{array}{l}\mathrm{s} \\
\mathrm{s}\end{array}$ & 964.98 & 953.96 \\
\hline & 642 & $\mathrm{~s}$ & 990.02 & 1013.60 & & 1375 & $\mathrm{~s}$ & 964.97 & 967. \\
\hline \multirow[t]{8}{*}{1.2} & 213 & $\mathrm{~s}$ & 972.24 & 1002.63 & \multirow[t]{11}{*}{2.4} & 558 & $\mathrm{~s}$ & 931.00 & 971. \\
\hline & $\begin{array}{r}293 \\
7360\end{array}$ & 5 & 977.58 & 1011.70 & & 742 & s & & \\
\hline & $\begin{array}{l}1369 \\
1395\end{array}$ & $\begin{array}{l}s \\
s\end{array}$ & $\begin{array}{l}903.19 \\
966.17\end{array}$ & 967.07 & & $\begin{array}{l}145 \\
796\end{array}$ & $\begin{array}{l}5 \\
5\end{array}$ & 899.79 & 933.36 \\
\hline & 1399 & $\mathrm{~s}$ & 968.23 & 962.17 & & 763 & 5 & 934.65 & 947.8 \\
\hline & 1470 & $\mathrm{~s}$ & 968.72 & 963.97 & & 663 & $\mathrm{~s}$ & 1010.18 & 1037.8 \\
\hline & 1477 & $\mathrm{~s}$ & 903.10 & 932.98 & & 265 & $\mathrm{~s}$ & 974.97 & 1006.6 \\
\hline & 1970 & $\mathrm{~s}$ & 905.95 & 1042.15 & & 1060 & $\mathrm{~s}$ & 967.08 & 964. \\
\hline & 2028 & $\mathrm{~s}$ & 942.03 & 946.05 & & 1108 & 5 & 909.00 & 935. \\
\hline \multirow[t]{3}{*}{1.3} & 725 & $s$ & 964.47 & 950.46 & & 1429 & 5 & 971.07 & 962.2 \\
\hline & 1426 & $s$ & 971.03 & 961.98 & & 1770 & $\mathrm{~s}$ & 920.71 & 1060.4 \\
\hline & 1770 & $s$ & 920.71 & 1060.44 & & 1871 & $\mathrm{~s}$ & 896.71 & 1042. \\
\hline \multirow{3}{*}{1.4} & 1532 & $\mathrm{~s}$ & 984.47 & 959.20 & \multirow{7}{*}{2.5} & 2191 & $\mathrm{~s}$ & 941.07 & 943.7 \\
\hline & 1879 & $s$ & 895.55 & 1039.88 & & 669 & $\mathrm{~s}$ & 1016.68 & 1048.8 \\
\hline & 2039 & $s$ & 945.16 & 949.72 & & 695 & $\mathrm{~s}$ & 696.71 & 652.7 \\
\hline 1.5 & 340 & $s$ & 975.40 & 1017.46 & & 787 & $\mathrm{~s}$ & 940.30 & 954.1 \\
\hline \multirow{22}{*}{2.1} & 824 & $s$ & 936.00 & 934.56 & & 1443 & $\mathrm{~s}$ & 970.29 & 968.6 \\
\hline & 233 & $\mathrm{~s}$ & 972.84 & 1003.83 & & 1770 & $\mathrm{~s}$ & 920.71 & 1060.4 \\
\hline & 321 & $\mathrm{~s}$ & 969.34 & 1014.29 & & 2440 & $s$ & 912.99 & 932 \\
\hline & 367 & $\mathrm{~s}$ & 937.70 & 1019.27 & \multirow[t]{2}{*}{3.1} & 1075 & $\mathrm{~s}$ & 907.62 & 93 \\
\hline & 626 & $\mathrm{~s}$ & 995.35 & 1011.59 & & 1433 & $\mathrm{~s}$ & 969.74 & 966 \\
\hline & 742 & $\mathrm{~s}$ & & & 3.2 & 753 & $\mathrm{~s}$ & 988.81 & \\
\hline & 763 & $s$ & 934.65 & 947.83 & & 769 & 5 & 929.38 & 94 \\
\hline & 764 & $\mathrm{~s}$ & 935.26 & 947.84 & & 820 & $\mathrm{~s}$ & 932.68 & \\
\hline & 1058 & $s$ & 966.46 & 963.44 & & 826 & $\mathrm{~s}$ & 937.61 & 93 \\
\hline & 1067 & $\mathrm{~s}$ & 909.40 & 939.14 & & 1076 & $\mathrm{~s}$ & 907.65 & 939.2 \\
\hline & 1135 & $s$ & 905.46 & 943.76 & & 1093 & $\mathrm{~s}$ & 908.94 & 936.8 \\
\hline & 1365 & $s$ & 961.99 & 961.59 & & 1384 & $\mathrm{~s}$ & 963.85 & 0 \\
\hline & 1378 & $\mathrm{~s}$ & 964.81 & 963.73 & & 1429 & $\mathrm{~s}$ & 971.07 & 962.2 \\
\hline & 1399 & $\mathrm{~s}$ & 968.22 & 962.17 & & 1442 & $\mathrm{~s}$ & 970.21 & 969.2 \\
\hline & 1407 & $s$ & 968.03 & 964.35 & 3.3 & 669 & $\mathrm{~s}$ & 1016.68 & 1048.8 \\
\hline & 1483 & $s$ & 899.86 & 939.44 & & 801 & $\mathrm{~s}$ & .54 & \\
\hline & 1815 & $\mathrm{~s}$ & 931.14 & 1066.37 & & 1444 & $\mathrm{~s}$ & 970.18 & 967.9 \\
\hline & 1816 & $s$ & 929.31 & 1065.03 & & 7780 & $\mathrm{~s}$ & 922.98 & 1061.6 \\
\hline & 1871 & $s$ & 896.71 & 1042.11 & 3.4 & 660 & $\mathrm{~s}$ & 1013.70 & 1028.5 \\
\hline & 1882 & $s$ & 894.92 & 1039.61 & 4.1 & 1036 & $\mathrm{~s}$ & 963.16 & 963.6 \\
\hline & 203 & $\mathrm{~s}$ & 943.22 & 948.13 & & 1054 & $\mathrm{~s}$ & 960.14 & 962.1 \\
\hline & 279 & $\mathrm{~s}$ & & & & & $\mathrm{~s}$ & 963.79 & \\
\hline 2.2 & 69 & $s$ & 969.93 & 953.51 & & 1432 & $\mathrm{~s}$ & 969.33 & 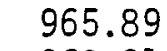 \\
\hline & 770 & $\mathrm{~s}$ & 926.56 & 942.68 & & 1385 & $\mathrm{~s}$ & 964.66 & 963.3 \\
\hline & 102 & $\mathrm{~s}$ & 962.07 & 963.47 & & 640 & $\mathrm{~s}$ & 991.99 & 1013.4 \\
\hline & .171 & $\mathrm{~s}$ & 907.37 & 936.17 & 4.2 & 1363 & $\mathrm{~s}$ & 961.23 & 962.6 \\
\hline & 142 & $\mathrm{~s}$ & 971.07 & 962.28 & & 1462 & $\mathrm{~s}$ & 965.50 & 966.4 \\
\hline & 153 & $\mathrm{~s}$ & 987.11 & 959.72 & & 1930 & $s$ & 893.79 & 1043.7 \\
\hline
\end{tabular}


Table 13.01 - Continued

Type \# FN SE East North

$\begin{array}{lllll}4.3 & 767 & s & 923.79 & 945.58\end{array}$

$\begin{array}{llll}810 & s & 913.30 \quad 929.48\end{array}$

$\begin{array}{llll}795 & s & 888.51 & 933.57\end{array}$

$\begin{array}{llll}1024 & s & 962.07 & 963.47\end{array}$

$\begin{array}{lllll}4.4 & 780 & s & 916.44 & 945.85\end{array}$

$\begin{array}{llll}850 & s & 919.21 & 961.47\end{array}$

$\begin{array}{rrrrr}4.5 & 738 & s & 1028.83 & 1077.70 \\ & 800 & s & 895.34 & 933.52\end{array}$

$\begin{array}{llll}1051 & s & 961.00 & 966.12\end{array}$

$1147 \mathrm{~s} \quad 908.97 \quad 932.63$

$\begin{array}{lllll}4.6 & 751 & s & 976.67 & 1009.89\end{array}$

$\begin{array}{lllll}5.0 & 1420 & s & 962.42 & 969.59\end{array}$

$\begin{array}{lllll}6.0 & 386 & \mathrm{~s} & 970.61 & 1017.94\end{array}$

437 s $\quad 974.51 \quad 1013.72$

620 s 990.831010 .18

$664 \mathrm{~s} \quad 988.051010 .18$

$\begin{array}{llll}706 & s & 977.11 & 853.80\end{array}$

$\begin{array}{llll}763 & s & 934.65 & 947.83\end{array}$

$\begin{array}{llll}771 & s & 925.47 & 948.48\end{array}$

$\begin{array}{llll}849 & s & 974.57 & 975.95\end{array}$

1003 s $\quad 960.98 \quad 960.16$

$\begin{array}{llll}1069 \mathrm{~s} & 909.21 & 939.48\end{array}$

$\begin{array}{llll}1108 \mathrm{~s} & 909.00 & 935.35\end{array}$

$\begin{array}{llll}1139 & \mathrm{~s} & 903.72 & 973.36\end{array}$

$\begin{array}{llll}1397 & s & 967.17 & 965.84\end{array}$

$\begin{array}{llll}1402 \mathrm{~s} & 970.29 & 961.49\end{array}$

$\begin{array}{llll}1421 & s & 962.31 & 969.48\end{array}$

$\begin{array}{llll}1424 & s & 969.60 & 964.97\end{array}$

1818 s $\quad 926.911062 .09$

$1930 \mathrm{~s} \quad 893.791043 .76$

2008 s $\quad 900.451039 .61$

$\begin{array}{llll}2181 & s & 943.41 & 950.65\end{array}$

$\begin{array}{llll}2180 & s & 918.88 & 957.73\end{array}$

$\begin{array}{llll}2184 & s & 944.95 & 945.77\end{array}$

$\begin{array}{llllll}7.1 & 204 & \mathrm{~s} & 974.26 & 1000.74\end{array}$

$1141^{s} \quad 903.94 \quad 936.20$

$\begin{array}{llll}1102 & s & 906.78 & 937.47\end{array}$

$1150 \mathrm{~s} \quad 905.43 \quad 934.31$

$\begin{array}{llll}1400 \mathrm{~s} & 963.98 & 962.28\end{array}$

$\begin{array}{llll}1426 & s & 971.03 & 961.98\end{array}$

$1427 \mathrm{~s} \quad 969.82: 963.49$

$\begin{array}{llll}1433 & s & 969.74 & 966.38\end{array}$

$1806 \mathrm{~s}$

$1816 \mathrm{~s}$

$1970 \mathrm{~s}$

$2314 \mathrm{~s}$

$7.2 \quad 836 \mathrm{~s}$

1135

$1102 \mathrm{~s}$
929.311065 .03

$905.95 \quad 1042.15$

$931.67 \quad 970.44$

945.51940 .96

$905.46 \quad 943.76$

$906.78 \quad 937.47$
Type $\frac{H}{\pi}$

FN SE East North $\begin{array}{llll}1416 & s & 964.02 & 966.84\end{array}$ $\begin{array}{llll}1885 & s & 896.62 & 1042.64\end{array}$ $\begin{array}{lllll}2001 & s & 904.87 & 1050.26\end{array}$

$\begin{array}{lllll}7.3 & 1097 & s & 908.94 & 935.76\end{array}$ $\begin{array}{llll}1486 & s & 908.73 & 937.96\end{array}$ $\begin{array}{llll}1828 \mathrm{~s} & 927.96 & 1067.44\end{array}$ $\begin{array}{llll}1922 & s & 895.54 & 1049.83\end{array}$

$\begin{array}{rrrrr}7.4 & 2497 & s & & \\ 731 & s & 948.44 & 955.24\end{array}$ $\begin{array}{llll}1828 & s & 927.96 & 1067.44\end{array}$ 


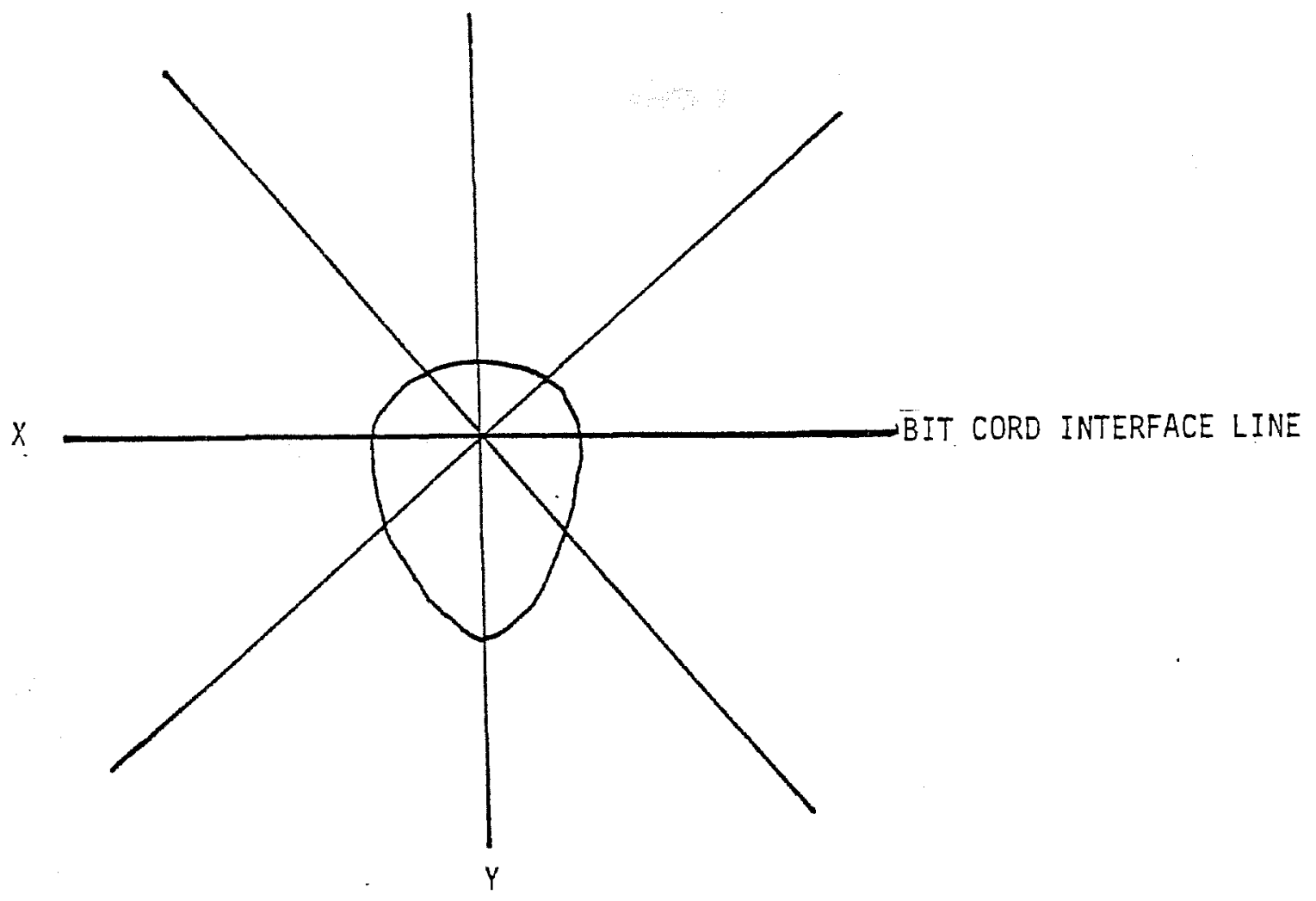

Figure 13.02. Polar Coordinate Grid for Uniface Measurement.

Uniface Descriptions

All 176 unifaces collected at Hop Hill, like the bifaces, were not "collector's pieces", although they provide important information for the archaeologist. A coding form was created to collect the maximum amount of information from each uniface. The shape of each uniface was then measured on a polar coordinate grid. The artifact was placed on the grid (Figure 13.02) with the bit toward the upper end of the grid so that the cord interface line (separation of bit from haft) is defined by axis $X$ and divided into two equal segments by axis $Y$.

After various runs of principle components analysis on the data, a principal components analys is of the polar coordinate measurements resulted in the separation of bit from haft. This study and a previous one indicate that the bit and haft of unifaces are the best discriminators between typological, and perhaps in the future functional, subgroups of unifaces. Based on these results, component scores were plotted along these two dimensions, with haft size ajong the $y$ axis and bit size along the $x$ axis (See Figure 13.03 ). This plotting aided in the separation of seven major groups of unifaces and twenty-four subgroups which served as the basis for the following typology.

\subsection{End Trimmed Unifaces $N=20$}

A11 specimens are trimmed at the end opposite from the bulb of percussion, typically the longest axix (Weir 1976:66). The specimens are further subdivided according to bit and haft size. 


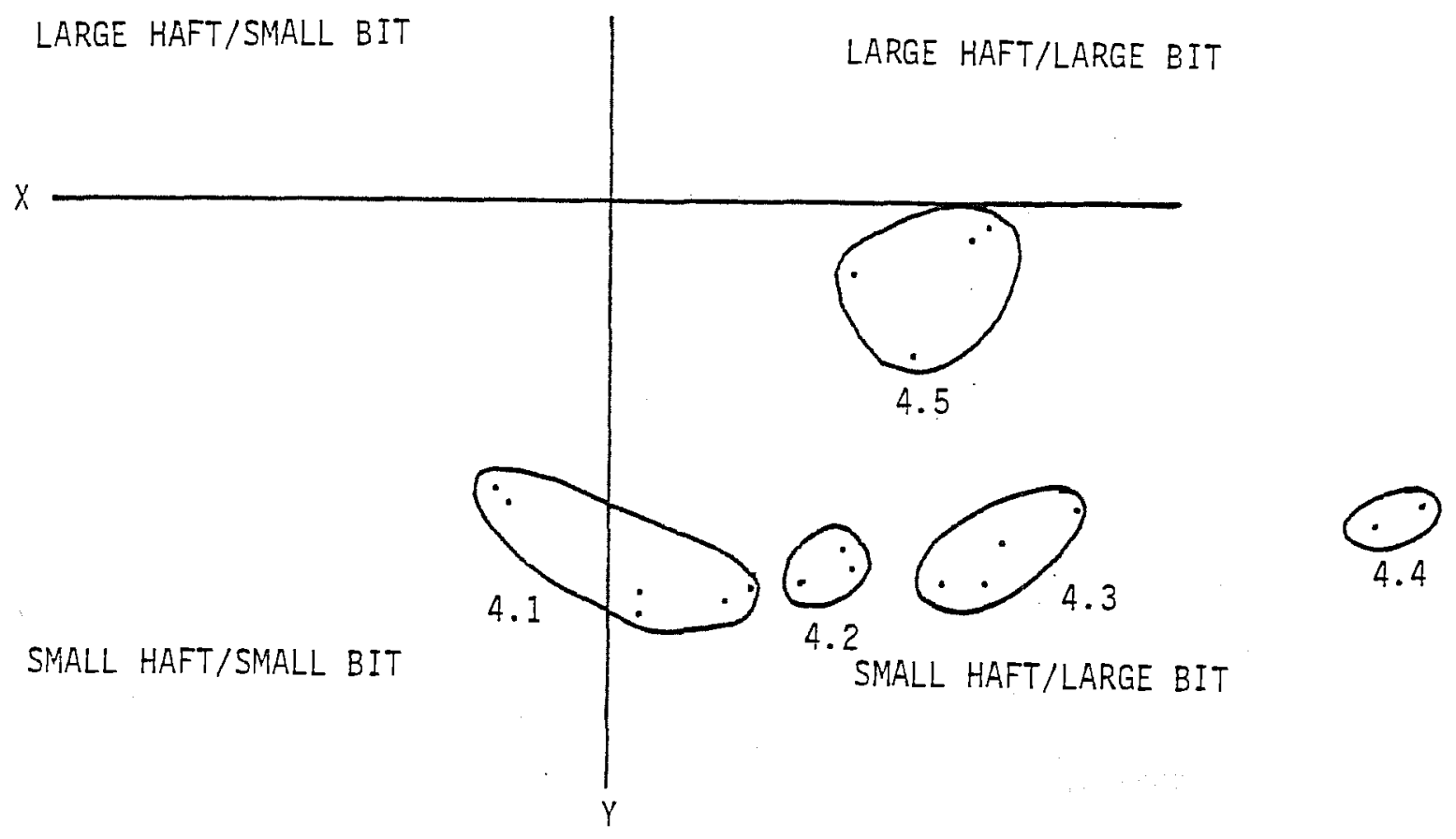

Figure 13.03. Example of Component Score Plotting Along Bit-Haft Dimensions.

1.1 End Trimmed Unifaces, Small to Med. Hajt/Small Bit

(N=3, L $\bar{x}=29 \mathrm{~mm}, W \bar{x}=37 \mathrm{~mm}$, Th $\bar{x}=7 \mathrm{~mm}$, Wt $\bar{x}=11 \mathrm{gm}, B C 1 \bar{x}=32$ )

See Table 13.01 for FN's and provenience. The members of this group have a straight to semi-concave edge and are subtriangular in shape (Figure 13.04). These specimens could have been hafted and are similar to, al though not as finely worked as, those specimens found in Coahuila, Mexico (Hester 1971).

1.2 End Trimmed Unifaces, Small to Med. Haft/Med. Bit

$(N=9, L \bar{x}=33 \mathrm{~mm}, W \bar{x}=29 \mathrm{~mm}$, Th $\bar{x}=12 \mathrm{~mm}$, Ht $\bar{x}=12 \mathrm{gm}, \mathrm{BCl} \quad \bar{x}=29)$

See Table 13.01 for FN's and provenience. Members of this group have a convex bit and vary in total outline. Uniess these tools were used on very soft or small items, it is also possible that they could be hafted since this process would increase potential force (Figure 13.04b). 


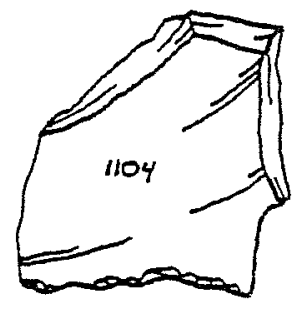

a

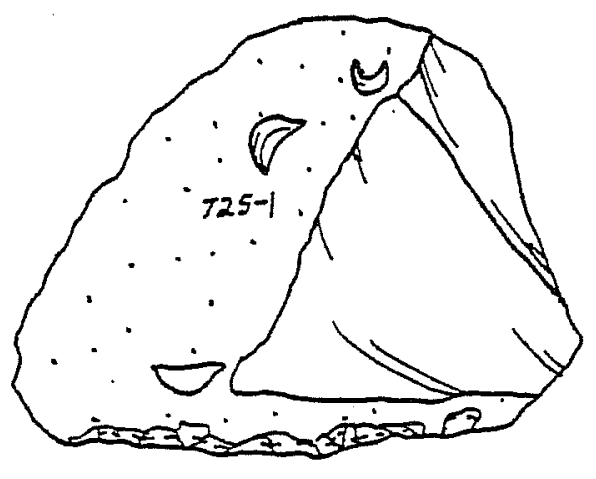

c

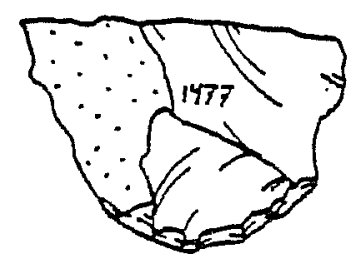

$b$
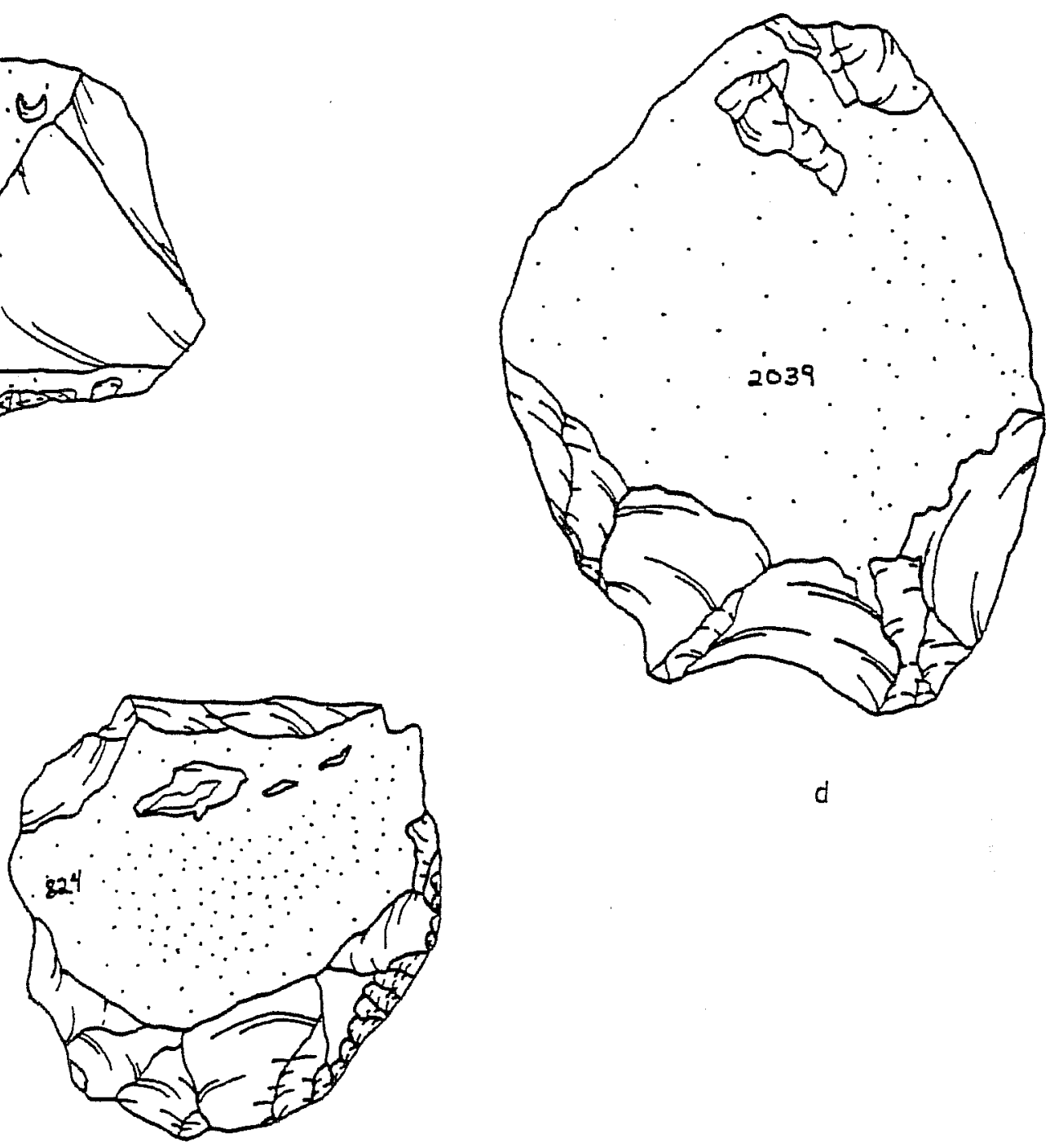

d e

Figure 13.04. End Trimmed Unifaces. a, group 1.1; b, group 1.2; c., group $1.3 ; d$, group $1: 4 ; e$, group 1.5 . 


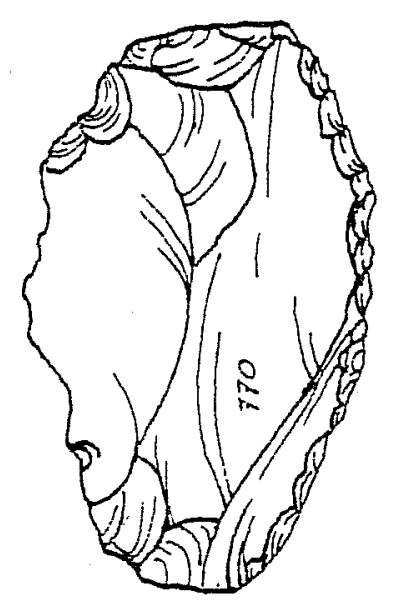

a

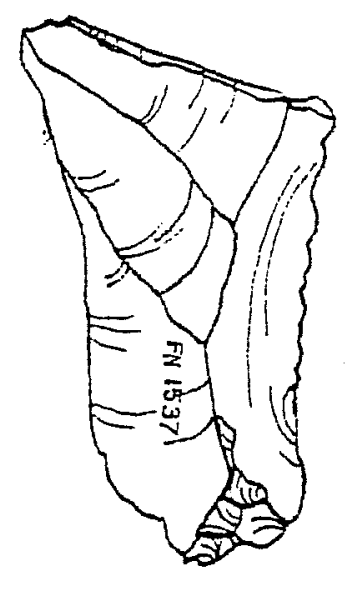

b
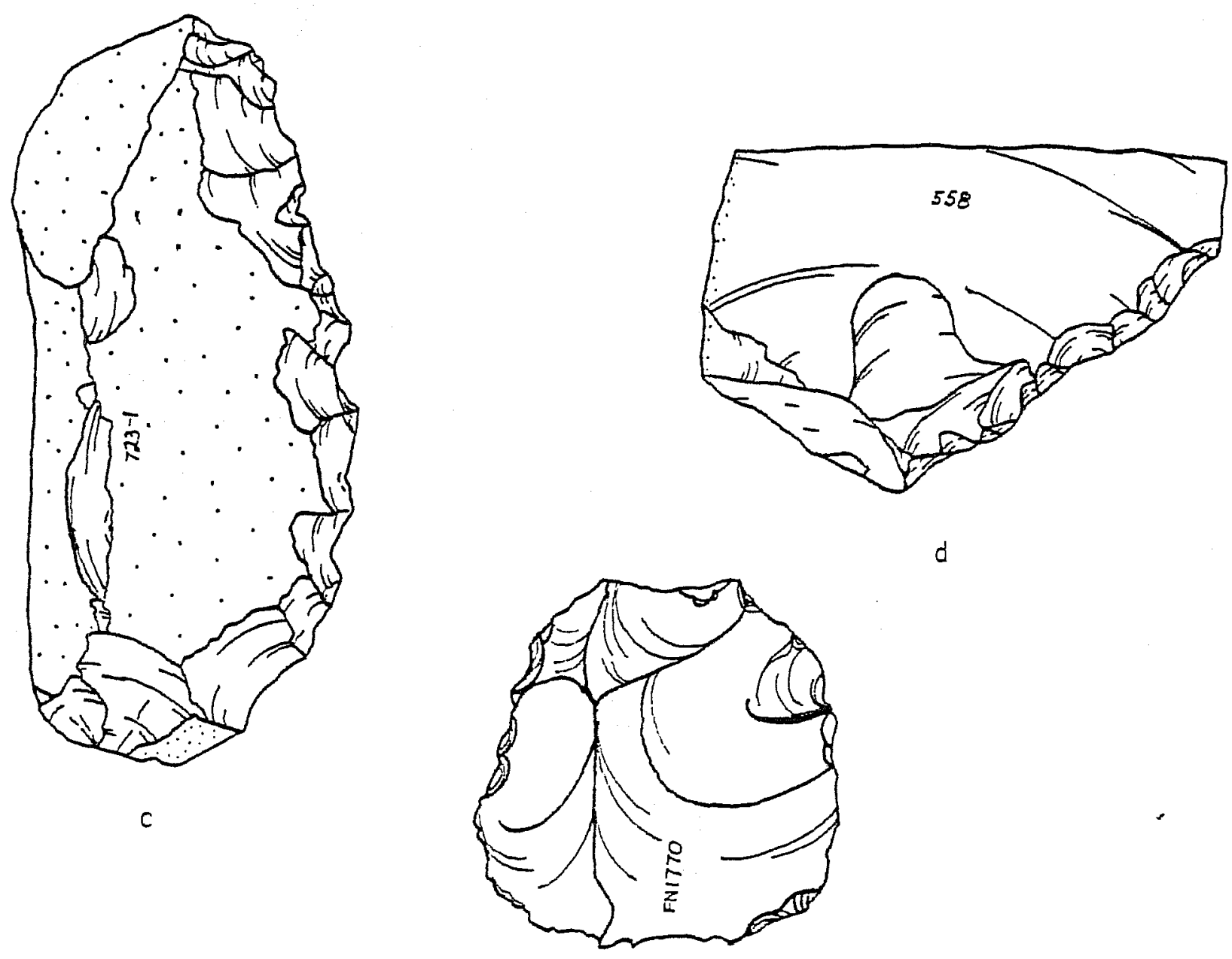

e

Figure 13.05. Unilaterally Trimmed unifaces. a, group 2.1; b, group 2.2; $c$, group 2.3 ; $d$, group 2.4 ; e, group 2.5 . 
1.3 End Trimmed Unifaces, Med. Hait/Med. Bit

( $N=3, L \bar{x}=43 \mathrm{~mm}, W \bar{x}=46 \mathrm{~mm}$, Th $\bar{x}=12 \mathrm{~mm}$, wt $\bar{x}=27 \mathrm{gm}, B \mathrm{cl} \bar{x}=40$ )

See Table 13.01 for FN's and provenience. Members of this group are similar to those from group 1.1 except that they are larger and self-hafted. They have straight bits and are triangular in outline (Figure 13.04c).

1.4 End Trimmed Unifaces, Large Haft/Med. Bit

$(N=3, L \bar{x}=88 \mathrm{~mm}, W \bar{x}=71 \mathrm{~mm}$, Th $\bar{x}=42 \mathrm{~mm}$, Wt $\bar{x}=275 \mathrm{gm}, B c 1 \bar{x}=53)$

See Table 13.01 for FN's and provenience. Members of this group are large, self-hafted tools, possibly cores rejuvenated into end trimmed unifaces (Figure 13.04d).

1.5 End Trimmed Unifiaces, Med. Haft/Large Bit

( $N=2, L \bar{x}=55 \mathrm{~mm}, W \bar{x}=55 \mathrm{~mm}$, Th $\bar{x}=12 \mathrm{~mm}, W t \bar{x}=55 \mathrm{gm}, B c l \bar{x}=55)$

See Table 13.01 for FN's and provenience. The two members in this group had bits that were larger than their hafts perhaps due to retouching (Figure 13.04e). They may be examples of exhausted and trimmed unifaces, or perhaps misgrouped due to sampling biases.

\subsection{Unilaterally Trimmed Unifiaces $(N=55)$.}

A11 specimens are trimmed on one lateral edge (Weir 1976:66). They vary from straight to concave bits and are made on both thin and crude flakes. These tools are usually thought to be cutting rather than scraping tools (Figure 13.05).

2.1 Unilaterally Trimmed Unifaces, Small to Med. Haft/Small Bit

$(N=25, L \bar{x}=29 \mathrm{~mm}, W \bar{x}=35 \mathrm{~mm}$, Th $\bar{x}=10 \mathrm{~mm}$, Wt $\bar{x}=11 \mathrm{gm}, \mathrm{Bc} 1 \bar{x}=29)$

See Table 13.01 for FN's and provenience. The members of this group have convex bits and are usually referred to as convex side scrapers (Figure 13.05a).

2.2 Unilaterally Trimmed Unifaces. Med. Hakt/Med. Bit

$(N=3, L \bar{x}=47 \mathrm{~mm}, W \bar{x}=53 \mathrm{~mm}$, Th $\bar{x}=22 \mathrm{~mm}$, Wt $\bar{x}=86 \mathrm{gm}$, Bcl $\bar{x}=55)$

See Table 13.01 for FN's and provenience. The members of this group have straight to semi-straight bits (Figure 13.05b).

2.3 Unilaterally Trimmed Unifaces, Med. Haft/Large Bit

$(N=3, L \bar{x}=47 \mathrm{~mm}, W \bar{x}=53 \mathrm{~mm}$, Th $\bar{x}=22 \mathrm{~mm}$, Wt $\bar{x}=86 \mathrm{gm}, \mathrm{Bcl} \bar{x}=55$ )

See Table 13.01 for FN's and provenience. Members of this group, like those from group 2.1, have convex bits but they are much 1arger (Figure 13.05c). 


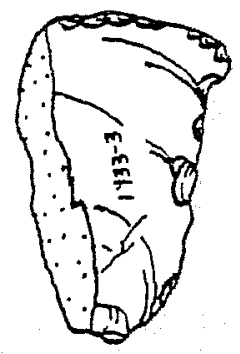

2

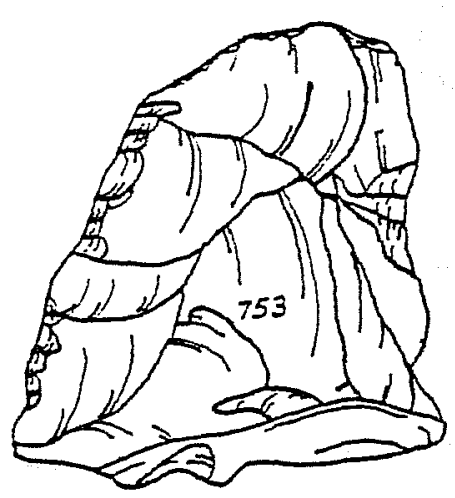

b

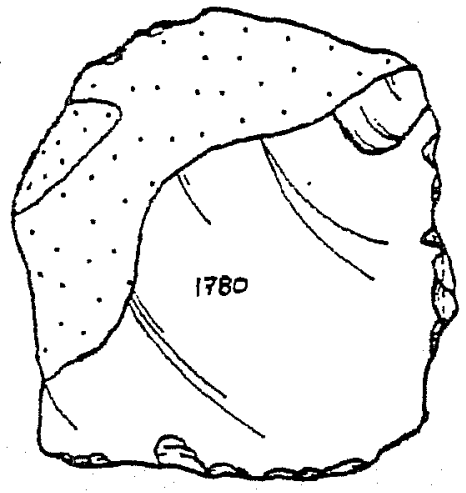

c

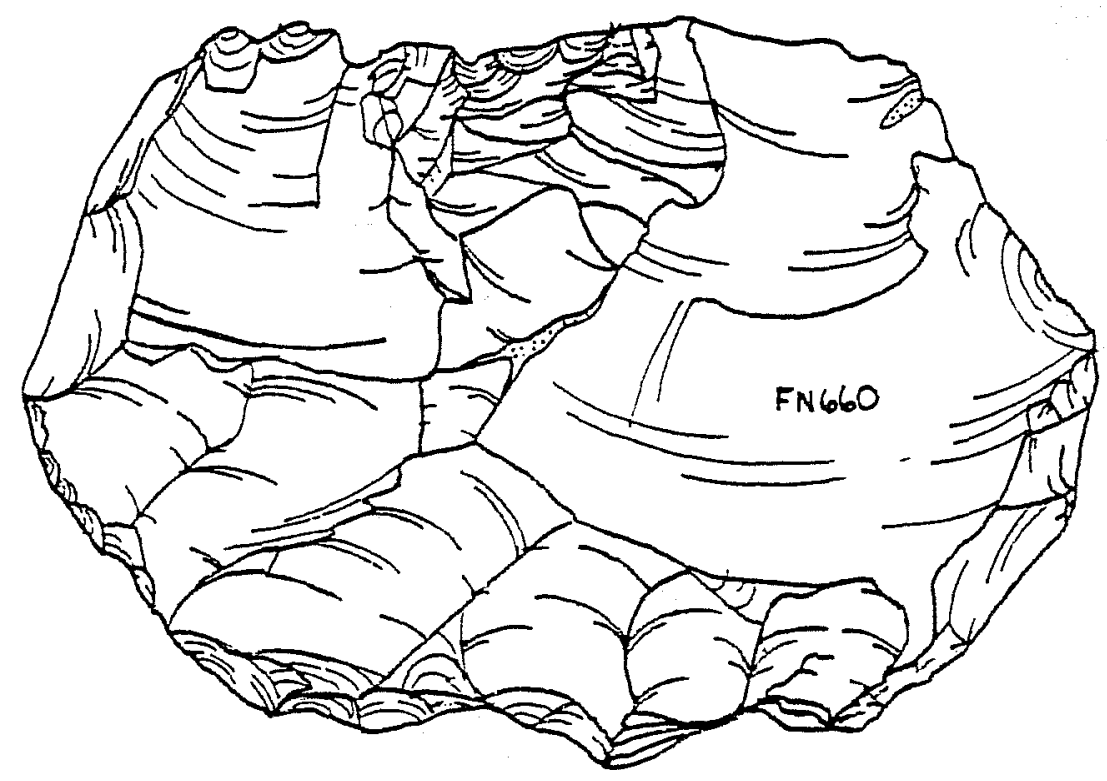

d

Figure 13.05. Unilateralcy and End Trimmed Unifaces. a, group 3.1; $\mathrm{t}$, group $3.2 ; c$, group $3.3 ; d$, group 3.4 . 
2.4 Unilaterally Trimmed Unifaces, Med. to Large Haft/Small to Med. Bit $(N=13, L \bar{x}=43 \mathrm{~mm}, W \bar{x}=53 \mathrm{~mm}$, Th $\bar{x}=12 \mathrm{~mm}, W \mathrm{t} \bar{x}=38 \mathrm{gm}, \mathrm{Bc} 1 \bar{x}=51$ )

See Table 13.01 for FN's and provenience. Members of this group have irregular outlines and straight bits (Figure 13.05d).

2.5 Unilaterally Trimmed Unifaces, Large Haft/Large Bit ( $N=6, L \bar{x}=17 \mathrm{~mm}, W \bar{x}=74 \mathrm{~mm}$, Th $\bar{x}=29 \mathrm{~mm}$, Wt $\bar{x}=69 \mathrm{gm}, B \mathrm{c} T \bar{x}=69$ )

See Table 13.01 for FN's and provenience. Members of this group have convex bits (Figure 13.05e).

3.0 Unilateraldy and End Trimmed Unifiaces $(N=18)$

A11 specimens are trimmed at the end opposite the bulb of percussion and along one lateral edge. They vary from the large core type to the small flake type.

3.1 Unilateraily and End Trimmed Unifaces, Small Hajt/Med. Bit

$(N=2, L \bar{x}=25 \mathrm{~mm}, W \bar{x}=29 \mathrm{~mm}$, Th $\bar{x}=8 \mathrm{~mm}$, Wt $\bar{x}=3 \mathrm{gm}, B c 7 \bar{x}=39$ )

See Table 13.01 for $\mathrm{FN}^{\prime} \mathrm{s}$ and provenience; also see Figure 13.06a)

3.2 Unilaterally and End Trimmed Unifaces, Med. Haft/Small to Med. Bit

( $N=9, L \bar{x}=38 \mathrm{~mm}, W \bar{x}=44 \mathrm{~mm}$, Th $\bar{x}=10 \mathrm{~mm}$, Wt $\bar{x}=24 \mathrm{gm}, B c 1 \bar{x}=42)$

See Table for FN's and provenience; also see Figure 13.06b.

3.3 Unilaterally and End Trimmed Unifaces. Large Haft/Small to Med. Bit

( $N=6, L \bar{x}=52 \mathrm{~mm}, W \bar{x}=48 \mathrm{~mm}$, Th $\bar{x}=14 \mathrm{~mm}, W t \bar{x}=48 \mathrm{gm}, B c 1 \bar{x}=36)$

See Table 13.01 for FN's and provenience; also see Figure 13.06c.

3.4 Unilaterally and End Trimmed Unifiaces, Large Hakt/Large Bit

$(N=1, L \bar{x}=95 \mathrm{~mm}, W \bar{x}=102 \mathrm{~mm}$, Th $\bar{x}=40 \mathrm{~mm}$, Wt $\bar{x}=310 \mathrm{gm}, \mathrm{Bcl} \bar{x}=36)$

See Table 13.01 for FN's and provenience; also see Figure 13.06d.

4.0 Bilaterally and End Trimmed Unifaces $(N=20)$

A11 specimens are trimmed at the end opposite the bulb of percussion and along two semi-parallel edges (Weir 1976:66). The specimens progress in a 7inear fashion from very sma17 "thumbnai1" unifaces to large ovates (Figure 13.07). 

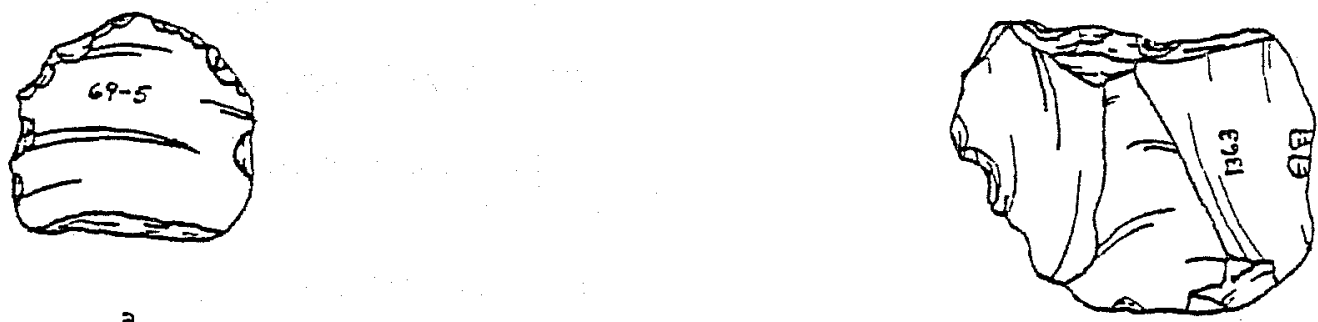

b
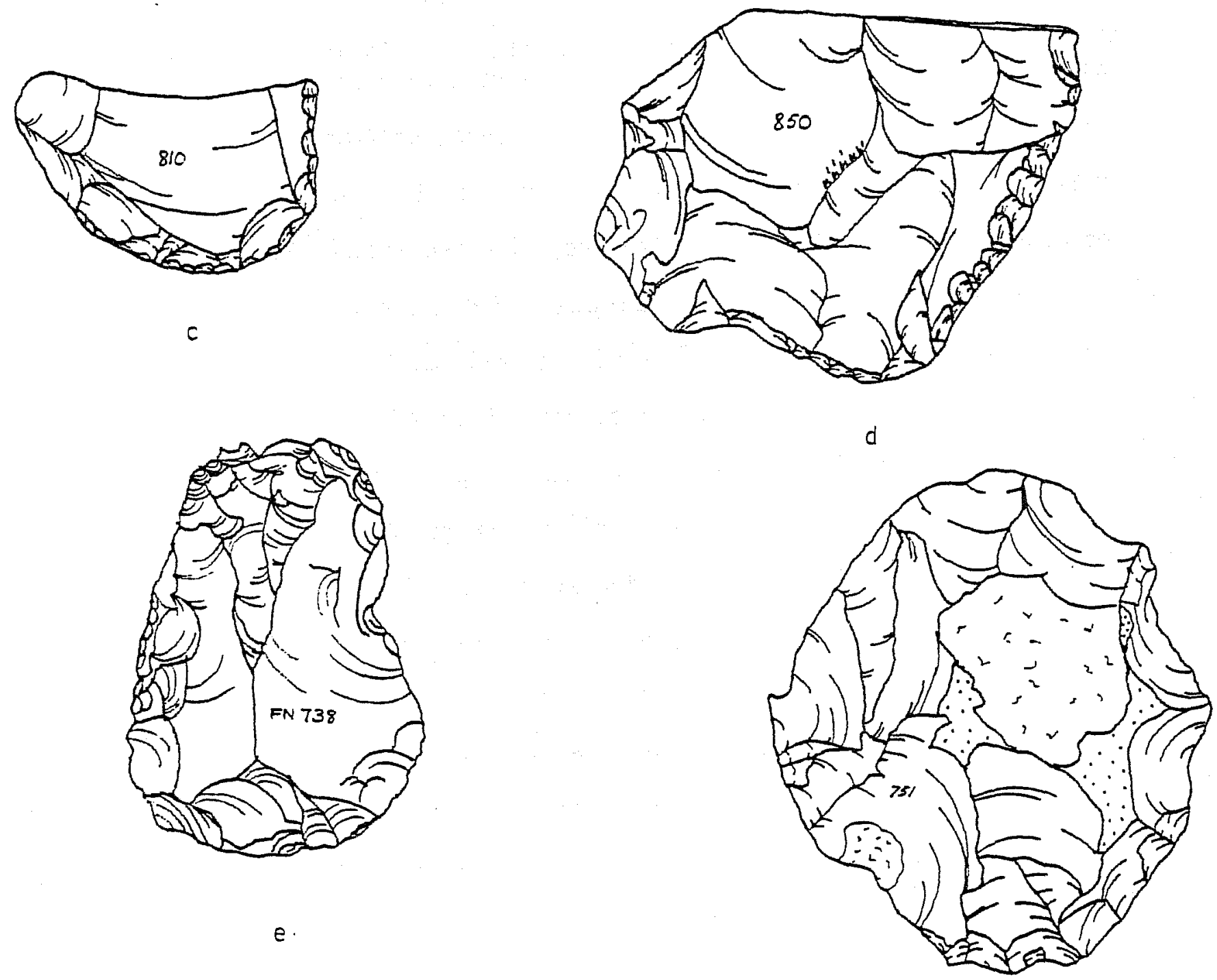

Finure 13.07. Bilaterally and End Trimmed Unifaces. a, group 4. $f$; b, group 4.2; c, group 4.3; d, group 4.4; e, group 4.5. 
4.1 Bilaterally and End Trimmed Unifaces, Small Haft/Small to Med. Bit ( $N=6, L \bar{x}=21 \mathrm{~mm}, W \bar{x}=19 \mathrm{~mm}$, Th $\bar{x}=4 \mathrm{~mm}$, Wt $\bar{x}=3 \mathrm{gm}, \mathrm{Bc} \overline{\mathrm{x}}=19$ )

See Table 13.01 for FN's and provenience. Members of this group are commoniy known as thumbnail scrapers. They are made on interior flakes (Figure 13.07a).

4.2 Bilaterally and End Trimmed Unifaces, Small Hajt/Med. Bit

( $N=3, L \bar{x}=20 \mathrm{~mm}, W \bar{x}=32 \mathrm{~mm}$, Th $\bar{x}=14 \mathrm{~mm}$, Wt $\bar{x}=16 \mathrm{gm}, \mathrm{Bcl} \bar{x}=24)$

See Table 13.01 for $\mathrm{FN}^{\prime} \mathrm{s}$ and provenience. A11 members of this group are made on interior flakes and have irregular retouch (Figure 13.07b).

\subsection{Bilaterally and End Trimmed Unifaces, Small Haft/Med. to Large Bit}

( $N=4, L \bar{x}=39 \mathrm{~mm}, W \bar{x}=46 \mathrm{~mm}$, Th $\bar{x}=25 \mathrm{~mm}$, Wt $\bar{x}=29 \mathrm{gm}, B \mathrm{cl} \overline{\mathrm{x}}=32$ )

See Table 13.01 for FN's and provenience. Members of this group are made in a manner that suggests that they were hafted (Figure 13.07c).

4.4 Bilaterally and End Trimmed Unifaces. Small Haft/Large Bit

( $N=2, L \bar{x}=64 \mathrm{~mm}, W \bar{x}=70 \mathrm{~mm}$, Th $\bar{x}=23 \mathrm{~mm}$, Wt $\bar{x}=112 \mathrm{gm}$, Bcl $\bar{x}=44)$

See Table 13.01 for FN's and provenience; also see Figure 13.07d.

4.5 Bilaterallu and End Trimmed Unifaces, Med. Hakt/Med. to Large Bit

$(N=4, L \bar{x}=44 \mathrm{~mm}, W \bar{x}=52 \mathrm{~mm}$, Th $\bar{x}=14 \mathrm{~mm}, W t \bar{x}=38 \mathrm{gm}, \mathrm{BC}] \bar{x}=50)$

See Table 13.01 for FN's and provenience. Members of this group have a very ovate outline and are finely worked (Figure 13.07e).

4.6 Bilaterallu and End Trimmed Unifaces, Large Haft/Large Bit

$(N=1, L \bar{x}=68 \mathrm{~mm}, W \bar{x}=72 \mathrm{~mm}$, Th $\bar{x}=27 \mathrm{~mm}$, Wt $\bar{x}=111 \mathrm{gm}, B c 1 \bar{x}=71)$

See Table 13.01 for FN's and provenience; also see Figure $13.07 \mathrm{f}$.

5.0 Circular Trimmed Unijace $(N=1)$

This was the only circular trimmed uniface recovered during the Hop Hill survey. It is from the same area of the specialized core group (Figure 13.08a)

\subsection{Miscellaneously Trimmed unifaces $(N=23)$}

These specimens were irregularly trimmed items which did not cluster with any specific subgroup of unifaces. These tools may be examples of the sporadically made tools. 


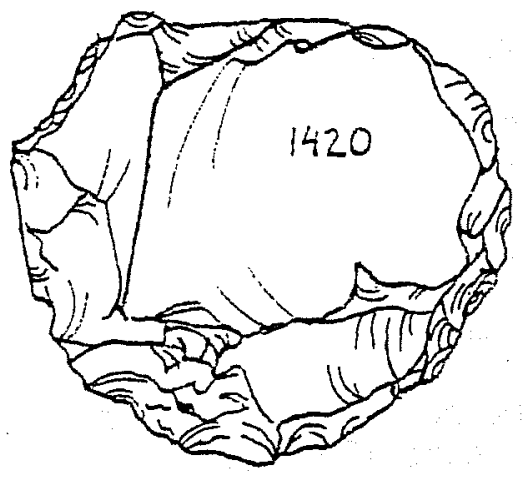

$a$

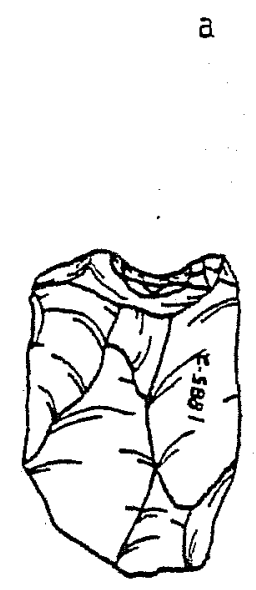

c

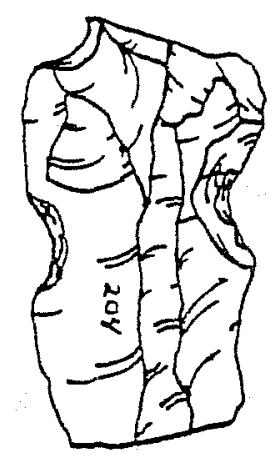

b

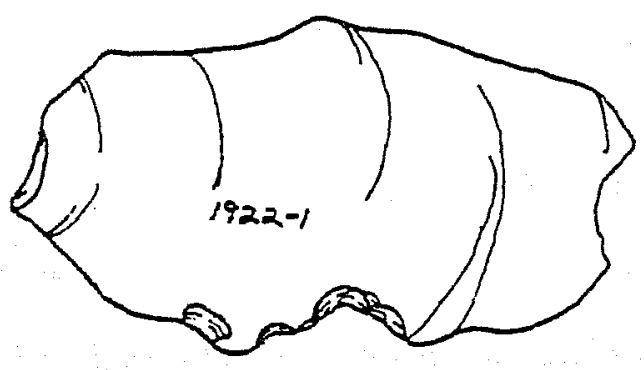

d

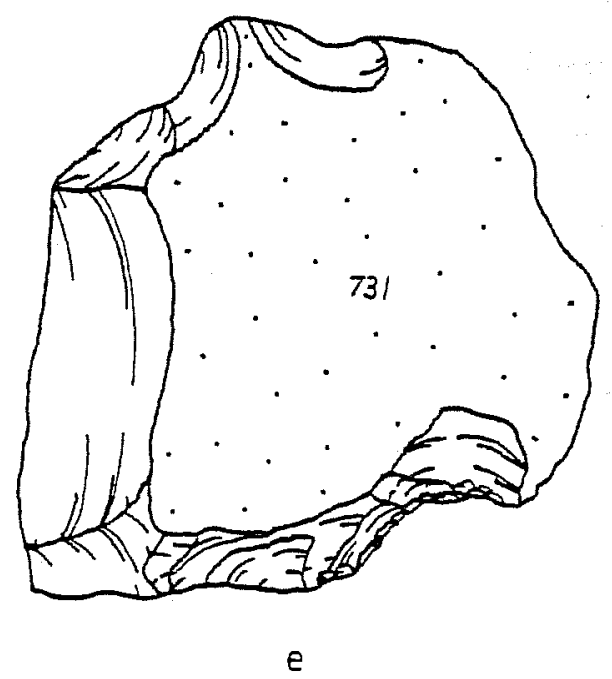

Figure 13.08 Circular Trimmed and Notched Unijaces. a, group 5.0; $t$, group $7.1 ; c$, group $7.2 ; d$, group $7.3 ; e$, group 7.4 . 


\subsection{Notched Trimmed Unifaces ( $N=28)$}

A11 specimens have a trimmed indention, usually made by pressure flaking (Crabtree 1972:79). The specimens vary from notched flake-blades to irregular notched pieces. Notched pieces have been thought to be woodworking tools.

7.1 Notched Trimmed Unifaces, Small to Med. Ha fit and Small Bit

( N=15, L $\bar{x}=25 \mathrm{~mm}, W \bar{x}=32 \mathrm{~mm}$, Th $\bar{x}=7 \mathrm{~mm}$, Wt $\bar{x}=41 \mathrm{gm}, \mathrm{Bcl} \bar{x}=29$ )

See Table 13.01 for FN's and provenience. Members of this group are typical of flake-blades and three members are double notched (Figure 13.08b).

7.2 Notched Trimmed Unifaces, Med. Hafit/Small Bit

$(N=6, L \bar{x}=33 \mathrm{~mm}, W \bar{x}=44 \mathrm{~mm}$, Th $\bar{x}=11 \mathrm{~mm}, W t \bar{x}=18 \mathrm{gm}, B c 1 \bar{x}=30)$

See Table 13.01 for FN's and provenience. Members of this group are made on small interior flakes and are single notched specimens (Figure 13.08c).

7.3 Notched Trimmed Unifaces, Med. to Large Hafit/Small Bit

$(N=5, L \bar{x}=40 \mathrm{~mm}, W \bar{x}=57 \mathrm{~mm}$, Th $\bar{x}=23 \mathrm{~mm}$, $W t \bar{x}=35 \mathrm{gm}, B c 7 \bar{x}=34)$

See Table 13.01 for $\mathrm{FN}^{\prime} \mathrm{s}$ and provenience. Members of this group are made on long flakes (Figure 13.08d).

7.4 Notched Trimmed Unifaces, Large Haft/Small Bit

$(N=2, L \bar{x}=53 \mathrm{~mm}, W \bar{x}=57 \mathrm{~mm}$, Th $\bar{x}=17 \mathrm{~mm}$, Wt $\bar{x}=62 \mathrm{gm}, B c 1 \bar{x}=32)$

See Table 13.01 for FN's and provenience. Members of this group are irregular in outline and tend to have larger notches (Figure 13.08e).

Conclusion

The purpose of this study was not to create a whole array of dangling categories, but to develop a tool which might facilitate future uniface research. This erivironmentaliy oriented method is not considered to be sufficient in and of itself. It must be implemented in conjunction with other techniques such as microwear analysis and the new information being discovered on extractions of organic materials from unifaces. Only then may the envirocultural associations begin to be drawn. 


\subsection{AN EXPERIMENT IN STONE BOILING (W. Max Witkind)}

During the survey and excavation of the Hop Hill locality, a large collection of fire-altered limestones, tentatively identified as "boiling stones", was recovered from what have been interpreted as cooking areas. The following report presents condensed data generated in a preliminary experiment endeavoring to produce stones exhibiting colors, textures, surface fissures and fracture angles similar to those boiling stones recovered from Hop $\mathrm{Hill}$.

\section{Experiment Procedures}

The test specimens used in conducting this experiment included dolomite stream cobbles, pieces of tabular dolomite and pieces of tabular limestone collected from four separate locations and divided into Groups A, B, C and $D$. The specimens ranged from six centimeters to twelve centimeters in diameter.

All firing was done in a medium-sized campfire of oakwood. Fire temperature was measured with Orton standard pyrometric cones \#21 and \#15. The temperature of the fire during the testing procedures reached $1140^{\circ} \mathrm{F}$ but did not exceed $1480^{\circ}$. Pre-firing and post-firing weights were measured on a singlebeam balance scale. Color coding was done with a Geological Society of America Rock-Color Chart. Boiling was done in a fifteen-quart, plastic dishpan.

Groups $A, B$ and $C$ were used primarily in test runs to discover obtainable temperatures and to establish control procedures for firing and boiling Group $D$. Data was derived from analys is of Group D specimens.

Group D

Group D included 34 tabular dolomite and tabular limestone specimens. The specimens were divided into two groups, designated as Series I and Series II, and numbered one through 34. Series I included specimens one through ten. Series II was selected and randomly and included specimens 11, 13, 14, 16, 17, $19,26,28,29$ and 30 (see Table 14.01). The remaining specimens were not fired but held for later comparison. The specimens in Series I were fired and boiled twice. The Series II specimens were fired and boiled once. 
TABLE 14.01 BOILING STONE DATA
GROUP D

Rock \# Pre-fire Weight Pre-fire Color Post-fire Weight Post-fire Color

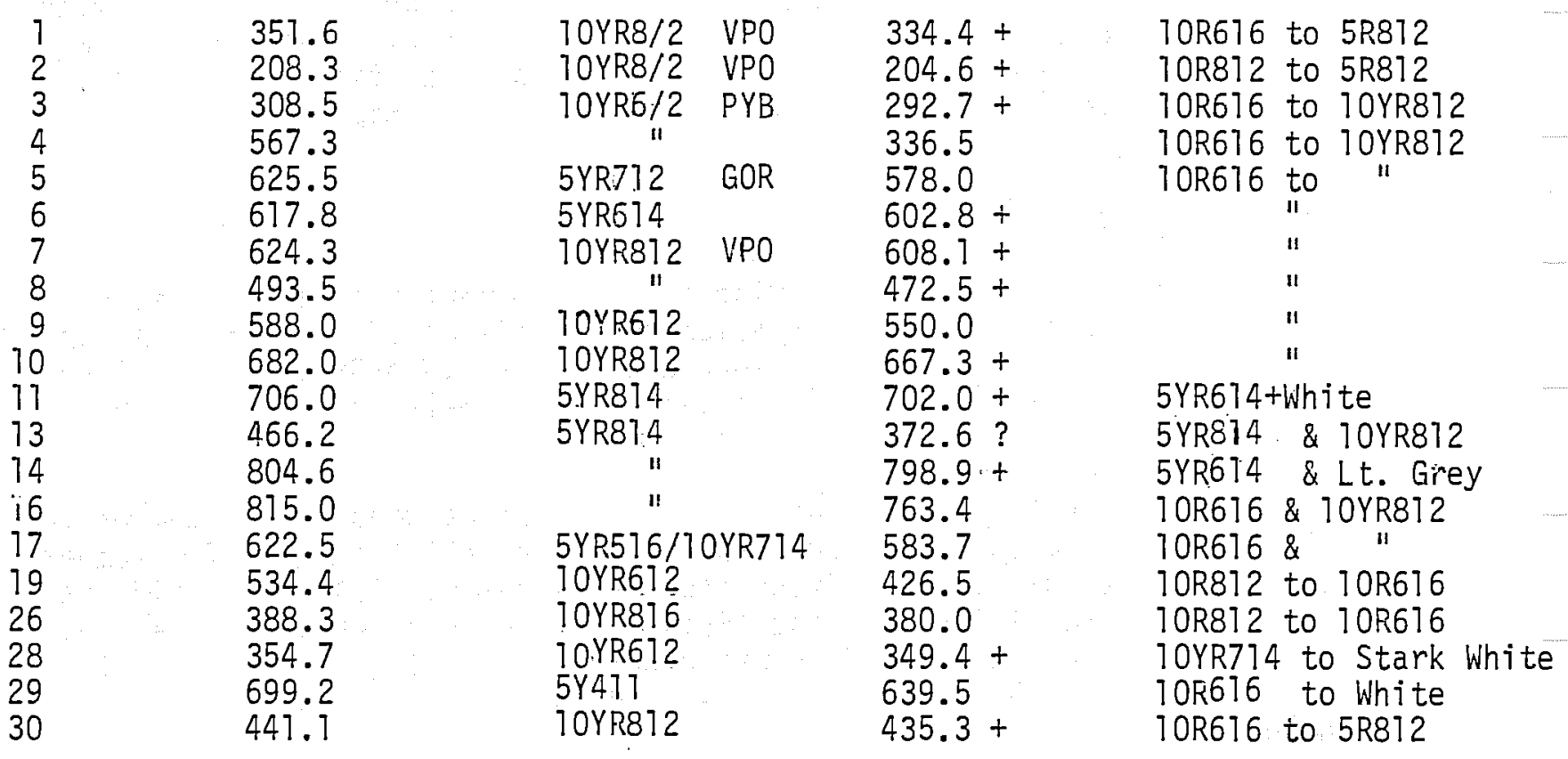

$+=$ Loss due to moisture release.

Before each of the three boiling procedures the specimens were heated for thirty-five minutes. During their first exposure to the water the Series I specimens raised the water's temperature from approximately $75^{\circ} \mathrm{F}$ to $212^{\circ} \mathrm{F}$ within two minutes. The boil was maintained for approximately four and one-half minutes. During their first exposure to the water the Series II specimens raised the water's temperature from approximately $195^{\circ} \mathrm{F}$ to $212^{\circ} \mathrm{F}$ within one minute and maintained a hard boil for approximately four and one-half minutes. During their second exposure to the water the Series I specimens required three minutes to raise the water's temperature from approximately $127^{\circ} \mathrm{F}$ to $215^{\circ} \mathrm{F}$. The boil was maintained for only about two minutes. The temperature had dropped to $170^{\circ} \mathrm{F}$ within 17 minutes.

It is assumed that by rotating freshly-heated stones for cooled stones that a rolling boil could be maintained indefinitely.

Analysis

Examination of Group $D$ specimens revealed a marked loss of weight during firing and boiling procedures. A small amount of weight was lost to fragmentation during firing. A major portion of the weight loss seems to have occurred through moisture release. 
Changes in color were highly discernible. A major portion of the specimens were pre-fire coded as very paie orange, pale yellowish orange and grayish orange. Post-fire coding revealed changes to moderate reddish orange, grayish orange pink or moderate orange pink. Other specimens changed from orange pink toward gradations of light brown mixed with very pale orange. A number became fringed with 1 ight gray or white.

James Pittman (personal communication, San Antonio College, Geology Department) states that the reddish to brown colors which developed during the firing procedures reflect the presence of iron in the limestone and resulted from the oxidizing atmosphere created by the fire.

Examination of the Group $D$ specimens also revealed a rind of material, displaying the various described colors, surrounding a major portion of each specimen. The interior stone toward the core of each specimen appears to have been less affected and altered to a lesser degree than the cortex material.

Each specimen exhibits hairline cracks running over its surface and deep into the stone. Five specimens fragmented along the cracks to produce sharp, angular fracture lines.

\section{Comments}

The experiment produced stones exhibiting similar fracture lines, surface texture and color shades to those often labeled as boiling stones by some Texas archaeologists. It should be noted, however, that limestones possibly used in cooking pits to roast or steam meat or vegetable foods might also exhibit similar characteristics. This conjecture is offered because the cracks and color similarities appear to be a product of fire exposure rather than exposure to boiling water. However, stones heated and then exposed to water may in some instances be characterized by a more friable surface texture than stones exposed only to fire. Future tests should be conducted to determine if a friable surface is the most diagnostic characteristic of a boiling stone.

The postulation that burned rock middens, to include the "boiling stones" so often associated with them, are a by-product of acorn-deer processing activities (Hester 1973, Weir 1976)

Richard Welch (personal communication, San Antonio College, Botany Department) states that lime released into boiling water being used to cook acorns would act to neutralize the distasteful tannic acid in various acorn species in South Central Texas. Limestones used in pits to steam acorns might also have similar results. (Experiments to test the neutralizing capability of lime on tannic acid are underway at UTSA.) Bearing this in mind, the possibility that the South Central Texas Indians used heated 7 imestones to boil or steam acorns seems highly possible. Such activity may account for the relative absence of acorn-leaching pits in South Texas such as those described in ethnographic accounts of acorn-gathering Indians of California. Also, leaching away the outer surface of 7 imestone cobbles explains the fossiliferous appearance of boiling stones, assuming the fossils are more resistant to acid. 
Visual examination of the rind exhibited by the Group $D$ specimens led to speculation that the boiling activity removed enough lime from the outer surface to render the stone unusable a third or fourth time in cooking acorns. However, casual chemical tests failed to show an appreciable difference between the lime content in the rind of stones boiled twice and the lime present in the surface material of unboiled stones.

The sharp drop in the water's temperature during the second boiling procedure of the Series I specimens suggests that stones used more than once lose heat more rapidly than stones being used for the first time.

The proclivity to fragment after several heating procedures may quite possibly have limited the re-use of stones as either boiling stones or cooking pit stones. This, coupled with the tendency of a reheated stone to lose heat more rapidly than a previously unheated stone, may have also encouraged the use of a stone only several times before it was discarded on a nearby midden. Thousands of stones, each used only a few times by people repeatedly visiting the same location for extended periods of time, could account for the numerous, and sometimes massive, burned rock middens common in South Central Texas. 
15.0 CLUSTERING OF TECHNOLOGICAL TYPES (Nontinear Technospatial Analys is, Resolv 4) (Joel Gunn and Douglas R. White)

In primitive societies the one reliable feature of technological structure is division of labor by sex. In archaeological remains, therefore, it should be the generating force behind a large proportion of the observable variation in archaeological assemblages. Several authors have used division of labor as an interpretive device (Binford and Binford 1966, Katz and Katz 1976:120-121, etc.). In this section we review a cross-cultural analysis of division of labor as it pertains to the problem of interpreting the prehistory of Central Texas. The discussion is set in the context of entailment theory and is therefore methodologically consistent with the nonlinear spatial analysis to follow.

The Division of Labor code (Murdock and Provost 1973) is composed of a crosscultural sample of 185 societies stratified by continents and randomly selected (Murdock and White 1969) from cultures in the Ethnographic Atlas (Murdock 1967). These 185 societies were coded for division of labor by sex on 50 technological traits. The cultural complexity of the 185 societies ranges from hunters and gatherers to non-industrial civilizations such as the Romans. On a scale of cultural complexity which ranges from 0 to 40 (Murdock and Provost 1973.), zero being the least complex, the societies are distributed as follows:

$\begin{array}{lcc}0-20 \text { (Hunters and Gatherers) } & \frac{\text { Number }}{122} & \frac{\text { Percent }}{66} \\ 21-33 \text { (Agricul tural ists) } & 38 & 21 \\ 34-40 \text { (Civilizations) } & 25 & 13 \\ \text { Total } & 185 & 100\end{array}$

As is apparent, a substantial portion of the standard cross-cultural sample is comparable to the level of cultural complexity we have presumed for all of prehistory in the Central Texas Archaic (see introduction; also Weir 1976). In order to analyze the entailment structure of the division of labor the data was recorded to $1=$ female activity and $0=$ male activity.

Entailment will be explained in greater detail later. For now let us say that it is an analytical process which determines the relationships between traits from a logical analysis of their presence or absence. If trait $X$ is present, then is trait $Y$ also always present? The technique was developed by White (White et al. 1975) for the analys is of cross-cultural data.

As a focus for discussion white et al. (1975: 4-7) define a formal theory of division of labor. Three assumptions are made at the outset which are derived exclusively from the fact that infants must be nursed in pre-industrial societies where no artificial feeding devices are used. It follows, then, that 1) women as a group will assume primary responsibility for offspring, 2) women (and their offspring) will not be routinely exposed to avoidable danger, 3) women will not travel long distances from home bases.

These assumptions have direct and constraining influences on division of labor. Productive sequences are composed of tasks which can be generalized under 
"collecting" and "processing" operations. Tasks for collectina raw materials are to varying degrees inherentiy dangerous (for example, hunting), while processing activities are not. Typically, then, males collect raw materials and females process them into finished products. Similarly, collecting often requires traveling over long distances while processing is done at the home base.

Since collecting tasks often require that males confront the environment with tough and heavy tools relative to the softer and lighter materials of processing, there is a correlative association of these attributes with the respective sexes.

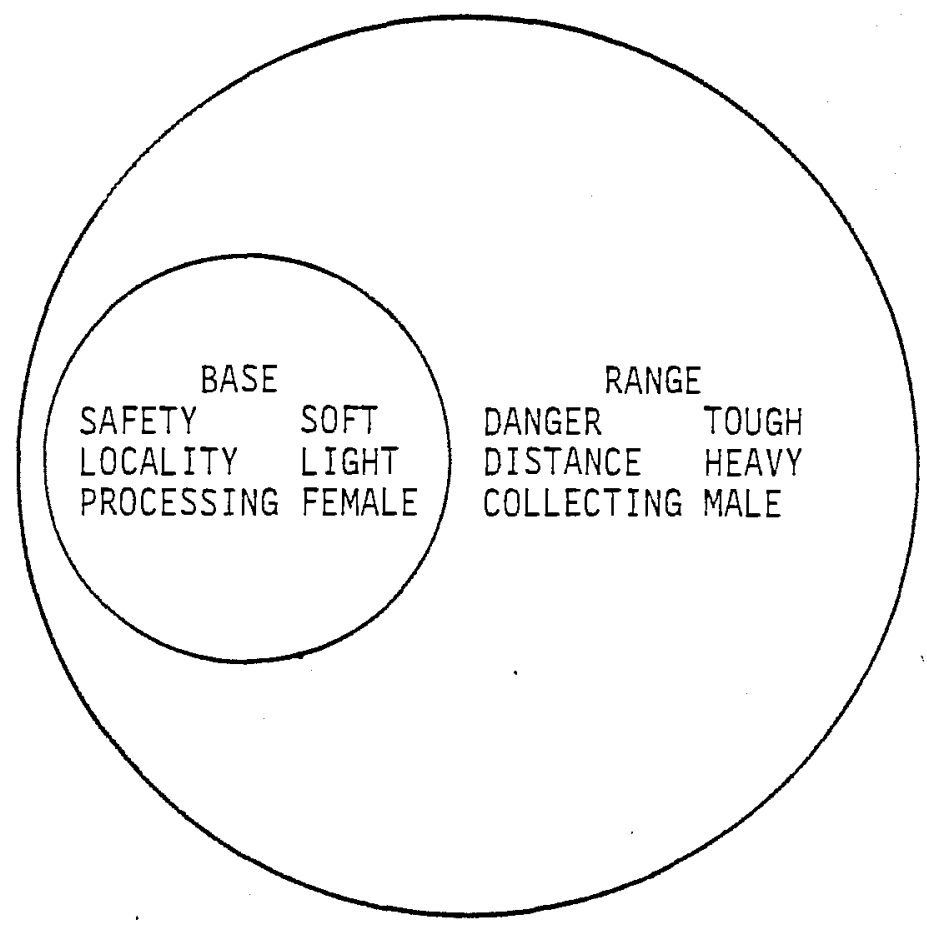

Figure 15.01. Base and Range Concept of Site Locality.

As a consequence of these assumptions about child care and productive sequences the habitat of primitive people should be divided into a "Base" and a "Range" as illustrated in Figure 15.01 with the inferred properties as they are assigned. With the site as the center of activities, the Base area is of an order of maynitude of about one mile radius. The Range is of the order of ten miles.

Entailment analysis of the division of labor code produced an entailment digraph (White et al. 1975:12) which contained five activity sets, two of which pertain to the hunter-and-gatherer mode of subsistence. Entailed activities form task sequences: up to five steps long and are generally of the form "if a civen sex does task $X$, and task $Y$ is present, then that sex also does task $Y$ " (White et al. 1975:13). 
Cluster I of the analysis (Figure 15.02) is a collection of tasks that can be performed in the Base area or adjacent areas.

$10 \%$ $80 \%$

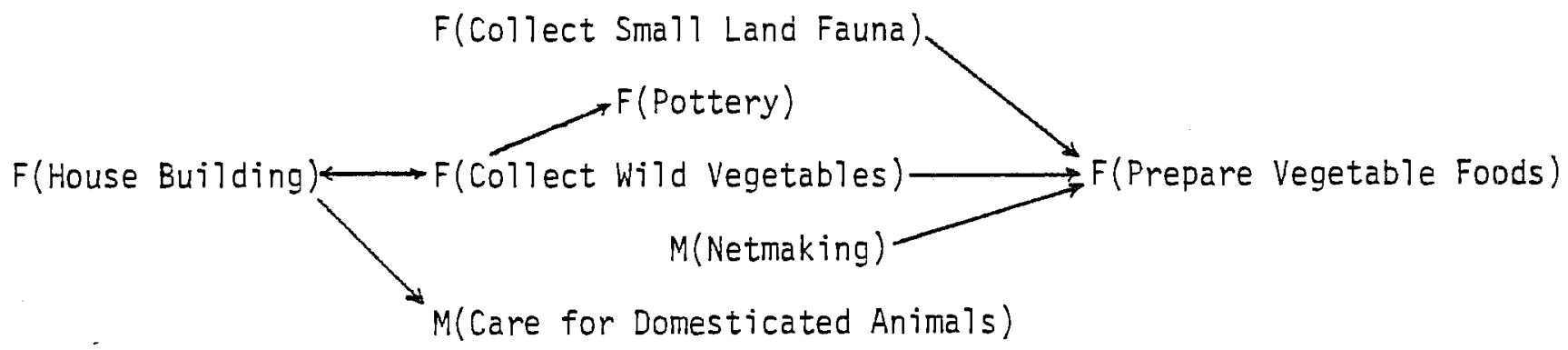

Figure 15.02. Food Collecting and Related Tasks. ( $F=$ female, $M=m a 7 e)$

In societies characterized by this pattern of tasks houses are temporary and sometimes portable and made of soft, pliable materials such as vegetation or skins. Thus, women collect vegetation for house building and food which is consumed in association with small land fauna which they also collected. When women tend to this task sequence men care for domestic animals. Women make pottery to facilitate food preparation while men make nets for hunting and fishing.

Cluster II is much more complex, consisting of 13 traits (Figure 15.03). The entailments imply task sequences related to processing of meat, skins and fibers, their use and maintenance.

$5 \%$ $-45 \%$ $-85 \%$

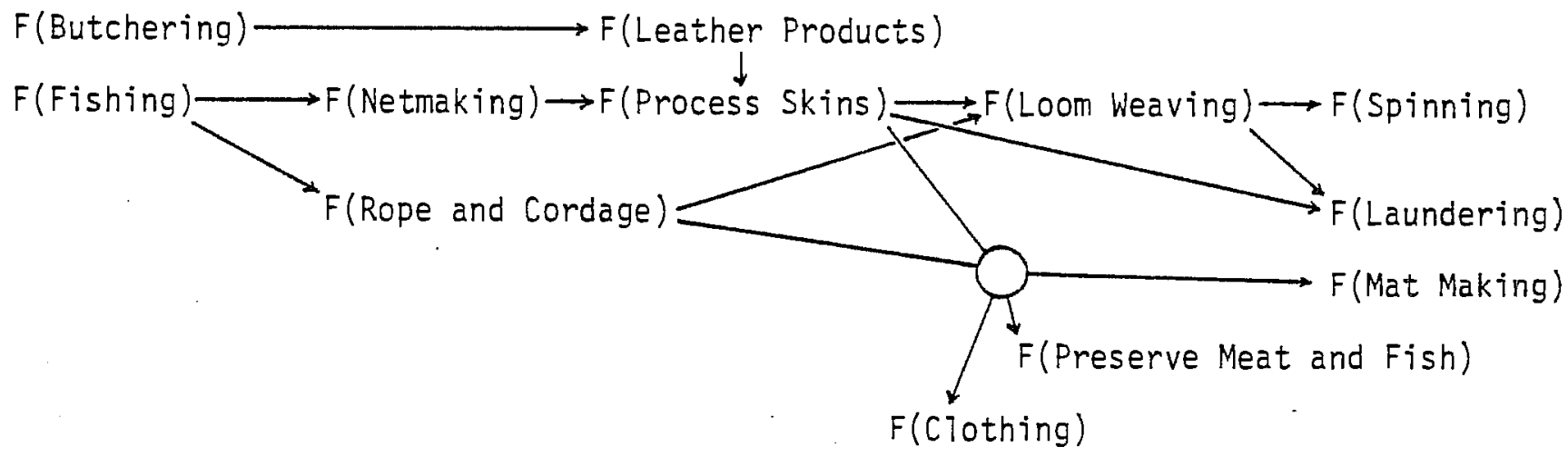

Figure 15.03. Meat, Skin and Fiber Processing ( $F=$ female). 
Table 15.01 Rotated Principal Components Matrix for Loci Matrix

I. II. III. IV. V. VI. VIII.

\begin{tabular}{|c|c|c|c|c|c|c|c|c|}
\hline $\begin{array}{l}1 . \\
2 .\end{array}$ & $\begin{array}{l}\text { Pointed Bifaces }(1.1,1.2) \\
\text { End Bifaces, Large Choppers } \\
\text { (2. } 1-2.3)\end{array}$ & $\begin{array}{r}.34 \\
-.10\end{array}$ & $\begin{array}{r}.33 \\
-.23\end{array}$ & $\begin{array}{r}-.22 \\
.72 \\
\end{array}$ &.$\frac{39}{.00}$ & $\begin{array}{r}.24 \\
-.07\end{array}$ & $\begin{array}{r}.21 \\
-.12\end{array}$ & $\begin{array}{l}-.26 \\
-.28\end{array}$ \\
\hline $\begin{array}{l}3 . \\
4 . \\
5 . \\
6 . \\
7 . \\
8 .\end{array}$ & $\begin{array}{l}\text { End Bifaces, Celt (2.4) } \\
\text { Side Bifaces (3.0) } \\
\text { Rectangular Bifaces }(4.0) \\
\text { Round Bifaces, T m }(5.7) \\
\text { Round Biface, Thick }(5.2) \\
\text { Oval Biface, Thick } \\
(6.7-6.4)\end{array}$ & $\begin{array}{r}.07 \\
.02 \\
.25 \\
.18 \\
.03 \\
-.14\end{array}$ & $\begin{array}{r}-.10 \\
.00 \\
.02 \\
.78 \\
-.05 \\
.80\end{array}$ & $\begin{array}{r}.00 \\
-.21 \\
.82 \\
-.02 \\
-.09 \\
-.09\end{array}$ & $\begin{array}{r}-.18 \\
-.70 \\
-.02 \\
-.02 \\
.07 \\
-.04\end{array}$ & $\begin{array}{l}-.75 \\
-.23 \\
-.12 \\
-.07 \\
-.08 \\
-.73\end{array}$ & $\begin{array}{r}-.07 \\
.14 \\
-.05 \\
-.25 \\
-.14 \\
.01\end{array}$ & $\begin{array}{r}.09 \\
-.12 \\
.12 \\
.05 \\
.85 \\
-.04\end{array}$ \\
\hline 9. & $\begin{array}{l}(6.1-6.4) \\
\text { Ovoid Biface, Smal1, } \\
\text { Thinned }(7.1)\end{array}$ & -.27 & .41 & .47 & -.00 & .05 & .15 & .48 \\
\hline 10. & $\begin{array}{l}\text { Ovoid Bifaces, Large, } \\
\text { Thick }(7.2)\end{array}$ & .02 & -.01 & -.19 &.$\underline{58}$ & -.28 & .32 & -.19 \\
\hline 11. & $\begin{array}{l}\text { Triangular Bifaces, } \\
\text { Smal1 }(8.0)\end{array}$ & -.73 & -.00 & -.04 & .14 & .70 & -.04 & -.15 \\
\hline $\begin{array}{l}12 . \\
13 . \\
14 . \\
15 . \\
16 . \\
17 . \\
18 . \\
19 .\end{array}$ & $\begin{array}{l}\text { Boiling Stones } \\
\text { Uniface-End Scraper } \\
\text { Uniface-Side Scraper } \\
\text { Uniface-End-Side Scraper } \\
\text { Uniface-Ovate Scraper } \\
\text { Uniface-Circular Scraper } \\
\text { Uniface-Thumbnail Scraper } \\
\text { Uniface-Edge Altered } \\
\text { Flake }\end{array}$ & $\begin{array}{l}. \frac{84}{.70} \\
. \frac{.55}{.26} \\
.28 \\
.07 \\
.75 \\
.70 \\
\end{array}$ & $\begin{array}{r}-.07 \\
.73 \\
.21 \\
-.31 \\
-.02 \\
-.08 \\
-.11 \\
-.32\end{array}$ & $\begin{array}{r}-.00 \\
.07 \\
-.00 \\
-.29 \\
-.26 \\
.08 \\
-.11 \\
.06\end{array}$ & $\begin{array}{r}.12 \\
.07 \\
.44 \\
. .59 \\
-.06 \\
.08 \\
.49 \\
.07\end{array}$ & $\begin{array}{r}-.01 \\
-.23 \\
.14 \\
-.14 \\
-.06 \\
-.05 \\
-.05 \\
.03\end{array}$ & $\begin{array}{r}.19 \\
-.09 \\
.06 \\
.27 \\
.74 \\
. \frac{.65}{.65} \\
.34\end{array}$ & $\begin{array}{r}.02 \\
-.19 \\
.04 \\
.15 \\
-.02 \\
-.02 \\
-.18 \\
.17\end{array}$ \\
\hline \multirow[t]{2}{*}{20.} & $\begin{array}{l}\text { Uniface-Notches } \\
\text { Variance Accounted for }\end{array}$ & .29 & $\begin{array}{r}-.00 \\
75 \% \\
\end{array}$ & $\begin{array}{c}.09 \\
9 \% \\
\end{array}$ &.$\frac{58}{17 \%}$ &.$- \frac{42}{9} \%$ & $\begin{array}{l}.15 \\
10 \%\end{array}$ & $\begin{array}{l}.10 \\
7 \%\end{array}$ \\
\hline & & $\begin{array}{l}\text { Unifaces } \\
\& \\
\text { Boiling }\end{array}$ & \multicolumn{2}{|r|}{ Biface } & $\begin{array}{l}\text { es } \\
\text { Uni } \\
\& \\
\text { Bif }\end{array}$ & $\begin{array}{l}\text { Bit } \\
\text { S }\end{array}$ & \multicolumn{2}{|c|}{${ }^{S}$ Unifaces } \\
\hline
\end{tabular}


Table 15.02 Hypothesized Tool Kits from Hop Hill

\section{BIFACES}

II. Round Bifaces, thin *Oval Biface, thick Ovoid Bifaces, smal?

VIII. *Round Bifaces, thick Ovoid Bifaces, smal1
III. *End bifaces, chopper Rectangular Bifaces Ovoid Bifaces, small

V. End Biface, Celt * Triangular Biface, small, thinned

-Notches

UNIFACES

I. *Boiling Stones

End Scraper

Side Scraper

Edge-Altered Flake
VI. Ovate Scraper

Circular Scraper

*Thumbnail Scraper

\section{BIFACES AND UNIFACES}

IV. Pointed Bifaces

Side Bifaces

Ovoid Bifaces, large, thick

Side Scraper

End-Side Scraper

Thumbnail Scraper

*Notches

* indicates pivotal tool 
The digraphs illustrated here represent half the analysis. The range of percentage points across the top of each digraph shows to what degree females manage the various tasks represented below them. Thus, in very few cultures do women butcher (5\%), but where they do butcher they manage all tasks subsequent to butchering in a task sequence, i.e., processing of skins, making leather items, preserving meat. The entailments clearly show that once females enter the task sequence they do not return control to males at any succeeding task. The other half of the analysis, then, is simply a story of males dropping out of the task sequence at varying junctures. If females butcher in $5 \%$ of the cultures, then males do so in $95 \%$. It is 7 argely a male task. When it comes time to produce leather products, on the other hand, the male component has dropped to $55 \%$ and females have taken over the sequence in $45 \%$ of the cultures. Finally, preserving meat is an almost exclusively female endeavor.

In terms of the Base-Range continuum this analysis is Base-oriented. A Range-oriented analysis need only reverse the process. Tasks which do not appear because of the base orientation are those tasks which are exclusively male, tasks which are carried out in the Range or are related to hard materials such as hunting, trapping, fowling, collecting honey, working in stone, wood, horn, bone and sher1, mining, quarrying, smelting of ore, metal working, etc.

Final1y, firemaking ( $F=28 \%)$, gathering shel1 fish $(F=69 \%)$ and fuel gathering $(F=71 \%)$ form no implications because they are not a part of any production sequence and therefore are not systematically related to collecting and processing variables.

Although we cannot observe the range of tasks practiced by the prehistoric inhabitants of Central Texas directly, we can reasonably assume that their daily activities formed a subset of the task sequences observed in a worldwide sample of human behavior at a comparable level of cultural complexity. While we presume to draw no direct analogies between these patterns and prehistoric populations we do take the patterns to be a rich source of hypotheses which can be tested by various means (Binford 1967).

In fact, we have very 1 ittle to build from by way of developing hypotheses of tool kit structure. Few ethnohistorical observations were collected on the pre-Apachean and Comanchean peoples of Central Texas. Virtually none were made on use of stone tools. We will, therefore, assume a very simple model of stone tool usage based on manufacturing technology. Scrapers are known for a number of reasons to be associated with (at least) skin preparation. We will generalize this and say that unifacial knapping techniques were a female tool technology. This is taken to mean that women generaily use unifaces whether they make and maintain them or not. Core and bifacing technologies, on the other hand, are presumed to be of male manufacture and use. Hunting implements, such as points for instance, are often bifaciat.

It should be stated at the outset that we know the hypothes is is true only in part. Unifaces are effective woodworking tools; Hester, Gilbow and Albee (1973) have demonstrated that Clear Fork tools were almost assuredly wood or bone working 
implements. Furthermore, there are time trends in the Central Texas Archaic (800-8000BP) which show that unifaces gradually are replaced through time by bifaces (Gunn and Weir 1976). The only argument we can make nere (and on tenous grounds) is that the site appears to have been occupied predominately in the Middle and Late Archaic time intervals, during which bifacing was the predominant stone tool manufacturing technique (Gunn and Weir 1976).

With the tenuousness of the uniface-biface pattern in mind we can only assume that it was the predominant trend in the tool kit structure and test the data for that pattern. When breaches of the pattern occur we will then attempt to determine their meaning on an ad hoc basis.

Additionalyy, one other tool class is large enough in sample size to affect the analysis and may be of help in factoring out various interpretations. Boiling stones are common on the site. As the cross-cultural data show cooking food is a virtual female preserve in societies of low complexity. Anything strongly associated with boiling stones should be female related.

Nonlinear Distribution Analysis Loci Matrix

Nonlinear distribution analys is as it is devised here is a strictly type oriented approach to the distribution, and association, of artifacts over a habitation space. Each artifact in that space is a type at a locus to which others types are potentially associated. A "loci matrix" is composed of a series of row vectors each of which represents a locus (artifact) in the habitation space (Base and Range) of a site. The columns in the matrix represent types of artifacts. This matrix is illustrated in Figure 15.04 where $T=$ type and $L=$ locus. There are $n$

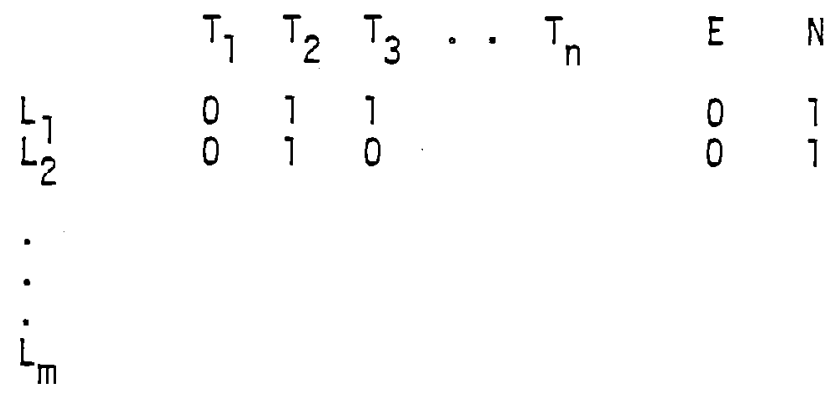

Figure 15.04. Loci Matrix for Nonlinear Distribution Analysis.

Types and $m$ Toci. E and $N$ are binary representations of east and north respectively. Two of the rows are filled in with $7^{\prime} s$ and $O^{\prime} s$ to $i l$ lustrate the content of the matrix. A "l" means that type occurs within a specified distance from the locus, let us say 5 meters for this example. A o (zero) means it does not. Suppose that the locus is a $T_{2}$ artifact. The locus itself is assigned a 1 in $L_{1}$ by $T_{2}$. The zero in $L_{1}$ by $T_{1}$ shows that there are no $T_{1}$ artifacts within 5 meters of Locus 1 . A 1 in $L_{1}$ by $T_{3}$, however, shows that at least one $T_{3}$ occurs within 5 meters. 
The following discussion is based on such a matrix constructed for 20 types and 347 loci. The artifacts were virtually all surface-collected from the Hop Hill locality in Central Texas. Since they represent deflated occupation floors the artifacts from different times are mixed together. We will assume, however, that the mixing process will not produce patterned association between artifact types resulting in artificial tool kits. It is important only that artifacts not be moved away from each other to preserve the integrity of the tool kits.

The program which determines the contents of the Loci Matrix does so by sweeping around each artifact within the limits of a criterion radius and determining the types of artifacts within that radius. For each artifact within the radius a 1 is entered in the appropriate type column. Before proceeding further an understanding of the importance of the criterion distance needs to be made explicit. The radius is set according to the level of social organization one wishes to test for patterned relations between types. llorking up the hierarchy, the individual, the nuclear family and the band each requires a minimum amount of space in which to operate comfortably. Although these opinions can be altered according to various researchers we have set the criterion radii as follows:

$\begin{array}{lr}\text { individual } & 1 \text { meter } \\ \text { family } & 5 \text { meters } \\ \text { band } & 20 \text { meters }\end{array}$

It would probably do more harm to set the criterion radii too low than too high. "Too high" will just pick up more random associations while "too low" will eliminate part of the pattern.

Once the nonlinear relations between types are represented in a loci matrix they can be analyzed by any standard technique. As we have seen, entailment is peculiarly well adapted for showing operational sequences. Ultimately, then, we will rely on entailment. For the sake of comparison, however, a brief discussion of a principal components analysis of the data follows.

The loci matrix analyzed below was constructed on the basis of 347 artifacts divided into 20 types. The 5 -meter radius criterion was used so the discussion involves the association of tools at the family level of social organization.

Principal components analysis extracted seven eigenvalues greater than 1.0 which accounted for $66 \%$ of the variance in the Loci Matrix. These seven components were rotated to varimax criteria. Table 15.01 shows the rotated components matrix.

Of the subsets of tools in the matrix components II, III, V, and VIII are bifacial. I and VI are unifacial and IV contains elements of both technologies. The combinations are displayed in Table 15.02 . 
Some suggestions as to the import of these clusters of tools can be drawn by using the "pivotal tool" concept (Gunn and Weir 1976). A pivotal tool is the tool in a numerically inferred tool kit which is best understood functionally. Pivotal tools are asterisked in Table 15.02 .

The biface tool kits are crosscut by sma11, ovoid bifaces with one exception. These are probably preforms for projectile points. Because of errors in the knapping procedure most are detectably incomplete and were discarded. They could have been used as knives. Pivotal tools other than ovoid bifaces have been marked because the invariant distribution of the smal1 ovoids detracts from their power as an indicator of functional variability. The pivotal elements in Tool Kits II and VIII suggest hifacial cores, so these tool combinations probably occur in 1 ithic workshop areas. Tool kit III, on the other hand, appears to be a functional association as the presence of the small end biface choppers suggests. Elsewhere it has been suggested that these are food-processing tools (Gunn and Weir 1976). However, their association here with two kinds of bifaces which require highly sophisticated knapping techniques probably implies male activities and calls that hypothesis into question.

Tool Kit $V$ is composed of two bifacial types. The negative loading for notches means they are never present with celts and smal1, triangular bifaces. Apparently small ovoid bifaces became associated with celts once they were successfully converted into small, thinned, triangular bifaces. The ubiquitous presence of small thinned bifaces of varying types in bifacial tool kits deserves future consideration.

There are two purely uniface tool kits: I and VI. The association of boiling stones with I implies a food preparation orientation. Edward Droste of San Antonio has studied the native uses of cactus and believes peoples of the Southwest would have used flakes and scrapers to remove spines from cactus during preparation for use as food. Whether the relationship is as direct as that, or indirect as in the case where food is prepared in a normal1y common space with hide preparation, the tool kit suggests a clearly female orientation.

Tool Kit VI is composed of morphologically well defined scraper types. It might be a female tool kit. Alternatively, it could be a woodworking tool kit made and used by males. Thumbnail scrapers are probably the remnants of hafted scrapers which have been resharpened nearly to extinction. Males are usually given to such elaborations ethnographically while females are not.

Tool Kits IV and $V$ are composed of both unifaces and bifaces. Tool Kit IV is a combination of source materials, heavy implements (pointed bifaces), scrapers and notches, Notches of the larger size implied here could be useful for preparing round objects such as shafts, bone points, etc. It is probably a tool preparation and maintenance kit of male usage.

The relationships between artifacts as tool kits and their consequent inferred functional and sexual associations can only be taken as hypotheses which suggest direction for future research. In the following analysis of the same 
data, however. Entailment Analysis which calculates statistical tests of these relationships will be used.

In addition to statistical inference, Entailment Analysis allows the researcher a detailed and complex view of relationships in the data. Entailment is at $i$ ts base the analysis of a $2 \times 2$ contingency table. Figure 15.05 shows such a contingency table for variables $X$ and $Y$. Within each cell is the logical implication of finding the relationship between $X$ and $Y$ in that position.

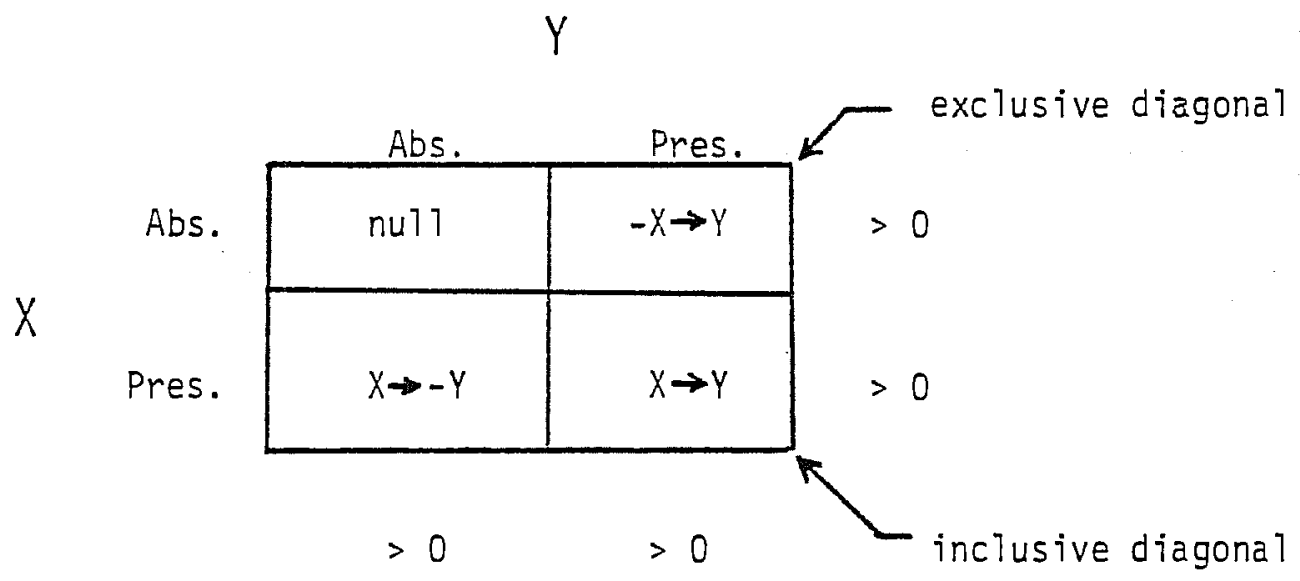

Figure 15.05. Fundamental Opcrators of Entailment Analusis.

In the lower right-hand cel1 ( $X$ and $Y$ present) there is the implication "if $X$, then $Y$ is found." This is equivalent to a Guttman scale which is also designed to locate positive implications. In addition, Entailment Analysis determines whether the absence of a trait implies the other variable. This feature is more important where both states of the binary variables are substantively

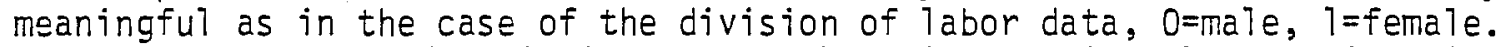
For pure pres.-abs. data it is somewhat less interesting although in this study presence implying absence could be used as an indicator of assemblage purity.

The celis in the contingency table are grouped according to their general properties. Cells diagonally across from each other share properties. The cells on the principal diagonal (upper left and lower right) are the "inclusive" cells. Everything that includes an $X$ includes a $Y$, or if there is no $X$ there 


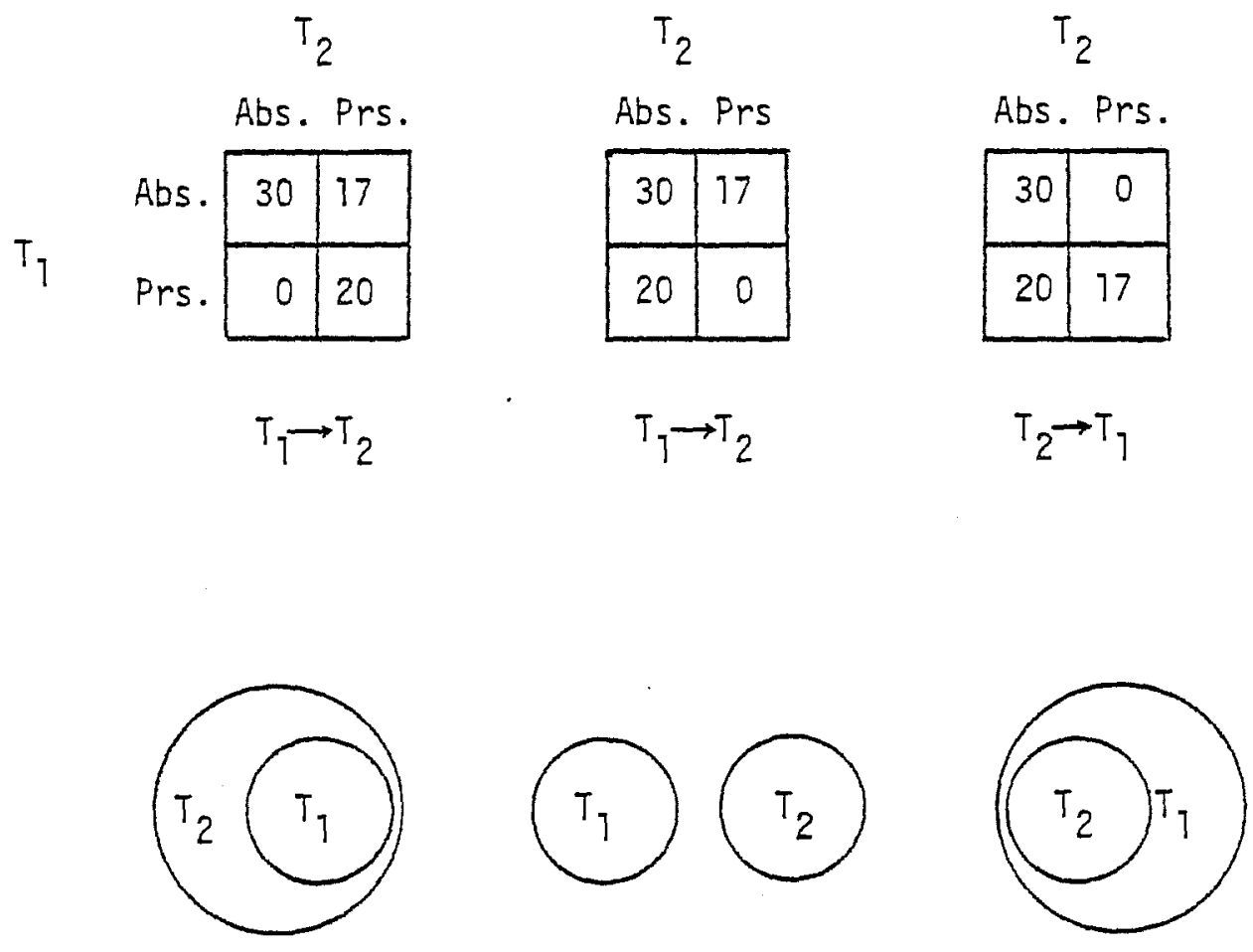

Figure 15.06. $T_{1}$ and $T_{2}$ in Three Constructed Contingency Tables. $a-c$, note différent locations of zero cells. 
is no $Y$. In contrast, the cells on the secondary diaganal (lower left and upper right) are the "exclusive" cells. If there is an $X$, then the $Y$ is excluded and vice versa.

Entailments are determined by searching for zero cells. The entailment is on the diagonal opposite the zero cell. Figure 15.06 a shows a case where $T \rightarrow T$ ( $T_{1}$ entails $T_{2}$ ), or, wherever a type 1 artifact is found a type 2 is also found. Figure 15.06b shows a zero on the inclusive diagonal implying the mutually exclusive or disjointed character of the distribution, $T_{1} T_{2}$ ( $T_{1}$ entails not $T_{2}$ ).

A zero in the null cell produces overlapping sets (not illustrated) and no solution or entailment. None of the marginals can be zero; only invariant variables would produce this condition. The probability characteristics of a $2 \times 2$ table are readily computed by Fischer's Exact Test.

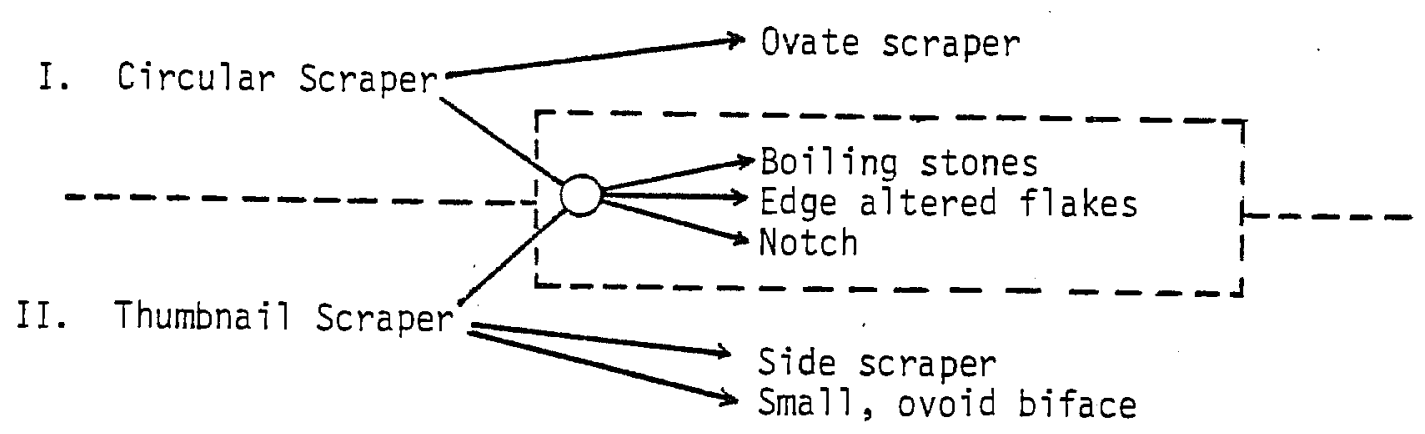

Ficure 15.07. Entailogram for Hop Hill Loci Matrix.

The entailment in Figure 15.07 shows the positive, significant relationships in the loci matrix. Sequence I reads, "if there are circular scrapers at a locus, then there are also ovate scrapers, edge-altered flakes, notches and boiling stones." Relative to the principle component analysis, the entailogram shows that Components I and VI are statisticaliy significant.

Series II shows that where there are thumbnail scrapers, there are also small, ovoid bifaces, boiling stones, edge-altered flakes and notches (mostiy component IV).

Arguing from the fresence of thumbnail scrapers and small ovoid bifaces in Series II, it seems likely this is a male task kit. This leaves Series I as a possible female tool kit. It seems likely that female tasks are represented because of the boiling stones but we know of no specific ethnohistorical incident or theoretical proposition that would link circular and ovate scrapers to women other than the generally demonstrated fact that women work skins and use some kind of scraper. 
The cluster of tools in the center is shared by both series, either by functional association or by normal but unrelated proximity of function. Edgealtered flakes, in particular, are expectably generalized tools that could be used for any number of tasks, whether they be male or female. 



\subsection{ANALYSIS OF OCCUPATION FLOORS (Joel Gunn)}

Burned rock middens are a standard and stubborn point of archaeological curiosity in Central Texas. Elton Prewitt (1974) has traced the development of midden studies from Pearce (1979) through later investigations such as Kelly and Campbel1 (1942), Suhm (1959), and Hester (1971), to mention a few. The function of the middens is so obscure it seems to be an instant topic of conversation on any occasion, and speculation as to function runs from steam baths to cactus-frying stations. Weir (1976) favors an acornand deer-processing station interpretation. This argument is supported by the fact that the heaviest concentration of burned rock middens is during a cool and presumably wet period in Texas prehistory (Gunn and Weir 1976). If oak woodlands expand westward during such times the acorn/deer-processing function is certainly indicated, as these were the traditional staples of the eastern woodlands. Probably people so adapted expanded their doma in westward during favorable climatic episodes.

One question which this hypothesis suggests is, "What is the archaeological feature east of the Edwards Plateau of Central Texas which is the functional equivalent of the burned rock midden?" Such processing areas are marked in Central Texas by the presence of burned limestone which is abundant in the region. Burned clay balls such as those found at Poverty Point may be equivalent to boiling stones. It may be that the peculiar resources of the Edwards Plateau brought forth a regional variation of an eastern adaptation which due to available resources of boiling objects and relatively dry climate preserved a unique record of those activities.

What then of the analysis of these sites has tended to show their actual function? Bouche de Perthe noted the camp-like structure of the Somme eoliths as early as the 1840s and reported on that fact before the Royal Society of England (Daniels 1967). If the mystery of burned rock middens is to be resolved it seems likely that it will be the product of observations similar to those of that most ancient of French archaeologists, i.e., details on the internal camp structure of middens.

Hypotheses developed to explain functions of middens have ranged through virtually the extent of prehistoric human activity. Excavation techniques on the other hand, have been limited to relatively thick arbitrary levels which represent a level of resolution comparable to and capable of testing only those hypotheses which suppose an unstructured utility such as refuse heaps (Hester 1971), or broad chronological occupation periods (Suhm 1959:23). As was described in Section 9.0, the Hop Hill midden was excavated by a modified microstratigraphic technique, which, it was hoped, would lend support to either a structured or unstructured hypothesis and, if it be structured, to delineate the functional character of that structure. 
Fortunately for comparative purposes, the upper two occupation floors in the Hop Hill midden appear to be camps that were coincidentally located on a midden. This is a relatively common occurrence (Suhm 1959:218). On1y the third and fourth floors are in the midden proper. In the following discussion, this coincidence will be capitalized upon as a device for studying changing camp patterns in Archaic Central Texas.

The nomadic nature of prehistoric people of Central Texas (see Section 1.0) indicates that they would have preferred portable containers such as baskets, and stone boiling in waterproof baskets would have been a common mode of food preparation. In fact, such activities are known ethnohistorically in Texas (Turner n.d.). It is reasonable to assume, therefore, that some part of the accumulation of middens can be accounted for by such activities and that the analys is of occupation floors in middens should show evidence of stone boiling. Given the usual jumble of debris in middens, however, some sort of analytical device is necessary to determine if such activities did transpire.

The elements that comprise stone boiling are fire, water, some rocks of appropriate size and texture and a basket or skin bag (see Section 14.0). The process is to heat the rocks, put them into the basket with the water and food to be cooked and when the food has been removed, discard the rocks. As far as identifying the process from the archaeological record is concerned, the fire and the discarded rocks will be the on $7 y$ evidence left in a wet site.
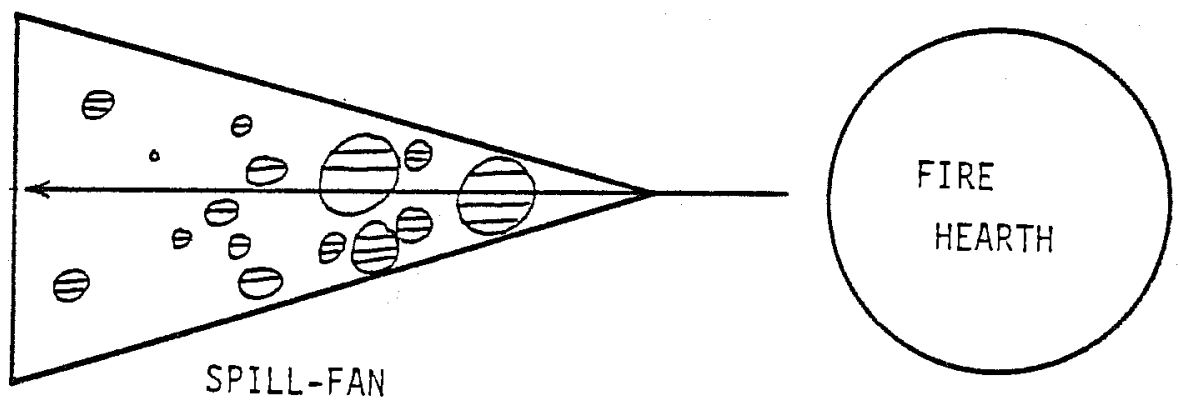

Figure 16.01. Spill-Fan Analytical Concept.

Some aspects of these two features may be helpful in identifying them. When rocks of varying sizes are discarded from a container, the sma 11 rocks should roll further than the larger rocks and rocks should splay out from the throwing point in a fan-shaped pattern. A typical "spilifan," then, should be triangular in shape with the larger rocks at one of the points of the triangle. Furthermore, the point of the triangle with the large rocks should point generally in the direction of the fireplace (Fig. 16.01) in which the rocks were heated since it would have been the point of origin of the person discarding the debris. Using these assumptions we can reduce the spill-fan and fire hearths to a number of activity units around which other tasks were organized. 
In the following discussion an attempt is made to use this device with the understanding that it is only an idea which may or may not be correct in a given instance. It should, however, account for some of the behavior which leads to the accumulation of a burned rock midden, and it provides a vector of orientation for those activity units which may be of use in analyzing complementary activity phenomena such as the chipping of stone tools.

Four definable occupation floors were sampled extensively enough to warrant analysis. Whether they were opened over a large enough area to represent a significant portion of living space is difficult to ascertain. Any conclusions drawn concerning camp habits will be distorted to the extent that a full camp is not represented. Perhaps it would be safest to say that the following discussion is predicated on less than full understanding. If a camp is a system of interactions between people and the camp environment, then, we are studying the camps at the subsystem level. The subsystems are cut short at the edge of the excavation and there is no way of knowing whether we have the whole system or not short of total excavation of the substratum. This abbreviation can only result in a bias of our view of occupation floor activities. How biased that view is depends on the amount of redundancy in the camp patterns per unit of area. If redundancy is frequent, the bias is reduced directly.

The floors are mapped in Figures $16.02,16.05,16.07$ and 16.08 . The rocks mapped on the living floors represent only those that could be picked up off the surface once a thin substratum was excavated to the bottom of the rocks nearest the surface. Perhaps the most starting result of picking up the midden in microlayers is that the normally cluttered appearance of a midden becomes a somewhat ordered and even relatively thin scatter of rocks. Furthermore, there are spaces which are relatively void of limestone debris; and though we make no claims for the patterns, the rocks seem to cluster or form outlines of ovals and circles. As often as not, concentrations of chert are associated with areas where rocks are less concentrated. All of this suggests a reasonable pattern of camp debris.

Midden Occupation 2.10 (Resolv 2.07--Fig. 16.02)

This occupation is the one closest to the surface. There were surface artifacts, redeposited materials and perhaps a few floor fragments above it. OF 2.10 was the first substratum that could be excavated with some confidence. It was opened in $15 \mathrm{~m}^{2}$. The bottom is well defined by a brown substratum but the top was eroded to an unknown degree. In the south margin a rill cut through part of the floor, as indicated in Fig. 16.02. The rill cut completely through $7 \%$ of the floor. If we assume a Resolv 2 floor, the resolution is 2.07 plus an unknown quantity of erosive activity to the upper surface of the substratum. 


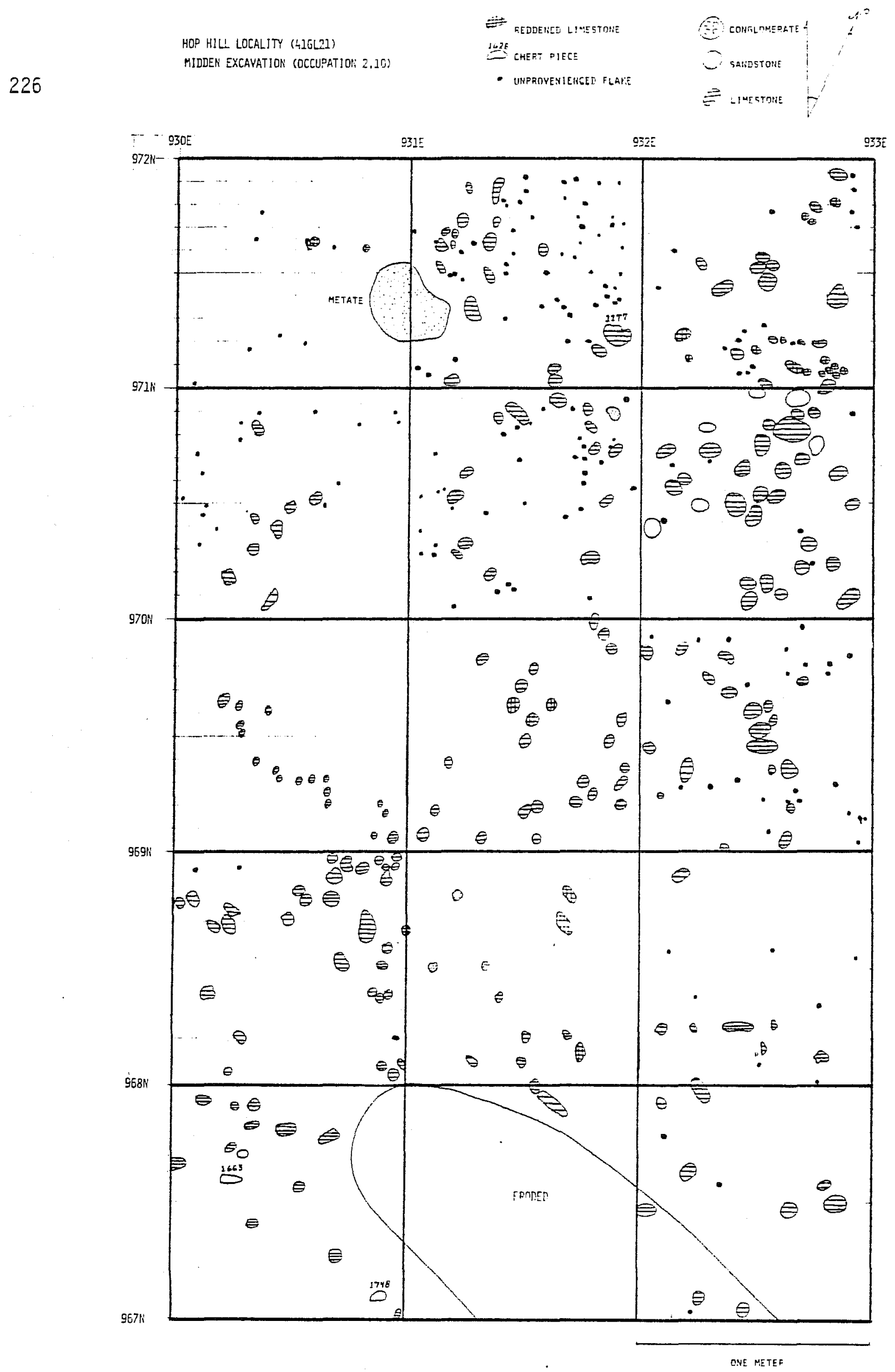

Figure 16.02. Occupation Floor 2.10. 
A metate, the one which drew our attention to the spot, is located in the northern portion of the floor. Also, most of the flaking debris is located near the metate. There are several reddened pieces of limestone to the east of the metate. A concentration of larger limestone pieces lies to the southeast of the metate about $7.5 \mathrm{~m}$. There is little lithic debris of any description to the west.

The association of these elements suggests a community of activities. The proximity of the metate to the empty space at the west suggests that the person doing the grinding faced east. A series of about a half dozen oblong stones within $.5 \mathrm{~m}$ east of the metate might have been manos and should be checked for smoothing. On the east the reddened stones indicate a fire. They are divided into two piles as would happen if someone divided the coals to put the fire out. The pile of stones to the south of the fire grade from large to small away from the fire. This would be expected if bolling stones were dumped from a container; the smaller stones roll farther than the larger ones. Judging by the relationship of the stones the trajectory of the throw was just west of grid south. The thrower, then, must have stood to the east of the fire. The stones in the throw-away pile are all larger than the reddened specimens. This suggests that the reason the reddened stones were left is because they were too small to trouble putting them in the container while the larger ones were rescued from the fire before their chemical structure was significantly oxidized. A replicative experiment could show to what temperature boiling stones were heated before they were used. The temperature may have been controlled to avoid scorching the food.

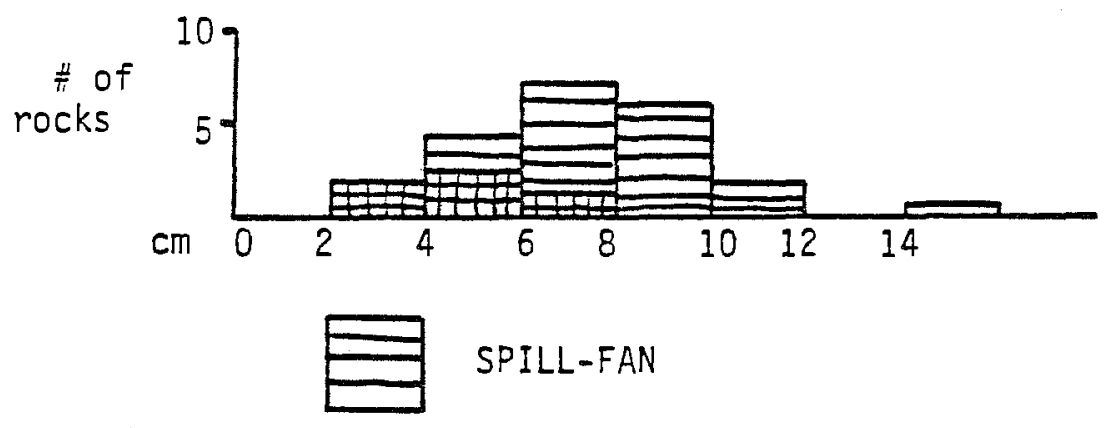

FIRE HEARTH

Figure 16.03. Histogram of Rack Sizes in Hearth and Spill-Fan. 
The longest diameters of reddened and throw-away rocks are graphed in Fig. 16.03. It suggests that the favored size of boiling stones was between 6 and $12 \mathrm{~cm}$. Some large pieces of chert in the throw-away pile indicates at least some use of chert as boiling stones.

Between the fire and the metate is a concentration of flaking debris. There seems to be nothing, however, which suggests the location of the knapper.

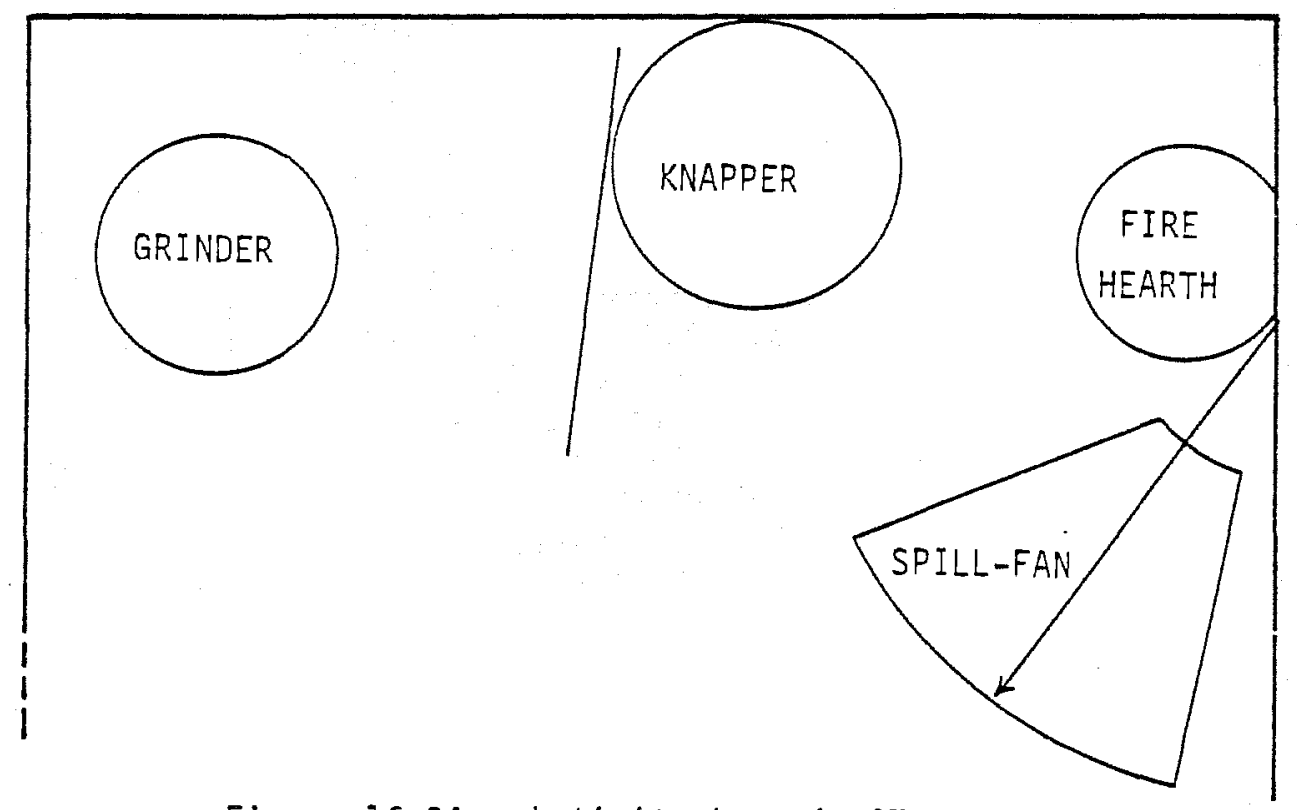

Figure 16.04. Activity Area in OF 2.10.

From the north part of the floor (Fig. 16.04), then, it can be surmised that we have a closely spaced set of camp activities, perhaps recovered at Resolv 1, judging by the generally uncluttered density of artifacts.

The southern $3 / 5$ of the floor offers little in the way of contrastive activity indicators. Virtually all of the debris is limestone. Various lines, circles and open places can be observed.

Midden Occupation 2.20 (Resolv 2.0, Fig. 16-05)

OF 2.20 was opened in $15 \mathrm{~m}^{2}$. The most striking feature of this floor is an apparent fire hearth in squares 930-968 and 930-969 (Fig. 16.05). A pit was apparently dug down into OF 2.30. The disturbed area in of 2.30 (Fig. 16.07) immediately below the feature is a product of this activity. The fire pit was lined with pieces of limestone which were burned until they were reddened. There are concentrations of unplatformed flakes in the NW and SE corners of the area. Most of the larger rocks are concentrated in the $\mathrm{SW} 1 / 2$ of the floor. Above the diagonal they tend to be randomly dispersed rather than clustered. Judging by the preferred size of bolling stones noted in the floor above, the rocks are at the proper 


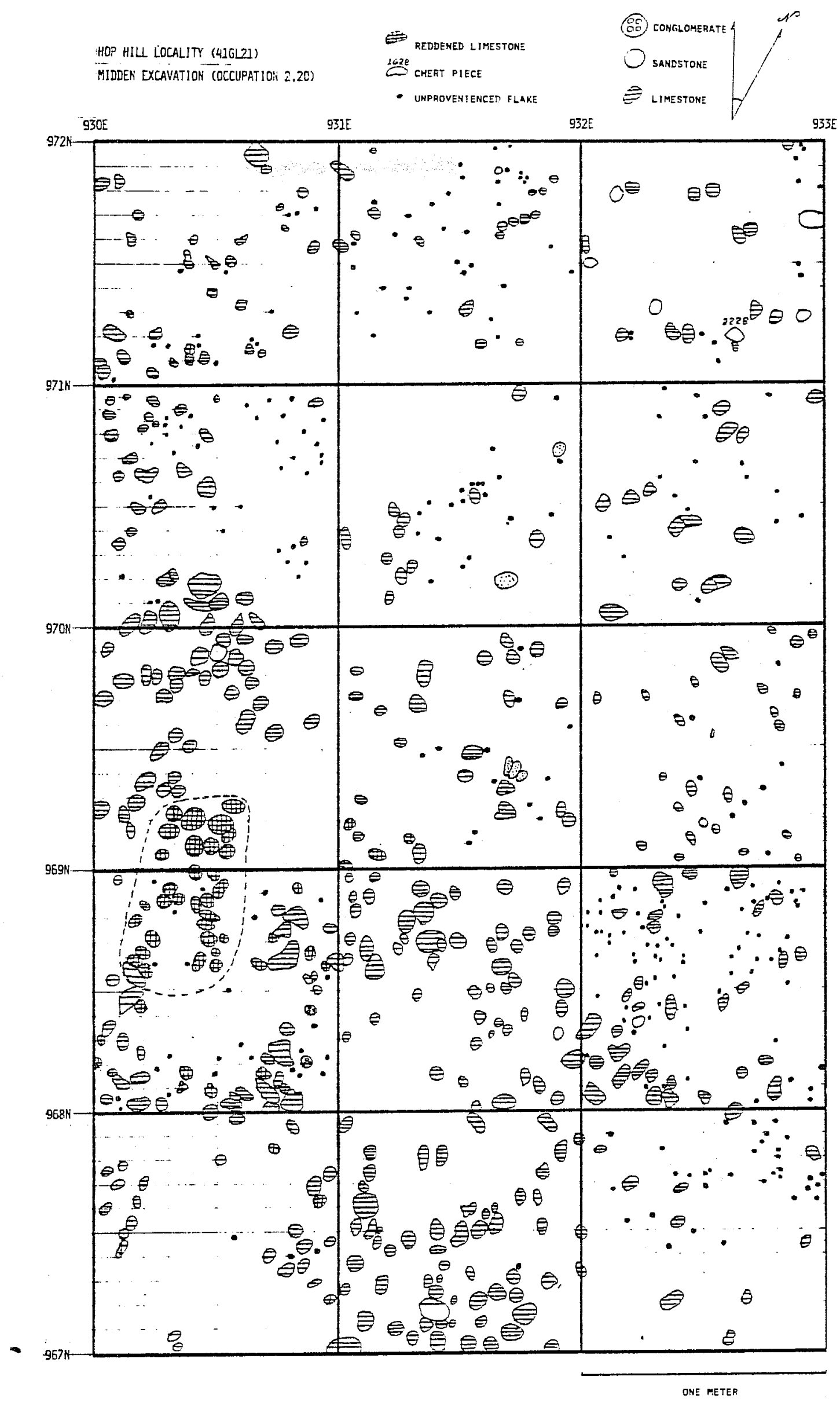

Figure 16.05. Occupation Floor 2.20. 
size to be used as boiling stones. If they are boiling stones, however, they are still in the heating pit. Also, if there was an attempt to control the temperature of boiling stones as noted above, these rocks were too hot. These two facts indicate that the rocks were either abandoned before use, or someone forgot them, did not recover them in time and did not use them. Alternatively and more probable is that the feature served another function, perhaps a roasting pit. Prewitt reports a vaguely similar one-sided pit (1974:76, Ash pit 1) from Loeve-Fox.

The cluster of rocks that centers at E930.5-N970 is fan shaped and has the larger pieces at the north and narrow end of the concentration. It may, then, represent another spill or throw-away incident.

Assuming the spill-fan should point to the fire from which the activity originates, then the reddened rocks on the south margin of square E930-N971 should represent the relevant fire place (Fig. 16.06).

There appears to be knapping to the south and east of this fire.

Midden Occupation 2.30 (Resolv 2.19, Fig 16.07).

OF 2.30 was opened in $12 \mathrm{~m}^{2}$. The resolution is relatively low because of two problems. Three percent of the floor was disturbed because the fire feature was excavated from the floor above. A $150,16 \%$ of the surface was not mapped because of confusion resulting from a rain storm. Not all of the information was lost because artifacts were located on maps, but the rocks and unplatformed flakes were not mapped within the two SW squares.

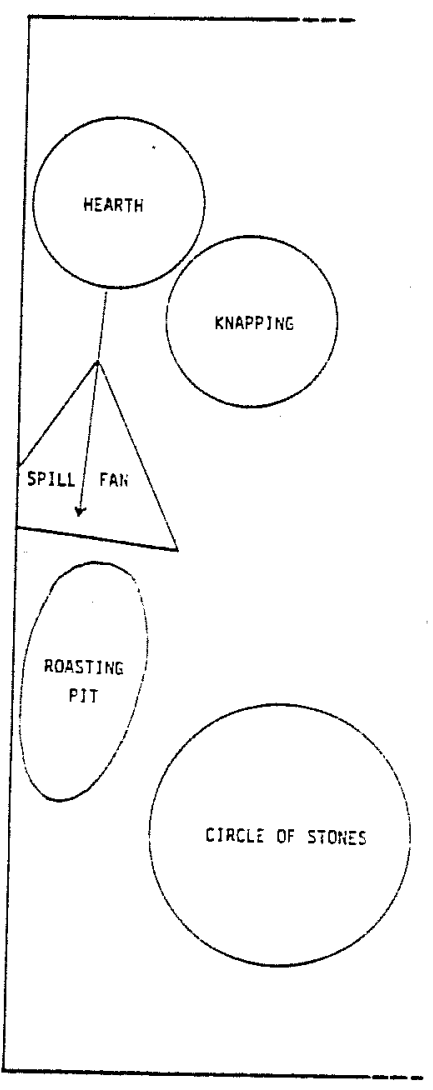

Figure 16.06. Schematic of Occupation Floor 2.20. 


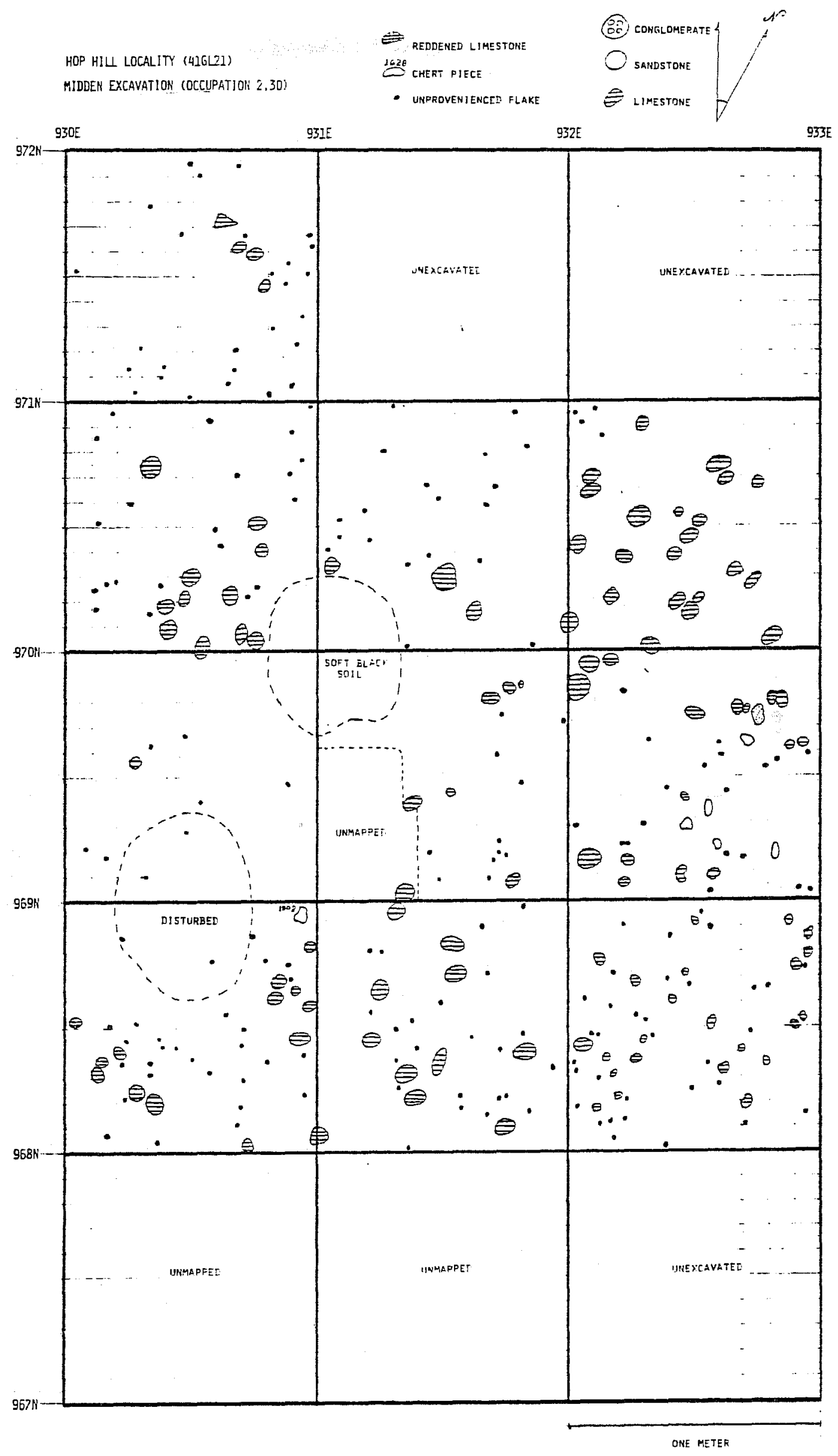

Figure 16.07. Occupation Floor 2.30 . 
The floor seems to be an undifferentiated scatter of 1 imestone and flakes so no interpretation is offered. The reason could be that the excavated part of the site was not used for a period of time so functional loci are elsewhere; the site was not used for food preparation for a period and the debris represent stopovers without usual features, etc.

Midden Occupation 2.40 (Resolv 2.0, Fig. 16.08)

OF 2.40 was opened in $10 \mathrm{~m}^{2}+$. It is the lowest of the floors and is associated with a point which dates to the Middle to Late Archaic. OF 2.40 is different from the above floors in a qualitative way. The limestone rocks are much bigger in numerous cases. The variety of rocks is noticeably broader with chunks of conglomerate and sandstone appearing.

The patterning of the rocks also appears to be different. There are some reddened rocks in the center square of the excavation. There is nothing, however, that can be securely identified as a spill-fan.

There is a distinct tendency for smaller rocks to occur to the south and knapping is to some extent confined to the area of small rocks, suggesting a division of work areas into south for knapping and north for food preparation. Whether the reddened rocks and the large flat rocks in E930N970 are associated in the food preparation task is a matter of pure conjecture without some sort of convincing analytical device. Since we have only one example to work from, an analytical device can only be posed as a very tenuous hypothesis, something to be watched for in the future. However, there is a notable barren strip between the flat rocks and the reddened rocks. It seems possible that the reddened rocks were part of some debris on top of the flat rocks. A fire was built on the flat rocks to heat them for food preparation purposes and then swept off to the southeast to clear them for use. They were swept far enough away to leave the barren strip. Since the smaller rocks were swept off with and remained in the hot coals for a substantial time, they were chemically altered enough to turn them red.

Since the sample of camp patterns is so small, no conclusions can be drawn concerning changing prehistoric lifeways on the Hop Hill midden A hypothesis can be tentatively advanced, however. In the absence of firm dates let us assume the midden is associated with the Round Rock Phase which implies deer and acorn processing as supported by Weir (1976), and that the occupation levels above represent San Marcos and/or Twin Sisters and/or Late Prehistoric occupations. This assumption is based on the generally accepted proposition that true burned rock middens are a predominantly Round Rock Phase phenomenon (Weir 1976, Prewitt 1974). 


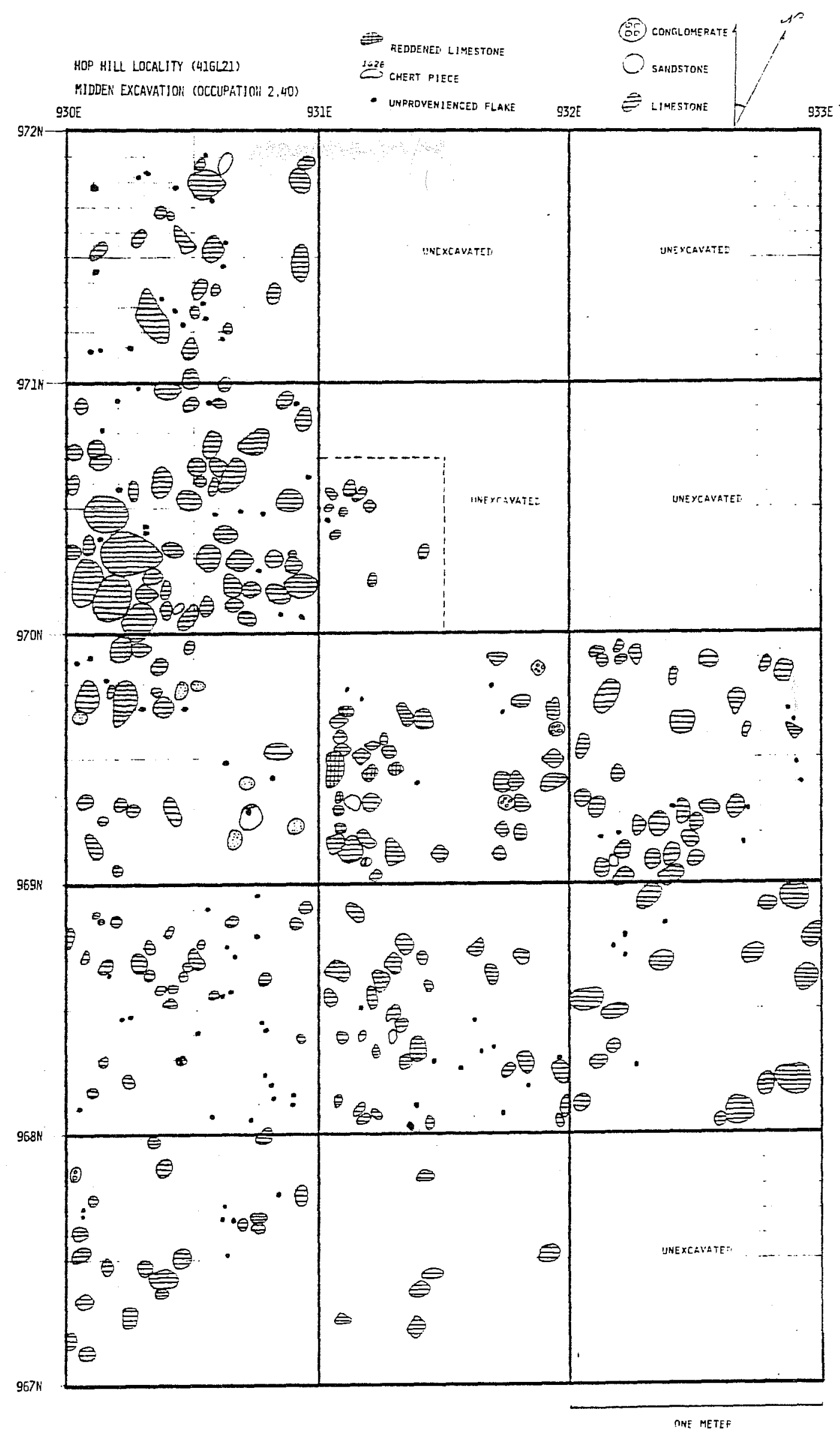

Figure 16.08. Occupation Floor 2.40. 
During the earlier period the flat and burned rock pattern, "burn-andsweep," implies a food preparation process which is still current in Texas. Rocks are heated, and vegetation, food and more vegetation are placed on the rocks. Finally water is poured into the mix and it is covered with soil (now aluminum foil) to retain the steam until the food is cooked (James Escobedo, personal communication). Such a technique would be appropriate for steaming deer meat along with auxiliary foods.

Subsequent to the Round Rock Phase the camp pattern changed. Stone boiling and stone-lined roasting pits became a part of camp activity. The archaeological evidence for stone boiling is a characteristic pattern of basketspilled rocks. This "boil-and-spill" technique seems like a reasonable pattern for more nomadic people such as those of the Western Desert tradition.

Based on this discussion, it is concluded that middens are of an at least somewhat structured nature. However, it is obvious that more work needs to be done using high-resolution recovery techniques to establish whether the patterns inferred from the Hop $\mathrm{Hill}$ midden are recurring or coincidental. 


\subsection{FLAKE TECHNOLOGY (Joel Gunn and Royce Mahula)}

In the field, excavators were instructed to open cultural unit formats on any flaking debris found in situ which retained a platform. In the fall of 1976 the flakes so mapped were measured and observed by the UTSA Anthropology Laboratory class. Thirteen coders made 42 observations on each flake twice (see Figure 17.02). As much as time would allow, these observations were checked and corrections entered. In other cases reljability tests were given. to each coder and the observations of the most reliable coder used.

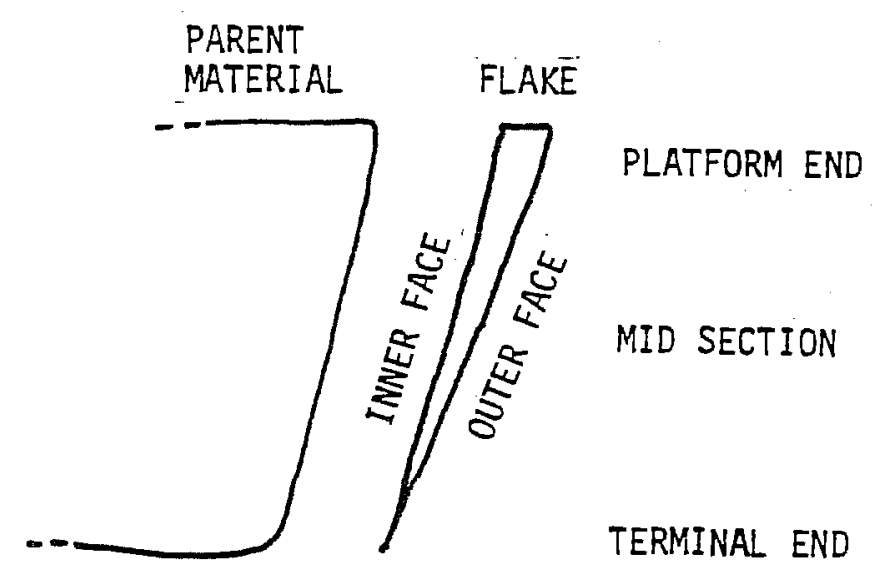

Figure 17.01. Terminology for Flake Technology

Fig. 17.01 shows the terminology which will be used relative to lithic technology. "Parent Material" implies the source of a flake whether it be a nodule, a core, a biface or another flake. The "Flake" is the object removed and it may be a decortication or secondary flake, bifacing flake, a flake-blade, a blade or a pressure flake. The "Platform End" is the end prepared and struck by a percussor instrument. The "Inner Face" is the surface of the flake toward the parent material which contains the bulb of percussion, ripple marks and fissure lines; the "Outer Face" is away from the parent material which exhibits cortex, patina or scalar evidence of previous removals. "Terminal End" is opposite the platform end.

Flake Technology Data

This section will describe the general data-collecting procedures for flakes excavated from Hop Hill. The data collected are intended to answer questions posed from several problems and therefore represent a relatively broad range of attributes. 
HOP HILL LITHICS

Flake and Blade Technology

ง. Gunn 24 AUG 76

Key punch each underscored digit in the next consecutive column. Zero=missing data;

blank=not done.

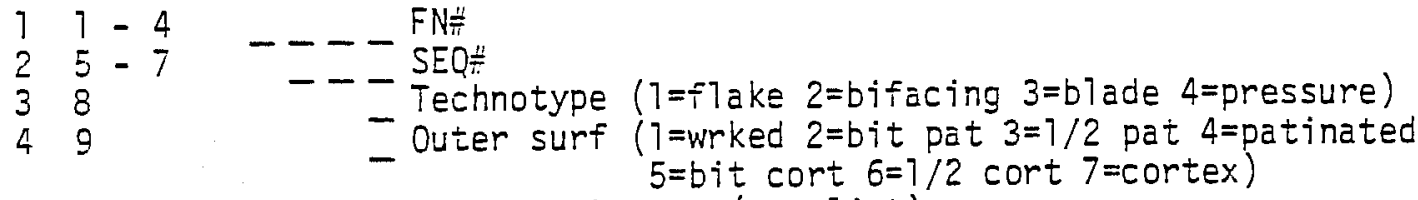

$510-12$ Functional type (see list)

513

714

815

916

1017

11 18-19

1220

$\begin{array}{ll}13 & 21\end{array}$

1422

1523

$16 \quad 24$

$1725-26$

18 27-28

$1929-30$

$\begin{array}{lll}20 & 37-33\end{array}$

$27 \quad 34-35$

22 36-37

23 38-40

24 47-42

25 43-46

$26 \quad 47$

27 48-52

28 53-57

29 58-62

3063

31 64-65

3266

3367

3468

3569

3670

3771

3872

3973

4074

4775

4276

$43 \quad 77-78$

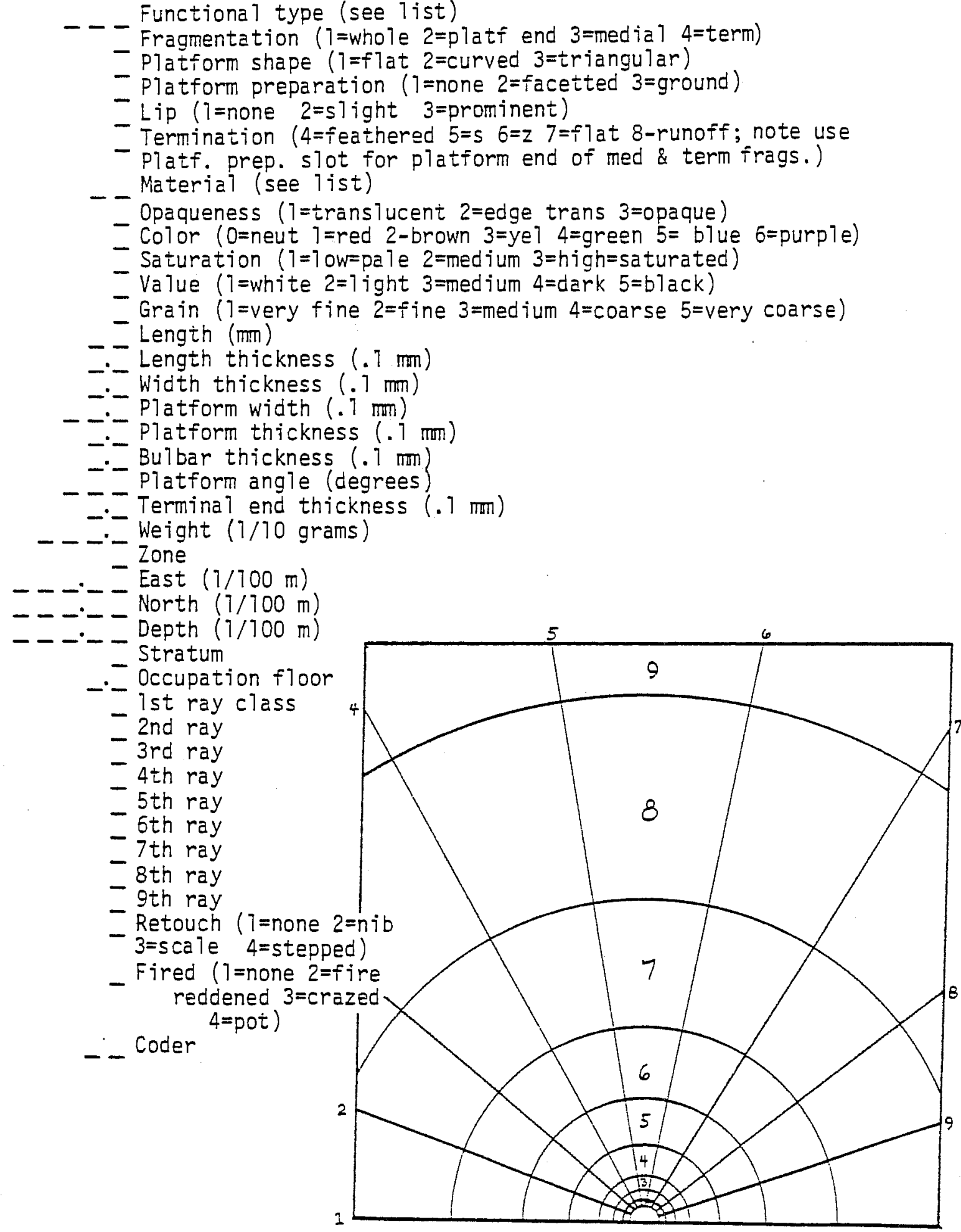


Some of the attributes are not completely non-redundant with others. This overlap is noted in the attribute description as a warning against analysis of non-independent variables. The reason for having some non-redundant variables in the list is for analytical convenience. Some analyses require different resolution, different scaling and slightly different points of view on the fundamentally same feature.

1. FN ( 4 Col.) -- Permanent field specimen number assigned by the Hop Hill project to an artifact. This number along with the sequence number and the site number, $41 \mathrm{GL} 021$, is inked onto the artifact.

2. Sequence $\#(3 \mathrm{Col}$.$) -. A number assigned to an artifact if a field$ number is assigned to more than one artifact.

3. Technotype (1 Col.) -- A nominal variable with four states which represents at the most superficial level the various methods by which flakes are removed from a parent block of material:

$$
1 \text { = flake, } 2 \text { = bifacing flake, } 3 \text { = blade, } 4 \text { = pressure flake. }
$$

If assumptions are made relative to the order in which each flaking technique is most probably used in a reduction sequence, the order is sequential from flakes to pressure flakes. Unspecialized flakes are produced in the initial stages of any flaking task. Bifacing flakes and blades appear as the process moves to more refined tool making. Finally, the pressure flakes come in the final stages of shaping and finishing and as a means of resharpening dulled tools subsequent to the reduction process proper. When it seems appropriate to invoke this assumption the variable is assumed to be a satisfactory linear, relative time scale.

There is some redundancy between technotype and functional type.

4. Outer Surface ( 1 Col.) -- A nominal variable with seven states which indicates the condition of the Outer Surface of a piece:

$$
\begin{aligned}
& 1=\text { worked, no patina or cortex, } 2=\text { bit patinated, only a trace, } \\
& 3=1 / 2 \text { patinated, } 4=\text { patinated, } 5=\text { bit cortex, only a trace, } \\
& 6=1 / 2 \text { cortex, } 7=\text { cortex. }
\end{aligned}
$$

Outer Surface is used in conjunction with Technotype to show the primary or secondary nature of a flake.

5. Functional Type $(3 \mathrm{Col}$.$) -- A nominal variable which indicates in some$ detail all of the types of lithic and non-lithic materials found at Hop Hill. This variable is redundant with Technotype, Outer Surface, Fragmentation, and Termination.

6. Fragmentation ( 1 Col.) -- A nominal variable which shows the portion of the flake or if it is a whole piece:

$$
1 \text { = whole, } 2 \text { = platiorm end, } 3=\text { mid-section, } 4=\text { terminal end. }
$$

This attribute is partially redundant with Functional Type. 
7. Platform Shape (1 Col.) -- A nominal variable with three states that shows the outline of the platform as the observer sees it looking at the lateral aspect of a flake:

$$
1=\text { flat, } 2 \text { = curved, } 3 \text { = triangular. }
$$

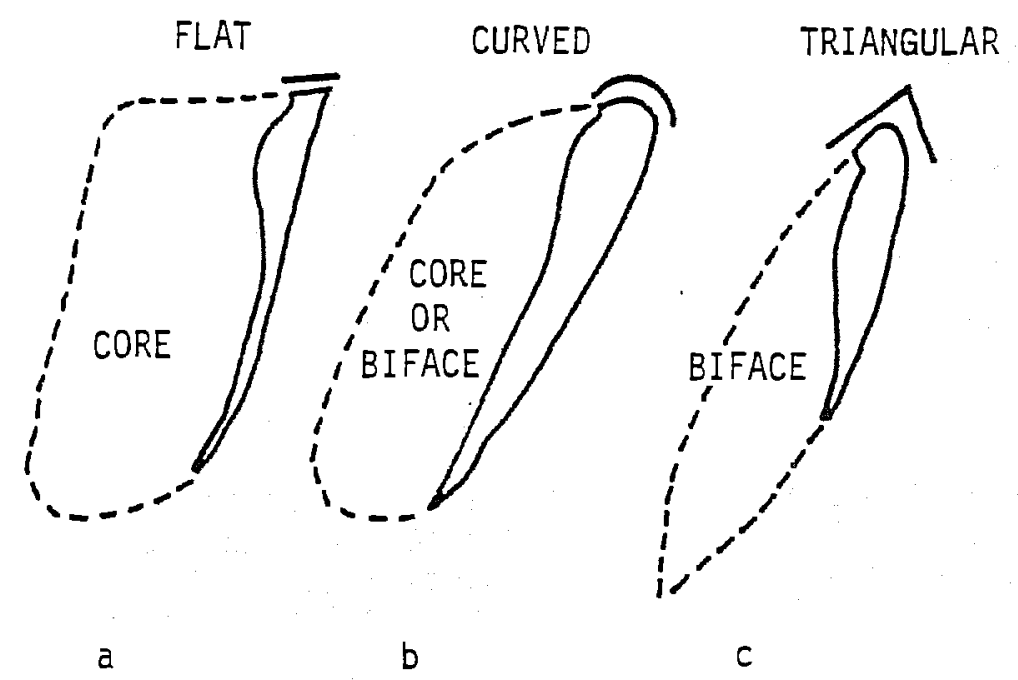

Figure 17.03. Various Platform Shapes.

Figure 17.03 illustrates the distinction that is being attempted with this attribute. The platforms of normal flakes and bifacing flakes are quite distinct in many cases. The reason is apparent when the relationship of the two types of flakes to their respective parent masses is examined. A core is something of a block (Fig. 17.03a) from which a flake is removed. The angle between the outer face and the platform is about $90^{\circ}$ or a little less. A biface, on the other hand, is a lenticular mass where edge angle becomes a striking platform. The result (Fig. 17.03 c) is a triangular platform.

8. Platform Preparation and Platform End Break ( 1 Col.) -- A nominal variable of three states designed to show the extent and kind of special effort that went into platform preparation:

$$
1 \text { = none, } 2 \text { = faceted, } 3 \text { = ground. }
$$

Faceting implies retouching the platform area in order to shape it to desired proportions. This process leaves the platform with a scalar, undulating appearance. Grinding is done by rubbing a coarse-grained rock along the platform. The result, as one might expect, is a rounded and roughed look at the platform. If the platform is missing the type of the break on the platform end of the piece is recorded in this column. See Termination for coding scheme. 
9. Lip (1 col.) -- A ordinal attribute of three states which shows the character of the lip on the inner edge of the platform:

$$
1 \text { = none, } 2 \text { = slight, } 3 \text { = prominent. }
$$

So-called "lipped flakes" have been mistakenly thought to be bifacing flakes. Virtually any flake can have a lip on it depending on how it was struck. Sollberger reports that any flake will have a lip whose cone is greater than $123^{\circ}$ (personal communication). The purpose of including this attribute in the list was to determine the exact relationship of lip frequencies to bifacing flakes.

10. Termination ( $1 \mathrm{Col}$. ) -- A nominal variable of five states designed to show the nature of the terminal end of a flake:

$$
\begin{aligned}
& 4 \text { = feathered, } 5=\text { s-termination, } 6=z \text {-termination, } 7=\text { flat } \\
& \text { termination, } 8=\text { runoff termination. }
\end{aligned}
$$

The states are numbered 4-8 so as to be numerically exclusive of the 1-3 used for Platform Preparation states. If the platform end is broken off the character of the break is recorded as state 5,6 , or 7 from this variable.

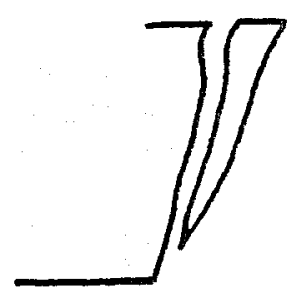

FEATHERED

a

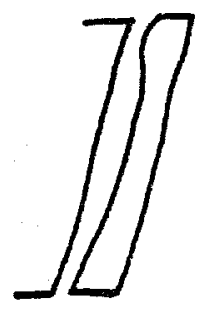

RUNOFF

b

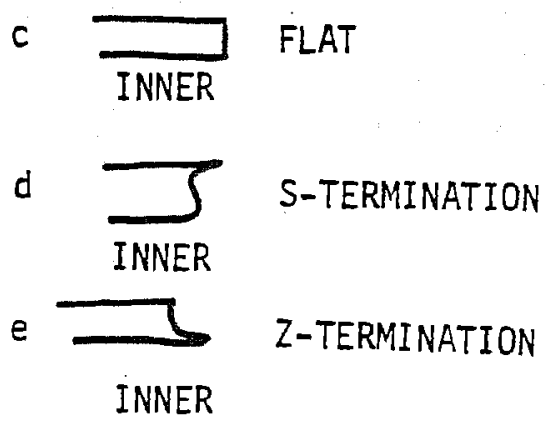

Figure 17.04. Various Terminations.

Figure 17.04a-e illustrates the various states of Termination. "Feathered" means the flake tapers to a fine edge at the terminal end (Fig. 17.04a). "Runoff" is the case where the terminal end would have feathered out had it not come to the end of the parent material first. Fig. 17.04c, d, and e illustrate various abnormal terminations and breaks.

Terminations and breaks are classified as follows: First, hold the piece in front of your eyes with the inner face down and the break to the right. 
Second, note if it is flat or if it protrudes at the top or bottom. If it is protruding more at the top it is an s-termination; if it is more at the bottom it is a z-termination. The tentative theory is that a flat end on the terminal end of a flake fragment is an intentional break or a step fracture An s-termination is a hinge fracture or an intentional break initiated from the inner face. A z-termination can only be a break initiated from the outer face. These distinctions will hopefully be useful for sorting functionally intended breaks from those which are a product of manufacture. As is immediately apparent, however, the model is partially indeterminant.

11. Material (2 Col.) -- A nominal attribute to distinguish material types. The motive for classifying material was not to assign pieces to quarry origins. An attempt was made to group tools and debris into clusters which represent a single block of parent material. If, for instance, a knapper brought a biface to the site and removed four flakes, those flakes should be distinguishable as a cluster from all other flakes on the site, and they should define a task areâ.

It is probably not possible to verbalize all of the dimensions of judgment that go into clustering one material with another. Two procedural guidelines were followed. First, classification was pursued after the fashion of a comparative collection. Each flake was compared with all the pieces previously clustered to see if it would fit into an established group. If it did not appear to be from the same parent block as any of those classes, a new group was established. Second, some of the criteria of classification were as follows. There are certain dominant traits that figure into the classification; the most important of these are color, texture, and character. color is the most prevalent hue in the material; texture is the graininess of the material. Character is something unique to the material. It may include banding, small fossils, specks and spots of various colors. Sometimes materials patinate in a special way due to metallic intrusions. Often cortex is very characteristic. Character is redundant with opaqueness, color, value and grain.

12. Opaqueness ( $1 \mathrm{Col}$.$) -- An ordinally scaled variable of three states$ designed to show the light-transmitting character of the material:

$$
1 \text { = translucent, } 2 \text { = edge translucent, } 3 \text { = opaque. }
$$

"Translucent" is the case where light comes through most of the piece when it is held to the light. "Edge translucent" materials allows the transmission of light only through the edge. No light passes through opaque objects. or at least very little. Translucence is a property imparted by the nature of inclusions in the predominantly silicate mass of flints and cherts and as such is an important part of defining the workability and quality of the material.

13. Color ( 1 Col.) -- An ordinally scaled variable of seven states. The scaling is based somewhat on the color light spectrum:

$$
\begin{aligned}
& 1=\text { red, } 2 \text { = yellow, } 3=\text { buff, } 4=\text { brown, } 5=\text { green, } 6=\text { blue, } \\
& 7=\text { purple. }
\end{aligned}
$$


14. Saturation ( 1 Col.) - An ordinal scale of three states which measures saturation of color. If color is neutral saturation must be zero:

$$
1 \text { = pale (low saturation), } 2 \text { = medium, } 3 \text { = high (saturated). }
$$

15. Value ( 1 Col.) -. An ordinal scale of four states intended to measure the gray scale of the material:

$$
1 \text { = 1ight, } 2 \text { = medium, } 3 \text { = dark, } 4 \text { = very dark. }
$$

16. Grain (1 Col.) -- An ordinal scale of four states which show the texture of material:

1 = very fine (about like glass), 2 = fine, 3 = medium, 4 = coarse.

17. Length $(2$ col.) -- A continuous scale measured perpendicular to the platform in $\mathrm{mm}$.

18. Length Thickness (2 Col.) -- A continuous scale measured in tenths of $\mathrm{mm}$ parallel to the length of a piece. A vernier caliper is closed over the piece so the platform and terminal ends touch one arm and the highest point on the outer surface touches the other arm. The measurement is illustrated in Fig. 17.05a.

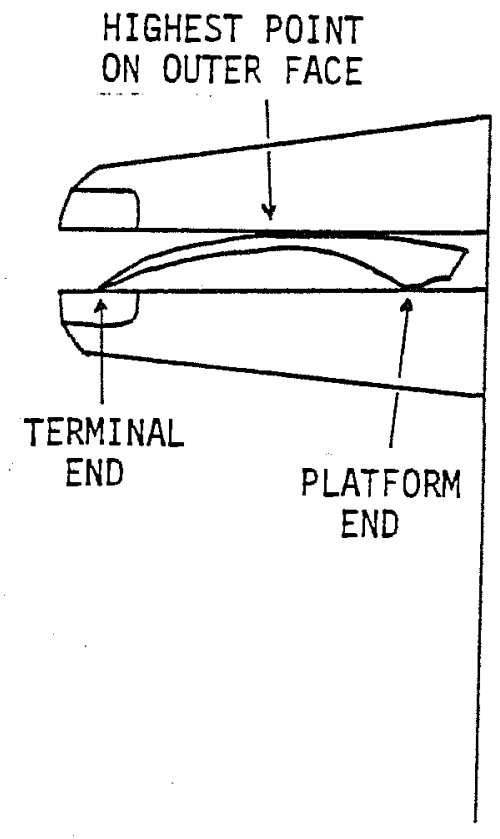

a. LENGTH THICKNESS

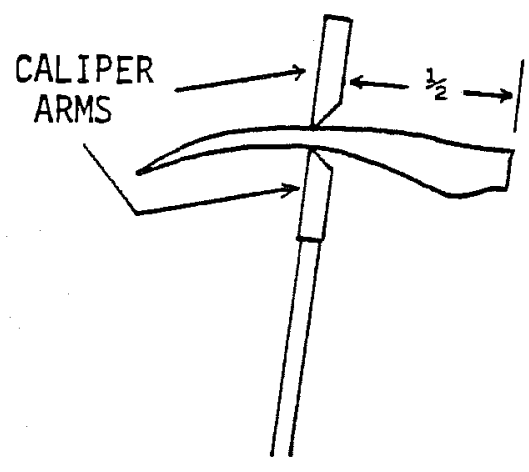

b. WIDTH THICKNESS

Figure 17.05. Measurement of Length and Width Thickness. 
19. Width Thickness (2 Col.) -- A continuous scale measured in tenths of $\mathrm{mm}$ about halfway down the length and perpendicular to the length. Use the fine end of the caliper arm to get the thinnest possible measurement. The measurement is illustrated in Fig. 17.05b.

20. Platform Width ( $3 \mathrm{Col}$. ) -- A continuous scale measured in tenths of $\mathrm{mm}$. Platform Width is measured across the platform parallel to the width of the flake.

21. Platform Thickness (2 Col.) -- A continuous scale measured in tenths of $\mathrm{mm}$. Platform Thickness is measured from the lip to the outer edge of the platform parallel to the thickness of the flake.

22. Bulbar Thickness (2 Col.) -- A continuous variable measured in tenths of $\mathrm{mm}$. The measurement is taken from the most salient point on the bulb of percussion to the outer surface parallel to the thickness.

23. Platform Angle ( $3 \mathrm{Col}$.$) -- A continuous variable measured in degrees.$ The measurement is taken between the inner surface as it is pressed against the arm of a goniometer and the aspect of the platform proximate to the inner face.

24. Terminal End Thickness (2 Col.) -- A continuous variable measured in tenths of $\mathrm{mm}$; the thickness of the terminal end whether it be feathered or a break.

25. Weight $(4 \mathrm{Col}$.$) -- A continuous variable measured in tenths of grams.$

26. Zone ( 1 Col.) -- Localith Zones 1-4.

27. East ( $5 \mathrm{Col}$.) -- A continuous variable measured in $\mathrm{cm}$ from the east baseline of the site. East is a horizontal Cartesian Coordinate. (Note: 3 digits, computer adds 1,000 meters if entry is below 800 meters.)

28. North (5 Col.) -- A continuous variable measured in $\mathrm{cm}$ from the south baseline of the site. North is a horizontal Cartesian Coordinate. (Note: on ly 3 digits, computer adds 1,000 meters if entry is below 800 meters.)

29. Depth (5 Col.) -- A continuous variable measured in $\mathrm{cm}$ from the vertical datum. Depth is a vertical Cartesian Coordinate.

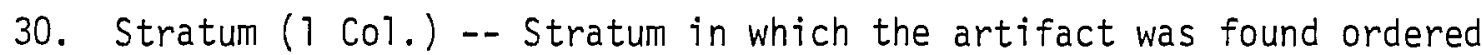
in sequence from top to bottom of the site.

31. Occupation Floor (2 Col.) -- Occupation floor designation number.

32-40. 1st-9th Ray Length (1 Col. each for 9 Col.) -- The nine rays represent a system of polar coordinates designed to represent the shape of a flake. The platform is centered at the bottom as indicated with the inner face down. Then a pencil line is drawn around the artifact. At each ray the class in which the outline crosses it is recorded in the appropriate column. The nine classes are scaled logarithmically to make measurement and 
recording as efficient as possible. Equivalents to the classes in $\mathrm{mm}$ are as follows:

$$
\begin{aligned}
& 0=0-2 \mathrm{~mm}, 1=2-3 \mathrm{~mm}, 3=5-7 \mathrm{~mm}, 4=7-12 \mathrm{~mm}, 5=12-20 \mathrm{~mm}, \\
& 6=20-30 \mathrm{~mm}, 7=33-55 \mathrm{~mm}, 8=55-90 \mathrm{~mm}, 9=>90 \mathrm{~mm} .
\end{aligned}
$$

41. Retouch ( $1 \mathrm{Col}$.$) -- Presence or absence of retouch:$

$$
1 \text { = none, } 2 \text { = nibbling, } 3 \text { = scalar retouch, } 4 \text { = stepped retouch } \text {. }
$$

42. Fired ( 1 Col.) -- Reflects the degree of firing:

1 = none, 2 = reddened, 3 = crazed, $4=$ pot 1 idded .

43. Coder (2 Col.) -- Coder person's $\#$

Force-Flake Model

It is generally believed by the community of modern flint knappers that increasing the amount of force in the process of making a flake increases the salience or prominence of the positive bulb of percussion, the curvature of the inner face and the tendency of the flake to fold around the end of the core or "runoff" the end opposite the platform. Controlled experiments such as Speth $(1972,1975)$ tend to support this point of view.

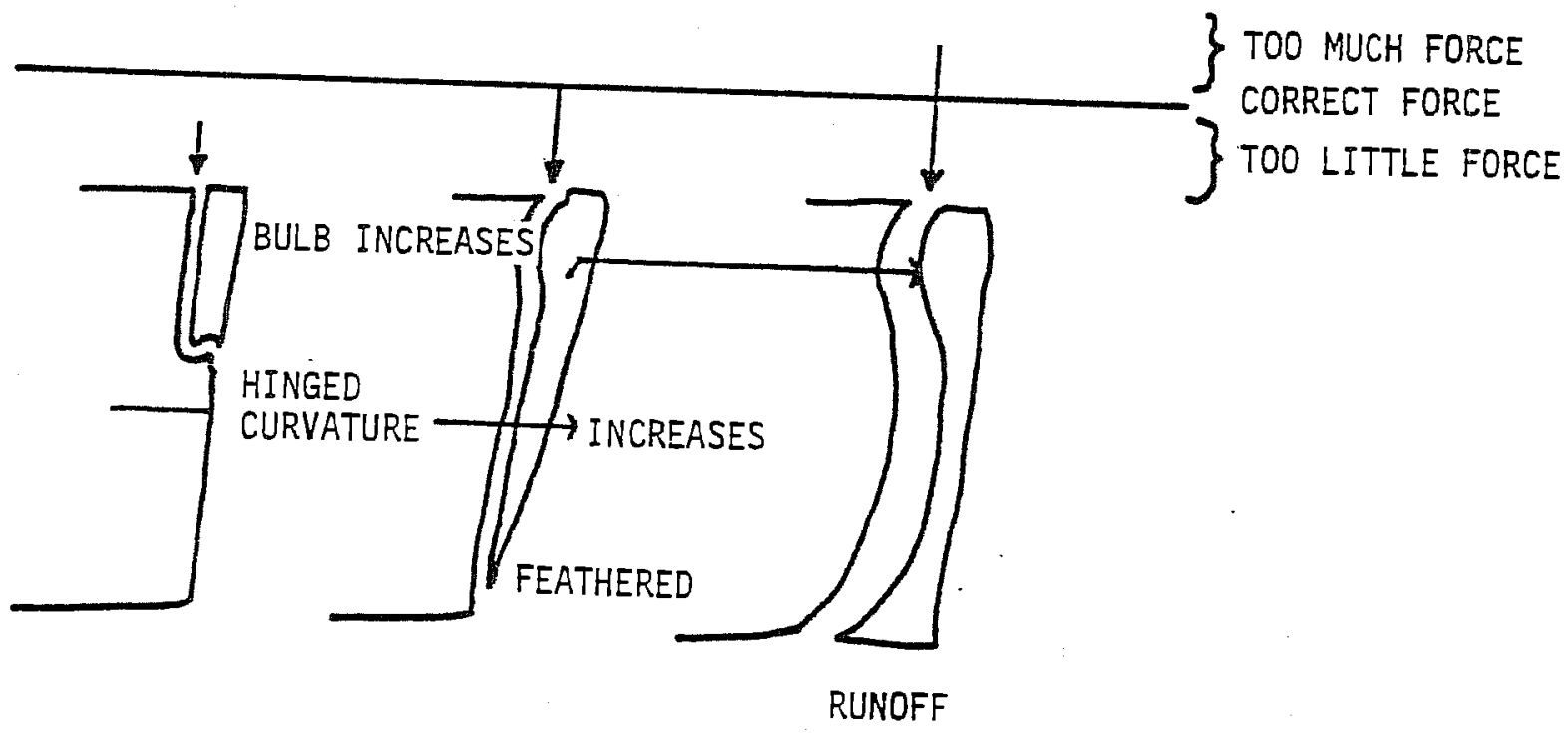

Figure 17.06. Effects of Varying Force on Termination. 
In addition, it is known that too little force results in a shortfall termination such as a hinge or step fracture. These various amounts of force can be arranged on a continuum, as in Fig. 17.06, in which the character and dimensions of terminal end thickness represent the accuracy with which the knapper of a particular flake applied force to his task. Too little force results in a hinge or step fracture. The correct amount of force produces feathered terminations, and too much force results in a runoff configuration.

A simple measurement of terminal end thickness should be a hyperbolic function. The inaccurate blows will have large measurements whether they be the result of too little or too much force. Accurate blows at the center of the continuum will produce the minutest measurements. In order to make the continuum a useful instrument for analys is by linear models all flakes with feathered, flat and s-terminations (see Termination, attribute 10, for implications of s-termination) were converted to minus values. Thus, larger minus values represent larger deviations toward too little force; large plus deviations are increasingly too much force.

The other indicators of force should correlate positively with accuracy once the effect of absolute mass is removed. These include bulbar salience which is calculated as:

$$
\text { Salience }=\text { Bulbar Thickness }- \text { Platform Thickness }
$$

Also, curvature is:

$$
\text { Curvature }=\text { Length Thickness - Width Thickness }
$$

Mass is controlled by the quantity Weight. The interaction of these variables as suggested above is illustrated in Fig. 17.07. The variables directly controlled by human ideas are to the left and the subsequent consequences of effecting those ideas are to the right.

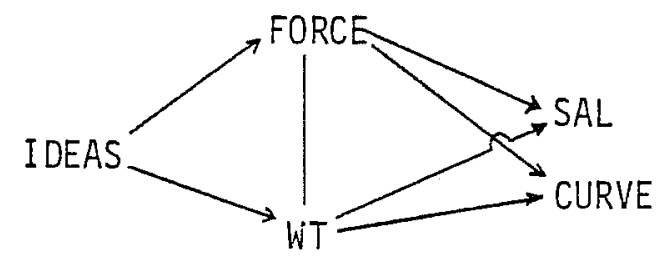

Figure 17.07. Interaction of FORCE and RELATED VARIABLES.

This process can be further elaborated since the material and the degree to which the material is heat treated also provide moderating influences on the effect FORCE has on shape (Fig. 17.08). Speth (1975) has demonstrated that platform angle influences the size of a flake. 


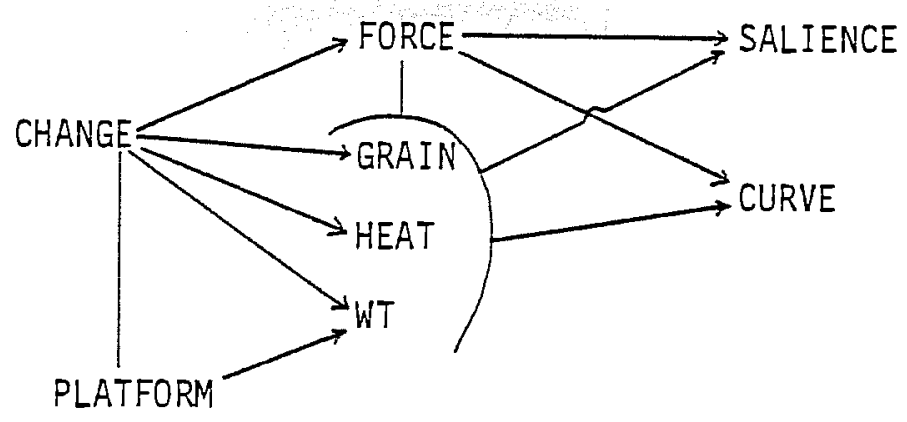

Figure 17.08. More Highly Specified Flake Production System.

To determine the configuration of these emphases, a principal components analys is was run on the flakes from each midden occupation floor and also an analysis was made of all the floors combined. All analyses were rotated to varimax criteria.

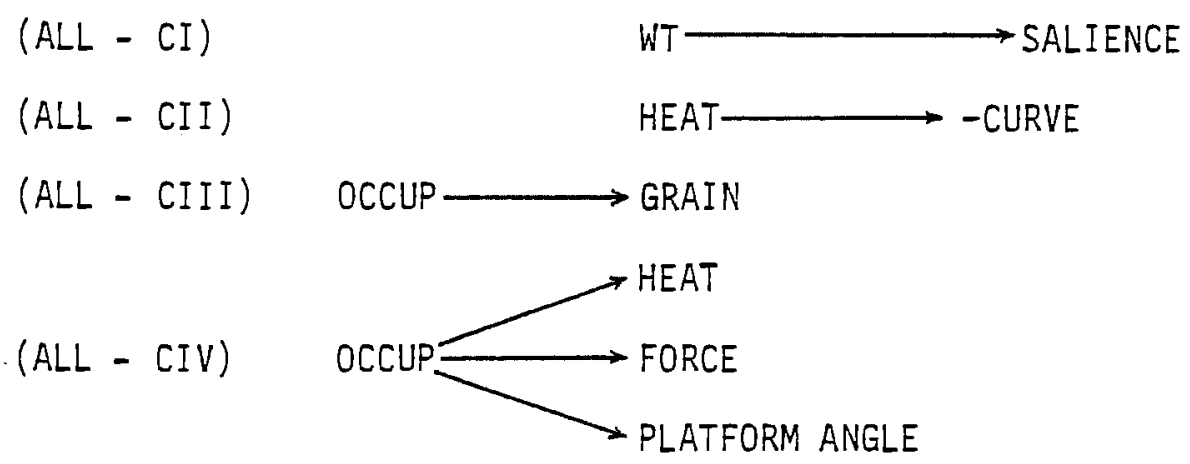

Figure 17.09. Universal Patterns in Hop Hill Flake Technology, Principal Components of All Floors (OFI, $N \approx 350-430$, Variance $=59 \%$ ).

Figure 17.09 shows the four principal components for all occupation floors. These are the universal trends in the assemblage. Weight affecting Salience is a dominant trend. Apparently the Hop Hill knappers simply struck larger pieces harder rather than applying any special techniques to reduce bulbar salience in larger items.

Component II shows that heat treating results in less curved inner faces. Since heat treating reduces the effort necessary to remove a flake it represents the extra force necessary to remove untreated flakes and the consequent frequent occurrence of more curved inner faces.

Components III and IV are both related to change through time. Grainier cherts were used during the early period (CIII). Also, earlier people heat treated more, prepared higher angle platforms and used more force in flake removal (CIV).

As will be shown below, some of these traits are not so universal because the sample is heavily weighted in favor of the two upper occupation floors. On the other hand, the time related variables are particularly important because 
they mark the most important trend in the data, a change from bifacing to core flaking over time. The meaning of the time related relationships will become apparent as the discussion progresses.

In the next four analyses, when these trends appear it will be kept in mind that they are universal and not peculiar to the time period represented by individual occupation floors. The association of Weight with Salience and Heat treating with straight inner faces are related to the mechanics of fijint knapping. Changes in Grain, along with changes in heat treating; platform preparation and the accuracy of force applied are related to time and changing ideals.

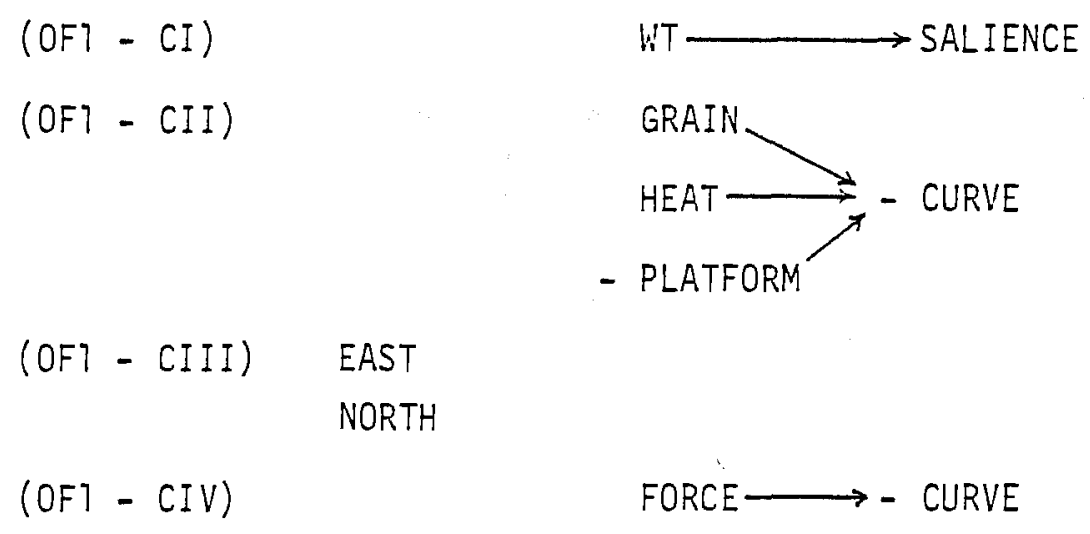

Figure 17.10. Knapping Pattern in Occupation Floor 2.10 10F1, $N=100-150$, Variance $=61 \%$.

Occupation Floor 2.10 (OFl, Fig. 17.10) has some of the universal characteristics, especially in Component I. However, in addition to heat treating reducing curvature of the inner face, curvature also decreases as graininess increases and Curvature decreases as Platform Angle decreases. Speth (1975) has determined experimental1y that outside platform angle influences the length of a flake. The OFl flakes show that reducing inner platform angle, perhaps by controlling the blow angle, decreases curvature; which is logical enough, as fig. 17.11 indicates.
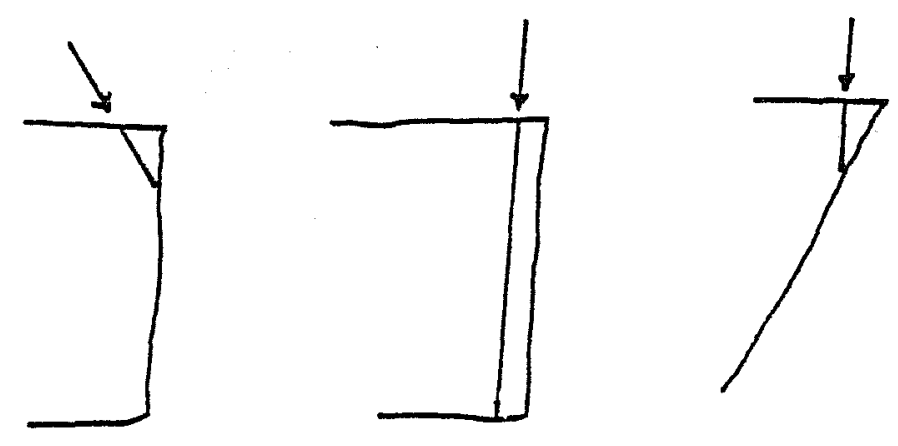

Figure 17.11. Curvature Decreases as Inner Platform Angle Decreases. 
East and North appear in CIII without relations to any attributes. CIII shows that the amount of force applied is inversely related to the amount of curvature produced. This is opposite of what the model predicts and it implies some kind of a special technique.

$\begin{array}{ll}(\text { OF2 - CI }) & \begin{array}{l}\text { EAST } \\ \text { NORTH }\end{array} \\ (\text { OF2 - CII }) & \\ (\text { OF3 - CIII }) & \text { GRAIN } \\ & \text { HEAT } \\ & \text { PLATFORM ANGLE SALIENCE }\end{array}$

$(\mathrm{OF} 4$ - CIV $) \quad$ FORCE $\longrightarrow$ CURVATURE

Figure 17.12. Knapping Patterns of Occupation Floor 2.20 10F2, $N \approx 1.25-155$, Variance $=64 \%$.

OF2 (Fig. 17.12) again shows the universal pattern of Weight-Salience relationship. It is, however, replaced as the component accounting for the most variance by the East-North patterning of artifacts on the occupation floor. While there is a clear tendency for artifacts to be aligned on a northeast-southwest axis, no attributes are related to the pattern.

Grain, heat treatment and platform angle (this time positive) are again on the same component. They, however, do not produce less curvature as they did in the floor above. Remarkably, the Force-Curvature relationship reverses itself to conform to the model prediction.

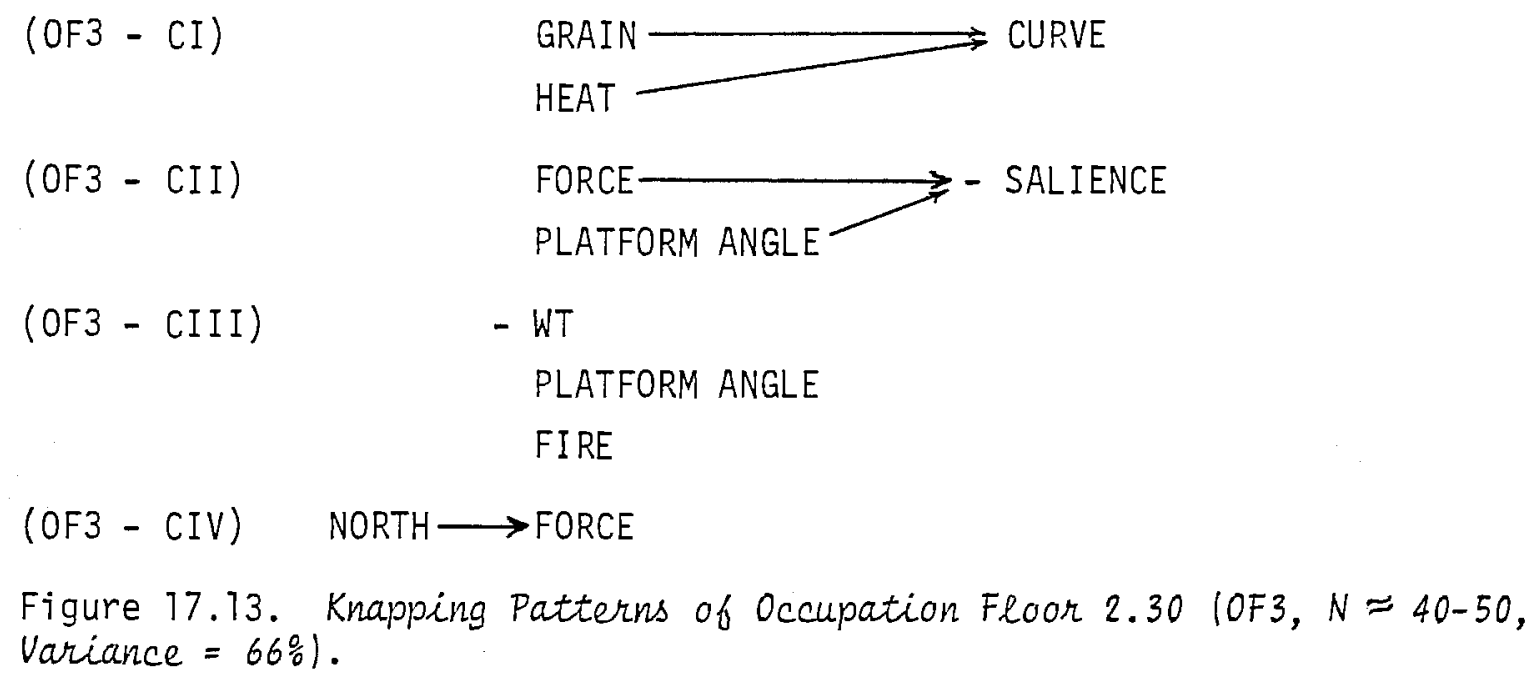

Figure 17.13. Knapping Patterns of Occupation Floor 2.30 10F3, $N \approx 40-50$, Variance $=66 \%$.

OF3 (Fig. 17.13) marks a departure from the floors above, and suggests the presence of an earlier and quite different knapping technique. The combinations of Weight-Salience, Force-Curvature and Grain-Heat treatment-Platform 
Angle disappear showing that their universality is a product of larger sample sizes in the upper floors. Knapping technique is dominated by a positive correlation between Grain. Heat treatment, and Curvature of the inner face (CI). Platform Angle is negatively related to Salience (CII). This suggests a bifacing flake technology since bifacing flakes have very high platform angles as they are measured in this study, and bifacing flakes have by necessity low saliency of the bulb of percussion. The force variable behaves negatively with Salience. The more salient the bulb the less likely there is to be a shortfall termination. This probably. indicates that exceptionally thin bifacing flakes tend to break off toward termination.

In CIII smaller items tend to be heat treated and have higher Platform Angles. Both characteristics are logical bifacing procedures.

\begin{tabular}{|c|c|c|c|}
\hline$(O F 4-C I)$ & EAST & & \\
\hline & NORTH & & \\
\hline$(O F 4-C I I)$ & & WT & \\
\hline & & FORCE & \\
\hline$($ OF4 - CIII) & & & SAL \\
\hline & & & \\
\hline$(O F 4-C I V)$ & & GRAIN & \\
\hline & & HEAT & \\
\hline
\end{tabular}

Figure 17.14. Knapping Patterns of Occupation Floor 2.40 10F4, $N \approx 65-77$, Variance $=66 \%$.

The striking feature of OF4 knapping is that none of the inferred causal relationships are effective. There are no arrows; also, Platform Angle drops out of the picture due to low communality. CII shows Weight and Force to be related. Larger pieces have more tendencies to be accurately or over-struck. Curvature is negatively related to Salience, certainly a bifacing characteristic, and finally (CIV) is the persistent Heat treating of coarser-grained material.

Fig. 17.15 illustrates the family tree of flint knapping at Hop Hill. Solid lines join components which share two or more variables (principal components recalculated without Time-Space variables). Dashed lines trace the wanderings of single attributes. As a composite it represents the trajectory of interaction between the various mechanical and decision-bound processes of the art much as Clarke (1968:104) does in his analysis of cultural subsystem interaction.

Grain and Heat treatment are consistently related early in time but fall apart late in the sequence. In contrast, the Weight-Salience combination appears in the third occupation floor and continues to the end of deposition. 


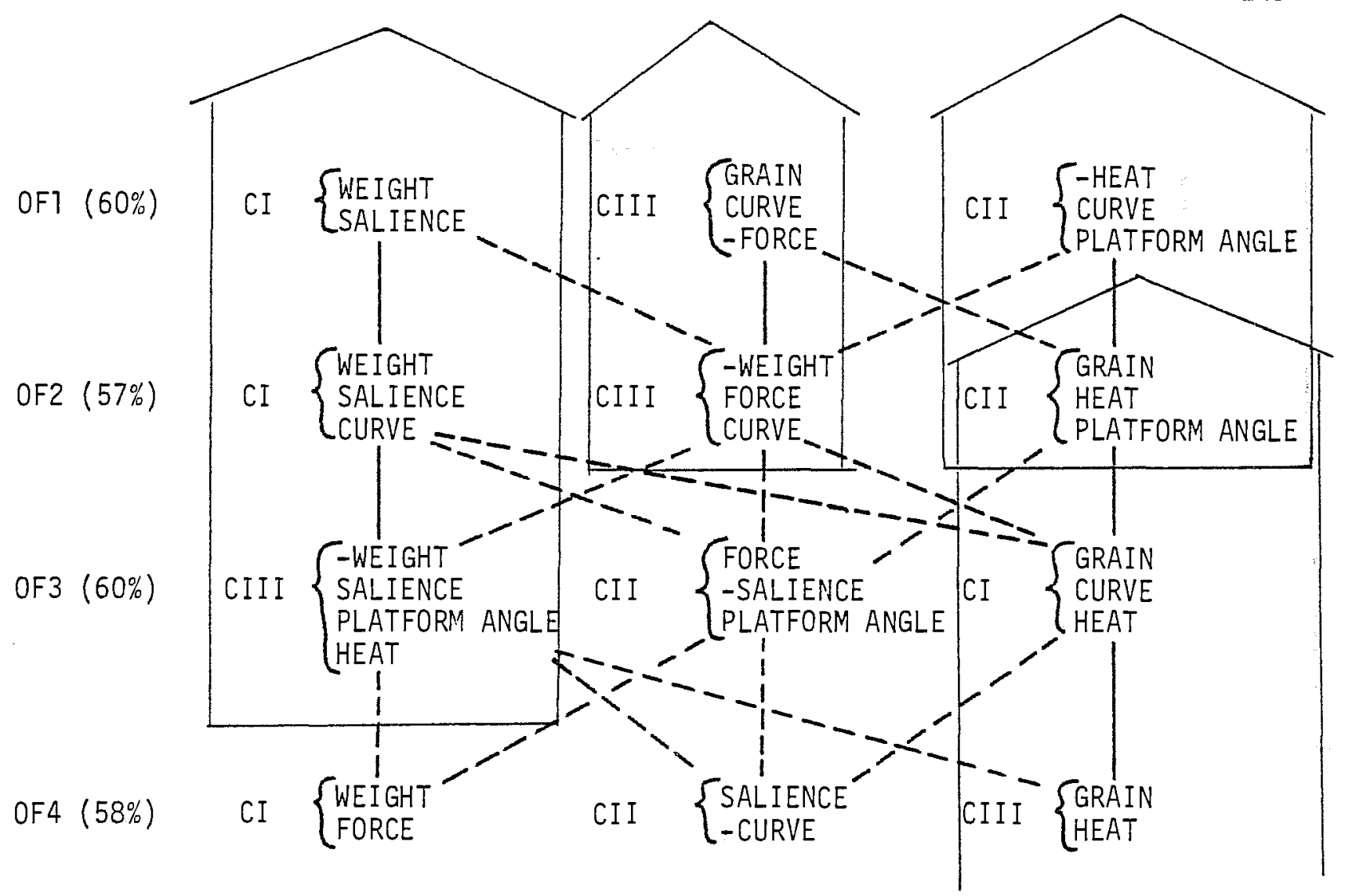

Figure 17.15 Trajectories of Interaction Between Attributes (Without SpaceTime Variables).

A close examination of the changes in use of grainy materials shows that the use of very fine-grained material increased monotonically, i.e., continuously, from $13 \%$ in the earliest floor to $35 \%$ in the latest floor. Meanwhile, the use of fine- and medium-grained materials dropped in a $73 \%-72 \%-60 \%-58 \%$ progression. Note that the biggest change is between the lower and upper two levels. The switch to more fine-grained materials probably explains the break-up in the Grain-Heat treating complex through time observed in the principal components analysis.

Probably the Heat treatment of grainy materials was to facilitate bifacing in the early period. As the technology changed to core flaking the size of the blank and its related saliency of the bulb of percussion became the most persistent trait.

By way of supporting these inferences it is of interest to note that flat platforms increase monotonically through time from $40 \%$ to $47 \%$ of the platforms observed while triangular platforms decrease from $50 \%$ to $44 \%$. This trend indicates a less frequent use of bifacing in later times. The highest percentage of feathered terminations is in OF 3 which supports a more bifacially oriented industry in OF3 than either before or after. This could explain the extreme aberrancy of OF3 which consistently appears across several analyses. Perhaps the most interesting case is platform angle which is so random of character in the lower levels it does not appear in the pattern because of low communality. In Occupation Floor 3 it begins to take form as a consistent 
trait related to two processes. Finaliy in mosi recent two floors it joins with heat treating as an important trait complex.

As shown above, the model proposed for flake production seems to apply to the later period because causally related attributes of ten appear on components together and thus can be assumed to represent true causal relationships. In the earlier period, however, the causal formation breaks down. All of the indications seem to be that the earljer floors represent bifacing while later knappers practiced another technique, probably core flaking.

It has been shown elsewhere that the general trend through the Holocene in Central Texas has been for bifacing to replace core flaking (Gunn and Weir 1976). However, in the San Marcos Phase (1800-2800 B.P.) there was a slight resurgence of core flaking technology, apparently associated with the re-entry of people associated with the Plains into the region.

The most plausible interpretation would seem to be that OF3 and OF4 are associated with the highly bifacial oriented Round Rock Phase of the Central Texas Archaic while the upper two occupation floors are related to the San Marcos Phase or to Late Prehisioric cultures practicing core flaking technology. The latter must be considered because of the high incidence of small arrow points recovered from the site.

Future research into the model appropriate for flake technology analysis should give emphasis to constructing a concept which will encompass both core and bifacing technology. The model used in this study seems to be appropriate to core flaking but all that can be said with assurance about the bifacing flakes is that some traits are logically related; mainly we just know it is not a core flaking technology.

\section{Flake Morphology}

In recent years it has become increasingly apparent that flakes are at the same time the most abundant and most neglected archaeological resource. The following is an attempt to distinguish variation in flake morphology using a method of analysis that is fast and reliable and would therefore be useful in characterizing large assemblages of debris.

The shape morphology of whole flakes was recorded by outlining them on a polar coordinate grid of nine rays and measuring from the origin of the polar coordinates to the point where flake outline intersects the rays (MontetWhite 1973). The platform is centered on the origin with the trend of the platform parallel to the bottom line of the grid (see Flake Technology Coding format). The rays are $18^{\circ}$ apart. Observations were made by classes defined in $\log \mathrm{mm}$, see attributes 32-40 for full discussion. The weight of flakes in .1 grams (.01 would be better) was also analyzed to get an estimate of thickness. Principal components were calculated on the data from the various occupation floors and rotated to varimax criteria. Two components generally appeared from each occupation floor, one for length and one for width. It was hoped that subtle variations in these component structures would show 

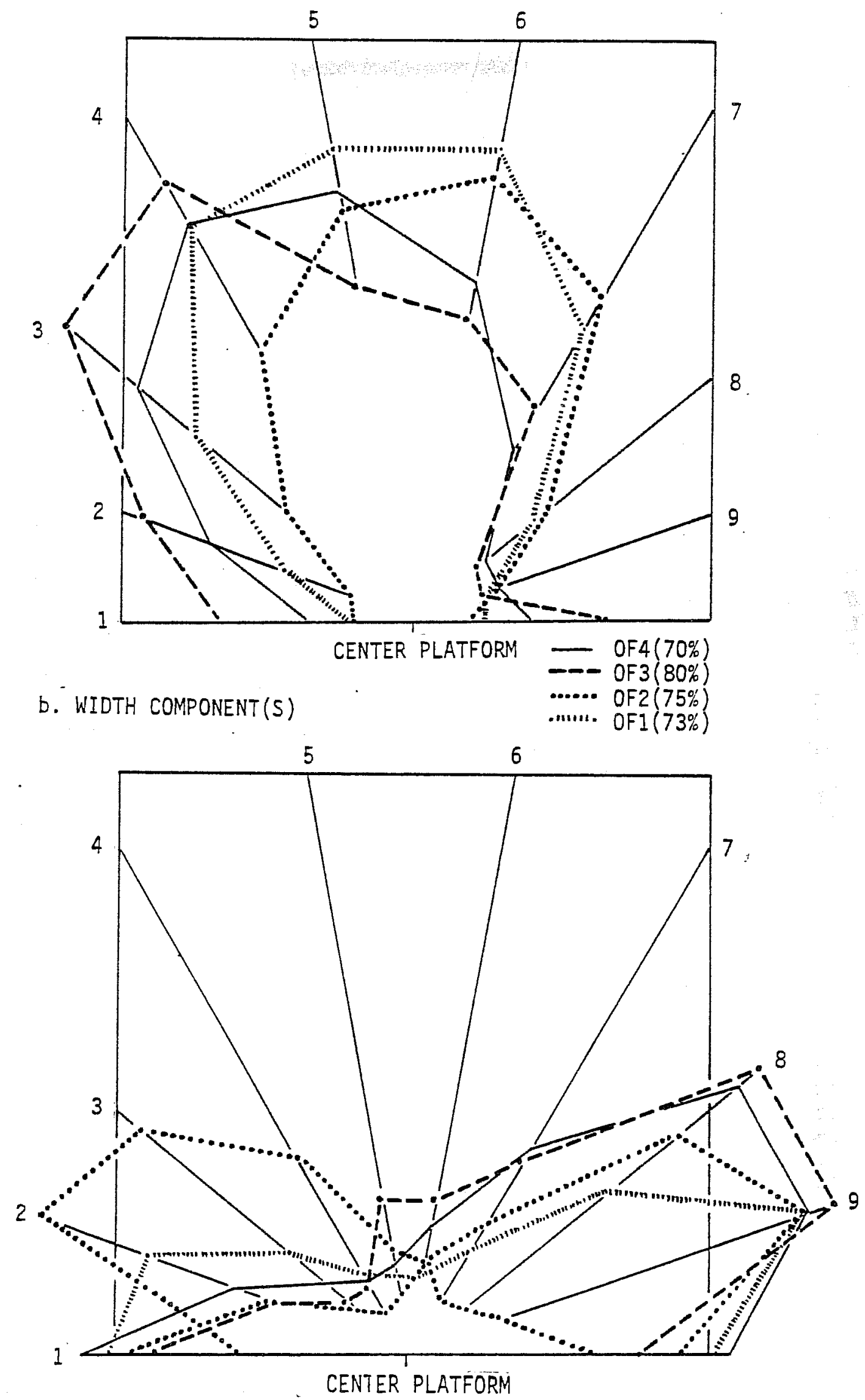

Figure 17.16. Length and Width Components of Hop Hill Flakes from Midden Occupation Floors. 
the characteristics of the various time periods with which the floors were associated.

Figure 17.16a-b are plots of the components. Distances from the origin are component loadings and therefore represent the degree of correlation between adjacent rays. (Component loadings are transformed by the antilog of the loading to emphasize larger values.)

In the length vectors, Occupation Floors 2.30 and 2.40 are asymmetrical to the left while Floors 2.10 and 2.20 are essentially symmetrical. Floor 2.30 is typically the most aberrant. The variability is probably a reflection of the change from bifacing to core flaking technology dealt with above, and could serve as the basis for characterizing assemblages.

There is apparently a tendency for bifacing flakes to be asymmetrical to the left while core flakes are symmetrical. Sollberger (1976) has pointed out that diagonal or chevron flaking is desirable and in some cases necessary to biface points. The degree of asymmetry and perhaps the direction of asymmetry could be used as an indicator of the core-biface flaking mix in an assemblage and as an indicator of period of manufacture. This would require reliably large collections of flakes from known time periods before assemblages of unknown chronology could be classified.

An index of asymmetry, the application of a goodness-of-fit test or the use of discriminant function as in Gunn and Prewitt (1975) could be used as classificatory devices.

\section{Conclusions}

The most important finding to come from analysis of the flakes on sequentially related occupation floors at the Hop Hill midden is the apparent shift from bifacing to core flaking technology through relative time. Both the causal forceflaking model and the Flake Morphology analysis suggest changes in attributes which could logically be attributed to such a technological shift.

While the finding does not provide a firm basis for placing the survey results in temporal perspective it does suggest that the core analysis pertains more to later occupations while the biface analysis is more important to earlier times. Based on lipped flakes, Hester found a similar diminution of bifacing at La Jita (1971:113).

As a consequence of the shift in techniques the inability of a commonly held model of flake mechanics to explain both core and biface flaking became apparent. More theoretical development is necessary in this area.

Finally, there seems to be variability in the symmetry of flakes depending on the technology used. Bifacing flakes are asymmetrical to the left while core flakes are symmetrical.

Since the two technologies have definite ups-and-downs in the region, an index of symmetry may prove useful as a means of roughly dating sites where no other chronological information is avajlable. 


\subsection{LITHIC RAW MATERIALS (James Escobedo)}

Rock samples collected at the Hop Hill locality were compared to artifacts representing the four occupation floors. The rock samples were broken open, exposing the interiors, then divided into five major types and one subtype (Table 17.1.01).

Three criteria were used to classify materials into types:

1. The amount of clay in the silicates as shown by translucence;

2. The range of grain structure; and

3. The color of the material which ranged from light red to yellow, brown, gray and black.

\section{Table 17.1.01 Chert Types From Hop Hi11 Quarry}

TYPE 1 Number sampled - 20, cobble size

Translucent-very fine grain--dense with banding--thin limestone cortex--dark

interiors--color range: black-dark/brown-dark/gray.

TYPE 2 Number sampled - 26, cobble size

Translucent-fine grain--dense with banding--thin limestone cortex--light

interior--color range: light/gray-reddish/brown-light/brown.

TYPE $2 a$ Number sampled - 13, cobble size

Translucent-medium grain fl.jnt--dense with white spots--thick limestone cortex-medium interiors--color range: brown-light/brown.

TYPE 3 Number sampled - 15, cobble size

Opaque-medium grain chert--dense with high amounts of clay--thin limestone cortex--medium to light interiors--color range: brown-brown/yellow.

TYPE 4 Number sampled - 17, cobble size

Edge translucent to opaque-coarse grain--medium dense with evidence of air pockets during crystallization--thin, rough limestone cortex--light interiors-color range: iight/brown-yellow/brown-gray.

TYPE 5 Number sampled - 9 chunks

Opaque-fine to medium grain--dense with white spots--thin, rough limestone cortex--light to dark interiors--color range: 1ight/gray-reddish/white-reddish/ brown.

The exotic category contained all those flakes that could not be classified according to the local core samples. In all there were 17 exotic items (Table 17.02). These had several common features, one being that they were red flint and chert flakes. Although there are reddened nodules in the local collection, none had the red saturation of the exotic pieces. 
Table 17.02 Breakdown of Artifacts to Rock Samples

LEVEL 190 Artifacts

Type $1-12$

Type 2- 46 Type 2a-7

Type 3- 7

Type 4- 8

Type 5- 3

exotic- 7

LEVEL $3 \quad 23$ Artifacts

Type 1- 2

Type 2- 12 Type 2a-2

Type 3- 4

Type 4- 2

Type 5- 0

exotic- 1
LEVEL $2 \quad 143$ Artifacts

Type 1- 10

Type 2- 90 Type 2a-6

Type 3- $\quad 15$

Type 4- 12

Type 5- 4

exotic- 6

LEVEL $4 \quad 65$ Artifacts

Type 1- 6

Type 2- 32 Type 2a-4

Type 3- 17

Type 4- 1

Type 5- 2

exotic- 3

of the most common materials found on the occupation floors, type 2 with 180 items, had the highest total of matches with source materials. The rest followed as shown:
Type $2-180$
Type $3-43$
Type $1-30$
Type $4-25$
Type 2a- 19
Type $5-9$

Type 5 contained in its description red color material. However, these were sma 11, exhausted cores. No nodules from the Hop Hi11 Quarry were found to contain this red material. Type 5 was also a category in which most of the flint was already exposed by previous knapping. 
This study shows that some outside lithic material may have been carried into the site during all levels of occupation. However, locally gathered materials were generally used in knapping. 


\subsection{ENVIROCULTURAL SYSTEM FOR CENTRAL TEXAS (Joe1 Gunn)}

The previous sections of this study have dealt with the various elements of the cultural and environmental system which could be observed or inferred for the inhabitants of the Hop Hill locality.

Ultimately, the goal of any such study is to conjoin elements into reasonable representation of the whole system, the ecosphere as it was discussed in the Introduction. Of the two benefits that come from such an exercise, two deserve emphasis here. First, a systems level examination of processes allows for broader and more insightful perception of the probable lifeways of prehistoric peoples whose lives we can only examine in general terms. Serendipity, (unexpected insights) is often the product of such undertakings. Second, at a site like Hop Hill, the observed appearances are often suggestive but may also be misleading due to pilfering and/or destruction of the record. Kelly, for instance, thinks that larger points were probably collected from the site because of their greater visibility to collectors. This would tend to bias estimates of earlier occupation frequencies downward since larger points are earlier points. A we1l-worked-out simulation which took into account factors of climate, population density, cultural adaptation, etc. could project probable occupation frequencies based on known cultural and climatic data and thus suggest alternatives to some of the more obstinate unknowns.

In this section, a system trajectory is adopted for the Hop Hill locality. The climatic aspects of that trajectory are derived from previous discussion in this study. The cultural trajectory adopted is that of Weir. While his sequence is not fully accepted, it does offer conceptual underpinnings in systems and information theory and is therefore infinitely more compatible to the approach taken here than chronologies founded in $7 i$ thomorphic change.

Further assumptions are made about the character and source of cultural adaptations under varying climatic regimes, and from these assumptions a system is derived which attempts to explain some of the observed variation in Archaic sett? ement patterns and occupation frequencies. Since time precludes programming of the more complex statistical properties of the system, the reader will have to be content with the serendipity of system building for now. A commitment has been made, however, to program a simulation of the system and a report will be issued in a later publication.

Central Texas Cultural Trajectory and Cultural Processes

The Central Texas Archaic has long been regarded as a very endurable and stable Holocene adaptation to a prairieland environment. Recent studies confirm that culture change in the nomal sense of transformation from technologically simple to technologically complex is indeed absent. There is every reason to believe, however, that Central Texas is environmentally unstable and required numerous adaptive responses on the part of the nomadic people who occupied it prehistorically. The evidence for this instability is accumulated in this volume and constitutes the empirical background for what follows. 
Frank Weir (1976) systemically analyzed the Central Texas Archaic which he defines as a period from $700 \mathrm{BP}$ to $8000 \mathrm{BP}$. It is divided into five Phases (Table 18.01).

Table 18.01 Weir's Central Texas Phases

\begin{tabular}{|c|c|c|}
\hline & Late Prehistoric & $\begin{array}{l}\text { Dates BP } \\
400-1500 \\
\end{array}$ \\
\hline $\begin{array}{l}\text { Central } \\
\text { Texas } \\
\text { Archaic }\end{array}$ & $\begin{array}{l}\text { Twin Sisters } \\
\text { San Marcos } \\
\text { Round Rock } \\
\text { Clear Fork } \\
\text { San Geronimo }\end{array}$ & $\begin{array}{l}7000-2000 \\
1800-2800 \\
2600-4200 \\
4000-5000 \\
4500-8000\end{array}$ \\
\hline & Paleo-Indian & $7000-?$ \\
\hline
\end{tabular}

Weir's phases are bounded periods of relative systemic stability separated by episodes of relative instability and adaptive adjustment. The goal of the investigation was to study "the complexity of those subsystems that regulated stability and effected change in the five phases of the Central Texas Archaic" $(1976: 5)$. Each phase is a period of stability fostered by negative feedback.

The mechanisms of positive feedback and "kickers," or unpredictable (by the system) events, are posed as the reasons for changes from one mode of systemization to the other. The interface between phases is bridged by a brief kicker induced period of positive feedback.

Three negative feedback configurations are adopted from Birdsell's array of demographic equilibrium processes as being applicable to Central Texas. The Density process provides that, given environmental restriction, any population deviation will be restored to its normal relationship to the environment. If the population is deflected downward it will be restored to normal by excessive human fertility. If it is deflected upward, it will be restored to normal by groups budding off to colonize areas of below normal population.

The Communications process maintains the size of dialectical tribes which are social units composed of local bands speaking the same dialect. Spacing of bands is again controlled by the environment since groups can only be as close as the food supply will allow. On the other hand, if groups are too far apart, they will lose their tribal identification and split into more dialectical tribes reducing the tribal size. If bands of a tribe are too few in number, their identity is overwhelmed by their neighbors and they lose their tribal coherence. The balance between too few and too many groups, therefore, has to be close to a normal value for the tribe to retain its dialectical integrity.

The Local Group process maintains the fundamental social unit at above 25 persons. A smaller unit is not demographically viable for a number of reasons. 
Among them is the fact that a group of less than 25 has precariously few women of reproductive age. Birdsel 1's original idea presumed that the size of the local groups is adjusted upward as resources allow for the gathering of larger numbers of people into smaller spaces. Some recent thinking by Sahiins (1972) and Wobst (1974) seems to suggest that the local group may in fact seek an equilibrium which tends downward toward 25 rather than upward toward carrying capacity. This point of view will be elaborated in subsequent discussions. Each of these processes controls key variables in the cultural system.

Table 18.02 Summary of Weir's (1976:120) Findings, Variable Trajectories

\begin{tabular}{|c|c|c|c|c|}
\hline $\begin{array}{l}\text { Late Prehistoric } \\
(400-1500)\end{array}$ & $\begin{array}{l}\text { SOCIO-POLITI } \\
\text { STRUCTURE }\end{array}$ & $\begin{array}{r}\text { CAL SPECIAL- } \\
\text { IZATION }\end{array}$ & $\begin{array}{l}\text { SPACIAL } \\
V \text { DELIMITA- } \\
\text { TIONS }\end{array}$ & CHARACTERISTICS \\
\hline $\begin{array}{l}\text { Twin Sisters } \\
(700-2000)\end{array}$ & unstruc & ired & undel imited & Tess population \\
\hline $\begin{array}{l}\text { San Marcos } \\
\quad(1800-2800)\end{array}$ & unstructured & specialized & undel imited & $\begin{array}{l}\text { pop. down, difused } \\
\text { economy, bison }\end{array}$ \\
\hline $\begin{array}{l}\text { Round Rock } \\
\qquad(2600-4200)\end{array}$ & $\begin{array}{l}\text { highly } \\
\text { structured. }\end{array}$ & specialized & undel imited & $\begin{array}{l}\text { coalescent, focal } \\
\text { economy, bifaces }\end{array}$ \\
\hline $\begin{array}{l}\text { Clear Fork } \\
\qquad(4000-5000)\end{array}$ & unstructured. & specialized & delimited & $\begin{array}{l}\text { acorns, burned rock } \\
\text { middens }\end{array}$ \\
\hline $\begin{array}{l}\text { San Geronimo } \\
(4500-8000)\end{array}$ & unstructured & unspecialized & undel imited & east-west influences \\
\hline
\end{tabular}

Table 18.02 summarizes the trajectories of the various cultural quanities Weir attempts to estimate in his analysis. He ultimately encapsulates these trends through time in the sequence "dissemination-coalescence-proliferation." During the early part of the Holocene the San Geronimo (4500-8000 BP) people subsisted in a generalized fashion on small game and gathering. They were probably organized very informally and unspecialized in their technological functions. No particular limits to their domain can be found. They seem to have been at the crossroads of the East and West as well as being influenced from the Southwest.

With the Clear Fork (4000-5000 BP) Phase structures began to move toward coalescence. Population appeared to increase, and social organization and technology became more complex and specialized, perhaps at the beckon of oak woodlands which supplied abundant acorns as a new source of energy. Burned rock 
middens increased in number and complexity and the Clear Fork Gouge was prominent in the tool inventory; it was probably a woodworking tool. There was only a hint of influence from the East. On the main the Central Texas Archaic seems to have taken a character and role of its own.

In the subsequent Round Rock Phase (2600-4200) these trends were carried to exorbitant extremes. All of the evidence indicates a tremendous, highly structured population, a focal economy and a completely local and strong character to the culture. Bifacing tools during this period were so dominant that they are the most characteristic feature of the lithic technology for the entire Archaic trajectory. Weir speculates that the Round Rock people may have been instrumental in expanding the potential of their environment by unintentionally spreading acorns to all parts of the countryside. Subsequent work (Gunn and Weir 1976) shows that this phase flourished in a cool, damp period which would have encouraged an eastern woodland life environment.

At the beginning of the San Marcos Phase (1800-2800 BP) bison reoccupied the southern plains and with them came influences and/or people of the Plains. Whether by barbarian invasion or some other means, the bison hunters appear to have shattered the Round Rock hegemony. Population dropped off and the economy became diffuse. Organization and local integrity of the culture disappeared.

By the time of the Twin Sisters Phase (700-2000 BP), the fickle bison returned to the north and the Central Texas Archaic was left as it began, small game hunters and gatherers. Weir believes that with the exception of bows and arrows this assemblage and pattern of life continue through the Late Prehistoric period.

The relationship between the succession of cultures in Archaic Texas, the lithic assemblages which mark their passage in the archaeological record, and progressive developments in climate are discussed in Gunn and Weir (1976). Numerical analysis shows that there is a general trend through time from a flake core lithic technology to bifacing. This trend is buffeted about, either hurried or retarded as the case may be, by periods of radical change in environment. Some time after the Round Rock Phase there seems to have been a slight return to core flaking technology (Hester 1971; and Section 17.0, Flake Technology, this volume).

\section{Modern Biota}

As has been suggested, these changes in technology can be interpreted as cultural responses to fluctuations in global climatic parameters which presumably act through the intermediary of biota to vary the available necessities of life. The basic outline of past biota can be best understood in terms of the modern biotic distribution. 


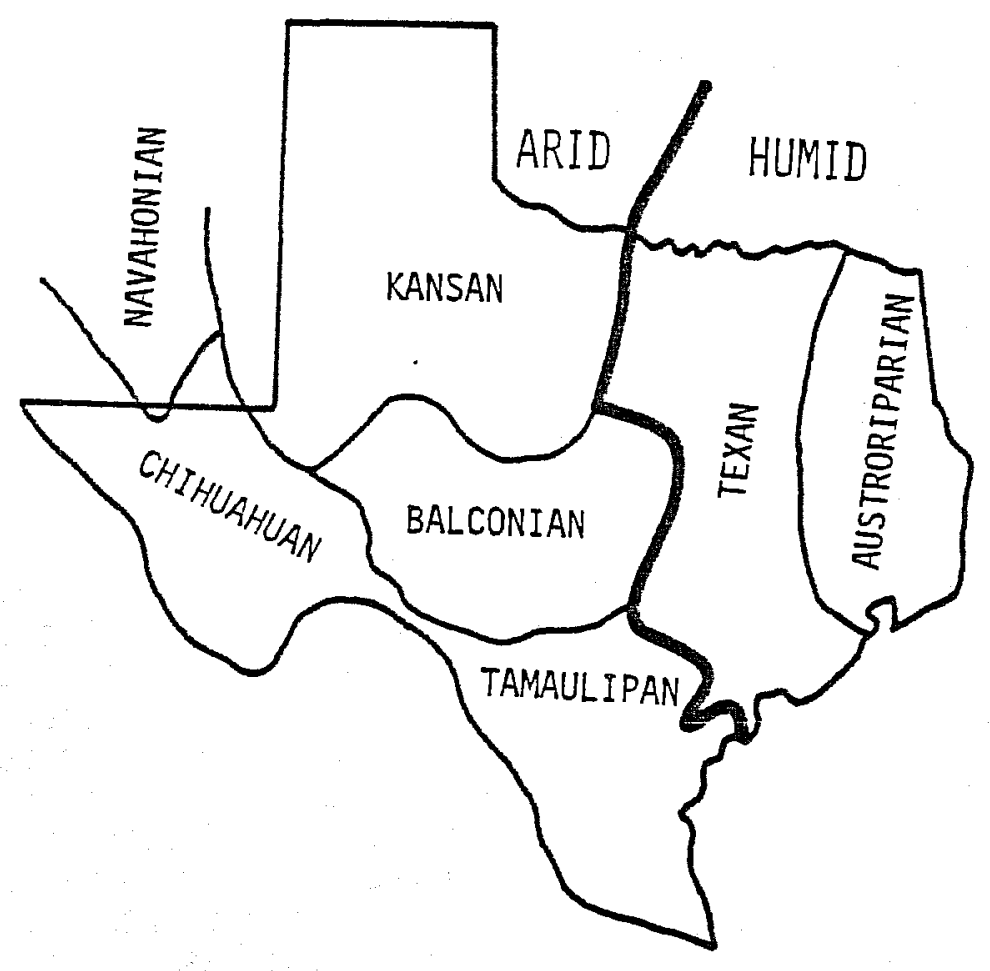

Figure 18.01. Biotic Provinces and Supraprovinces of Texas. (Adapted from B7air 1950) 
The classic work on biotic provinces of Texas was published by Blair (1950) and is probably the most cited publication in the archaeological literature of the region. Blair compiled his assessment of Texas biotic provinces from various surveys of plant and animal $1 \mathrm{ife}$ made in 190i, 1905, 1931, 1938, 1939, $1940,1942,1943,1944,1946,1947,1948$ and 1949. There was notable change in global temperatures during these five decades (see Section 4.0). However, the bulk of the work appears to have been done in the 1930 s and 1940s. In a way, this is ideal because these two decades represent the apex of global temperatures during the present cold-warm-cold cycle. Being at the top of a cycle mitigates to some extent the problem of claibrating the present regime with those of the past.

According to Blair,

A great area in central Texas, from the Pecos on the west to the western boundary of the Austroriparian forest on the east, is principally a region of transition between the Sonoran and Austroriparian biotas. In this area the two major biotas intermix or interdigitate... Two biotic provinces, the Balconian and Texan, are recognized in this transition area... Several obvious factors influence the distribution of plants and animals in the transition area, or great ecotone, that comprises much of the area of Texas. These factors are principally edaphic or physiographic ones. Austroriparian species may be expected to range westward in this area wherever local conditions result in more mesic environment than the regional average. Western species may be expected to occur eastward in this area where local conditions produce a more xeric environment than the regional average... The stream systems of Texas provide important routes for the westward distribution of Austroriparian species into comparatively arid, generally treeless environments... Rock outcrops on dissected piateaus and on escarpments provide avenues for dispersal of... Chihuahuan province species...eastward (B1air 1950:95-96).

Settlement Patterns

Recent work by Valdez (n.d.) shows that when the biota of these zones are reduced by multivariate procedures and plotted, the Austroriparian and Texan Zones, wet provinces to the east, are very close together and form a group which contrasts strongly with the Kansan, Balconian and Tamaulipan Zones, dryer provinces to the west. Since the grosser biotic characteristics of a given piece of land determine the general character of cultural adaptations to it, in the following study Texas will be assumed to be composed of two culturally relevant biotic supraprovinces, the Humid and the Arid Zones. Cultural adaptations to these zones are taken to be analogs of the Desert Archaic for the Arid Zone and the Eastern Archaic Tradition for the Humid Zone. While both adaptations involve a certain amount of annual movement in the quest for subsistence resources, the Eastern woodland tradition is more of a transhumant process while the western tradition is more of a nomadic seasonal round with a number of collecting stations of relatively equal importance. Without being too specific I would suggest Steward's (1938) Great Basin Study of the Shoshone as an extreme example of western nomadism and Winters' (1969) Riverton Cuiture as an example of the Eastern Archaic Tradition. The Desert Tradition should be reflected in the archaeological record as sites of similar and small size while the Eastern Tradition 
sites would be found to contrast in size between more permarient settlements and seasonal, transhumant collecting stations.

An important distinction to be made between the settlement patterns of the far western Desert Archaic and the probable semi-arid Archaic of western Texas is this. The systems of the desert are oriented to the exploitation of broad, unbroken stretches of ground which approach Christaler's ideal of an unbounded plain with all resources equally distributed (Hester et al. 1977). As Blair's description shows, however, west Texas is not of such a nature. Relatively rich riparian zones infested with the dense fauna of the eastern woodlands finger out onto an otherwise sparsely vegetated plain. Resources are clearly not equally distributed. Under such conditions base camps can be expected to be located near these richer areas. On the other hand, such river valleys are not broad enough to provide a stable existence and frequent exploitation camps can be expected in the open areas as well, evidence of the systematic exploitation of the interriparian areas. As a hypothesis, it might be suggested that the uplands would be less intensively exploited as floodplains become broader and richer to the east.

The nomadic and transhumant processes associated with these settlement patterns can likewise be inferred to imply somewhat different cognitive approaches to coping with the environment. Relative to the Desert Tradition, recent anthropological studies of nomadic hunters and gatherers have virtually reversed everything that has been thought about them for millenia. Empirical studies such as those by Lee and Devore (1973) show that theywere not pitiably hard pressed victims of circumstance. More importantly Sahlins (1972) has demonstrated that they probably had a view of their economic world which was diametrically opposed to our own and whichwas founded in the realities of their technology, population density and resource availability. That economic philosophy is most readily summed up as "do as little as you can and carry as little as you can with you." This sounds like the western definition for laziness but in primitive cultures it is a viable strategy for survival which the most ambitious people cleverly follow. (Sahlins thinks the concept of laziness was probably a neolithic invention and I am sure Childe would have said it was the invention of priests in order to subvert the masses.) The full impact of these ideas is difficult to realize until they are implemented into our systems formulations of how primitive cultures operate. Wobst (1974) has shown that the "magic number 25," which seems to an almost ubiquitous band size, represents the minimum demographic unit which can be expected to survive stochastic fluctuations in demographic variables.

It has always been assumed before that primitive cultures, like our own, were continually pressing against the carrying capacity of the land and no little effort has been expended groping about for an elusive formula which shows how much below carrying capacity primitives tried to maintain themselves in order to avoid problems of overpopulation. The present trend of research seems to show that primitives did exactly the opposite. They tried to see how small they could make their population without getting caught by the "fickle finger" of stochastic, demographic fate. They are trying to depress rather than increase 
their population. The reasons are quite tenable within the context of huntergatherer philosphy. The more people there are in a group the harder everyone has to work. This is easily demonstrated by the fact that the more people there are in a band the sooner the resources are used up around a camp and the sooner everyone has to move to a new location. Therefore, keep population of the band to a bare minimum. In the case of primitive cultures, then, the equilibrium is not between population and carrying capacity; the equilibrium is between demography and chance. They gamble with the fate of the band rather than the fate of the individuals who would starve if the carrying capacity were overrun. When it is considered that bands are loosely organized and if a band proves inviable its members simply disband and go to live with relatives in other groups, the gamble seems remarkably palatable. As Birdsell (1973) has pointed out, their loyalty is with the dialectic tribe rather than the band.

It seems to me that this orientation has the potential to bring order out of chaos of thinking that has revolved around primitive society for the last few years.

The next question has to be, "How does carrying capacity fit into the picture?" It obviously has an influence or people would be spaced over the world in an even distribution regardless of the density of resources. In principle carrying capacity increases the number of people that can be clustered in an area without causing more work for everyone.

Increasing density can be effected by packing bands closer together or by increasing band size. The characteristics of the two approaches are radically different. Band packing can be accomplished with no fundamental changes in the social organization of the band. Band enlarging, on the other hand, requires an elaboration of the social structure to keep the increased unit size functioning in an orderly manner. As Flannery (1972) has pointed out, elaboration of the social structure requires that everyone pay overhead to ceremonialism or to those appointed to position of power. In terms of primitive economic philosophy this is causing everyone more work and therefore would not be an acceptable alternative.

Band enlargement requires a different philosophy of life. For one thing the populace has to be willing to give up the principle of least work and pay the overhead to maintain ceremonial or governmental mechanisms to direct the order of social progress. Furthermore, such organization appears to be inextricably bound to the ability to materialize the symbols of power and order. People of the primitive order could carry elaborate symbols about only with difficulty. Thus, band enlargement and social elaboration implies some form of sedentariness.

So far as we can guess, Central Texas was generally occupied by people of the Desert Archaic variety except for periods of lower global temperature during which the eastern woodland and presumably eastern woodland-like people would have dominated the biotic and the cultural milieu. Exceptionally large sites during the Round Rock Phase of the Archaic which was during a cold interval tend to confirm this (Weir 1976; Prewitt 1974 ; Gunn and Weir 1976). Based 
on the foregoing discussion the assumption is made that these people were of a very different philosophy of 1 ife and would have been adverse to their Desert Archaic contemporaries to the west. This diversity of subsistence and economic operation would have dampened serious interaction across the ecotone between the Humid and Arid Zones.

In summary, then, general explanation of prehistoric demography and site distributions in Central Texas can be encompassed by adequate modeling of three processes. The first is the shift of the broad Arid-Humid ecotone in concordance with global temperatures. The second is the nomadic, labor reducing, broad spectrum subsistence adaptations of the Desert Archaic people of the Arid Zone. The third is the transhumant, labor inducing, focal economy of the Eastern Archaic Tradition. In the following pages an attempt will be made to reduce these processes to mathematic and statistical relationships. The effort is superficial and tentative, a first step intended to explore the potential of the approach as a tool for the study of prehistoric Central Texas.

\section{Envirocultural Model for Occupation Frequency}

It should be noted that in the following discussion "A model... is a description of subsystems within the system being studied, each having its own properties and al1--interacting together according to their individual properties--being responsible for observed appearances" (Powers 1973:14). Thus, concepts such as density equilibrium, communications equilibrium, local group equilibrium as per Birdsell and Weir, and labor equilibria, subsistence spectrum equilibria, mobility equilibria, etc., are observed properties of socio-cultural groups or subsystems. They are the variables which control the subsystems and result in the observable archaeological record.

The first and perhaps easiest problem to deal with is a deterministic mapping of climatic conditions in Texas at different global temperature levels. The formulas that follow are systems of equations which conjoin climatic, temporal relationships. The driving force is a climatic curve of average Northern Hemispheric temperatures (National Academy of Science 1975; Section 3.0, this volume).

During the 1931-1969 period, Texas received an annual average of $1422 \mathrm{~mm}$ of precipitation in the East and $200 \mathrm{~mm}$ in the West. A study of Texas weather patterns (Bielser and Gunn this volume) suggests resolution of precipitation into two vectors representing two processes. Spring and summer rain is predominantly from a monsoon effect which draws supersaturated air off the Gulf of Mexico and deposits it on land as rain, due either to cooling in the spring or convection cells during the summer. The effect of monsoon and convection circulation spreads westward with cooling of global temperatures. Fall and winter precipitation appears to be the product of a peculiar relationship to the mountains in Western North America. Warm global temperatures drive the westerlies northward so most of the moisture is combed out of the clouds in California and in other high mountainous regions. Global cooling, however, general7y moves weather systems southward (Sanchez and Kutzbach 1974). To the west of central and south Texas, the mountains of northern Mexico are relatively low and they allow moisture to pass into the southern Plains. Here northern 


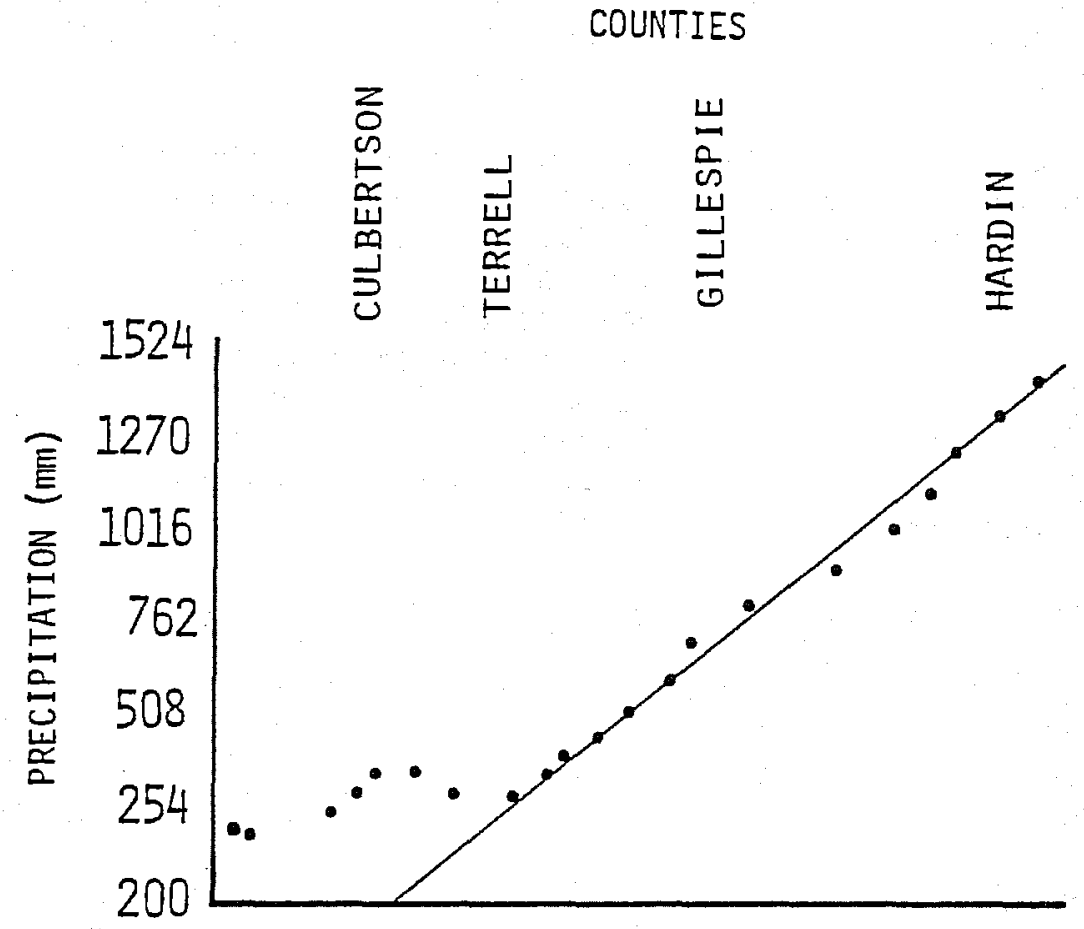

Figure 18.01. Monsoonal Precipitation Gradient on an East-West Transect Through Study Area. 


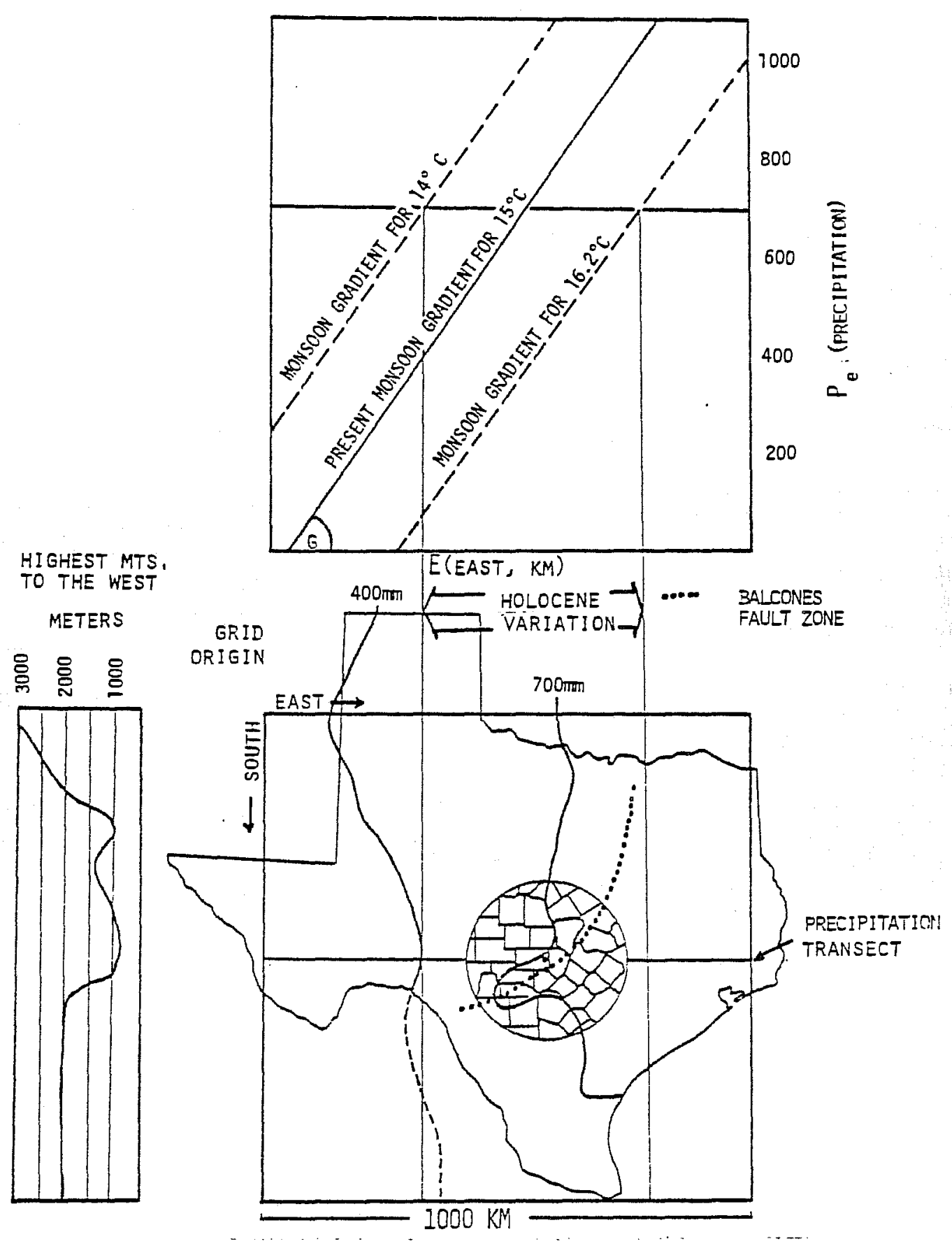

Figure 18.03. Climatic Variation During the Holocene. 
cold fronts collide with the moist westerlies causing spectacular increases in precipitation during colder winters.

Estimating how far changing global temperatures displace the monsoon circulation is something of an inexact science right now. However, Jose Enrique de la Pena who traveled with Santa Anna's army to the Alamo in the winter of 1936 reported a very hard journey due to weather conditions which do not resemble those in south Texas and northern Mexico today. He saw cypress trees in northern Mexico about 300 kilometers west of their present range which is in areas of greater than $700 \mathrm{~mm}$ of rainfall (Figure 18.02). This would effectively move the $700-\mathrm{mm}$ rainfall 1 ine out to the present $400-\mathrm{mm}$ line. 1836 was preceded by a half -century of lower than usual sunspot activity (Eddy 1977); and judging by several graphs such as those published by the National Academy of Science (1975), the temperature of the Northern Hemisphere was probably depressed about $1.5^{\circ} \mathrm{C}$. Until better evidence is found a figure of $200 \mathrm{~km}$ displacement per $1^{\circ} \mathrm{C}$ of climatic change wi 17 be assumed. $\left(300 \mathrm{~km} \div 1.5^{\circ} \mathrm{C}\right)$

The $1^{\circ}: 200 \mathrm{~km}$ ratio is somewhat questionable because of the simultaneous southward movement of the westerlies. The westerlies, however, affect fall and winter precipitation and the mesic cypress would have to be sustained through the summer by monsoonal activity. It may, therefore, be at least of the correct order of magnitude. It is a conservative estimate because we do not know how far cypress extended west beyond the place where de la Pena saw them.

To simplify the problems of calculating monsoonal displacement, a 1000 meter square was superimposed on Texas as is illustrated in Figure 18.03. Figure 18.02 shows the present monsoonal precipitation gradient on an east-west transect that goes across Gillespie County where Hop Hill is located. If the precipitation is $305 \mathrm{~mm}$ at $250 \mathrm{~km}$ east on the grid and $1397 \mathrm{~mm}$ at $100 \mathrm{~km}$ east, the moisture gradient across the grid is like the triangle at the top of Figure 18.03. The trigonometric formula to find the opposite leg of a triangle when the angle and adjacent leg are known is:

$$
\frac{P}{E}=\tan G
$$

Since we know $P$ (precipitation in $\mathrm{mm}$ ) and $E$ (distance east in $\mathrm{km}$ ) in both cases we can solve for angle $G$ by subtracting the unknown displacement distance (D) from both equations and setting them equal to each other. Cross multiplying and solving for D obtains:

$$
\tan G=\frac{P}{E-D}=\frac{305 \mathrm{~mm}}{250 \mathrm{~km}-D}=\frac{1397 \mathrm{~mm}}{1000 \mathrm{~km}-D} \therefore D=40.5 \mathrm{~km} .
$$

Substituting $D=40.5$ we find that $\tan G=1.45$.

If we want to find out what the precipitation is at any point on the east-west gradient $\left(\mathrm{Pe}_{\mathrm{e}}\right)$, say $560 \mathrm{~km}$ east, it can be obtained by reformulating equation 18.02: 


$$
P_{e}=\tan G(E-D)
$$

Entering the Values:

$$
\mathrm{Pe}=1.45 \quad(650-40.5)=884 \mathrm{~mm} .
$$

If it is assumed that the gradient stays the same when global temperatures chane, then changes in precipitation can be represented as changes in the length of $D$. It was decided earlier that 1 C would represent a $200-\mathrm{km}$ shift in the precipitation regime. Since the present displacement is $400 \mathrm{~km}$ and the present mean temperature of the Northern Hemisphere is $15 \mathrm{C}$ a displacement factor can be calculated as:

$$
D=[(C-15) 200]+40.5,
$$

where $C$ equals the new mean hemispheric temperature. Calculations for $14^{\circ} \mathrm{C}$ and $16.2^{\circ} \mathrm{C}$, the lowest and highest Holocene temperatures, were made and entered on Figure 18.03 as dashed lines. Lines projected from where these estimates intersect the $700-\mathrm{mm}$ rain line on the graph onto the map show a rough estimate of the range of variation of the Humid-Arid ecotone in post-Pleistocene times. The Hop Hill locality in Gillespie County is in the center of the zone of variation.

Estimating values for the north-south or westerlies gradient is even more difficult. Warmer winters bring less rain to both south and north Texas because the westerlies move out of south Texas and points west capture the rain that might have come to the central and northern sections of the region. As the westerlies move south, the south receives unbelievable increases in rainfall. The fall of 1976 saw as much as 50 inches of rain in a previously semi-arid region. Water tables rose to higher than any point on record and springs appeared that had been dormant within the scope of living memory.

For now let us assume that the area down specified jet stream tracks receives rain in inverse proportion to the height of the mountains to the west and in inverse proportion to the distance from the center of the jet stream. The center of the jet stream and accompanying westerlies will move southward in inverse proportion to the mean November temperature. In 1976 the mean November temperature was $8.33^{\circ} \mathrm{C}\left(47^{\circ} \mathrm{F}\right)$ which is $2.00^{\circ} \mathrm{C}$ below $19 x \mathrm{x}$ and it moved the jet stream $400 \mathrm{~km}$ south.

$$
\frac{400 \mathrm{~km}}{2}=200 \mathrm{~km}
$$

As equation 18.06 shows, we can take a $200 \mathrm{~km}$ per ${ }^{\circ} \mathrm{C}$ shift as a working figure. This rate of change implies a $5^{\circ} \mathrm{C}$ potential from north to south in the $1000 \mathrm{~km}$ grid.

Since the jet stream tracks are northwesterly in origin the location of the jet stream in 1976 cannot be directly to the west of point E-S (Figure 18.03). If.a storm track (T) $22^{\circ}$ north of west is assumed, the relevant point on the west 
margin of the grid is $\mathrm{J} \mathrm{km}$ south of grid origin, where

$$
J=S-[\tan T(E)] \text {. }
$$

Thus, for a point at $E=500, S=700$,

$$
J=498 \mathrm{~km}=700-[.404(500)] .
$$

The location of the jet stream for a given year is calculated as

$$
J=[(C-8.33) 200]+498 \text {. }
$$

The height of the mountains at point $J$ is the quantity which is inversely related to rainfal1. Judging by 1976 the jet stream coming through at the lowest point has the potential to increase fa 11 precipitation about $760 \mathrm{~mm}$. The mountains range from 900 to 3000 meters, a range of 2100 meters. Dividing precipitation potential by elevation range shows that a meter of elevation influences $.36 \mathrm{~mm}$ of precipitation. Thus, if mountain height is 2300 meters, westerly precipitation at the center of the jet stream is,

$$
P_{s}=252 \mathrm{~mm}=[2100-(H-900)] .36 .
$$

While it makes sense that the amount of precipitation would decrease away from the center of the jet stream I have not studied the rate of diminution. Just as a working figure the distance from the jet stream on the western grid margin was divided by 50 and the inverse of that number multiplied times precipitation at the center of the jet stream. If a site were $200 \mathrm{~km}$ from the storm track, then the influenced track calculated in (18.09) would be:

$$
\begin{aligned}
& P_{S}=\frac{1}{(200 / 50)} \quad 252 \mathrm{~m}=63 \mathrm{~mm} \\
& \text { (note: distance to the storm track must be }>50 \mathrm{~km} \text { ) }
\end{aligned}
$$

For any given location:

$$
P=P_{i s}+P_{e} .
$$

On the left Figure 18.03 shows the elevations of mountains directly to the west. Since weather systems veer slightly south of east it is south rather than central Texas which receives the precipitation. Because the precipitation is in the fall and winter one might question the utility of modeling this source of moisture. Our research, however, has shown that summer temperatures are affected far more than winter temperatures. Over the last two decades summer monthly means in the vicinity of Hop $\mathrm{H} i 11$ have fallen nearly $3^{\circ} \mathrm{C}$. Such reduced temperatures result in reduced evaporation and an increase in effective moisture, higher water tables, etc., for the rest of the year. Hopefully, the curves developed here will reflect in part the increased effective moisture.

Within the context set by the calculations of moisture the settlement processes of the two cultural systems need to be specified. Thomas (1974) has devoted 
considerable effort to testing Julian Steward's (1938) model of Desert Archaic subsistence patterns. Also, Prewitt (1974:1-20) briefly outlines a system designed to explain site location during the Texas Archaic. In a derived form it could go far toward delineating the processes which explain the observed appearances. Prewitt assumes that his prehistoric subjects were responsive rather than active toward the environment. Next he distinguishes between

"exploitive" systems and "management" systems. Exploitive systems gather resources in a location until they reach a point of diminishing returns. Management systems consciously manipulate resources in a manner designed to conserve them, as in the case of some modern cattle ranchers. I take this to mean that a hunter-gatherer band would leave "seed," whether floral or faunal, so that the environment can assuredly and rapidly replenish itself after the band departs. In practice, then, management probably consists of leaving too early, before the perceived rate of returns is fully diminished. I have seen ethnographic reports which indicate management is within the behavioral repertoire of primitive people, though there is no reason to think that it was universally practiced.

Whichever system is active, site locations are determined by surface topography and by proximity to unequally valued resources. Unequally valued resources imply an element of immediacy. Locations, for instance, are more likely to be proximate to food than to raw materials for tools.

NORMAL SYSTEM

ALTERNATE SYSTEM

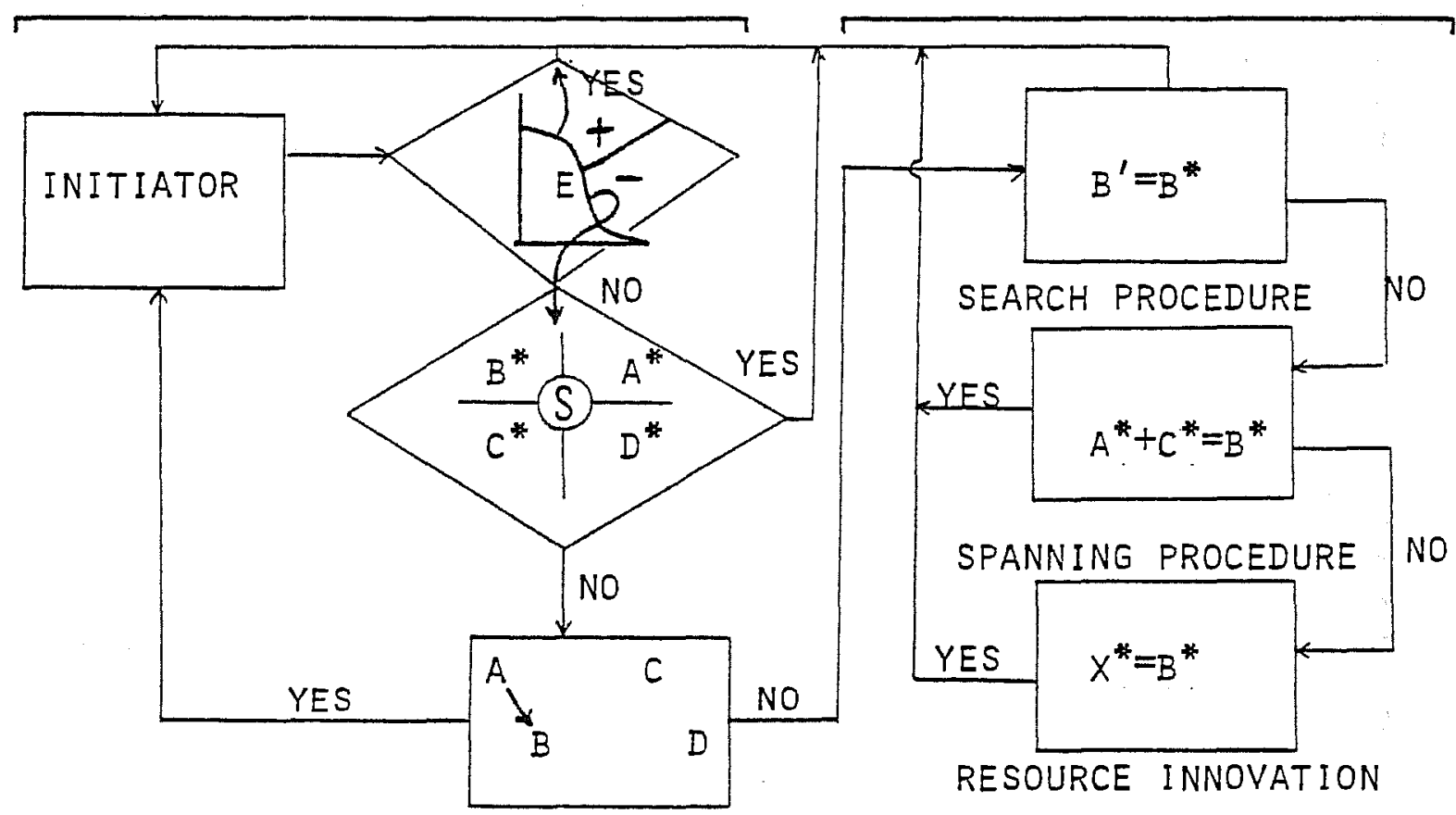

Figure 18.04. Nomadic Seasonal Round. A - site A, etc., $A^{*}=$ Resource at site $A$, etc., $A^{\prime}=A 7$ ternate site with resource $A^{*}, X=$ new site with new resource $X^{*}$. 
Repeated occupation of a given site is governed in theory by foreknowledge of the location of desired resources, or in the case where different cultures have located at the same spot, resources that were coincidentally valued in all cases were available at the same place over long periods of time.

When the resources of a given locality were depleted to a determined point of efficiency in energy-versus-yield, movement to a new locality was initiated on a course drawn from foreknowledge of the next scheduled resource and its location. Seasonal variation would be a limiting factor on the time of movement. As Figure 18.04 shows, this model can be flow charted so it could be satisfactorily simulated with a system of resources, seasonal availability schedules, area maps, climatic change effects, potential but unused resources, etc. The flow chart shows that as a group exploits resource $A$ it checks the energy-expended/energy-returned ration (E) and the progress of the seasons (S). As long as these quantities are positive, checking is initiated on a patterned or stochastic basis but no action is taken relative to a new site: When energy status changes or the season changes a cognitive map of the resource area is checked and the move from $A$ to $B$ effected.

If the expected resources are found at $B$ the process of checking as it is done in the "normal" system is resumed. If $B$ is not found a series of procedures are initiated. I have arranged these procedures on the basis of conservatism. The first procedure is the "search" for a substitute site B' that has the same resource, $B^{*}$. If the search procedure is successful the activity goes back to the nomal system with $B^{\prime}$ as a known alternative which may eventualiy replace $B$ on the seasonal round if it is reliable.

If search is not successful another alternative such as "spanning" may be attempted. Spanning is, or is something like, trying to make resource $A$ last in its waning hours until a skimpy bit of $C$ becomes available. Thus, the tails of $A$ and $C$ span the duration of missing resource $B$. This might also be called "making ends meet."

A third alternative is "resource innovation," looking for something that has not been used before. The Chinese eating stewed mud in The Good Earth would be a desperate example of resource innovation. This is symbolized as a resource $X$ which will replace $B$ in nutrition and scheduling characteristics. It would be the underlying process in primary forest efficiency as defined by Caldwell (1958)

Any number of procedures could be entered in the Alternate System, and the procedures in the alternate system are subject to innovation. It is an expandable list. Also, in reality alternative procedures would probably be used in combinations rather than singularly as the simulation flow chart suggests.

Two things should be noted about this model. First, it is an attempt to escape the purely stimulus-response function which has been a large part of archaeological modeling in the past. It allows either patterned or innovative movement in the environment. The groundwork for these arguments is in psychological studies of brain function such as those by Ross-Ashby (1960) and Powers (1973) and of cultural function (Flannery 1972; Gunn 1977). Second, the model explains the 
behavior of an entity with recognizable boundary condition which exists in a world of similarly conceived entities.

Given this system, what are the probabilities that a given location will be occupied in a given year? The phenomenon of break in the archaeological record is relatively common and can probably be best explained in terms of band life span (Wobst 1974:174). A site as we have described it is a station on a cognitively mapped seasonal round. It is a member of a finite number of loci chosen from a large number of potential stations within a band territory which forms a regular and predictable part of a band's annual life cycle. If a band dies out due to any of the stochastic or systematic phenomena which tend to end such organizations, the site will not be reoccupied again until a system search determines its suitability, and that suitability is routinized. While certain highly desirable loci may have a higher probability of being chosen. there is no reason to think that selection of any site is not a matter of probability and combinations of diverse selective pressures.

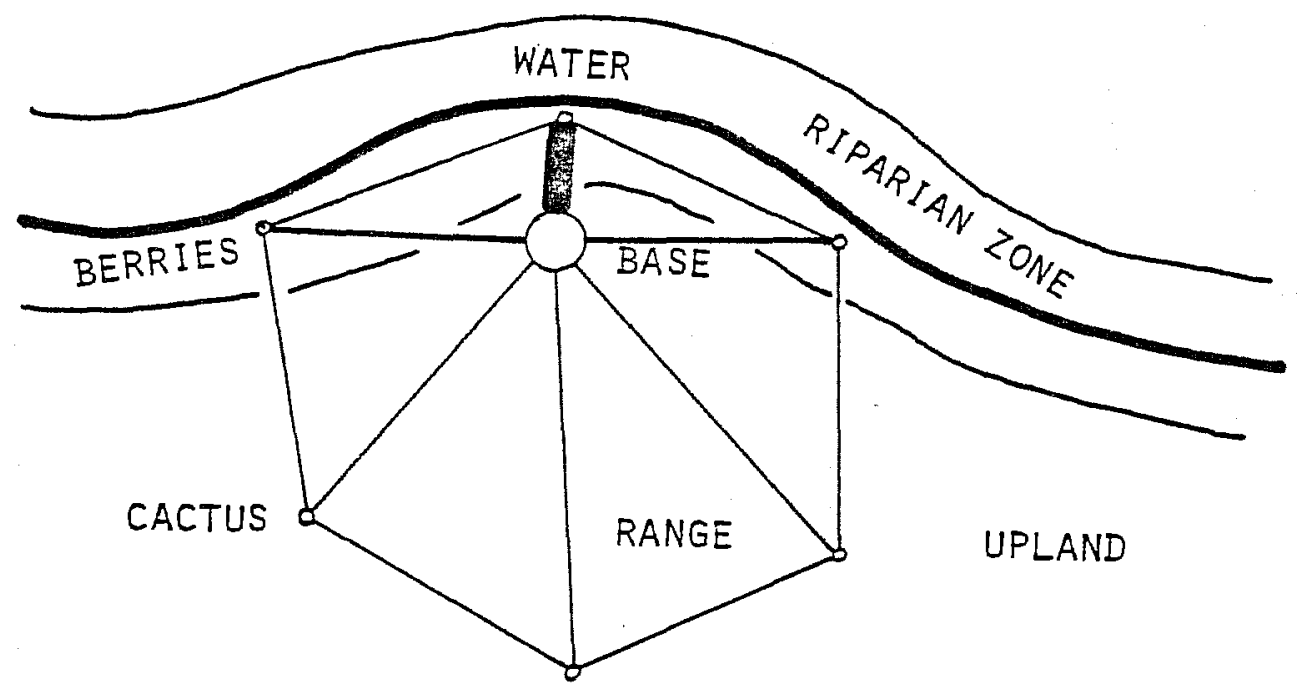

Figure 78.05. 'Load Length to Various Substations.

Implementing such a statistical scheme requires an exact model of land use patterns. Central Place theory provides the basis for such a scheme. Previous work (Hester et al. 1977) has shown that in broad apen desert conditions a pattern of unpacked hexagons is an at least interesting potential model. That is, hexagons are desirable to gatherers for the standard reason of equal access to substations from base stations. These hexagons, however, would not be packed together because it would only result in walking to an area which was already half gathered.

An additional consideration needs to be made in an area like Central Texas which is richly supplied with water courses. Most main sites appear to be on upland margins, valley slopes, or floodplains. Such locations were, no doubt, chosen because of access to water, richer riparian habitats and 
observability, lithic material, even perhaps for issues of prevailing winds and other camp comfort considerations. Such camps are probably parts of hexagonal systems which are distorted as the one in Figure 18.05. A possible line of research might be to show that each of the rays from station to substations has a "load length." Water and riparian resources have relatively heavy concentrations and are frequently required. The camp is, therefore, located to minimize the distance relatively "heavy" loads must be carried. Upland resources, in contrast, are sparse and light, both suggesting a longer acceptable load length. In Figure 18.05 the paths are darkened to show relative load length ratios. Presumably the heavier paths should always be shorter.

Such a model provides a rationale for saying that the margins of stream courses are more probably occupied than upland substations and the occupation of such stations is contingent on occupation of streamside stations.

Other influences might act to increase the probability of a site being occupied on the stream side such as good vantage points. Figure 18.06 shows the relative probabilities of various locations along ar occupied stream. Locations on curves in the stream are highly probable even more so if they are at the juncture of two streams. Straight runs are less probable. Uplands away from the stream margin are low probability. Again, working quantities are set:

$$
V H=.8, H=.7, M=.4, L=.1
$$

In a simulation, when the research area is showing values for arid environment, each site cell adjacent to a stream would be tested for occupation during a given season according to its probability structure as modified by the needs of the local band. These needs are worked out on the basis of proximity to the band, rate of change in the environment which might cause them to look for new sites, band half life, etc. Over the years the debris is cumulated in relative terms posited on the number of visits.

The third and final process to be made explicit is the one of the transhumant, labor inducing, focal economy of the Eastern Archaic. In this case, and in contrast to the previous process, labor intensification is taken to be a desirable alternative to mobility intensification. They would rather "fight than move." Since there is no reason to think we are dealing with agriculturalists in the strict sense, effort will be lavished on broadening the resource spectrum if the availability of primary resources is diminished by climate or overuse. Accumulation of goods and balance of population with environment probably become matters of concern since carrying infants is no longer a convincing influence to limit family size. Also, the inception of desire for large families may have its roots in the need for a large labor force to collect food and deal with a potentially competitive social milieu.

In this scheme the initiator does not consider the diminishing returns as an indicator for mobility (Figure 18.07). The seasons are checked and the labor force is dispatched to $A^{*}$ working at home and/or working away, $B^{*}$. At the transhumant stations food is collected and, if sufficient, carried home.. If not sufficient, other localities are tested and collected until sufficiency is 


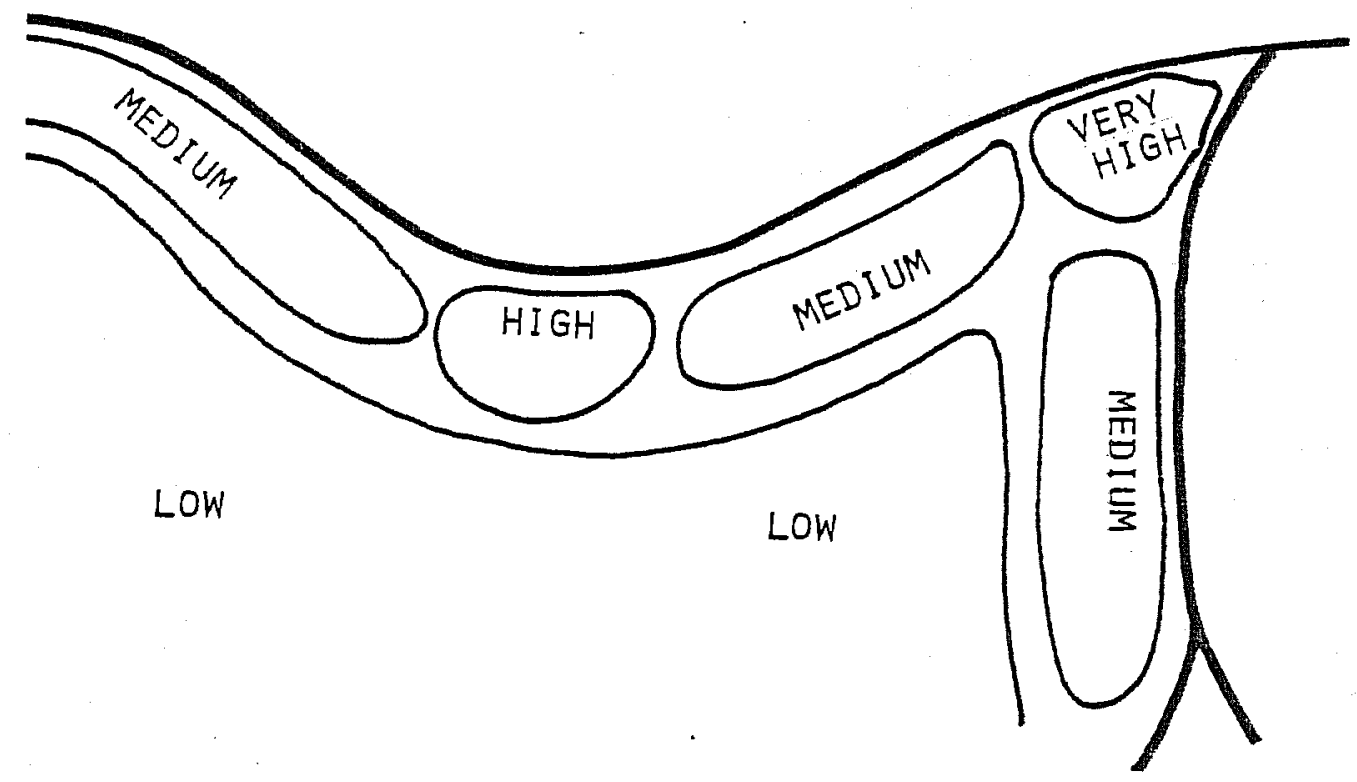

Figure 18.06. Relative Probability of Occupation at various Geographic Localities. 
reached. At the village the iransported and local resources are tested for sufficiency. If sufficiency is not reached trading, raiding and migration are alternatives in order of caste to the community. In certain circumstances trade and raid may be reversed depending on value systems. Migration, however, is undesirable.

Note that the system is posited on a very broad and rich riparian environment or woodland regime. The settlement pattern is modified to reflect a broader emphasis in a richer environment. Sites are more probably occupied in direct proportion to water available not only for household tasks but also for fishing and transportation $(U H=.9, H=.6, M=.4, L=.1)$. A group has a bigger territory and is willing to transport large quantities of food by improved transportation methods longer distances to a permanent village.

NORMAL SYSTEM

ALTERNATIVE SYSTEM

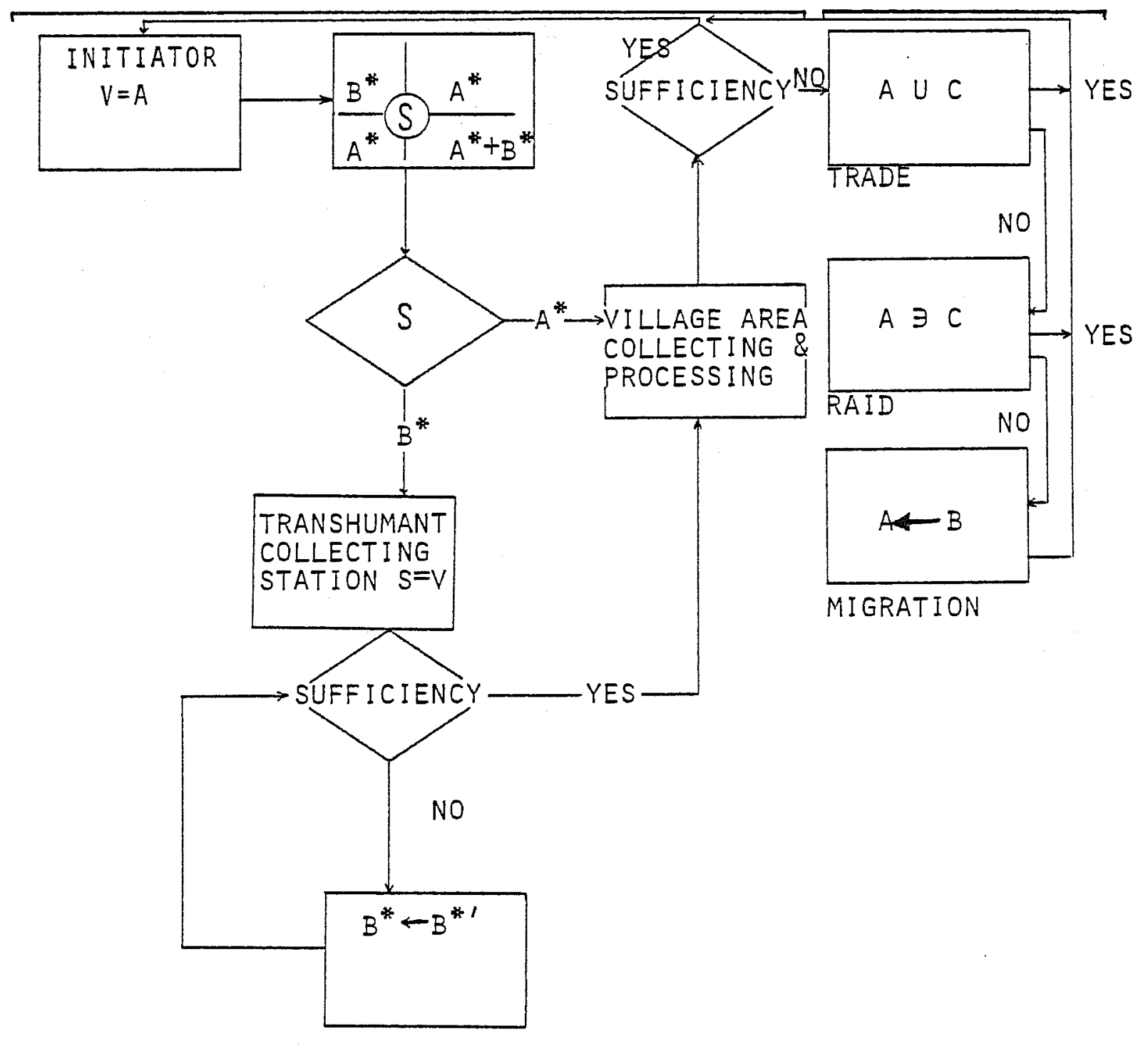

Figure 18.07. Transhumant Seasonal Activities. 


\subsection{CONCLUSIONS (Joe1 Cunn and Royce Mahula)}

The foregoing sections of this report have dealt with a broad range of information as viewed by a relatively large number of individuals. It is the purpose of this section to summarize the findings of these analyses and draw them together into the broader picture. It may appear that these discoveries are stated in a somewhat over-definite manner. It would indeed be scientifically unsound to presume that all of what follows is "conclusions" in the sense of firmly established truths. The conclusion of each individual study represents an hypothesis which the author felt was supported by his data. This conclusion section represents the final systematic hypothesis which in part sums the efforts and discoveries of those involved. This is not to imply that all of the work included here is conjecture. Some of the analyses were preceded by years of careful study and as a result are relatively sophisticated. Reference should be made to the character of the background studies preceding each analysis to determine the degree of certainty with which a given remark is offered.

In the introduction the various aspects of the Hop Hill research project were generally organized around a set of concepts centering on an ecosphere, with relevant variables grouped according to whether they pertained to the atmosphere, geosphere or ground plane of the system. The summary which follows is similarly organized. The key variables and the trajectory of those variabies are discussed in each case. The order taken is atmosphere - geosphere - ground plane reflecting the idea that causal processes are predominantly ordered in that sequence.

\section{Atmosphere}

The atmosphere is an unstable aspect of the ecosphere which responds to a complex set of factors as ultimate as the sun's heat radiation and as finite as local altitude. One of the major indices of atmospheric variation appears to be average annual Northern Hemispheric temperature which is controlled to some large degree by secular variations in the Earth's orbital relationship to the sun. Also, solar heat production, which is reflected in sun spot activity and volcanic eruptions, appears to have significant effects on global temperatures. The variations engendered by these larger causes are reflected in the atmosphere as shifts in the tracts and behaviors of weather systems which deliver the local weather. Specifically, lowering of global temperatures causes weather systems to move southward and an extension of monsoonal moisture to the west which in sum produces more precipitation. Lower temperatures also reduce evaporation which increases effective moisture.

As a consequence, under colder climatic regimes, the humid eastern biota extend westward to occupy areas which are arid during wamer periods. Based on calculated displacement of the humid-arid ecotone, we are of the opinion that the Hop Hill locality would have experienced changes from essentially arid to essentially humid conditions within the global temperature variation known to have occurred during the Holocene. As of this writing, evidence has been obtained from the study mollusks, ethnohistorical records, and inferred from cultural adaptations to the effect that such climatic changes did occur. Further evidence is being sought to substantiate the hypothesis of climatic instabilities. 


\section{Geosphere}

The geopshere is a quasi-stable medium below the ground plane which in large part reacts to more active agents of the atmosphere. Examination of the geology of the Hop Hill region shows the general area to be underlain by a permeable limestone formation which is richly coursed with underground waterways. An underlying impermeable clay acts to facilitate an accessible and reliable water table. This favorable hydrological situation acts to foster springs and rivers which appear to be consistently reliable sources of water even under relatively severe drought conditions such as those in the 1930's. Thus, while uplands between water courses might be subject to drying under more arid conditions, valleys and spring localities would provide a reliable source of water for human populations. The width of riparian zones, however, could vary directly with the amount of precipitation and would therefore be an inconstant source of subsistence requiring frequent adjustments in adaptation.

\section{Ground Plane}

The ground plane bisects the ecosphere and is composed of biotic and cultural processes which respond to the atmosphere, alter the upper surface of the geosphere resulting in soil, and leave a record of those responses in a biocultural residue within the soil.

Findings in various studies show that within the Hop Hill locality, the soils themselves respond post-depositionally to active agents in the atmosphere. The tougher Pedernales soil in the eastern half is erosion resistant. Relative to preservation of biocultural residues, this is a mixed blessing. The remains that were deposited there were apparently well preserved. However, prehistoric people came to the locality to gather lithic materials eroding out of the more erodable Hensley soils to the west and as a consequence tended to also leave cultural materials in greater quantities in that area.

Predominately within the permeable Hensley soil area of the locality, human activity divides into three relatively discrete areas. On the valley slope is the quarry area. On the upland margin there is an occupation-workshop complex with occupation activities concentrated more to the west and workshop pursuits more to the east. Simultaneous occupation of the quarry and occupation-workshop is indicated by the use of quarry materials in the occupation-workshop area with only rare exotic introductions from other sources. That there was differential use of the areas is shown by the fact that decortication flakes appear in the quarry while shaping and sharpening is more prevalent in the occupation-workshop complex.

The locality was utilized on occasion from the Early Archaic to the present. A major focus of prehistoric activity was during the Late Archaic, according to projectile point frequencies, and also the presence of a burned rock midden implies some sort of Archaic association.

On the upland margin several lines of evidence suggest an occupation-workshop and a division of labor within the heavily utilized area. Specialized cores, large choppers, heat treating, etc., are localized to the east, while the midden, as well as choppers, picks, metate, manos, boiling stones, etc., are to the west. 
The fact that these tendencies show up in an eroded site bespeaks a relatively long lived tradition of arranging living patterns in this way. The reason for maintaining such a tradition over a long period of time suggests an inherent relationship between camp patterns and topography. Since the site is on a prominence which extends out into the Pedernales floodplain, the arrangement may have related to an advantageous position from which to observe game and intruders.

\section{Subsistence}

Recovered evidence of subsistence of people who visited the locality is limited to fresh water mollusks. Inferences from patterns of food preparation suggested by patterns of limestone scatter on successive occupation floors may support a deer and acorn processing phase earlier followed by a change; perhaps to a more broad spectrum "Desert Culture" type of subsistence. The pioneer vegetation observed in the abandoned stock pens probably reflects the character and range of seed plants which would have been available annualiy to prehistoric inhabitants in the Pedernales floodplain after floods destroyed more xeric grasses. These include various chenopods, etc., which have been standard fare for western arid zone cultures. We are inclined to believe that 1 imestone cobbles 6-12 cm long, and which are 1 imy-grey or red in color and are unusually fossiliferous in appearance and have angular breaks on an otherwise smooth surface are boiling stones. The key to this complex of traits is the acid in acorns which must be leached before consumption. Using limestone boiling stones would neutralize the acid while simultaneously eating away the outside rind of the stones. The more acid resistant fossils in the limestone would be left as prominances on the otherwise leached away surface. Breakage due to frequent heating resulted in the ultimate discard of the boiling stone. Large limestone fragments $(>12)$ probably represent other food preparation processes.

\section{Ethnohistory}

Ethnohistorically, European-derived inhabitants of the Fredericksburg area appeared during the relatively cold period of the "Little Ice Age." The tall prairie grasses used in the construction of thatched roofs attests to the Texan biotic province character of the vegetation at the time. Also typical of the eastern habitat is the abundance of bears which the Indians hunted extensively in order to obtain bear grease to trade to German settlers. It is of interest to note that early European inhabitants first adopted bear grease and corn to insure survival and only later were able to experiment with European crops and integrate them into the local economy.

During the first hundred years of intensive European settlement material culture slowly incorporated the realities of the Pedernales River basin into an increasingly characteristic modified German culture. Subsistence changed from an almost exclusive reliance on native crops and wildlife for subsistence level agriculture and collecting to an increasingly market-oriented, non-native domesticant orientation. In other words, native habitat and culture profoundly affected early settlers and it was only after a time that the flow of the population was reintegrated into the Euro-American cultural milieu. 


\section{Recommendations}

The various numerical analyses in this report should be refined in two ways. Both are related to auto correlaijon. The seriousness of the auto correlation problem did not become apparent until the various papers were in final form and the completion deadline for the report was near. Auto correlation was removed from time series such as global climatic parameters and local Fredericksburg climatic indicators. It might also be helpful to remove auto correlation from polar coordinate data. The procedure would be executed by rows to remove auto correlation effects from individual artifacts. A special computer program would have to be written but would not be complicated.

Also, spatial auto correlation appears to be thwarting our efforts to determine directional tendencies in artifact distributions (linear technospatial analysis). The degree to which auto correlation is effective appears to be directly related to sample size. Linear technospatial analysis worked well with 27 cores, not so well with 121 bifaces and hardly at a11 with 176 unifaces. Removal of spatial auto correlation would probably clarify much relative to distributions of artifact classes containing large numbers of artifacts.

With respect to future excavation, the 1976 field season showed that there was little to be gained by excavation in the Pedernales soil area. The Hensley soil was much more productive yielding several thousand artifacts. Future archaeological activity at the Hop Hill locality would be most profitably concentrated in the midden excavation area in this Hensley soil. Due to the level of recovery deemed necessary to retrieve the maximum amount of cultural information on camp deposition and patterning, only the top $10 \mathrm{~cm}$ of the midden were excavated. Soundings to obtain soil samples show that there are at least $34 \mathrm{~cm}$ of deposits in the midden. Provided that future excavation is continued at the level of resolution initiated during the 1976 field season, the Hop Hill midden could provide the basis for a different order of approach to the problem of excavation and interpretation of middens. However, though further midden excavation would no doubt prove valuable additional collecting would probably change the statistical characteristics of the collection very little, and we have only begun to scratch the surface of the study potential of the materials already recovered. For example, to further maximize recovery of cultural information, soils from microlevels could be processed by froth flotation to determine if carbonized microbiotic materials can be obtained. A feasibility study is planned under the present project, but has not yet been conducted. 


\section{ACKNOWLEDGMENTS}

The successful completion of a project of the scope of Hop Hill naturally owes much to numerous persons who graciously lent the support of their time, energies and expertise. We wish to thank these many individuals who have contributed to this undertaking.

David Ing and George Kegley of Texas Parks and Wildlife and Elton Prewitt of the Texas Archeological Survey lent invaluable assistance in the initial stages of research design. Parks Department personnel Richard Fosse and Harold Woods were likewise primary sources of information about the local area as wel1 as providers of a base of operations during field work.

Paul Katz aided the field supervisor in the practical aspects of logistical planning and Anne and Henry Fox greatly contributed to the comfort of the crew by generously providing their farm house and surrounding property for the lodging of personnet.

The people of Stonewall were most hospitable and helpful, in particular, grocery store proprietor, Mr. Levi Deike and Mr. and Mrs. Kermit Hahne, owners of the Stonewall Motel. Local residents for many years, Mr. Hugo Weinheimer and Mr. Eugene Lindig spent many an hour imparting their knowledge of the history of the area.

The office of the USDA Soil Conservation Service, Fredericksburg, Texas offered pertinent information and provided soil surveys of Gillespie County as well as various maps of the locality. The staff of the Fredericksburg Times provided weather data for the climatic analyses.

Many valuable insights into the interpretation of various aspects of analysis were offered by consultants Richard McGehee and John Morgan, Division of Earth Sciences, UTSA, and James Wadington, Division of Social Sciences, UTSA. Dr. E. Mott Davis, Radiocarbon Laboratory, Balcones Research Center, University of Texas, Austin, handled carbon samples and offered advice on sample collection and processing. Philip Bandy of Texas Tech University offered detailed information on the construction and operation of a portion of the apparatus. Vicki $R$. Holloway as photographic consultant recorded a11 aspects of the project on fiTm.

Several people volunteered their talents to work at the site. Debbie Gunn worked tirelessly in many areas -- purchasing of groceries and supplies, mea and chore planning, in addition to taking part in excavation and recording at the site. RaTph Robinson and Max Witkind volunteered as excavators and Tom Kelly performed an additional field survey around the site proper.

In addition to the above mentioned persons heartfelt thanks go to the members of the field school and laboratory analys is class who put in long hours of work and whose unceasing interest and enthusiasm for the goals of archaeology were always in evidence. 
FIELD SCHOOL PERSONNEL

Dr. Joel Gunn, Director

Sarah Bielser David 0. Brown Beth Byrnes Elizabeth Cantu Waynne Cox Clinton Davis James Escobedo Annamaria Ferraro Vicki R. Holloway Arthur Keiper
Royce A. Mahula Jane (Lynn) Marshal1 Logan McNatt Evan O'Hara Laura Patton Elaine Hoke Saunders Edwin Lang Scruggs Alma Sollinger Ellen Sue Turner Richard Yoe

\section{LABORATORY ANALYSIS CLASS}

\author{
Mary Bechen \\ Sarah Bielser \\ Beth Byrnes \\ Waynne Cox \\ James Escobedo \\ Annamaria Ferraro \\ Ray Fisher \\ Carol Graves \\ Diane Hayden \\ Lynn Highley \\ Vicki R. Holloway \\ James E. Ivey
}

\author{
Jules Jaquier \\ Arther Keiper \\ Thomas Kelly \\ Dan Lopez \\ Royce A. Mahula \\ A7 McGraw \\ Evan O'Hara \\ Laura Patton \\ Elaine Hoke Saunders \\ Edwin Lang Scruggs \\ Kathy Thomas \\ Ellen Sue Turner
}

Sincere thanks go to the Center for Archaeological Research, its Director, Dr. Thomas R. Hester for his advice and support, to the Center secretary, Kathy McCauley for her able liajson between the Center and the field and to the Center typists Jeanette Burch, Mary Ellis, Rebecca Hettler and Gina McAvoy.

Thanks are also extended to Dr. Thomas Greaves, Director, Division of Social Sciences who was likewise supportive of the project, as was the division staff. Rae Ann Capps and Elaine Saunders volunteered their typing skills to expedite publication, and Kay Simpson aided in editing the manuscript. Technical editing was done by Royce Mahula, Elizabeth Branch and Thomas R. Hester. 


\section{REFERENCES CITED}

Alexander, Tom

1974 Ominous Changes in the World's Weather. Fortune 89(2):90-95.

Allison, John E., Glen W. Dittmar and James L. Hensell

1975 Soil Survey of Gillespie County, Texas. Soil Conservation Service, United States Department of Agriculture.

Arbingast, Stanley A., Lorin G. Kennamer, Robert H. Ryan, Alice Lo, David L. Karney, Charles P. Zlatkovich, Michae] E. Bonnie and Robert G. Steele

1973 Atlas of Texas. University of Texas, Austin, Bureau of Business Research.

Bandy, Philip A.

1975 Report and Test of a New Technique for Mapping and Recovery of "Exposed" Archaeological Sites. Paper presented to the 46th Annual Meeting of the Texas Archeological Society, San Antonio, Texas.

Barnes, Virgi1 E.

1965a Geology of the Hye Quandrangle, Blanco and Gillespie Counties, Texas. University of Texas, Austin, Bureau of Economic Geology, Quadrangle Map 27.

1965b Geology of the Rocky Creek Quadrangle, Blanco and Gillespie Counties, Texas. University of Texas, Austin, Bureau of Economic Geology, Quadrangle Map 29.

1966a Geology of the Cave Creek School Quadrangle, Gillespie County, Texas. University of Texas, Austin, Bureau of Economic Geology, Quadrangle Map 32.

1966b Geology of the Stonewall 2uadrangle, Gillespie and Kendall Counties, Texas. University of Texas, Austin, Bureau of Economic Geology, Quadrangle Map 31.

Barry, R. G.

1975 Climate Models in Paleoclimatic Reconstruction. Paleogeography, Paleoclimatology, Paleoecology $17(2): 123-137$. 
Binford, Lewis R.

1967 Smudge Pots and Hide Smoking: The Use of Analogy in Archaeological Reasoning. American Antiquity 32(1):1-12.

Binford, Lewis R. and Sally R. Binford

1966 A Preliminary Analys is of Functional Variability in the Mousterian of Levallois Facies. American Anthropologist $68(2): 238-295$

Birdse11, Joseph B.

1973 A Basic Demographic Unit. Current Anthropology 4:337-356.

Blair, W. Frank

1950 The Biotic Provinces of Texas. The Texas Journal of Science 2(1):93-117.

Bridges, E. M. and D. M. Harding

1971 Micro-Erosion Processes and Factors Affecting Slope

Development in the Lower Swansen Valley. In: Slopes

Form and Process. Compiled by D. Brunsden. Institute of

British Geographers, London, Special Publication 3:65-79.

Brose, David S. and John F. Scarry

1976 The Boston Ledges Shelter: Comparative Spatial Analyses of Early Late Woodland Occupations in Summit County, Ohio. Mid-Continental Journal of Archaeology 1(2):179-228.

Brune, Gunnar

1975 Major and Historical Springs of Texas. Texas Water Development Board, Report 189.

Butzer, Karl W.

1974 Environment and Archaeology. Aldine Publishing Company, Chicago.

1976 Early Hydraulic Civilization in Egypt. University of Chicago Press, Chicago.

Carr, John T., Jr.

1967 The Ciimate and Physiography of Texas. Texas water Development Board, Report 53. 
Carson, M. A. and M. J. Kirkby

1972 Hileslope Form and Process. Cambridge University Press, Cambridge.

Cheatum, E. P. and R. W. Fullington

1973 The Aquatic and Land Mollusca of Texas, Part 2: The Recent and Pleistocene Members of the Pupillidae and Urocoptidae (Gastropoda) in Texas. Dallas Museum of Natural History, Bulletin 1.

Clarke, David L.

1968 Analytical Archaeology. Methuen and Company, Ltd., London. Connor, Seymour V.

1971 Texas: A History. Thomas Y. Crowell Company, New York. Cook, H. M.

1967 Suspended-Sediment Load of Texas Streams. Texas Water Development Board, Report 45.

Crabtree, Don E.

1972 An Introduction to Flintworking. In: Occasional Papers of the Idaho State University Museum 28.

Daniels, Glyn

1967 The Origins and Growth of Archaeology. Galahad Books, New York.

de la Pena, Jose Enrique

1975 With Santa Anna in Texas. Translated and edited by Carmen Perry. Texas A \& M University Press, College Station.

Denten, G. H. and W. Karlen

1973 Holocene Climatic Variations, Their Pattern and Possible Cause. Quaternary Research 13(2):155-205.

Douglas, John $H$.

1975 Climate Change: Chilling Possibilities. Science News $107(9): 138-740$. 
Eddy, John A.

1977 The Case of the Missing Sunspots. Scientific American $236(5): 80-92$.

Eidt, Robert C.

1973 A Rapid Chemical Test for Archaeological Site Surveying. American Antiquity 38(2):206-215.

Emmett, W. W.

1965 The Vigil Network: Methods of Measurement and a Sampling of Data Collected. International Association of Scientific Hiydrology 66:89-106.

Ferguson, Walter Keene

1969 Geology and Politics in Frontier Texas, 1845-1909. University of Texas Press. Austin and London.

Flannery, Kent $V$.

1972 The Cultural Evolution of Civilizations. Annual Review of Ecology and Systematics 3.

Follett, C. R.

1973 Ground-Water Resources of Blanco County, Texas. Texas water Development Board, Report 174.

Girard, Rose1le

1972 Texas Rocks and Minerals. Bureau of Economic Gealogy, Guidebook 6. Lhiversity of Texas, Austin.

Gould, F. W.

1975 Texas Plants--An Ecological Summary. Texas Agriculture Experimental Station, Texas A \& M Lhiversity, College Station.

Green, L. M. and Thomas R. Hester

1973 The Finis Frost Site: A Toyah Phase Occupation in San Saba County, Central Texas. Bulletin of the Texas Archeological society 44:69-88.

Gregory, K. J. and D. E. Walling

1973 Drainage Basin Form and Process. Halstead Press, New York. 
Greig-Smith, P.

1964 Quantitative Plant Ecology. London.

Gunn, Joel

1970 The Use of Computer Programs for Mapping Archaeological Data. Plains Anthropologist 15(49):219-228.

1971 Research in Theory and Method of Functional Classification for Paleolithic Burins. Unpublished Master's thesis, University of Kansas, Department of Anthropology.

Gunn, Joel, Royce Mahula and J. B. Sollberger

1976 The Sollberger Distribution. La Tierra 3(4):2-8.

Gunn, Joel and Elton Prewitt

1975 Automatic Classification: Projectile Points from West

Texas. Plains Anthropologist 20(68):139-149.

Gunn, Joel and Frank Weir

1976 Tool Kit Hypotheses: A Case of Numerical Induction. Newsletter of Lithic Technology 5(3):137-135. Center for Archaeological Research, The University of Texas at San Antonio.

Hendricks, Leo

1952 Correlation Between Surface and Subsurface Sections of the Ellenburger Group of Texas. University of Texas, Austin. Bureau of Economic Geology, Report of Investigations 11.

Hester, Thomas

1971 Archeological Investigations at the La Jita Site, Uvalde, Texas. Bulletin of the Texas Archeological Society $42: 51-148$.

1973 The Formation of a "Burned Rock Midden": A California Example. The Records 29(3):4 (Dallas Archaeological Society).

1975 Chipped Stone Industries on the Rio Grande Plain, Texas: Some Preliminary Observations. The Texas Journal of Science 26(1-2):213-222. 
1976 The Archaic Southern Texas. In the Texas Archaic: A Symposium. Thomas R. Hester, ed., Center for Archaeological Research. The University of Texas at San Antonio, Special Report 2.

Hester, Thomas R., et al.

1977 An Archaeological Survey of the Radium Springs area, Southern New Mexico. Thomas R. Hester, et., Center for Archaedogical Research, The University of Texas at San Antonio, Archaeological Survey Report 26.

Hester, Thomas Roy, Delbert Gilbow and Alan D. Albee

1973 A Functional Analys is of "Clear Fork" Artifacts from the Rio Grande Plain, Texas. American Antiquity 38(1):90-96.

Hester, Thomas R. and Harry J. Shafer

1975 An Initial Study of Blade Technology on the Central and Southern Texas Coast. Plains Anthropologist 20(69):175-186.

Hole, Frank and Richard G. Wilkinson

1973 Shell Point: A Coastal Camp and Burial Site in Brazoria County. Bulletin of the Texas Archeological Society $44: 5-50$.

Holland, Pat H. and Leon S. Hughes

1964 Base-Flow Studies Pedernales River, Texas. Texas water Commission Bulletin 6407.

Hudson, N.

1971 Soil Conservation. Cornell University Press, Ithaca, New York.

Johnsen, S. J., W. Dansgaard and H. B. Clausen

1972 Oxygen Isotope Profiles Through the Antarctic and Greenland Ice Sheets. Nature 235:429-435.

Johnson, Leroy, Jr.

1967 Toward a Statistical Overview of the Archaic Cultures of Central and Southwestern Texas. University of Texas, Austin, Texas Memorial Museum Bulletin 12.

Jordan, Terry G.

1966 German Seed in Texas Soil. University of Texas Press, Austin. 
Katz, Paut R.

1976 A Technological Analysis of the Kansas City Hopewell Chipped Stone Industry. Unpublished Ph.D. dissertation, University of Kansas.

Katz, Susanna R. and Paul R. Katz

1976 Archeological Investigations in Lower Tule Canyon, Briscoe County, Texas. Texas Historical Foundation, Office of the State Archeologist, Austin, Archeological Survey Report 16.

Kelly, J. Charles and T. N. Campbell

1942 What Are the Burned Mounds of Texas? American Antiquity 7(38):319-322.

Kelly, Thomas C. and Thomas R. Hester

1975 Archaeological Investigations at Four Sites in the Dry Comal Watershed, Comal County, South Central Texas. Center for Archaeological Research. The University of Texas at San Antonio, Archaeological Survey Report. 15.

King, Irene Marsha 11

1967 John O. Meusebach. University of Texas Press, Austin.

Knowles, Hershel $T$.

1972 A Central Texas Synoptic Climatology Forecast Tool. University of Texas Press, Austin.

Kukla, George $\mathrm{J}$.

1975 Around the Ice Age World. Natural History 4:56-61.

Ladd, Boyd

1957 The Southwest Resources Handbook, Volumes 1-4. Department of Industrial Economics, Southwest Research Institute, San Antonio, Texas

Lamarche, V. C., Jr. and H. C. Fritts

1971 Anomaly Patterns of Climate Over the Western United States, 1700-1930, Derived from Principal Components Analys is of Tree-Ring Data. Monthly Weather Review 99(2):138-142.

Lamarche, Valmore C., Jr.

1974 Paleoclimatic Inferences from Long Tree Ring Records. Science 183(4129):1043-1048. 
Lamb, H. H.

1972 Climate: Present, Past \& Future. Methuen \& Company, Ltd, London.

Lee, Richard B. and Irven Devore (eds.)

1973 Man the Hunter. Aldine Publishing Company, Chicago.

Lindig, Otto

1970100 years: Historical Recollections of Gillespie County, 1870-1970. Private printing, Fredericksburg.

Matthews, Samue $\mathrm{W}$.

1976 What's Happening to Our Climate? National Geographic $150(9): 576-615$

Marwe 11, Ross A.

1970 Geologic and Historic Guide to the State Parks of Texas. University of Texas, Austin. Bureau of Economic Geology, Guidebook 10.

Mount, J. R.

1963 Investigation of Ground-Water Resources Near Fredericksburg, Texas. Texas water Commission Memorandum Report 63-03.

Murdock, George Peter

1967 Ethnographic At1as: A Sumnary. Ethnology 6(2):109-236

Murdock, George P. and Caterina Provost

1973 Factors in the Division of Labor by Sex: A Cross-Cultural Analysis. Ethnology 12(2):203-223.

Murdock, G. P. and D. R. White

1969 Standard Cross-Cultural Sample. Ethnology 8(4):329-369.

National Academy of Sciences

1975 Understanding Climatic Change: A Program for Action. Washington, D.C.

Newcomb, W. W., Jr.

1951 The Indians of Texas. University of Texas Press, Austin. 
Nie, Norman H., et al.

1975 Statistical Package for the Social Sciences, 2nd Edition. McGraw-Hi11, New York.

Orton, Robert B.

1960 Climates of the States; Texas. U. S. Department of Commerce, Washington, D.C.

1964 The Climate of Texas and Adjacent Gulf waters. U. S. Department of Commerce, Washington, D.C.

Palmer, Erik and C. W. Newton

1969 Atmospheric Circulation Systems. Academic Press, New York.

Patterson, James L.

1969 Floods in Texas. Texas Water Commission Bulletin 6311.

Patterssen, Suerre

1969 Introductory Meteorology. McGraw-Hill, New York.

Pearce, J. E.

1919 Indian Mounds and Other Relics of Indian Life in Texas. American Anthropologist 21(3):223-234.

Pearson, Emerson L.

1974 Soil Characteristics of an Archaeological Deposit:

Randall County, Texas. Bulletin of the Texas Archeological Society $45: 157-189$.

Pilsbry, Henry A.

1948 Land Mollusca of North America (North of Mexico), Volumes 1 and 2. The Academy of Natural Sciences of Philadelphia Monograph 2.

Plummer, F. B.

1943 Carboniferous Rocks of the Llano Region of Central Texas. University of Texas, Austin. Bureau of Economic Geology 4329.

Powers, William T.

1973 Behavior: The Control of Perception. Aldine Publishing Company, Chicago. 
Prewitt, Elton R.

1974 Archeological Investigations at the Leve-Fox Site, Williamson County, Texas. Texas Archeological Survey, The University of Texas, Austin, Research Repart 49.

Rainwater, E. H. and R. P. Zinguia

1962 Geology of Gulf Coast and Central Texas. Houston Geologic Society.

Ray, C. N.

1938 The Clear Fork Culture Complex. Texas Archeological and Paleontological Society Bulletin 10:193-207.

Roemer, Ferdinand (Oswald Mueller, Translator)

1935 Roemer's Texas: With Particular Reference to German Immigration and Physical Appearance of the Country. Standard Printing Company, San Antonio.

Ross-Ashby, Wi11iam

1960 Design for a Brain. Chapman and Ha11, Ltd., and Science Paperbacks.

Sahlins, Marsha11

1972 Stone Age Economics. Aldine-Atherton, Chicago.

Sanchez, W. A. and J. E. Kutzbach

1974 Climate of the American Tropics and Subtropics in the 1960s and Possible Comparisons with Climatic Variations of the Last Millenium. Quaternary Research 4(2):128-135.

Sellards, E. H. et al.

1932 The Geology of Texas, Volume 1, Stratigraphy. University of Texas, Austin, Bureau of Economic Geology 3232.

Shafer, Harry $\mathrm{J}$.

1976 The Consideration of Lithic Refuse at Archeological Sites. La Tierra 3(2):8-10.

Shapley, Harlow (ed.)

1970 Climatic Change, Evidence, Causes, and Effects. Harvard University Press, Cambridge. 
Singh, Jagjit

1966 Information Theory Language and Cybernetics. Dover Publications, Inc., New York.

Sjoberg, Andree F.

1953 The Culture of the Tonkawa, A Texas Indian Tribe. The Texas Journal of Science. 5(3):280-304.

Skee1s, Lydia Lowndes Maury

1972 An Ethnohistorical Survey of Texas Indians. Texas Historical Commission, Ofjice of the State Archaeologist Report 12. Austin, Texas.

Sokal, R. O. and F. J. Rolph

1969 Biometry. W. H. Freeman and Company, San Francisco.

Sollberger, J. B.

1968 A Partial Report on Research Work Concerning Lithic Typology and Technology. Bulletin of the Texas Archeological Society 39:95-109.

1976 Bifacing Patterns on Prismatic Flakes. Bulletin of the Texas Archeological Society 47:261-267.

Sollberger, J. B. and L. W. Patterson

1976 Prismatic Blade Replication. American Antiquity $41(4): 517-531$.

Sorrow, William M., Harry J. Shafer and Richard E. Ross

1967 Excavations of Stillhouse Hollow Reservoir. Texas Archeological Salvage Project. University of Texas Papers 11.

Speth, John D.

1972 Mechanical Basis of Percussion Flaking. American Antiquity 37(1):34-60.

1975 Miscellaneous Studies in Hard-Hamer Percussion Flaking: The Effects of Oblique Impact. American Antiquity 40(2):203-207.

Stenzel, H. B.

1937 Field Trip Through the Llano Uplift. University of Texas, Austin, Southwestern Society. Bureau of Economic Geology. 
Steward, J. H.

1938 Basin-Plateau Aboriginal Sociopolitical Groups. Bureau of American Ethnology. Bulletin 120.

Stonewall Centennial History Committee

1960 Stonewall Centennial; 1860-1960.

Suhm, Dee Ann

1959 The Williams Site and Central Texas Archeology. The Texas Journal of Science 6(2):218-250.

Suhm, Dee Ann and Edward B. Jelks

1962 Handbook of Texas Archeology: Type Descriptions.

Texas Archeological Society, Special Publication 1.

Texas Almanac

1964-65 A. H. Belo Corporation, Dallas Morning News.

Thomas, Alfred $B$.

1935 After Coronado: Spanish Exploration Northeast of New Mexico, 1696-1727. University of Ok1ahoma Press, Norman.

Thomas, David Hurst

1974 Am Empirical Test for Steward's Model of Great Basin Settlement Patterns. American Antiquity 38(2):155-176.

Turner, Ellen Sue

Exploring the Possibilities of Acorn Utilization in Texas. Unpub7 ished manuscript.

Valdez, Fred

Biodistributions of Texas. Unpublished manuscript.

Wallace, Earnest and E. Adamson Hoebel

1952 The comanches: Lords of the South Plains. University of Oklahoma Press, Norman.

Weinheimer, Hugo

1973 Excerpts from Memoirs of Mr. Hugo weinheimer. Copied by Evelyn Klein with consent of Mr. Weinheimer. 
Weir, Frank A.

1976 The Central Texas Archaic. Unpublished Ph.D. dissertation, Washington State Uriversity, Department of Anthropology.

Wenland, Wayne M. and Reid A. Bryson 1974 Dating Climatic Episodes of the Holocene. Quaternary
Research $4(1): 9-24$.

Wha11 on, Robert, Jr.

1974 Spatial Analysis of Occupation Floors II: The Application of Nearest Neighbor Analysis. American Antiquity 39(1):16-34.

White, D., M. Burton, L. Brudner and J. Gunn

1975 Implicational Structures in the Sexual Division of Labor. School of Social Sciences, Unviersity of California, Irvine, Social Science working Papers 83.

Wilmsen, Edwin N.

1970 Lithic Analysis and Cultural Inference: A Paleo-Indian Case. University of Arizona Press, Tucson.

Winters, Howard D.

1969 The Riverton Culture. Illinois Archaeological Survey, Monograph 1.

Wischmeier, W. H., D. D. Smith and R. E. Uhland

1958 Evaluation of Factors in the Soil-Loss Equation. Agricultural Engineering 39(8):458-462.

Wisseman, C. I. (Translator for German Text)

1869 Festival for the 50th Anniversary Jubilee of the Founding of the City of Fredericksburg. Published by Robert Penniger, Editor of the Fredericksburg Wachenblatt, Fredericksburg.

Wobst, H. Martin

1974 Boundary Conditions for Paleolithic Social Systems: A Simulation Approach. American Antiquity 39(2):147-178.

Young, A.

1972 Slopes. 01 iver and Boyd, Edinburgh. 